\title{
Talking to chromatin : polycomb function in gene environment interactions
}

Citation for published version (APA):

Prickaerts, P. (2012). Talking to chromatin : polycomb function in gene environment interactions. [Doctoral Thesis, Maastricht University]. Maastricht University. https://doi.org/10.26481/dis.20120622pp

Document status and date:

Published: 01/01/2012

DOI:

10.26481/dis.20120622pp

Document Version:

Publisher's PDF, also known as Version of record

\section{Please check the document version of this publication:}

- A submitted manuscript is the version of the article upon submission and before peer-review. There can be important differences between the submitted version and the official published version of record.

People interested in the research are advised to contact the author for the final version of the publication, or visit the DOI to the publisher's website.

- The final author version and the galley proof are versions of the publication after peer review.

- The final published version features the final layout of the paper including the volume, issue and page numbers.

Link to publication

\footnotetext{
General rights rights.

- You may freely distribute the URL identifying the publication in the public portal. please follow below link for the End User Agreement:

www.umlib.nl/taverne-license

Take down policy

If you believe that this document breaches copyright please contact us at:

repository@maastrichtuniversity.nl

providing details and we will investigate your claim.
}

Copyright and moral rights for the publications made accessible in the public portal are retained by the authors and/or other copyright owners and it is a condition of accessing publications that users recognise and abide by the legal requirements associated with these

- Users may download and print one copy of any publication from the public portal for the purpose of private study or research.

- You may not further distribute the material or use it for any profit-making activity or commercial gain

If the publication is distributed under the terms of Article $25 \mathrm{fa}$ of the Dutch Copyright Act, indicated by the "Taverne" license above, 
Talking to Chromation polyoomb function in qene envionment interactions 
ISBN 978-90-9026812-5

Cover design: Jo Havenith

Cover lay-out: Leon van Dorp

List of Abbreviations: Yvonne Prickaerts

Design invitations \& chapter pages: Jos Opdekamp

(C) copyright Peggy Prickaerts, Maastricht 2012

Printed by Drukkerij Gijsemberg bvba | Maasmechelen BE 


\title{
Talking to Chromatin
}

Polycomb function in gene environment interactions

\author{
PROEFSCHRIFT
}

ter verkrijging van de graad van doctor

aan de Universiteit Maastricht,

op gezag van de Rector Magnificus,

Prof. mr. G.P.M.F. Mols,

volgens het besluit van het College van Decanen,

in het openbaar te verdedigen

op vrijdag 22 juni 2012 om 12.00 uur

door

Peggy Prickaerts

geboren te Heerlen op 30 september 1983 


\section{Promotores}

Prof. dr. J.P.M. Geraedts

Prof. dr. B.G. Wouters

\section{Copromotor}

Dr. ir. J.W. Voncken

\section{Beoordelingscommissie}

Prof. dr. F.C.S. Ramaekers (voorzitter)

Prof. dr. R. van Driel (UvA, Amsterdam, Nederland)

Prof. dr. G. de Haan (UMCG, Groningen, Nederland)

Prof. dr. J.C.S. Kleinjans

Dr. R.C.J. Langen

The research described in this thesis was carried out at the department of Molecular Genetics as part of the research School GROW and the Faculty of Health, Medicine and Life Sciences of Maastricht University.

Financial support for the studies herein was obtained from grants by the transnational University Limburg, NWO/ZonMW and from granting institutions acknowledged in chapters 2 through 6.

Printing costs were in part defrayed by a stipend from Bodinco and Greiner Bio-One.
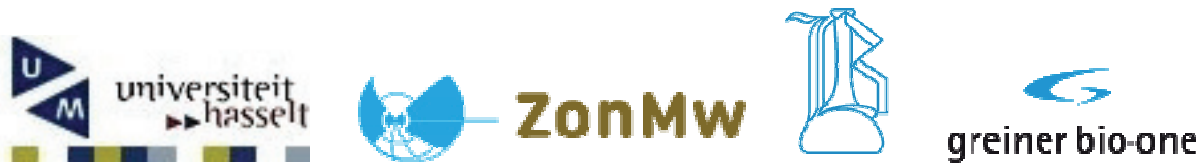


\section{CONTENTS}

Chapter 1 General Introduction 7

Aim of this thesis

Chapter $2 \quad$ MK3 controls replicative life-span through interaction with $\quad 27$ BMI1

Chapter $3 \quad$ MK3 controls Polycomb-target gene expression via negative $\quad 57$ feedback on ERK

Chapter 4 Signaling-induced degradation of BMI1 by chromatin-associated 87 KAP1

Chapter 5 Hypoxia induces bivalent chromatin domains by specific gain of 139 H3K27me3

Chapter 6 Capturing ChIP-seq profiles of H3K27me3 in dynamic biological 185 systems

$\begin{array}{lll}\text { Chapter } 7 & \text { General Discussion } & 201\end{array}$

Conclusions \& Future Perspectives

Summary 233

$\begin{array}{ll}\text { Samenvatting } & 239\end{array}$

$\begin{array}{ll}\text { Dankwoord } & 245\end{array}$

Curriculum Vitae 256

List of Publications $\quad 257$

$\begin{array}{ll}\text { List of Abbreviations } & 259\end{array}$ 



\section{CHAPTER 1 \\ General Introduction}




\section{Genetics and Epigenetics - a brief historical overview}

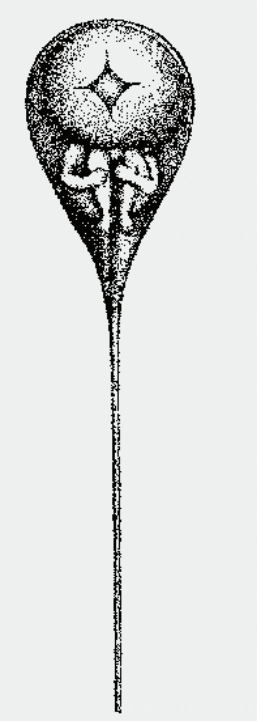

Inheritance in organisms occurs by means of transfer of discrete traits, called genes, from one generation to the next. Over the last two centuries a number of scholars have significantly contributed to the study of heredity. Mendel, who is now considered the founder of modern genetics, first published his systematic analysis on segregation of heritable traits in pea plants in 1866 (Druery and Bateson, 1901; Mendel, 1866). DNA had been discovered by Miescher, a contemporary of Mendel; he had extracted a substance from nuclei that differed from lipids and proteins, which he called nuclein. Miescher noted an increase in nuclear substances, among which nuclein, in preparation for cell division in proliferating tissues. As to its function, he speculated about a role for nuclein in transferring a "motion stimulus" to the oocyte, which was thought to be required for fertilization and embryonic development. This notion was partly based on his observation that nuclein was a major constituent of salmon sperm cells. However, Miescher had dismissed the idea that the multi-based acid nuclein played a role of any significance in heredity, as he deemed it unlikely that one single chemical substance could explain the diversity in animals he had isolated nuclein from (Dahm, 2008). Although the nature of the inherited material was not known at the time, Boveri and Sutton proposed that normal and abnormal development were closely linked to chromosomes (Boveri, 1904; Satzinger, 2008; Sutton, 1903). For a long time the belief was held that the variation in the composition of proteins was responsible for genetic variation, until early experiments on bacterial transformation, originally by Griffith and later by Avery and colleagues, established that it was DNA that carried heritable information (Avery et al., 1944; Griffith, 1928). Less than a decade later, Franklin, Wilkins, Watson and Crick discovered the double helix structure of DNA (Franklin and Gosling, 1953a, b; Watson and Crick, 1953a, b). Their collective studies showed that genetic information exists in the sequence of nucleotides and provide a simple modus for replication: new daughter strands can be synthesized on separated original strands of the double helix, thus reconstructing the nucleotide sequence of the old strand. The quote: "This structure has novel features which are of considerable biological interest" has become one of the most famous understatements in science (Watson, 2003; Watson and Crick, 1953a, b). Research in many labs has revealed what has become known as the central dogma - the translation of a macromolecular nucleic acid code into a protein/amino acid code; i.e. DNA is transcribed into 
RNA which is translated into protein, and laid the basis for our current understanding of genetic inheritance.

In 1990, a world-wide effort to map the entire human genome was initiated: the premise of the Human Genome Project (HGP) was to determine the base pair sequence of the entire human DNA, and to physically and functionally identify and map all genes encoded by the human genome. Although the project was extremely successful and a rough outline of the genome was already available in 2000 , it became clear that the mere presence of a DNA blueprint was not sufficient to explain the different ways in which the genetic code is expressed in time and space during differentiation, development and functional adaptation. There had to be another layer of regulation which controlled the utilization of genetic information. Similarly, not all genetic disease can be explained by genetic predisposition or the acquisition of a genetic mutation. For example, it has been known for many years that Mendelian genetics also depends on environmental factors. The most direct evidence for this was found in the discordance in susceptibility to diseases frequently observed in genetically identical twins (Bell and Spector, 2011).

Our current understanding of the function of chromatin (i.e. the macro-molecular structure in which nuclear DNA is organized) originated with work that argued against the long held belief that DNA was 'simply' encased in protein, showing instead that the DNA and chromatin-associated histone proteins display a periodic organization, resembling 'beads on a string'. Double-stranded DNA is wrapped around an octamer of 4 core histone proteins, H2A, H2B, H3 and H4 (Kornberg, 1974; Kornberg and Thomas, 1974; Olins and Olins, 1974; Sheehan and Olins, 1974). This nucleosome theory represented a major step forward in our understanding of the role of chromatin in controlling DNA-templated processes like transcription. Although it is currently clear that nuclear DNA is compacted at several higher order levels, most of the underlying mechanisms are currently incompletely understood.

Over the last two decades, it was recognized that chromatin serves a more extensive functional purpose than simply packing DNA into the confined nuclear space: chromatin provides a molecular interface on which many DNA-templated processes are coordinated. Other than DNA, histone and additional chromatin-associated proteins, RNA molecules are also involved in the packing and unpacking of DNA in a cells' nucleus (Kornberg and Lorch, 1999). Collectively, the regulatory processes that control shutdown (silencing) and utilization (activation) of DNA (genic and non-genic elements) are currently referred to as epigenetic control mechanisms. Scientists recently recognized that epigenetic information controls phenotypic plasticity (including predisposition to disease) and, remarkably, displayed transgenerational heritability (Gluckman et al., 2007; Nadeau, 2009). Clearly, these novel concepts posed new challenges for scientific 
research. The excitement surrounding the novel awareness that chromatin may possibly be the most physiologically relevant substrate, was perhaps most accurately phrased by Watson: "The major problem, I think, is chromatin... you can inherit something beyond the DNA sequence. That's where the real excitement of genetics is now" (Watson, 2003).

\section{Epigenetic regulation of chromatin structure}

During development, cells acquire a specific morphology and function in a spatio-temporal manner, i.e. depending on their differentiation status (time) and the tissue (position) in which they reside. Positional information is defined in many different ways, and include gradients in specific developmental morphogens, nutrients, oxygen or waste products as well as locally released soluble factors and signaling through cell-cell contacts (Ashe and Briscoe, 2006; Davidson, 1990). The cellular phenotype is determined by its genotype and the influence it receives from its microenvironment. Consequently, it is a matter of seed and soil, the combination of genes that are activated (transcribed) and silenced in response to the information available in the microenvironment. Although all genetic information is encoded by DNA, cells depend on specific gene expression profiles for the acquisition and maintenance of cellular identity. Formally, the study of these heritable, phenotypical changes (i.e. gene expression profiles), which are stable during meisosis and mitosis and occur without any alteration within the underlying DNA sequence, is referred to as epigenetics (Goldberg et al., 2007; Kelly and Trasler, 2004).

Epigenetic regulation occurs at the level of DNA (DNA methylation), RNA (noncoding RNAs) and protein (covalent histone modifications), which ultimately affects chromatin structure and hence, DNA-templated processes like transcription, replication and repair (Murr, 2010; Vermaak et al., 2003). The smallest functional unit of chromatin is the nucleosome, which comprises an octameric core of 4 histone protein pairs and almost two full turns of associated double-stranded DNA (Figure 1). Epigenetic processes influence both access and binding of regulatory factors to chromatin as a result of changed chromatin-compaction, repositioning of nucleosomes and/or recruitment of regulatory factors (i.e. by acting as a scaffold).

DNA can be methylated at cytosine bases (resulting in 5-methylcytosine or $5 \mathrm{mC}$ ) within CpG sites by DNA methyltransferases (DNMTs) (Bird, 2002; Feinberg and Tycko, 2004; Goll and Bestor, 2005). CpG-methylation is generally associated with recruitment of methyl binding domain proteins (MBDs) and subsequent chromatin compaction, and besides at repetitive elements throughout the genome, occurs at genic regions (Collas, 2009; Meissner et al., 2008; Pastor et al., 2011). DNA methylation is reversible, either 
through passive removal (i.e. cell division and inactivation of responsible de novo DNA methylases), or by active removal (which combines chemical conversion and DNA repair) (Rai et al., 2008). At the RNA level, perhaps the best known example of epigenetic regulation in mammals is the coating of the inactive X-chromosome in female cells by the long non-coding RNA (IncRNA) Xist, resulting in chromatin condensation (Brockdorff, 2011). The presence of ncRNA and/or DNA methylation is commonly associated with gene silencing.

Histone proteins carry N-terminal tails which protrude from nucleosomes and that are subject to various post-translational modifications among which methylation, acetylation, phosphorylation, ubiquitylation, SUMOylation and ADP-ribosylation. These signaling-generated, often transient covalent modifications, are recognized and bound by chromatin adaptor proteins (Bernstein et al., 2007; Kouzarides, 2007). In contrast to DNA methylation, histone modifications can promote chromatin compaction (i.e. ubiquitylation) as well as relaxation (i.e. acetylation, phosphorylation), resulting in gene silencing and active transcription respectively (Kouzarides, 2007; Winter and Fischle, 2010).

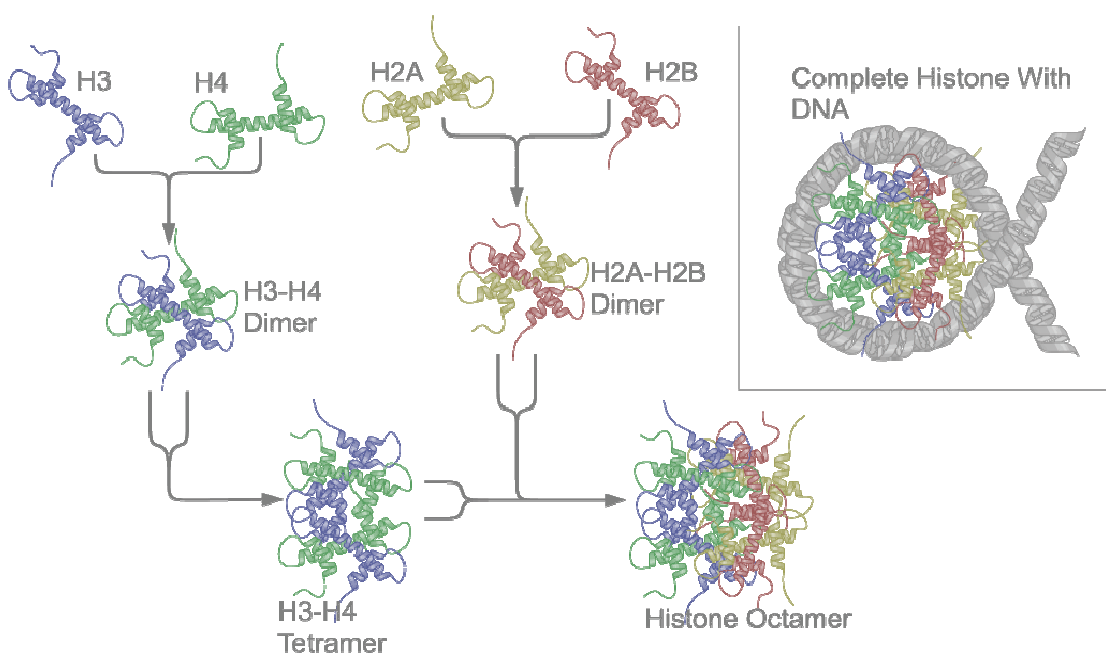

Figure 1: Schematic representation of a nucleosome core particle.

The nucleosome is comprised of a core particle of the histones $\mathrm{H} 2 \mathrm{~A}, \mathrm{H} 2 \mathrm{~B}, \mathrm{H} 3$ and $\mathrm{H} 4$ around which approximately 147 base pairs (bp) of DNA is wrapped, and a linker region which varies in length from about $10 \mathrm{bp}$ to about $80 \mathrm{bp}$, depending on the species and the tissue. Linker histones, like $\mathrm{H} 1$, can be associated with the linker region to accomplish chromatin compaction (not shown here). Within the core particle $\mathrm{H} 3$ dimerizes with $\mathrm{H} 4$ and $\mathrm{H} 2 \mathrm{~A}$ forms a dimer with $\mathrm{H} 2 \mathrm{~B}$, together they constitute a histone core octamer. Source picture http://en.wikipedia.org/wiki/File:Nucleosome_structure.png 
Combined, epigenetic regulation at these three macro-molecular levels controls virtually all chromatin-based processes. For all intents and purposes, studies on epigenetics currently entail all structural adaptations of chromosomal regions which register, signal or perpetuate altered activity states. This includes, besides chemical modification of DNA itself (e.g. cytosine-methylation), complex, post-translational modifications on histones, all transcriptional effects controlled by non-coding RNAs (RNA interference) processes and their effects on higher-order structure chromatin, chromosomes \& nuclear organization (Berger et al., 2009).

\section{"Histone code"; an epigenetic register}

Combinations of histone modifications constitute a local epigenetic register of chromatin state, also referred to as a "histone code" (Jenuwein and Allis, 2001; Strahl and Allis, 2000). The writers of this register are enzymes that install post-translational modifications (PTMs) on histone proteins (i.e. "write histone marks"), such as histone methyltransferases (HMTs), histone acetyltransferases (HATs), kinases, E2/E3-ubiquitin conjugases \& ligases, E3-SUMOylases and ADP-ribosylases (Bernstein et al., 2007; Kouzarides, 2007; Winter and Fischle, 2010).

Histone modifications can either favor gene silencing or gene activation: for instance acetylation of $\mathrm{H} 3$ and $\mathrm{H} 4$ is generally correlated with transcriptional activity, whereas histone methylation has been associated with gene activation as well as repression depending on the exact lysine $(K)$ or arginine $(R)$ involved (i.e. molecular context; see below) and the number of methyl marks present. Some methyl marks, mainly trimethylation of lysine residues (Kme3), have been extensively studied and their correlation with gene expression is well understood (Table 1).

The interpreters, or readers, of covalent histone modifications recognize and bind specific PTMs (i.e. "read histone marks") through specific domains, like chromo-domain (trimethyl mark), WD40 domains (dimethyl mark) bromodomain (acetyl mark), PHD fingers (phospho-mark), SIM domain (SUMO mark), etc. The final important players in maintenance of epigenomic homeostasis are the erasers: enzymes that remove PTMs (i.e. "erase histone marks"); they generally reverse the action of "writers" and change the local chromatin-interactome. Erasers include: histone demethylases (HDMs), histone deacetylases (HDACs), protein phosphatases (PPase), deubiquitylating/ SUMOylating enzymes (DUBs; isopeptidases). 
Table 1: Histone methyl marks in relation to transcriptional activity

\begin{tabular}{|c|c|c|c|}
\hline Methyl mark & & Writer & Eraser \\
\hline H3K4me1 & $\begin{array}{l}\text { associated with activated gene state } \\
\text { present at enhancers }\end{array}$ & KMT2F/SET1 & LSD1 \\
\hline H3K4me3 & $\begin{array}{l}\text { associated with gene transcription; } \\
\text { present at transcription start sites (TSS) }\end{array}$ & MLL; ASH1L/ASH2 & JARID/SMCX \\
\hline H3K9me2 & $\begin{array}{l}\text { associated with transcriptional repres- } \\
\text { sion; present within heterochromatin }\end{array}$ & GLP/G9a & LSD2/KDM1b/AOF1 \\
\hline HЗK9me3 & $\begin{array}{l}\text { associated with transcriptional repres- } \\
\text { sion; present within heterochromatin }\end{array}$ & SUV39H1 & JMJD1/2 \\
\hline H3K27me3 & $\begin{array}{l}\text { associated with transcriptional repres- } \\
\text { sion; present within gene body }\end{array}$ & $\mathrm{EZH} 2$ & JMJD3/UTX/UTY \\
\hline H3К36me3 & $\begin{array}{l}\text { associated with transcriptional elonga- } \\
\text { tion; present within gene body }\end{array}$ & SETD/HYPB & JMJD \\
\hline
\end{tabular}

Indicated are mammalian examples of histone methyltransferases and demethylases

\section{Chromatin states}

The term "heterochromatin" was introduced by Heitz in 1928 to describe localized areas of the chromosomes of the scale moss Pellia, which remained condensed and visible in early "interkinesis" nuclei (Heitz, 1928). Later, the same word was used to describe functional properties of chromosomes, such as, gene inactivity and late DNA replication (Brown, 1966; Schmid and Leppert, 1969). Thereafter a distinction was made between constitutive and facultative heterochromatin.

Depending on the genomic location, heterochromatin is subdivided into constitutive heterochromatin (present in all cells), which is found at centromeres and telomeres, where it prevents ectopic expression of transposable elements and maintains chromosome structure thereby ensuring genome stability and integrity respectively. Facultative heterochromatin (only present in some cells) is localized within euchromatic regions of the genome where it stably represses target genes (e.g. at the inactive X-chromosome (Beisel and Paro; Rosenfeld et al., 2009). We now know that heterochromatin is often associated with DNA methylation (on CpG-dinucleotides), hypoacetylation of histone proteins (i.e. transcriptionally inactive DNA), histone $\mathrm{H} 3$ lysine 9 trimethylation (H3K9me3) and the presence of heterochromatin protein (HP1) (Figure 2, c.f. Table 1) (Rosenfeld et al., 2009). Euchromatin, in contrast to heterochromatin, typically has a high gene-density. 


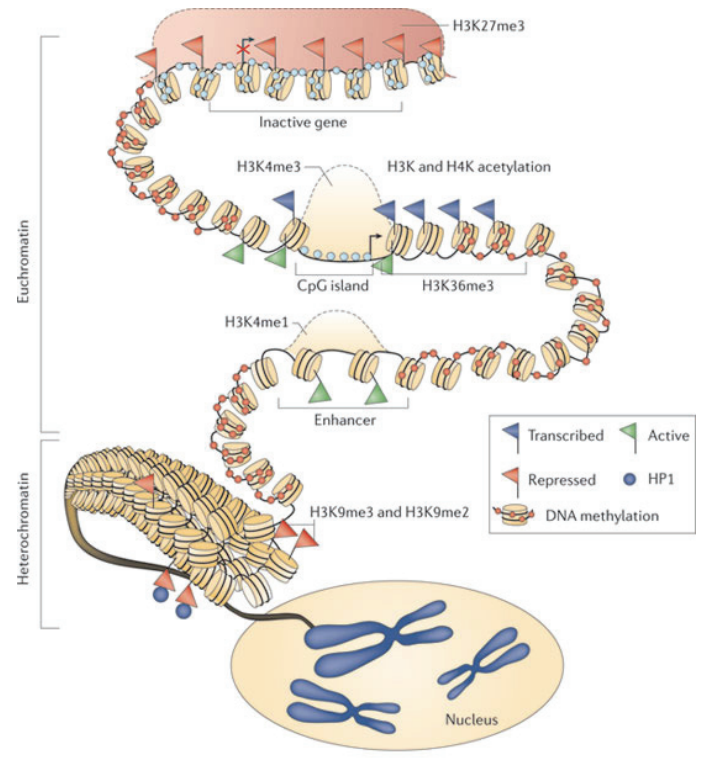

Figure 2: Schematic representation of chromatin state within human cells. In the lower right corner the cell nucleus is indicated containing chromosomes which are composed of hetero-chromatin (mainly located at the centromeres and telomeres) and euchromatin. Heterochromatic regions are associated with H3K9 methylation (me2/3), HP1 binding and DNA methylation. Euchromatic regions contain inactive genes, marked by H3K27me3, and active genes, marked by H3K4me3 (TSS), H3K36me3 (gene body) and $\mathrm{H} 3 / \mathrm{H} 4$ acetylation. Enhancer regions are usually associated with H3K4me1. Image: (Baylin and Jones, 2011).

A large part of euchromatic genes ( $60 \%$ ) contains CpG-rich regions at their 5-prime (5') ends known as CpG-islands (Figure 2).CpG-islands are usually relatively hypomethylated (at the DNA level), but instead are marked by trimethylation on histone 3 lysine 4 (H3K4me3) or lysine 27 (H3K27me3) (Azuara et al., 2006; Byun et al., 2009; Choi et al., 2011; Thomson et al., 2010). These marks correspond to gene transcription and silencing respectively. Transcriptionally silenced genes usually contain low levels of histone acetylation, display nucleosomes positioning over the transcription start site (TSS) and H3K27me3 covering the entire gene body. Active genes, in contrast, contain high levels of acetylation (of histone 3 and 4), a TSS which is relatively devoid of nucleosomes (i.e. enables access of transcription factors to DNA) and H3K36me3-enrichment across the gene body to facilitate transcriptional elongation. Thus, despite using a common post-translational modification to mark the chromatin (histone lysine trimethylation) and often very similar molecular contexts (amino acid contexts surrounding K4, K9 and K27 in the N-terminal tail of histone H3: $\mathrm{ARTK}_{4}, \mathrm{ARK}_{9} \mathrm{~S} ; \mathrm{ARK}_{27} \mathrm{~S}$ ) such epigenetic histone marks control transcription in different ways: $\mathrm{ARTK}_{4} / \mathrm{H} 3 \mathrm{~K} 4 \mathrm{me} 3$ is associated with gene activation, and $\mathrm{ARK}_{9} \mathrm{~S} / \mathrm{H} 3 \mathrm{~K} 9 \mathrm{me} 3$ and $\mathrm{ARK}_{27} \mathrm{~S} / \mathrm{H} 3 \mathrm{~K} 27 \mathrm{me} 3$ with silencing (Min et al., 2003). H3K9me3 and H3K27me3-marks are associated with (constitutive \& facultative) heterochromatin and Polycombmediated silencing (see below) respectively. These two forms of silencing show a high degree of mechanistic similarity and are both involved in genomic stability, yet they regulate spatially distinct elements and impose functionally distinct chromatin states (Barski et al., 2007; Filion et al., 2010). 


\section{Polycomb Group proteins}

Polycomb Group (PcG) proteins were first discovered in the fruit fly Drosophila melanogaster (D.mel) as transcriptional silencers of Homeotic genes (Biggin and Tjian, 1988; Blastyak et al., 2006; Brown et al., 2005; Dejardin et al., 2005). The evolutionarily conserved Homeotic genes (Hom-C cluster in D.mel; HOXA-D cluster in mice and human) are critical for the proper determination of segment structures of animals during early embryonic development. PcG-mutant flies show defects in anteriorposterior (AP) positioning resulting in characteristic homeotic defects in body patterning. Typically, single gene-mutations in the Hom-C genes may result in dramatic positioning defects, for example: mutations in the bithorax gene cluster results in transformation of the third thoracic segment, which normally carries halteres (a balancing organ), into the second thoracic segment (which carries the wings), generating a fly with a double set of wings (Figure 3 ). Likewise, a mutation in the antennapedia gene cluster transforms antennae into legs as a result of misexpressing of these homeotic genes (Figure 3) (Grier et al., 2005; Lappin et al., 2006).
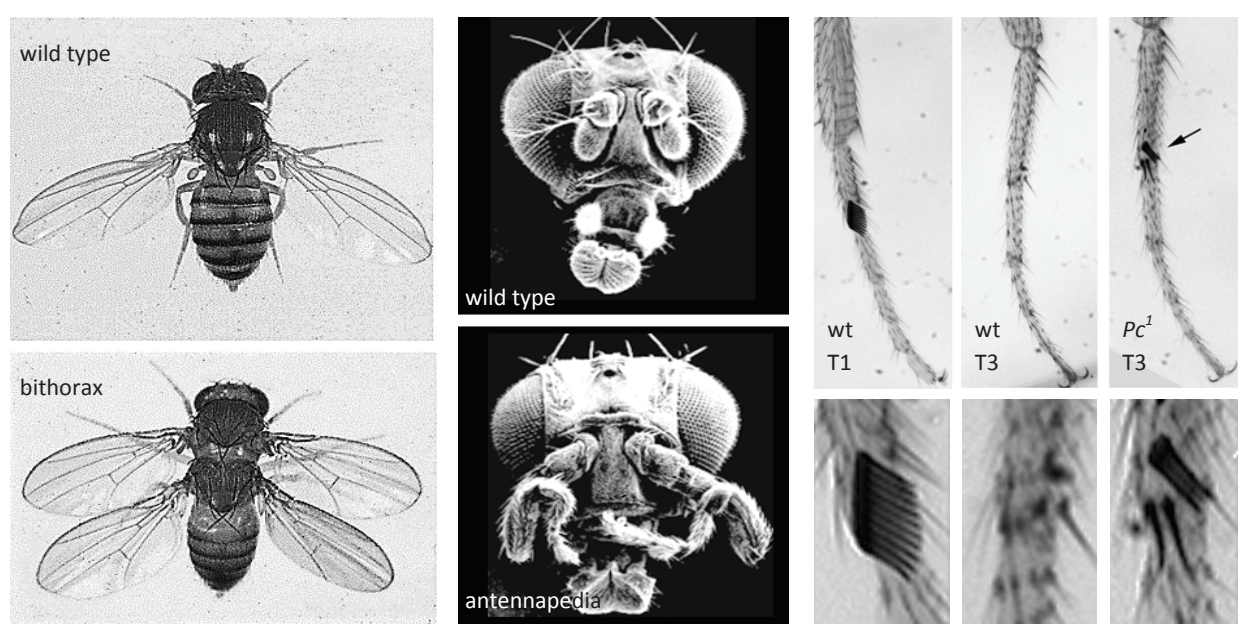

Figure 3: Drosophila melanogaster wild type (wt) and mutant flies.

left panels, top: Drosophila wild type fly showing one pair of wings on the second thoracic segment and balancer organs on the third thoracic segment; bottom homeotic mutant fly (bithorax) in which the third thoracic segment has transformed into the second segment, generating an extra set of wings. Source: http://www.bioinfo.org.cn/book/biochemistry/chapt27/bio9.htm Middle panels, top: wild type Drosophila head, with normal antennae; bottom: homeotic mutant fly (antennapedia) antennae are replaced by legs. Right panels, top - left to right: sex comb on the prothoracic (T1) leg in male flies (wt), no sex comb on wt metathoracic leg (T3), $P c^{1}$ mutant fly in which sex combs are also present on the meso- (T2), and metathoracic (T3) leg (shown); bottom panels: enlarged sections of corresponding images (top). Courtesy: F. Peronnet. 
Starting from the assumption that homeotic phenotypes are caused by deregulation of Hom-C cluster genes, it was soon discovered that PcG act on Hom-C genes: Polycomb Group proteins maintain homeotic gene expression boundaries: in an over-simplified model, the combination of active homeotic genes (within the Hom-C cluster) at any given location in the fly embryo, determines the nature of the segment, including its extremities (antennae, wings, legs etc). Loss of PcG-function leads to defects in repressive maintenance of Hom-C genes and results in the ectopic formation of (sometimes multiple) sex combs on the meso- (T2), and metathoracic (T3) leg in male flies, which normally only carry one sexcomb on the prothoracic (T1) leg. It is from mutation-associated sex combs phenotypes that the Polycomb Group family of genes derives its name; the first Polycomb-phenotype described in flies was caused by a mutant polycomb-gene allele: $P c^{1}$; an example of an abnormal $P c^{1}$-sex comb-phenotype is illustrated in Figure $\mathbf{3}$.

The biochemical counterparts of PcG-proteins, the Trithorax group (TrxG) proteins, were originally identified as suppressors of PcG-mutations in the fly (Brock and van Lohuizen, 2001; Kennison, 1995). The name Trithorax, like Polycomb, is derived from a phenotypic change caused by gene mutations; the founding member of the TrxG-genes in Drosophila is trithorax (TRX; Mm/Hs: MLL); TrxG-proteins are involved in maintenance of active gene transcription, thereby functionally antagonizing PcGfunction (Ringrose, 2006; Schuettengruber et al., 2007; Simon and Tamkun, 2002).

Beside the conservation of homeotic gene structure, function and organization in eukaryotic organisms (from plants to human), PcG and TrxG function and structure have also been conserved throughout evolution. Polycomb mutations in mice induce vertebral identity shifts, which are ontogenically akin to the segment identity shift found in Drosophila (Deschamps, 2004; Duboule, 1995; Simon, 2010; Simon and Kingston, 2009; Soshnikova and Duboule, 2009; van Lohuizen, 1998). Thus, Polycomb and Trithorax genes are implicated in the control of spatially restricted gene-expression patterns and as such provide a cellular memory system throughout the lifetime of an organism. Besides a role in AP-axis development, Polycomb and Trithorax gene function have been linked to stem cell biology, and cancer. Hence these genes fulfill important roles in both normal and abnormal development (Mills; Richly et al.; Geutjes et al., 2011).

\section{Molecular mechanisms in PcG-function}

PcG proteins act in multimeric complexes, which have been assigned initiation or maintenance functions based on observations in Drosophila. The initiation complex, also referred to as Polycomb repressive complex 2 (PRC2), is involved in epigenetic indexing of histone tails, such as $\mathrm{H} 3 \mathrm{~K} 27$ me3. The PRC2 complex comprises four core- 
subunits; EZH2 (D.mel: Enhancer of zeste, E(z)) which contains the catalytic SET domain, and the three non-catalytic subunits SU(Z)12 (D.mel: Suppressor of zeste, SU(Z)12), EED (D.mel: extra sex combs, ESC) and RBAP48 (D.mel: NURF55) (Figure 4, left complex; Table 2) (Cao and Zhang, 2004; Ketel et al., 2005; Nekrasov et al., 2005; Pasini et al., 2004). The maintenance complex, PRC1, is able to recognize and bind the H3K27me3mark via its chromo domain, in addition to a H2AK119ub1 motif (mono-ubiquitin) (Min et al., 2003) (Wang et al., 2004). The four core-subunits of PRC1 include CBX4 (D.mel: polycomb, PC), RNF2 (D.mel: dRING), BMI1 (D.mel: posterior sex combs, PSC) and PH1 (D.mel: polyhomeotic, PH) (Figure 4, right complex) (Francis et al., 2001; Lavigne et al., 2004; Levine et al., 2002; Mohd-Sarip et al., 2005; Niessen et al., 2009). CBX4 is a SUMO-E3 ligase; the RINGfinger domain PCG-proteins, of which RNF2 and BMI1 are examples, act as ubiquitin-E3-ligases; these catalytic activities were shown to be required for transcriptional repression by PRC1-complexes (Table 2). (Francis et al., 2001; Lavigne et al., 2004; Levine et al., 2002; Mohd-Sarip et al., 2005; Niessen et al., 2009; Simon and Kingston, 2009).

PRC2

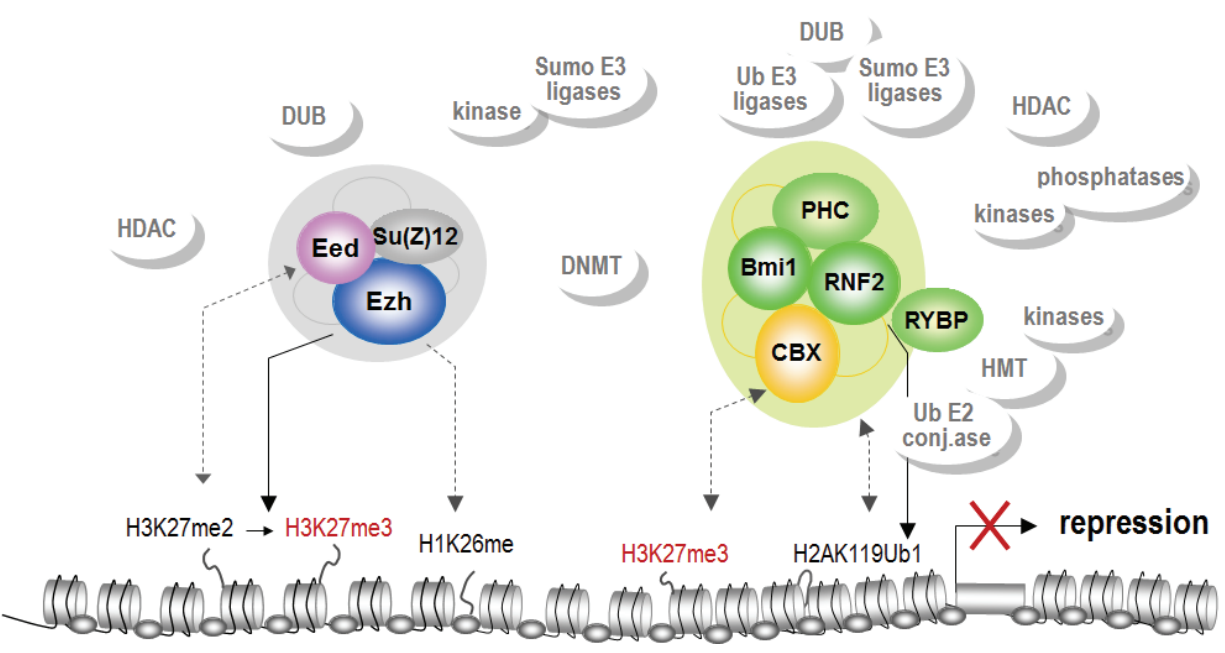

Figure 4: Repressive Polycomb complexes.

The PRC2 complex contains the histone methyl-transferase EZH, which establishes trimethyl marks on histone 3 lysine 27. H3K27me3 is bound by the chromo-domain of CBX subunits of the PRC1 complex. The combined action of PRC2 and PRC1 result in transcriptional repression of underlying target genes. Adapted from Niessen \& Voncken, 2008; Epigenetics in cancer, CRC press 
Table 2: insect and mammalian Polycomb orthologs

\begin{tabular}{lllll}
\hline $\begin{array}{l}\text { PcG } \\
\text { complex }\end{array}$ & Drosophila & Human & $\begin{array}{l}\text { Protein } \\
\text { domains }\end{array}$ & Biochemical activity \\
\hline PRC2 & E(z) & EZH1 & SET & Histone methyl \\
& & EZH2 & WD40 & transferase for H3K27 \\
& EsC & EED & & Cofactor for E(z); methyl- \\
& & & lysine binding motif?
\end{tabular}

Due to the large number of PcG orthologs, this table is limited to core complex members. Gene names according to HUGO gene nomenclature are shown in bold. BMI, B lymphoma mo-MLV insertion region; CBX, chromobox homologue; EDR, early development regulator; EED, embryonic ectoderm development; ESC, extra sex combs; $\mathrm{E}(\mathrm{z})$, enhancer of zeste; HPC, homolog of polycomb; HPH, homolog of polyhomeotic; Pc, polycomb; PCGF, polycomb group ring finger; Ph, polyhomeotic; PHC, polyhomeotic-like; Pho, pleiohomeotic; Psc, posterior sex combs; RING, really interesting new gene; RNF, ring finger protein; SAM, self association motif; Sce, sex combs extra; Su(z), suppressor of zeste; YY, yin-yang transcription factor.

Both PRC1 and PRC2 are known to interact with additional regulatory factors (i.a. non-coding RNAs and DNA methyltransferases, histone demethylases, histone deacetylases) thereby acting as convergence points of epigenetic regulation at all three macromolecular levels (i.e. DNA, RNA, protein) (Figure 4) (Niessen et al., 2009). In addition, PRC1 complexes exert direct effects on chromatin-structure, a property for which catalytic activity appears dispensable (Francis et al., 2004; Shao et al., 1999; Simon and Kingston, 2009).

\section{The micro-environment and epigenetic regulation}

Cellular phenotypes (e.g. morphology, physiology, function) are determined by which genes are active and silenced (genotype; nature; seed); the cells' transcriptome is controlled by intrinsic factors as well as by information a cell receives (microenvironment; nurture; soil). Information available through the environment of a cell 
may include growth and/or differentiation factors, nutrition, oxygenation, inflammatory molecules and/or exposure to other cell stressors; all information is ultimately integrated at the level of chromatin, where gene activity is controlled by epigenetic processes. Whereas activity of most epigenetic regulators is likely to be controlled by post-translational modification(s) downstream of signaling pathways, specific epigenetic regulators are direct sensors of, for instance, a cells' energy status or the environment. Many known proteins (including histone) deacetylases hydrolyze acetyl-lysine residues, yet the Sirtuin-class deacetylases, like SIRT1, couple lysine deacetylation to nicotinamide adenine dinucleotide (NAD)-hydrolysis (Imai et al., 2000). Thus, the intracelullar NAD:NADH ratio directly links the activity of NAD-dependent Sirtuins to the cells' energy status (Vaquero and Reinberg, 2009). SIRT1 recruits and deacetylates (and thereby activates) SUV39H1, an H3K9 methyl-transferase associated with facultative heterochromatin formation and hence controls heterochromatin formation (Vaquero et al., 2007). Likewise histone demethylation is directly coupled to cell oxygenation. Two classes of protein demethylases remove methyl groups from histones (see Table 1): the flavin adenine dinucleotide (FAD)-dependent amine oxidases remove mono-/di-methylation; the Jumonji C-terminal domain containing histone demethylases (JHDM) family uses Fe2+ and $\alpha$-ketoglutarate as cofactors and removes all methylation states by converting the methyl groups in the methyllysine to hydroxymethyl groups (Hou and Yu; Shi et al., 2004). Alpha-ketoglutarate and oxygen act as a co-substrates; as such $\alpha$-ketoglutarate-dependent oxygenases, like the prolylhydoxylase (PHD) which targets hypoxia-inducible factor 1 a (HIF1a), act as $\mathrm{O}_{2}$-sensors (Ivan et al., 2001; Jaakkola et al., 2001). Also histone and DNA methylation respond to oxygen via induction of corresponding epigenetic activity (Heddleston et al., 2011; Kang et al., 2011). The H3K27me3-demethylases belong to the JHDM-family; however, their role in hypoxia-dependent epigenomic modeling is relatively unknown. A steadily growing number of studies suggest that PcG-proteins are subject to control by intrinsic and external factors: protein phosphorylation was shown to accompany PcG/chromatin dissociation during cell-cycle progression, and the identification of several protein kinases associated with PRC1 and/or PRC2 suggests direct links to the microenvironment (Niessen et al., 2009; Voncken et al., 2005; Voncken et al., 1999) (Cha et al., 2005; Merrill et al., 2010; Sanchez et al., 2007). PcG- and TrxG-proteins are important regulators of stem cell maintenance (Lessard and Sauvageau, 2003a, b) (Ono et al., 2005). In keeping with this, H3K27me3 and H3K4me3 marks are both present in embryonic stem cells (ES cells). Remarkably however, both marks co-occur at key developmental genes (Azuara et al., 2006; Barski et al., 2007; Bernstein et al., 2006; Mikkelsen et al., 2007); during differentiation, at many of these key controller loci bivalent marking is changed into monovalent or no marking at all. 


\section{Aim of this thesis}

Despite significant progress that has been made over the last decennium, which significantly enhanced our understanding of PcG-function, the establishment and maintenance of repressive chromatin, the dynamic interplay with TrxG mediated chromatin dynamics, and heritability of PcG-mediated silencing, numerous questions remain. Among these issues are: How is Polycomb/chromatin-association dynamically regulated during development and differentiation? What is the effect of posttranslational modification (PTM) of PRC proteins and/or histone proteins? Does PTM act on chromatin recruitment, complex composition or regulation of catalytic activity? How is PRC-mediated silencing and TrxG-mediated gene activation balanced? How does the micro-environment control PRC-function and how does this contribute to development, differentiation and disease? Can any of these mechanisms be exploited for therapeutic purposes?

Many epigenetic processes and regulators, among which the PcG and TrxGproteins, are implicated in malignant cancer progression (Geutjes et al., 2011). Hence it is important to increase our understanding of how intrinsic and external factors act together to control chromatin. This thesis work focuses on environment-mediated control of PcG-biology (Figure 5). The studies in this thesis started from the general hypothesis that Polycomb-function is dynamically controlled by environmental factors. Chapters 2-4 take a candidate-gene approach: we started from PcG-interactor proteins previously identified by our group and studied their functional relevance in the context of mitogen- or stress-signaling, whereas in chapters $\mathbf{5}$ and $\mathbf{6}$ we take an unbiased approach: we used chromatin-immunoprecipitation/deep-sequencing to quantitatively and qualitatively measure Polycomb-mediated epigenetic remodeling in response to oxygen stress.

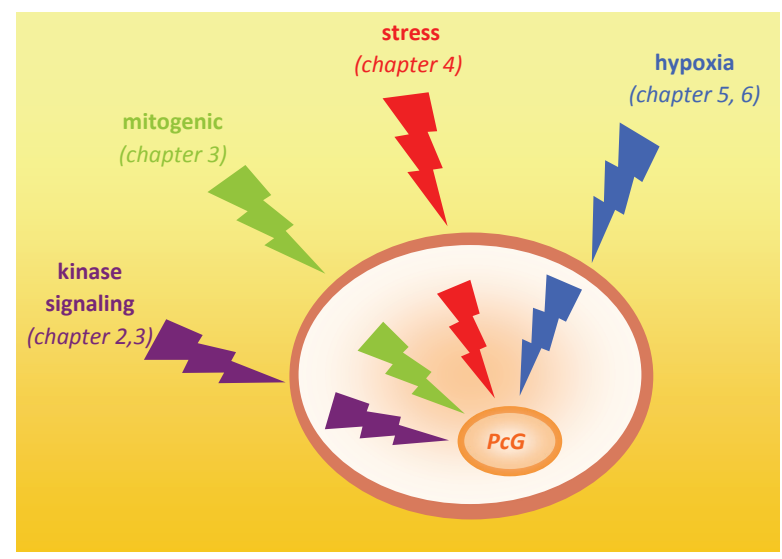

Figure 5: Effect of changes in the microenvironment on Polycomb function. Mitogenic and oncogenic (kinase) signaling to Polycomb (chapter 2 \& 3), Polycomb regulation in response to cell stress (chapter 4 ) and lack of oxygen (hypoxia; chapter 5 \& 6). 
Other workers from the same laboratory had previously identified MAPKAP kinase 3 (MK3) as a binding partner of PRC1 (Voncken et al., 2005). However, the exact role of MK3 within the Polycomb complex remained elusive. Therefore we have studied the role of MK3 on Polycomb function and cell phenotype either by increasing/reducing the expression of the kinase (chapter $\mathbf{2}$ ), or by modulating its catalytic activity (chapter $\mathbf{3}$ ). Furthermore, we studied the role of M/SAPK-signaling in release and re-establishment of PcG-mediated repression in the context of mitogenic stimulation of primary human fibroblasts (chapter 3). We previously identified a number of novel PcGphosphorylation sites (Niessen et al., 2009); currently, nearly 120 different PcGphospho-sites have been documented, most of which have not been functionally defined. We here hypothesized that signaling through phosphorylation cascades affects the PcG-interactome. To this end, we used the mammalian PcG-protein BMI1 to identify novel interaction partners in the context of cell stress (chapter 4). This led to the identification of KAP1 as a novel interaction partner of BMI1; we show that BMI1 is targeted by KAP1 for proteolysis in response to environmental stress. We discuss the repercussions of our findings in the context of DNA-damage control and transcriptional regulation.

In the final chapters, we charted the effects of acute changes in cell oxygenation on epigenomic distribution of PcG- and TrxG-mediated marking. Based on the biochemical dependency of the Jumonji-lass histone demethylases on oxygen, we hypothesized (chapter 5) that genome-wide histone trimethylation will be affected by changes is cell oxygenation. We determined the relationship between epigenomic and transcriptomic reprogramming in a model for fluctuating oxygen tension within the tumor microenvironment. To this end we combined chromatin-immunoprecipitation and deepsequencing analysis with expression array data. We describe a novel standardized method to reliably identify H3K27me3 enriched regions based on chromatin-immunoprecipitation/deep-sequencing data (chapter 6), and we established that in response to oxygen deprivation, global histone trimetyl-marking at genic regions is dramatically altered and that, as a consequence, the chromatin acquires characteristics of more a primitive epigenomic state (chapter 5 ). The relevance of our findings is discussed in the context of cancer maintenance and progression.

Taken together these studies firmly establish a role for extracellular cues and signaling pathways in regulation of PcG-function and provide a solid basis for future research into specific processes and molecular mechanisms in epigenetic regulation of PcG-associated chromatin. 


\section{REFERENCES}

Ashe, H.L., and Briscoe, J. (2006). The interpretation of morphogen gradients. Development 133, 385-394.

Avery, O.T., Macleod, C.M., and McCarty, M. (1944). Studies on the Chemical Nature of the Substance Inducing Transformation of Pneumococcal Types : Induction of Transformation by a Desoxyribonucleic Acid Fraction Isolated from Pneumococcus Type lii. J Exp Med 79, 137-158.

Azuara, V., Perry, P., Sauer, S., Spivakov, M., Jorgensen, H.F., John, R.M., Gouti, M., Casanova, M., Warnes, G., Merkenschlager, M., and Fisher, A.G. (2006). Chromatin signatures of pluripotent cell lines. Nat Cell Biol 8, 532-538.

Barski, A., Cuddapah, S., Cui, K., Roh, T.Y., Schones, D.E., Wang, Z., Wei, G., Chepelev, I., and Zhao, K. (2007). High-resolution profiling of histone methylations in the human genome. Cell 129, 823-837.

Baylin, S.B., and Jones, P.A. (2011). A decade of exploring the cancer epigenome - biological and translational implications. Nat Rev Cancer 11, 726-734.

Beisel, C., and Paro, R. (2011). Silencing chromatin: comparing modes and mechanisms. Nat Rev Genet 12, 123-135.

Bell, J.T., and Spector, T.D. (2011). A twin approach to unraveling epigenetics. Trends Genet 27, 116-125.

Berger, S.L., Kouzarides, T., Shiekhattar, R., and Shilatifard, A. (2009). An operational definition of epigenetics. Genes Dev 23, 781-783.

Bernstein, B.E., Meissner, A., and Lander, E.S. (2007). The Mammalian Epigenome. Cell 128, 669-681.

Bernstein, B.E., Mikkelsen, T.S., Xie, X., Kamal, M., Huebert, D.J., Cuff, J., Fry, B., Meissner, A., Wernig, M., Plath, K., et al. (2006). A bivalent chromatin structure marks key developmental genes in embryonic stem cells. Cell 125, 315-326.

Biggin, M.D., and Tjian, R. (1988). Transcription factors that activate the Ultrabithorax promoter in developmentally staged extracts. Cell 53, 699-711.

Bird, A. (2002). DNA methylation patterns and epigenetic memory. Genes Dev 16, 6-21.

Blastyak, A., Mishra, R.K., Karch, F., and Gyurkovics, H. (2006). Efficient and specific targeting of Polycomb group proteins requires cooperative interaction between Grainyhead and Pleiohomeotic. Mol Cell Biol 26, 1434-1444.

Boveri, T. (1904). Ergebnisse über die Konstitution der Chromatischen Substanz des Zellkerns. Gustav Fischer, Jena, 1904.

Brock, H.W., and van Lohuizen, M. (2001). The Polycomb group--no longer an exclusive club? Curr Opin Genet Dev 11, 175-181.

Brockdorff, N. (2011). Chromosome silencing mechanisms in X-chromosome inactivation: unknown unknowns. Development 138, 5057-5065.

Brown, J.L., Grau, D.J., DeVido, S.K., and Kassis, J.A. (2005). An Sp1/KLF binding site is important for the activity of a Polycomb group response element from the Drosophila engrailed gene. Nucleic Acids Res 33, 5181-5189.

Brown, S.W. (1966). Heterochromatin. Science 151, 417-425.

Byun, H.-M., Siegmund, K.D., Pan, F., Weisenberger, D.J., Kanel, G., Laird, P.W., and Yang, A.S. (2009). Epigenetic profiling of somatic tissues from human autopsy specimens identifies tissue- and individualspecific DNA methylation patterns. Human Molecular Genetics 18, 4808-4817.

Cao, R., and Zhang, Y. (2004). SUZ12 is required for both the histone methyltransferase activity and the silencing function of the EED-EZH2 complex. Mol Cell 15, 57-67. 
Cha, T.L., Zhou, B.P., Xia, W., Wu, Y., Yang, C.C., Chen, C.T., Ping, B., Otte, A.P., and Hung, M.C. (2005). Aktmediated phosphorylation of EZH2 suppresses methylation of lysine 27 in histone H3. Science 310, 306-310.

Choi, S.H., Heo, K., Byun, H.-M., An, W., Lu, W., and Yang, A.S. (2011). Identification of preferential target sites for human DNA methyltransferases. Nucleic Acids Research 39, 104-118.

Collas, P. (2009). Epigenetic states in stem cells. Biochimica et Biophysica Acta (BBA) - General Subjects 1790, 900-905.

Dahm, R. (2008). Discovering DNA: Friedrich Miescher and the early years of nucleic acid research. Hum Genet 122, 565-581.

Davidson, E.H. (1990). How embryos work: a comparative view of diverse modes of cell fate specification. Development 108, 365-389.

Dejardin, J., Rappailles, A., Cuvier, O., Grimaud, C., Decoville, M., Locker, D., and Cavalli, G. (2005). Recruitment of Drosophila Polycomb group proteins to chromatin by DSP1. Nature 434, 533-538.

Deschamps, J. (2004). Developmental biology. Hox genes in the limb: a play in two acts. Science 304, 16101611.

Druery, C.T., and Bateson, W. (1901). Experiments in plant hybridization (English translation origian manuscript Mendel, J.G.). Journal of the Royal Horticultural Society 26, 1-32.

Duboule, D. (1995). Vertebrate Hox genes and proliferation: an alternative pathway to homeosis? Curr Opin Genet Dev 5, 525-528.

Feinberg, A.P., and Tycko, B. (2004). The history of cancer epigenetics. Nat Rev Cancer 4, 143-153.

Filion, G.J., van Bemmel, J.G., Braunschweig, U., Talhout, W., Kind, J., Ward, L.D., Brugman, W., de Castro, I.J., Kerkhoven, R.M., Bussemaker, H.J., and van Steensel, B. (2010). Systematic Protein Location Mapping Reveals Five Principal Chromatin Types in Drosophila Cells. Cell 143, 212-224.

Francis, N.J., Kingston, R.E., and Woodcock, C.L. (2004). Chromatin compaction by a polycomb group protein complex. Science 306, 1574-1577.

Francis, N.J., Saurin, A.J., Shao, Z., and Kingston, R.E. (2001). Reconstitution of a functional core polycomb repressive complex. Mol Cell 8, 545-556.

Franklin, R.E., and Gosling, R.G. (1953a). Evidence for 2-chain helix in crystalline structure of sodium deoxyribonucleate. Nature 172, 156-157.

Franklin, R.E., and Gosling, R.G. (1953b). Molecular configuration in sodium thymonucleate. Nature 171, 740741.

Geutjes, E.J., Bajpe, P.K., and Bernards, R. (2011). Targeting the epigenome for treatment of cancer. Oncogene.

Gluckman, P.D., Hanson, M.A., and Beedle, A.S. (2007). Non-genomic transgenerational inheritance of disease risk. Bioessays 29, 145-154.

Goldberg, A.D., Allis, C.D., and Bernstein, E. (2007). Epigenetics: a landscape takes shape. Cell 128, 635-638.

Goll, M.G., and Bestor, T.H. (2005). Eukaryotic cytosine methyltransferases. Annu Rev Biochem 74, 481-514.

Grier, D.G., Thompson, A., Kwasniewska, A., McGonigle, G.J., Halliday, H.L., and Lappin, T.R. (2005). The pathophysiology of HOX genes and their role in cancer. J Pathol 205, 154-171.

Griffith, F. (1928). The Significance of Pneumococcal Types. J Hyg (Lond) 27, 113-159.

Heddleston, J.M., Wu, Q., Rivera, M., Minhas, S., Lathia, J.D., Sloan, A.E., Iliopoulos, O., Hjelmeland, A.B., and Rich, J.N. (2011). Hypoxia-induced mixed-lineage leukemia 1 regulates glioma stem cell tumorigenic potential. Cell Death Differ. 


\section{Chapter 1}

Heitz, E. (1928). Das Heterochromatin der Moose I. Jahrb Wiss Bot 69, 762-818.

Hou, H., and Yu, H. (2010). Structural insights into histone lysine demethylation. Curr Opin Struct Biol 20, 739748.

Imai, S., Armstrong, C.M., Kaeberlein, M., and Guarente, L. (2000). Transcriptional silencing and longevity protein Sir2 is an NAD-dependent histone deacetylase. Nature 403, 795-800.

Ivan, M., Kondo, K., Yang, H., Kim, W., Valiando, J., Ohh, M., Salic, A., Asara, J.M., Lane, W.S., and Kaelin, W.G., Jr. (2001). HIFalpha targeted for VHL-mediated destruction by proline hydroxylation: implications for $\mathrm{O} 2$ sensing. Science $292,464-468$.

Jaakkola, P., Mole, D.R., Tian, Y.M., Wilson, M.I., Gielbert, J., Gaskell, S.J., Kriegsheim, A., Hebestreit, H.F., Mukherji, M., Schofield, C.J., et al. (2001). Targeting of HIF-alpha to the von Hippel-Lindau ubiquitylation complex by O2-regulated prolyl hydroxylation. Science 292, 468-472.

Jenuwein, T., and Allis, C.D. (2001). Translating the histone code. Science 293, 1074-1080.

Kang, K., Zhang, R., Kim, G., Bae, S., and Hyun, J. (2011). Epigenetic changes induced by oxidative stress in colorectal cancer cells: methylation of tumor suppressor RUNX3. Tumor Biology, 1-10.

Kelly, T.L., and Trasler, J.M. (2004). Reproductive epigenetics. Clin Genet 65, 247-260.

Kennison, J.A. (1995). The Polycomb and trithorax group proteins of Drosophila: trans- regulators of homeotic gene function. Annu.Rev.Genet. 29, 289-303.

Ketel, C.S., Andersen, E.F., Vargas, M.L., Suh, J., Strome, S., and Simon, J.A. (2005). Subunit contributions to histone methyltransferase activities of fly and worm polycomb group complexes. Mol Cell Biol 25, 6857-6868.

Kornberg, R.D. (1974). Chromatin structure: a repeating unit of histones and DNA. Science 184, 868-871.

Kornberg, R.D., and Lorch, Y. (1999). Twenty-five years of the nucleosome, fundamental particle of the eukaryote chromosome. Cell 98, 285-294.

Kornberg, R.D., and Thomas, J.O. (1974). Chromatin structure; oligomers of the histones. Science 184, 865868.

Kouzarides, T. (2007). Chromatin modifications and their function. Cell 128, 693-705.

Lappin, T.R., Grier, D.G., Thompson, A., and Halliday, H.L. (2006). HOX genes: seductive science, mysterious mechanisms. Ulster Med J 75, 23-31.

Lavigne, M., Francis, N.J., King, I.F., and Kingston, R.E. (2004). Propagation of silencing; recruitment and repression of naive chromatin in trans by polycomb repressed chromatin. Mol Cell 13, 415-425.

Lessard, J., and Sauvageau, G. (2003a). Bmi-1 determines the proliferative capacity of normal and leukaemic stem cells. Nature $423,255-260$.

Lessard, J., and Sauvageau, G. (2003b). Polycomb group genes as epigenetic regulators of normal and leukemic hemopoiesis. Exp Hematol 31, 567-585.

Levine, S.S., Weiss, A., Erdjument-Bromage, H., Shao, Z., Tempst, P., and Kingston, R.E. (2002). The core of the polycomb repressive complex is compositionally and functionally conserved in flies and humans. Mol Cell Biol 22, 6070-6078.

Meissner, A., Mikkelsen, T.S., Gu, H., Wernig, M., Hanna, J., Sivachenko, A., Zhang, X., Bernstein, B.E., Nusbaum, C., Jaffe, D.B., et al. (2008). Genome-scale DNA methylation maps of pluripotent and differentiated cells. Nature $454,766-770$.

Mendel, J.G. (1866). Versuche über Pflanzenhybriden. Verhandlungen des naturforschenden Vereines in Brünn Bd. IV für das Jahr, 1865, Abhandlungen:3-47. 
Merrill, J.C., Kagey, M.H., Melhuish, T.A., Powers, S.E., Zerlanko, B.J., and Wotton, D. (2010). Inhibition of CtBP1 Activity by Akt-mediated Phosphorylation. Journal of Molecular Biology 398, 657-671.

Mikkelsen, T.S., Ku, M., Jaffe, D.B., Issac, B., Lieberman, E., Giannoukos, G., Alvarez, P., Brockman, W., Kim, T.K., Koche, R.P., et al. (2007). Genome-wide maps of chromatin state in pluripotent and lineage-committed cells. Nature $448,553-560$.

Mills, A.A. (2010). Throwing the cancer switch: reciprocal roles of polycomb and trithorax proteins. Nat Rev Cancer 10, 669-682.

Min, J., Zhang, Y., and Xu, R.M. (2003). Structural basis for specific binding of Polycomb chromodomain to histone H3 methylated at Lys 27. Genes Dev 17, 1823-1828.

Mohd-Sarip, A., Cleard, F., Mishra, R.K., Karch, F., and Verrijzer, C.P. (2005). Synergistic recognition of an epigenetic DNA element by Pleiohomeotic and a Polycomb core complex. Genes Dev 19, 1755-1760.

Murr, R. (2010). Interplay between different epigenetic modifications and mechanisms. Adv Genet 70, 101141.

Nadeau, J.H. (2009). Transgenerational genetic effects on phenotypic variation and disease risk. Hum Mol Genet 18, R202-210.

Nekrasov, M., Wild, B., and Muller, J. (2005). Nucleosome binding and histone methyltransferase activity of Drosophila PRC2. EMBO Rep 6, 348-353.

Niessen, H.E., Demmers, J.A., and Voncken, J.W. (2009). Talking to chromatin: post-translational modulation of polycomb group function. Epigenetics Chromatin 2, 10.

Olins, A.L., and Olins, D.E. (1974). Spheroid chromatin units (v bodies). Science 183, 330-332.

Ono, R., Nosaka, T., and Hayashi, Y. (2005). Roles of a trithorax group gene, MLL, in hematopoiesis. Int J Hematol 81, 288-293.

Pasini, D., Bracken, A.P., Jensen, M.R., Denchi, E.L., and Helin, K. (2004). Suz12 is essential for mouse development and for EZH2 histone methyltransferase activity. Embo J 23, 4061-4071.

Pastor, W.A., Pape, U.J., Huang, Y., Henderson, H.R., Lister, R., Ko, M., McLoughlin, E.M., Brudno, Y., Mahapatra, S., Kapranov, P., et al. (2011). Genome-wide mapping of 5-hydroxymethylcytosine in embryonic stem cells. Nature 473, 394-397.

Rai, K., Huggins, I.J., James, S.R., Karpf, A.R., Jones, D.A., and Cairns, B.R. (2008). DNA Demethylation in Zebrafish Involves the Coupling of a Deaminase, a Glycosylase, and Gadd45. CELL 135, 1201-1212.

Richly, H., Aloia, L., and Di Croce, L. (2011). Roles of the Polycomb group proteins in stem cells and cancer. Cell Death Dis 2, e204.

Ringrose, L. (2006). Polycomb, trithorax and the decision to differentiate. Bioessays 28, 330-334.

Rosenfeld, J.A., Wang, Z., Schones, D.E., Zhao, K., DeSalle, R., and Zhang, M.Q. (2009). Determination of enriched histone modifications in non-genic portions of the human genome. BMC Genomics 10, 143.

Sanchez, C., Sanchez, I., Demmers, J.A., Rodriguez, P., Strouboulis, J., and Vidal, M. (2007). Proteomic analysis of Ring1B/Rnf2 interactors identifies a novel complex with the Fbxl10/ Jmjd1B histone demethylase and the BcoR corepressor. Mol Cell Proteomics.

Satzinger, H. (2008). Theodor and Marcella Boveri: chromosomes and cytoplasm in heredity and development. Nat Rev Genet 9, 231-238.

Schmid, W., and Leppert, M.F. (1969). Rates of DNA synthesis in heterochromatic and euchromatic segments of the chromosome complements of two rodents. Cytogenetics 8, 125-135

Schuettengruber, B., Chourrout, D., Vervoort, M., Leblanc, B., and Cavalli, G. (2007). Genome Regulation by Polycomb and Trithorax Proteins. Cell 128, 735-745. 
Shao, Z., Raible, F., Mollaaghababa, R., Guyon, J.R., Wu, C.T., Bender, W., and Kingston, R.E. (1999). Stabilization of chromatin structure by PRC1, a Polycomb complex. Cell 98, 37-46.

Sheehan, D.M., and Olins, D.E. (1974). The binding of nuclear non-histone protein to DNA. Biochim Biophys Acta $353,438-446$.

Shi, Y., Lan, F., Matson, C., Mulligan, P., Whetstine, J.R., Cole, P.A., Casero, R.A., and Shi, Y. (2004). Histone Demethylation Mediated by the Nuclear Amine Oxidase Homolog LSD1. Cell 119, 941-953.

Simon, J.A. (2010). Chromatin compaction at Hox loci: a polycomb tale beyond histone tails. Mol Cell 38, 321322.

Simon, J.A., and Kingston, R.E. (2009). Mechanisms of Polycomb gene silencing: knowns and unknowns. Nat Rev Mol Cell Biol 10, 697-708.

Simon, J.A., and Tamkun, J.W. (2002). Programming off and on states in chromatin: mechanisms of Polycomb and trithorax group complexes. Curr Opin Genet Dev 12, 210-218.

Soshnikova, N., and Duboule, D. (2009). Epigenetic temporal control of mouse Hox genes in vivo. Science 324, 1320-1323.

Strahl, B.D., and Allis, C.D. (2000). The language of covalent histone modifications. Nature 403, 41-45.

Sutton, W.S. (1903). The chromosomes in heredity. Biol. Bull. 4, 231-251.

Thomson, J.P., Skene, P.J., Selfridge, J., Clouaire, T., Guy, J., Webb, S., Kerr, A.R.W., Deaton, A., Andrews, R., James, K.D., et al. (2010). CpG islands influence chromatin structure via the CpG-binding protein Cfp1. Nature 464, 1082-1086.

van Lohuizen, M. (1998). Functional analysis of mouse Polycomb group genes. Cell Mol Life Sci 54, 71-79.

Vaquero, A., and Reinberg, D. (2009). Calorie restriction and the exercise of chromatin. Genes Dev 23, 18491869.

Vaquero, A., Scher, M., Erdjument-Bromage, H., Tempst, P., Serrano, L., and Reinberg, D. (2007). SIRT1 regulates the histone methyl-transferase SUV39H1 during heterochromatin formation. Nature 450, 440-444.

Vermaak, D., Ahmad, K., and Henikoff, S. (2003). Maintenance of chromatin states: an open-and-shut case. Curr Opin Cell Biol 15, 266-274.

Voncken, J.W., Niessen, H., Neufeld, B., Rennefahrt, U., Dahlmans, V., Kubben, N., Holzer, B., Ludwig, S., and Rapp, U.R. (2005). MAPKAP kinase 3pK phosphorylates and regulates chromatin-association of the polycombgroup protein Bmi1. J Biol Chem 280, 5178-5187.

Voncken, J.W., Schweizer, D., Aagaard, L., Sattler, L., Jantsch, M.F., and van Lohuizen, M. (1999). Chromatinassociation of the Polycomb group protein BMI1 is cell cycle- regulated and correlates with its phosphorylation status. J Cell Sci 112, 4627-4639.

Wang, H., Wang, L., Erdjument-Bromage, H., Vidal, M., Tempst, P., Jones, R.S., and Zhang, Y. (2004). Role of histone H2A ubiquitination in Polycomb silencing. Nature 431, 873-878.

Watson, J.D. (2003). Celebrating the genetic jubilee: a conversation with James D. Watson. Interviewed by John Rennie. Scientific American 288, 66-69.

Watson, J.D., and Crick, F.H. (1953a). Genetical implications of the structure of deoxyribonucleic acid. Nature 171, 964-967.

Watson, J.D., and Crick, F.H. (1953b). Molecular structure of nucleic acids; a structure for deoxyribose nucleic acid. Nature $171,737-738$.

Winter, S., and Fischle, W. (2010). Epigenetic markers and their cross-talk. Essays Biochem 48, 45-61. 


\section{CHAPTER 2}

MK3 controls replicative life-span through interaction with BMI1

Peggy Prickaerts*, Hanneke EC Niessen*, Vivian EH Dahlmans, Juliette Salvaing, Frank Spaapen, Jolien Vanhove, Claudia Geijselaers, Stefanie JJ Bartels, Yoshihiro Takihara, Bradly G Wouters, Ulf R Rapp, Jan Willem Voncken

* equal contribution

Status: submitted 


\section{ABSTRACT}

The mitogen-activated protein kinase-activated protein kinase 3 (MAPKAPK3/ $3 \mathrm{pK} / \mathrm{MK} 3)$ was originally proposed as a potential tumor-suppressor protein. Paradoxically, MK3 integrates signaling through mitogen- as well as stress-activated protein kinases (M/SAPKs). The exact relevance of signaling via MK3 in oncogenesis is insufficiently clear. We here show that overexpression of MK3 induces a cell-cycle arrest that is DNA-damage dependent and features characteristics of oncogeneinduced senescence (OIS). Consistent with our recent identification of MK3 as a binding partner of Polycomb Repressive Complex 1 (PRC1) proteins, we here reveal interaction between MK3 and PRC-function at several functional levels. In MK3-OIS cells, increased expression of $p C D K N 2 A / I N K 4 A$, a locus known to be epigenetically controlled by Polycomb Group complexes, correlates with reduced expression level of the Polycomb Group protein EZH2 and concomitant loss of epigenetic H3K27me3-marking and CBX8/chromatin-occupation. Co-expression of the Polycomb Group oncoprotein BMI1 bypasses MK3-induced senescence and restores global H3K27me3 levels. Surprisingly, loss of $M K 3$ induces similar effects as $M K 3$-overexpression on replication and morphology in primary human fibroblasts. We establish that cells overexpressing or lacking $M K 3$ exhibit altered expression and phosphorylation kinetics of MAPKs and SAPKs in response to mitogenic signaling. This MAPK/SAPK signaling-imbalance results in aberrant expression of the immediate early response gene product EGR1, a crucial factor in control of senescence. Taken together, our data supports a role for MK3 in control of proliferation and replicative life-span through concerted action with BMI1.

Keywords: MAPKAPK3, MK3, Polycomb, BMI1, senescence, signaling-imbalance 


\section{INTRODUCTION}

The mitogen-activated protein kinase-activated protein kinase 3 (MK3) is a member of the MAPKAPK family, including RSK1-4, MSK1/2, MNK1/2 and MK2/3/5; despite their high homology, the three MKs display distinct spatio-temporal expression profiles and act in different biological processes $(1,2)$. MK3 was originally referred to as 3pK (for chromosome $3 p$ kinase); as it was often found homozygously deleted in small cell lung cancer cell lines, it was viewed as a potential tumor-suppressor gene (3). MK3 was identified as the first kinase activated through all three mitogen- and stress-activated protein kinase (M/SAPK) cascades. Therefore MK3 was considered a novel convergence point of the M/SAPK pathways that functioned as an integrative element in signaling of mitogen and stress responses (4). Sequential activation of kinases within the canonical mitogen-activated protein kinase cascades is a common and evolutionary-conserved signal transduction mechanism. These cascades cooperate in transmitting various extracellular signals and thus control a large number of distinct and even opposing cellular processes such as proliferation, differentiation, survival, development, stress response, and apoptosis (5). Mitogenic signaling in eukaryotic cells involves activation of the small GTPase $\mathrm{p} 21^{\text {Ras }}$. In cancer, oncogenic RASV12-signaling initiates a strong proliferative response, which ultimately triggers a senescence response as part of a fail-safe mechanism against oncogenic transformation (6); this protective mechanism includes a hyperreplication-induced DNA-damage response (DDR) (7). The collective biological responses to activated oncogenes culminate in oncogene-induced senescence (OIS) (8-11).

Polycomb Group repressive complexes (PRC1 and PRC2) act as part of a cellular epigenetic memory and play an important role in the determination of cell fate (12). Both complexes are associated with epigenetic catalytic activity and are subject to posttranslational modification themselves $(13,14)$. PRC proteins have been linked to oncogenesis: high expression of several PRC members has been etiologically implicated in onset and malignant progression of cancer (15).

We previously reported that MK3 associates with PRC1-complexes through direct interaction with the Polyhomeotic orthologs PHC1 and PHC2 (16). As PRC1-protein phosphorylation correlates with chromatin-dissociation $(16,17)$, it is tempting to speculate on a role for signaling through MK3 in regulation of PRC1-mediated transcriptional repression. Given its crucial positioning at convergent mitogenic and stress signals, it is conceivable that MK3 fine-tunes signaling in cell cycle regulation. We therefore set out to investigate the importance of the MK3/PRC1-association in the context of mitogenic signaling. 


\section{RESULTS}

\section{MK induces TP53 and CDKN2/INK4A-dependent senescence}

To study the role of MK3 in cellular processes related to cell division, we forced MK3 overexpression (MK3OE) in different cell models using retroviral expression systems and followed cell proliferation over time. MK3OE in primary human TIG3 fibroblasts significantly diminishes their proliferative capacity (Figure 1A). In good agreement with decreased proliferation, reduced BrdU-incorporation in TIG3/MK3OE cells reveals diminished de novo DNA synthesis (Figure 1B). In addition, TIG3/MK3OE cells display enlarged cell morphology, a characteristic feature of senescent cells (Figure 1C). Senescence is confirmed by the observation that large flat cells express the senescence-associated beta-Galactosidase (SA-bGal) marker protein (Figure 1C). MK3 overexpression also negatively affects cell division in immortal TIG ${ }^{\text {hTERT }}$ cells, indicating that MK3 acts downstream or independent of hTERT in proliferative control (Supplementary Figure 1A). Importantly, U2-OS/ MK3OE and HeLa/MK3OE cultures also show an enhanced flat cell morphology upon MK3OE (Figure 1E). Further analyses of U2-OS/MK3OE cells display both reduced proliferation and BrdU-incorporation, as well as increased G1-arrest and expression of SA-bGal, demonstrating that these effects occur in primary and cancer cell lines (Figure 1D; Supplementary Figure 1B,C).

The above findings show that MK3 overexpression triggers accelerated senescence. Oncogenic signaling is known to induce senescence and this is often associated with replication-induced DNA damage (7-9). To determine whether the MK3OE-induced response is similar to that observed in oncogene-induced senescence (OIS), we studied known hallmarks of OIS, including the occurrence of double strand DNA breaks (DSBs), activation of a DNA damage response (DDR) marked by activation of the TP53-pathway including its transcriptional target $\mathrm{p} 21^{\mathrm{CIP} 1 / \mathrm{WAF} 1}$, and expression of the senescence marker CDKN2A/INK4A which encodes the cell cycle inhibitors p16 and p14 ${ }^{\text {ARF }}$.

Figure 1 MK3-overexpression induces senescence in primary human fibroblasts and cancer cells. (a) Growth curve of primary human TIG3 fibroblasts transduced with a murine Bmi1 expression vector (open triangles), an $M K 3$ expression vector (MK3OE; open circles) or an empty vector (con; filled circles). (b) Corresponding representative DNA profiles (BrdU pulse-labeling and S-phase quantification by FACS) of TIG3 cells transduced with an MK3-expression vector (MK3OE; lower panel) or control cells (con; upper panel). (c) Flat cell morphology in TIG3/MK3OE cells (phase contrast; right upper panel); Senescence-Associated betaGalactosidase (SA-bGal) staining in TIG3/MK3OE cells (right lower panel). (d) Growth curve of established human U2-OS cells transduced with an $M K 3$ expression vector (MK3OE; open circles) or an empty vector (con; filled circles). (e) Flat cell morphology in U2-OS/MK3OE and HeLa/MK3OE cells (phase contrast; right panels); arrows indicate circumference of one flat cell. 

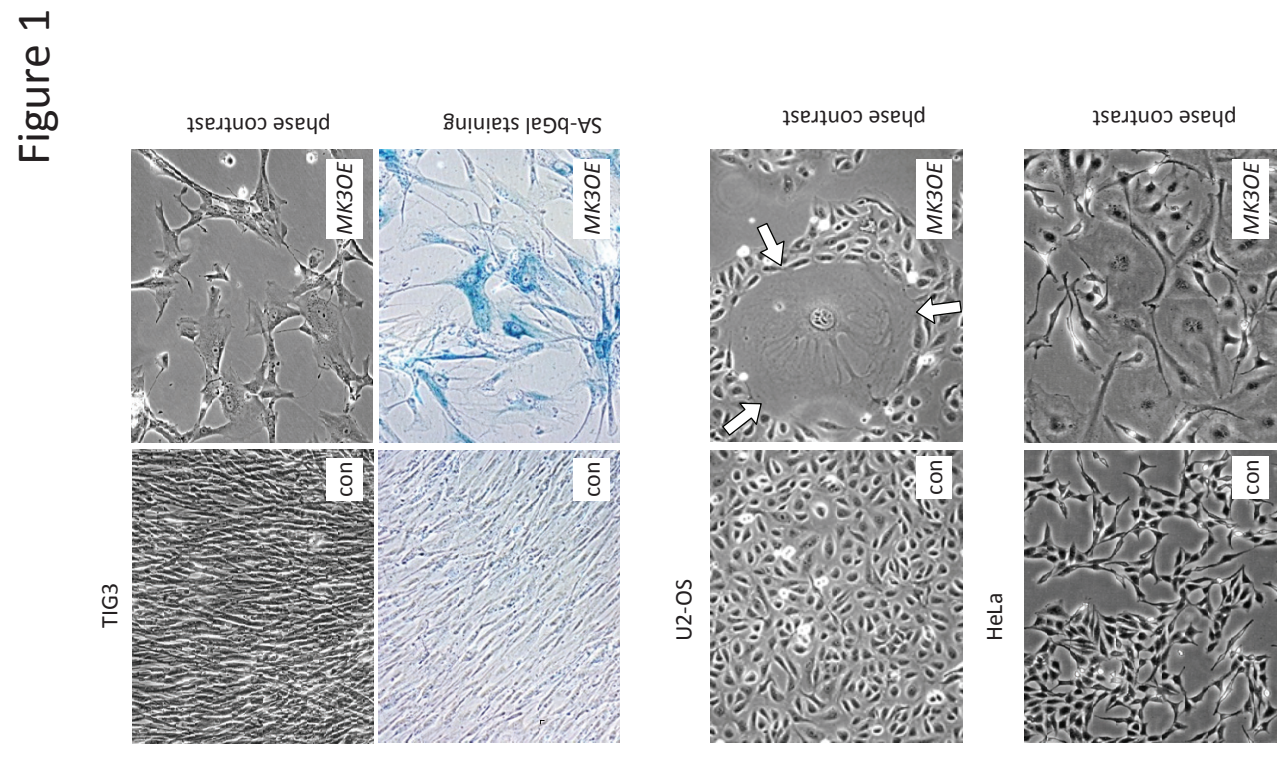

$\cup$

(1)
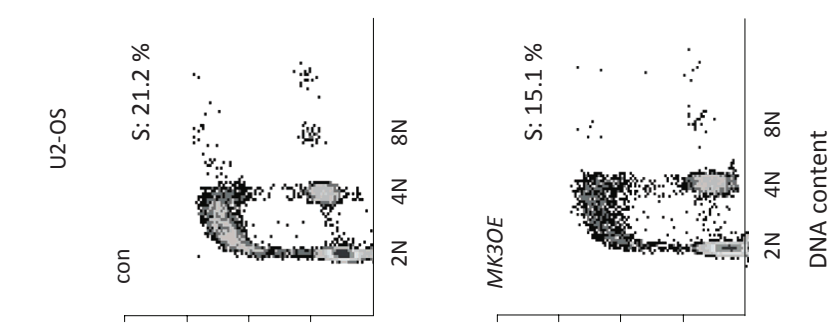

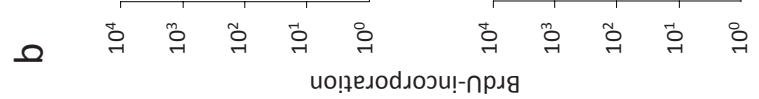
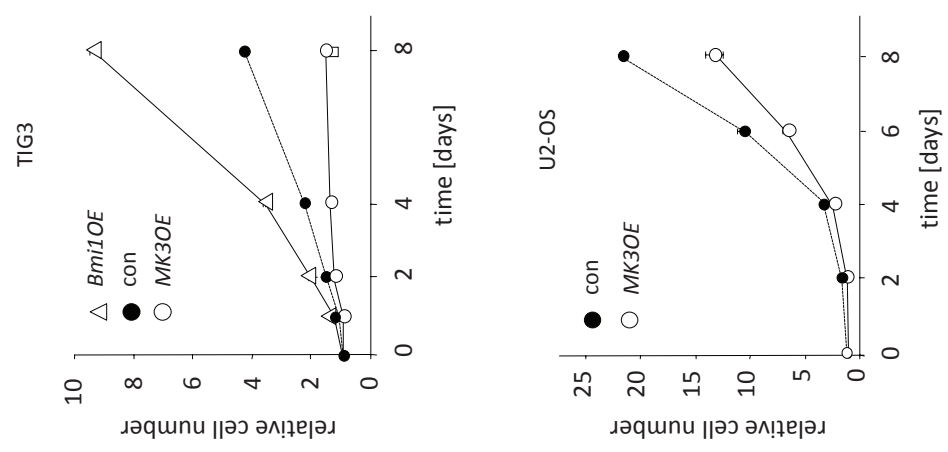

ర 
Chapter 2 |

ำ
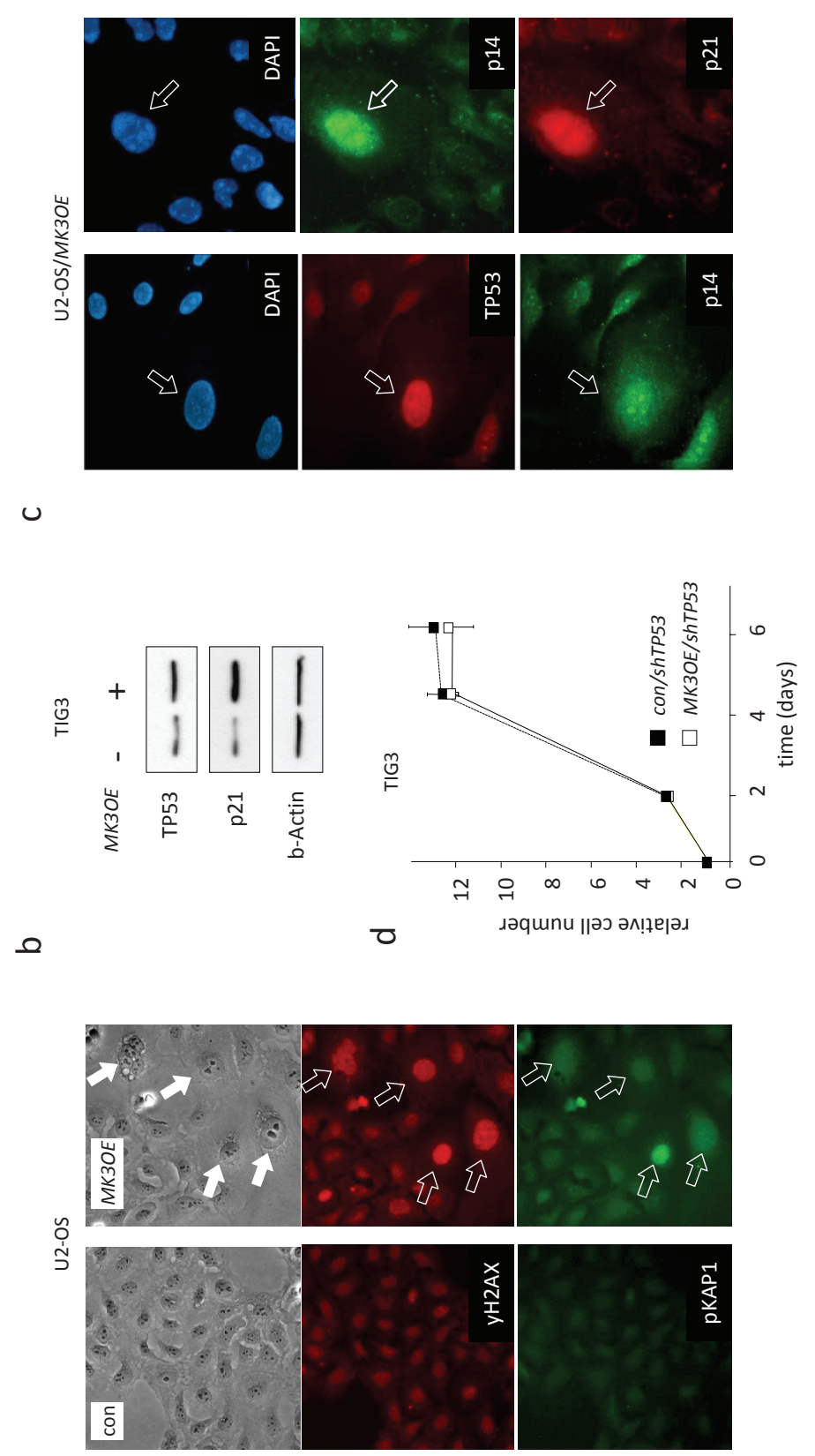

$\sigma$ 
Figure 2 MK3 evokes a TP53-dependent oncogene-induced senescence. (a) Phosphorylated H2A.X ( $\gamma H 2 A . X)$ and phosphorylated KAP1pSer824 (pKAP1) in senescent U2-OS/MK3OE cells (arrows); control (left panels) or

MK3OE (right panels). (b) Increased protein levels of the check-point regulator proteins TP53 and p21 ${ }^{\text {CIP1/WAF1 }}$ (p21) in U2-OS/MK3OE cells; b-Actin detection was used as loading control. (c) TP53 (TP53) and p14 ${ }^{\text {ARF }}$ (p14) co-staining (left panels) or $\mathrm{p} 14^{\mathrm{ARF}}(\mathrm{p} 14)$ and $\mathrm{p} 21^{\text {CIP1/WAF1 }}$ (p21) co-staining (right panels) in senescent U2-OS/MK3OE cells; DAPI was used to counterstain nuclei. (d) Growth curves of TIG3 fibroblasts deficient for TP53 (shTP53) show insensitivity to MK3OE-induced cell cycle arrest (open squares: MK3OE + shTP53; solid squares: empty vector control + shTP53; for comparison proliferation profiles cf. Figure 1a).

Similar as in OIS, U2-OS/MK3OE cells show increased DNA damage, measured as increased phosphorylation of histone variant H2A.X ( $\gamma \mathrm{H} 2 \mathrm{~A} . \mathrm{X}$; Figure $2 \mathrm{~A})$. Parallel staining for DSB-associated phosphorylated KAP1 (pKAP1) confirms activation of DDR (Figure 2A). The occurrence of OIS-associated DNA damage is typically associated with an initial intra S-phase arrest. We therefore probed for increased S-phase accumulation as an early response to $M K 3 O E$. Indeed, within days after overexpression of MK3 (as opposed to weeks; Figure 1), U2-OS/MK3OE and HeLa/MK3OE cells show a nearly 1.5 fold increase in S-phase cells (Supplementary Figure S2A and not shown). A prominent feature of intra S-phase arrest is its TP53-dependency. Global expression of TP53 is elevated in MK3OE cells, as is its downstream target $\mathrm{p} 21^{\mathrm{CIP} 1 / \mathrm{WAF} 1}$ (Figure 2B); indeed many enlarged nuclei of senescent U2-OS/MK3OE and TIG3/MK3OE cells are positive for both TP53 and p21 ${ }^{\text {CIP1/WAF1 }}$ (Figure 2C; Supplementary Figure S2B,C). TP53 is stabilized by $\mathrm{p} 14^{\mathrm{ARF}}$, through preventing its MDM2-mediated proteolytic degradation. In line with this notion, flat cell nuclei show increased co-staining of p14 ${ }^{\text {ARF }}$ and TP53 in both osteosarcoma cells and in fibroblasts (Figure 2C; Supplementary Figure S2C). Some senescent TIG3/MK3OE nuclei display co-staining for both gene-products encoded by the CDKN2/INK4A locus: $\mathrm{p} 14^{\mathrm{ARF}}$ and P16 ${ }^{\mathrm{INK} 4 \mathrm{~A}}$ (Supplementary Figure S2C). To obtain functional evidence that TP53 is involved in the MK3OE-induced senescence, we applied short hairpin RNAs to reduce cellular TP53 levels (shTP53). Depletion of cellular TP53 is predicted to prevent DDR and bypass intra S-phase arrest. Indeed, when TIG3 co-express MK3 and shTP53, cells evade the MK3-induced proliferative block and show proliferation dynamics indistinguishable from TIG3/shTP53 (Figure 2D cf. Figure 1A). Hence, the MK3OE-induced senescent response is bypassed by disabling TP53.

\section{Aberrant MK3-levels cause signaling-imbalance}

Thus far the combined results are compatible with an OIS-response in cells overexpressing $M K 3$. This is somewhat counter-intuitive, as MK3-deletion in different forms of cancer suggests MK3 may act as a tumor-suppressor. Since MK3 is a down- 
stream target in both stress- (p38, JNK) and mitogen-signaling (ERK) cascades, and both are implicated in senescence programming (see discussion), we probed the effect of reduced mitogenic-signaling on the anti-proliferative action of $M K 3 O E$. Reduced mitogenic signaling from ERK to MK3 by serum reduction enhances the percentage of enlarged cells in both TIG3/MK3OE and U2-OS/MK3OE cultures (Supplementary Figure $3 \mathrm{~A}, \mathrm{~B})$. In U2-OS/MK3OE cells, the proliferation rate was substantially more decreased by mitogen reduction than in control cells: growth in $2 \%$ serum reduced proliferation to approximately $70 \%$ of control cells (vs. $\pm 20 \%$ at $10 \%$ serum; Supplemental Figure 3C), suggesting that mitogenic-signaling counterbalances the senescent program elicited by $M K 3 O E$. We next studied the effect of reducing MK3 levels on cell cycling using RNA-interference. Expression of shMK3 induces a senescentlike morphology and results in reduced proliferation in TIG3 fibroblasts (Figure 3A, B). We hypothesized that forced modulation of MK3-signaling results in signalingimbalance and altered cell proliferation. Of note, we have recently found that modulation of MK levels in cells had significant effects on ERK and p38: mitogeninduced signaling to ERK resulted in sustained ERK-phosphorylation in the absence of $\mathrm{MK}$, whereas $M K 3 O E$ delayed the induction of pERK. In contrast p38 protein was largely lost from U2-OS/shMK (knock-down for both MK2 and MK3) cells, whereas MK3OE stabilized p38 levels; pp38 levels were reduced in response to mitogen stimulation whether MK levels were increased or reduced (18). We here show that also JNK and pJNK levels are affected by MK-signaling: MK3OE marginally increases overall JNK levels and, consequently, pJNK levels, yet leaves phosphorylation kinetics unchanged (Figure $3 C$ ). ShMK, in contrast, abrogates the pJNK response to mitogenic signaling almost completely (Figure 3C).

Figure 3 Altered MK3-levels cause signalling imbalance. (a) shRNA-mediated knockdown of MK3 (shMK3) induces an arrest in TIG3 cells. Flat cell morphology in TIG3/shMK3 cells (shMK3, lower panel; phase contrast) versus control cells (con, upper panel). (b) Corresponding representative DNA profiles (BrdU pulse-labeling and S-phase quantification by FACS) of TIG3 cells transduced with a shMK3-vector (shMK3; lower panel) versus control cells (con; upper panel). (c) pJNK and JNK levels in U2-OS/MK3OE (MK3OE; upper panels) cells versus control cells (con) and in U2-OS/shMK2/3 (shMK) versus control (con; lower panels) c ells. Cells were starved for $48 \mathrm{hrs}$ and then stimulated with mitogens; (d) Exogenous MK3 and endogenous MK2 levels in U2-OS/MK3OE (MK3OE; lower panels) or control cells (con; upper panels), and in U2-OS/shMK2/3 (shMK) and control cells (con; lower panels); AKT and beta-Actin (b-Actin): loading control; $\left({ }^{*}\right)$ indicates loading controls for corresponding genotypes in ( $c, \mathrm{~d}$ and $\mathrm{e}$ ); $\mathrm{t}(\mathrm{p}$-st; $\mathrm{min}$ ) indicates time elapsed since stimulation (minutes) as in (c). (e) Endogenous EGR1 in U2-OS/MK3OE (MK3OE) cells, in U2-OS/shMK2/3 (shMK) and in U2-OS/MKi (10 $\mu \mathrm{M}$ MK2a) versus control cells; (f) Endogenous TP53 in U2-OS/shMK2/3 (shMK) versus control cells. (c-f) $\mathrm{t}(\mathrm{min})$ indicates time elapsed since mitogenic stimulation (minutes); antibodies used are indicated. 
$m$
인
$\frac{1}{3}$
문
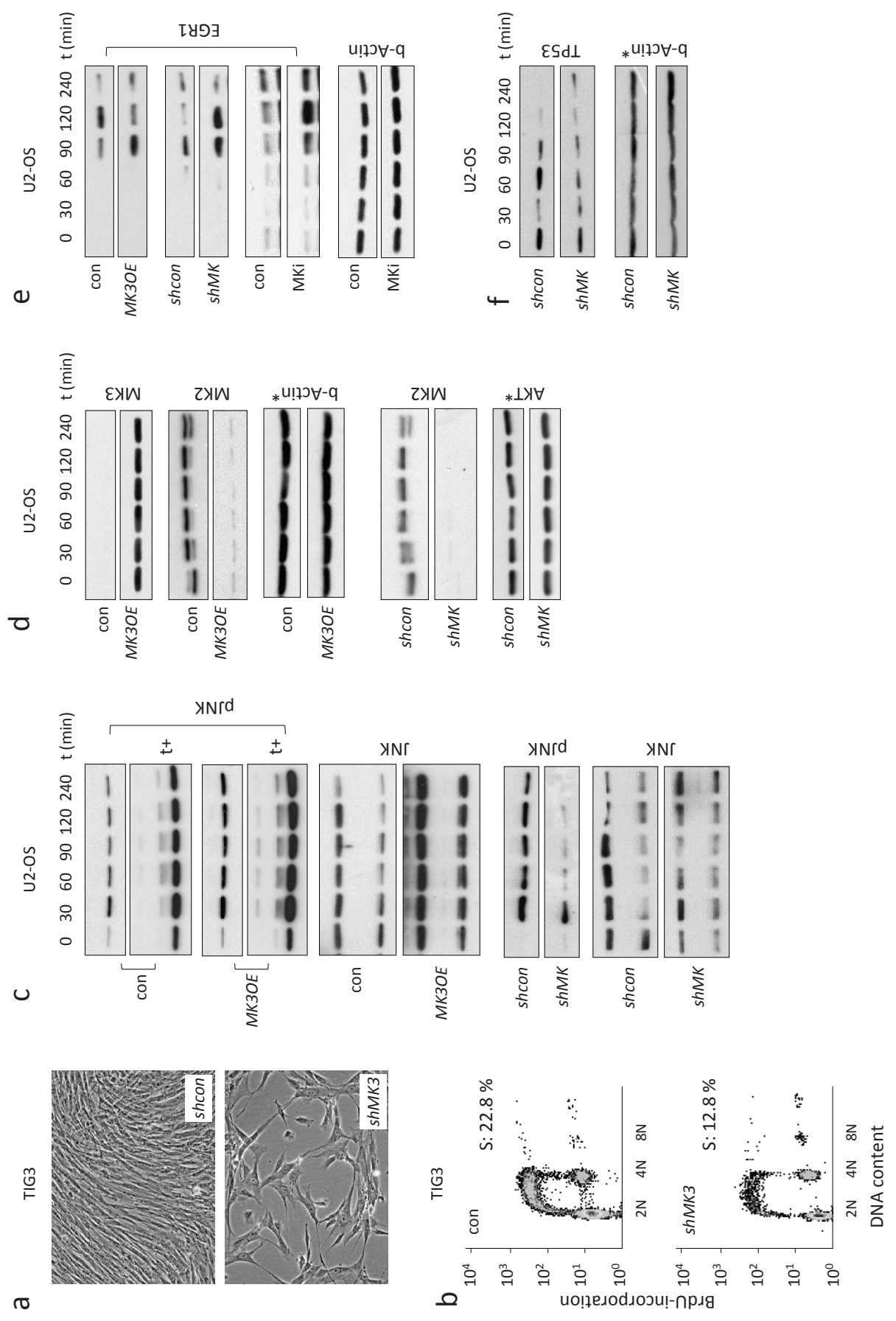
Overexpression of MK3 reduces endogenous MK2 and MK3 levels (Figure 3D and data not shown). To determine the potential contribution of MK2 to the MK3-induced proliferation phenotype, we treated cells with either shMK3 or shMK2/3. Both shMK3 and shMK2/3 comparably increase the proportion of cells in $\mathrm{G} 1$ at the expense of replicating cell numbers (S-phase; Supplementary Figure S4A), suggesting that MK3 is crucial in relaying proliferative signals from affector kinases in fibroblasts. In addition, we find that shMK3 cells show a normal arrest in response to UV-induced DNA damage (Supplementary Figure S5), whereas MK2 was found to be indispensable for UVinduced cell cycle arrest (19); these data support independent functions for MK3 and MK2 in cell cycle control. Taken together, the above findings are in agreement with several defined modes of functional interaction between MKs and their upstream affector kinases ERK, p38 and JNK, which, when disturbed, likely result in abnormal mitogenic responses. Indeed cells with gain- (GOF) or loss-of-function (LOF) of MK3, display altered expression kinetics of the immediate early response gene (IEG) product EGR1 in response to mitogenic signaling; this finding was independently confirmed by pharmacological inhibition of MK function (MKi; Figure 3E). In addition, the induction of TP53 in shMK cells in response to starvation $(t=0)$ and to stimulation (subsequent time points) is abnormal (Figure 3F). Combined, these findings suggest that altered stimulatory and inhibitory signaling lies at the bases of the proliferation phenotype induced by $M K 3 O E$ and $M K k d$.

\section{Functional links between $M K 3$ and Polycomb in senescence}

Senescent cells are known to release CDKN2A/INK4A repression by loss of repressive Polycomb-associated histone H3 Lys27 trimethylation (H3K27me3). The responsible histone lysine methyltransferase is $\mathrm{EZH2}$, a member of the PRC2-class of Polycomb Group proteins (20). In keeping with this, senescent TIG3/MK3OE cells express less EZH2 and show concomitant loss of global H3K27me3; reduced EZH2 and H3K27me3 correlate with increased $P 16^{I N K 4 A}$ expression (Figure 4A,B; Supplemental Figure S6A,B; $c f$. Figure $2 \mathrm{C}, \mathrm{S} 2 \mathrm{C}$ ). We used chromatin immunoprecipitation to study chromatin-associated changes at the CDKN2/INK4A locus. This locus was charted in detail before in respect to H3K27me3 marking and PRC1-occupation (21). We used a number of previously identified PRC1-negative (p15exon1; p14exon1) and positive (HOXA10; HOXA11) regions as methodological controls. Absence or presence of H3K27me3 correlates perfectly with absence or presence of CBX8 (Figure 4C), an epigenetic regulator protein which binds the me3-mark through its chromobox domain. Interestingly, MK3-overexpression enhances chromatin-occupation of MK3 at all loci probed (Figure 4C), and in parallel, enrichment of its direct PRC1-binding partner, PHC1, 
appears to increase as well, except in the $p 16^{I N K 4 A}$ coding region. Consistent with increased $P 16^{I N K 4 A}$ expression, TIG3/MK3OE cells show loss of H3K27me3 enrichment at p16-exon1, along with reduced $\mathrm{CBX} 8$ and $\mathrm{PHC} 1$. This data demonstrates that $M K 3 O E$ leads to reduced PRC1-occupation and increased INK4A/ARF expression.

The $M K 3 O E$-associated senescence phenotype resembles that induced by loss-ofPRC1-function: knockdown of the PRC1-proteins BMI1 (shBMI1) or PHC2 (shPHC2) induces a flat-cell phenotype in TIG3 as well as U2-OS cells (Supplementary Figure S7A). Hence, knockdown of PRC1 function is expected to aggravate the MK3OE-induced senescence. Indeed, combined shBMI1 has a strong additive effect on the induction of flat-cell morphology in U2-OS/MK3OE cells (Supplementary Figure S7B). Conversely, in accordance with our previous observation that $M K 3 O E$ reduces the amount of chromatin-associated BMI1 (16), we predicted that Bmi1-overexpression may antagonize the negative effect of $M K 3 O E$ on cell proliferation. To this end, TIG3 fibroblasts were serially transduced with retroviral vectors expressing murine Bmi1 and/or a GFP reporter, followed by a MK3 and/or a puromycin resistance marker (empty vector control). Whereas TIG3/Bmi1OE display similar cell morphology as control cells, and $M K 3 O E$ alone induces the senescent morphology, co-expression of both Bmi1 and $M K 3$ in primary human fibroblasts bypasses the senescence-associated morphological changes induced by $M K 3 O E$ and restores proliferation rate (Figure 4D; cf. Figure 1A). Relevantly, serially transduced fibroblast cultures overexpressing both Bmi1 and $M K 3$ select for increased BMI1 protein levels, compared to cultures overexpressing Bmi1 alone (Figure 4E). Furthermore, in good correlation with sustained proliferation, Bmi1OE/MK3OE cells display normal global H3K27me3 (Figure 4F). This data suggests that cells utilize BMI 1 to overcome the adverse effects of $M K 3 O E$ on cell cycling. Combined our findings convincingly support a functional interaction between MK3 and Polycomb-function in the context of proliferative control.

Figure 4 Functional interactions between MK3 and Polycomb Group proteins. (a) MK3, TP53, p16 ${ }^{\text {INK4A }}$ (p16), EZH2 levels in TIG3/MK3OE (MK3OE) and control (con) cell lysates. (b) MK3-overexpressing HeLa cells show reduced global H3K27me3. Quantification of $\mathrm{H} 3 \mathrm{~K} 27 \mathrm{me} 3$ in $\mathrm{HeLa} / \mathrm{MK} 3 \mathrm{OE}$ (MK3OE) or control (con) cell lysates; expression vectors indicated below figure. (c) ChIP of CBX8, PHC1, MK3 and H3K27me3, and negative control HA in MK3OE (MK3OE) expressing and control (con) TIG3 cells; PRC1-target loci (p16 promoter, p16exon1, HOXA10, HoXA11) and non-target loci (p15exon1, p14exon1) are indicated below the figure. Enrichments are presented as percentages of total input. (d) Comparative morphology of TIG3 cells that were sequentially transduced with either Bmi1.ires.GFP (Bmi1OE) or GFP (con) virus, and MK3/puromycin (MK3OE) or control puromycin virus (con) at $48 \mathrm{hr}$ intervals. (e) Serially transduced TIG3 cultures (expression vectors indicated below figure) were grown for 28 days and analysed for BMI1, MK3, TP53 expression at the indicated time points; antibodies indicated in figure. (f) Overexpression of Bmi1 in $M K 3 O E$ cells corrects $\mathrm{H} 3 \mathrm{~K} 27 \mathrm{me} 3$ levels. Quantification of H3K27me3 levels in HeLa/MK3OE, Bmi1OE, Bmi1OE/MK3OE and control cell lysates; expression vectors indicated below figure. H3K27me3 levels in (b, f) are normalized to beta-Actin (b-Actin). 
Chapter 2 |

ป
는
몬
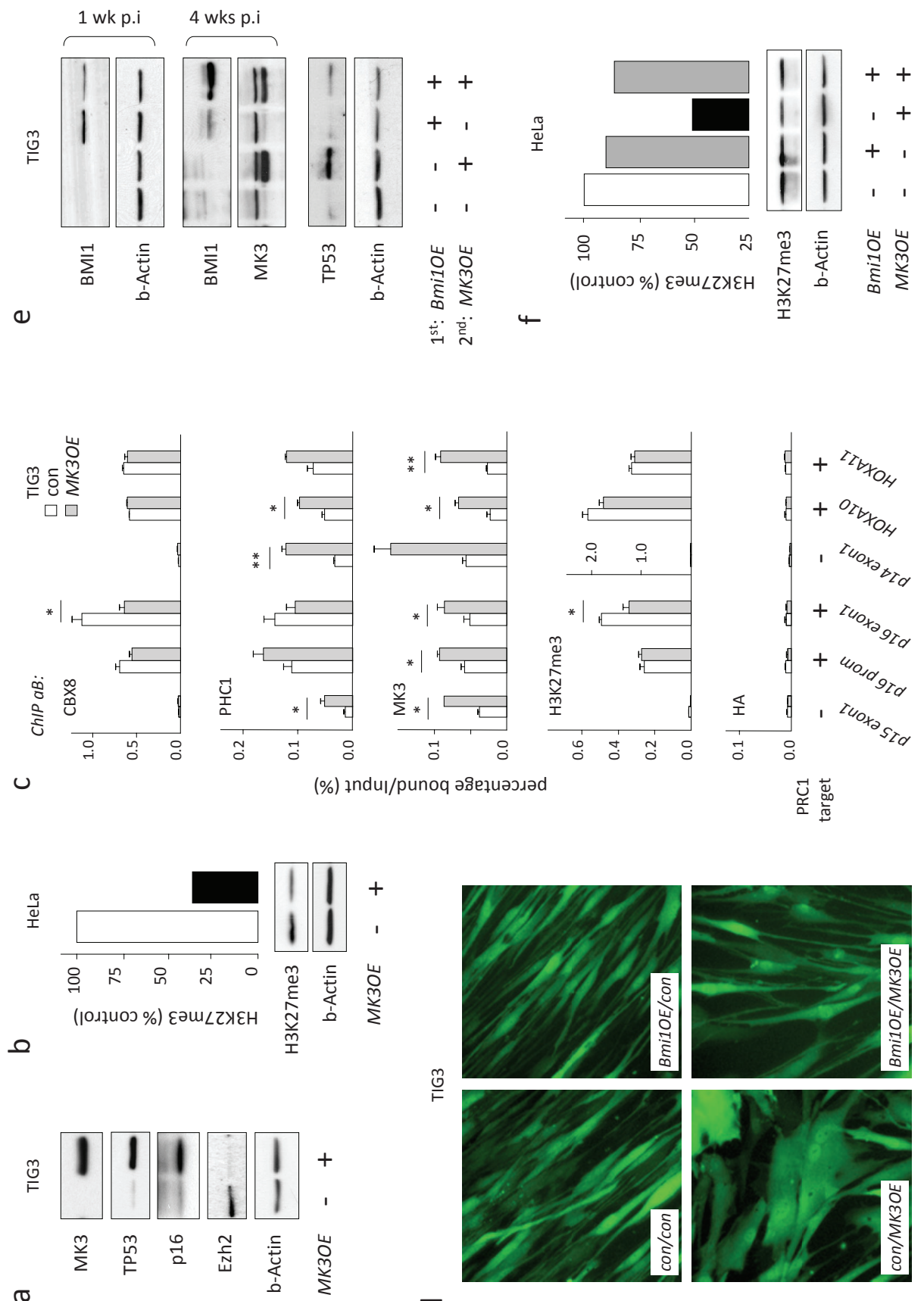

은

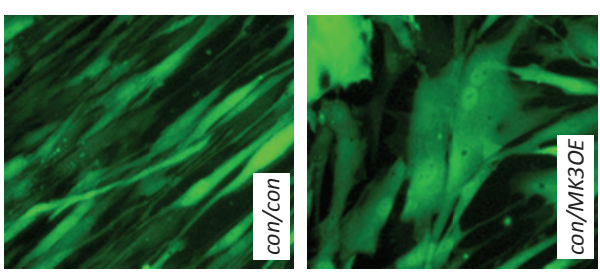




\section{DISCUSSION}

We here report that overexpression of MK3 induces a senescence state similar to loss-of-Polycomb-associated senescence. Relevantly, the oncogene Bmi1 rescues the anti-proliferative and senescence-accelerative effects induced by $M K 3 O E$, demonstrating that MK3/PRC1 together control proliferative life-span.

MK3 was originally proposed as a potential tumor-suppressor gene, as it resides with a number of other genes in chromosomal region 3p21.3, which often shows homozygous deletion in cancer (4). However, our findings suggest MK3-overexpression evokes a phenotype similar to oncogene-induced senescence (OIS), as evidenced by accumulation of DNA damage, intra S-phase arrest, activation of a DNA damage response and ultimately, proliferative arrest accompanied by $\mathrm{p} 16^{\text {INK4 }}$ and SA-bGal expression and morphological alterations typical of senescent cells. RASV12-mediated OIS involves TP53 signaling (6). We show that TP53 and $\mathrm{p} 21^{\text {CIP1/WAF1 }}$ levels are both increased in $M K 3 O E$ cells in accordance with TP53/p21 $1^{\text {CIP1/WAF1 }}$-dependency of early senescence (22). In addition MK3OE senescent cells express $\mathrm{p} 14^{\mathrm{ARF}}$ which, like $\mathrm{p} 16^{\mathrm{INK} 4 \mathrm{~A}}$, is associated with replication check-points in human cells (23). Although p14 ${ }^{\text {ARF }}$ stabilizes TP53 (24), we cannot formally rule out independent roles for TP53, p14 ${ }^{\text {ARF }}$ and $\mathrm{p} 21^{\mathrm{CIP} 1 / \mathrm{WAF} 1}$ in MK3-induced proliferation arrest $(25,26)$. The increased expression of $p 16^{\text {INK4a }}$ upon sustained $M K 3$ overexpression is in good agreement with its crucial role in establishing irreversible senescence (27-29). Of interest, $M K 3$ was recently suggested to harbour potentially oncogenic (driver) mutations, in contrast to MK2 (30). Although the exact role of these mutations in tumorigenesis awaits further analysis, these and other observations suggest that these highly homologous proteins have acquired divergent cellular-context dependent functions $(1,31)$. Our data contribute to this notion in several ways: we find no additional effect of $M K 2$-depletion in addition to shRNA-mediated loss of MK3, and, in contrast to MK2, MK3 appears not to be required for UV-induced arrest (19). Also MK5 (PRAK), although required for RASV12-mediated OIS, is by itself not capable of inducing senescence and is not required for damage-induced responses (32).

The observation that both MK3-GOF and LOF limit proliferation appears paradoxical. However, MK3 was shown to be targeted by all three canonical M/SAPkinases ERK, JNK and p38 (4). These unique properties position MK3 at the convergence point of potentially conflicting input. In addition, we provide evidence that MK3 provides regulatory feedback to M/SAPKs at several functional levels: JNKphosphorylation and synthesis and/or stability are dependent on MK2/3 (this study), p38 protein levels are enhanced by MK3 (18), and MK3 negatively regulates ERK phosphorylation (18). Given the fact that all three kinases have been implicated in senescence $(33,34)$ it is perhaps not surprising that both MK3-GOF and LOF elicits a 
similar effect on cell proliferation. Our data support the idea that signalling-imbalance is responsible for $M K 3$-dependent proliferative effects. Studies on $N$-MYC suggested that the outcome of oncogenic signalling-imbalance, i.e. proliferation (oncogenic), arrest or apoptosis (anti-oncogenic), is dependent on critical threshold N-MYC expression levels and, equally relevant, on the cells' intrinsic (i.e. genetic) state and its microenvironment (35). Likewise, and as is the case for M/SAPKs, relative concentrations of MK2, MK3, and MK5 and cellular context have been proposed to dictate protein-protein interactions, and thus signalling events and outcome $(2,36)$. Relative MK2/3/5 expression levels differ among various cells and tissues; hence, the effect of overexpression and loss-of function is expected to be cell type dependent, and may help explain the large variation in karyotypic events (heterozygous and homozygous deletions, loss of heterozygocity) involving chromosome $3 p$ in cancer (3).

We recently showed that MK3 associates with PRC1 via direct interaction with the alpha helix-rich SAM domains of PHC-proteins and that overexpression and activation of MK3 correlates with phosphorylation of PRC1-member proteins and PRC1/chromatin-dissociation (16). In light of the PRC1-LOF induced premature senescence in several human cell systems $(37,38)$, it is tempting to view $M K 3 O E$, via phosphorylation-mediated chromatin-release of PRC1, as a functional equivalent of PRC1-LOF. This view may explain why overexpression of the Polycomb Group oncoprotein BMI1 counterbalances the adverse effects of MK3 on proliferation. Senescence is known to be associated with loss of EZH2, H3K27me3, and, conversely, overexpression of either $\mathrm{EZH} 2$ or $\mathrm{CBX} 8$ act tumorigenic (39-42). In addition, the intricate functional relationships between EZH2, BMI1, TP53 and pRB in the context of senescence and their effect on H3K27me3-marking of the CDKN2/INK4A locus fit well with the effects of $M K 3$-overexpression reported herein $(21,40,43,44)$. Consistent with this we find reduced EZH2 levels and H3K27me3-marking besides loss of $\mathrm{CBX} 8 /$ chromatin-occupation in $M K 3 O E$ senescent cells. In addition, we here show that mitogen-induced expression of an important immediate-early response gene (IEG) EGR1 shows an abnormal response pattern in MK3OE and MKkd cells. EGR1 was shown to control senescence through regulation of TP53/p21 ${ }^{\mathrm{CIP} 1 / \text { WAF1 }}$ (45). Of note, MK2 was recently suggested to control murine haematopoietic stem-cell renewal through $\mathrm{p} 19^{\text {ARF }}$ (46). IEGs represent a standing response mechanism to a variety of triggers and their activation is closely linked to M/SAPK and MK action $(47,48)$. Polycomb Group proteins control IEG expression through regulation by MK (Bracken et al., 2006; Prickaerts et al., submitted). Thus, MK3/PRC1-mediated control over IEG likely plays a pivotal role in preventing oncogenic responses in cells. Global MK3/chromatin co-association analysis and PcG phospho-site mutation will help clarify the underlying molecular effects of MK3-to-Polycomb signaling in detail. 
In summary, our findings clearly demonstrate a direct functional interaction between PRC1 and MK3 and reveal a joined role in control over senescence, i.a. via IEG transcription. MK3-overexpression appears to reactivate 'dormant' senescence checkpoints in cancer cells with compromised pRB, TP53 or CDKN2-function (e.g. CDKN2A/INK4A locus methylation in U2-OS cells c.q. constitutive HPV-E6/E7 mediated inactivation of PRB and TP53 in HeLa cells) $(49,50)$. This is remarkable in itself and warrants further investigation into MK3-dependent epigenomic regulation of gene transcription in cancer.

\section{MATERIALS AND METHODS}

Cell lines Human U2-OS osteosarcoma cells were kindly provided by D. Shvarts (Utrecht Medical Center, Utrecht, The Netherlands), HeLa cervical carcinoma cells by M. Koritzinsky (MAASTRO, Maastricht, The Netherlands), BJ-hTERT and primary human TIG3 fibroblasts expressing the murine ecotropic receptor: courtesy D. Peeper (Netherlands Cancer Institute, Amsterdam, The Netherlands).

Tissue culture and expression systems Cells were cultured at $37^{\circ} \mathrm{C}, 5 \% \mathrm{CO}_{2}, 100 \%$ humidity in Dulbecco's Modified Eagle's medium (DMEM; 41966; Gibco/Invitrogen; Carlsbad, CA, USA) supplemented with $10 \%$ fetal calf serum (FCS Bodinco, Alkmaar, the Netherlands) and antibiotics (100 units/ml penicillin and $100 \mu \mathrm{g} / \mathrm{ml}$ streptomycin; Gibco). Serum starvation was accomplished at 0.05\% FCS for cancer cell lines and at $0.1 \%$ for human fibroblasts for $48 \mathrm{hrs}$. Mitogenic stimulation was achieved by supplementing 15\% FCS/100ng/ml tetradecanoyl phorbol acetate (TPA; Sigma-Aldrich, St. Louis, MO, USA) for the indicated duration. Retroviral expression vectors were used to maximize the percentage of expressing cells and to minimize integration effects (28, 51, 52). Retroviral vectors (pBABE-PURO, pBMN-LZRS.ires.GFP, pBMN-LZRS.ires.NEO) expressing murine Bmi1 and human MK3 have been described (16). Expression vectors encoding the murine ecotropic receptor or hTERT were kindly provided by R. Bernards (Amsterdam, The Netherlands). Criteria used for shRNA-sequence design and the retroviral expression system were as described before (53). Targeting sequences are listed in Supplementary Table 1. Transduced cells were selected for 1 week on 4-16 $\mu \mathrm{g} / \mathrm{ml}$ puromycin (Sigma). Production of infectious viral particles and growth curves were performed as described (6). For pharmacological inhibition, cells were preincubated for 30 min with 10 MM MK inhibitor (MK2a Inhibitor; Calbiochem/Merck, Darmstadt, Germany); control cells were incubated with solvent. 
BrdU-pulse labeling \& DNA profiling TIG3 cells were pulse-labeled with $10 \mu \mathrm{M}$ BrdU (Sigma) for $45 \mathrm{~min}$. Cells were fixed in cold methanol overnight at $-20^{\circ} \mathrm{C}$ immediately after labeling, DNA was denatured by incubation in $0.4 \mathrm{mg} / \mathrm{ml}$ pepsin (Sigma) in $0.1 \mathrm{~N} \mathrm{HCl}$ for 30 min followed by a 30 min incubation with $2 \mathrm{~N} \mathrm{HCl}$ at $37^{\circ} \mathrm{C}, \mathrm{pH}$ was readjusted to 7-8 with $0.1 \mathrm{M}$ sodium borate buffer $\mathrm{pH}$ 8.5. Cells were incubated with $5 \mu \mathrm{l}$ anti-BrdU mAb (IIB5, courtesy B. Schutte, Maastricht University, The Netherlands) for 1-1.5 h at RT. Cells were washed and incubated with $5 \mu$ FITCconjugated anti-mouse pAb (rabbit-anti-mouse FITC, Dako, Glostrup, Denmark) over night at $4^{\circ} \mathrm{C}$, washed and resuspended in PBS containing $100 \mu \mathrm{g} / \mathrm{ml} \mathrm{RNase}$ and $20 \mu \mathrm{g} / \mathrm{ml}$ PI for 2-3 hours at RT in dark environment, immediately prior to FACS-analysis (Becton Dickinson; CellQuest software).

For DNA-damage response measurements, after UV-irradiation $\left(20 \mathrm{~J} / \mathrm{m}^{2}\right), \mathrm{U} 2-\mathrm{OS}$ cells were incubated in $50 \mathrm{ng} / \mathrm{ml}$ nocodazole-containing medium (Fluka, Buchs, Switserland) for 16 hours and fixed in $70 \%$ ethanol overnight at $-20^{\circ} \mathrm{C}$. Fixed cells were permeabilized with $0.2 \%$ Triton X-100, $2 \% \mathrm{FCS} / \mathrm{PBS}$ for $20 \mathrm{~min}$ at $4^{\circ} \mathrm{C}$, and incubated with anti-phospho-Ser10 histone H3 (pAb, 06-570; Upstate, Upstate Biotechnology/ Millipore, Billerica, MA, USA) for $60 \mathrm{~min}$ at RT. Cells were washed and incubated with FITC-conjugated goat anti-rabbit antibody (diluted 1:500; Southern Biotech) for $30 \mathrm{~min}$ at RT, washed, and resuspended in PBS containing $100 \mu \mathrm{g} / \mathrm{ml}$ RNase and $20 \mu \mathrm{g} / \mathrm{ml} \mathrm{PI}$ for 2-3 hours at RT in dark environment, immediately prior to FACS analysis.

Immuno-histochemistry and cell staining Cell were seeded at 5-10\% confluence on glass slides, infected at low MOI $( \pm 1: 1)$ and subjected to a selection (i.e. 8 (Tig3) $-16 \mu \mathrm{g}$ (U2-OS) puromycin/ml) where applicable. Cells were fixed for 10 minutes at RT in $2 \%$ formaldehyde/PBS solution, permeabilized in $0,2 \%$ Triton X/PBS or directly fixed and permeabilized for 20 minutes in $100 \%$ methanol at $-20^{\circ} \mathrm{C}$. Antibody dilution was done in blocking buffer ( $5 \%$ normal goat serum, $5 \%$ FCS, $0.02 \%$ Triton X in PBS). Polyclonal (pAb) rabbit antiserum glutathione S-transferase (GST) and monoclonal antiserum (mAb) against MK3 were kindly provided by S. Ludwig (Münster, Germany), anti-2Py-tag mAb (MMS-115R; Babco, Richmond, California); anti-p14ARF/p16ß pAb (Ab-1; LabVision corp., Fremont, California), anti-p16INKA mAb (E6H4, MTM CINtec laboratories, Heidelberg, Germany), anti-p21 pAb (C-19; Santa Cruz Biotechnology, Santa Cruz, CA, USA), anti-P53 mAb (DO-7; M7001; DAKO), anti- $ү$ H2A.X mAb (JBW301, 05-636, Upstate Biotechnology/Millipore), anti-pKAP1S824 pAb (A300-767A; Bethyl Laboratories, Montgomery, TX, USA). Primary mAbs were detected with goat-anti-mouse TexasRed (TXRD, Southern Biotech, Birmingham, LA, USA), primary pAbs with goat-anti-rabbit fluorescein isothiocyanate (FITC, Southern Biotech). 4'-6-diamidino-2-phenylindole 
(DAPI) was co-incubated with secondary conjugated antibodies to counterstain cell nuclei. SA-bGal assays were performed as previously described (54).

Chromatin immunoprecipitation (ChIP) assays TIG3 cells were transduced with pBABE-puro-GST or GST-3pK and selected with $4 \mu \mathrm{g} / \mathrm{ml}$ puromycin. Fixation and ChIP procedures were essentially as described before (55). Samples were immunoprecipitated overnight at $4^{\circ} \mathrm{C}$ with antibodies against either HA (sc-805; Santa Cruz), H3K27me3 (07-449; Upstate), an equal mix of anti-CBX8 "LAST" and "GALD" (55), antiMK3 (4) or anti-PHC1 (56). Crosslinks were reversed overnight at $65^{\circ} \mathrm{C}$ followed by a $2 \mathrm{~h}$ digestion with RNase A and protease K. DNA fragments were recovered using QIAquick PCR purification columns (Qiagen, Hilden, Germany), according to manufacturers' instructions. Samples were eluted in $75 \mu$ lelution buffer and then further $1 / 5$ diluted in TE buffer. The immune-precipitated DNA was quantified by real-time qPCR. The sequences of the primers used are listed in Supplementary Table 1. Each experiment was performed three times. Results of one representative experiment are shown.

Western analysis Cells were lysed in RIPA buffer containing $5 \mathrm{mM}$ Benzamidine, 5 $\mu \mathrm{g} / \mathrm{ml}$ Antipain, $5 \mu \mathrm{g} / \mathrm{ml}$ Leupeptin, $5 \mu \mathrm{g} / \mathrm{ml}$ Aprotinin, $1 \mathrm{mM}$ Sodium Vanadate, $10 \mathrm{mM}$ Sodium Fluoride, $10 \mathrm{mM}$ Pyrophosphate, $10 \mathrm{mM}$ ß-glycerophosphate, $0.5 \mathrm{mM}$ DTT and $1 \mathrm{mM}$ PMSF. Protein concentration was determined using a BCA protein assay kit (Pierce/Thermo Fisher Scientific, Rockford, IL, USA). Proteins were immobilized on polyvinylidene fluoride (PVDF) membranes. After blocking with $5 \%$ non-fat dry milk/PBS/0.1\% Tween-20, membranes were incubated $\mathrm{o} / \mathrm{n}$ at $4^{\circ} \mathrm{C}$ with antibodies detecting: beta-Actin mAb (C4, 691001, MP Biomedicals, Solon, OH, USA), CBX8 (courtesy K.Hansen, Copenhagen, Denmark), Ezh2 mAb (BD4) and p16 mAb (DCS50; courtesy A. Bracken, BRIC, Copenhagen, Denmark), histone H3 pAb (Ab1791, Abcam, Cambridge, UK), p21 pAb (C-19; Santa Cruz Biotechnology), TP53 mAb (DO-7; M7001; DAKO), anti Bmi1 mAb (F6; courtesy M. van Lohuizen; Amsterdam, The Netherlands), anti MK3 mAb (3pK; clone 3p8-1, S. Ludwig, Münster, Germany), MK3 pAb (2362 3pK; U. Rapp, Würzburg, Germany), H3K27me3 pAb (07-449, Upstate), 2Py-tag mAb (MMS115R; Babco), AKT (9272, Cell Signaling, Danvers, MA, USA), EGR1 (sc-110, Santa Cruz), pJNK (Thr183/Tyr185) pAb (9251, Cell Signaling), JNK pAb (9252, Cell Signaling) and MK2 pAb (3042, Cell Signaling).

Quantification of mRNA levels by real-time (rt) PCR Total RNA was isolated using Tri-Reagent (Sigma). Quantity and quality of the RNA were checked on the nanodrop (Witec AG, Luzern, Switserland) according to the manufacturer's instructions. RNA (500 ng) was converted into cDNA using the iScript ${ }^{\mathrm{TM}}$ cDNA synthesis kit (Bio-Rad, Herculus, 
CA, USA) according to the manufacturer's protocol. MylQ analysis was performed on 10 ng of $\mathrm{CDNA}$ using the $\mathrm{qPCR}_{\mathrm{iQ}}{ }^{\mathrm{TM}}$ Custom $\mathrm{SYBR}^{\circledR}$ Green Supermix (Bio-Rad) and $300 \mathrm{nM}$ primer. Analysis was performed on an $\mathrm{iCycler}^{\circledR}$ thermal cycler (Bio-Rad). Cyclophillin A was used for normalization. The sequences of the primers used are listed in Supplementary Table 1.

\section{ACKNOWLEDGEMENTS}

We thank many colleagues for research materials and support of the study (please refer to Materials and Methods section). We thank Frédérique Peronnet, Ronit Sverdlov and Joep Geraedts for critically reading the manuscript and members of the MAASTRO and Molecular Genetics departments for helpful discussions. Financial support for these studies: European Molecular Biology Organization (Germany) 186.00-06 (URR); Dutch Science Organization (ZonMW-NWO): Research Support grant 908-02-040 (JWV) and VIDI grant 016.046.362 (JWV); Netherlands Genome Initiative (NGI) fellowship 050-72$422(\mathrm{HN})$. 


\section{REFERENCES}

1. Gaestel M. MAPKAP kinases - MKs - two's company, three's a crowd. Nat Rev Mol Cell Biol. 2006 Feb;7(2):120-30.

2. Shiryaev A, Moens U. Mitogen-activated protein kinase p38 and MK2, MK3 and MK5: menage a trois or menage a quatre? Cell Signal. 2010 Aug;22(8):1185-92.

3. Sithanandam G, Latif F, Duh F, Bernal R, Smola U, Li H, et al. 3pK, a new mitogen-activated protein kinase-activated protein kinase located in the small cell lung cancer tumor suppressor gene region [published erratum appears in Mol Cell Biol 1996 Apr;16(4):1880]. Mol Cell Biol. 1996 March 1, 1996;16(3):868-76.

4. Ludwig S, Engel K, Hoffmeyer A, Sithanandam G, Neufeld B, Palm D, et al. 3pK, a novel mitogen-activated protein (MAP) kinase-activated protein kinase, is targeted by three MAP kinase pathways. Mol Cell Biol. 1996 Dec;16(12):6687-97.

5. Keshet $Y$, Seger R. The MAP kinase signaling cascades: a system of hundreds of components regulates a diverse array of physiological functions. Methods Mol Biol. 2010;661:3-38.

6. Serrano M, Lin AW, McCurrach ME, Beach D, Lowe SW. Oncogenic ras provokes premature cell senescence associated with accumulation of p53 and p16INK4a. Cell. 1997;88(5):593-602.

7. Mallette FA, Gaumont-Leclerc M-F, Ferbeyre G. The DNA damage signaling pathway is a critical mediator of oncogene-induced senescence. Genes Dev. 2007 January 1, 2007;21(1):43-8.

8. Di Micco R, Fumagalli M, d'Adda di Fagagna F. Breaking news: high-speed race ends in arrest - how oncogenes induce senescence. Trends in Cell Biology. 2007;17(11):529-36.

9. Halazonetis TD, Gorgoulis VG, Bartek J. An oncogene-induced DNA damage model for cancer development. Science. 2008 Mar 7;319(5868):1352-5.

10. Prieur A, Peeper DS. Cellular senescence in vivo: a barrier to tumorigenesis. Curr Opin Cell Biol. 2008 Apr;20(2):150-5.

11. Rodier F, Coppe JP, Patil CK, Hoeijmakers WA, Munoz DP, Raza SR, et al. Persistent DNA damage signalling triggers senescence-associated inflammatory cytokine secretion. Nat Cell Biol. 2009 Jul 13;11:973-9.

12. Prezioso C, Orlando V. Polycomb proteins in mammalian cell differentiation and plasticity. FEBS Letters. 2011;585(13):2067-77.

13. Niessen HE, Demmers JA, Voncken JW. Talking to chromatin: post-translational modulation of polycomb group function. Epigenetics Chromatin. 2009;2(10):10.

14. Simon JA, Kingston RE. Mechanisms of Polycomb gene silencing: knowns and unknowns. Nat Rev Mol Cell Biol. 2009;10(10):697-708.

15. Sparmann A, van Lohuizen M. Polycomb silencers control cell fate, development and cancer. Nat Rev Cancer. 2006;6(11):846-56.

16. Voncken JW, Niessen H, Neufeld B, Rennefahrt U, Dahlmans V, Kubben N, et al. MAPKAP kinase 3pK phosphorylates and regulates chromatin association of the polycomb group protein Bmi1. J Biol Chem. 2005 Feb 18;280(7):5178-87.

17. Voncken JW, Schweizer D, Aagaard L, Sattler L, Jantsch MF, van Lohuizen M. Chromatin-association of the Polycomb group protein BMI1 is cell cycle- regulated and correlates with its phosphorylation status. J Cell Sci. 1999;112(Pt 24):4627-39.

18. Prickaerts P, Niessen H, Mouchel-Vielh E, Dahlmans V, Geijselaers C, Adriaens M, et al. MK3 controls Polycomb-target gene expression via negative feedback on ERK submitted.

19. Manke IA, Nguyen A, Lim D, Stewart MQ, Elia AE, Yaffe MB. MAPKAP kinase-2 is a cell cycle checkpoint kinase that regulates the $\mathrm{G} 2 / \mathrm{M}$ transition and $\mathrm{S}$ phase progression in response to UV irradiation. Mol Cell. 2005 Jan 7;17(1):37-48. 
20. Cao R, Zhang $Y$. The functions of $E(Z) / E Z H 2$-mediated methylation of lysine 27 in histone H3. Current Opinion in Genetics \& Development. 2004;14(2):155-64.

21. Bracken AP, Kleine-Kohlbrecher D, Dietrich N, Pasini D, Gargiulo G, Beekman C, et al. The Polycomb group proteins bind throughout the INK4A-ARF locus and are disassociated in senescent cells. Genes Dev. 2007 Mar 1;21(5):525-30.

22. Chen J, Huang X, Halicka D, Brodsky S, Avram A, Eskander J, et al. Contribution of p16INK4a and p21CIP1 pathways to induction of premature senescence of human endothelial cells: permissive role of p53. Am J Physiol Heart Circ Physiol. 2006 April 1, 2006;290(4):H1575-86.

23. Sherr CJ. The INK4a/ARF network in tumour suppression. Nat Rev Mol Cell Biol. 2001 Oct;2(10):731-7.

24. Pomerantz J, Schreiber-Agus N, Liegeois NJ, Silverman A, Alland L, Chin L, et al. The Ink4a tumor suppressor gene product, p19Arf, interacts with MDM2 and neutralizes MDM2's inhibition of p53. Cell. 1998;92(6):713-23.

25. Sherr CJ. Divorcing ARF and p53: an unsettled case. Nat Rev Cancer. 2006;6(9):663-73.

26. Rowland BD, Peeper DS. KLF4, p21 and context-dependent opposing forces in cancer. Nat Rev Cancer. 2006;6(1):11-23.

27. Beausejour CM, Krtolica A, Galimi F, Narita M, Lowe SW, Yaswen P, et al. Reversal of human cellular senescence: roles of the p53 and p16 pathways. Embo J. 2003 Aug 15;22(16):4212-22.

28. Campisi J. Senescent cells, tumor suppression, and organismal aging: good citizens, bad neighbors. Cell. 2005 Feb 25;120(4):513-22.

29. Collado M, Blasco MA, Serrano M. Cellular senescence in cancer and aging. Cell. 2007 Jul 27;130(2):22333.

30. Greenman C, Stephens P, Smith R, Dalgliesh GL, Hunter C, Bignell G, et al. Patterns of somatic mutation in human cancer genomes. Nature. 2007 Mar 8;446(7132):153-8.

31. Ronkina N, Kotlyarov A, Gaestel M. MK2 and MK3--a pair of isoenzymes? Front Biosci. 2008;13:5511-21.

32. Sun P, Yoshizuka N, New L, Moser BA, Li Y, Liao R, et al. PRAK is essential for ras-induced senescence and tumor suppression. Cell. 2007 Jan 26;128(2):295-308.

33. Debacq-Chainiaux F, Boilan E, Dedessus Le Moutier J, Weemaels G, Toussaint O. p38(MAPK) in the senescence of human and murine fibroblasts. Adv Exp Med Biol. 2010;694:126-37.

34. Maruyama J, Naguro I, Takeda K, Ichijo H. Stress-activated MAP kinase cascades in cellular senescence. Curr Med Chem. 2009;16(10):1229-35.

35. Murphy DJ, Junttila MR, Pouyet L, Karnezis A, Shchors K, Bui DA, et al. Distinct Thresholds Govern Myc's Biological Output In Vivo. Cancer Cell. 2008;14(6):447-57.

36. Murphy LO, Blenis J. MAPK signal specificity: the right place at the right time. Trends in Biochemical Sciences. 2006;31(5):268-75.

37. Isono K, Fujimura Y, Shinga J, Yamaki M, J OW, Takihara Y, et al. Mammalian polyhomeotic homologues phc2 and phc1 act in synergy to mediate polycomb repression of hox genes. Mol Cell Biol. 2005 Aug;25(15):6694-706.

38. Itahana K, Zou Y, Itahana Y, Martinez JL, Beausejour C, Jacobs JJ, et al. Control of the replicative life span of human fibroblasts by p16 and the polycomb protein Bmi-1. Mol Cell Biol. 2003 Jan;23(1):389-401.

39. Bachmann IM, Halvorsen OJ, Collett K, Stefansson IM, Straume O, Haukaas SA, et al. EZH2 expression is associated with high proliferation rate and aggressive tumor subgroups in cutaneous melanoma and cancers of the endometrium, prostate, and breast. J Clin Oncol. 2006 Jan 10;24(2):268-73. 
40. Bracken AP, Pasini D, Capra M, Prosperini E, Colli E, Helin K. EZH2 is downstream of the pRB-E2F pathway, essential for proliferation and amplified in cancer. Embo J. 2003 Oct 15;22(20):5323-35.

41. Dietrich N, Bracken AP, Trinh E, Schjerling CK, Koseki H, Rappsilber J, et al. Bypass of senescence by the polycomb group protein CBX8 through direct binding to the INK4A-ARF locus. Embo J. 2007 Mar 21;26(6):1637-48.

42. Kheradmand Kia S, Solaimani Kartalaei $P$, Farahbakhshian E, Pourfarzad F, von Lindern M, Verrijzer CP. EZH2-dependent chromatin looping controls INK4a and INK4b, but not ARF, during human progenitor cell differentiation and cellular senescence. Epigenetics \& Chromatin. 2009;2(1):16.

43. Kotake $Y$, Cao R, Viatour P, Sage J, Zhang Y, Xiong Y. pRB family proteins are required for H3K27 trimethylation and Polycomb repression complexes binding to and silencing p16INK4a tumor suppressor gene. Genes Dev. 2007 January 1, 2007;21(1):49-54.

44. Tang X, Milyavsky M, Shats I, Erez N, Goldfinger N, Rotter V. Activated p53 suppresses the histone methyltransferase EZH2 gene. Oncogene. 2004 Jun 21.

45. Krones-Herzig A, Adamson E, Mercola D. Early growth response 1 protein, an upstream gatekeeper of the p53 tumor suppressor, controls replicative senescence. Proc Natl Acad Sci U S A. 2003 Mar 18;100(6):3233-8.

46. Schwermann J, Rathinam C, Schubert M, Schumacher S, Noyan F, Koseki H, et al. MAPKAP kinase MK2 maintains self-renewal capacity of haematopoietic stem cells. Embo J. 2009 Apr 16.

47. Plotnikov A, Zehorai E, Procaccia S, Seger R. The MAPK cascades: Signaling components, nuclear roles and mechanisms of nuclear translocation. Biochimica et Biophysica Acta (BBA) - Molecular Cell Research. 2011;1813(9):1619-33.

48. Ronkina N, Menon MB, Schwermann J, Arthur JS, Legault H, Telliez JB, et al. Stress induced gene expression: a direct role for MAPKAP kinases in transcriptional activation of immediate early genes. Nucleic Acids Res. 2010 Apr;39(7):2503-18.

49. Park YB, Park MJ, Kimura K, Shimizu K, Lee SH, Yokota J. Alterations in the INK4a/ARF locus and their effects on the growth of human osteosarcoma cell lines. Cancer Genet Cytogenet. 2002 Mar;133(2):105-11.

50. Scheffner M, Whitaker NJ. Human papillomavirus-induced carcinogenesis and the ubiquitin-proteasome system. Seminars in Cancer Biology. 2003;13(1):59-67.

51. Kinsella TM, Nolan GP. Episomal vectors rapidly and stably produce high-titer recombinant retrovirus. HumGene Ther. 1996 8/1/1996;7(12):1405-13.

52. Morgenstern JP, Land $\mathrm{H}$. Advanced mammalian gene transfer: high titre retroviral vectors with multiple drug selection markers and a complementary helper-free packaging cell line. Nucleic Acids Res. 1990 6/25/1990;18(12):3587-96.

53. Brummelkamp TR, Bernards R, Agami R. A system for stable expression of short interfering RNAs in mammalian cells. Science. 2002;296(5567):550-3.

54. Narita M, Nunez S, Heard E, Lin AW, Hearn SA, Spector DL, et al. Rb-mediated heterochromatin formation and silencing of E2F target genes during cellular senescence. Cell. 2003 Jun 13;113(6):703-16.

55. Bracken AP, Dietrich N, Pasini D, Hansen KH, Helin K. Genome-wide mapping of Polycomb target genes unravels their roles in cell fate transitions. Genes Dev. 2006 May 1;20(9):1123-36.

56. Takihara Y, Tomotsune D, Shirai M, Katoh-Fukui Y, Nishii K, Motaleb MA, et al. Targeted disruption of the mouse homologue of the Drosophila polyhomeotic gene leads to altered anteroposterior patterning and neural crest defects. Development. 1997 Oct;124(19):3673-82. 
Figure S1

a

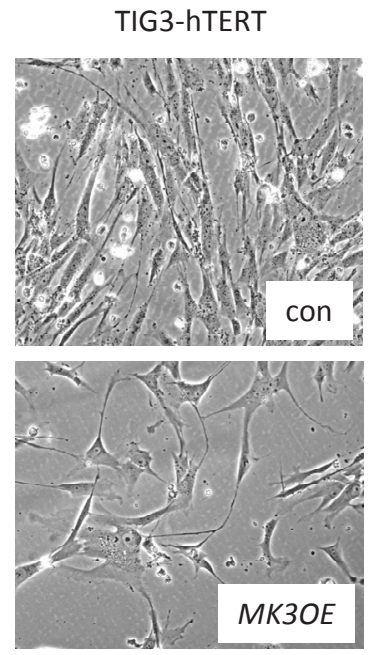

b

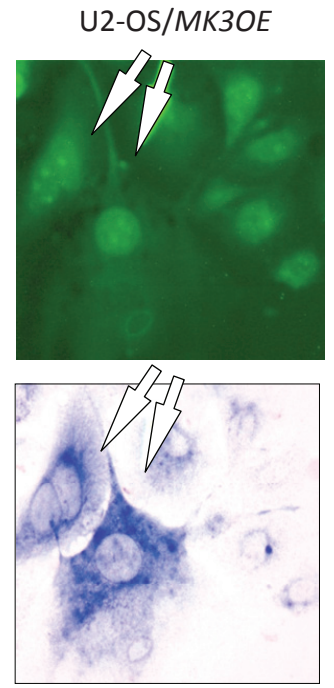

TIG3

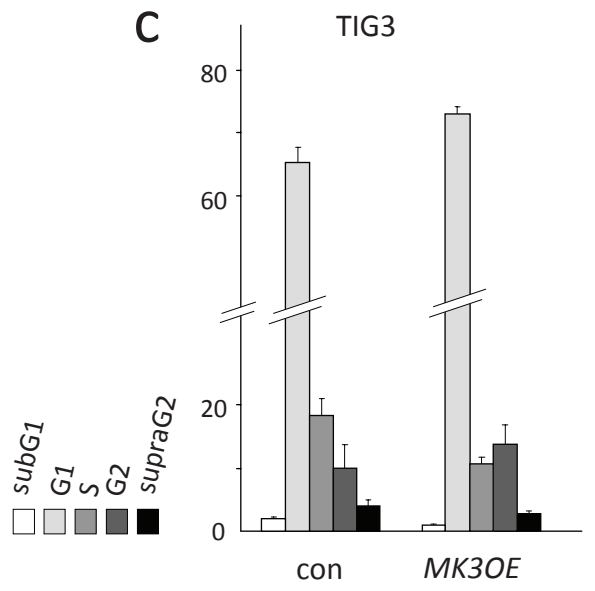

Figure S1 MK3-overexpression induces senescence in primary human fibroblasts and cancer cells. (a-c) Morphological changes of MK3-overexpressing (a) immortalized TIG3 cells (TIG3 ${ }^{\text {hTERT }}$ ) and $\mathrm{BJ}^{\text {hTERT }}$ fibroblasts (data not shown), (b) U2-OS/MK3.ires.GFPOE cells display senescent morphology; arrows demarcate flat cells positive for GFP (upper panel) and SA-bGal (lower panel). (c) Representative DNA profiling of TIG3/control and TIG3/MK3OE cells shows increased cell numbers in G1-phase and reduced S-phase (BrdU pulse-labeling and quantification of S-phase cells by FACS. 
Figure S2

a

\begin{tabular}{lll} 
phase & con & $M K 3 O E$ \\
\hline G0/G1 & $49.4 \%$ & $31.9 \%$ \\
S & $22.9 \%$ & $36.4 \%$ \\
G2/M & $27.7 \%$ & $31.7 \%$ \\
4n & $\pm 1.0 \%$ & $\pm 2.5 \%$
\end{tabular}

b

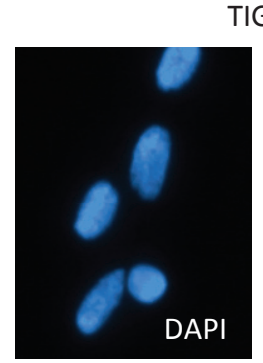

TIG3

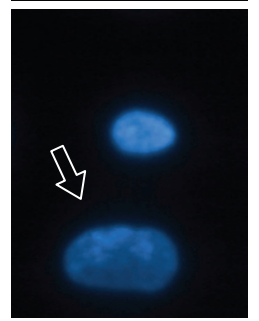

C
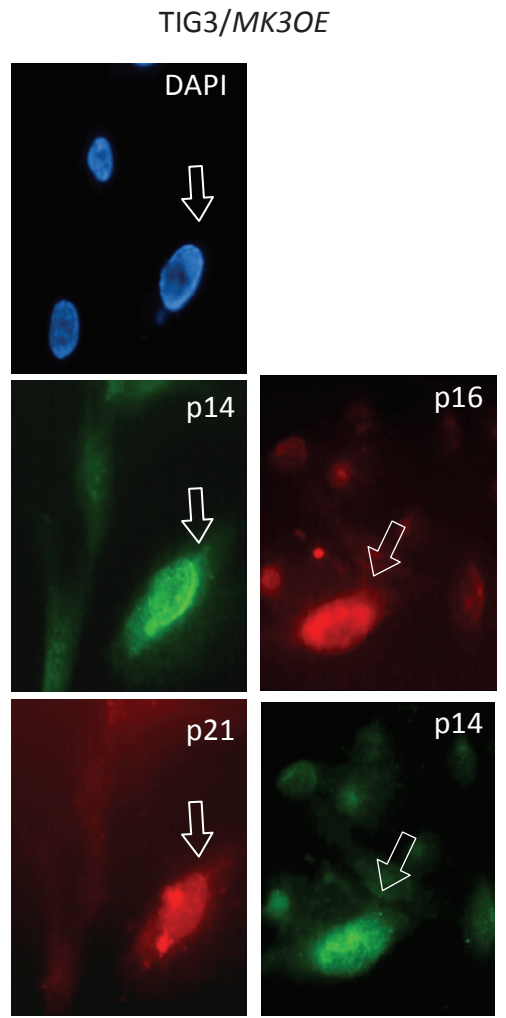

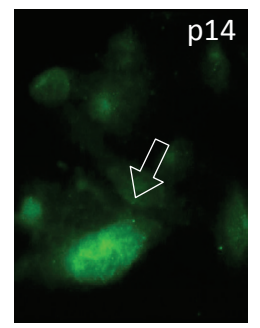

Figure S2 (above) MK3 evokes a TP53-dependent OIS. (a) MK3OE elicits initial intra S-phase arrest: representative DNA profile (BrdU-incorporation analysis) of U2-OS/MK3OE versus control cells (4-6 days post-transduction). (b) Increased expression of TP53 in primary human TIG3/MK3OE fibroblasts (MK3OE; lower panel) versus control (con; upper panel) cells. (c) Co-expression of p21 $1^{\text {CIP1/WAF1 }}$ and p14 ${ }^{\text {ARF }}$ (p21, p14; left panel, and $\mathrm{p} 16^{\mathrm{INK} 4 \mathrm{~A}}$ and $\mathrm{p} 14^{\mathrm{ARF}}$ ( $\mathrm{p} 16$, $\mathrm{p} 14$; right panel) in primary human TIG3/MK3OE fibroblasts. Successful transduction was confirmed by GFP-fluorescence prior to methanol fixation; all enlarged cells were GFP-positive; empty vector control TIG3 fibroblasts did not show the senescent phenotype.

Figure S3 (next page) Altered MK3-levels cause signalling imbalance. Low serum concentrations substantially increases flat cell formation induced by $M K 3 O E$. (a,b) Morphological changes in (a) low PDL (30-35) TIG3/MK3OE cultures and in (b) U2-OS cells under reduced serum conditions ( $2 \%$ and $1 \%$, respectively); empty vector control cells (con) (c) The effect of $M K 3 O E$ is dependent on the intensity of mitogenic stimulation: decreased serum concentrations reduce proliferation in U2-OS/MK3OE cells (squares: 10\% FCS; circles: 5\% FCS; triangles: $2 \%$ FCS; filled symbols: control; open symbols: MK3OE). Indicated (brackets) is percentage growth reduction relative to control cells at each serum concentrations. 
Figure S3

a

TIG3; 2\% FCS
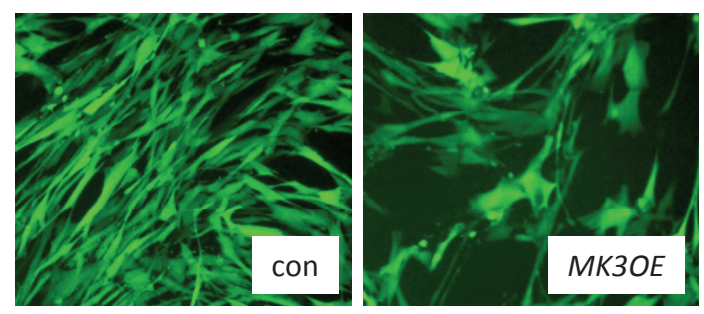

b

U2-OS; 1\% FCS
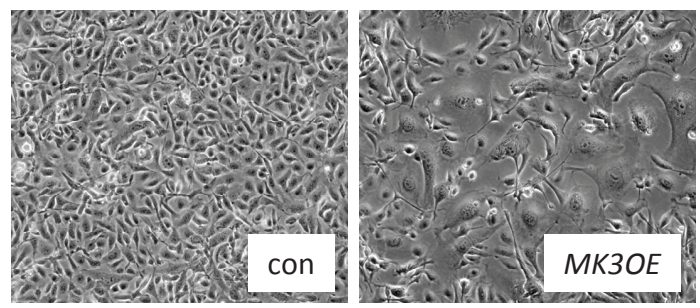

U2-OS

C

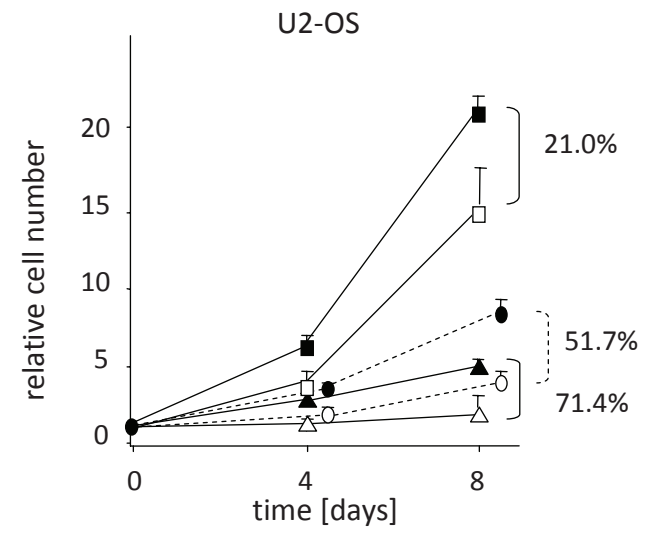

\begin{tabular}{lll|l}
10 & 5 & 2 & $\% \mathrm{FCS}$ \\
\hline $\mathbf{\square}$ & $\bullet$ & $\mathbf{\Delta}$ & $\mathrm{CON}$ \\
\hline & $\bigcirc$ & $\triangle$ & $M K 3 O E$
\end{tabular} 
Figure S4

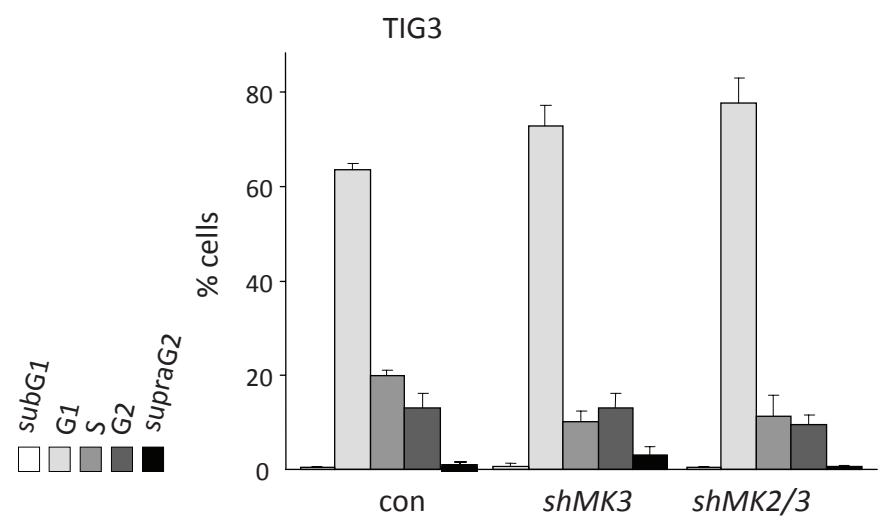

Figure S4 (above) Altered MK3-levels cause signalling imbalance. shRNA-mediated knockdown of MK3 (shMK3) induces a replication arrest in TIG3 cells; corresponding quantitative analysis of representative DNA profile of TIG3/shMK3 cultures versus control show increased G1 and reduced S-phase. shMK3 and shMK2/3 represent two independent shMK3-target sequences; shMK2/3 targets both MK2 and MK3 mRNA.

Figure S5 (next page) MK3 does not control UV-induced cell cycle arrest. MK3 is not required for G2/M arrest following UV-induced DNA damage. (a) Western blot analysis of cell lysates prepared from U2-OS cells transduced with shMK3 or control shGFP. (b,c) shGFP (con; b) or shMK3 (c) expressing U2-OS cells were exposed to $20 \mathrm{~J} / \mathrm{m}^{2} \mathrm{UV}$ (+UV) or sham treated (-UV) and then placed in $50 \mathrm{ng} / \mathrm{ml}$ nocodazole-containing media for an additional 16 hrs. Cells were analyzed by flow cytometry, for DNA content by PI staining and for mitotic entry by H3S1Oph. 
Figure S5

a

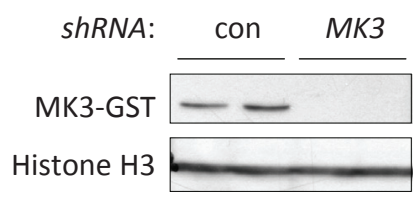

U2-OS

b
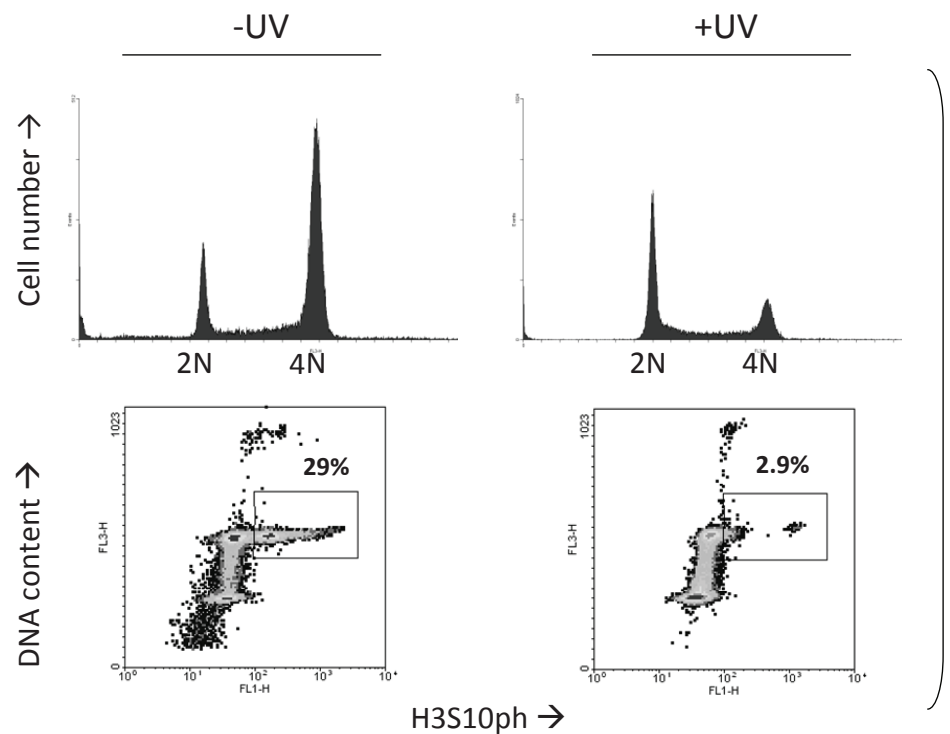

shcon

C
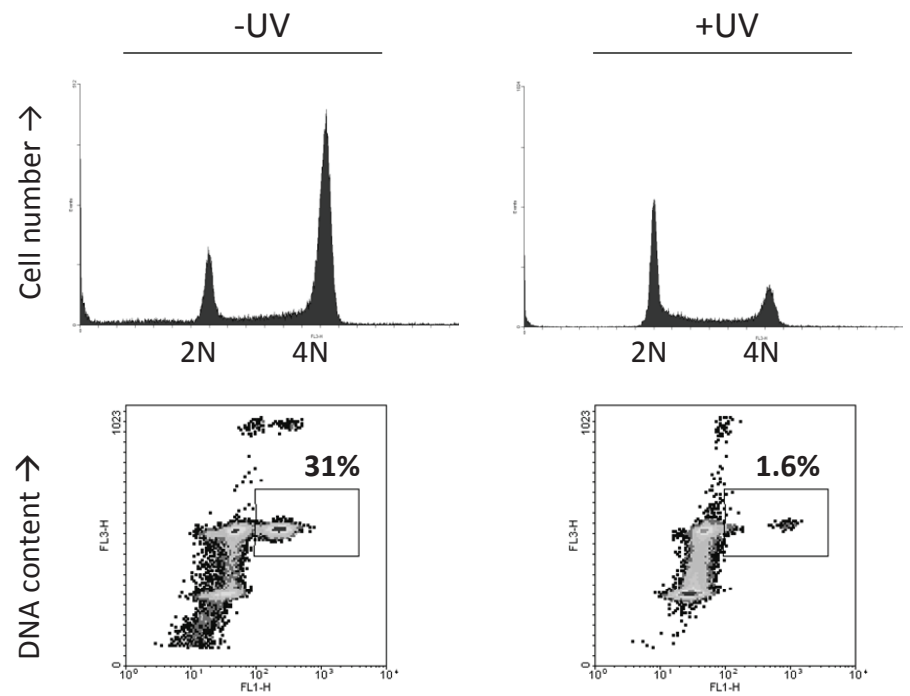

shMK3

H3S10ph $\rightarrow$

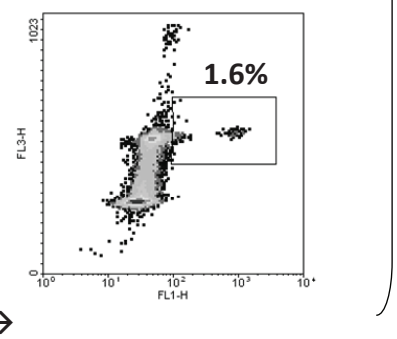


Figure S6

a

TIG3
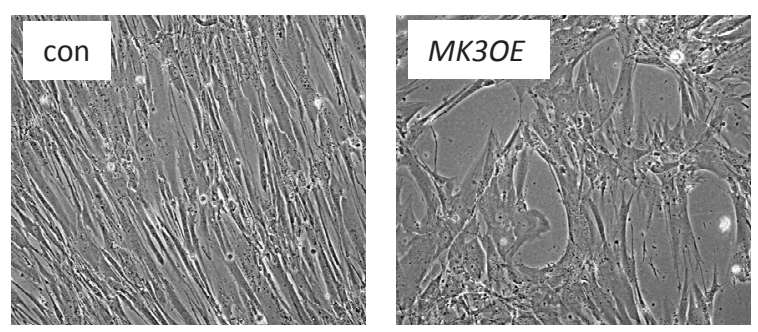

b
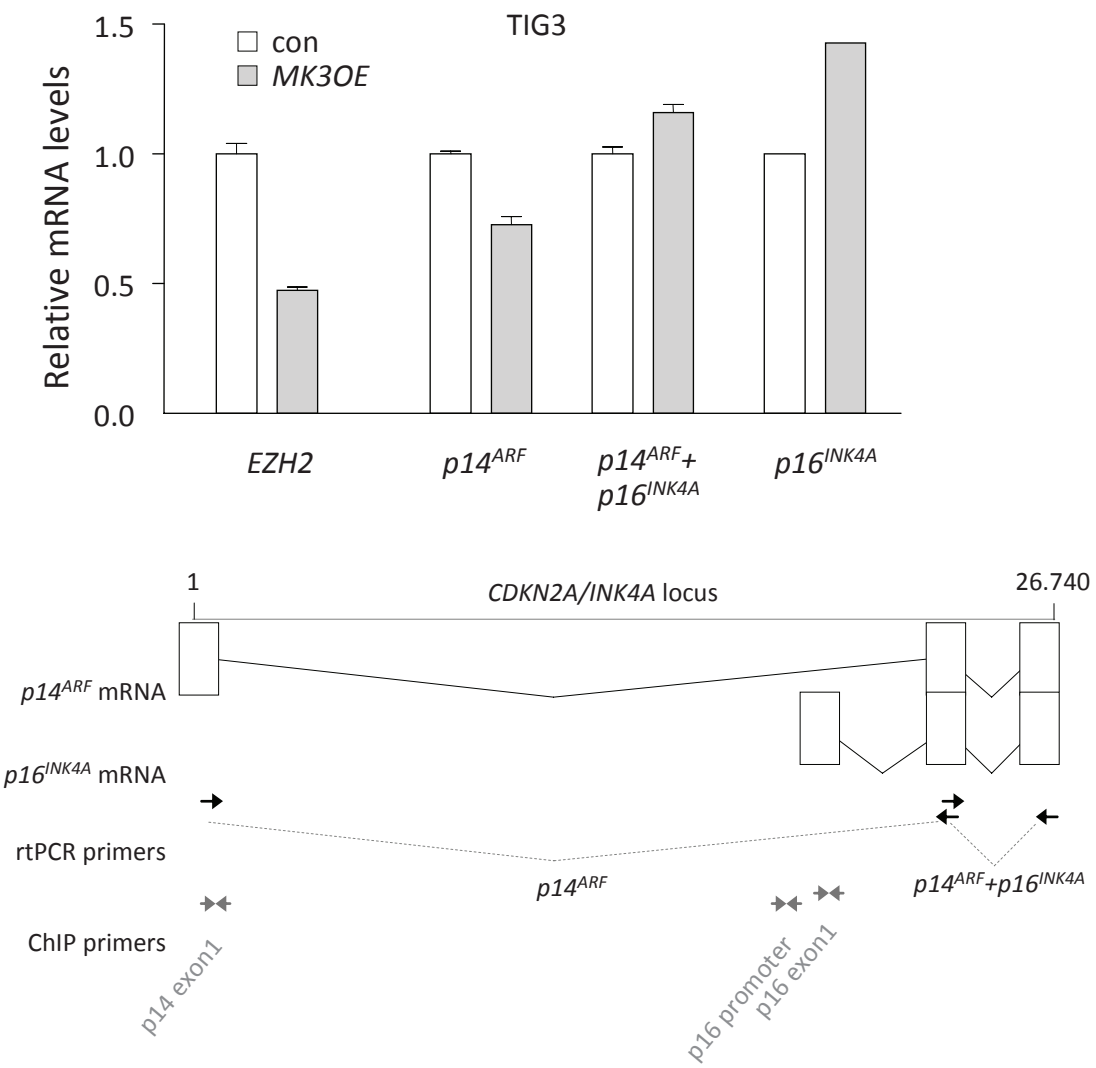

Figure S6 Functional interactions between MK3 and Polycomb Group proteins. (a) Morphology of cell cultures corresponding to experiments in Figure 4; phase contrast images reveal flat cell phenotype in TIG3/MK3OE cells. (b) real-time PCR analysis of EZH2 and INK4A/ARF mRNA levels in TIG3/MK3OE and control cells; bottom: schematic overview of human INK4A/ARF locus and primers used in this study. Due to overlap in mRNA sequences of $p 14^{A R F}$ and $p 16^{I N K 4 A}$, levels of $p 16^{I N K 4 A}$ mRNA were deduced by subtraction. 
$a$

TIG3
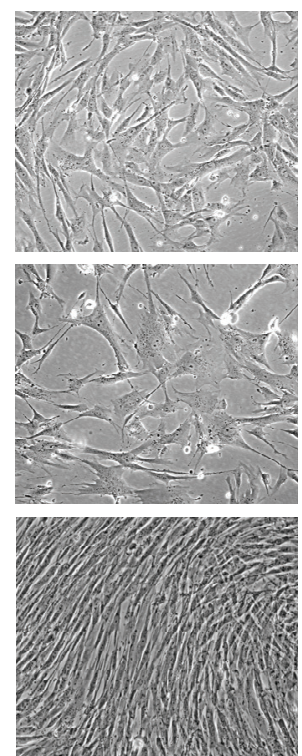

U2-OS

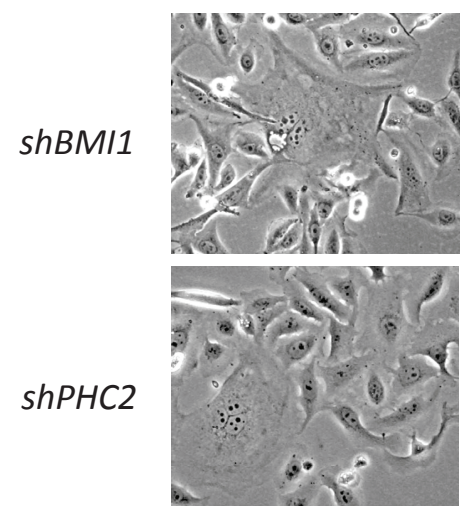

shcon

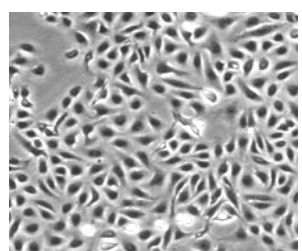

Figure S7

\section{b}

U2-OS

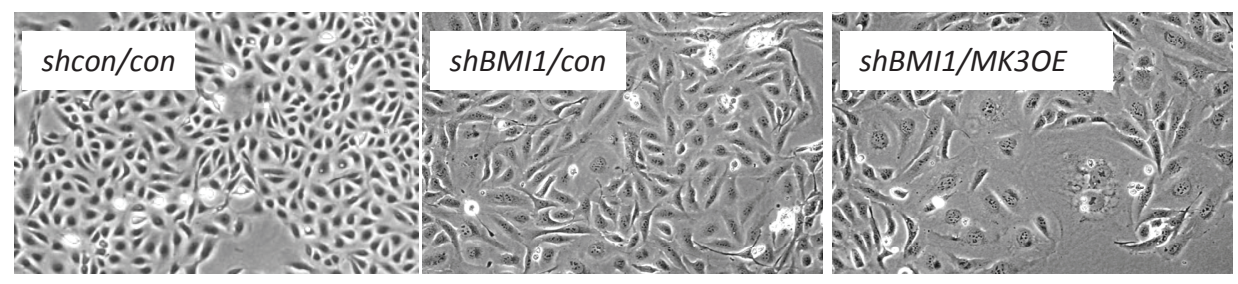

Figure S7 Functional interactions between MK3 and Polycomb Group proteins. (a) Induction of a senescent state in TIG3 (left panel) and U2-OS (right panel) cells via stable shRNA-mediated knockdown of BMI1 (upper panels) and PHC2 (middle panels) versus control cells (lower panels). Enlarged cells are positive for SA-bGal (not shown). (b) Simultaneous knockdown of BMI1 (shBMI1) and overexpression of $M K 3$ (MK3OE) increases senescent phenotype in U2-OS cells. 
Supplemental Table 1: Primers used for ChIP and mRNA expression analysis

$\begin{array}{ll}\text { ChIP primers } & \\ \text { Gene } & \\ \text { p14ARF exon1 } & \text { Forward } \\ & \text { Reverse } \\ \text { p16INK4A promoter } & \text { Forward } \\ \text { p16INK4A exon1 } & \text { Reverse } \\ & \text { Forward } \\ \text { p15 exon1 } & \text { Reverse } \\ \text { HOXA10 } & \text { Forward } \\ \text { HOXA11 } & \text { Reverse } \\ & \text { Forward } \\ & \text { Reverse } \\ & \text { Forward } \\ & \text { Reverse }\end{array}$

Primers quantitative expression analysis

Gene

$E Z H 2$

p14ARF

p14ARF+p16INK4A

cyclophillin A

shRNA target sequences

shMK3

shMK2/3

shBMI1

shPHC2

shTP53

$\begin{array}{ll}\text { Forward } & \text { GGGACAGTAAAAATGTGTCCTGC } \\ \text { Reverse } & \text { TGCCAGCAATAGATGCTTTTTG } \\ \text { Forward } & \text { CCCTCGTGCTGCTGATGCTACTG } \\ \text { Reverse } & \text { CCCATCATCATGACCTGGTCTT } \\ \text { Forward } & \text { GAAGGTCCCTCAGACATCCCC } \\ \text { Reverse } & \text { CCCTGTAGGACCTTCGGTGAC } \\ \text { Forward } & \text { TTCCTGCTTTCACAGAATTATTCC } \\ \text { Reverse } & \text { GCCACCAGTGCCATTATGG }\end{array}$

CGGCAAAGTGCTGGAGTGC

GGAGGAGATGACCAGTGCC

GAATGGTCCACTTCCATTG

GTTCAAGCGTCCAAGCGC

GACTCCAGTGGTAATCTAC 
Chapter 2 


\section{CHAPTER 3}

\section{MK3 controls Polycomb-target gene expression via negative feedback on ERK}

Peggy Prickaerts, Hanneke EC Niessen*, Emmanuèle Mouchel-Vielh*, Vivian EH Dahlmans, Claudia Geijselaers, Michiel Adriaens, Frank Spaapen, Yoshihiro Takihara, Ulf R Rapp,

Frédérique Peronnet, Jan Willem Voncken

* equal contribution

Status: submitted 


\section{ABSTRACT}

Background: Gene-environment interactions are mediated by epigenetic mechanisms. Polycomb Group proteins constitute part of an epigenetic cellular transcriptional memory system that is subject to dynamic modulation during differentiation. Molecular insight in processes that control dynamic chromatinassociation and dissociation of Polycomb repressive complexes during and beyond development is limited. We recently showed that MK3 interacts with Polycomb Repressive Complex-1 (PRC1). The functional relevance of this interaction, however, remained poorly understood. MK3 is activated downstream of mitogen- and stress activated protein kinases (M/SAPKs), all of which fulfill crucial roles during development. We here use activation of the immediate-early response gene ATF3, a bona fide PRC1-target gene, as a model to study how MK3 and its affector-kinases MAPK/ERK and SAPK/p38 are involved in regulation of PRC1-dependent ATF3 transcription.

Results: Our current data show that mitogenic signaling through ERK, p38 and MK3 regulates $A T F 3$-expression by $\mathrm{PRC1/chromatin-dissociation} \mathrm{and} \mathrm{epigenetic} \mathrm{modulation.}$ Mitogenic stimulation results in transient p38-dependent H3S28-phosphorylation and ERK-driven PRC1/chromatin-dissociation at PRC1-targets. H3S28-phosphorylation by itself appears not sufficient to induce PRC1/chromatin-dissociation, nor ATF3transcription, as inhibition of MEK/ERK-signaling blocks BMI1-dissociation and ATF3expression, despite induced H3S28-phosphorylation. In addition, we establish that concomitant loss of local H3K27me3 promoter-marking is not required for ATF3acivation. We identify pERK as a novel signaling-induced binding partner of PRC1, and provide evidence that MK3 controls ATF3-expression in cultured cells via negative regulatory feedback on M/SAPKs. Dramatically increased ectopic wing-vein formation in the absence of Drosophila MK in a Drosophila ERK gain-of-function wing vein patterning-model, supports the existence of MK-mediated negative feedback regulation on $\mathrm{PERK}$.

Conclusion: We here identify and characterize important actors in a PRC1dependent epigenetic signal/response-mechanism, some of which appear to be nonspecific global responses, whereas others provide modular specificity. Our findings provide novel insight into a Polycomb-mediated epigenetic mechanism that dynamically controls gene transcription and support a direct link between PRC1 and cellular responses to changes in the microenvironment. 


\section{INTRODUCTION}

Mitogen and stress-activated protein kinase (M/SAPK) signaling pathways relay environment-to-gene information and enable physiologically appropriate cellular responses [1]. MK3 is an interaction partner of ERK and p38 and is targeted by all three M/SAPK signaling cascades [2]; these phosphorylation cascades induce multiple responses among which altered gene transcription $[3,4]$.

We previously demonstrated that mitogen-activated protein kinase-activated protein kinase 3 (MK3/3pK/MAPKAPK3) binds Polycomb repressive complex 1 (PRC), via the SAM-domain (self-association motif) of PHC [5]. Polycomb Group and Trithorax Group proteins maintain transcriptionally repressed and activated epigenetic states respectively, and as such are part of an important cellular transcriptional memory system. Although PRC1-mediated transcriptional repression has long been considered a stable repressive state, increasing evidence indicates that PRC1-repression is a dynamic process [6, 7]. Genome-wide chromatin-association studies have revealed changes in Polycomb/chromatin-distribution and transcriptional re-programming during lineage commitment and differentiation [8-10]. As M/SAPKs and MKs also play important roles in differentiation, development and cell proliferation, the physical association of PRC1 and MK3 suggests a functionally relevant connection. We have previously established that PRC1/chromatin-dissociation correlates with their phosphorylation during cell cycle progression [11]. Subsequently, we showed that acute mitogenic and stresssignaling also results in PRC1/chromatin-dissociation [5]. The molecular mechanisms that underlie this dynamic relocation of PRC1 and the exact role of MK3 in signaling to chromatin remained unknown. We here hypothesized that M/SAPK-MK signaling imposes a molecular mechanism by which cells regulate $\mathrm{PRC1/chromatin-association}$ and PRC1-target gene expression in response to environmental cues. We used the previously identified PRC1-target gene ATF3 to study PRC1-mediated transcriptional regulation [12]. ATF3 is an immediate-early response gene (IEG) [13]; relevantly, ERK and MK have been implicated in IEG-activation [14]. Therefore mitogen-induced ATF3activation represents a suitable model to study the biological relevance of ERK/MK3/PRC1-signaling. Our current data establish that ATF3-expression is dynamically controlled by MAPK/MK/PRC1, and identifies important players in an epigenetic switch-module in response to changes in the cellular microenvironment. 


\section{RESULTS and DISCUSSION}

\section{M/SAPK signaling controls PRC1-target gene transcription.}

To determine how M/SAPKs are implicated in Polycomb-mediated repression, transcription of the PRC1-target gene ATF3 was measured in the presence or absence of kinase inhibitors. All cells were serum-starved (G0/G1)-arrest prior to stimulation throughout this study, to avoid interference by late S/G2-associated PRC1/chromatindissociation [11]. ERK, p38 and JNK are all phosphorylated in response to mitogenic stimulation (Fig S1A). Consistent with immediate-early response kinetics, ATF3 mRNA levels rapidly increase with a peak-expression at 60 minutes after stimulation (Fig $1 A$ ). P38-inhibition (p38i) and MEK/ERK inhibition (MEKi) both substantially and reproducibly reduce $A T F 3$-induction; the effect of ERK inhibition is consistently stronger than $\mathrm{p} 38 \mathrm{i}$ ( \pm 3-4 fold versus \pm 1.5 -2 fold, respectively); combining both inhibitors blocks ATF3-expression completely; JNK-inhibition (JNKi) slightly enhances ATF3 induction (Fig 1B). Various PRC1-proteins cluster to pericentromeric heterochromatin-associated Polycomb Group nuclear bodies (PcG-NB) in several cancer cell lines [11, 15, 16]. Using PCG-NB/chromatin-dissociation in U2-OS cells as a read-out for PRC1/chromatinbinding $[5,11]$, we find that PcG-NB/chromatin-dissociation correlates well with increased histone $\mathrm{H} 3$ is rapidly phosphorylated at Ser28 (H3S28ph) in mitogenstimulated control cells, in concordance with previous observations [5], and that combined MEKi/p38i blocks both events (Fig 1C). H3S28 is phosphorylated by numerous triggers and MAPKAPK-family kinases, including RSKs and MSKs are involved $[17,18]$. Of note, immediate-early response genes (IEG) are controlled by MKs and local H3S28-phosphorylation [14, 19]. In the context of mitogenic stimulation, we find that P38i alone blocks H3S28ph and chromatin-dissociation of the PRC1-protein BMI1, whereas BMI1/chromatin-binding is largely retained when ERK-signaling is interrupted (MEKi), without affecting H3S28ph (Fig S1B). These results support the notion that p38 targets $\mathrm{H} 3 \mathrm{~S} 28$ and that ERK-signaling may target PRC1/chromatin-association, and possibly PRC1-protein function and/or interaction.

Fig 1 | PRC1-target gene expression is controlled by M/SAPKs. (A,B) PRC1-target gene ATF3-expression (mRNA) in mitogen-stimulated (FCS/TPA) TIG3 cells, in the absence or presence of kinase inhibitors; data \pm SD. (C) H3S28ph and BMI1 staining in starved or mitogen-stimulated U2-OS cells; DAPI: nuclear counterstain. (D) Sub-cellular PRC1-protein distribution in starved and stimulated U2-OS cells; nuclear soluble and chromatin-bound fractions were proportionally loaded, cytoplasmic fractions 4-fold less; AKT, TUB, H3: fractioning and loading controls. (E) Interaction of pERK and BMI 1 in response to mitogens (stim) or selenite (stress) in U2-OS cells; +/-: transfected BMI1; (IB: immunoblot; IP: immunoprecipitation). 

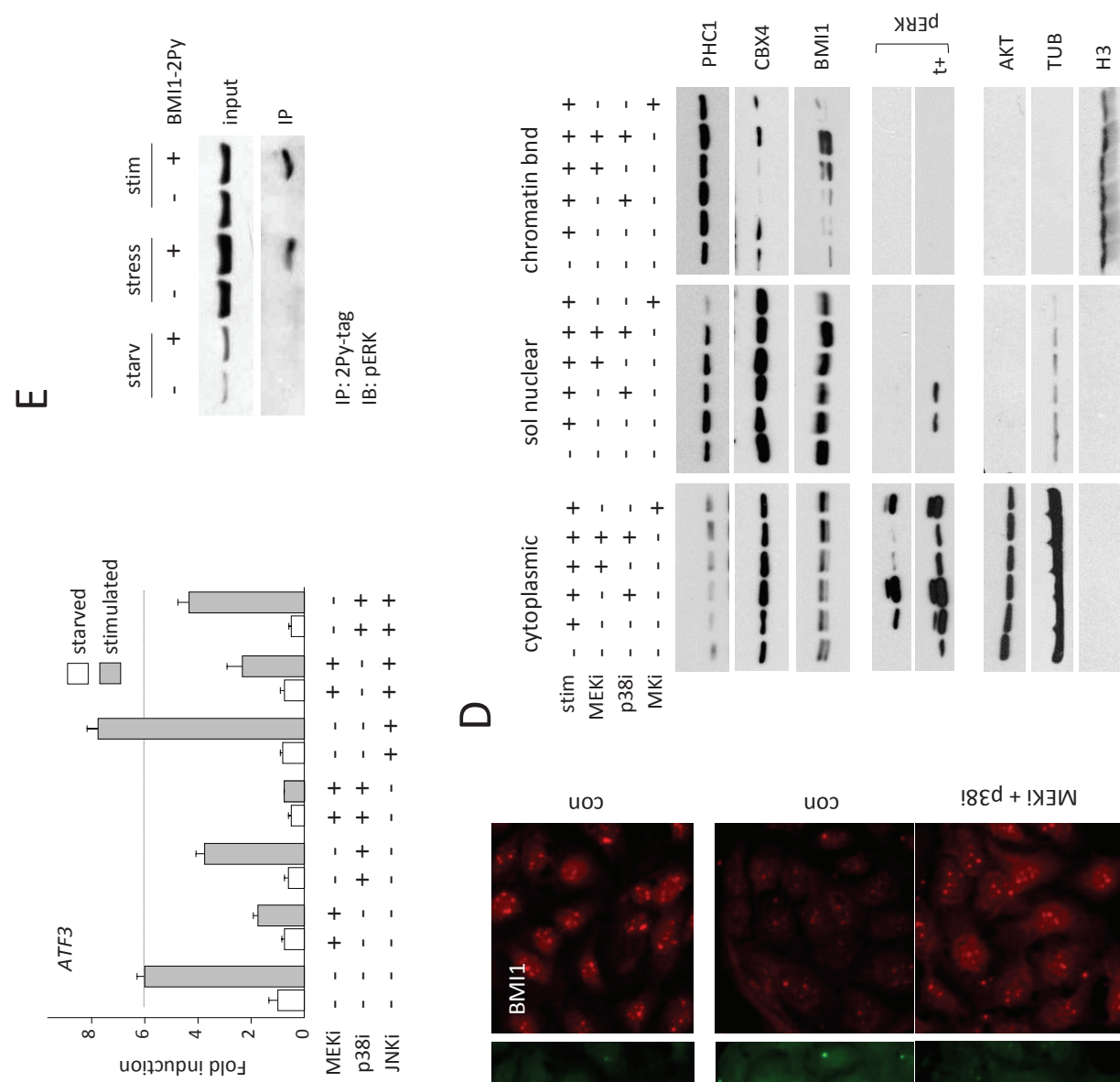

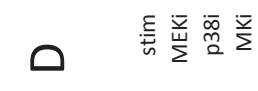
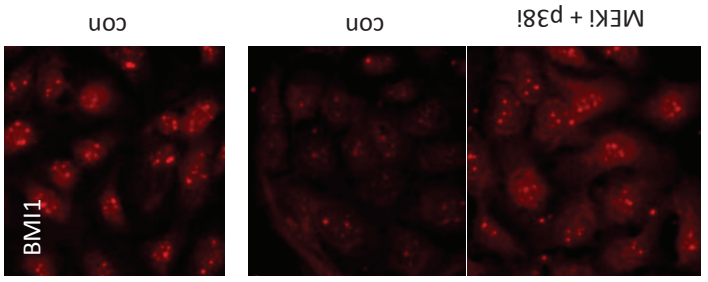

$\infty$

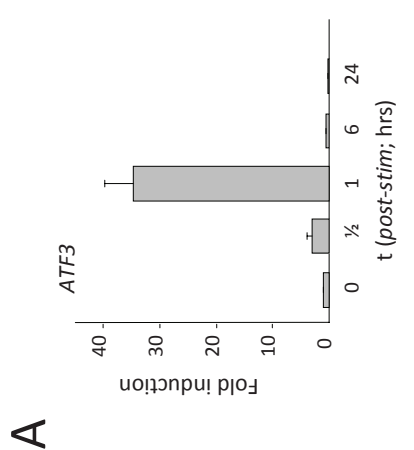

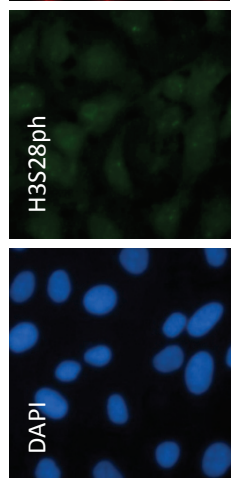

рамлетs
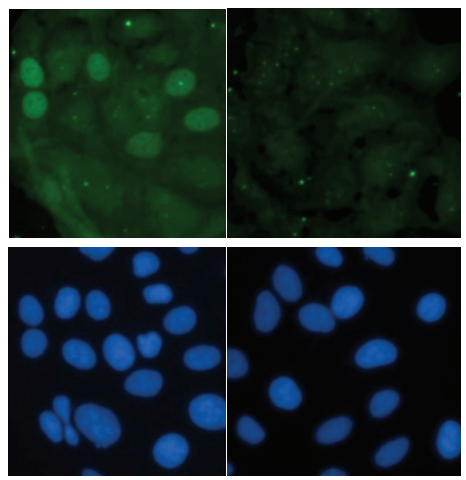

рәтејnu!ts

$\cup$ 
To examine the effect of mitogenic-stimulation on sub-nuclear distribution of PRC1proteins (in the presence or absence of kinase inhibitors), cells were differentially extracted to yield cytoplasmic, soluble nuclear and chromatin-bound fractions. We previously found that the PRC1-protein PHC1 undergoes a global chromatinredistribution in response to cell stress in contrast to some other PRC1-members [5]; as such determination of total (i.e. PcG-NB and global) PRC1-protein/chromatinassociation is pivotal. Of note: mitogenic stimulation differentially affects global PHC1/, CBX4/ (both increased) and BMI1/chromatin-association (decreased), suggesting differential regulation of PRC1 members by M/SAPKs. Consistent with this, single inhibitor-treatment ( $\mathrm{p} 38 \mathrm{i}$ or MEKi) clearly affects chromatin-association of these proteins in distinct ways (Fig 1D); the CBX4/chromatin-association analysis suggests a direct requirement for $\mathrm{CBX} 4$-phosphorylation in relation to chromatin-association. Combined inhibitor-treatment of stimulated cells shows that more BMI 1 remains chromatin-associated, consistent with the immunofluorescence (IF) analysis (Fig 1D, C); also CBX4 and PHC1/chromatin-binding are increased under p38i/MEKi-conditions. Besides differential phospho-regulation of PRC1-proteins by M/SAPKs, the above findings predicted an interaction between MAPK and PRC1, downstream of mitogenic signaling. Indeed, co-immunoprecipitation analyses confirm a signaling-induced interaction between pERK and PRC1-complexes (Fig 1E). The PRC1/pERK-interaction is counteracted by $\mathrm{p} 38$ signaling, as more pERK co-precipitates in a PRC1-directed IP in p38i cells (Fig S1C cf Fig 1C), in support of cross-talk between kinases at multiple levels. The combined data demonstrates that mitogenic signaling, via both activated p38 and ERK, converges at the level of PRC1/chromatin-regulation and target-gene activation, and establish a relevant functional interaction between these signal transducers and PRC1.

\section{Transcription requires PRC1 dissociation, not loss of H3K27me3.}

We next studied changes in PRC1 (CBX8, PHC1) chromatin-occupation and H3K27me3-marks at established PRC1-target genes [12]. CBX8 and PHC1 show distinct chromatin-occupation profiles: whereas $\mathrm{CBX} 8$ is exclusively enriched at PRC1-target loci and is released upon serum stimulation at most targets, PHC1 appears present also at non-target genes and shows opposing dissociation-dynamics at targets (decreased; ATF3; HOXA11) and non-targets (increased; $p 15$ exon1, CCNA2, p14 ${ }^{A R F}$ exon1) upon stimulation (Fig 2). The observed changes in PHC1-occupation are consistent with enhanced chromatin-association in response to mitogens ( $c f$. Fig 1D). We find a clear correlation between simultaneous chromatin-dissociation of CBX8 and PHC1, and activation of expression at the ATF3-locus (Fig 2). 
A

\section{Figure 2}
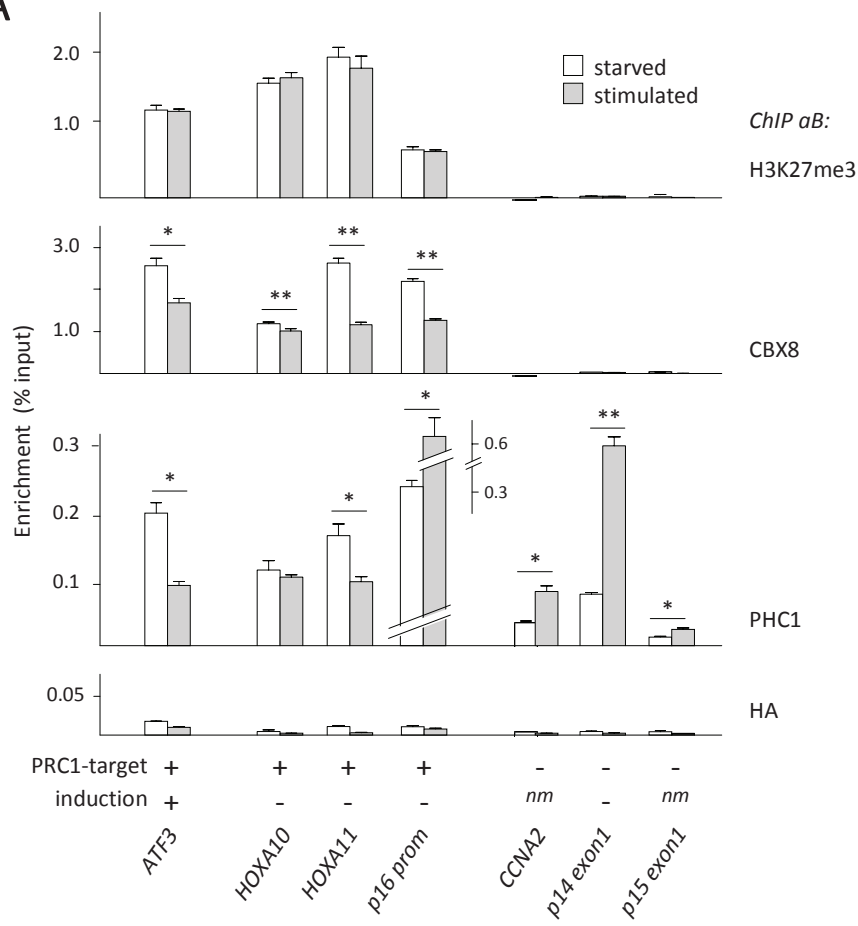

B

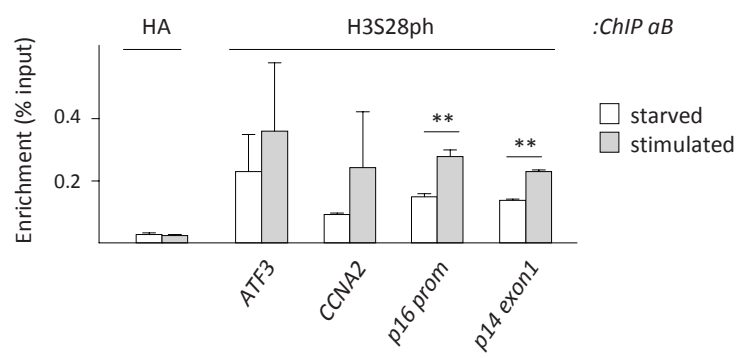

Fig 2 | PRC1/chromatin-dissociation, not loss of H3K27me3, correlates with transcription. (A,B) ChIP analysis of PRC1-protein binding, histone H3K27me3 (A) and H3S28ph-marking (B) at known PRC1-target and nontarget loci in G1-arrested (starved) and mitogen-stimulated TIG3 cells. ChIP-antisera are indicated in the figures (ChIP aB:). (Significance: ${ }^{*} \mathrm{p}<0.05,{ }^{* *} \mathrm{p}<0.01$; t-test).

The reduced $\mathrm{CBX} 8 /$ chromatin-asscociation is not caused by reduced cellular $\mathrm{CBX} 8$ protein, since this remains unaltered throughout the duration of the experiment (Fig S2B). In contrast to CBX8, PHC1 increases at all non-induced loci (Fig 2, S2A). The contrasting chromatin-binding dynamics of $\mathrm{CBX} 8$ and $\mathrm{PHC} 1$ in response to signaling are 
in good agreement with our previous observations that PHC1/chromatin-binding appears to increase globally in the context of cell cycle progression or cell stress, whereas detection of other PRC1-members in PcG-NB (like BMI1, CBX4 and RNF2) is reduced $[5,11]$. Of note, $\mathrm{PHC} / \mathrm{PRC} 1$-core complex-association was reported to be relatively weak [20]. In addition, purified PHC1 is known to reside in non-PRC complexes [21]. Combined, these results indicate that PHC1-occupation occurs without the need for simultaneous CBX8-binding, and further supports the notion of differential regulation of PRC1-complex members.

Consistent with a global mitogen-induced increase of H3S28ph, ChIP-analysis shows increased H3S28ph-enrichment at PRC1-targets as well as non-targets; this epigenetic change occurs independent of transcriptional induction (Fig 2B, S2A). Remarkably, the unaltered H3K27me3-occupation at the ATF3-promoter suggests that transcriptional activation of PRC1-target genes occurs without concomitant loss of H3K27me3 (Fig 2A). Thus, although PcG-NB/chromatin-dissociation and H3S28ph correlate well [5, 22, 23], H3S28ph by itself does not determine transcriptional status. In addition, our findings argue that local maintenance of $\mathrm{H} 3 \mathrm{~K} 27 \mathrm{me} 3$ does not obstruct transcriptional reactivation of PCR1-targets. The recently reported presence of H3K27me3-marks on active promoters supports this notion [24]. Whether or not the H3K27me3-mark needs to be removed at reactivated genes remains contradictory at this point [22-24], and may, besides on models and tools used, depend on the sub-genic location of epigenetic modifications (possibly in conjunction with other modifications). Consistent with this idea, repressive $\mathrm{H} 3 \mathrm{~K} 27$ me3-marking correlates best with transcription at loci that display 'blanketing'-type H3K27me3-enrichment, i.e. gene body-wide (down-stream of the transcription start site; TSS) [24]. It is conceivable that H3K27me3/promotermarking defines a specific class of response factors, which are dynamically controlled by rapid removal of $\mathrm{PRC1}$-complexes. Thus far, our H3S28ph analyses suggest that H3S28ph, like CBX8-dissocciation, is a global event; despite being essential for PRC1target gene activation, it is clearly not the sole determining factor and as such is unlikely to specify regulation only at PRC1-target genes. Co-occurrence of methylphosphoryl modifications on adjacent histone lysines and serines was proposed to act as a binary epigenetic switch-mechanism, by affecting chromobox-domain binding to histone-trimethyl marks [22, 25-27]; the simultaneous detection of local H3S10ph/HP1binding seemingly challenges the strictness of the methyl-phosphoryl switch concept [28]. Irrespective of the exact mechanism, our combined molecular epigenetic analyses suggest PRC1-target gene activation does not require loss of H3K27me3 and point to chromatin-dissociation of multiple PRC1-proteins as an important event in transcription initiation. 
产

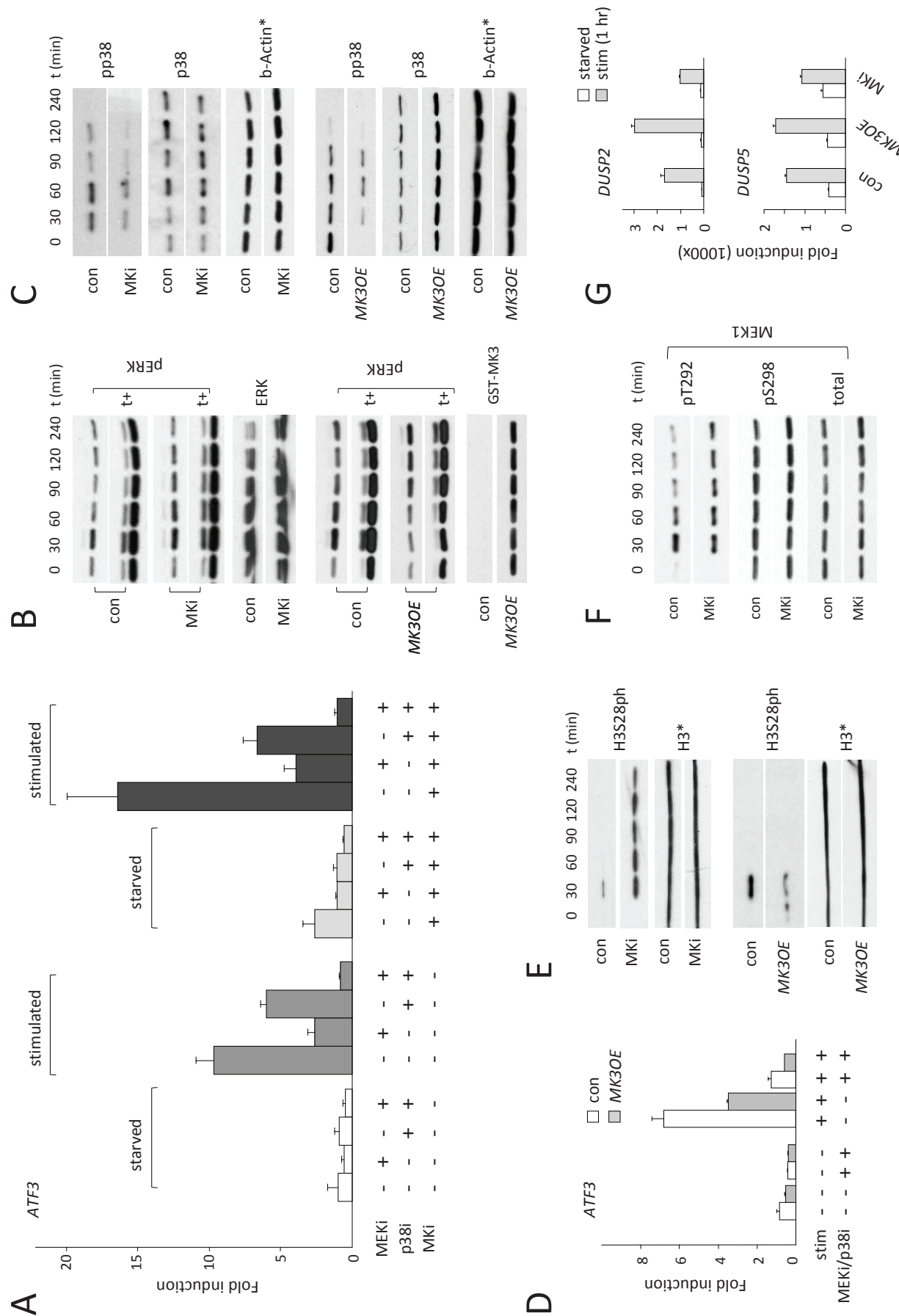


Fig 3 | MK3 is a negative regulator of ERK. (A, D) mRNA Expression of PRC1-target gene ATF3 as a function of kinase-inhibition (A) or MK3OE (D) in TIG3 cells. (B,C, E, F) pERK (B), pp38 (C), H3S28ph (E) and pMEK (F) levels in starved and stimulated U2-OS cells in relation to MK-function; $\mathrm{t}(\mathrm{min})$ : time post-stimulation in minutes; ${ }^{*}$ : loading controls for corresponding panels; $\mathrm{t}+$ : longer exposure. (G) Mitogen-induced DUSP2 and DUSP5mRNA in control, MK3OE and MKi TIG3 cells.

\section{MK3 negatively regulates ERK signaling.}

To determine the role of MK in PRC1-target gene regulation, we next studied the effects of MK-inhibition or MK protein level modulation on ATF3 gene expression in TIG3 and U2-OS cells. MK-inhibition (MKi) releases basal repression of ATF3 in starved MKi cells and allows for more robust ATF3 mRNA induction; in contrast, treatment with MEKi or P38i, both suppress transcription (Fig 3A; Fig S3A). Of note: MKi does not show an added effect on ATF3 mRNA synthesis in combination with other single or multiple kinase inhibitors, suggesting that MKs exert their effect upstream of ERK and p38signaling (Fig 3A). Thus, our data suggests a negative regulatory role for MKs in mitogenic signaling through MEK/ERK. In good agreement with this data, $M K$-deficient cells (by MKi or shRNA-mediated knockdown, shMK) display sustained ERK1/2:T202/T204-phosphorylation (pERK; up to 90 to 120 minutes, compared to control cells) upon stimulation (Fig 3B; Fig S3B). Conversely, MK3-overexpression ( $M K 3 O E)$ reduces both basal and induced ATF3 transcription, and, in combination with MEKi/p38i, further represses expression (Fig 3B). As predicted, MK3OE delays ERKphosphorylation, which is consistent with an inhibitory action of MK3 on ERK activity and reduced target gene induction (Fig 3D, B). MK2 was recently found to stabilize p38 in a kinase domain-independent manner, i.e. physical association of MK2 to p38 controls p38-stability [29]. Analogous with this report, we detect a rise in p38 levels in MK3OE cells, and reduced p38 levels in shMK cells; relevantly, p38 levels remain unaltered in MKi cells (Fig 3C; Fig S3C). Contrary to pERK, p38:T180/T182phosphorylation (pp38) is reduced in MK3OE cells as well as in shMK and MKi cells. Also JNK:T183/T185-phosphorylation (pJNK) is reduced by MKi, whereas MK3OE did not pJNK levels (Fig S3D; data not shown). Although the full extent of factors and mechanisms controlling M/SAPK-phosphorylation is not clear at this point, these findings are in keeping with multiple complex regulatory cross-interactions between MKs and M/SAPKs [3, 30].

Consistent with our IF-analyses ( $c f$ Fig 1, S1), H3S28 shows rapid and transit phosphorylation kinetics in response to mitogens (Fig 3E). Relevantly, global H3S28ph is increased and sustained in MKi cells, whereas it is blunted in MK3OE cells (Fig 3E), in line with altered PRC1-target gene expression under these respective conditions ( $c f$ Fig 1B). This data demonstrates that H3S28-phosphorylation kinetics respond to 
MK3-activity, and support a regulatory role for MK3 in epigenetic modulation of cell responses to environmental stimuli.

To gain further insight into the molecular mechanisms by which MK3 controls M/SAPK-activity, we studied MEK, an ERK affector-kinase. MEKpT292 and MEKpS298 were examined, as these two phospho-residues are part of a mechanism that controls MEK activity [31]. Like pERK, also MEKpT292 is sustained in MK-deficient cells, suggesting that MK3 controls phosphatase-activity directed toward upstream kinases (Fig 3F).

A

B

Figure 4

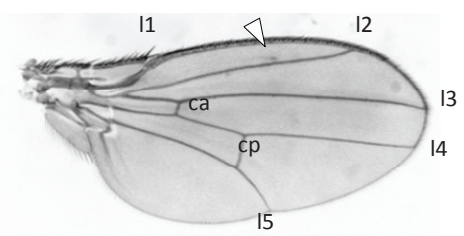

a) $s d:: G a l 4 /+$

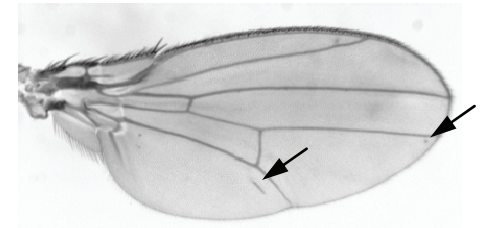

b) $s d:: G a l 4>U A S:: D-p 38 b$

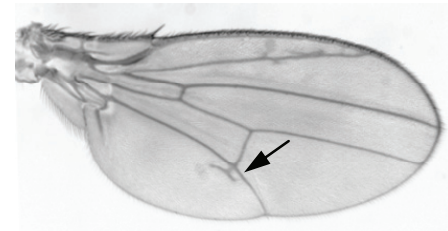

c) $s d:: G a l 4>$ UAS::rolled, v3171

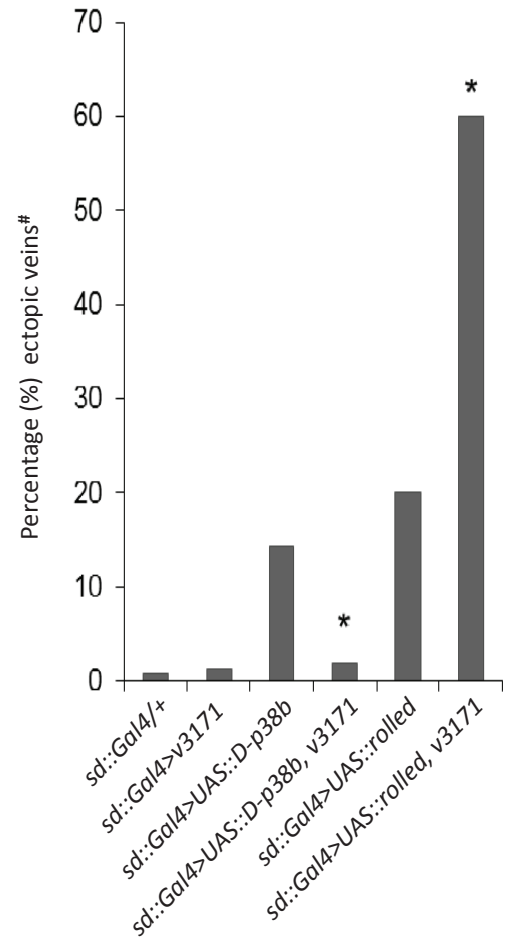

Fig 4 | Negative feedback by $d M K 2$ on $d E R K /$ rolled in ectopic wing-vein development (A, B) Representative ectopic vein patterns (black arrows) in control (a) and transgenic lines (b,c); percentages flies with ectopic wing veins in different genetic contexts (indicated below graph; B); \#: sd::Gal4 driver-induced ectopic veins nearby vein 2 ( 12 ; white arrow head cf $A)$, were not scored; *: significantly different from corresponding genotype without $d M K 2-L O F(z-$ test; $\mathrm{p}<0.001)$. 
Dual specificity phosphatases (DUSPs) play important roles in feedback-loops on phosphorylation cascades [32]. DUSP2 and DUSP5-induction meet the criteria of being dependent on mitogenic signaling and on $\mathrm{MK}$, as $M K 3 O E$ and $\mathrm{MKi}$, enhance and decrease DUSP mRNA induction, respectively (Fig 3G). Combined, the above findings support a negative regulatory role for MKs in MEK/ERK signaling, at least in part, via induction and/or activation of phosphatases.

To obtain independent proof for the functional relationships between MKs and M/SAPKs, we examined kinase interactions in Drosophila melanogaster. We first reproduced a signaling-induced interaction between $\mathrm{PH}$ ( $\mathrm{PHC} 1 / 2$ ortholog) and dMK2 (the only $M K$ ortholog in D.mel) in cultured insect cells (Fig S4A). We next exploited Drosophila wing vein patterning to probe for M/SAPK-MK interaction in vivo. Wing vein formation in the fruitfly is dependent on MAPKs [33-35]. As expected, gain-of-function (GOF) flies for dERK/rolled (sd::Gal4>UAS::rolled; D.mel ERK ortholog) induce ectopic wing veins during wing imaginal disc development (Fig 4A, B). We here report that flies overexpressing D-p38b (sd::Gal4>UAS::D-p38b; one of the D.mel p38 orthologs) also display enhanced ectopic vein formation (Fig 4A, B). Hence the ectopic vein phenotype can be used to gauge the effects of MK loss-of-function (LOF) on ERK/p38 pathway activity. To determine the effect of dMK2 on dERK and D-p38b, we crossed a transgenic RNAi-mediated $d M K 2-k n o c k d o w n$ line (v3171) to $d E R K$ - and D-p38b-GOF lines (see: Materials and Methods section; Fig S4B). dMK2-LOF enhances the $d E R K$-GOF ectopic vein phenotype (Fig 4A, B). In contrast, $d M K 2$-LOF suppresses the ectopic vein phenotype induced by $D-p 38 b$-GOF (Fig 4A, B). Importantly, this data is fully congruent with our in vitro findings in mammalian cells and supports the existence of a complex genetic network between MAPKs, SAPKs and MKs. In conclusion, our in vivo experiments substantiate a negative regulatory role of $d M K 2$ on $d E R K$-signaling in wing vein development.

\section{CONCLUSIONS}

In summary, we here identify and carefully characterize a number of actors in PRC1-dependent gene regulation. Signaling through p38 has direct implications for local chromatin-modulation (i.e. H3S28-phosphorylation). We propose that ERK/PRC1/MK3 acts as a molecular dimmer-switch that enables rapid and reversible transcriptional activation of ATF3. This mechanism defines negative-feedback on Polycomb-mediated repression as an integral process in the regulation of appropriate cellular responses to environmental changes (Fig 5). 
Figure 5

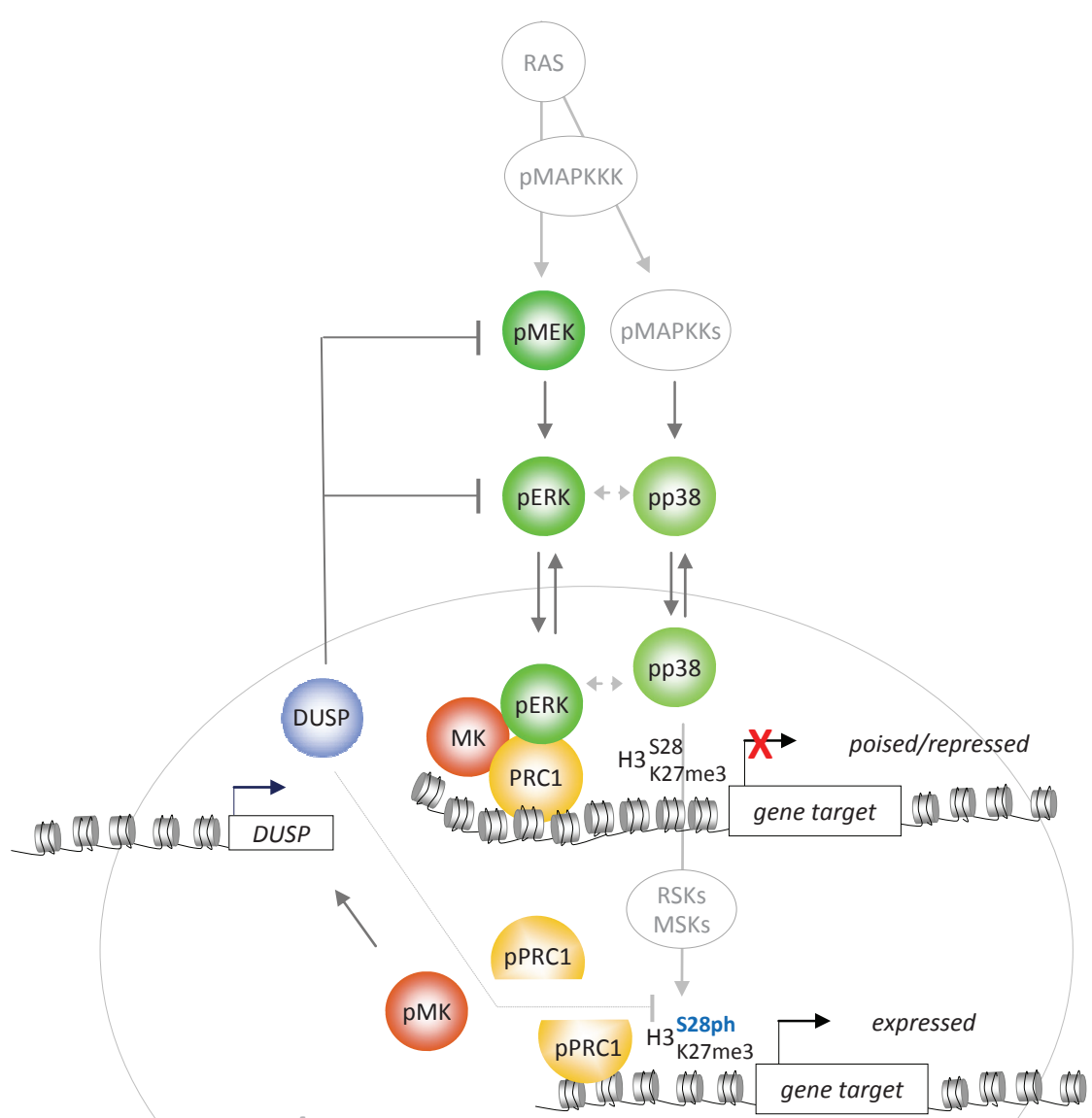

nucleus

Fig 5 | Model for MAPK/MK-regulation of PRC1-target genes. The PRC1/MK/ERK module represents a molecular switch mechanism: fine-tuning of converging p38 and ERK-signals at the chromatin level triggers a potential methyl-phosphoryl switching module and a signaling-dependent disruption of the PRC1/chromatinassociation which permits gene activation. Transient H3S28ph, but not removal of H3K27me3, accompanies gene expression. Concomitant MK-activation initiates DUSP-dependent negative regulatory feed-back on MEK/ERK (and possibly also on H3S28ph; dashed lines reflect potential functional relationships). 
Whereas part of the responses appears global, like H3S28-phosphorylation and CBXdissociation, the presence of PRC1/MK3 likely contributes to chromatin-targeting of PERK and provides specific local regulation. PRC-complex members are emerging as proteins harboring many different functions and binding partners [7]. We recently categorized \pm 120 known phosphorylation-sites on a limited number of PRC-proteins; many of these sites are predicted M/SAPK target-sites and are likely to cooperate in gene-regulation [36-39]. Although some phospho-sites have been linked to PRC-protein function [40-45], the biological relevance of the vast majority of these phospho-events is currently unknown. Our findings pave the way for detailed mutational analysis of the functional consequences of post-translational modification of Polycomb-proteins. The findings presented herein provide novel insight into differential regulation of PRC1members in response to signaling and add to the growing recognition that PRC1mediated repression is a highly dynamic process.

\section{MATERIALS AND METHODS}

Cell Culture, viral infections Human U2-OS osteosarcoma cells and TIG3 primary human fibroblasts expressing the murine ecotropic receptor were kindly provided by Dr. D. Shvarts (Utrecht Medical Center, Utrecht, The Netherlands) and Dr. D. Peeper (Netherlands Cancer Institute (NKI), Amsterdam, The Netherlands), respectively. Expression vectors encoding the murine ecotropic receptor were a courtesy of R. Bernards (NKI, Amsterdam, The Netherlands). All human cell lines were cultured under standard conditions in DMEM/10\% fetal calf serum (FCS). Retroviral expression vectors were used to maximize percentages of expressing cells and to minimize integration effects. Production of infectious viral particles was carried out as described previously [46]. Briefly, ecotropic retroviral supernatants were produced by transfection of producer cells using calcium-phosphate co-precipitation. 40-48 hours post-transfection, the supernatants were harvested, filtered and stored at $-80^{\circ} \mathrm{C}$ until further use. Viral titers were sufficiently high to achieve near $100 \%$ infection. Cells were transduced with retrovirus in the presence of $4 \mu \mathrm{g} / \mathrm{ml}$ polybrene (Sigma-Aldrich, St. Louis, MO, USA) at around $25 \%$ confluency for $6-8$ hours and then allowed to recover for 48 hours on fresh medium before selection pressure was applied. Transduced cells were grown for \pm 1 week on $1-4 \mu \mathrm{g} / \mathrm{ml}$ puromycin (Sigma) preceding experiments. Expression of all plasmid constructs was verified by immunoblotting.

Cells were serum-starved at $0.1 \%$ FCS for $48 \mathrm{hrs}$. Mitogenic stimulation was achieved by supplementing $15 \% \mathrm{FCS} / 100 \mathrm{ng} / \mathrm{ml}$ tetradecanoyl phorbol acetate (TPA; Sigma) for 45 minutes or as indicated. Cells were pretreated with kinase inhibitors: 30 
min $10 \mu \mathrm{M}$ MEK inhibitor (U0126; Promega, Madison, WI, USA), 30 min $10 \mu \mathrm{M}$ MK inhibitor (MK2a Inhibitor; Calbiochem/Merck, Darmstadt, Germany), 30 min 20 MM p38 inhibitor (SB202190; Calbiochem), 30 min 25 MM JNK inhibitor (SP600125; Biomol, Plymouth Meeting, PA, USA), 30 min $85 \mu \mathrm{M}$ Etoposide (ETP; Calbiochem). MK2a was confirmed to inhibit MK3 (not shown). Control cells were incubated with solvent. Sodium selenite (Sigma) treated cells ( $90 \mathrm{~min} 0.2 \mathrm{mM}$ ) were used as a positive control. Drosophila S2 cells were cultured at $25^{\circ} \mathrm{C}$ in Schneider medium with $10 \%$ FCS. The day before transfection, $5.10^{6}$ cells were transferred in medium with $0.1 \%$ FCS. For

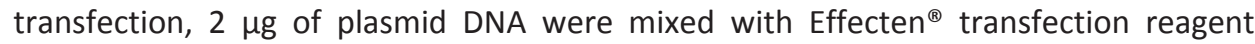
(Qiagen; Hilden, Germany) according to manufacturer's instructions (1/10 DNAEffecten ${ }^{\circledR}$ ratio). $48 \mathrm{~h}$ later, cells were either untreated, stimulated with $10 \%$ FCS/TPA $(100 \mathrm{ng} / \mathrm{ml})$ or sodium-meta-arsenite $(0.5 \mathrm{mM})$ for $2 \mathrm{hrs}$ at $25^{\circ} \mathrm{C}$. For Co-immunoprecipitation (IP)-experiments, plasmids Act::Myc-PH and Act::EGFP-MAPKAP were cotransfected into S2 Drosophila cells grown in 0.1\% FCS. IPs were performed as described (Mouchel-Vielh et al., 2011); IP antibodies are listed in Supplementary Table S2.

Expression constructs Retroviral vectors (pBMN-LZRS.ires.GFP, pBMNLZRS.ires.NEO) expressing murine BMI1-2Py or GST-MK3 have been described elsewhere [5, 11]. Retroviral systems were used as published [47, 48]. RNA interfering MK3 sequences were cloned into stable shRNA vectors [49]; targeting sequences: Supplementary Table S1). The $d M K 2$ cDNA was amplified from clone SD05481 (Drosophila Genomics Resource Center; for oligonucleotides: see Supplementary Table S1). The resulting amplicon was introduced into pENTR/D-TOPO ${ }^{\circledR}$ (Invitrogen, Carlsbad, CA, USA), then transferred through LR-recombination into T. Murphy's vectors pAWG (https://dgrc.cgb.indiana.edu/vectors). Similarly, a cDNA corresponding to the C-terminal part of Drosophila Polyhomeotic (PH) was amplified introduced in pENTR/D-TOPO ${ }^{\circledR}$, and subsequently transferred in PAMW (for oligonucleotides: see Supplementary Table S1).

Chromatin immunoprecipitation (ChIP) assays ChIPs on primary human fibroblasts were performed and analyzed essentially as described [12]. Cells were fixed for 10 minutes in $1 \%$ formaldehyde/phosphate buffered saline (PBS) and stopped by 5 minutes incubation in glycine (final concentration: $0.125 \mathrm{M}$ ). Fixed cells were washed twice with PBS and harvested in SDS buffer $(50 \mathrm{mM}$ Tris, $\mathrm{pH} 8.1 ; 0.5 \%$ SDS, $100 \mathrm{mM}$ $\mathrm{NaCl}$, 5mM EDTA), supplemented with protease inhibitors (Aprotinin, Antipain and Leupeptin all at $5 \mu \mathrm{g} / \mathrm{ml}$ and $1 \mathrm{mM}$ PMSF). Cells were pelleted by centrifugation, and suspended in IP buffer (100 mM Tris at $\mathrm{pH} 8.6,100 \mathrm{mM} \mathrm{NaCl}, 0.3 \%$ SDS, $1.7 \%$ Triton X-100, and 5mM EDTA), containing protease inhibitors. Cells were disrupted by 
sonication, yielding genomic DNA fragments with a bulk size of 200-500bp. For each IP, $1 \mathrm{ml}$ of lysate was pre-cleared by adding of $35 \mu \mathrm{l}$ of blocked protein A beads (Protein ASepharose/CL-4B, GE Healthcare, Piscataway, NJ, USA); $0.5 \mathrm{mg} / \mathrm{ml}$ fatty acid-free BSA, Sigma; and $0.2 \mathrm{mg} / \mathrm{ml}$ herring sperm DNA in TE), followed by centrifugation. $10 \mu \mathrm{l}$ aliquots of pre-cleared suspension were put aside as input DNA and kept at $4^{\circ} \mathrm{C}$. Samples were IP'ed overnight at $4^{\circ} \mathrm{C}$. HA antiserum was used as negative control. Immune complexes were recovered by adding $40 \mu \mathrm{l}$ of blocked protein $\mathrm{A}$ or $\mathrm{G}$ beads (GE Healthcare) and incubated for 4 hours at $4^{\circ} \mathrm{C}$. Beads were washed three times in 1 $\mathrm{ml}$ of Mixed Micelle Buffer (20 mM Tris at pH 8.1, $150 \mathrm{mM} \mathrm{NaCl} 5 \mathrm{mM}$ EDTA, 5\% w/v sucrose, $1 \%$ Triton X-100, and 0.2\% SDS), twice in $1 \mathrm{ml}$ of Buffer 500 (50 mM HEPES at $\mathrm{pH} 7.5,0.1 \% \mathrm{w} / \mathrm{v}$ Sodium deoxycholate, $1 \%$ Triton X-100, and $1 \mathrm{mM}$ EDTA), twice in $1 \mathrm{ml}$ of LiCl Detergent Wash Buffer (10 mM Tris, pH 8.0; 0.5\% Sodium Deoxycholate, 0.5\% NP-40, $250 \mathrm{mM} \mathrm{LiCl}$, and $1 \mathrm{mM}$ EDTA), and once in $1 \mathrm{ml} \mathrm{TE}$. Immune complexes were eluted from beads in $250 \mu$ l elution buffer (1\% SDS; and 0.1M NaHCO3) for 2 hours at $65^{\circ} \mathrm{C}$ (continuous shaking at $1000 \mathrm{rpm}$ ), and after centrifugation, supernatants were collected. $250 \mu \mathrm{l}$ elution buffer was added to input DNA samples and these were processed in parallel with eluted samples. Crosslinks were reversed overnight at $65^{\circ} \mathrm{C}$, followed by a 2 hours digestion with RNase $A$ at $37^{\circ} \mathrm{C}$ and 2 hours proteinase $\mathrm{K}(0.2$ $\mu \mathrm{g} / \mu \mathrm{l})$ at $55^{\circ} \mathrm{C}$. DNA fragments were recovered using QIAquick PCR purification columns, according to manufacturers' instructions (Qiagen, Hilden, Germany). Samples were eluted in $75 \mu \mathrm{l} \mathrm{EB}$ buffer and then diluted 1:5 in TE buffer. IP'ed DNA was quantified by real-time PCR (for ChIP antisera, primers: see Supplementary Tables S1, S2). Each experiment was performed in triplicate. Results of one representative experiment are shown.

RNA isolation, cDNA synthesis, real-time ( $r$ t)PCR analysis For rtPCR analysis, total RNA was isolated using Tri-Reagent (Sigma) according to the manufacturers' protocol. Quantity and quality of the RNA were determined by 260/280 nm and 260/230nm absorbance measurements, respectively, using the Nanodrop (Witec AG, Luzern, Switzerland). Total RNA (1 $\mu \mathrm{g})$ for each sample/replicate was converted into first strand cDNA using the iScript ${ }^{\mathrm{TM}}$ cDNA synthesis kit (Bio-Rad, Herculus, CA, USA) according to the manufacturers' instructions. Gene expression was determined by rtPCR using the MyiQ $^{\text {TM }}$ Thermal Cycler (Bio-Rad) in combination with the IQ5 v2 software (Bio-Rad). rtPCR was performed on $25 \mathrm{ng}$ of $\mathrm{cDNA}$ using the $\mathrm{qPCR}^{\mathrm{iQ}}{ }^{\mathrm{TM}}$ Custom SYBR ${ }^{\circledR}$ Green Supermix with fluorescein (Bio-Rad) and 300nM primer in 96 well plates (Bio-Rad). For each primer pair a standard curve was generated with a serial dilution of a cDNA pool. rtPCR data was analyzed according to the relative standard curve method. All values were normalized to either beta-Actin (Fig S2) or cyclophillin A (Figs 1, 3, S1, S3). 
The control condition is used as a reference. Primer sets for the selected genes were developed with Primer Express version 2.0 (Applied Biosystems, Foster City, CA, USA) using default settings (Supplementary Table S1).

Immunofluorescence Cells were grown on 6- or 12-well culture plates (Greiner Bio-One; Alphen aan de Rijn; The Netherlands) to $\pm 60-80 \%$ confluency, pre-treated when applicable, washed twice with PBS, and either fixed for 15 min in $2 \%$ formaldehyde/PBS at room temperature (RT) followed by a 15 min incubation in chilled $100 \%$ methanol $(\mathrm{MetOH})$ at $-20^{\circ} \mathrm{C}$, or directly fixed in $100 \% \mathrm{MetOH}$ for $15 \mathrm{~min}$ at $-20^{\circ} \mathrm{C}$. Fixed plates were stored at $4^{\circ} \mathrm{C}$ in $70 \%$ ethanol (EtOH) or directly washed 3 times with PBS and used for immunocytochemistry (ICC). For detection of PRC1-proteins and histone modifications cells were first permeabilized for $10 \mathrm{~min}$ at RT in $0.2 \%$ Triton $X$ (TrX) in PBS. After extensive washing in PBS/0.02\% TrX, cells were incubated with primary antibody (Supplementary Table S2) for 1.5-2.5 hours in a pre-warmed humidified chamber at $37^{\circ} \mathrm{C}$, washed 5 times in PBS $/ 0.02 \% \operatorname{TrX}$ and incubated with fluorescently labeled secondary antibody for $60 \mathrm{~min}$ at $37^{\circ} \mathrm{C}$. $4^{\prime}-6$-Diamidino-2phenylindole (DAPI) was co-incubated with secondary conjugated antibodies to counterstain cell nuclei. Plates were washed in PBS/0.02\% $\mathrm{TrX}$, rinsed in PBS and subsequently dehydrated: $1 \mathrm{~min}$ in $70 \% \mathrm{EtOH}, 2$ times $1 \mathrm{~min}$ in $100 \% \mathrm{EtOH}$ and air-dried. Cells were mounted in Vectashield (Vector Laboratories, Inc. Burlingame, CA, USA), analyzed using a NIKON TE200 Eclipse fluorescence microscope and photographed using a NIKON DXM1200 digital camera in combination with NIS Elements 3.0 Imaging software. All antibodies were diluted in blocking buffer (1\% BSA, $5 \%$ FCS, 5\% normal goat serum (NGS), in PBS/0.02\% TrX). Secondary antisera used were goat-anti-mouse Texas Red ${ }^{\circledR}$ (TXRD, 1:100; Southern Biotech, Birmingham, LA, USA) and goat-anti-rabbit fluorescein isothiocyanate (FITC, 1:100; Southern Biotech), to detect monoclonal and polyclonal primary antibodies respectively.

Protein isolation, Differential extraction, Immunoprecipitation (IP), Immunoblotting (IB) Protein extraction and immunoblotting (IB) were performed and analyzed as described previously with minor adjustments [2, 5]. For extraction, cells were washed twice with cold PBS and lysed in RIPA buffer $(150 \mathrm{mM} \mathrm{NaCl}, 1 \% \mathrm{NP}-40,0.5 \%$ w/v Sodium Deoxycholate, 0.1\% SDS, 50 mM Tris at pH 8.0, 5 mM EDTA) supplemented with protease and phosphatase inhibitors $(5 \mathrm{mM}$ Benzamidine, $5 \mu \mathrm{g} / \mathrm{ml}$ Antipain, 5 $\mu \mathrm{g} / \mathrm{ml}$ Leupeptin, $5 \mathrm{\mu g} / \mu \mathrm{L}$ Aprotinin, $1 \mathrm{mM}$ Sodium Vanadate, $10 \mathrm{mM}$ Sodium Fluoride, $10 \mathrm{mM}$ Pyrophosphate, $10 \mathrm{mM}$ ß-glycerophosphate, $0.5 \mathrm{mM}$ DTT and 1mM PMSF). Lysates were subjected to two freeze-thaw cycles in liquid nitrogen, followed by sonication on ice with a probe sonicator (Soniprep 150; MSE, London, UK) for 12 cycli 
( $1 \mathrm{sec}$ ON, $1 \mathrm{sec}$ OFF) with amplitude 5 . After 10 min centrifugation at $13200 \mathrm{rpm}\left(4^{\circ} \mathrm{C}\right.$; Eppendorf centrifuge), the supernatant was transferred to a fresh tube and protein concentration was determined using a BCA protein assay kit (Pierce/Thermo Fisher Scientific, Rockford, IL, USA) according to the manufacturer's protocols on a Benchmark 550 Micro-plate Reader (Bio-Rad).

For differential extraction, cells were washed $2 x$ with cold PBS and scraped in lysis buffer (0.5\% Triton X-100, 1 mM EDTA, $150 \mathrm{mM} \mathrm{NaCl}$, Tris $\mathrm{HCl}$ pH 7,5; supplemented with inhibitors). After 30 minutes incubation on ice, nuclei are collected in the pellet by centrifugation ( $8000 \mathrm{rpm} ; 4^{\circ} \mathrm{C}$ ); the supernatant is the cytoplasmic fraction. Nuclei are washed in lysis buffer and suspended in ELB-buffer and incubated on ice for 10 minutes. Nuclear soluble (supernatant) and chromatin bound fractions (pellet) are separated by centrifugation (13200 rpm; $4^{\circ} \mathrm{C}$ ). After an additional wash in ELB buffer, the pellets were suspended in ELB and sonicated.

For IP, cells were either cross-linked followed by cell lysis, or cells were lysed directly in ELB buffer (250 mM NaCl, 0.1\% Igepal (NP40), 5 mM EDTA, 50 mM HEPES pH 7.0) supplemented with $5 \mathrm{mM}$ Benzamidine, $5 \mu \mathrm{g} / \mathrm{ml}$ Antipain, $5 \mu \mathrm{g} / \mathrm{ml}$ Leupeptin, 5 $\mu \mathrm{g} / \mathrm{ml}$ Aprotinin, $1 \mathrm{mM}$ Sodium Vanadate, $10 \mathrm{mM}$ Sodium Fluoride, 10mM Pyrophosphate, $10 \mathrm{mM}$ ß-glycerophosphate, $0.5 \mathrm{mM}$ DTT and $1 \mathrm{mM}$ PMSF. For crosslinking, cells were incubated in 1\% formaldehyde (in PBS) for $10 \mathrm{~min}$ at RT, fixation was stopped by addition of $2 \mathrm{M}$ glycin to a final concentration of $0.125 \mathrm{M}$. After $5 \mathrm{~min}$ incubation, cells were washed twice in cold PBS and lysed in ELB buffer. IP was carried out as described $[11,16]$. Briefly, extracts were sonicated on ice, centrifuged for 10 minutes at $4^{\circ} \mathrm{C}$ at $13200 \mathrm{rpm}$ and supernatants were transferred to a pre-cooled tube; $10 \%$ of the supernatant was taken as input and stored at $-80^{\circ} \mathrm{C}$. Appropriate amount of antiserum (Supplementary Table S2) was added and tubes were rotated for $1 \mathrm{hr}$ at $4^{\circ} \mathrm{C}$ on a spinning wheel. To precipitate immune complexes, washed protein-G beads (Protein G Sepharose/4 Fast Flow; GE Healthcare) were added to the extracts and rotated for $3-4$ hours at $4^{\circ} \mathrm{C}$, followed by $3 \mathrm{~min}$ centrifugation at $3000 \mathrm{rpm}$ at $4^{\circ} \mathrm{C}$. Supernatant was collected as depleted fraction and stored at $-80^{\circ} \mathrm{C}$. Beads were washed $4 \mathrm{x}$ in ELB buffer (with supplements) and stored dry at $-80^{\circ} \mathrm{C}$ until IB analysis.

For IB, equal protein amounts were boiled in sample buffer for $5 \mathrm{~min}$ and loaded on 9-15\% polyacrylamide gels. Following separation by SDS-PAGE, proteins were transferred onto polyvinylidene fluoride (PVDF) membranes (GE Healthcare). Ponceau $S$ (Sigma) staining was used to check protein transfer. PVDF membranes were blocked with $3.4 \%$ non-fat dry milk (Protifar; Nutricia, Zoetermeer, the Netherlands) in PBS containing $0.1 \%$ Tween-20 $(\mathrm{pH} 7.5)$ for 1 hour at RT, followed by an overnight incubation at $4^{\circ} \mathrm{C}$ with the primary antibody (Supplementary Table S2; anti-CBX4 [50]). After extensive washing with PBS/0.2\% Tween-20, membranes were probed with 
corresponding horseradish peroxidase conjugated secondary antibodies for 1 hour at RT: goat-anti-rat (7077; 1:2.000; Cell Signaling, Danvers, MA, USA), rat-anti-mouse (P0260; 1:5000; DAKO, Glostrup, Denmark) and donkey-anti-rabbit (711035-152; 1:15.000; Jackson Lab, Bar Harbor, ME, USA), to detect monoclonal rat, monoclonal mouse and rabbit polyclonal primary antibodies respectively. Signals were detected on autoradiograms using enhanced chemoluminescence (ECL; Pierce). Intensity of the bands was quantified with Quantity One software (Bio-Rad).

Drosophila lines Drosophila melanogaster stocks and crosses were kept on standard media at $25^{\circ} \mathrm{C}$. For crosses, 5 females were mated with 5 males; they were transferred each $48 \mathrm{~h}$ in new tubes. The $w^{1118}$ line was used as control line. The UAS::rolled line was a gift from Dr. Kevin Moses (University of Cambridge, UK); the $U A S:: D-p 38 b$ was a gift from Jean-Michel Gibert (University of Geneva, Switzerland); UAS::rolled and UAS::D-p38b allow $d E R K$ and $D-p 38 b$ overexpression, respectively [33, 51]. The v3171 line $\left(w^{1118} ;\right.$ MAPk-Ak2 $\left.{ }^{\mathrm{GD} 1597}\right)$ that downregulates $d M K 2$ by RNAinterference, was purchased from the Vienna Drosophila RNAi Center (VDRC) [52]; it does not present any known off-target effects. Downregulation of $d M K 2$ in third instar larvae was verified by RT/real-time PCR using primers located outside the v3171 repeats (Fig S4B) as described [38]. Results were normalized against rp49 or spt6 (Supplementary Table S1). Transgene overexpression was achieved using the wingspecific Gal4 transgenic driver scalloped $s d^{29.1}$ (called sd::Gal4; BL-8609 line Bloomington Drosophila Stock Center (BDSC; Bloomington, Indiana, USA). All the transgenic lines display Gal4-independent mini-white expression that allows tracing transgene transmission. $d M K 2$-LOF and $h M K 3-$ GOF transgenic flies were also crossed to mutant Polycomb lines to study a potential interaction of MK and PcG in vivo. Neither hMK3-GOF nor $d M K 2$-LOF induce a discernable sexcomb-phenotype by themselves or in combination with established $P C^{1}$ and $S c m^{D 1}$ alleles in heterozygote crosses (data not shown). The most likely explanation for this lack of phenotype is that $d M K 2$ may not play a role in anterior-posterior (AP) patterning. In support of the latter, AP-axis abnormalities have not been reported in single or double MK2/3 knockout mice [30]. 


\section{ACKNOWLEDGEMENTS}

We thank S. Bloyer, K. Hansen, M. Inagaki, S. Ludwig, M. Mooij, A. Otten, M. Schepens, R. Sverdlov, M. van Lohuizen, J. Geraedts and the MAASTRO and MolGen departments for research materials, technical support and scientific discussion. Financial support: Dutch Science Organization (ZonMW-NWO): RS grant 908-02-040 (JWV); VIDI grant 016.046.362 (JWV), joined vanGogh grant (JWV, FP); tUL Grant (JWV); VSBfonds scholarship (PP); Netherlands Genome Initiative (NGI) fellowship 050-72-422 (HN); UPMC and CNRS (FP). 


\section{REFERENCES}

1. Keshet $\mathrm{Y}$, Seger R: The MAP kinase signaling cascades: a system of hundreds of components regulates a diverse array of physiological functions. Methods Mol Biol 2010, 661:3-38.

2. Ludwig S, Engel K, Hoffmeyer A, Sithanandam G, Neufeld B, Palm D, Gaestel M, Rapp U: 3pK, a novel mitogen-activated protein (MAP) kinase-activated protein kinase, is targeted by three MAP kinase pathways. Mol Cell Biol 1996, 16:6687-6697.

3. Gaestel M: Specificity of signaling from MAPKs to MAPKAPKs: kinases' tango nuevo. Front Biosci 2008, 13:6050-6059.

4. Shiryaev A, Moens U: Mitogen-activated protein kinase p38 and MK2, MK3 and MK5: menage a trois or menage a quatre? Cell Signal 2010, 22:1185-1192.

5. Voncken JW, Niessen H, Neufeld B, Rennefahrt U, Dahlmans V, Kubben N, Holzer B, Ludwig S, Rapp UR: MAPKAP kinase 3pK phosphorylates and regulates chromatin-association of the polycomb-group protein Bmi1. J Biol Chem 2005, 280:5178-5187.

6. Prezioso C, Orlando V: Polycomb proteins in mammalian cell differentiation and plasticity. FEBS Letters 2011, 585:2067-2077.

7. Simon JA, Kingston RE: Mechanisms of Polycomb gene silencing: knowns and unknowns. Nat Rev Mol Cell Biol 2009, 10:697-708.

8. Azuara V, Perry P, Sauer S, Spivakov M, Jorgensen HF, John RM, Gouti M, Casanova M, Warnes G, Merkenschlager M, Fisher AG: Chromatin signatures of pluripotent cell lines. Nat Cell Biol 2006, 8:532538.

9. Bernstein BE, Mikkelsen TS, Xie X, Kamal M, Huebert DJ, Cuff J, Fry B, Meissner A, Wernig M, Plath K, et al: A bivalent chromatin structure marks key developmental genes in embryonic stem cells. Cell 2006, 125:315-326.

10. Mikkelsen TS, Ku M, Jaffe DB, Issac B, Lieberman E, Giannoukos G, Alvarez P, Brockman W, Kim TK, Koche RP, et al: Genome-wide maps of chromatin state in pluripotent and lineage-committed cells. Nature 2007, 448:553-560.

11. Voncken JW, Schweizer D, Aagaard L, Sattler L, Jantsch MF, van Lohuizen M: Chromatin-association of the Polycomb group protein BMI1 is cell cycle- regulated and correlates with its phosphorylation status. J Cell Sci 1999, 112:4627-4639.

12. Bracken AP, Dietrich N, Pasini D, Hansen KH, Helin K: Genome-wide mapping of Polycomb target genes unravels their roles in cell fate transitions. Genes and Development 2006, 20:1123-1136.

13. Tarn C, Bilodeau ML, Hullinger RL, Andrisani OM: Differential Immediate Early Gene Expression in Conditional Hepatitis B Virus pX-transforming Versus Nontransforming Hepatocyte Cell Lines. Journal of Biological Chemistry 1999, 274:2327-2336.

14. Ronkina N, Menon MB, Schwermann J, Arthur JS, Legault H, Telliez JB, Kayyali US, Nebreda AR, Kotlyarov A, Gaestel M: Stress induced gene expression: a direct role for MAPKAP kinases in transcriptional activation of immediate early genes. Nucleic Acids Res 2010, 39:2503-2518.

15. Saurin AJ, Shiels C, Williamson J, Satijn DP, Otte AP, Sheer D, Freemont PS: The human polycomb group complex associates with pericentromeric heterochromatin to form a novel nuclear domain. J Cell Biol 1998, 142:887-898.

16. Alkema MJ, Bronk M, Verhoeven E, Otte A, van't Veer LJ, Berns A, van Lohuizen M: Identification of Bmi1-interacting proteins as constituents of a multimeric mammalian polycomb complex. Genes Dev 1997, 11:226-240.

17. Perez-Cadahia B, Drobic B, Davie JR: H3 phosphorylation: dual role in mitosis and interphase. Biochem Cell Biol 2009, 87:695-709. 
18. Sassone-Corsi P, Mizzen CA, Cheung P, Crosio C, Monaco L, Jacquot S, Hanauer A, Allis CD: Requirement of Rsk-2 for epidermal growth factor-activated phosphorylation of histone H3. Science 1999, 285:886891.

19. Drobic B, Perez-Cadahia B, Yu J, Kung SK, Davie JR: Promoter chromatin remodeling of immediate-early genes is mediated through $\mathrm{H} 3$ phosphorylation at either serine 28 or 10 by the MSK1 multi-protein complex. Nucleic Acids Res 2010, 38:3196-3208.

20. Cao R, Tsukada YI, Zhang Y: Role of Bmi-1 and Ring1A in H2A Ubiquitylation and Hox Gene Silencing. Mol Cell 2005, 20:845-854.

21. Hodgson JW, Argiropoulos B, Brock HW: Site-specific recognition of a 70-base-pair element containing $\mathrm{d}(\mathrm{GA})(\mathrm{n})$ repeats mediates bithoraxoid polycomb group response element-dependent silencing. Mol Cell Biol 2001, 21:4528-4543.

22. Lau PN, Cheung P: Histone code pathway involving H3 S28 phosphorylation and K27 acetylation activates transcription and antagonizes polycomb silencing. Proc Natl Acad Sci U S A 2011, 108:2801-2806.

23. Gehani SS, Agrawal-Singh S, Dietrich N, Christophersen NS, Helin K, Hansen K: Polycomb group protein displacement and gene activation through MSK-dependent H3K27me3S28 phosphorylation. Mol Cell 2010, 39:886-900.

24. Young MD, Willson TA, Wakefield MJ, Trounson E, Hilton DJ, Blewitt ME, Oshlack A, Majewski IJ: ChIPseq analysis reveals distinct H3K27me3 profiles that correlate with transcriptional activity. Nucleic Acids Res 2011, 39:7415-7427.

25. Fischle W, Tseng BS, Dormann HL, Ueberheide BM, Garcia BA, Shabanowitz J, Hunt DF, Funabiki H, Allis CD: Regulation of HP1-chromatin binding by histone H3 methylation and phosphorylation. Nature 2005, 438:1116-1122.

26. Hirota T, Lipp JJ, Toh BH, Peters JM: Histone H3 serine 10 phosphorylation by Aurora B causes HP1 dissociation from heterochromatin. Nature 2005, 438:1176-1180.

27. Dormann HL, Tseng BS, Allis CD, Funabiki H, Fischle W: Dynamic regulation of effector protein binding to histone modifications: the biology of HP1 switching. Cell Cycle 2006, 5:2842-2851.

28. Vermeulen M, Eberl HC, Matarese F, Marks H, Denissov S, Butter F, Lee KK, Olsen JV, Hyman AA, Stunnenberg HG, Mann M: Quantitative Interaction Proteomics and Genome-wide Profiling of Epigenetic Histone Marks and Their Readers. Cell 2010, 142:967-980.

29. Ronkina N, Kotlyarov A, Dittrich-Breiholz O, Kracht M, Hitti E, Milarski K, Askew R, Marusic S, Lin LL, Gaestel M, Telliez JB: The mitogen-activated protein kinase (MAPK)-activated protein kinases MK2 and MK3 cooperate in stimulation of tumor necrosis factor biosynthesis and stabilization of p38 MAPK. Mol Cell Biol 2007, 27:170-181.

30. Ronkina N, Kotlyarov A, Gaestel M: MK2 and MK3--a pair of isoenzymes? Front Biosci 2008, 13:55115521.

31. Eblen ST, Slack-Davis JK, Tarcsafalvi A, Parsons JT, Weber MJ, Catling AD: Mitogen-Activated Protein Kinase Feedback Phosphorylation Regulates MEK1 Complex Formation and Activation during Cellular Adhesion. Mol Cell Biol 2004, 24:2308-2317.

32. Patterson KI, Brummer T, O'Brien PM, Daly RJ: Dual-specificity phosphatases: critical regulators with diverse cellular targets. Biochem J 2009, 418:475-489.

33. Adachi-Yamada T, Nakamura M, Irie K, Tomoyasu Y, Sano Y, Mori E, Goto S, Ueno N, Nishida Y, Matsumoto K: p38 mitogen-activated protein kinase can be involved in transforming growth factor beta superfamily signal transduction in Drosophila wing morphogenesis. Mol Cell Biol 1999, 19:2322-2329.

34. De Celis JF: Pattern formation in the Drosophila wing: The development of the veins. Bioessays 2003, 25:443-451. 
35. Martin-Blanco E: p38 MAPK signalling cascades: ancient roles and new functions. Bioessays 2000, 22:637-645.

36. Niessen HE, Demmers JA, Voncken JW: Talking to chromatin: post-translational modulation of polycomb group function. Epigenetics Chromatin 2009, 2.

37. Rao PS, Satelli A, Zhang S, Srivastava SK, Srivenugopal KS, Rao US: RNF2 is the target for phosphorylation by the p38 MAPK and ERK signaling pathways. Proteomics 2009, 9:2776-2787.

38. Mouchel-Vielh E, Rougeot J, Decoville M, Peronnet F: The MAP kinase ERK and its scaffold protein MP1 interact with the chromatin regulator Corto during Drosophila wing tissue development. BMC Dev Biol 2011, 11:17.

39. Srivastav RK, Schwede S, Klaus M, Schwermann J, Gaestel M, Niedenthal R: Monitoring protein-protein interactions in mammalian cells by trans-SUMOylation. Biochem J 2011, 438:495-503.

40. Wei Y, Chen Y-H, Li L-Y, Lang J, Yeh S-P, Shi B, Yang C-C, Yang J-Y, Lin C-Y, Lai C-C, Hung M-C: CDK1dependent phosphorylation of EZH2 suppresses methylation of H3K27 and promotes osteogenic differentiation of human mesenchymal stem cells. Nat Cell Biol 2011, 13:87-94.

41. Kaneko S, Li G, Son J, Xu CF, Margueron R, Neubert TA, Reinberg D: Phosphorylation of the PRC2 component Ezh2 is cell cycle-regulated and up-regulates its binding to ncRNA. Genes Dev 2010, 24:26152620.

42. Chen S, Bohrer LR, Rai AN, Pan Y, Gan L, Zhou X, Bagchi A, Simon JA, Huang H: Cyclin-dependent kinases regulate epigenetic gene silencing through phosphorylation of EZH2. Nat Cell Biol 2010, 12:1108-1114.

43. Roscic A, Moller A, Calzado MA, Renner F, Wimmer VC, Gresko E, Ludi KS, Schmitz ML: Phosphorylationdependent control of Pc2 SUMO E3 ligase activity by its substrate protein HIPK2. Mol Cell 2006, 24:7789.

44. Fujisaki S, Ninomiya $\mathrm{Y}$, Ishihara $\mathrm{H}$, Miyazaki M, Kanno R, Asahara T, Kanno M: Dimerization of the Polycomb-group protein Mel-18 is regulated by PKC phosphorylation. Biochem Biophys Res Commun 2003, 300:135-140.

45. Hatano A, Matsumoto M, Higashinakagawa T, Nakayama KI: Phosphorylation of the chromodomain changes the binding specificity of Cbx2 for methylated histone H3. Biochem Biophys Res Commun 2010, 397:93-99.

46. Serrano M, Lin AW, McCurrach ME, Beach D, Lowe SW: Oncogenic ras provokes premature cell senescence associated with accumulation of p53 and p16INK4a. Cell 1997, 88:593-602.

47. Morgenstern JP, Land $\mathrm{H}$ : Advanced mammalian gene transfer: high titre retroviral vectors with multiple drug selection markers and a complementary helper-free packaging cell line. Nucleic Acids Res 1990, 18:3587-3596.

48. Kinsella TM, Nolan GP: Episomal vectors rapidly and stably produce high-titer recombinant retrovirus. HumGene Ther 1996, 7:1405-1413.

49. Brummelkamp TR, Bernards R, Agami R: A system for stable expression of short interfering RNAs in mammalian cells. Science 2002, 296:550-553.

50. Hamer KM, Sewalt RG, den Blaauwen JL, Hendrix T, Satijn DP, Otte AP: A panel of monoclonal antibodies against human polycomb group proteins. Hybrid Hybridomics 2002, 21:245-252.

51. Kumar JP, Moses K: EGF receptor and Notch signaling act upstream of Eyeless/Pax6 to control eye specification. Cell 2001, 104:687-697.

52. Dietzl G, Chen D, Schnorrer F, Su KC, Barinova Y, Fellner M, Gasser B, Kinsey K, Oppel S, Scheiblauer S, et al: A genome-wide transgenic RNAi library for conditional gene inactivation in Drosophila. Nature 2007, 448:151-156. 
Figure S1

A

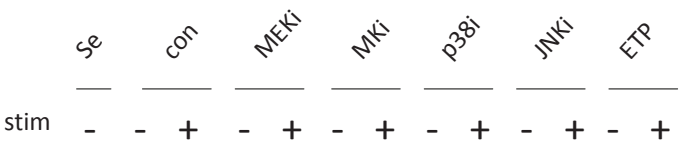

pERK

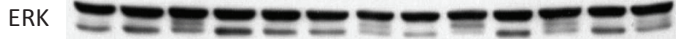

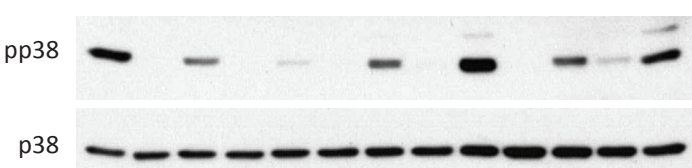

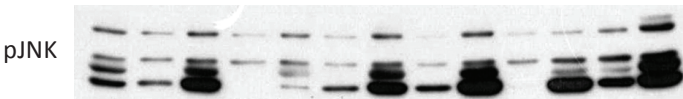
JNK

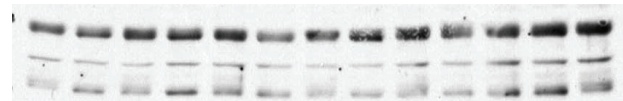

C

P: 2 Py

IB: pERK

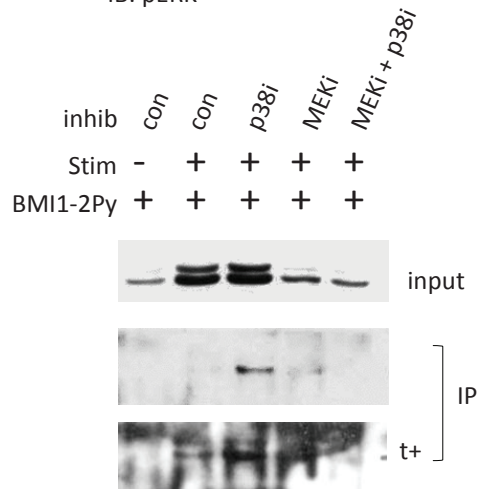

B

starved
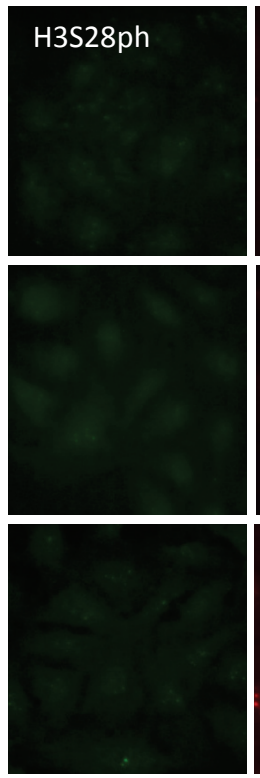

BMI1

H3S28ph

stimulated

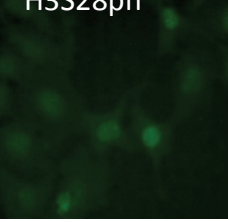

BMI1

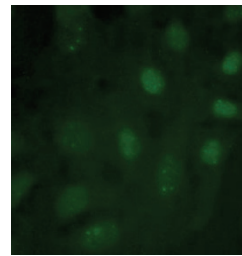

$\therefore \frac{\overline{⿱ 亠}}{\Sigma}$

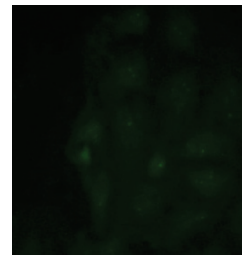

ㅇํ

$\stackrel{\infty}{m}$ 
Figure S2

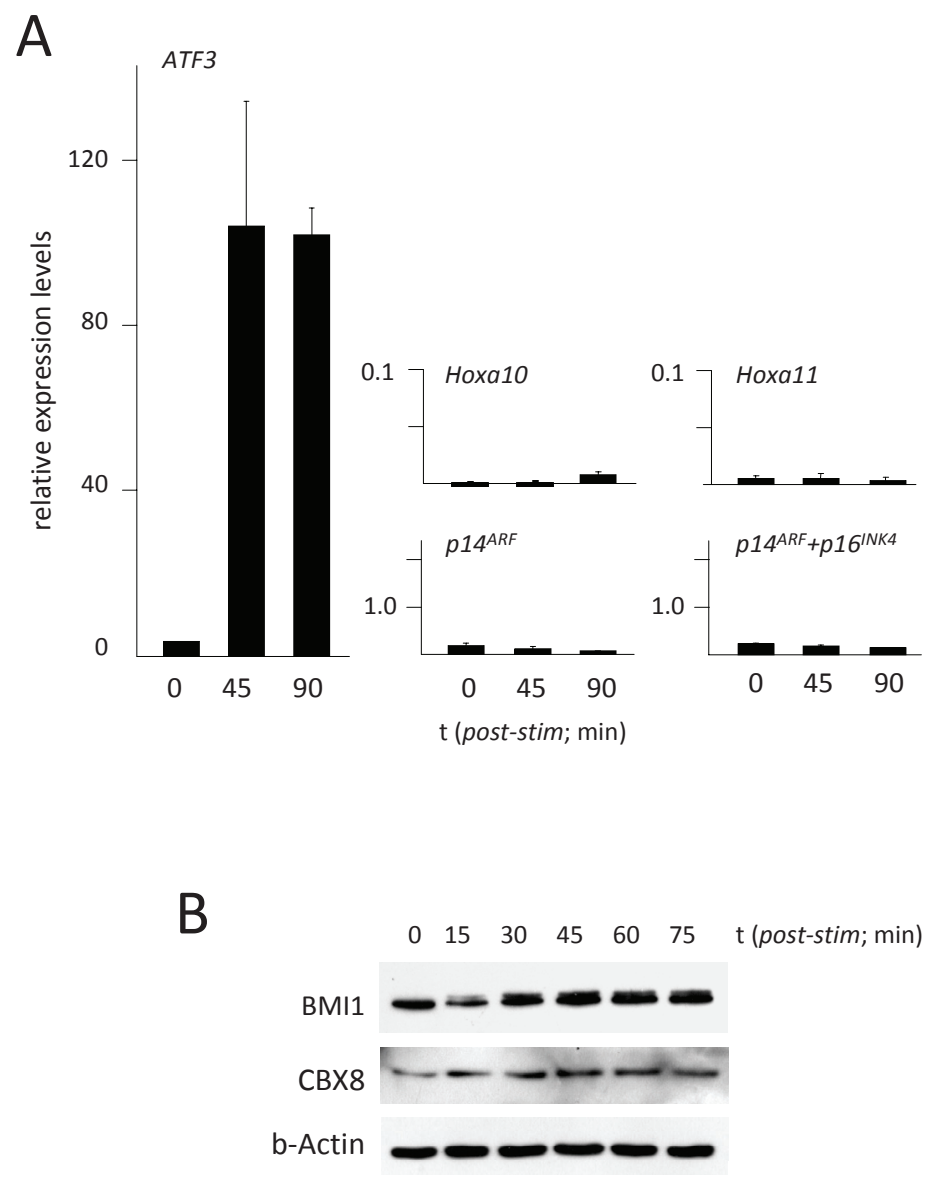

Fig S2 (above) | PRC1/chromatin dissociation, not loss of H3K27me3, correlates with transcription. (A) mRNA expression of PRC1-target and non-target genes in TIG3 cells upon 0, 45 and 90 min of mitogen-stimulation. (B) Mitogenic stimulation induces BMI- phosphorylation (upper panel) in TIG3 cells; cellular BMI1 and CBX8 protein (lower panel) levels remain unchanged under these conditions in support of changed PRC1/chromatinassociation rather than loss of protein (e.g. degradation).

Fig S1 (on the left) | PRC1-target gene expression is controlled by ERK and P38. (A). MAPK and SAPK phosphorylation in response to mitogenic stimulation (stim) in U2-OS cells; specificity of response was supported by distinctive phosphorylation profiles induced by two different stressors (ETP: etoposide, Se: selenite). (B) H3S28ph and PRC1-protein BMI1 staining in G1-arrested (starved) or mitogen-stimulated U2-OS cells. (C) Interaction of pERK and BMI1; U2-OS/BMI2Py cells were stimulated with mitogen (stim) prior to IP; $\mathrm{t+}$ : longer exposure (IB: immunoblot; IP: immunoprecipitation). Similar experiments with pp38 were inconclusive due to IB-detection issues with antisera applied. 


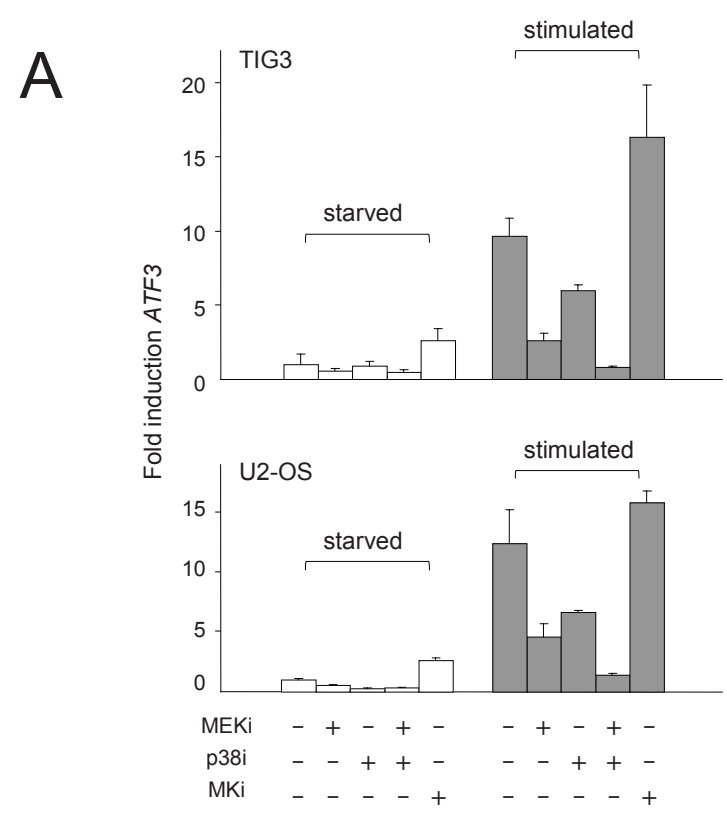

Figure S3

B

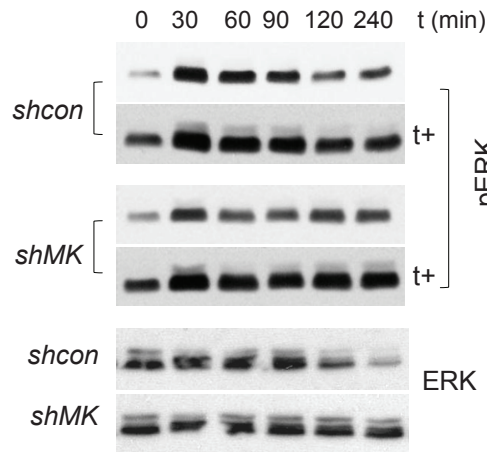

C

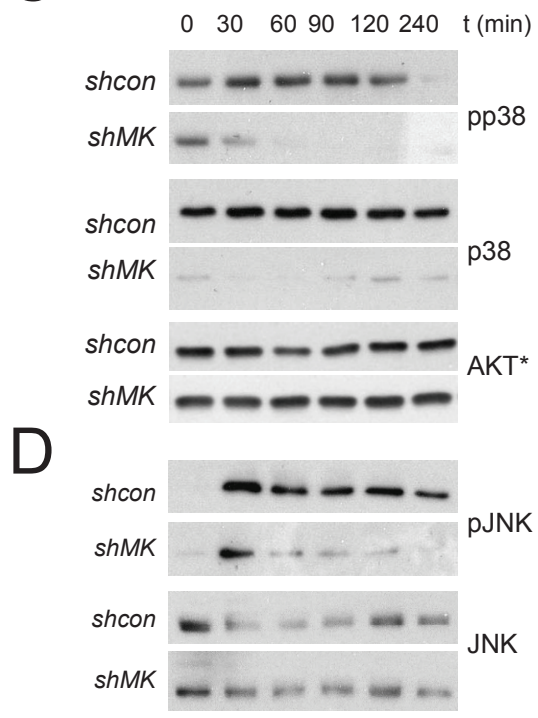

Fig S3| MK3 is a negative regulator of ERK. (A) mRNA-expression of PRC1-target gene ATF3 as a function of kinase inhibition in TIG3 cells (data as in Fig 3A) and U2-OS cells. (B-D) IB-analysis of pERK (B), pp38 (C), pJNK (D) in resting or mitogen-stimulated (stim) control and MK-knockdown (shMK) cells; $t$ (min): time poststimulation in minutes; *: loading controls for all corresponding panels; $\mathrm{t}+$ : longer exposure. 
Figure S4

A
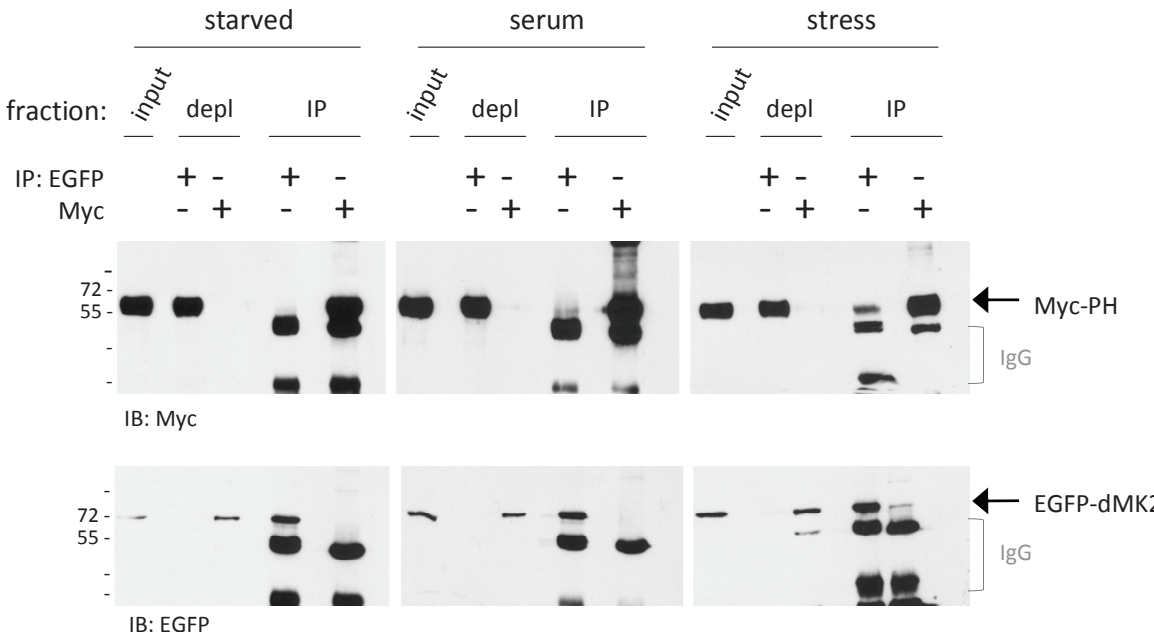

B: EGFP

B

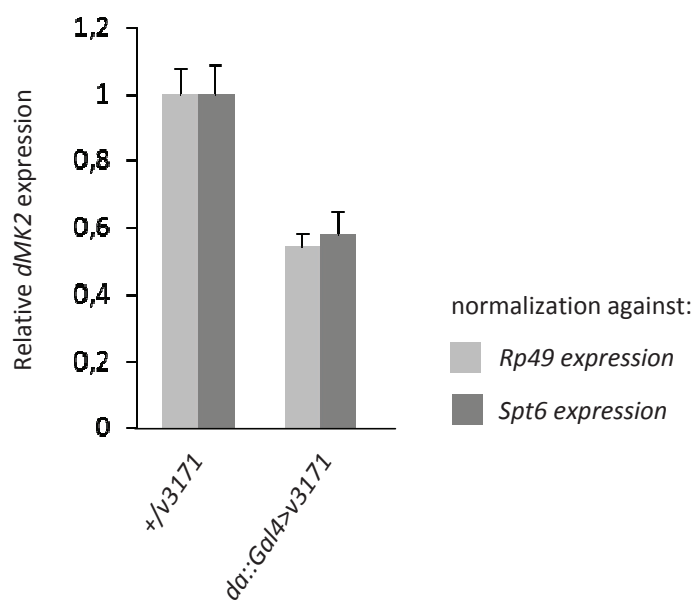

Fig S4 | PRC1/MK/ERK module represents a molecular switch mechanism. (A) Functional interaction between dMK2 and Polyhomeotic in vitro: stress-signaling induced interaction of EGFP-tagged dMK2 (Drosophila ortholog of MK2/3) and Myc-tagged PH (Drosophila ortholog of PHC1/2) in S2 cells. Conditions tested: starved, mitogen-stimulated (FCS/TPA) and stressed (arsenite). IgG represents IgG-heavy and light chains. (B) Reduced dMK2 expression in third instar v3171 larvae; normalization indicated. 
Table S1: primer sequences and targeting sequences

\begin{tabular}{|c|c|c|}
\hline Cloning primers -Taqman & Fw/Rev & sequence $\left(5^{\prime} \rightarrow 3^{\prime}\right)$ \\
\hline$d M K 2$ & $\begin{array}{l}\text { Forward } \\
\text { Reverse }\end{array}$ & $\begin{array}{l}\text { CACCATGCTTTCTCTGCAGAATCAACG } \\
\text { GTTGCGCGTCGCATTGGCCATGTATAACTC }\end{array}$ \\
\hline$P H$ & $\begin{array}{l}\text { Forward } \\
\text { Reverse }\end{array}$ & $\begin{array}{l}\text { CACCATGACAACGATCACCAATGG } \\
\text { CTGCGCTCCTGGATGCTTGGCCTC }\end{array}$ \\
\hline shRNA target sequences & Number & sequence $\left(5^{\prime} \rightarrow 3^{\prime}\right)$ \\
\hline MK3 & $\# 3$ & GGAGGAGATGACCAGTGCC \\
\hline PCR primers for ChIP analysis & $\mathrm{Fw} / \mathrm{Rev}$ & sequence $\left(5^{\prime} \rightarrow 3^{\prime}\right)$ \\
\hline p14ARF exon 1 & $\begin{array}{l}\text { Forward } \\
\text { Reverse }\end{array}$ & $\begin{array}{l}\text { GTGGGTCCCAGTCTGCAGTTA } \\
\text { CCTTTGGCACCAGAGGTGAG }\end{array}$ \\
\hline $15 \mathrm{~kb}$ downstream of ARFpromoter & $\begin{array}{l}\text { Forward } \\
\text { Reverse }\end{array}$ & $\begin{array}{l}\text { GCACTTGCCCTTCCAGGTATA } \\
\text { TGATAGTTCAAGGCCCTATGCC }\end{array}$ \\
\hline p16INK4A promoter & $\begin{array}{l}\text { Forward } \\
\text { Reverse }\end{array}$ & $\begin{array}{l}\text { ACCCCGATTCAATTTGGCAG } \\
\text { AAAAAGAAATCCGCCCCCG }\end{array}$ \\
\hline p16INK4A exon1 & $\begin{array}{l}\text { Forward } \\
\text { Reverse }\end{array}$ & $\begin{array}{l}\text { AGAGGGTCTGCAGCGG } \\
\text { TCGAAGCGCTACCTGATTCC }\end{array}$ \\
\hline \multirow[t]{2}{*}{ CCNA2 } & Forward & TGACGTCATTCAAGGCGACAG \\
\hline & Reverse & GCTCAGTTTCCTTTGGTTTACCC \\
\hline p15 exon 1 & $\begin{array}{l}\text { Forward } \\
\text { Reverse }\end{array}$ & $\begin{array}{l}\text { GGAACCTAGATCGCCGATGTAG } \\
\text { TGTTTTACGCGTGGAATGCAC }\end{array}$ \\
\hline ATF3 & $\begin{array}{l}\text { Forward } \\
\text { Reverse }\end{array}$ & $\begin{array}{l}\text { TGTTTTTTCTTTTGCGTTTGGC } \\
\text { TCGTGGCAACCAAATCTAAACAG }\end{array}$ \\
\hline HOXA10 & $\begin{array}{l}\text { Forward } \\
\text { Reverse }\end{array}$ & $\begin{array}{l}\text { CCCGAGCTGATGAGCGAGTC } \\
\text { GCCAAATTATCCCACAACAATGTC }\end{array}$ \\
\hline HOXA11 & $\begin{array}{l}\text { Forward } \\
\text { Reverse }\end{array}$ & $\begin{array}{l}\text { AATCTATCCCCATCCTTAGCAGG } \\
\text { TTGTCAATTTCAACATCGGGTC }\end{array}$ \\
\hline primer sequences for rtPCR analysis & $\mathrm{Fw} / \mathrm{Rev}$ & sequence $\left(5^{\prime} \rightarrow 3^{\prime}\right)$ \\
\hline p14ARF & $\begin{array}{l}\text { Forward } \\
\text { Reverse }\end{array}$ & $\begin{array}{l}\text { CCCTCGTGCTGCTGATGCTACTG } \\
\text { CCCATCATCATGACCTGGTCTT }\end{array}$ \\
\hline$p 14 A R F+p 16 I N K 4 A$ & $\begin{array}{l}\text { Forward } \\
\text { Reverse }\end{array}$ & $\begin{array}{l}\text { GAAGGTCCCTCAGACATCCCC } \\
\text { CCCTGTAGGACCTTCGGTGAC }\end{array}$ \\
\hline cyclophillin A & $\begin{array}{l}\text { Forward } \\
\text { Reverse }\end{array}$ & $\begin{array}{l}\text { TTCCTGCTTTCACAGAATTATTCC } \\
\text { GCCACCAGTGCCATTATGG }\end{array}$ \\
\hline ATF3 & $\begin{array}{l}\text { Forward } \\
\text { Reverse }\end{array}$ & $\begin{array}{l}\text { TCACTGTCAGCGACAGACCC } \\
\text { CTACCTCGGCTTTTGTGATGG }\end{array}$ \\
\hline HOXA10 & Forward & GCCCTTCCGAGAGCAGCAAAG \\
\hline \multirow[t]{2}{*}{ HOXA11 } & Forward & TGCCAAGTTGTACTTACTACGTC \\
\hline & Reverse & GTTGGAGGAGTAGGAGTATGTCA \\
\hline CCNA2 & $\begin{array}{l}\text { Forward } \\
\text { Reverse }\end{array}$ & $\begin{array}{l}\text { CGCTCCAAGAGGACCAGGA } \\
\text { СCCTCCTGCAGATATCCCG }\end{array}$ \\
\hline$b$-Actin & $\begin{array}{l}\text { Forward } \\
\text { Reverse }\end{array}$ & $\begin{array}{l}\text { CCTGGCACCCAGCACAAT } \\
\text { GCCGATCCACACGGAGTACT }\end{array}$ \\
\hline$d M K 2$ & $\begin{array}{l}\text { Forward } \\
\text { Reverse }\end{array}$ & $\begin{array}{l}\text { CCTGAGGTTCTGGGCCCGGA } \\
\text { AGAACGGCGGGAATCCGCAC }\end{array}$ \\
\hline \multirow[t]{2}{*}{ rp49 } & Forward & CCGCTTCAAGGGACAGTATC \\
\hline & Reverse & GACAATCTCCTTGCGCTTCT \\
\hline \multirow[t]{2}{*}{ spt6 } & Forward & CGGAGGAGCTCTTCGATATG \\
\hline & Reverse & GACAGCTCTGGGAAGTCGTC \\
\hline
\end{tabular}


Table S2: antibodies used for ChIP, ICC, IB and IP

\begin{tabular}{|c|c|c|c|}
\hline ChIP antisera & Species & $\mu \mathrm{l} / \mathrm{ChIP}^{\#}$ & Source \\
\hline CBX8 & rabbit & 1 & $\begin{array}{l}\text { Equal mix of } \alpha \text {-CBX8 “LAST" and "GALD"; } \\
\text { courtesy Klaus Hansen, Copenhagen, DK }\end{array}$ \\
\hline H3K27me3 & rabbit & 4 & $\begin{array}{l}\text { 07-449; Upstate Biotechnology/Millipore, Waltham, MA, } \\
\text { USA }\end{array}$ \\
\hline H3S28ph & rat* & 40 & HTA28; courtesy Masaki Inagaki, Aichi, JP \\
\hline $\mathrm{HA}$ & rabbit & 20 & sc-805; Santa Cruz Biotechnology, Santa Cruz, CA, USA \\
\hline Rae28/PHC1 & rabbit & 4 & Rae28; Yoshihiro Takihara, Hiroshima, JP \\
\hline ICC antisera & Species & Dilution & Source \\
\hline BMI1 & mouse & $1: 250$ & $\begin{array}{l}\text { F6; courtesy Maarten van Lohuizen, } \\
\text { Amsterdam, NL }\end{array}$ \\
\hline H3S28ph & rat* & $1: 20$ & See: ChIP antisera \\
\hline IB antisera & Species & Dilution & Source \\
\hline 2PY-tag & mouse & $1: 300$ & MMS-155R; Babco/Covance, Princeton, NJ, USA \\
\hline BMI1 & mouse & $1: 250-1000$ & See: ICC sera \\
\hline CBX4 & mouse & $1: 25$ & Clone M9; courtesy Arie Otte, Amsterdam, NL \\
\hline $\mathrm{CBX8}$ & rabbit & 1:5000 & See: ChIP antisera \\
\hline MK3 & mouse & $1: 500$ & 3p8-1; gift from Stephan Ludwig, Münster, GE \\
\hline b-Actin & mouse & $1: 200.000$ & C4, 69100, MP Biomedicals, Solon, OH, USA \\
\hline AKT & rabbit & 1:1000 & 9272; Cell Signaling, Danvers, MA, USA \\
\hline pERK (Thr202/Tyr204) & rabbit & 1:1000 & 9101; Cell Signaling, Danvers, MA, USA \\
\hline ERK1/2 & rabbit & 1:1000 & 9102; Cell Signaling, Danvers, MA, USA \\
\hline pp38 (Thr180/Tyr182) & rabbit & 1:1000 & 9211; Cell Signaling, Danvers, MA, USA \\
\hline p38 & rabbit & 1:1000 & 9212; Cell Signaling, Danvers, MA, USA \\
\hline pJNK (Thr183/Tyr185) & rabbit & 1:1000 & 9251; Cell Signaling, Danvers, MA, USA \\
\hline JNK & rabbit & 1:1000 & 9252; Cell Signalling, Danvers, MA, USA \\
\hline pMEK1 (T292) & rabbit* & 1:1000 & GTX61817; Gene Tex, Irvine, CA, USA \\
\hline pMEK1 (S298) & mouse & $1: 100.000$ & $\begin{array}{l}\text { 558375; BD Pharmingen }{ }^{\mathrm{TM}} \text { /BD Biosciences, Franklin } \\
\text { Lakes, NJ, USA }\end{array}$ \\
\hline MEK1,2 & rabbit & 1:2000 & ABIN192427; Antibodies-online, Aachen, GE \\
\hline H3 & rabbit & $1: 5000$ & ab1791; Abcam, Cambridge, UK \\
\hline EGFP & mouse & 1:5000 & Roche Diagnostics, Basal, $\mathrm{CH}$ \\
\hline MYC & mouse & $1: 2500$ & 9E10; Santa Cruz Biotechnology, Santa Cruz, CA, USA \\
\hline H3S28ph & rat* & $1: 500$ & See: ChIP antisera \\
\hline Tubulin & mouse & 1:10.000 & T6074, Sigma-Aldrich, St. Louis, MO, USA \\
\hline IP antisera & Species & $\mu \mathrm{l} / \mathrm{IP}$ & Source \\
\hline 2Py-tag & mouse & 3 & See: IB antisera \\
\hline EGFP & mouse & $3 \mu \mathrm{g}$ & See: IB antisera \\
\hline MYC & mouse & $3 \mu \mathrm{g}$ & See: IB antisera \\
\hline
\end{tabular}

${ }^{*} \mathrm{mAb} ;{ }^{\#} \sim 4 \mu \mathrm{g}$ 
Chapter 3 | 
Signaling-induced degradation of BMI1 by chromatin-associated KAP1

Peggy Prickaerts*, Hanneke EC Niessen*, Vivian EH Dahlmans, Frank Spaapen, Claudia Geijselaers, Sheng Chung Lee, Bradly G Wouters, Frédérique Peronnet, Jan Willem Voncken

* equal contribution

Status: in preparation 


\section{ABSTRACT}

Chromatin-associated processes are controlled by establishing and disrupting protein-partnerships. Polycomb Repressive Complex (PRC) proteins are implicated in numerous DNA-templated processes, including transcription, replication and DNA repair. In the context of mitogen response, we recently identified MK3 and PERK as PRC1 interaction partners. We here identify a stress-induced association between BMI1 and KAP1, a heterochromatin factor involved in transcriptional regulation and DNA repair. KAP1/BMI1-interaction is induced by arsenite and selenite and is mediated by the KAP1 RINGfinger/BB-box/coiled-coil domain. Both p38 and ATM are required in the context of double strand DNA damage (selenite) to establish the interaction, which, in addition, is subject to complex regulation by several other signaling pathways, including PKC, JNK and GSK3ß. Overexpression of full length-KAP1 induces ubiquitin-mediated proteolytic degradation of BMI1, whereas a KAP1-deltaRingfinger protein does not. Although the interaction is also detected in the soluble nuclear compartment, BMI1proteolysis appears dependent on chromatin-association of KAP1 and BMI1. Drosophila bonus, the fly ortholog of KAP1, displays genetic interaction with Polycomb in anteriorposterior patterning, which is manifested as reduced expression of the $P C^{1}$-associated sexcomb phenotype. Finally we show that KAP1-depletion allows enhanced mitogeninduced expression of the PRC1-target gene ATF3. Relevantly, p38-inhibition prevents chromatin-association of KAP1 and enhances KAP1/BMI1-interaction in mitogenstimulated cells. Combined, our findings suggest a model in which stress-signaling enables KAP1 to target BMI1 for ubiquitin-mediated breakdown in the context of chromatin-binding. To the best of our knowledge, this is the first report on a functional interaction of Polycomb Repressive Complex 1 with the heterochromatin factor KAP1. 


\section{INTRODUCTION}

Polycomb Repressive Complexes (PRCs) contribute to an epigenetic memory system that maintains gene expression profiles during development; as such, PRCs play an crucial role in stem cell self-renewal, differentiation and cell plasticity (Boyer et al., 2006; Bracken et al., 2006; Lee et al., 2006b; Prezioso and Orlando, 2011). Although PRC-composition is known to change within spatio-temporal context (i.e. during development), two main functionally distinct classes are recognized: PRC1 and PRC2 (Prezioso and Orlando, 2011). Mammalian PRC2 contains EZH2, a histone lysine methyltransferase (HKMT) which associates with EED and Suz12 to form the functional core of PRC2; EZH2 catalyzes Polycomb-associated repressive histone $\mathrm{H} 3$ lysine 27 trimethylation (H3K27me3) (Cao et al., 2002; Kuzmichev et al., 2002). PRC1 comprises Polycomb Group proteins BMI1, PHC, CBX and RING1. Numerous homologs of these 4 core-components exist and are known to interact with each other (Levine et al., 2002; Simon and Kingston, 2009). PRC1 recognizes and binds H3K27me3-marks through chromobox domains ( $\mathrm{CBX}$ ) and harbors several catalytically active components (i.e. Ubiquitin E3 ligases BMI1, RNF2; E3 SUMO-protein ligase CBX4) (Buchwald et al., 2006; Cao et al., 2005; de Napoles et al., 2004; Fischle et al., 2003; Min et al., 2003; Wang et al., 2004). Both PRC1 and PRC2 are known to interact with additional regulatory factors (i.a. non-coding RNAs and DNA methyltransferases, histone demethylases, histone deacetylases) thereby acting as convergence points of epigenetic regulation at all three macromolecular levels (i.e. DNA, RNA, protein) (Niessen et al., 2009). In addition, PRC1 complexes exert direct effects on chromatin-structure, a property for which catalytic activity appears dispensable (Francis et al., 2004; Shao et al., 1999; Simon and Kingston, 2009).

Polycomb function was first described in Drosophila: Polycomb Group mutations cause a range of anterior-posterior (AP) patterning defects through defective maintenance of HOX (HOM-C) gene expression boundaries (Lewis, 1978). During Drosophila development, PRC2 consolidates transcriptionally repressed gene states installed by maternal repressive factors by H3K27me3-marking; PRC1 maintains repression as PRC2 proteins disappear early in fly development (Simon and Tamkun, 2002; Struhl, 1981). PRC structure and function are evolutionarily conserved; as such, both insect and mammalian PRCs control establishment of segment identity (Muller et al., 1995).

PRC1 and PRC2 proteins undergo a wide range of post-translational modifications (PTMs). PRC/chromatin-occupation (i.e. target-gene association) in embryonic and induced pluripotent progenitor cells is different from that in differentiated cells (Boyer et al., 2006; Bracken et al., 2006; Lee et al., 2006b; Mikkelsen et al., 2007). This raises 
the question how this dynamic PRC-based epigenomic remodeling is orchestrated at the molecular level. We previously showed that PRC1/chromatin-association is subject to regulation induced by changes in the cellular microenvironment: mitogenic-stimulation or cell-stress induce PTMs on PRC1-proteins, which correlate with chromatin/dissociation and/or redistribution (Voncken et al., 2005; Voncken et al., 1999). PTMs, like phosphorylation, affect individual proteins and processes at the level of activity, interaction, subcellular re-localization and stability. Our understanding of how PTMs affect PRC function is growing, but far from complete (Niessen et al., 2009; Voncken et al., 2005).

Recent observations indicate that beside transcription, Polycomb-function directly controls replication and DNA-repair (Alchanati et al., 2009; Chagraoui et al., 2011; Ginjala et al., 2011; Ismail et al., 2010; O'Dor et al., 2005). Numerous protein factors appear functionally linked to transcription, replication as well as DNA-repair; the protein complexes involved are thought to be required for coordinated regulation of these DNA-templated processes (Dinant et al., 2008; Dinant et al., 2009). Whether and how these chromatin-based Polycomb-functions relate to one another and how these diverse roles are coordinated is insufficiently clear.

We previously identified MK3/MAPKAPK3/3pK and active ERK as functional binding partners of PRC1 downstream in the context of mitogenic signaling (Prickaerts et al., submitted; Voncken et al., 2005). To gain more insight into the role of PRC1 in stresssignaling, we set out to determine how cell-stress regulates PRC1-complex composition and binding of associated partners. To this end cells were exposed to arsenic (As) and a tagged-BMI1 construct was employed to purify and examine the BMI1-associated proteome by mass spectrometry. We identified the chromatin-associated KRABassociated protein, KAP1 (also known as TRIM28/TIF1ß/KRIP-1) as a stress-induced interaction partner of BMI1. The possible biological relevance of KAP1/PRC1 interaction is discussed in the context of current insights in chromatin biology.

\section{RESULTS}

\section{Cell stress-induced interaction of BMI1 and KAP1}

To identify changes in Polycomb Repressive Complex 1 (PRC1) composition upon cell stress, mass-spectrometric analysis of immune-precipitated (IP/MS) BMI1 was performed on control and stressed U2-OS osteosarcoma cells. To reduce interference by cell cycle-dependent phosphorylation of PRC1-proteins, all cells were serum-starved to induce G0/G1-arrest prior to stimulation throughout this study, unless indicated otherwise (Voncken et al., 1999). 
Figure 1

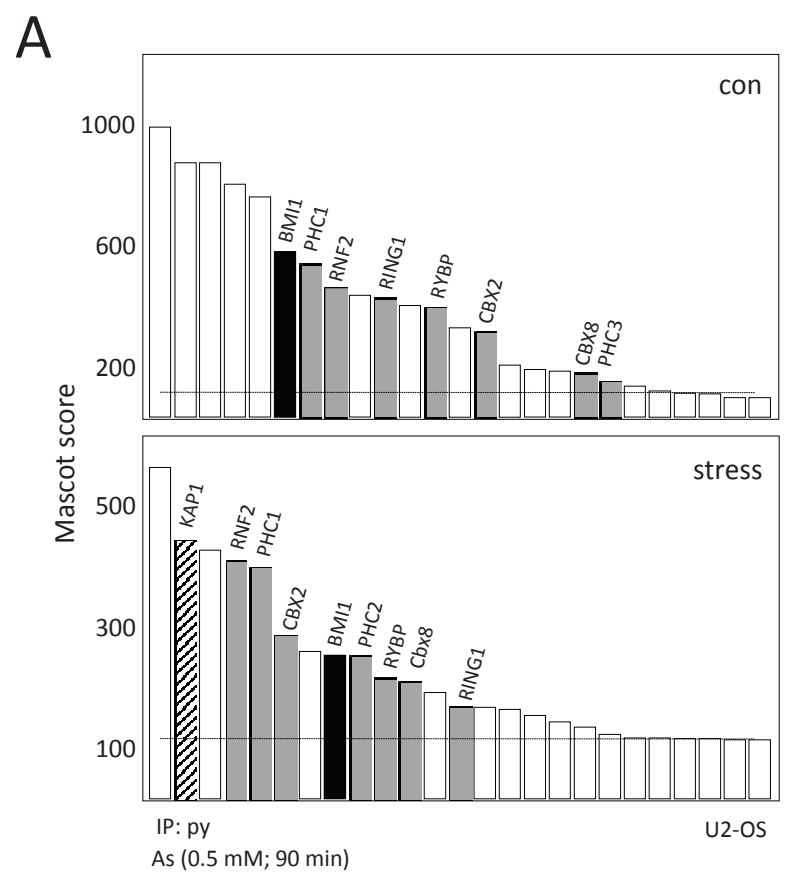

B
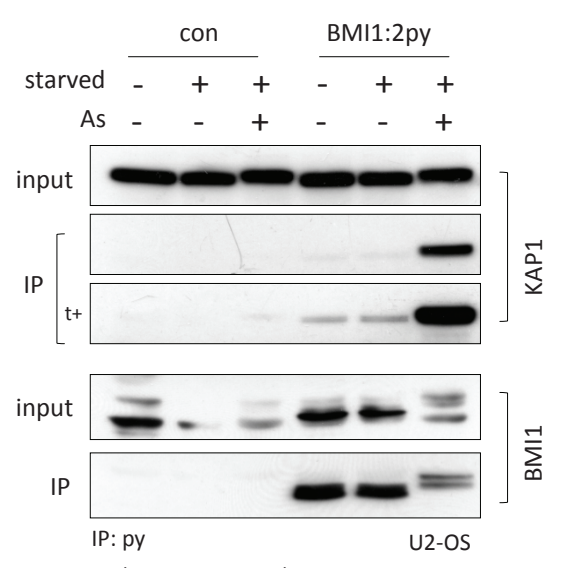

As (0.5 mM; $75 \mathrm{~min})$

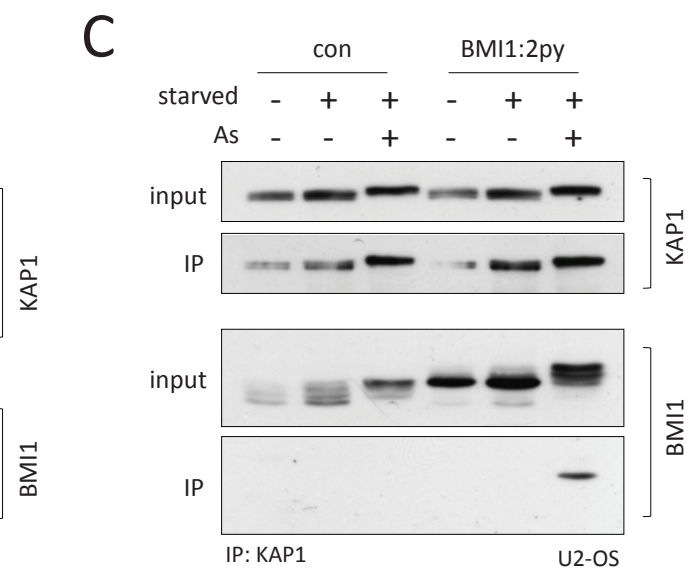

As (0.5 mM; $75 \mathrm{~min})$

Figure 1 Stress induced KAp1/BMI1 interaction. A: representative illustration of 1 of 3 IP/MS results; Mascot scores of < 100 were not included. B-C: reciprocal immunoprecipitation (IP)/immunoblot (IB) analysis confirms KAP1/BMI1-interaction in U2-OS cells expressing Bmi1:2Py. Cells were treated with Arsenic (As) as indicated; IP/IB was performed as indicated; $t+$ : longer apposition of IB membranes to photo-sensitive film. 
To induce stress-signaling PRC1-proteins cells were exposed to arsenite (As), a stimulus that induces rapid phosphorylation of BMI1 and other PRC1-proteins (Voncken et al., 2005). We used polyoma virus antigen-tagged murine BMI1 (BMI1:2Py) to purify BMI1, to detect phosphoryl-residues in BMI1 and BMI1-associated proteins and to identify potential novel stress-induced interactors of PRC1. Consistent with published data, most known mammalian PRC1-members including PHC, RING, RYBP and CBX proteins interacted with tagged BMI1, in control and stimulated cells, which validated the MS-IP approach (Fig. 1A). We identified 11 novel phospho-sites on CBX8, PHC1, PHC2, PHC3, RING1 and BMI1, in addition to a number of previously reported phospho-sites. $A$ number of additional phosphorylated peptides were detected, for which the exact phosphorylation sites could not be determined (not shown). As the applied detection method was non-quantitative, any relative changes in phosphorylation at the detected residues could formally not be excluded. In addition, many PRC1 members contain potential phosphorylation sites in relatively arginine- $(R)$ and lysine (K)-rich stretches; these were typically lost during the tryptic digestion. We published a full overview of currently known and novel PRC-related phosphoryl-residues elsewhere (Niessen et al., 2009). The above data confirms that PRC-proteins are phosphorylated and identifies several phosphoryl-sites on a number of PRC1 members.

Although IP/MS-detection of PRC1-complex members remained largely unchanged upon As treatment, KAP1 (TRIM28/TIF1ß/KRIP-1) was consistently detected as a novel strongly-associated factor in stressed cell (Fig. 1A; suppl Table S1). The As-induced KAP1/PRC1-association was confirmed by co-immunoprecipitation (co-IP) (Fig. 1B). The interaction was validated by reciprocal IP against KAP1, which co-precipitated BMI (Fig. 1C). In addition, the stress-induced KAP1/BMI1-association was reproduced in cervical carcinoma cells (HeLa) and primary human fibroblasts (TIG3), using other expression vectors, different tags and a different PRC1-gene; combined these experiments confirmed the KAP1/BMI1-interaction in multiple cell lines and excluded potential interference by peptide tags (suppl Fig. S1A-E). The higher Molecular Weight (hMW) forms of BMI1 and KAP1 in As-stressed cells represent phosphorylated proteins, as we reported before (Fig. 1B,C) (Voncken et al., 2005). As-induced phosphorylation and KAP1/BMI1-association increased over time and as a function of stressorconcentration. Next to As, Selenium (Se) also potently induced the KAP1/BMI1association (Fig. 2A; suppl Fig. S2A).

Figure 2. Stress-induced post-translational modification of KAP1 and BMI1. A, IP/IB-analysis of the KAP1/ PRC1-interaction and stress markers in Se-stressed cells. B-E, detection of KAPp824 and BMI1-modification in cells exposed to ionizing radiation (IR; B), UV light (C), Se (D) and As (E). Cells were treated with as indicated. IP/IB was performed as indicated. 
Figure 2

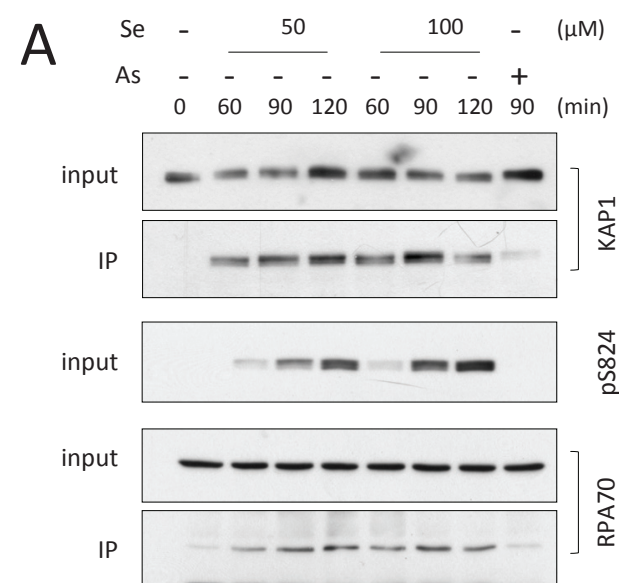

B
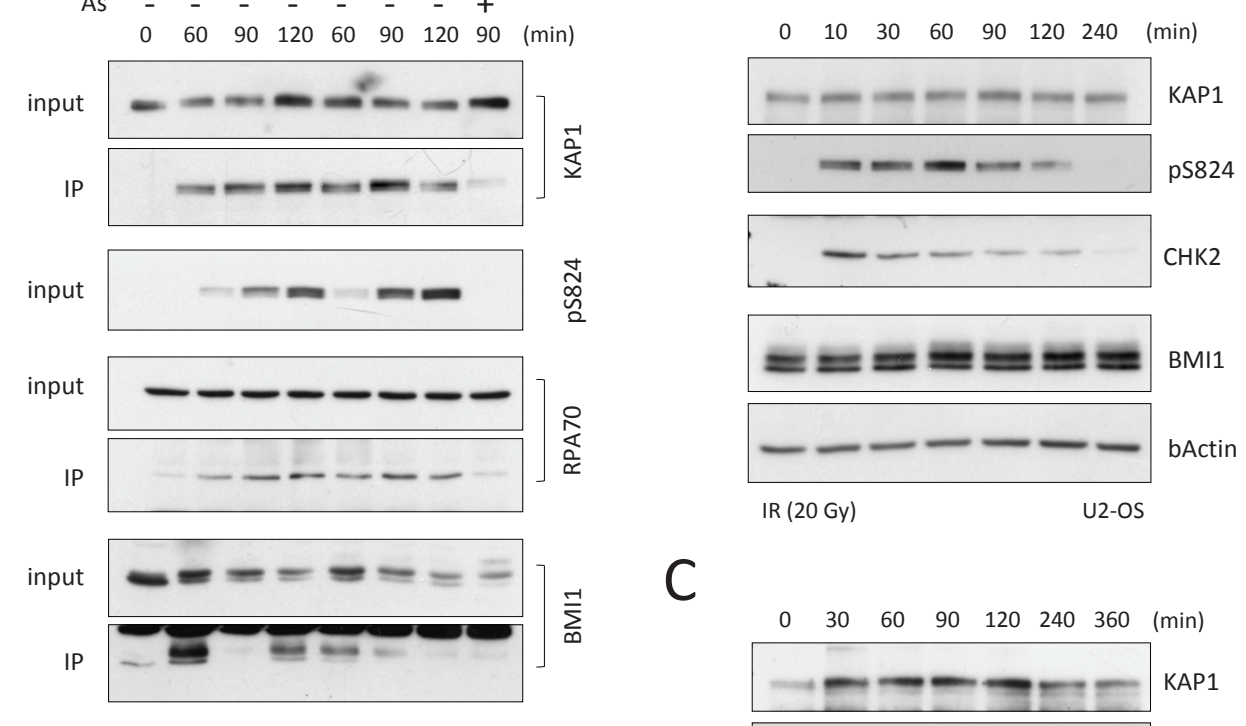

C
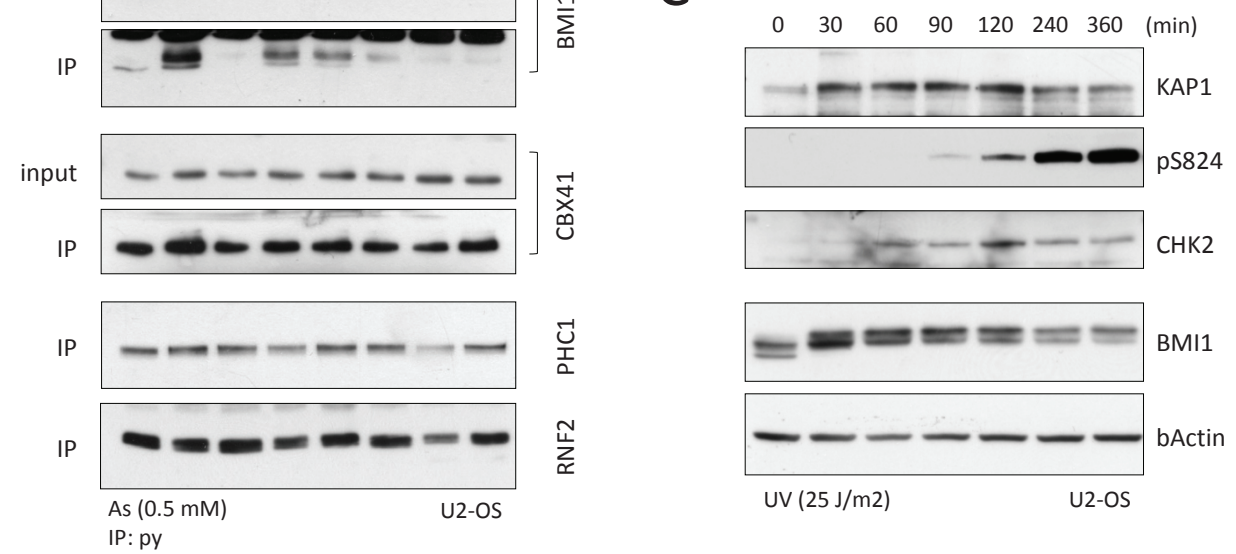

D

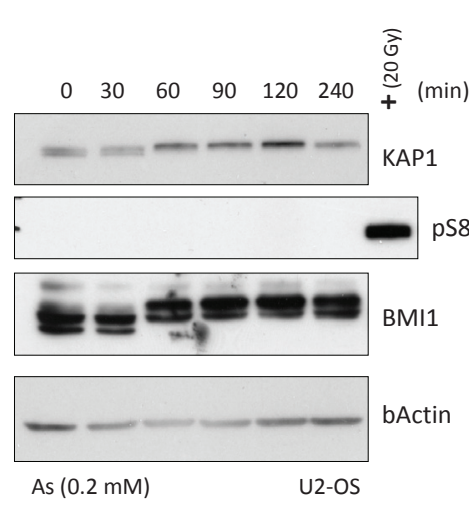

E

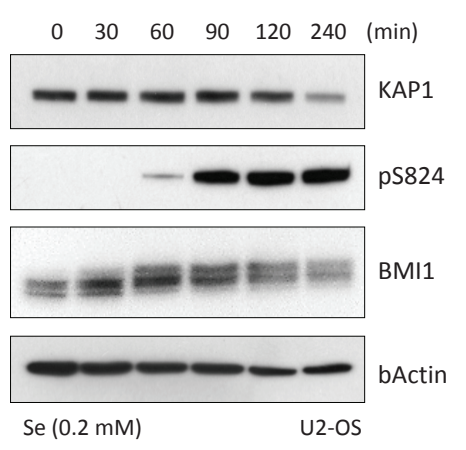


The Se-induced association between BMI1 and KAP1 was time- and concentrationdependent, as was the case for As-induced interaction (suppl Fig. 2B). In good agreement with our IP/MS findings and our previous observations (Voncken et al., 2005), the association of BMI1 with CBX4 appeared largely unaffected, although PRC1protein stability appeared to become compromised at higher As- or Se-concentrations and after longer exposures ( $c f$. Fig. 2A, suppl Fig. S2A, B). Taken together, this data demonstrates that PRC1 and KAP1 associate upon in stressed cells and that this correlates with post-translational modification of the proteins involved.

\section{KAP1/BMI1-interaction correlates with post-translational modification}

To gain insight into the relevance of phosphorylation for the PRC1/KAP1association, co-IPs were subjected to protein phosphatase (PP-ase)-treatment, vigorously washed and then loaded for analysis. Dephosphorylation restored the As-related slower migration of BMI, KAP1, CBX4 and PHC1 to that observed in non-stressed cell extracts (suppl Fig. S2C), confirming that phosphorylation was the major visible PTM under these conditions and validating catalytic PP-ase activity. Analogously, the Se-induced KAP1/BMI1-association was not disrupted by PP-ase treatment (suppl Fig. S2D). Of note, removal of phosphoryl-groups by PP-ase treatment of the co-immunoprecipitated (co-IP) complexes did not affect interaction between BMI1 and KAP1 or other PRC1 members (suppl Fig. S2C).

To chart physiological conditions that induced PRC1 and KAP1 phosphorylation in more detail, U2-OS cells were subjected to several stressors, including arsenite (As), selenite (Se), ionizing radiation (IR) or ultra violet (UV) treatment. Exposure to As, Se or UV induced multiple phosphoryl-BMI1 subspecies, whereas IR did not (Fig. 2B-E). Whereas As-exposure readily induced KAP1-phosphorylation (detectable as hMW KAP1-protein), this was less evident for UV, Se and IR treatment. Se, IR and UV, but not As, specifically induced phosphorylation of KAP1 at Ser824 (KAP1pS824) (Fig. 2B-E). This site was previously shown to be phosphorylated by ATM in response to double strand DNA breaks (DSBs) (Goodarzi et al., 2008; White et al., 2006; Ziv et al., 2006). Consistent with this, $\mathrm{PCHK} 2$ is readily detectable in response to IR and UV treatment (Fig. 2B, C). In contrast to As, Se induced KAP1pS824 (suppl Fig. 2A; cf. suppl Fig. S6B-D); Of note, a concentration-dependent association of BMI1 with RPA70, a molecule that binds single- stranded DNA (sSDNA), was observed in response to Se and As (Fig. 2A). KAP1pS824 detection in co-IPs was not consistent and the signal was usually very weak ( $c f$. suppl Fig. S2A, D; Fig. 4A). This suggested that KAPpS824 is neither the main KAP1-form that interacts with BMI1, nor a prerequisite for KAP1/BMI1-interaction (suppl Fig. S3A). Exposure to IR, UV or camptothecin (CPT) did not produce the KAP1/BMI1-association (suppl Fig. S3E-G) under the experimental 
conditions used. This meant that either the appropriate response-signal may have not been activated, or that the interaction was too weak to detect. KAP1-phosphorylation at Ser473 (KAP1pS473) relates directly to the transcriptional repressive function of KAP1 (Chang et al., 2008). Consistent with this notion, KAP1pS473 was potently induced by UV; the kinetics of S473-phosphorylation paralleled the decrease in migration speed of total KAP1 (Fig. 2C). KAP1pS473 was induced to a lesser extent by As and Se, compared to UV, and virtually not by IR within the time-span of the experiment (suppl Fig. S3A-D). Thus, our data confirmed that both BMI1 and KAP1 proteins are phosphorylated at multiple residues, that phosphorylation is stimulus-specific.

We next studied the contribution of signaling pathways to the stress-induced BMI1/KAP1-association. In the context of cell stress, including DNA damage responses (DDR), ATM activation is a crucial early event (Ditch and Paull, 2011). Our recent studies showed that the stress-induced phosphorylation of BMI1 is p38-dependent (Prickaerts et al., submitted; Voncken et al., 2005). We therefore used inhibitors of p38 (SB202190; p38i) and ATM (caffeine; ATMi) to probe for any effect of interruption of signaling via these kinases on the KAP1/BMI1-interaction. Arsenite or selenite-induced phosphorylation of BMI1 was dependent both on p38 and ATM-signaling, as the protein migrated faster in inhibitor pre-treated extracts (Fig. 3A,B); combined p38i/ATMipretreatment further reduced the migration-size of $B M I 1$, suggesting that these pathways function independently in BMI1-phosphorylation. Combined kinase inhibitor pre-treatment also affected KAP1-migration, independent of the stressor used. P38i and ATMi had no negative effect on the As-induced BMI/KAP1-association (Fig. 3B). In sharp contrast, p38i and/or ATMi strongly reduced the BMI1/KAP1-association in Se-stressed cells (Fig. 3A). Pretreatment of cells with additional kinase inhibitors revealed different kinase-dependency profiles in regards to Se or As-stress induced association. Inhibitors of PKCi (staurosporin), JNKi (SP600125) reduced BMI1- and KAP1phosphorylation in response to As as well as Se (suppl Fig. S4A, B). Treatment with PKCi or JNKi enhanced the stress-induced association between BMI1 and KAP1 (suppl Fig. S4A, B); GSK3ßi ( $\mathrm{LiCl}$ ) reduced KAP1/BMI1-association independent of the stressor used. Inhibitors for NFkB and PI3K-AKT/PKB neither affected BMI1 or KAP1 phosphorylation nor interaction (data not shown). Consistent with the absence of pS824 in co-IPs, S824 phosphorylation and KAP1/BMI1 co-IP inversely correlate (suppl Fig. S4Aa). Thus phosphorylation-dependent association of BMI1 and KAP1 in As or Se-stressed cells, showed an apparent differential dependency on p38 and ATM, supporting an involvement of one or more phosphorylation-events in regulating the BMI1/KAP1-interaction induced by As or Se. 


\section{Figure 3}

A

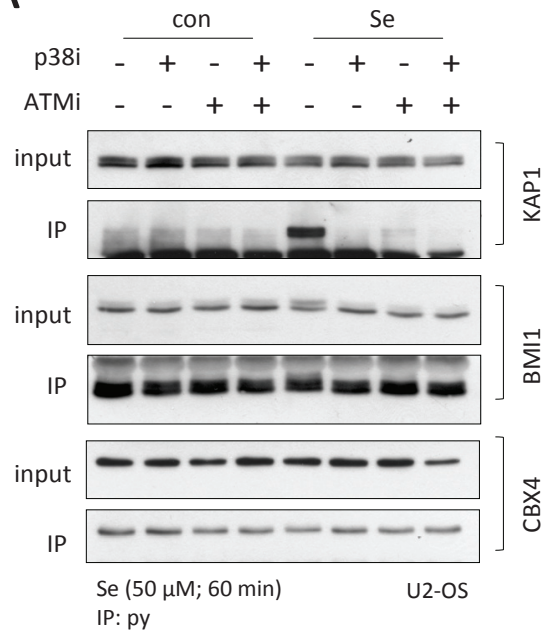

B
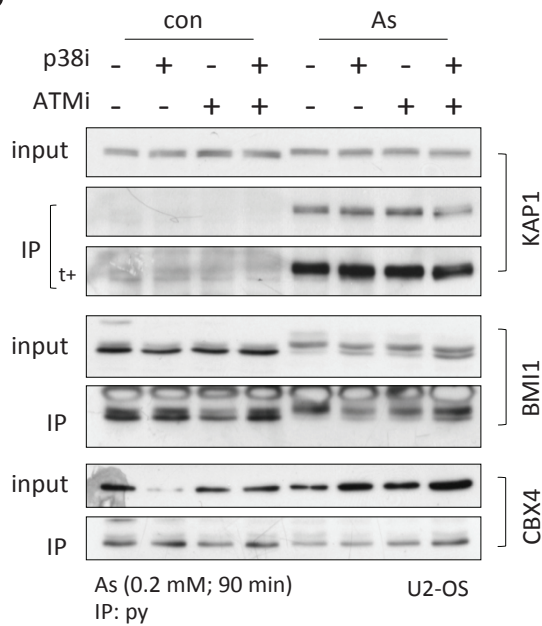

C

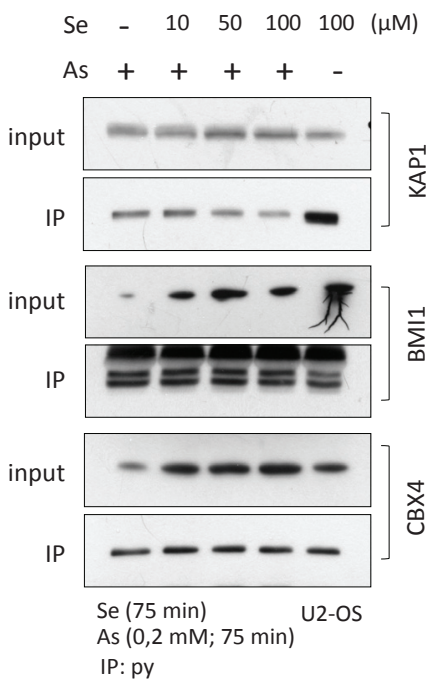

Figure 3. Se, not As-induced KAP1/BMI1-interaction is mediated by SAPK/p38 and ATM. A, Reduced KAP1/ BMI1-interaction by inhibition of p38/SAPK and ATM in Se-stressed cells. B, p38/SAPK or ATM-inhibition do not affect KAP1/BMI1-association in As-exposed cells. C, As counteracts Se-induced KAP1/BMI1-association. Cells were treated with As or Se as indicated. IP/IB was performed as indicated; $t+$ : longer apposition of IB membranes. 
Arsenite and selenite have been reported to mutually counteract their effects on cells via antagonistic effects on cellular signaling (Zeng et al., 2005). Of note, simultaneous exposure of cells to As and Se reduced the intensity of the BMI/KAP1-association compared to that observed with As only (Fig. 3C). This data is consistent with an inhibitory action of As on Se-induced signaling. Remarkably, we did not detect any consistent effect, neither by stressor nor by kinase-inhibitor pretreatment, on the interaction between PRC1 core-complex members BMI, CBX4, PHC1 and RNF2. The collective data above point toward a complex, differential involvement of various stress-defined signaling cascades and PTM-events in initiation of the PRC1/KAP1association and suggest that the maintenance of interaction between PRC1 and KAP1, once established, may not be not solely dependent on phosphorylation, in vitro.

\section{KAP1 and BMI1 are recruited to chromatin in stressed cells}

The enhanced interaction between PRC1 and KAP1 prompted us to determine in which cellular compartment the PRC1/KAP1-association occurs. To this end separate cytoplasmic, nuclear-soluble and chromatin-bound fractions were isolated in resting, Se-stressed U2-OS cells; mitogen-stimulation was taken along to determine stimulus-specific responses. Nucleoplasmic and KAP1 and BMI1 were predominantly found in the cytoplasmic and the soluble nuclear fraction in G0/G1-arrested (starved) cells; a proportion of KAP1 and BMI1 were associated with chromatin at rest (Fig. 4Aa). Compared to the mitogen-enhanced chromatin-binding of KAP1, Se-exposure triggered massive KAP1/chromatin-accumulation; the simultaneous depletion of cytoplasmic KAP1 suggested that KAP1 was recruited to the nuclear compartment upon signaling (Fig. 4Aa); alternatively, KAP1 may become targeted for degradation in the cytoplasm. KAP1S824-phosphorylation occurred only in response to Se, whereas pS473 increased in response to both Se-stress and mitogenic stimulation (Fig. $4 \mathbf{A b}, \mathbf{c}$ ). The near complete absence of cytoplasmic KAP1pS824 in Se-stressed cells is consistent with KAP1S824-phosphorylation being a nuclear event, and is in line with its role in DNArepair. Chromatin-accumulation of both phosphoryl-forms of KAP1 correlated well with the robustly increased total KAP1/chromatin-binding in response to Se (Fig. 4Aa-c). The comparatively lower KAP1pS473/chromatin-accumulation in mitogen-stimulated cells, despite comparable KAP1pS473 levels in the cyto- and nucleoplasmic fractions, pointed to the requirement of additional events for KAP1pS473/chromatin-binding in Se-stressed cells (Fig. 4Ac). Parallel to KAP1/chromatin-binding, a strong increase of chromatin-bound BMI1 was observed in Se-stressed nuclei; BMI1, like KAP1, appeared to be recruited from cytoplasmic stores (Fig. 4Ad). The relatively hyper-phosphorylated fraction of BMI1 was found to reside in the nucleus, also in starved cells, suggesting 
that phosphorylation is linked to nuclear residence (Fig. 4Ad). In As-exposed cells similar observations were made: KAP1 and BMI1 accumulated on chromatin in response to stress (suppl Fig. S5A). 90 minutes following Se-exposure, when apparent phosphorylation of BMI1 and KAP1 reached a maximum (cf. Fig. 2E), KAP1 and BMI1 were found to interact in the soluble and bound nuclear fraction (Fig. 4Aa) suggesting the interaction is a nuclear event. BMI1 and CBX4 interacted mainly in the nuclear fractions; in line with above findings, the BMI/CBX4-interaction was only marginally affected by Se or mitogen-exposure (Fig. 4Ae). Consistent with our previous observations, KAP1pS824 appeared excluded from interaction, whereas the KAP1pS473 co-immunoprecipitated with BMI1 (Fig. 4Ab, c). These observations suggest that multiple functionally distinct nuclear KAP1-compartments exist: KAP1pS473 appears to engage PRC1, KAP1pS824 does not. Combined these findings suggested that KAP1 and BMI1 are recruited from the cytoplasm to the nuclear compartment, where interaction occurs and chromatin-binding of both proteins increases.

\section{KAP1-RINGfinger/B-box/coiled-coil domain mediates interaction with PRC1; KAP1- Ringfinger mediates BMI1-degradation}

We next asked which of the KAP1-domains was responsible for the association with BMI1. To this end we constructed various FLAG-tagged KAP1-mutants: a RINGfinger mutant (lacking the RF-domain; KAP1-deltaRF), a N-terminal mutant (harboring $\mathrm{N}$-terminal sequences including the $\mathrm{RF}$, a double B-box and the coiled-coil domain; KAP1-RBCC), and a C-terminal mutant (expressing only the PHD and BROMO-domain: KAP1-PHDBROMO) (suppl Fig. S6A). Apparently, a putative nuclear localization sequence $(n / s)$ in the region between the $C C$ and the $P H D$ domains was inadvertently removed from some constructs, as the truncated proteins were only expressed in the cytoplasm, not in the nucleus (suppl Fig. S6C; $c f$. Materials and Methods section). To permit interaction studies, we fused a synthetic nls to the FLAG-tag (cf. Materials and Methods section); all Flag:nls proteins were expressed in the nucleus at their expected size (suppl Fig. S6D). Although the synthetic-n/s overexpression approach was likely to interfere with studies on regulated translocation of KAP1, it enabled us to ask which KAP1-domain(s) participates in protein-interaction.

Figure 4. KAP1 and BMI1 are recruited to chromatin in stressed cells. A, G0/G1-arrested cells were stimulated with mitogens or selenium for the indicated amount of time. Cells were fractionated in cytoplasmic (cyto), soluble nuclear (sol nuc) and chromatin bound (chrom) fractions used for co-immunopreciptation analysis. Soluble nuclear and chromatin-bound fractions were proportionally loaded; cytoplasmic fractions 3-4x less. Panels a-e correspond to grouped input and IP samples; panel f, shows loading controls. IP/IB was performed as indicated; $\mathrm{t}+$ : longer apposition of IB membranes. 
Figure 4

A
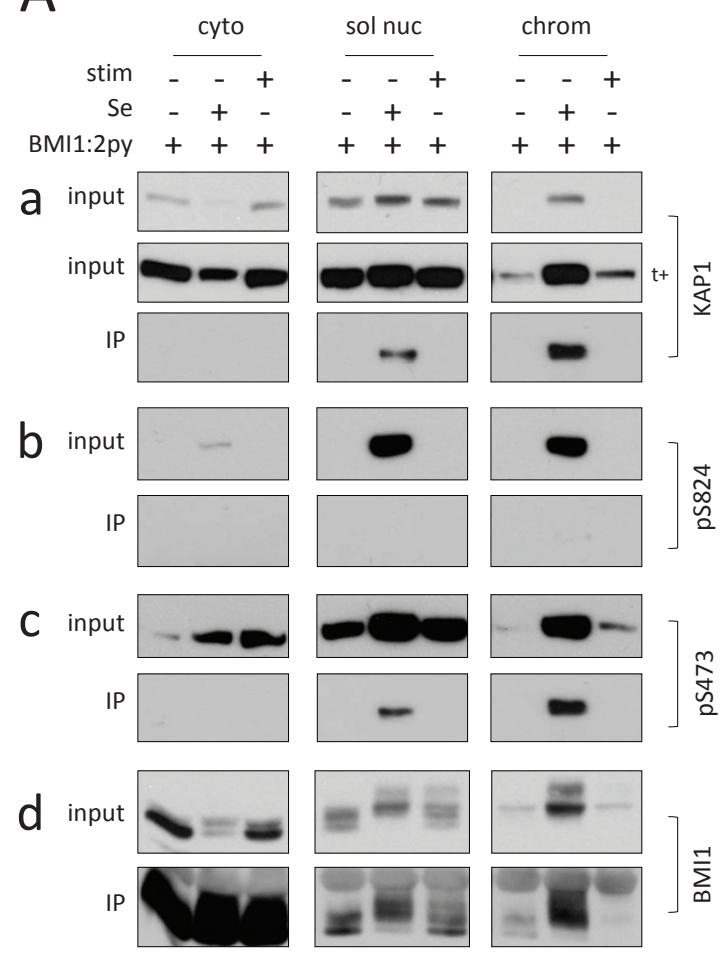

e input
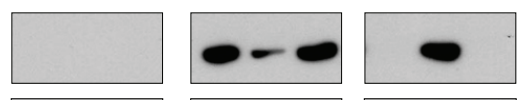

IP
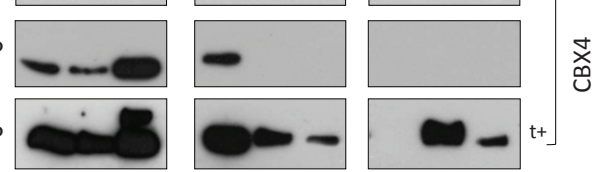

f

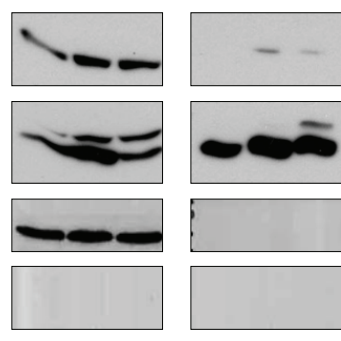

stim (serum/TPA, $45 \mathrm{~min}$ ) Se $(50 \mu M, 60 \mathrm{~min})$
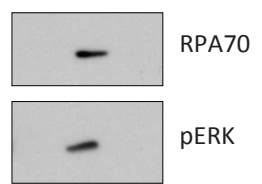

IP: py

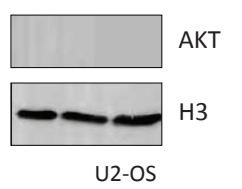


Flag:nls/KAP1-RBCC co-precipitated endogenous KAP1 (endoKAP1), as did Flag:KAP1deltaRF, confirming that KAP1 homodimerization is dependent on sequences included in the RBCC-domain (suppl Fig. S6D; panel: IP: Flag/IB: KAP1). Interestingly, the isolated KAP1-PHDBROMO-domain interacted with endoKAP1, but only in the context of cellstress (suppl Fig. S6D; panel: IP: Flag/IB: KAP1). This suggests that the PHDBROMOdomain provides an additional stress-induced protein-binding interface, and that multiple KAP1-domains may be involved in KAP1-multimerization. The isolated PHDBROMO-domain, which comprises the S(824)QE-motif, was subject to spontaneous S824-phosphorylation under control (non-stressed) conditions (suppl Fig. S6D, B; panel: input/IB: KAP1pS824), suggesting $\mathrm{N}$-terminal KAP1 sequences may function in regulation of S824-phosphorylation. Likewise, overexpression of Flag.flKAP1 appeared sufficient for S473-phosphorylation, even in resting cells (suppl Fig. S6D; panel: input/IB: KAP1pS473).

All full-length or truncated Flag:KAP1-proteins, except Flag:nls/KAP1-PHDBROMO, co-precipitated with BMI1:2Py, demonstrating that the RBCC-domain is likely also important for KAP1/BMI1-interaction (suppl Fig. S6B; panel: IP: Py/IB: Flag). Se induced the expected association between BMI1 and endogenous KAP1 (endoKAP) in control BMI1.2Py cells (suppl Fig. S6D; panel: IP: Py/IB: KAP1). Overexpression of full-length (fI) Flag:fIKAP1 and Flag:nls/KAP1-RBCC interfered with the stress-induced BMI1/endoKAPassociation, whereas Flag:KAP1-deltaRF appeared not to (suppl Fig. S6D; panel: IP: Py/IB: KAP1). Of note: hMW forms of BMI1 were undetectable in the presence of Flag:fIKAP1 (suppl Fig. S6D; panel: input/IB: BMI1; asterisk). These observations suggested some level of competitive binding of RBCC-mediated KAP1 self-association with KAP1/BMI1-binding and/or reduced interaction through loss of interactionpartner.

Given the reduced BMI1 levels in response to Se (cf. Fig. 2A; suppl Fig. S6D) and the fact that KAP1 is a recognized ubiquitin (Ub) E3-ligase (Wang et al., 2005), we next pursued the possibility that BMI1 is degraded by KAP1 in a KAP1/RINGfinger-dependent manner.

Figure 5. KAP1 degrades BMI1 in a RINGfinger-dependent manner. A, Se-induced PTM of BMI1 occurs relatively late; cells were pre-incubated with MG132 (p.i.); NEM was added during cell extraction. B, overexpression of KAP1 induces rapid degradation of chromatin-associated BMI1. White arrowheads point to chromatin-fractions of stressed cells; blocked-out sections correspond to immunoglobulin heavy chains (IgH). C, KAP1 and BMI1 do not depend on each other for chromatin-recruitment. Soluble nuclear and chromatinbound fractions in $(B, C)$ were proportionally loaded; cytoplasmic fractions 3-4x less. D, endogenous KAP1 and BMI1 interact in Se-stressed cells at early time points. Complete nuclear fractions were used for IP; cells were treated with As or Se as indicated; IP/IB was performed as indicated; $t+/ t++$ : longer appositions of IB membranes. 
Figure 5

A

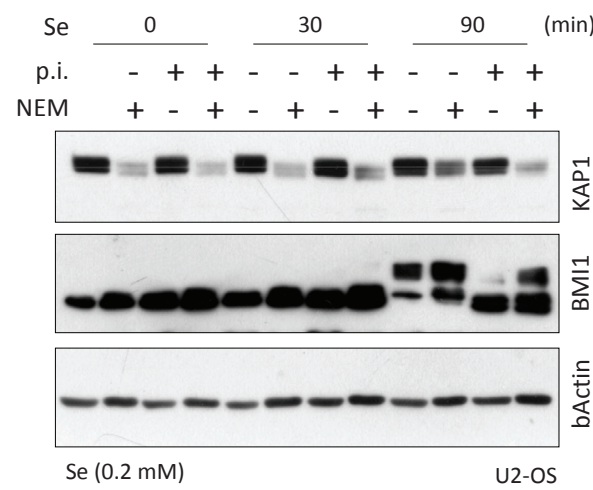

C
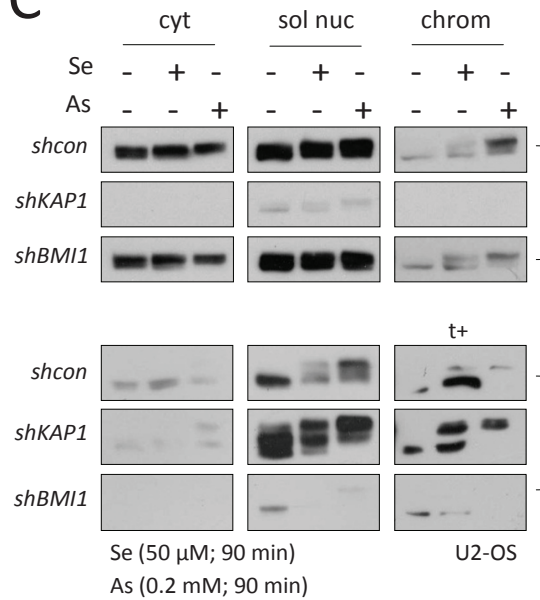

D

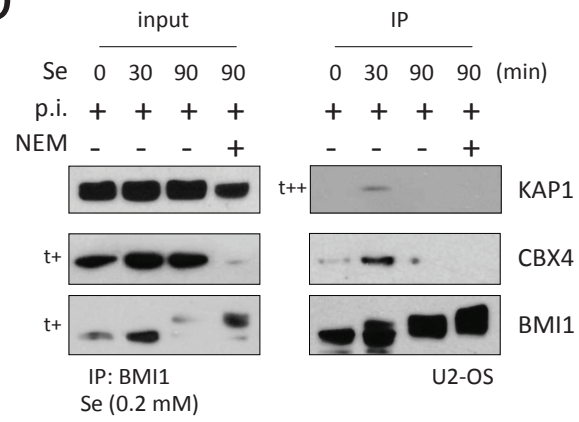

B Flag:full-length KAP1
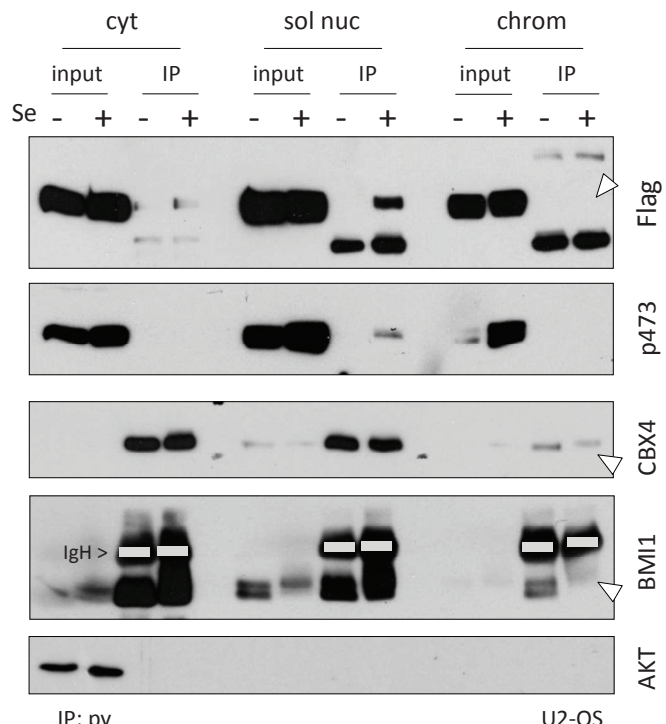

Se $(50 \mu \mathrm{M} ; 90 \mathrm{~min})$

Flag:KAP1-deltaRF
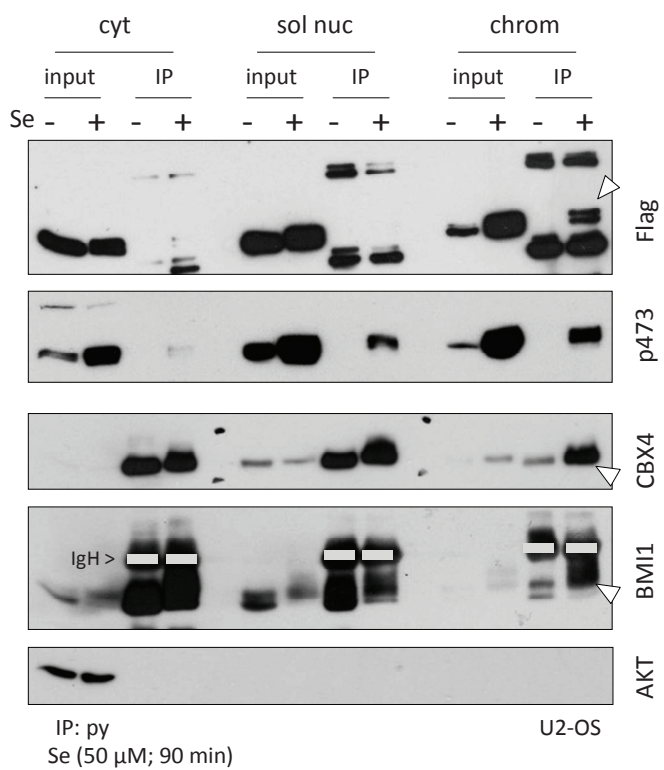
To obtain evidence for the existence of ubiquitylated BMI1-states, cells were stressed in the presence or absence of a proteasome inhibitor (p.i.; MG132) and extracted with lysis-buffers containing a deubiquitylase/desumoylase blocker ( $N$-ethylmaleimide; NEM). Relevantly, Se and UV-treatment induced prominent PTM of BMI1, which are stabilized by inhibition of proteolysis and/or blocking cleavage of the covalently linked Ub/SUMO-moieties; this strongly suggested that BMI1 becomes ubiquitylated in response to Se (Fig. 5A; suppl Fig. S4B). BMI1-ubiquitylation is detected at 90 minutes after Se-exposure or, not at an earlier time-point (30 min; Fig. 5A); given the kinetics of protein-phosphorylation and protein-interaction in response to Se (cf. Fig. 2), this suggested that although interaction is an early event, BMI1-ubiquitylation peaks at a later time-point. We then investigated in which cellular compartment the KAP1/BMI1interaction was established. Cellular fractionation revealed that both Flag:fIKAP1 and Flag:KAP1-deltaRF interacted with BMI1 in the nucleus (Fig. 5B; IP: Py; IB: Flag). Notably: only in Flag:KAP1-deltaRF showed a prominent Se-induced interaction with BMI1 in the chromatin-bound fraction, whereas no interaction was detectable in the presence of exogenous Flag:fIKAP1. Overexpression of Flag:flKAP1 correlated with strongly reduced chromatin-bound BMI1 levels; Flag:flKAP1 and BMI1 were instead found to interact in the nucleoplasm (note the equal BMI1-input levels in the soluble nuclear fraction; Fig. 5B). Relevantly, Flag:KAP1-deltaRF did not affect BMI1 levels, consistent with a role for KAP1 as an ubiquitin-E3 ligase in BMI1 proteolysis. In keeping with KAP1-RF-mediated BMI1 degradation, BMI1-associated CBX4 levels were strongly reduced in stressed Flag:fIKAP1 nuclei, but not in Flag:KAP1-deltaRF (Fig. 5B). The combined above data are consistent with loss of endoKAP1/BMI1-interaction by KAP1mediated BMI1 degradation. To investigate the possibility that KAP1 and/or BMI1 were required for each other's chromatin-recruitment (cf. Fig. 4), stress-induced protein chromatin-binding was studied in the context of RNA-interference-mediated depletion of either one of the interaction partners. Although a clear effect of KAP1 depletion on overall BMI1-levels is observed, neither KAP1 nor BMI1 depletion affected chromatinrecruitment of the other component in response to cell stress (Fig. 5C). In light of above findings, we predicted that interaction between endogenous KAP1 and BMI1 proteins should be detectable in the nucleus, at early time points following Se-induction, in the presence of MG132. Indeed, under these conditions, KAP1/BMI1 interaction was reproduced with endogenous factors (Fig. 5D).

Figure 6. Co-localization of KAP1pS824 and BMI1. A, Immunofluoresence (IF) analysis shows that KAP1pS824 and BMI1-NBs do not colocalize in pan-nuclear staining KAP1pS824 cells; lower panel: representative IF sample used for quantitation. B, KAP1 and BMI1 colocalize in stressed cells at relatively early time points. 
Figure 6

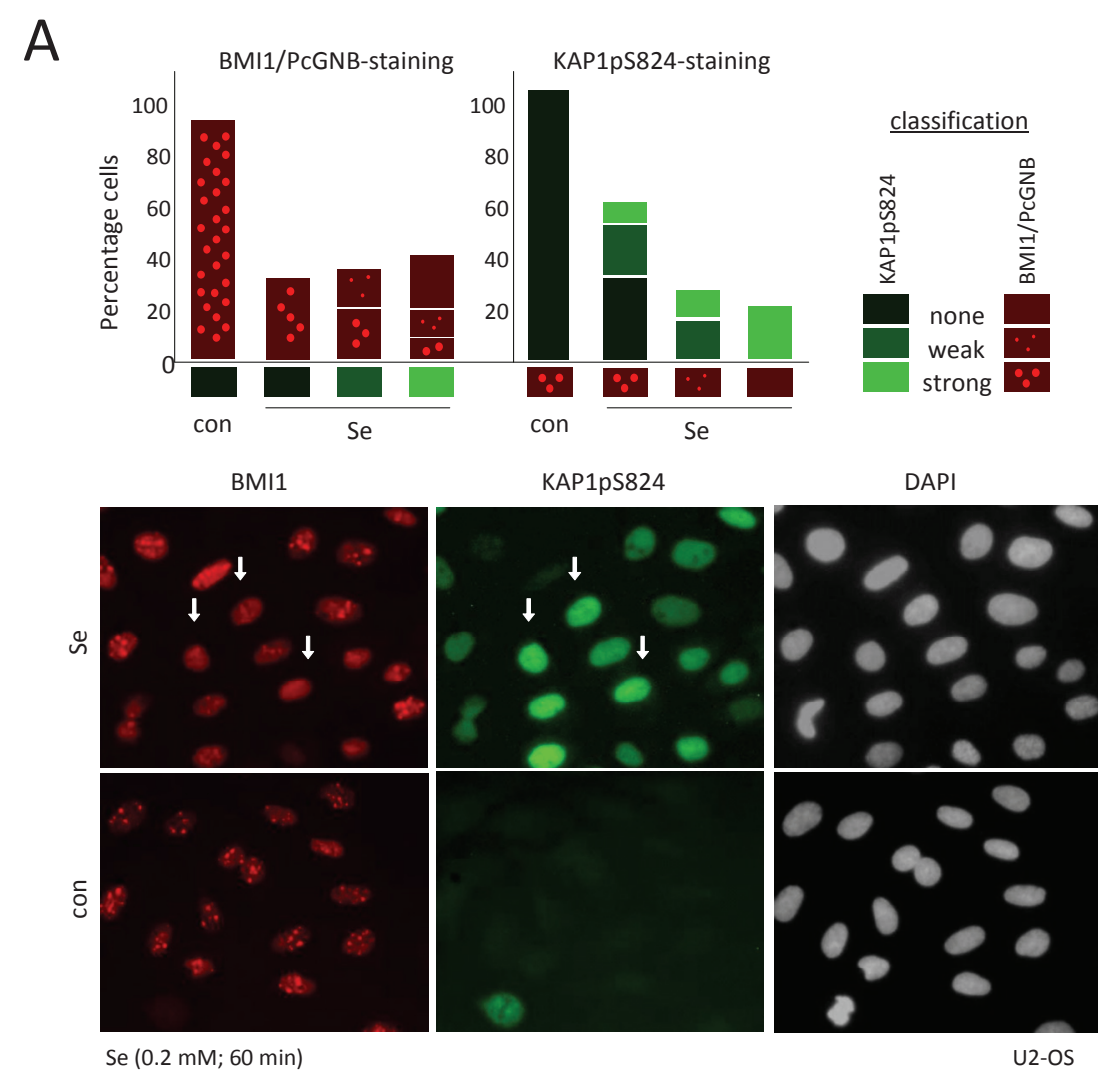

B

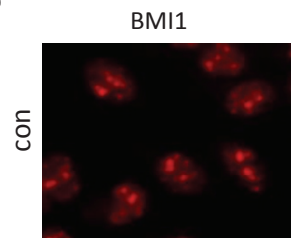

merge

panKAP1

phase contrast
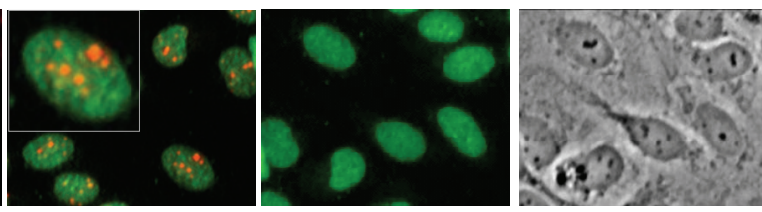

PHC1

merge
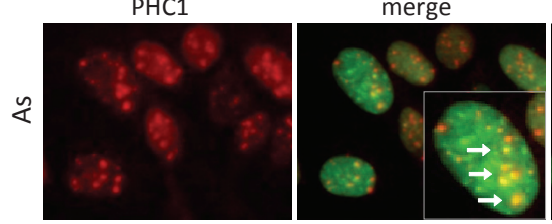

panKAP1

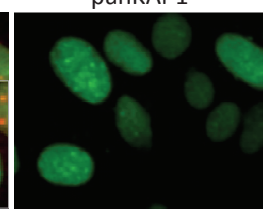

phase contrast

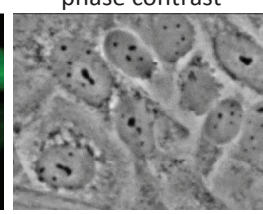

U2-OS 
Importantly this data confirmed the KAP1/BMI1-interaction using non-transduced cells and validated our IP/MS-findings.

\section{Stress selectively targets Polycomb Nuclear Bodies for degradation}

We next used immunofluorescence (IF) analysis to examine global changes in PRC1 and (phosphorylated) KAP1/chromatin-association in respect to each other. Of relevance: we studied endogenous proteins for this purpose. To selectively probe chromatin-associated proteins, cells were fixed in chilled methanol (i.e. all non-bound protein is lost). Control cells showed the characteristic nuclear PRC1 aggregation patterns (Polycomb nuclear bodies; PcG NBs), whereas KAP1 showed global chromatinassociation (Fig. 6A); from this staining pattern no direct association could be inferred under standard growth conditions.

KAP1pS824 and H2A.XS139-phosphorylation ( $\mathrm{HH} 2 \mathrm{~A} . \mathrm{X}$ ) co-occurred (suppl Fig. S7A) in stressed nuclei, consistent with both being ATM-catalyzed events in response to DNA damage (Modesti and Kanaar, 2001; Noon et al., 2010). Cells displaying less intensive pan-nuclear staining for KAP1 were positive for Se-induced KAP1pS824 and yH2A.X, confirming these cells had activated a DDR (suppl Fig. S7A,B). As expected, KAP1pS824 and were blocked by ATM-inhibition (suppl Fig. S7A,B). P38-inhibition partially blocked PRC1-dissociation, but had no discernible effect on KAP1pS824 or $\mathrm{YH} 2 \mathrm{~A} . \mathrm{X}$ (suppl Fig. S7C) (Voncken et al., 2005). Arsenite did not evoke a classic DNA-damage response (i.e. absence of KAP1pS824 and $\mathrm{yH} 2 \mathrm{~A} . \mathrm{X})$ under these conditions. Interestingly, simultaneous exposure of cells to As and Se blocked Se-induced KAP1pS824 and $\mathrm{\gamma H} 2 \mathrm{~A} . \mathrm{X}$ as efficiently as ATMi did, suggesting that As possibly counteracts Se through a mechanism involving ATM (suppl Fig. S7A,B). This notion is consistent with the dominant-inhibitory effect of As over Se ( $c f$. Fig. 3C) and the negative effect of ATMi on Se-induced KAP1/BMI1interaction (cf. Fig. 3A). Relevantly, As-exposed cells also displayed lower P53-levels, in contrast to Se-treated cells; combined p38i/ATMi nearly restored P53-levels in As-stressed cells, whereas it had little/no effect in combination with Se (cf. suppl Fig. S3A,B). This data further supported a central role for p38 and ATM in As- and Se-mediated cellular responses.

In U2-OS cells exposed for 90 minutes to As, disappearance of BMI1-containing PcG NBs correlated with decreased pan-nuclear staining of KAP1 (suppl Fig. S7D). Similar results were obtained in Se-stressed (90 min) nuclei: detection of PcG NBs and KAP1pS824 inversely correlated in Se-stressed cells: approximately half of PcG NBpositive nuclei were weakly/not stained for KAP1pS824, whereas approximately half of the KAP1pS824-positive nuclei lacked PcG NBs (Fig. 6A; cf. suppl Fig. S7E). Of note: a number of nuclei, including those with reduced PcG NBs, showed increased global BMI1/chromatin-associated staining (Fig. 6A,B), consistent with increased BMI1- 
detection in the chromatin fraction (cf. Fig. 4Ad). Relevantly, also global PHC1/chromatin-binding was increased in Se-stressed nuclei, indicating that the Se-induced changes applied to more PRC1-core components (suppl Fig. S7F,G; $c f$. Fig. 4Ad,e). Conversely, the reduced IF-detection of pan-chromatin associated KAP1, despite enhanced KAP1pS824-staining and increased KAP1/chromatin-binding in these cells ( $c f$. Fig. 4Aa-c), suggested that KAP1 may no longer be detectable in its native configuration with the KAP1 antiserum (i.e. due to epitope masking) and/or that most KAP1 is progressively lost from chromatin and that the chromatin-based KAP1/BMI1interaction may become undetectable over time. The data was fully consistent with the above described KAP1-mediated degradation of BMI1. Indeed, at shorter periods after stress-induction, co-localization of endogenous PRC1-complexes with KAP1 was observed in U2-OS cultures (Fig. 6B): numerous cells with relatively high KAP1-staining intensity also showed high global BMI1-chromatin-staining and KAP1/BMI1 co-localization; conversely, cells that displayed less PCG NB/BMI1 and panchromatin/KAP1-association, also showed less co-localization (Fig. 6B). Combined with the cellular fractionation/IP findings (Fig. 4A), these results indicated that the KAP1/BMI1-association is transiently detectable at the chromatin level at early time points following stress, but that at later time points the chromatin KAP1/BMI1association is no longer measureable.

Combined our findings indicated that KAP1 and BMI1 both accumulate on chromatin in response to different cell stressors. Although global PRC1/chromatin binding is initially increased, the typical subnuclear PRC1/chromatin-aggregations, PcG NBs, selectively disappear in response to As or Se stress.

\section{KAP1 co-regulates PRC1-target genes in vivo and in vitro}

To improve our understanding of the biological relevance of KAP1/BMI1interaction, we examined its potential role as a co-regulator of PRC1-mediated transcriptional repression. We first probed a transgenic Drosophila melanogaster model for interaction between Polycomb and bonus (the D.mel KAP1-ortholog) alleles. To this end, $P c^{1}$ and $b o n^{249}$ alleles were inter-crossed to generate a double heterozygous condition for both mutant alleles (Beckstead et al., 2001); in this cross, the effect of $b_{o n}{ }^{249}$ on the classical $P c^{1}$-sexcomb phenotype was evaluated. Typically, wild-type male flies carry one sexcomb on the most proximal tarsal segment of the prothoracic (T1) leg; Polycomb mutants, like $P C^{1}$ display multiple and/or ectopic sexcombs, including on the meso- (T2), and metathoracic (T3) leg (Fig. 7A,B). Bon ${ }^{249} /+$ flies did not show a Polycomb-associated sexcomb phenotype. Notably, however, we found that $P c^{1} / b o n^{249}$ flies showed significantly less penetrance of the sexcomb phenotype than their $P c^{1} /+$ counterparts, indicating that loss of bonus compensates for loss of $P c$. 
Figure 7

A
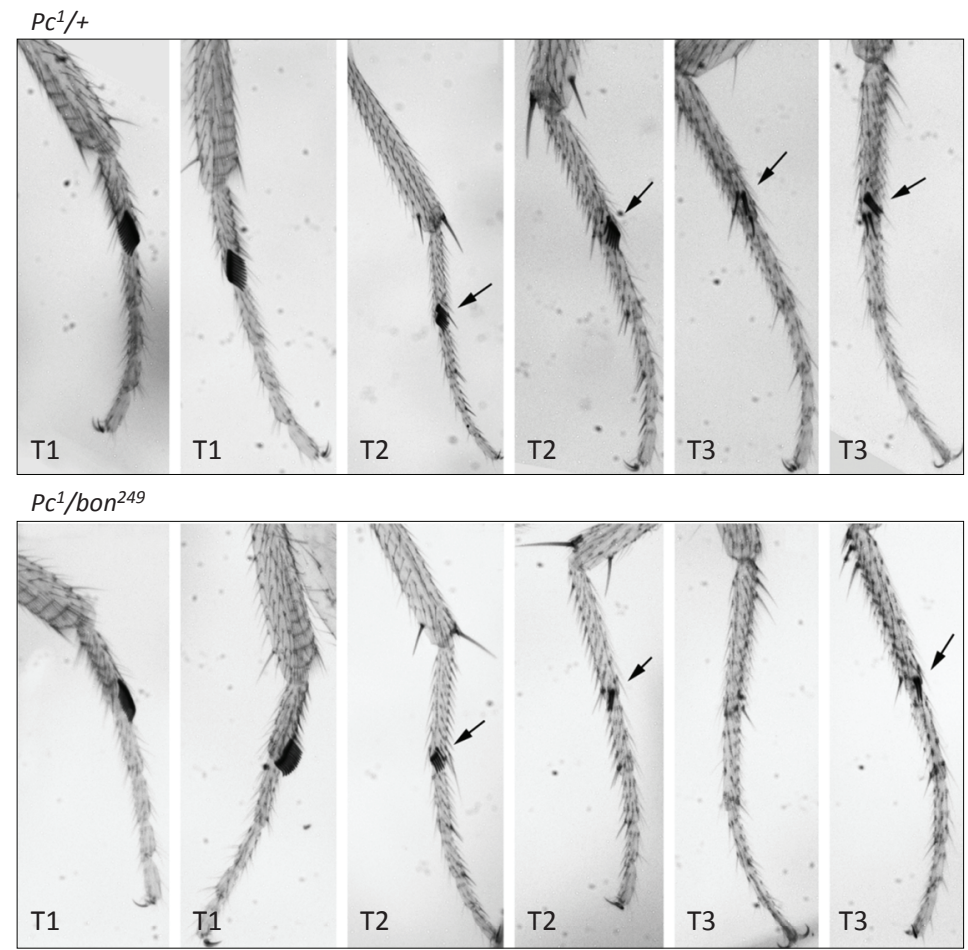

B
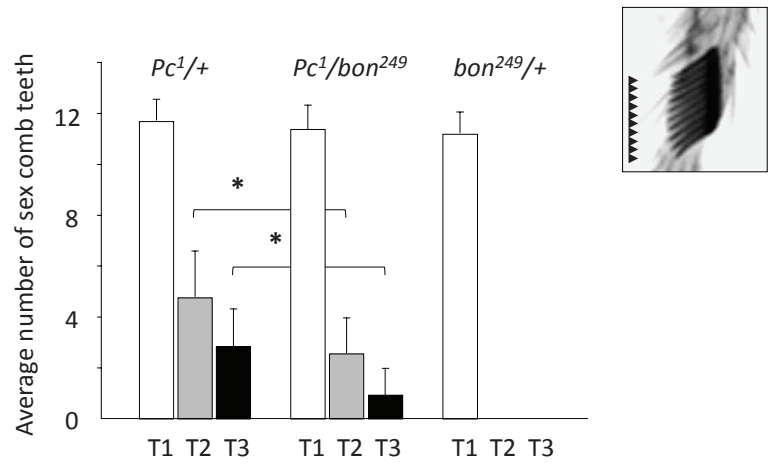

Figure 7. Genetic interaction between bonus and Polycomb in vivo. A, sexcomb phenotype in $P c^{1}$ heterozygous and $P c^{1} / b^{249}$ double-heterozygous flies. B, quantitative representation of the number of sexcomb teeth (inset) displayed on T1, T2 and T3 legs of $P c^{1} /+(n=30), P c^{1} / b o n^{249}(n=22)$ and $b o n^{249} /+$ flies $(n=29) ; .^{*}: P<0,001$; confidence threshold $<0.05$ (Student T-test). 
These data support a genetic interaction between Polycomb and dKAP-function in vivo and are compatible with a potential negative regulatory role for bonus in PRC1-function during AP- patterning (Fig. 7A,B).

We then turned to signaling-induced expression of a PRC1-repressed target-gene as a potential measure for functional PRC1/KAP1 interaction\}. To probe for a possible KAP1-involvement in PRC1-mediated transcriptional repression, we studied the effect of KAP1-depletion on mitogenic induction of ATF3, an immediate early gene (IEG) ATF3 is controlled by PRC1 (Bracken et al., 2006; Prickaerts et al., submitted), using short hairpin RNA (shKAP1). Mitogen-stimulation in KAP1-depleted cells reproducibly increased ATF3-induction approximately 2 fold (Fig. 8A); this finding implicated KAP1 directly in the regulation of PRC1-target gene expression. We have recently found that ATF3-expression in response to mitogenic signaling in primary human TIG3 fibroblasts is controlled by ERK and p38-dependent PRC1/chromatin-dissociation (Prickaerts et al., submitted). KAP1-phosphorylation at S473 abolishes HP1-binding and KAP1-repression at target genes (Chang et al., 2008). In good correlation with this, a rapid, transient increase in KAP1pS473 was observed in our model (Fig. 8B); KAP1-phosphorylation was p38 and MEK/ERK-dependent, as p38i or MEKi substantially reduced, and p38i/MEKi abolished pS473 in response to mitogenic stimulation completely (Fig. 8B). We next examined the effect of inhibitors on ATF3-induction, in the presence or absence of KAP1. P38i or MEKi significantly reduced ATF3-induction in mitogen-stimulated control cells, whereas combined inhibitor treatment blocked ATF3-induction completely (Fig. 8C). MEKi or p38i/MEKi cells both showed a dominant inhibitory action over shKAP1, indicating MEKi affects most likely a process upstream or parallel to KAP1, yet crucial for ATF3-transcription. In contrast shKAP1 compensated for p38i-mediated ATF3induction inhibition, suggesting that p38 is involved in control of transcriptional repression by KAP1. Combined, these findings suggested that p38 may control KAP1/chromatin-association. Indeed, p38-inhibition substantially reduced KAP1S473phosphorylation and KAP1/chromatin-recruitment in mitogen-stimulated cells (Fig. 8D). Basal KAP1/BMI1:2Py-interaction (i.e. non-stressed conditions $c f$. Fig. 1) was increased by mitogen-stimulation (Fig. 8E). Relevantly, p38i further enhanced KAP1/BMI1interaction; similar to the Se-stress situation, this suggested that the KAP1/BMI1interaction took place in the nucleoplasm, where it did not induce proteolytic cleavage. MEKi or P38i/MEKi respectively reduced or nearly completely blocked their association BMI1 clearly accumulated on chromatin when p38 and ERK were both inhibited (Fig. 8D) (Fig. 8E). Combined these results are consistent with a role for ERK and p38 in regulating KAP1/BMI1-interaction and chromatin-recruitment in the context of PRC1target gene ATF3 expression. 
A

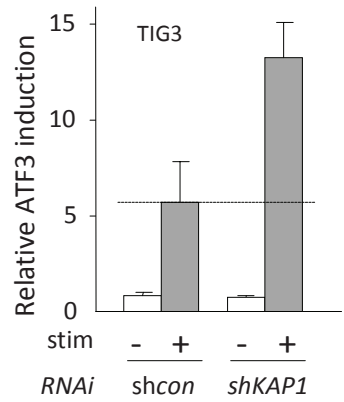

D
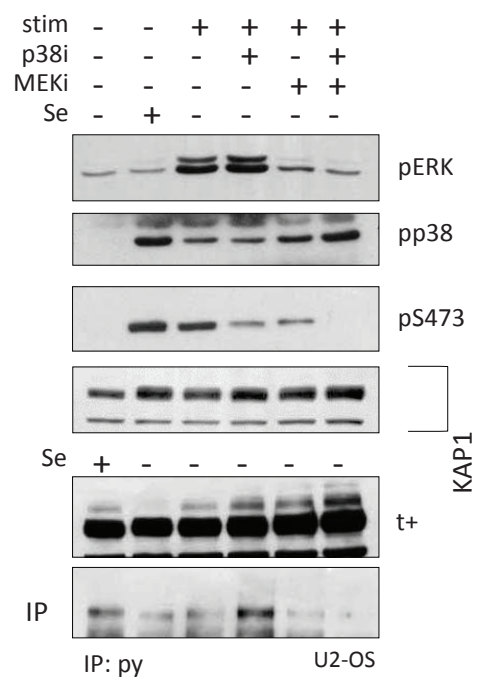

C
B

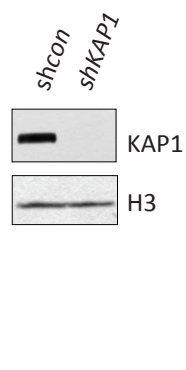

Figure 8

$E$
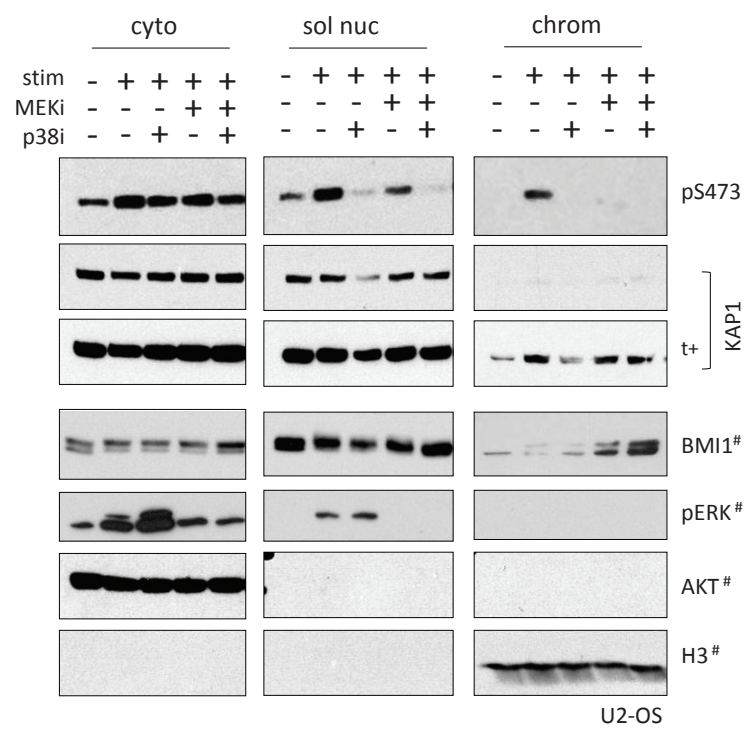

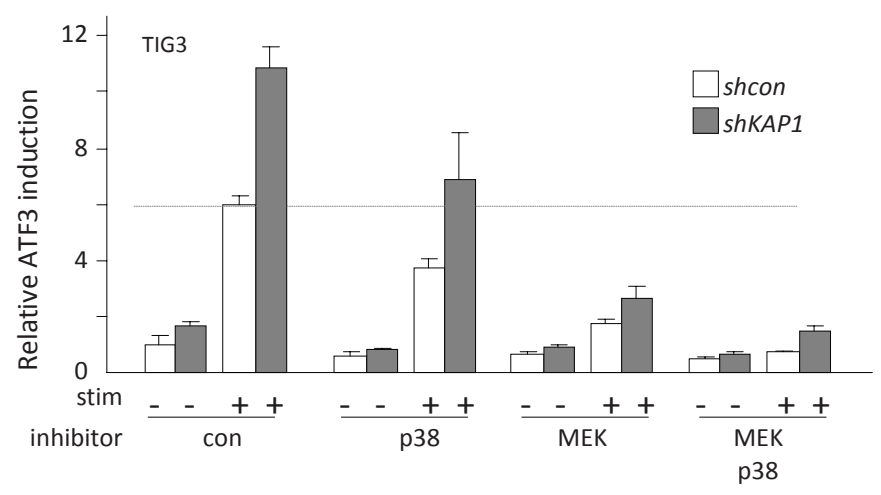


Figure 8. KAP1 co-regulates mitogen-induced expression of the PRC1-target gene ATF3. A, mitogenic signaling-induced ATF3-expression is elevated in shKAP1 TIG3 cells. B, mitogen signaling induces KAP1pS473. C, shKAP1 compensates inhibited p38-signaling in mitogen-induced ATF3-expression. D, p38 and ERK control mitogen-induced KAP1/BMI1-interaction. E, p38 controls chromatin recruitment of KAP1. Mitogen-stimulated cells were fractionated and analyzed for the proteins indicated; \#: data used elsewhere (Prickaerts et al., submitted). Quantitative mRNA measurements were done by real time PCR; cells were treated with serum/TPA for 45 minutes.

\section{DISCUSSION}

We here identify KAP1 (TRIM28/TIF1ß/KRIP-1) as a novel interaction partner of $B M I$. The stress-induced interaction is regulated by several stress-response pathways and leads to chromatin-recruitment of KAP1 and BMI1. We show that minimally the KAP1-RBCC domain is required for the KAP1/BMI1-nteraction and that KAP1 degrades BMI1 in a RINGfinger-dependent manner in the context of cell stress. We provide evidence for genetic interaction of KAP1 and BMI1 in a Drosophila developmental model and show that KAP1 co-regulates mitogen-induced expression of the PRC1target gene ATF3.

\section{KAP1-RBCC domain mediates KAP1/BMI1 interaction}

Tripartite motif (TRIM) family proteins share an N-terminal tripartite motif, the RBCC-domain, which harbors a RINGfinger domain, one or two B-box zinc finger domains and a coiled-coil region. KAP1 harbors several catalytically active, regulatory or binding motifs in its mid and C-terminal portion: a HP1-interaction motif, a PHDBROMO-domain, and a number of functionally defined phosphorylation and SUMOylation sites ( $c f$. suppl Fig. S6A). The RBCC-domain is crucial for KRAB1/KAP1interaction, and for KRAB1/KAP1-dependent transcriptional repression (Friedman et al., 1996; Peng et al., 2000). In addition, RBCC mediates self-association of KAP1 into 'homo' or 'hetero'trimers with truncated KAP1-proteins, as long as the RBCC-domain is included (Peng et al., 2000). Our findings are consistent with these reports and show that KAP1/BMI1 interaction also requires an intact $R B C C$-domain. Although the exact molecular details of KAP1/BMI1-interaction are not completely understood, enhanced detection of KAP1 with overexpressed BMI1 (in our IP/MS- and co-IP-setting) and the resistance of the KAP1/BMI1-association to high stringency washing $(1.5 \mathrm{M} \mathrm{NaCl})$ supported a direct interaction. The phosphatase-resistance of the interaction suggests that once established the interaction may be sustained by additional PTMs and/or structural features. RINGfinger modules are interaction domains (Peng et al., 2007). Based on current data, we cannot formally define the role of the RF in BMI1-binding, as BMI1, in analogy to KRAB1, may bind 'hetero'multimers of endoKAP1 and Flag:KAP1- 
deltaRF. Nonetheless, the observation that KAP1 degrades BMI1 in a KAP1-RINGfingerdependent fashion, suggests that the respective RINGfinger domains partake in interaction. It is interesting to note that we detected two additional BMI1-associated TRIM motif proteins by IP/MS: TRIM21/RO52 and TRIM25/Efp (data not shown). Both proteins are UbE3-ligases with established roles in cell cycle regulation, apoptosis and other processes (Espinosa et al., 2006; Nalepa and Wade Harper, 2003; Sabile et al., 2006; Ueyama et al., 2010). In addition, binding between PcG-proteins and a TRIM protein was described before. RET finger protein TRIM27/RFP interacts via its coiled-coil region with Enhancer of Polycomb (EPC) (Shimono et al., 2000). Although it is tempting to speculate that PRC1-association with this protein family may more widespread, whether and how these TRIM proteins connect to PRC1-function requires further investigation.

\section{Stress-induced KAP1/BMI-interaction}

The semi-metallic elements Arsenic (As) and Selenium (Se) are required in (ultra)trace amounts in humans; both compounds form toxic oxoanions which induce cell-stress and damage (Zeng, 2001; Zeng et al., 2005). The cellular effects of As and/or Se greatly depend on exposure concentrations (Zeng, 2001; Zeng et al., 2005). Although As and Se are carcinogenic, both compounds have long been known for their anticarcinogenic effects and exhibit clinical efficacy in cancer treatment (see discussion below) (Cui et al., 2008; Schrauzer, 1976; Valko et al., 2006; Zhu et al., 2002). Moreover, As and Se have been shown to neutralize each other's cytotoxic effects (Zeng, 2001). We here report that As and Se both induce KAP1/BMI1-interaction and that this interaction leads to KAP1-mediated BMI1 degradation. Our experimental findings also suggest that the interplay between As and Se affects the KAP1/BMI1-interaction in a stress-signaling dependent manner. Remarkably, Se-induced $\mathrm{YH} 2 \mathrm{~A} . \mathrm{X}$ and KAP1pS824 are blocked by co-incubation with As (suppl Fig. 6) suggesting that As counteracts Se in a manner that involves inhibition of ATM-activation.

Arsenic forms numerous radicals in the cell, it produces reactive oxygen species (ROS) which cause direct molecular damage or release bivalent metal ions via thiol-oxidation and it binds vicinal cysteines; anyone of these processes may contribute to altered protein function in our setting. Since ATM-function can be regulated by oxidation, it is conceivable that As targets oxidative ATM-activation (Guo et al., 2010a; Guo et al., 2010b; Yamamoto et al., 2006); however, it is currently not clear whether and how As blocks the ability of ATM to respond to DNA-damage. Se-stress enhanced cellular P53-protein levels, whereas As did the opposite suggesting an involvement of induced proteolysis. In agreement with this idea, KAP1 was shown to enhance MDM2mediated P53 ubiquitylation and subsequent degradation (Wang et al., 2005). 
We observed that combined pre-incubation with p38i and ATMi reversed the effect of As on P53 levels, whereas these inhibitors exerted little effect on Se-induced P53 levels alone or in combination; whether pre-treatment with kinase inhibitors directly interfered with chemical protein modification by As (Jagdeo and Brody, 2011) or affected pathways downstream of ATM, these findings supported the observation that p38 and ATM signaling contribute to the differences in cellular responses between As and Se. The absence of any overt DNA-damage markers in As-stressed cells would at minimum suggest that the As-induced KAP1/BMI1-interaction is independent of KAP1pS824 and/or $y$ H2A.X. Nevertheless, the As or Se-induced nuclear accumulation of RPA70, an indicator of exposed ssDNA, suggests that DNA-damage is implicated in both types of cell-stress responses. In addition, several kinase inhibitors (PKCi, JNKi, GSKB3i) had similar effects on the KAP1/BMI1-interaction in Se or As-stressed cells. Thus, in regards to the stress-induced KAP1/BMI1-interaction, the most prominent differences between Se and As-induced center on p38 and ATM signaling.

\section{Post-translational modification defines interactomes}

KAP1 interacts with numerous factors, many of which are associated with repressive chromatin. These interactions are subject to regulation by PTMs. KAP-1 interacts with the nucleosome remodeling and deacetylase (NuRD) complex via direct binding of CHD3 (Schultz et al., 2001). KAP1/CHD3-dissociation is controlled by S824phosphorylation: it disrupts the SIM-mediated binding of CHD3 to KAP1-SUMO (Goodarzi et al., 2011). SUMOylation of KAP1 is essential for its transcriptionally repressive properties (Ivanov et al., 2007; Lee et al., 2007; Mascle et al., 2007), and is required for HDAC, SETDB1 and $\mathrm{CHD3/Mi2}$ recruitment (Ivanov et al., 2007; Mascle et al., 2007). Phosphorylation of KAP1 on S824 by ATM was shown to correlate with reduced KAP1-SUMOylation and an increased expression of several KAP1-repressed genes such as p21 and Gadd45 $\alpha$ ( $\mathrm{Li}$ et al., 2007). KAP1S824-phosphorylation is a DDR/ATM-promoted event, required for DSB-repair in heterochromatin. Dissociation of KAP1pS824/CHD3-binding was proposed to disrupt a self-reinforcing heterochromatin assembly mechanism (involving CHD3, HDAC, HKMT, and adapter-molecules like HP1) which results in heterochromatin decondensation (Goodarzi et al., 2011). S473 phosphorylation was shown to disrupt KAP1/HP1-binding (Chang et al., 2008).

Like the RBCC-domain, the PHDBROMO-domain is required for transcriptional silencing. Several of our observations point to potential auto-regulatory properties within KAP1. The isolated KAP1-PHDBROMO domain is spontaneously phosphorylated, the full-length protein is not, suggesting that KAP1-structure provides negative control on S824-phosphorylation (suppl Fig. 7D). In addition, the KAP1-PHDBROMO interacts with endogenous KAP1 under cell-stress conditions. Although it is currently unclear 
how this apparently $R B C C$-independent interaction is established, we observed that overexpression of Flag:fIKAP1 or the Flag:nls/KAP1-RBCC domain reduced endogenous KAP1pS824, suggesting in involvement of additional KAP1-domains in, for instance, competition for kinase-binding or subcellular localization. Likewise, overexpression of KAP1 induces pS473 (suppl Fig. 7D); the sparse presence of pS473 in Flag:KAP1-deltaRF extracts suggested that KAP1 requires an intact $R F$-domain to become spontaneously phosphorylated under resting conditions.

The Se-induced KAP1/BMI1-interaction is dependent on phosphorylation events, as inhibition of several kinases prevented interaction (Fig. 2; 3). Our data indicate that it is KAP1pS473, not KAP1pS824, which interacts with BMI1. A number of observations support this idea: i) in contrast to KAP1pS473, KAP1pS824 is not detected in co-IPs with BMI1, ii) signaling-interference that increases KAP1pS824 tends to reduce KAP1/BMI1 interaction, iii) arsenite-induced KAP1/BMI1-interaction occurs independent of pS824, iv) S824-phosphorylated PHDBROMO-domains do not bind BMI1, and v) pan-nuclear pS824-staining inversely correlates with PcG NB-detection within our experimental setting. Whereas pS824 appears to be an exclusively DDR-associated event, pS473 is detectable in the context of both DDR and mitogenic responses (this report, (White et al., 2011)). Of note: pS473 induced by overexpression of full-length KAP1 is clearly not sufficient to induce KAP1/BMI1 co-IP under resting conditions, suggesting that the interaction is dependent on additional, likely PMT-driven and/or subcellular localization-dependent, events.

\section{Distinct chromatin functions}

We and others have observed that a portion of KAP1 is chromatin-bound at rest. Consistent with a recent report, we observe a global increase of KAP1pS473 in response to cell stress and/or DNA-damage (Goodarzi et al., 2011; Noon et al., 2010; White et al., 2011) and an increased recruitment of KAP1pS473 to chromatin. Whereas KAP1pS824 initially localizes directly to sites of DSBs, it spreads genome-wide shortly after. This pan-KAP1pS824 staining pattern dissipates within hours of DNA damage-induction, leaving KAP1pS824 foci at slow-repairing $\mathrm{YH} 2 \mathrm{~A}$.X-positive heterochromatin regions (Noon et al., 2010; White et al., 2006). At this stage no co-localization is observed between pS824 and PRC1-proteins in response to any stress stimulus (UV, IR, CPT, hydroxyurea, aphidicolin; unpublished observations). Although it is currently unclear whether pS824 and pS473 occur simultaneously or in succession on a single KAP1 molecule, the isolated interaction of BMI1 with KAP1pS473 contributes to the idea of distinct chromatin-associated functions for the two phospho-KAP1 forms. BMI, like KAP1pS824, was recently implicated in DNA damage repair and independently shown to rapidly accumulate at induced DSBs (Ginjala et al., 2011; Ismail et al., 2010) hence, 
it is likely that these proteins co-localize to DSBs. Part of cellular BMI1, like KAP1, is chromatin-resident in the form of PcG NBs in resting cells. Although we cannot rule out a potential KAP1pS824/BMI1-interaction at these early time-points, at later timepoints BMI accumulates at chromatin with KAP1 and interacts with KAP1p473. The opposing movement of resident and recruited BMI1 supports differential regulation and distinctive roles in stress responses. The functional implication of global recruitment may be rapid re-establishment of chromatin re-condensation/repression in euchromatic regions. It has been proposed that KAP1 fulfills a dual role in this respect: whereas the initial global and subsequent focal accumulation of KAP1pS824 at heterochromatin-rich areas is required for DSB repair in highly condensed chromatin, its pan-genomic recruitment in the pS473-form may relate to transcriptional repression or establishment of another chromatin-condition required for DNA repair inside a damaged cell. The finding that PRC1-core complex members are quantitatively retrieved in co-IPs at early time points collectively accumulate at chromatin in response to stress, suggests that PCR1-core complex interactions are not compromised by KAP1binding and/or that BMI1 is part of more than one complex. Thus, based on the analysis of phosphorylation profiles and nuclear movements by our and other research groups, KAP1 and BMI1 appear to occupy multiple spatially and functionally separated nuclear niches.

\section{Biological relevance of KAP1/BMI1-interaction}

BMI1 and other PCR1-core protein levels decline as a function of Se or As exposure time and/or concentration (Fig. 2), suggesting cell-stress induces proteolytic degradation of one or more PRC1-members. We could show that multiple hMW forms of BMI1 are stabilized in the presence of MG132 and/or NEM, a deubiquitylase/ desumoylase inhibitor (Fig. 6). Exogenous fIKAP1 induces rapid BMI1 degradation, which is dependent on the presence of a KAP1-RINGfinger domain. KAP1-dependent BMI1 degradation is detectable within hours after Se-stress; as the Flag:fIKAP1/BMI1:2Py-interaction was detectable in the soluble nuclear fraction (Fig. 6), this suggests that the KAP1/BMI1-interaction may be established in the nucleoplasm and/or that KAP1-dependent BMI1-ubiquitilation may not be solely dependent on KAP1, but requires an additional chromatin-associated factor(s) or event(s). Based on the data presented herein, two opposing aspects of PRC1/chromatin-association are revealed: PcG NBs are lost from chromatin in response to As or Se-stress, whereas globally, PRC1/chromatin-association increases dramatically. It is at this stage not clear whether PCG NBs are targeted for proteolysis and/or disrupted and redistributed; the quantitative gain of PRC1/chromatin-binding, however, is not explained by PcG NBredistribution only (Fig. 4). Although PCG NBs have been proposed as potential 
nucleation-sites for transcriptional repression, in analogy to telomere proximitysilencing in yeast, their function remains elusive (Chopra and Mishra, 2005). PcG NBs co-localize with $\alpha$-satellite-rich pericentromeric heterochromatin in human cancer cells; their existence in primary human cells is controversial (Saurin et al., 1998; Voncken et al., 1999). PML NB formation of the TRIM protein PML was recently shown to be mediated by reactive oxygen species (ROS): arsenic trioxide $\left(\mathrm{As}_{2} \mathrm{O}_{3}\right)$ induces ROSmediated PML and PML/RAR multimerization into NBs and induces degradation of PML/RAR NBs, providing a biochemical rational for its clinical efficacy in acute promyelocytic leukemia (Jeanne et al., 2010). As the SUMO E3 ligase PML SUMOylates and binds numerous partner proteins via SUMO and SIMs, the authors suggested a role for oxidative cell-stress in controlling SUMO-based processes (Jeanne et al., 2010). In light of these findings, and given the recently shown dependency of PRC1-biology on SUMO and $\mathrm{Ub}$, it is tempting to speculate about a similar role for oxidative stress in Polycomb-dependent processes (i.e. transcriptional silencing, DNA damage repair) as well. The exact effect of ROS on the biogenesis and/or degradation of PcG NBs, on the PRC1-interactome and on cell death/survival requires detailed future study.

\section{Chromatin analogies in mitogen-induced transcriptional responses and DDR}

Our observation that the bon ${ }^{249}$ allele exhibits a compensatory effect on the $P C^{1}$-associated sexcomb phenotype in Drosophila double heterozygotes could be explained by a direct repressive effect of bonus on HOX-genes; thus far no homeotic transformations have been reported for bonus alleles. Alternatively, bonus loss of function may act on preservation of PcG-function through reduced degradation of PRC1-components during larval development. Consistent with this idea, we observed elevated PRC1-protein levels in shKAP1 cells ( $c f$. Fig. 5C). Many proteins have been associated with more than one DNA-templated process, such as transcription, DNA repair or replication (Dinant et al., 2008; Dinant et al., 2009), suggesting that these factors fulfill analogous roles in related processes. In the context of transcriptional regulation, we note a number of resemblances between mitogen-induced immediate-early responses (MIR) and DDR: both reveal increased KAP1/chromatinaccumulation; in addition, mitogen-induced transcription of the IEG-factor ATF3 is sensitive to KAP1-depletion, as is DDR. Both DDR and MIR are accompanied by KAP1S473-phosphorylation. The observation that Se-induced KAP1/BMI1-interaction in DDR and KAP1/chromatin-association in MIR are blocked by inhibition of the stressactivated protein kinase p38, reinforces the notion that both controlled by stresssignaling pathways. Interestingly, we observed that RPA70 translocates to the nucleus in DDR as well as in MIR, suggesting that SSDNA-signaling also plays a role in immediateearly transcriptional responses. 
Both PRC1 and HP1-associated chromatin share repressive characteristics. A study on steady-state KAP1/promoter-occupation in human fibroblasts did not identify ATF3 as a KAP1-target, thus the finding that KAP1-depletion reproducibly enhances ATF3induction in response to MIR seems paradoxical (Fig. 8) (O'Geen et al., 2007). We note, however, that ATF3-induction is controlled by rapid ERK and p38-mediated PRC1/chromatin-release (Prickaerts et al., submitted). In addition, p38-signalling is required for local chromatin phosphorylation (Drobic et al., 2010; Dyson et al., 2005; Lee et al., 2006a; Lee and Shukla, 2007; Prickaerts et al., submitted; Soloaga et al., 2003). It is interesting to note a potential functional parallel between the role of KAP1 in DDR and MIR: KAP1-depletion (or KAP1S824D) obviates the need for ATM-signaling in repair of heterochromatic DSBs; similarly, KAP-depletion removes the requirement for p38-mediated chromatin-modification in the context of rapid transcriptional responses (Fig. 8). Although we have not explored the possibility that IEG-induction would require local PRC1-degradation, it is conceivable that KAP1-recruitment and S473-phosphorylation in MIR is required to release broad PRC1/chromatin-occupation over the gene body. KAP1-phosphorylation at Ser473 (KAP1pS473) was shown to release transcriptional repression at stress-response genes (Chang et al., 2008). Of relevance, interaction between PcG and HP1-related transcriptional silencing has been observed (Sewalt et al., 2002). Notably, it is the 'blanketing-type' Polycomb-associated histone H3K27me3-methylation profiles that correlates with transcriptional repression, whereas promoter and/or TSS-associated H3K27me3 is permissive of transcription (Young et al., 2011). Alternatively, KAP1-recruitment in MIR, perhaps through S473phosphorylation, may relate to re-establishment of repressive chromatin, and not be primarily related to PRC1-release. The ATF3-gene locus is likely to be embedded in euchromatic regions; as such this notion would be in agreement with a role for KAP1/BMI1 in re-establishing epigenetic homeostasis after cell stress (Drobic et al., 2010; Knoepfler et al., 2006). In depth chromatin-immunoprecipitation analysis will be important to provide insight into the role and movements of KAP1 and PRC1 in transcriptional responses.

\section{Summary}

We have identified a novel stress-induced functional interaction between BMI1 and KAP1, two proteins which are both involved in gene repression and DNA repair via epigenetic mechanisms. Both proteins are recruited to chromatin in response to various stress-stimuli. We show that in the context of DDR, KAP1 mediates proteolytic degradation of BMI. KAP1 also collaborates with PRC1-function in the transcriptional regulation of the immediate early gene ATF3 and plays a role in Polycomb-dependent 
establishment of segment identity in flies in vivo. Our data demonstrate that changes in a cells' microenvironment contribute to modulation of the interactome of KAP1 and BMI1, suggesting that these epigenetic regulators move between distinct chromatinbased functions. This is consistent with the growing body of experimental evidence that positions epigenetic regulator proteins in control of multiple DNA-templated processes. Further characterization of circumstances under which novel associations occur, the exact molecular nature of novel interactions, the PTMs involved and their biological significance is clearly needed. This will provide valuable new insights in regulation and coordination of PRC1-function in DNA-based processes that accompany chromatinbased aspects of development, differentiation and tumorigenesis.

\section{MATERIALS AND METHODS}

Tissue culture, expression systems and cell treatment Human osteosarcoma cells (U2-OS; courtesy D. Shvarts, Utrecht, The Netherlands, human cervix carcinoma cells (HeLa; courtesy M. Koritzinsky, Maastricht, The Netherlands)) and human primary fibroblasts (TIG3; courtesy D Peeper, Amsterdam, The Netherlands) were cultured at $37^{\circ} \mathrm{C}, 5 \% \mathrm{CO} 2,100 \%$ humidity in Dulbecco's modified Eagle's medium supplemented with $10 \%$ fetal calf serum (FCS), antibiotics (100 units/ml penicillin and $100 \mu \mathrm{g} / \mathrm{ml}$ streptomycin), $200 \mathrm{mM} \mathrm{L-glutamine} \mathrm{and} 4.5 \mathrm{~g} /$ liter L-glucose. All cells were serum starved at $0.1 \%$ FCS for $48 \mathrm{hrs}$, unless stated otherwise. Retroviral expression vectors were used throughout these studies to maximize percentages of expressing cells and to minimize integration effects. Expression constructs encoding murine Bmi1-2Py, murine PHC2-Flag-TAP and Bmi1-TAP were constructed in PBABE-PURO, pBMN-LZRS.ires.GFP or pBMN-LZRS.ires.NEO). The pRETRO-SUPER shRNA system used for KAP1 (or GFP control) knockdown was obtained from A. Goodarzi (Goodarzi et al., 2008), with permission of Ziv et al. (Ziv et al., 2006). The full-length Flag:KAP1 expression construct (courtesy David Ann, Los Angeles, CA) was recloned into pBluescript (pKS). The Flag-tag and the synthetic nls (PKKKRKV) were cloned in frame in pKS by PCR (Makkerh et al., 1996). Cloning of deltaRF was done by introduction of an extra Bbe1 REN site at the most C-terminal sequences encoding the RINGfinger domain was generated by removal of a newly generated internal N-terminal Bbe1-fragment. The RBCC- and the PHDBROMO domain were generated by removal of a large C-terminal Stu1-fragment and by Bbe1-Kpn1 linker fusion, respectivelty. Detailed information on the cloning procedures is available upon request (JWV). Sodium (meta)arsenite (S7400, Sigma), sodium selenite (S5261, Sigma) and 15\% FCS and $100 \mathrm{ng} / \mathrm{ml}$ tetradecanoyl phorbol acetate (TPA) were used to stimulate cells. Pretreatment with kinase inhibitors prior to 
stimulation was performed as follows: $30 \mathrm{~min} 20 \mu \mathrm{M}$ SB202190 (Calbiochem), $60 \mathrm{~min}$ $5 \mathrm{mM}$ caffeine (C8960, Sigma), $30 \mathrm{~min} 1 \mu \mathrm{M}$ Staurosporin (S4400, Sigma), 60 min 25 $\mu \mathrm{M}$

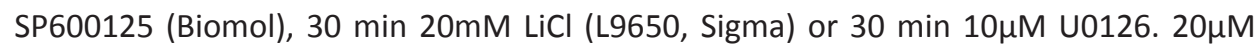
Proteasome inhibitor (539160, Calbiochem) or solvent (EtOH) was added 135 min prior to collection of cellular extracts.

Proteolytic digestion and mass spectrometric analysis Beads were washed three times with a $50 \mathrm{mM}$ Tris buffer ( $\mathrm{pH}$ 8.0). Trypsin was added to the beads suspension in a $1: 20$ ratio to the substrate and the suspension was then incubated $\mathrm{o} / \mathrm{n}$ at $37^{\circ} \mathrm{C}$ while shaking. Beads were spun down and the supernatant was used for mass spectrometry analysis. Phosphorylated peptides were selectively enriched for in an offline chromatographic manner using a titanium dioxide (TiO2, Titansphere) packed fused silica capillary that is used as a trap and which acts as an first-dimension separation step in a two-dimensional chromatography system (Pinkse et al., 2004). Phosphorylated peptides were separated from non-phosphorylated peptides by trapping them under acidic conditions on the TiO2 column and ultimately desorbed under alkaline conditions, dried and dissolved in $0.1 \mathrm{M}$ formic acid. Subsequently, nanoflow LC-MS/MS was performed on an 1100 series capillary LC system (Agilent Technologies) coupled to an LTQ-Orbitrap mass spectrometer (Thermo) operating in positive mode and equipped with a nanospray source. Peptide mixtures were trapped on a ReproSil C18 reversed phase column ( $\mathrm{Dr}$ Maisch GmbH; column dimensions $1.5 \mathrm{~cm} \times 100 \mu \mathrm{m}$, packed in-house) at a flow rate of $8 \mu \mathrm{l} / \mathrm{min}$. Peptide separation was performed on ReproSil C18 reversed phase column (Dr Maisch $\mathrm{GmbH}$; column dimensions $15 \mathrm{~cm} \times 50 \mu \mathrm{m}$, packed in-house) using a linear gradient from 0 to $80 \% \mathrm{~B}(\mathrm{~A}=0.1 \mathrm{M}$ formic acid; $\mathrm{B}=80 \%(\mathrm{v} / \mathrm{v})$ acetonitrile, $0.1 \mathrm{M}$ formic acid) in $70 \mathrm{~min}$ and at a constant flow rate of $200 \mathrm{nl} / \mathrm{min}$ using a splitter. The column eluent was directly sprayed into the ESI source of the mass spectrometer. Mass spectra were acquired in continuum mode; fragmentation of the peptides was performed in data-dependent mode. Peak lists were automatically created from raw data files using the Mascot Distiller software (version 2.1; MatrixScience). The Mascot search algorithm (version 2.2, MatrixScience) was used for searching against the NCBInr database (release 20070617; taxonomy: Mus musculus and Homo sapiens). The peptide tolerance was set to $10 \mathrm{ppm}$ and the fragment ion tolerance to $0.8 \mathrm{Da}$. A maximum number of 2 missed cleavages by trypsin were allowed and carbamidomethylated cysteine and oxidized methionine were set as fixed and variable modifications, respectively. The Mascot score cut-off value for a positive protein hit was set to 60 . Individual peptide MS/MS spectra with Mowse scores below 40 were checked manually and either interpreted as valid identifications or discarded. 
Protein isolation, Differential extraction, Immunoprecipitation (IP), Immunoblotting (IB) Cells were lysed in RIPA buffer (150 mM NaCl, 1\% NP-40, 0.5\% DOC, 0.1\% SDS, $50 \mathrm{mM}$ Tris (pH 8), $5 \mathrm{mM}$ EDTA) supplemented with $5 \mathrm{mM}$ Benzamidine, $5 \mu \mathrm{g} / \mathrm{ml}$ Antipain, $5 \mu \mathrm{g} / \mathrm{ml}$ Leupeptin, $5 \mu \mathrm{g} / \mathrm{ml}$ Aprotinin, $1 \mathrm{mM}$ Sodium Vanadate, $10 \mathrm{mM}$ Sodium Fluoride, $10 \mathrm{mM}$ Pyrophosphate, $10 \mathrm{mM}$ ß-glycerophosphate, $0.5 \mathrm{mM}$ DTT and $1 \mathrm{mM}$ PMSF. 10mM N-ethylmaleimide (NEM, E38760, Sigma Aldrich) or solvent (EtOH) was added to the cell lysis buffer. Lysates were subjected to two freeze-thaw cycles in liquid nitrogen, followed by sonication on ice with a probe sonicator (Soniprep 150; MSE, London, UK) for 12 cycli ( 1 sec ON, 1 sec OFF) with amplitude 5 . After 10 min centrifugation at $13200 \mathrm{rpm}\left(4^{\circ} \mathrm{C}\right.$; Eppendorf centrifuge), the supernatant was transferred to a fresh tube and protein concentration was determined using a BCA protein assay kit (Pierce/Thermo Fisher Scientific, Rockford, IL, USA) according to the manufacturer's protocols on a Benchmark 550 Micro-plate Reader (Bio-Rad). For cellular fractionation, cells were washed $2 x$ with cold PBS and scraped in lysis buffer (0.5\% Triton X-100, 1 mM EDTA, $150 \mathrm{mM} \mathrm{NaCl}$, Tris $\mathrm{HCl} \mathrm{pH} \mathrm{7,5;} \mathrm{supplemented} \mathrm{with}$ inhibitors). After 30 minutes incubation on ice, nuclei are collected in the pellet by centrifugation ( $8000 \mathrm{rpm} ; 4^{\circ} \mathrm{C}$ ), the supernatant is the cytoplasmic fraction. Nuclei are washed in lysis buffer and suspended in ELB-buffer and incubated on ice for 10 minutes. Nuclear soluble (supernatant) and chromatin bound fractions (pellet) are separated by centrifugation (13200 rpm; $4^{\circ} \mathrm{C}$ ). After an additional wash in ELB buffer, the pellets were suspended in ELB and sonicated. For immunoprecipitations cells were lysed in ELB buffer (250mM NaCl, 0.1\% Igepal (NP40), 5mM EDTA, 50mM HEPES pH 7.0) supplemented with $5 \mathrm{mM}$ Benzamidine, $5 \mu \mathrm{g} / \mathrm{ml}$ Antipain, $5 \mu \mathrm{g} / \mathrm{ml}$ Leupeptin, $5 \mu \mathrm{g} / \mathrm{ml}$ Aprotinin, $1 \mathrm{mM}$ Sodium Vanadate, $10 \mathrm{mM}$ Sodium Fluoride, $10 \mathrm{mM}$ Pyrophosphate, 10 $\mathrm{mM}$ ß-glycerophosphate, $0.5 \mathrm{mM}$ DTT and 1mM PMSF. Extracts were sonicated on ice and centrifuged for $10 \mathrm{~min}$ at $4^{\circ} \mathrm{C}$ at $13.2 \mathrm{Krpm}$. 1/10th of the supernatant was taken as input. Appropriate amount of antibody was added and incubated minimally $1 \mathrm{hr}$ at $4^{\circ} \mathrm{C}$ on a spinning wheel. Antibodies used were directed against 2Py-tag mAb (MMS-115R; Babco), KAP1 pAb (A300-275A, Bethyl laboratories, Montgomery TX, USA), FLAG mAb (M2, F3165, Sigma, Saint Louis, MI, USA), Bmi1 mAb (F6; courtesy M. van Lohuizen, Amsterdam), Bmi1 mAb (AF27) (Bracken et al., 2007), or no primary antibody for TAP constructs. Appropriately washed beads were added (ProtA, ProtG or IgG beads) and incubated for minimally $4 \mathrm{hrs}$ at $4^{\circ} \mathrm{C}$ on a spinning wheel. The mixture was centrifuged $2 \mathrm{~min}, 4^{\circ} \mathrm{C}, 3000 \mathrm{rpm}$ and supernatant was collected as depleted fraction. Beads were washed $4 \mathrm{x}$ in ELB buffer with supplements and stored in $-80^{\circ} \mathrm{C}$ until analysis. For phosphatase treatment, IPs were washed an additional $2 \mathrm{x}$ in phosphatase buffer, phosphatase was added and IPs were incubated for $30 \mathrm{~min}$ at $37^{\circ} \mathrm{C}$. Afterwards beads were washed $4 \mathrm{x}$ in ELB buffer with supplements and stored in $-80^{\circ} \mathrm{C}$ until analysis. IPS 
for mass spectrometry were performed differently. After taking input samples, cell lysates were precleared for $30 \mathrm{~min}$ at $4^{\circ} \mathrm{C}$ on a spinning wheel with an equal mix of ProtA and ProtG sepharose and subsequently incubated with $\alpha$-Py resin (affinity matrix AFC-115P, Covance) for minimally $4 \mathrm{hrs}$ at $4^{\circ} \mathrm{C}$ on a spinning wheel. The mixture was centrifuged $2 \mathrm{~min}, 4^{\circ} \mathrm{C}, 3000 \mathrm{rpm}$ and supernatant was collected as depleted fraction. Beads were washed $4 \mathrm{x}$ in ELB buffer with supplements and subsequently $4 \mathrm{x}$ washed in modified ELB buffer with supplements, containing $1.5 \mathrm{M} \mathrm{NaCl}$, and stored in $-80^{\circ} \mathrm{C}$ until analysis. Immunoblotting was performed with antisera against: bActin mAb (C4, 691001, MP Biomedicals), 2Py-tag mAb (MMS-115R; Babco), FLAG mAb (M2, F3165, Sigma, Saint Louis, MI, USA), KAP1 pAb (A300-275A, Bethyl laboratories, Montgomery TX, USA), KAP1pS824 pAb (A300-767A, Bethyl laboratories), KAP1pS473 pAb (courtesy S.-C. Lee, Taipei, Taiwan), Bmi1 mAb (F6; courtesy M. van Lohuizen, Amsterdam, The Netherlands); CBX4 (M9, courtesy A. Otte, Amsterdam), PHC1 pAb (Rae28, courtesy Y Takihara), PHC2 mAb (courtesy H. Koseki, RIKEN Yokohama Institute, Yokohama, Japan), and RNF2 pAb (Ring1B/dinG; courtesy M. Dyer, Leicester, UK). MK3 pAb (2362 3pK; courtesy Ulf Rapp, Würzburg, Germany), RPA70 pAb (Abcam, Eching, Germany), pERK pAb (9101; Cell Signaling, Danvers, MA, USA), pp38 pAb (9211; Cell Signaling), pCHK2 pAb (2661 Cell Signaling), P53 mAb (DO-7; M7001; DAKO), AKT pAb (Cell Signaling), Histone H3 (ab 1791-100, Abcam).

Immunofluorescence and cell staining Cell were grown on 6W culture plates and either fixed for 10 minutes at ambient temperature in 1-2\% formaldehyde/PBS solution followed by permeabilization in $0,2 \%$ Triton X/PBS or directly fixed and permeabilized for 20 minutes in $100 \%$ methanol at $-20^{\circ} \mathrm{C}$. Antibody dilution was done in blocking buffer ( $5 \%$ normal goat serum, $5 \%$ fetal bovine serum, $0.02 \%$ Triton $X$ in PBS). Antibodies used were: gamma H2A.X mAb (JBW301, Upstate), Bmi1 (F6) mAb (courtesy M. van Lohuizen, Amsterdam); KAP1 pAb (A300-275A, Bethyl laboratories), pKAP1S824 pAb (A300-767A, Bethyl laboratories). Primary mAbs were detected with goat-antimouse TexasRed, primary pAbs with goat-anti-rabbit FITC.

Quantification of mRNA levels by $q$ PCR Total RNA was isolated using Tri-Reagent (Sigma). Quantity and quality of the RNA were verified using a Nanodrop (Witec AG, Germany). RNA (500ng) was converted into cDNA using the iScript ${ }^{\mathrm{TM}}$ cDNA synthesis kit (Bio-Rad) according to the manufacturer's protocol. MylQ analysis was performed on 10 ng of cDNA using the qPCR iQ $^{\text {TM }}$ Custom SYBR ${ }^{\circledR}$ Green Supermix (Bio-Rad) and 300nM primer. Analysis was performed on an iCycler $^{\circledR}$ thermal cycler (Bio-Rad). Cyclophillin A was used as a control gene for normalization. Primers sequences are: ATF3: Forward 
TCACTGTCAGCGACAGACCC; Reverse CTACCTCGGCTTTTGTGATGG; Cyclophillin A: Forward TTCCTGCTTTCACAGAATTATTCC; Reverse GCCACCAGTGCCATTATGG.

Drosophila lines Drosophila melanogaster stocks and crosses were kept on standard media at $25^{\circ} \mathrm{C}$. For crosses, 5 females were mated with 5 males; they were transferred each $48 \mathrm{~h}$ in new tubes. The $b^{2}{ }^{249}$ line (representing the P-element allele bonS024912) was a kind gift from Hugo J Bellen (Illkirch-Cedex, France; (Beckstead et al., 2001)). The $P C^{1}$ line was obtained from the Bloomington Drosophila Stock Center (BDSC; Bloomington, Indiana, USA). $P C^{1}$ and $b o n^{249}$ lines were crossed with a Gal4independent mini-white marker that allows tracing of the mutant alleles. $P c^{1}$ and $b o n^{249}$ alleles were used in heterozygote crosses as both homozygotes are lethal (Beckstead et al., 2001). The sexcomb phenotype was scored in $P c^{1 /+}(n=30), P c^{1 /} b_{0}^{249}(n=22)$ and bon $^{249} /+(\mathrm{n}=29)$ heterozygotes by counting the individual number of sexcomb teeth, as they appear on the prothoracic (T1), meso- (T2) and metathoracic (T3) legs. Statistical significance was established by Student t-testing.

\section{ACKNOWLEDGEMENTS}

We thank many colleagues for research materials and support of the study (please refer to Materials and Methods section). We thank Penelope Jeggo, Joep Geraedts, Huawei Zeng for critically reading the manuscript and members of the MAASTRO and Molecular Genetics departments for helpful discussions. Financial support: Dutch Science Organization (ZonMW-NWO): RS grant 908-02-040 (JWV); VIDI grant 016.046.362 (JWV), joined vanGogh grant (JWV, FP); tUL Grant (JWV); VSB scholarship (PP); Netherlands Genome Initiative (NGI) fellowship 050-72-422 (HN); UPMC and CNRS (FP). 


\section{REFERENCES}

Alchanati, I., Teicher, C., Cohen, G., Shemesh, V., Barr, H.M., Nakache, P., Ben-Avraham, D., Idelevich, A., Angel, I., Livnah, N., et al. (2009). The E3 ubiquitin-ligase Bmi1/Ring1A controls the proteasomal degradation of Top2alpha cleavage complex - a potentially new drug target. PLoS One 4, e8104.

Beckstead, R., Ortiz, J.A., Sanchez, C., Prokopenko, S.N., Chambon, P., Losson, R.g., and Bellen, H.J. (2001).

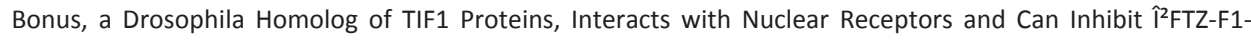
Dependent Transcription. Molecular Cell 7, 753-765.

Boyer, L.A., Plath, K., Zeitlinger, J., Brambrink, T., Medeiros, L.A., Lee, T.I., Levine, S.S., Wernig, M., Tajonar, A., Ray, M.K., et al. (2006). Polycomb complexes repress developmental regulators in murine embryonic stem cells. Nature $441,349-353$.

Bracken, A.P., Dietrich, N., Pasini, D., Hansen, K.H., and Helin, K. (2006). Genome-wide mapping of Polycomb target genes unravels their roles in cell fate transitions. Genes Dev 20, 1123-1136.

Bracken, A.P., Kleine-Kohlbrecher, D., Dietrich, N., Pasini, D., Gargiulo, G., Beekman, C., Theilgaard-Monch, K., Minucci, S., Porse, B.T., Marine, J.C., et al. (2007). The Polycomb group proteins bind throughout the INK4AARF locus and are disassociated in senescent cells. Genes Dev 21, 525-530.

Buchwald, G., van der Stoop, P., Weichenrieder, O., Perrakis, A., van Lohuizen, M., and Sixma, T.K. (2006). Structure and E3-ligase activity of the Ring-Ring complex of polycomb proteins Bmi1 and Ring1b. Embo J 25, 2465-2474.

Cao, R., Tsukada, Y., and Zhang, Y. (2005). Role of Bmi-1 and Ring1A in H2A ubiquitylation and Hox gene silencing. Mol Cell 20, 845-854.

Cao, R., Wang, L., Wang, H., Xia, L., Erdjument-Bromage, H., Tempst, P., Jones, R.S., and Zhang, Y. (2002). Role of histone H3 lysine 27 methylation in Polycomb-group silencing. Science 298, 1039-1043.

Chagraoui, J., HÃ@bert, J.e., Girard, S., and Sauvageau, G. (2011). An anticlastogenic function for the Polycomb Group gene Bmi1. Proceedings of the National Academy of Sciences.

Chang, C.W., Chou, H.Y., Lin, Y.S., Huang, K.H., Chang, C.J., Hsu, T.C., and Lee, S.C. (2008). Phosphorylation at Ser473 regulates heterochromatin protein 1 binding and corepressor function of TIF1beta/KAP1. BMC Mol Biol 9, 61 .

Chopra, V.S., and Mishra, R.K. (2005). To SIR with Polycomb: linking silencing mechanisms. Bioessays 27, 119121.

Cui, X., Kobayashi, Y., Akashi, M., and Okayasu, R. (2008). Metabolism and the paradoxical effects of arsenic: carcinogenesis and anticancer. Curr Med Chem 15, 2293-2304.

de Napoles, M., Mermoud, J.E., Wakao, R., Tang, Y.A., Endoh, M., Appanah, R., Nesterova, T.B., Silva, J., Otte, A.P., Vidal, M., et al. (2004). Polycomb group proteins Ring1A/B link ubiquitylation of histone H2A to heritable gene silencing and $\mathrm{X}$ inactivation. Dev Cell 7, 663-676.

Dinant, C., Houtsmuller, A.B., and Vermeulen, W. (2008). Chromatin structure and DNA damage repair. Epigenetics Chromatin 1, 9.

Dinant, C., Luijsterburg, M.S., Hofer, T., von Bornstaedt, G., Vermeulen, W., Houtsmuller, A.B., and van Driel, R. (2009). Assembly of multiprotein complexes that control genome function. J Cell Biol 185, 21-26.

Ditch, S., and Paull, T.T. (2011). The ATM protein kinase and cellular redox signaling: beyond the DNA damage response. Trends Biochem Sci.

Drobic, B., Perez-Cadahia, B., Yu, J., Kung, S.K., and Davie, J.R. (2010). Promoter chromatin remodeling of immediate-early genes is mediated through $\mathrm{H} 3$ phosphorylation at either serine 28 or 10 by the MSK1 multiprotein complex. Nucleic Acids Res 38, 3196-3208. 
Dyson, M.H., Thomson, S., Inagaki, M., Goto, H., Arthur, S.J., Nightingale, K., Iborra, F.J., and Mahadevan, L.C. (2005). MAP kinase-mediated phosphorylation of distinct pools of histone H3 at S10 or S28 via mitogen- and stress-activated kinase 1/2. J Cell Sci 118, 2247-2259.

Espinosa, A., Zhou, W., Ek, M., Hedlund, M., Brauner, S., Popovic, K., Horvath, L., Wallerskog, T., Oukka, M., Nyberg, F., et al. (2006). The Sjögren's Syndrome-Associated Autoantigen Ro52 Is an E3 Ligase That Regulates Proliferation and Cell Death. The Journal of Immunology 176, 6277-6285.

Fischle, W., Wang, Y., Jacobs, S.A., Kim, Y., Allis, C.D., and Khorasanizadeh, S. (2003). Molecular basis for the discrimination of repressive methyl-lysine marks in histone H3 by Polycomb and HP1 chromodomains. Genes Dev 17, 1870-1881.

Francis, N.J., Kingston, R.E., and Woodcock, C.L. (2004). Chromatin compaction by a polycomb group protein complex. Science 306, 1574-1577.

Friedman, J.R., Fredericks, W.J., Jensen, D.E., Speicher, D.W., Huang, X.P., Neilson, E.G., and Rauscher, F.J., 3rd (1996). KAP-1, a novel corepressor for the highly conserved KRAB repression domain. Genes Dev 10, $2067-$ 2078.

Ginjala, V., Nacerddine, K., Kulkarni, A., Oza, J., Hill, S.J., Yao, M., Citterio, E., van Lohuizen, M., and Ganesan, S. (2011). BMI1 is recruited to DNA breaks and contributes to DNA damage-induced H2A ubiquitination and repair. Mol Cell Biol 31, 1972-1982.

Goodarzi, A.A., Kurka, T., and Jeggo, P.A. (2011). KAP-1 phosphorylation regulates CHD3 nucleosome remodeling during the DNA double-strand break response. Nat Struct Mol Biol 18, 831-839.

Goodarzi, A.A., Noon, A.T., Deckbar, D., Ziv, Y., Shiloh, Y., Lobrich, M., and Jeggo, P.A. (2008). ATM signaling facilitates repair of DNA double-strand breaks associated with heterochromatin. Mol Cell 31, 167-177.

Guo, Z., Deshpande, R., and Paull, T.T. (2010a). ATM activation in the presence of oxidative stress. Cell Cycle $9,4805-4811$.

Guo, Z., Kozlov, S., Lavin, M.F., Person, M.D., and Paull, T.T. (2010b). ATM activation by oxidative stress. Science 330, 517-521.

Ismail, I.H., Andrin, C., McDonald, D., and Hendzel, M.J. (2010). BMI1-mediated histone ubiquitylation promotes DNA double-strand break repair. J Cell Biol 191, 45-60.

Ivanov, A.V., Peng, H., Yurchenko, V., Yap, K.L., Negorev, D.G., Schultz, D.C., Psulkowski, E., Fredericks, W.J., White, D.E., Maul, G.G., et al. (2007). PHD domain-mediated E3 ligase activity directs intramolecular sumoylation of an adjacent bromodomain required for gene silencing. Mol Cell 28, 823-837.

Jagdeo, J., and Brody, N. (2011). Complementary antioxidant function of caffeine and green tea polyphenols in normal human skin fibroblasts. J Drugs Dermatol 10, 753-761.

Jeanne, M., Lallemand-Breitenbach, V., Ferhi, O., Koken, M., Le Bras, M., Duffort, S., Peres, L., Berthier, C., Soilihi, H., Raught, B., and de The, H. (2010). PML/RARA oxidation and arsenic binding initiate the antileukemia response of As2O3. Cancer Cell 18, 88-98.

Knoepfler, P.S., Zhang, X.Y., Cheng, P.F., Gafken, P.R., McMahon, S.B., and Eisenman, R.N. (2006). Myc influences global chromatin structure. Embo J 25, 2723-2734.

Kuzmichev, A., Nishioka, K., Erdjument-Bromage, H., Tempst, P., and Reinberg, D. (2002). Histone methyltransferase activity associated with a human multiprotein complex containing the Enhancer of Zeste protein. Genes Dev 16, 2893-2905.

Lee, E.R., McCool, K.W., Murdoch, F.E., and Fritsch, M.K. (2006a). Dynamic changes in histone H3 phosphoacetylation during early embryonic stem cell differentiation are directly mediated by mitogen- and stress-activated protein kinase 1 via activation of MAPK pathways. J Biol Chem 281, 21162-21172. 
Lee, T.I., Jenner, R.G., Boyer, L.A., Guenther, M.G., Levine, S.S., Kumar, R.M., Chevalier, B., Johnstone, S.E., Cole, M.F., Isono, K., et al. (2006b). Control of developmental regulators by Polycomb in human embryonic stem cells. Cell 125, 301-313.

Lee, Y.J., and Shukla, S.D. (2007). Histone H3 phosphorylation at serine 10 and serine 28 is mediated by p38 MAPK in rat hepatocytes exposed to ethanol and acetaldehyde. Eur J Pharmacol 573, 29-38.

Lee, Y.K., Thomas, S.N., Yang, A.J., and Ann, D.K. (2007). Doxorubicin down-regulates Kruppel-associated box domain-associated protein 1 sumoylation that relieves its transcription repression on p21WAF1/CIP1 in breast cancer MCF-7 cells. J Biol Chem 282, 1595-1606.

Levine, S.S., Weiss, A., Erdjument-Bromage, H., Shao, Z., Tempst, P., and Kingston, R.E. (2002). The core of the polycomb repressive complex is compositionally and functionally conserved in flies and humans. Mol Cell Biol $22,6070-6078$.

Lewis, E.B. (1978). A gene complex controlling segmentation in Drosophila. Nature 276, 565-570.

Li, X., Lee, Y.K., Jeng, J.C., Yen, Y., Schultz, D.C., Shih, H.M., and Ann, D.K. (2007). Role for KAP1 serine 824 phosphorylation and sumoylation/desumoylation switch in regulating KAP1-mediated transcriptional repression. J Biol Chem 282, 36177-36189.

Makkerh, J.P., Dingwall, C., and Laskey, R.A. (1996). Comparative mutagenesis of nuclear localization signals reveals the importance of neutral and acidic amino acids. Curr Biol 6, 1025-1027.

Mascle, X.H., Germain-Desprez, D., Huynh, P., Estephan, P., and Aubry, M. (2007). Sumoylation of the transcriptional intermediary factor 1beta (TIF1beta), the Co-repressor of the KRAB Multifinger proteins, is required for its transcriptional activity and is modulated by the KRAB domain. J Biol Chem 282, 10190-10202.

Mikkelsen, T.S., Ku, M., Jaffe, D.B., Issac, B., Lieberman, E., Giannoukos, G., Alvarez, P., Brockman, W., Kim, T.K., Koche, R.P., et al. (2007). Genome-wide maps of chromatin state in pluripotent and lineage-committed cells. Nature $448,553-560$.

Min, J., Zhang, Y., and Xu, R.M. (2003). Structural basis for specific binding of Polycomb chromodomain to histone H3 methylated at Lys 27. Genes Dev 17, 1823-1828.

Modesti, M., and Kanaar, R. (2001). DNA repair: spot(light)s on chromatin. Curr Biol 11, R229-232.

Muller, J., Gaunt, S., and Lawrence, P.A. (1995). Function of the Polycomb protein is conserved in mice and flies. Development 121, 2847-2852.

Nalepa, G., and Wade Harper, J. (2003). Therapeutic anti-cancer targets upstream of the proteasome. Cancer Treatment Reviews 29, Supplement 1, 49-57.

Niessen, H.E., Demmers, J.A., and Voncken, J.W. (2009). Talking to chromatin: post-translational modulation of polycomb group function. Epigenetics Chromatin 2, 10.

Noon, A.T., Shibata, A., Rief, N., Lobrich, M., Stewart, G.S., Jeggo, P.A., and Goodarzi, A.A. (2010). 53BP1dependent robust localized KAP-1 phosphorylation is essential for heterochromatic DNA double-strand break repair. Nat Cell Biol 12, 177-184.

O'Dor, E., Beck, S.A., and Brock, H.W. (2005). Polycomb group mutants exhibit mitotic defects in syncytial cell cycles of Drosophila embryos. Dev Biol.

O'Geen, H., Squazzo, S.L., Iyengar, S., Blahnik, K., Rinn, J.L., Chang, H.Y., Green, R., and Farnham, P.J. (2007). Genome-wide analysis of KAP1 binding suggests autoregulation of KRAB-ZNFs. PLoS Genet 3, e89.

Peng, H., Begg, G.E., Schultz, D.C., Friedman, J.R., Jensen, D.E., Speicher, D.W., and Rauscher, F.J., 3rd (2000). Reconstitution of the KRAB-KAP-1 repressor complex: a model system for defining the molecular anatomy of RING-B box-coiled-coil domain-mediated protein-protein interactions. J Mol Biol 295, 1139-1162.

Peng, H., Gibson, L.C., Capili, A.D., Borden, K.L., Osborne, M.J., Harper, S.L., Speicher, D.W., Zhao, K., Marmorstein, R., Rock, T.A., and Rauscher, F.J., 3rd (2007). The structurally disordered KRAB repression 
domain is incorporated into a protease resistant core upon binding to KAP-1-RBCC domain. J Mol Biol 370, 269-289.

Pinkse, M.W., Uitto, P.M., Hilhorst, M.J., Ooms, B., and Heck, A.J. (2004). Selective isolation at the femtomole level of phosphopeptides from proteolytic digests using 2D-NanoLC-ESI-MS/MS and titanium oxide precolumns. Anal Chem 76, 3935-3943.

Prezioso, C., and Orlando, V. (2011). Polycomb proteins in mammalian cell differentiation and plasticity. FEBS Letters 585, 2067-2077.

Prickaerts, P., Niessen, H., Mouchel-Vielh, E., Dahlmans, V., Geijselaers, C., Adriaens, M., Spaapen, F., Takihara, Y., Rapp, U.R., Peronnet, F., and Voncken, J.W. (submitted). MK3 controls Polycomb-target gene expression via negative feedback on ERK

Sabile, A., Meyer, A.M., Wirbelauer, C., Hess, D., Kogel, U., Scheffner, M., and Krek, W. (2006). Regulation of p27 Degradation and S-Phase Progression by Ro52 RING Finger Protein. Molecular and Cellular Biology 26, 5994-6004.

Saurin, A.J., Shiels, C., Williamson, J., Satijn, D.P., Otte, A.P., Sheer, D., and Freemont, P.S. (1998). The human polycomb group complex associates with pericentromeric heterochromatin to form a novel nuclear domain. J Cell Biol 142, 887-898.

Schrauzer, G.N. (1976). Selenium and cancer: a review. Bioinorg Chem 5, 275-281.

Schultz, D.C., Friedman, J.R., and Rauscher, F.J., 3rd (2001). Targeting histone deacetylase complexes via KRAB-zinc finger proteins: the PHD and bromodomains of KAP-1 form a cooperative unit that recruits a novel isoform of the Mi-2alpha subunit of NuRD. Genes Dev 15, 428-443.

Sewalt, R.G., Lachner, M., Vargas, M., Hamer, K.M., den Blaauwen, J.L., Hendrix, T., Melcher, M., Schweizer, D., Jenuwein, T., and Otte, A.P. (2002). Selective interactions between vertebrate polycomb homologs and the SUV39H1 histone lysine methyltransferase suggest that histone H3-K9 methylation contributes to chromosomal targeting of Polycomb group proteins. Mol Cell Biol 22, 5539-5553.

Shao, Z., Raible, F., Mollaaghababa, R., Guyon, J.R., Wu, C.T., Bender, W., and Kingston, R.E. (1999). Stabilization of chromatin structure by PRC1, a Polycomb complex. Cell 98, 37-46.

Shimono, Y., Murakami, H., Hasegawa, Y., and Takahashi, M. (2000). RET finger protein is a transcriptional repressor and interacts with enhancer of polycomb that has dual transcriptional functions. J Biol Chem 275, 39411-39419.

Simon, J.A., and Kingston, R.E. (2009). Mechanisms of Polycomb gene silencing: knowns and unknowns. Nat Rev Mol Cell Biol 10, 697-708.

Simon, J.A., and Tamkun, J.W. (2002). Programming off and on states in chromatin: mechanisms of Polycomb and trithorax group complexes. Curr Opin Genet Dev 12, 210-218.

Soloaga, A., Thomson, S., Wiggin, G.R., Rampersaud, N., Dyson, M.H., Hazzalin, C.A., Mahadevan, L.C., and Arthur, J.S. (2003). MSK2 and MSK1 mediate the mitogen- and stress-induced phosphorylation of histone H3 and HMG-14. Embo J 22, 2788-2797.

Struhl, G. (1981). A gene product required for correct initiation of segmental determination in Drosophila. Nature 293, 36-41.

Ueyama, K., Ikeda, K., Sato, W., Nakasato, N., Horie-Inoue, K., Takeda, S., and Inoue, S. (2010). Knockdown of Efp by DNA-modified small interfering RNA inhibits breast cancer cell proliferation and in vivo tumor growth. Cancer Gene Ther 17, 624-632.

Valko, M., Rhodes, C.J., Moncol, J., Izakovic, M., and Mazur, M. (2006). Free radicals, metals and antioxidants in oxidative stress-induced cancer. Chemico-Biological Interactions 160, 1-40. 
Voncken, J.W., Niessen, H., Neufeld, B., Rennefahrt, U., Dahlmans, V., Kubben, N., Holzer, B., Ludwig, S., and Rapp, U.R. (2005). MAPKAP kinase 3pK phosphorylates and regulates chromatin association of the polycomb group protein Bmi1. J Biol Chem 280, 5178-5187.

Voncken, J.W., Schweizer, D., Aagaard, L., Sattler, L., Jantsch, M.F., and van Lohuizen, M. (1999). Chromatinassociation of the Polycomb group protein BMI is cell cycle-regulated and correlates with its phosphorylation status. J Cell Sci 112 ( Pt 24), 4627-4639.

Wang, C., Ivanov, A., Chen, L., Fredericks, W.J., Seto, E., Rauscher, F.J., 3rd, and Chen, J. (2005). MDM2 interaction with nuclear corepressor KAP1 contributes to p53 inactivation. Embo J 24, 3279-3290.

Wang, H., Wang, L., Erdjument-Bromage, H., Vidal, M., Tempst, P., Jones, R.S., and Zhang, Y. (2004). Role of histone H2A ubiquitination in Polycomb silencing. Nature 431, 873-878.

White, D., Rafalska-Metcalf, I.U., Ivanov, A.V., Corsinotti, A., Peng, H., Lee, S.-C., Trono, D., Janicki, S.M., and Rauscher, F.J. (2011). The ATM substrate KAP1 controls DNA repair in heterochromatin: Regulation by HP1 proteins and Serine 473/824 phosphorylation. Molecular Cancer Research.

White, D.E., Negorev, D., Peng, H., Ivanov, A.V., Maul, G.G., and Rauscher, F.J., 3rd (2006). KAP1, a novel substrate for PIKK family members, colocalizes with numerous damage response factors at DNA lesions. Cancer Res 66, 11594-11599.

Yamamoto, K., Kobayashi, M., and Shimizu, H. (2006). ATM, a paradigm for a stress-responsive signal transducer in higher vertebrate cells. Subcell Biochem 40, 327-339.

Young, M.D., Willson, T.A., Wakefield, M.J., Trounson, E., Hilton, D.J., Blewitt, M.E., Oshlack, A., and Majewski, I.J. (2011). ChIP-seq analysis reveals distinct H3K27me3 profiles that correlate with transcriptional activity. Nucleic Acids Res 39, 7415-7427.

Zeng, H. (2001). Arsenic suppresses necrosis induced by selenite in human leukemia HL-60 cells. Biol Trace Elem Res 83, 1-15.

Zeng, H., Uthus, E.O., and Combs, G.F., Jr. (2005). Mechanistic aspects of the interaction between selenium and arsenic. J Inorg Biochem 99, 1269-1274.

Zhu, J., Chen, Z., Lallemand-Breitenbach, V., and de The, H. (2002). How acute promyelocytic leukaemia revived arsenic. Nat Rev Cancer 2, 705-714.

Ziv, Y., Bielopolski, D., Galanty, Y., Lukas, C., Taya, Y., Schultz, D.C., Lukas, J., Bekker-Jensen, S., Bartek, J., and Shiloh, Y. (2006). Chromatin relaxation in response to DNA double-strand breaks is modulated by a novel ATM- and KAP-1 dependent pathway. Nat Cell Biol 8, 870-876. 


\section{suppl Figure S1}

A

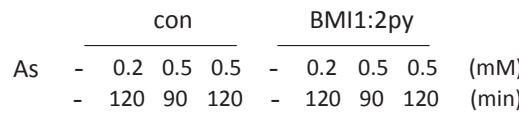
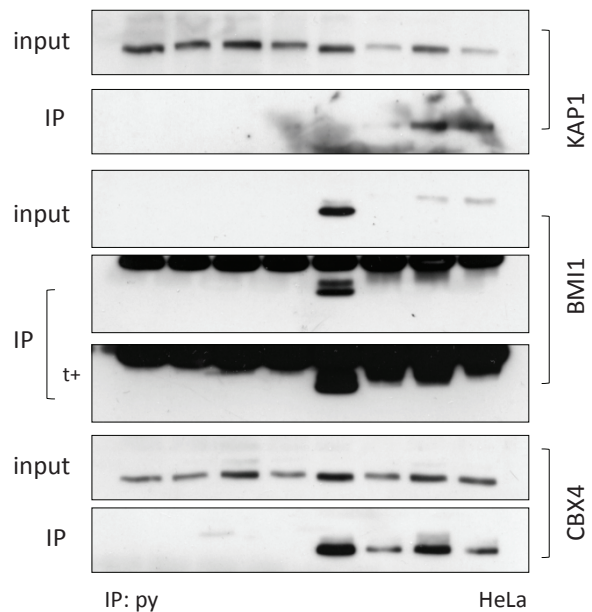

B
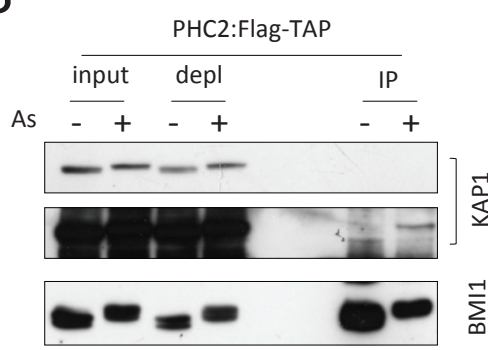

$\sum_{\infty}^{-1}$
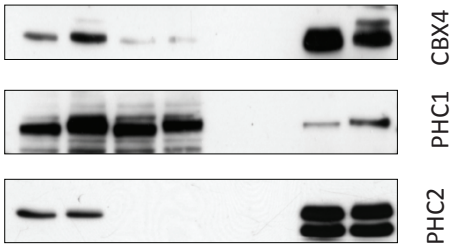

IP: Flag

U2-OS

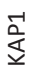

$\sum_{\infty}^{+1}$

As (0.5 mM; $75 \mathrm{~min}$ )

E

BMI1:TAP

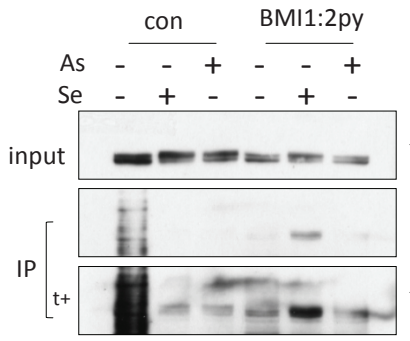

D

$-\frac{\text { con }}{+-}-\frac{\text { BMI1:2py }}{+-}$
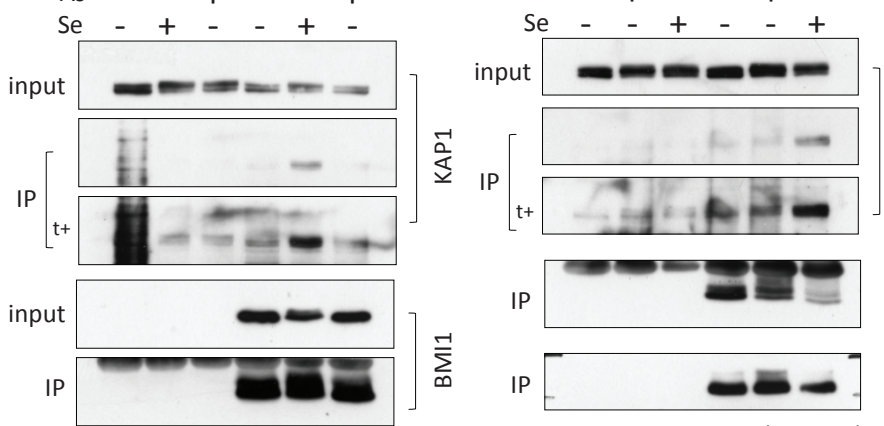

$\sum_{\infty}^{-1}$

IP

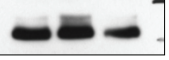

TIG3 (IRES.neo)

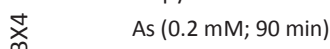

Se $(50 \mu M ; 90 \mathrm{~min})$

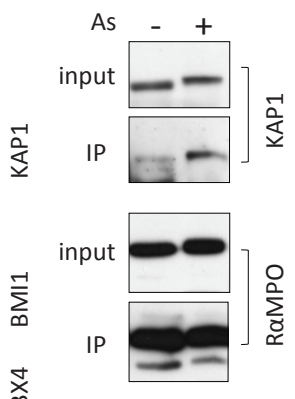

IP: IgG beads

As (0.2 mM; $90 \mathrm{~min}$ )

U2-OS 


\section{suppl Figure S2}

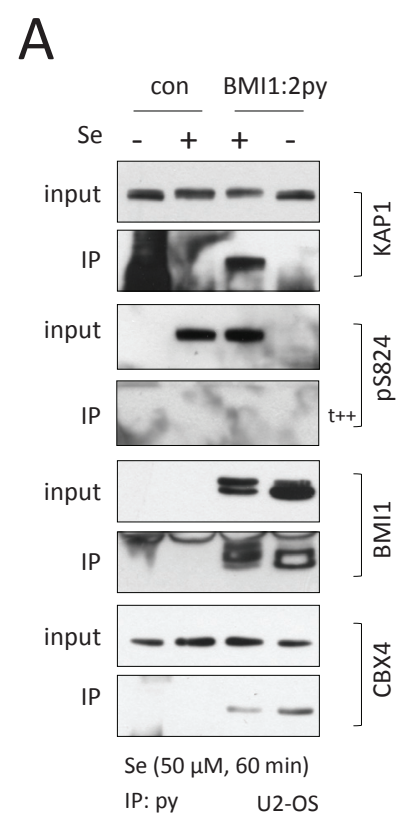

B

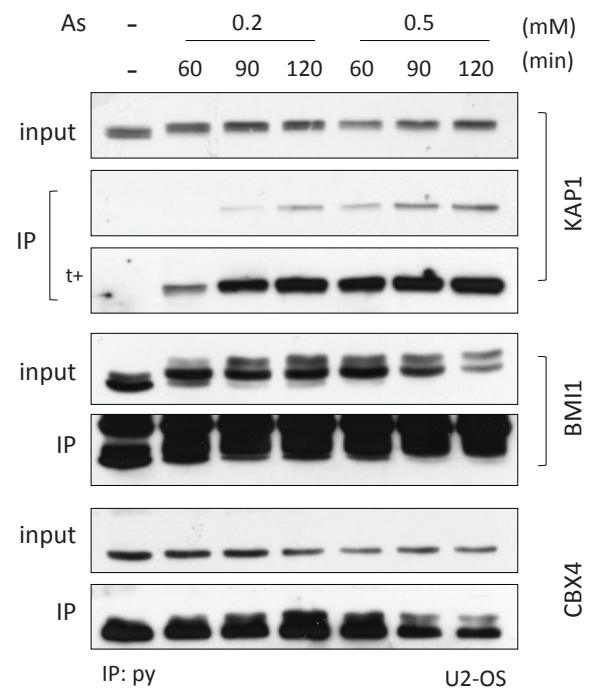

Figure S2. (above) Dose and time-responsive KAP1/BMI1-interaction. A, Selenium induces KAP1/BMI1association. B, As-induced KAP1/BMI1-iteraction is time and dose-responsive. (next page) C-D, phosphatase treatment does not disrupt KAP1/BMI1-interaction in vitro in co-immunoprecipitated proteins complexes from As (C) or Se-stressed (D) cell extracts. Cells were treated with As or Se as indicated. IP/IB was performed as indicated; $\mathrm{t}+$ : longer apposition of IB membranes.

Figure S1. (on the left) KAP1/BMI1-association occurs independent of cell-type and protein-tag. IP/IB-analysis of KAP1/BMI1-interactio in A, HeLa/BMI1:2Py cells, B, U2-OS/PHC2:Flag-TAP cells, C, D, TIG3/BMI:2Py cells transduced with BMI1:2Py construct expressed in conjunction with different marker genes (GFP, C; neomycin resistance, translational stop-sequences (3 ORF) included at 5-prime of BMI1-sequences D), E, U2-OS/ BMI1:TAP. Cells were treated with $\mathrm{As}$ and $\mathrm{Se}$ as indicated. IP/IB was performed as indicated; $\mathrm{t}+$ : longer apposition of IB. 


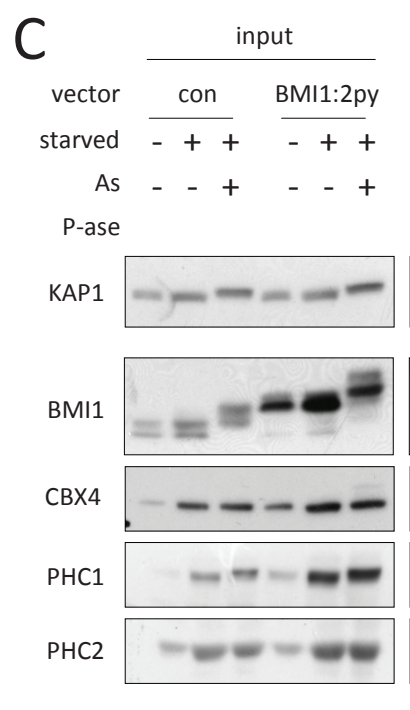

D

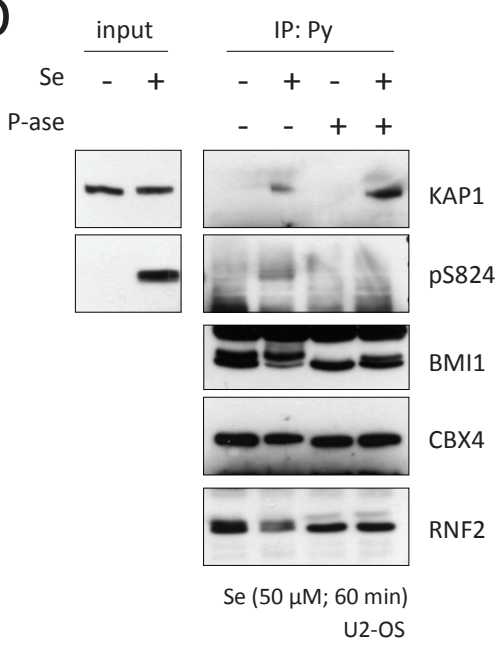

Figure S3. (on the right) Stress-induced post-translational modification of KAP1 and BMI1. A-D, IB-analysis of indicated stress-markers in As (A), Se (B), IR (C), UV stressed cells (D); MK3 was used as a loading control. E-G, absence of KAP1/BMI1-association in cells exposed to UV light (UV; E), ionizing radiation (IR; F) or camptothecin (CPT; G). Cells were treated with as indicated. IP/IB was performed as indicated. 


\section{suppl Figure S3}

A

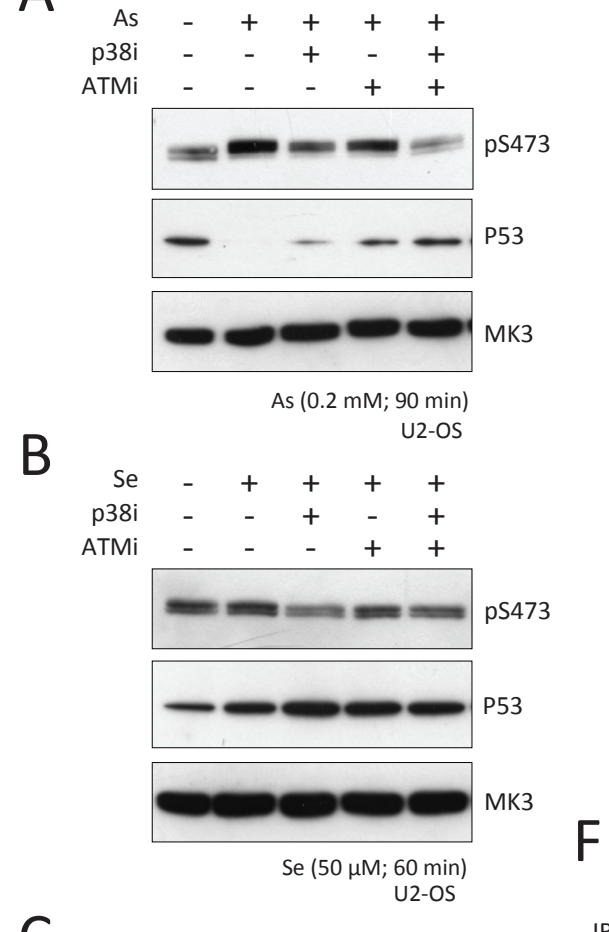

C

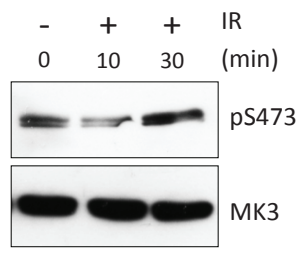

$$
\text { IR (20 Gy) }
$$$$
\text { U2-OS }
$$

D

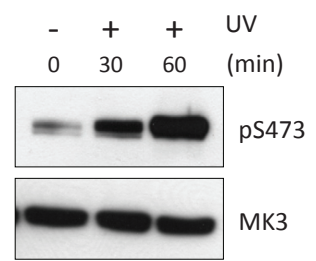

UV (25 J/m2)

U2-OS
$E$
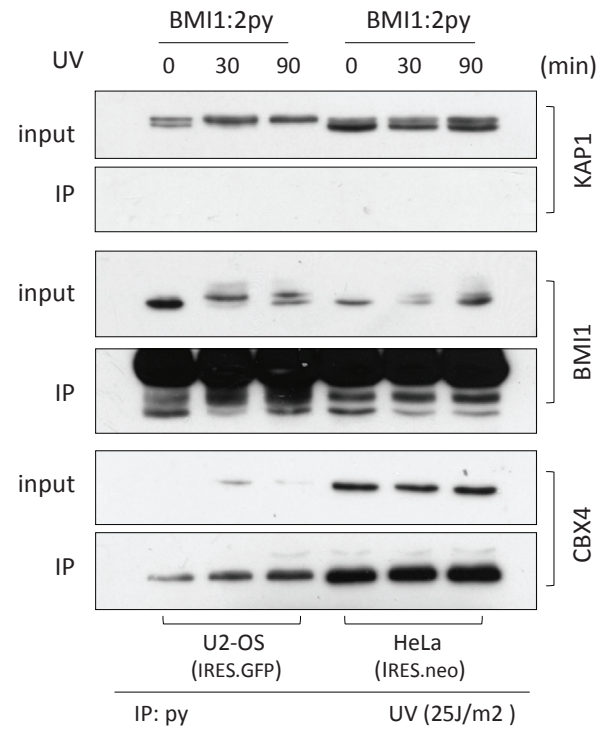

F
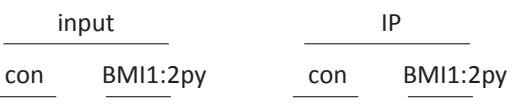

$\mathrm{IR}-+-+$
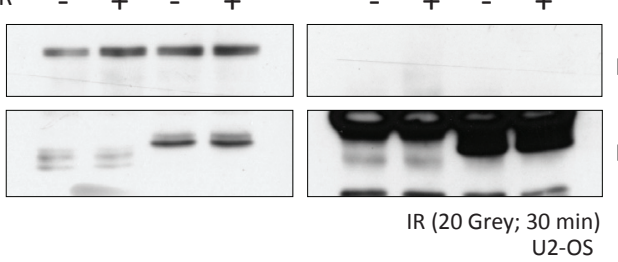

KAP1

BMI1

$G$
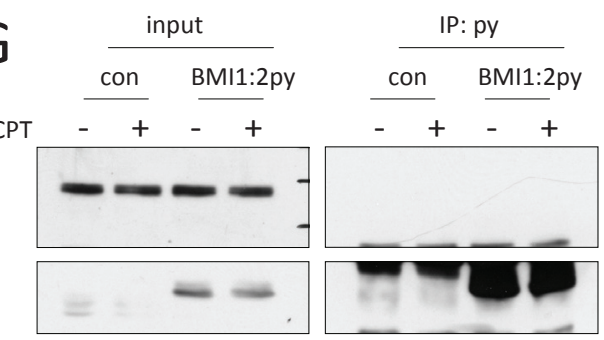

KAP1

CPT (100 mM; $60 \mathrm{~min}$ )

U2-OS 


\section{suppl Figure S4}

A
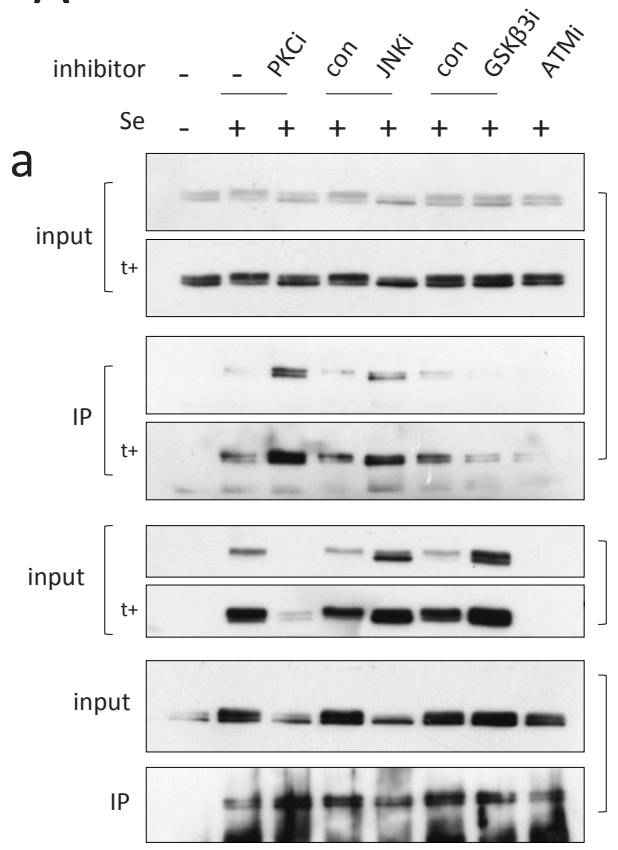

b
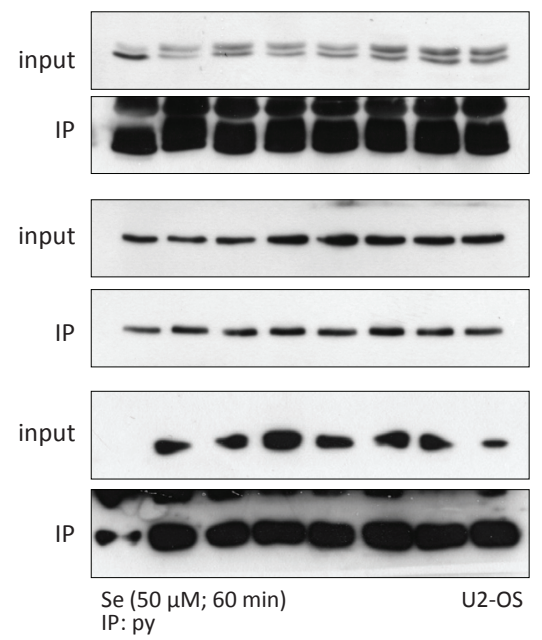

B
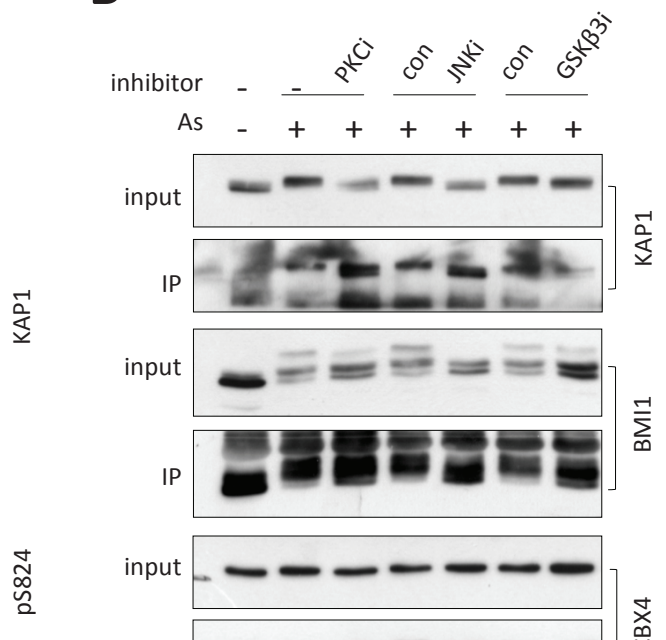

站

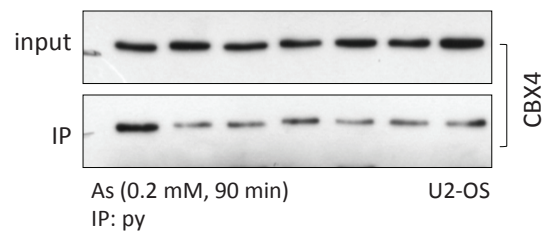

$\sum_{\infty}^{-1}$

艾

$\underset{\sim}{N}$ 


\section{suppl Figure S5}

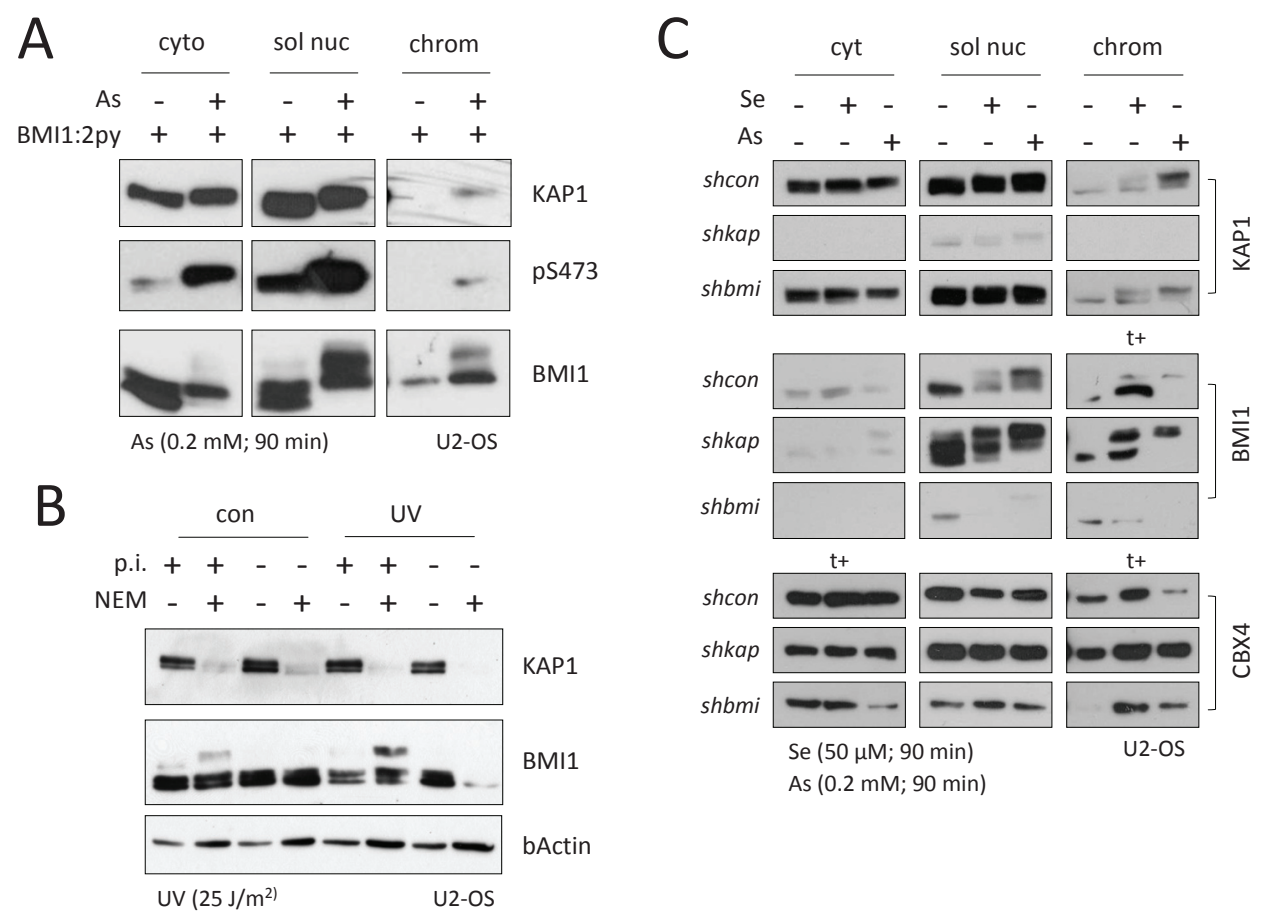

Figure S5. (above) KAP1/BMI1-interaction in the nucleus. A, KAP1 and BMI1 are recruited to chromatin in stressed cells. G0/G1-arrested cells were stimulated with arsenic for the indicated amount of time. Cells were fractionated in cytoplasmic (cyto), soluble nuclear (sol nuc) and chromatin bound (chrom) fractions used for co-immunoprecipitation analysis. Soluble nuclear and chromatin-bound fractions were proportionally loaded; cytoplasmic fractions 3-4x less. B, UV-induced PTM of BMI1; cells were preincubated with MG132 (p.i.); NEM was added during cell extraction. C, KAP1 and BMI1 do not depend on each other for chromatin-recruitment (cf. Figure $5 \mathrm{C}$ ). Cells were treated with $\mathrm{As}$ or Se as indicated; fractionation and IP/IB were performed as indicated; $\mathrm{t}+/ \mathrm{t}++$ : longer appositions of IB membranes.

Figure S4. (on the left) KAP1/BMI1-interaction is mediated by several kinases. Se (A) or As-stressed (B) cells pretreated with a PKC-inhibitor (staurosporin; con: water), a JNK-inhibitor (SP600125; con: DMSO) or a GSK3ß inhibitor ( $\mathrm{LiCl}$; con: $\mathrm{NaCl}$ ). Cells were treated with As or Se as indicated. IP/IB was performed as indicated; $\mathrm{t}+$ : longer apposition of IB membranes. 


\section{suppl Figure S6}
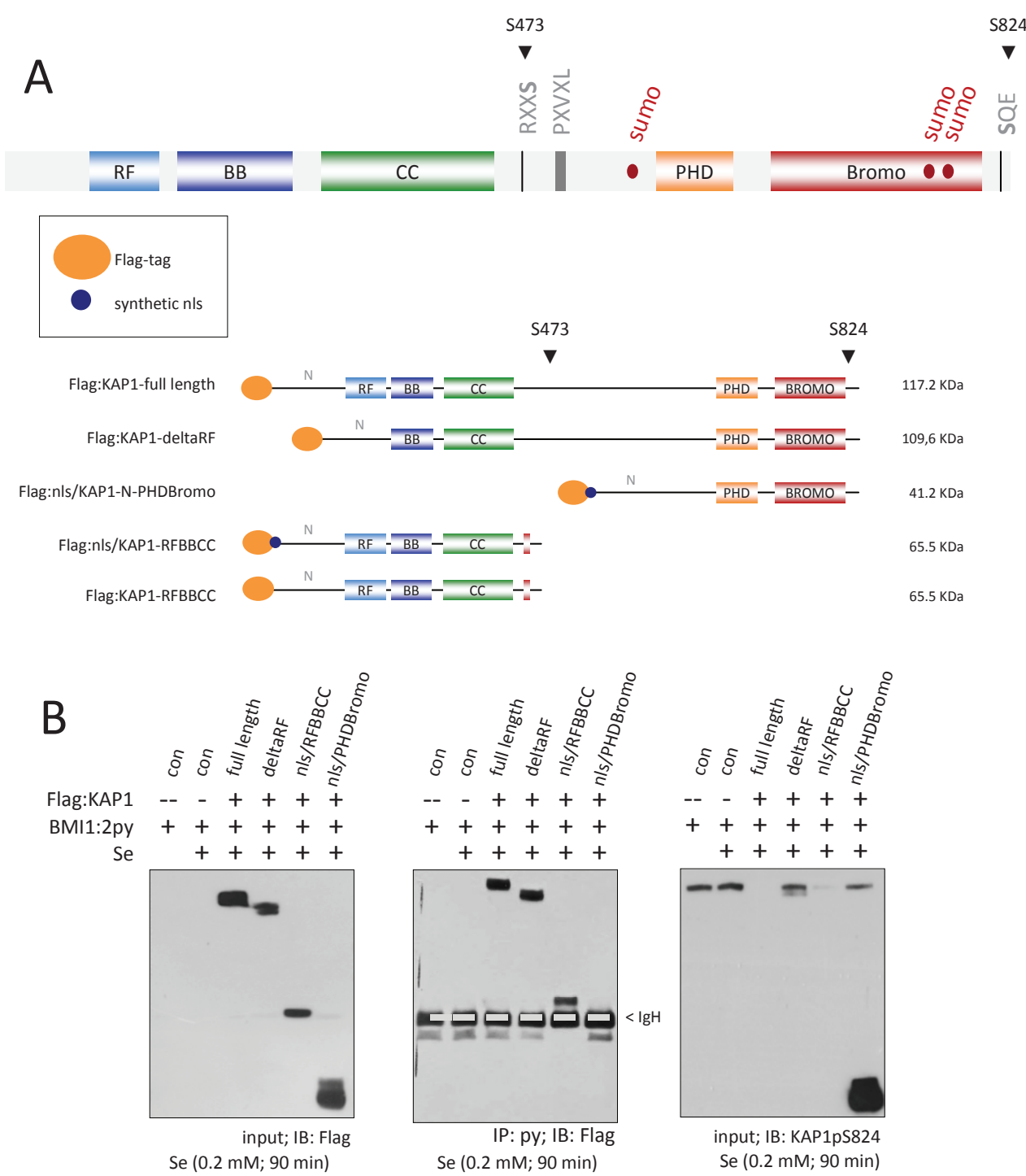

U2-OS

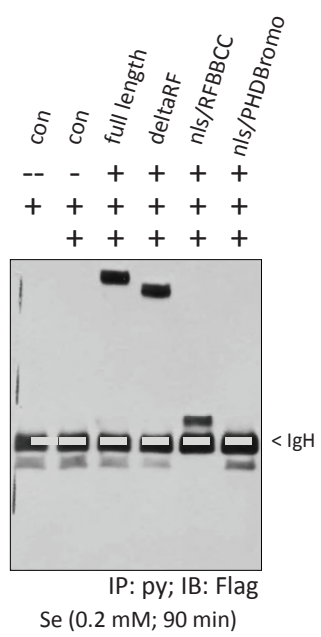

U2-OS

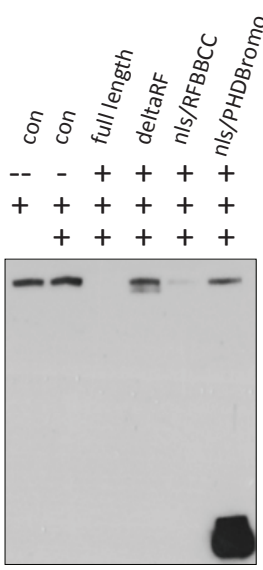

input; IB: KAP1pS824

Se $(0.2 \mathrm{mM} ; 90 \mathrm{~min})$

U2-OS

Figure S6. (above/on the right) KAP1/BMI1-interaction is mediated by the RBCC-domain. A, schematic representation of KAP1 protein; indicated are: RINGfinger (RF), double B-box (BB), Coiled-coil (CC), PHD and BROMO domains, the HP1-interaction motif ( $P x V x L)$ the pS473 and pS824-sites and three SUMOylation sites. Lower panel: Flag-tagged KAP1-constructs used in this study; synthetic nuclear localization sequence (nls) indicated. B,D IP/IB analysis of Flag:KAP1 expression. Cells were treated with As or Se as indicated; IP/IB were berformed as indicated: blocked-out sections corresnond to immunoglobin heavv chains (IgH). C. IF analvsis 


\section{suppl Figure S6}

C
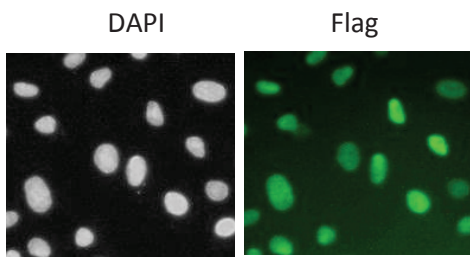

Flag:fIKAP1
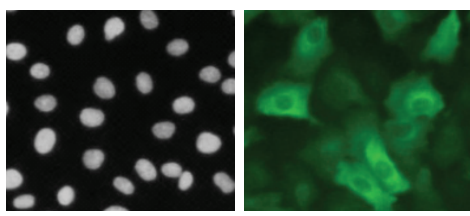

Flag:KAP1-RFBBCC
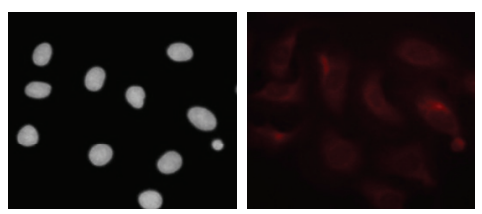

not transfected
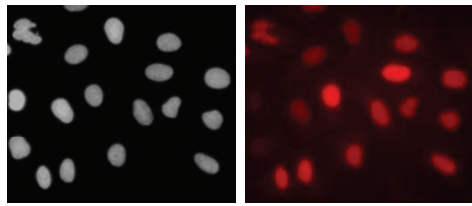

Flag:KAP1-deltaRF
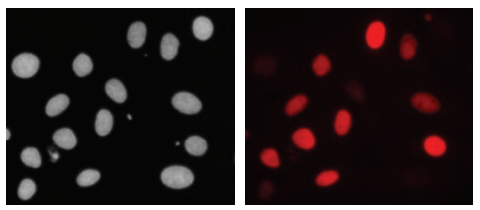

Flag:nls/KAP1-RFBBCC
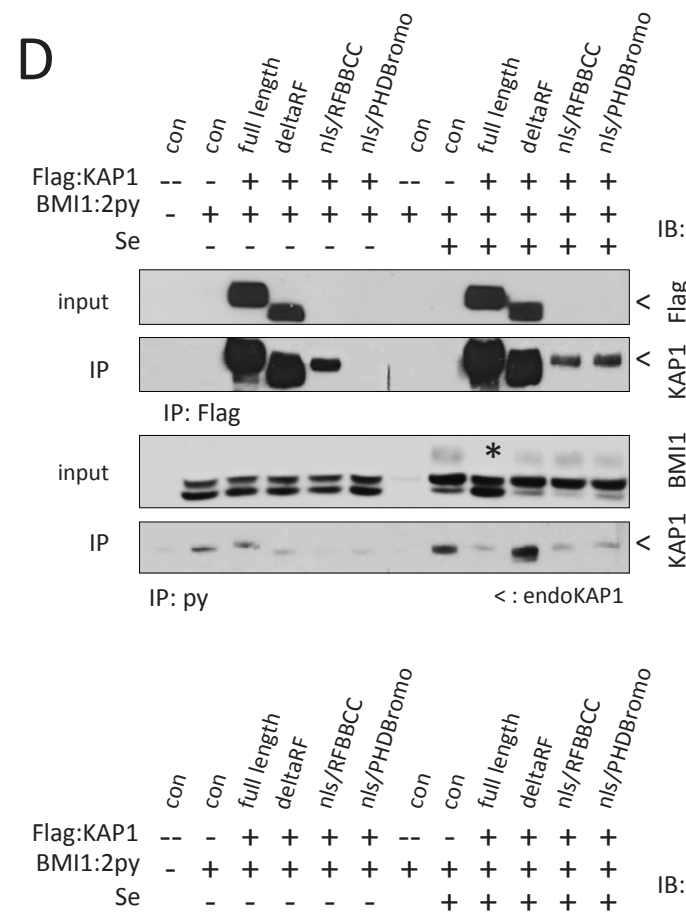

input

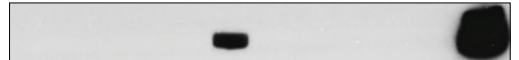

pS824

input

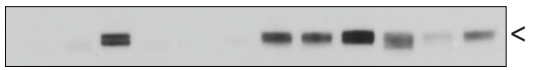

IP

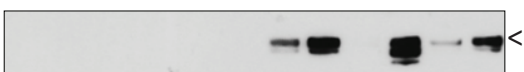

孚

IP

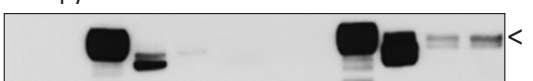

IP: Flag

$<$ : endoKAP1

Se (0.2 mM; $90 \mathrm{~min}$ )

U2-OS $\frac{\pi}{4}$ 迹

플

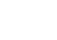


suppl Figure S7

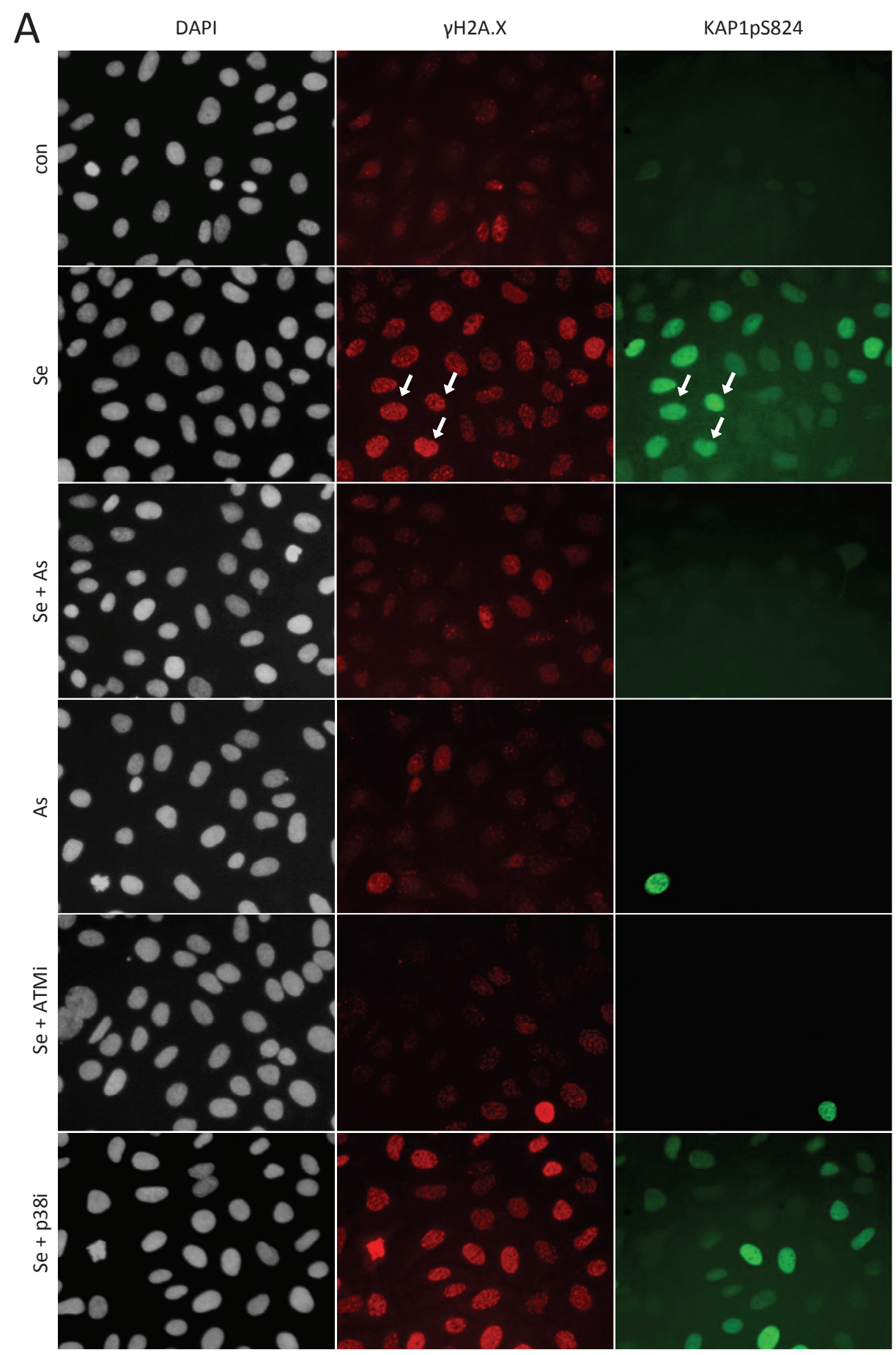




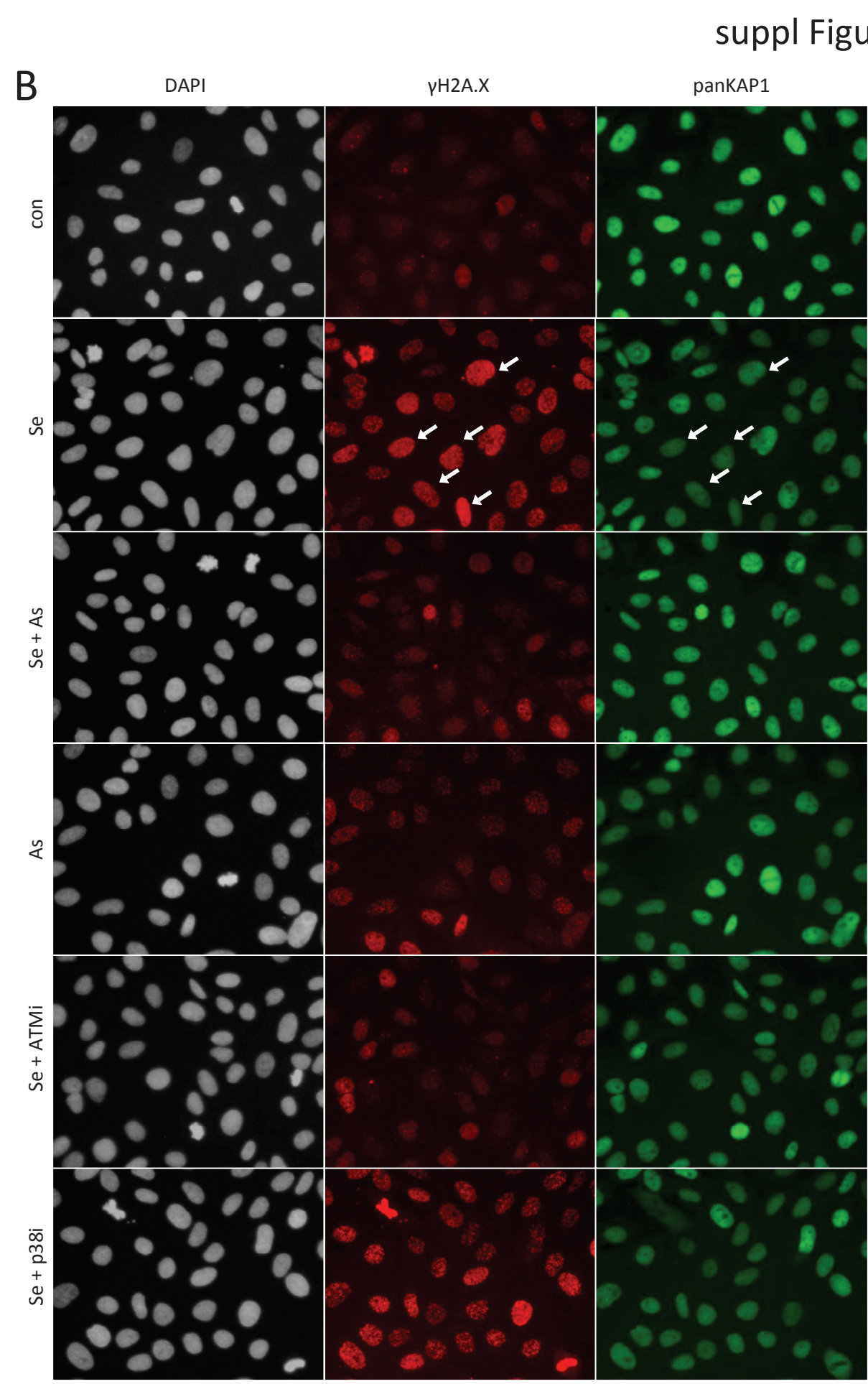


C

DAPI

BMI1

KAP1pS824

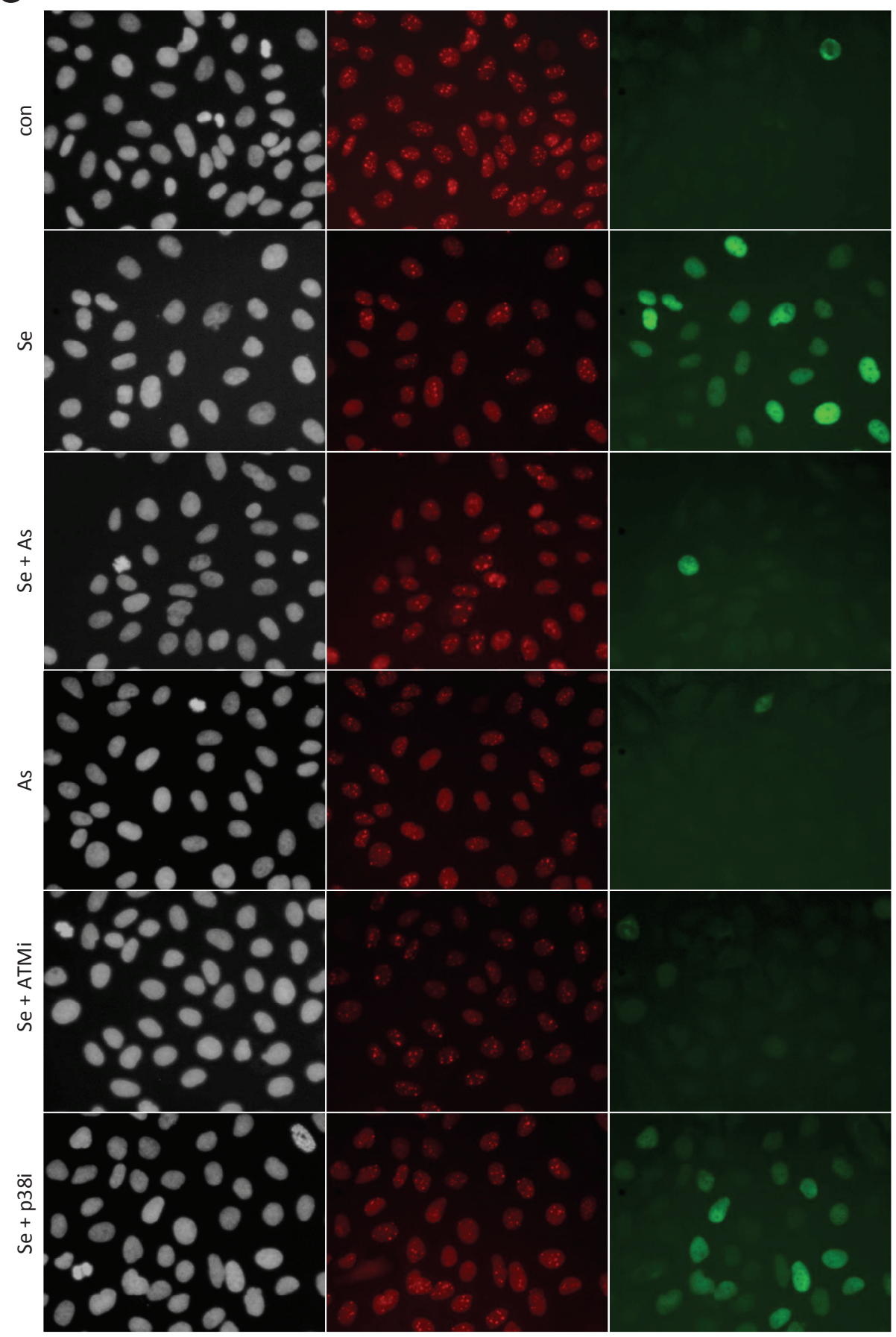


D

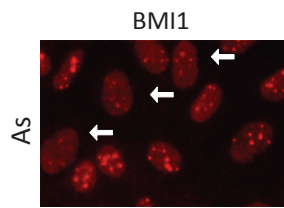

¿

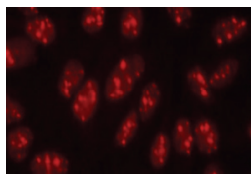

As (0.2 mM; $90 \mathrm{~min})$

E

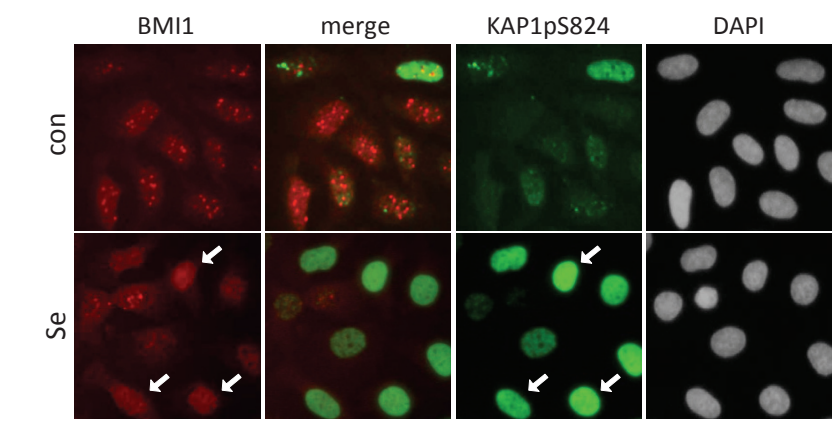

F
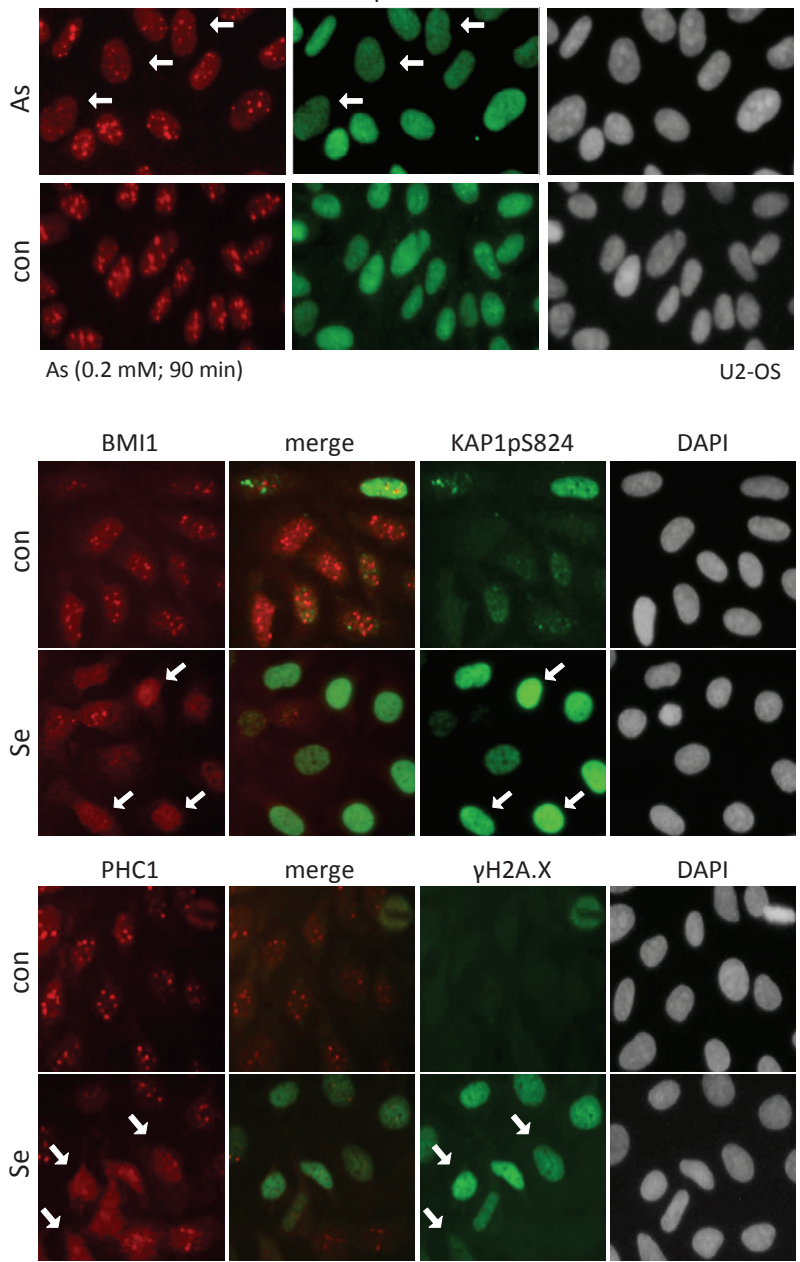

U2-OS

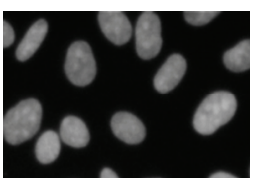

$\mathrm{PI}$ 
Chapter 4 
Hypoxia induces bivalent chromatin domains by specific gain of H3K27me3

Peggy Prickaerts*, Michiel Adriaens*, Twan van den Beucken, Caroline Gits, Vivian EH Dahlmans, Philip Zuzarte, John McPherson, Bradly G Wouters*, Jan Willem Voncken*

* equal contribution

Status: in preparation 


\section{ABSTRACT}

Trimethylation at histone $\mathrm{H} 3$ lysine 4 (H3K4me3) and lysine 27 (H3K27me3) has been linked to gene activity and repression, respectively. Distinctive H3K4me3 and H3K27me3-enrichment at specific target-genes in embryonic stem cells (ES cells) and more committed cell lineages reflects spatio-temporal epigenetic control over developmental processes and demonstrates that genomic distribution of both epigenetic marks is subject to dynamic change. How H3K4me3 and H3K27me3 respond to changes in the microenvironment is relatively unknown. Based on the biochemical dependency of the Jumonji-class histone demethylases, we hypothesized that genome-wide histone trimethylation enrichment will be dynamically affected by changes in cell oxygenation. We have determined the relationship between epigenomic and transcriptomic reprogramming in a model for fluctuating oxygen tension within the tumor micro-environment. To this end, we have combined chromatinimmunoprecipitation and deep-sequencing analysis of H3K4me3 and H3K27me3enrichment with expression array data of MCF7 breast cancer cells subjected to changes in oxygen tension (i.e. acute hypoxia, chronic hypoxia and reoxygenation). We observed a rapid global increase of both H3K4me3 and H3K27me3-marks at specific sites throughout the genome, which was reversed upon reoxygenation. Normoxic H3K4me3-profiles at marked genes were only marginally affected by hypoxia. Acquisition or loss of $\mathrm{H} 3 \mathrm{~K} 4 \mathrm{me} 3$ at target genes correlated with increased or reduced gene expression, respectively. In sharp contrast, de novo genic H3K27me3-marking was found to accumulate around transcription start sites (TSS) during hypoxia, was transitory in nature and did not correlate with transcriptional repression. Thus, under conditions of oxygen deprivation, H3K4me3-occupation was identified as the most important epigenetic marker of transcriptional regulation. As many TSS loci were already H3K4me3-marked, de novo acquisition of H3K27me3 resulted in increased bivalent marking. Hypoxia-induced bivalency revealed substantial overlap with embryonal stem cell-associated bivalent marking and was retained at numerous loci upon reoxygenation; this suggests strict control over histone (de)methylation at these sites. Our data show for the first time that oxygen availability dynamically regulates the epigenetic state of the genome. The possible repercussions of hypoxia-induced bivalency in the context of acquisition of stem cell-like epigenomic marking and tumor plasticity is discussed.

\section{Keywords:}

Deep sequencing, hypoxia, reoxygenation, epigenetics, bivalent, H3K4me3, H3K27me3 


\section{INTRODUCTION}

Cancer cells in solid tumors are often exposed to fluctuating oxygen tension resulting from inadequate blood supply due to poorly developed vasculature (Brown, 1999). Transcriptional changes in hypoxic cancer cells are influenced through several well understood hypoxia response pathways, including stabilization ad activation of hypoxia-inducible factor 1a (HIF-1a) (Kenneth and Rocha, 2008; Semenza, 2003). Transcriptional modulation of genes involved in glycolysis, angiogenesis, $\mathrm{pH}$ homeostasis and apoptosis (i.e. anti-apoptotic genes) enable cancer cells to survive and adapt to the hypoxic environment. Repeated oxygen deprivation and reoxygenation also has been hypothesized to promote tumor stem cell properties, metastasis, and patient prognosis. The phenotypic changes induced by adaptive responses to oxygen deprivation, in combination with other mutational changes in cancer, severely decrease the effectiveness of both ionizing radiation and chemotherapy (Brizel et al., 1997; Hockel et al., 1996).

Epigenetic regulation of gene expression is coordinated at the level of DNA methylation, covalent histone modifications and expression of non-coding RNAs. Their concerted action affects transcriptional regulation by influencing both access and binding of regulatory factors to chromatin as a result of changed chromatincompaction, repositioning of nucleosomes and/or recruitment of regulatory factors (i.e. by acting as a scaffold). These principles also apply to other DNA-templated processes such as replication and repair (Berger, 2007; Kouzarides, 2007). Recent data has demonstrated that epigenetic regulation can also mediate adaptation to changes in the micro-environment and this feature constitutes a major underlying mechanism in development, maintenance of cellular diversity, phenotypic plasticity and homeostasis (Bernstein et al., 2007).

The epigenetic status of the genome, including all chemical modifications of DNA and histone proteins, is often referred to as the epigenome (Esteller, 2007). Histone methylation, acetylation and phosphorylation are well-documented covalent chemical modifications that occur on N-terminal tails of core histones that constitute the nucleosomal units in chromatin (Kouzarides, 2007). Histone acetylation is firmly connected to gene activation. In contrast, histone methylation is associated with active gene transcription as well as gene repression: H3 lysine 9 (H3K9), H3K27 and H4K20 trimethylation (me3) are generally associated with gene silencing, whereas H3K4, H3K36 and H3K79 me3 are linked to transcriptional activation (Berger, 2007; Kouzarides, 2007).

H3K27me3 and H3K4me3 states have been intensely studied in the context of development in relation to transcriptional regulation (Barski et al., 2007; Mikkelsen et 
al., 2007). H3K27 is trimethylated by Polycomb Repressive Complex 2 (PRC2), which comprises the histone methyltransferase EZH2 (Cao et al., 2002). The reverse process (i.e. H3K27-demethylation) is accomplished by the histone demethylases UTX and JMJD3 (Swigut and Wysocka, 2007). H3K4me3 marks are installed by MLL-proteins that belong to the Trithorax Group (TrxG) of epigenetic modifiers, which functionally counteract PRCs (Brock and Fisher, 2005; Schuettengruber et al., 2007). JARID1A-D catalyse H3K4 demethylation (Cloos et al., 2008). Although in terminally differentiated cells most $\mathrm{H} 3 \mathrm{~K} 4 \mathrm{me} 3$ and $\mathrm{H} 3 \mathrm{~K} 27 \mathrm{me} 3$ marks appear mutually exclusive, bivalent marking at key developmental control genes is observed in embryonic stem cells (ES cells) (Azuara et al., 2006; Barski et al., 2007). Epigenomic comparison between ES cells and committed and/or fully differentiated cell types has shown that bivalent marking is resolved to monovalency (or loss of both marks) at some stage during lineage commitment and differentiation. Eventually this results in lineage-specific H3K27me3 and H3K4me3-profiles, which are stably transmitted during cell division (Hansen et al., 2007; Mikkelsen et al., 2007). As such, PRCs and TrxG play a fundamental role in the establishment and maintenance of lineage-specific gene expression (Brock and Fisher, 2005; Schuettengruber et al., 2007).

Current knowledge on the distribution dynamics of H3K27me3 and H3K4me3 beyond embryonic development is relatively limited. We therefore studied the dynamics of histone methylation in the context of micro-environmental change. As a relevant physiological model, we used cellular adaptation to hypoxia and subsequent reoxygenation. Acute and chronic hypoxia are known to induce major transcriptomic changes (Chan and Giaccia, 2007). Furthermore, hypoxia was reported to induce global changes in histone methylation (Johnson et al., 2008). However, a systematic profiling of H3K27me3 and H3K4me3 in relation to hypoxia induced gene expression is lacking. Relevantly, removal of histone trimethylation states is directly coupled to cell oxygenation: the Jumonji C-terminal domain containing histone demethylases (JHDM) use Fe2+, $\alpha$-ketoglutarate and oxygen as co-substrates to remove all methylation states by hydroxylation (Hou and Yu, 2010; Shi et al., 2004; Selak et al., 2005; Smith et al., 2008; Lohse et al., 2011); as such the regulation of $\alpha$-ketoglutarate-dependent demethylases under low oxygen is expected to be very similar, if not identical to that of the prolylhydoxylase HIF-PHD which targets hypoxia-inducible factor 1a (HIF1a) (Ivan et al., 2001; Jaakkola et al., 2001). We therefore hypothesized that oxygen affects the activity of JHDMs and will increase global repressive and/or activating histone trimethylation-marking. Since HIF1a targets transcription of a subset of responsive genes, it is expected that constitutive transcriptional activity is required at corresponding genic areas and that these need to be exempted from silencing. 
To study the relation between epigenomic and transcriptomic changes, we employed chromatin- immunoprecipitation (ChIP) followed by deep-sequencing (ChIP-seq) (Park, 2009; Robertson et al., 2007) and combined this with expression array analysis. We charted the distribution of both histone marks as a function of time cultured under low oxygen and correlated their distribution profiles to our gene expression data. Of relevance, we also included reoxygenation in our measurements, as cancer cells are subject to constantly changing oxygen tension in the tumor microenvironment. As H3K27me3 is known to cover large chromosomal regions instead of sharp defined peaks (Bracken et al., 2006; Pauler et al., 2009), we also developed a standardized protocol to define and summarize H3K27me3-enrichment using deepsequencing analysis. A detailed description of the protocol and the scripts used are published elsewhere (Adriaens et al., submitted).

\section{RESULTS}

\section{Reversible oxygen-dependent global changes of H3K4me3 and H3K27me3 levels}

To determine whether histone trimethyl-states are dependent on the availability of molecular oxygen we exposed MCF7 breast cancer cells to severe hypoxia $(<0.02 \%)$ and measured global changes in $\mathrm{H} 3 \mathrm{~K} 4 \mathrm{me} 3$ and $\mathrm{H} 3 \mathrm{~K} 27 \mathrm{me} 3$ levels by immunoblotting at 8 and 24 hours of hypoxia compared to normoxic cells $(t=0)$. Cancer cells are subject to repetitive fluctuation of oxygen availability in situ (i.e. hypoxia and reoxygenation); reoxygenation may affect cells through specific stress responses that that involve epigenetic change. For this reason we also determined H3K4me3 and H3K27me3 levels in response to reoxygenation. Trimethylation of H3K4 (1.7 fold) and H3K27 (2.2 fold) was increased after 8 hours culturing at low oxygen tension; the elevated trimethylstate was sustained up until 24 hours of culturing under hypoxic conditions (Figure 1A). We observed this in multiple cell lines, suggesting that the epigenomic remodeling was dictated by oxygen-status and occurred independent of cellular context (Figure S1A). Importantly these initial observations are consistent with our original hypothesis. Conversely, restoration of oxygenation induced an initial sharp decline of global histone H3K4 and K27-trimethylation that transiently dropped below levels in normoxic cells and returned to baseline at approximately 12-24 hours after reoxygenation, depending on the cell type used; concomitantly, H3K9/K14-acetlyation (H3K9/K14ac) increased in response to oxygen stress. (Figure 1A, Figure S1A).

To establish that demethylation activity is reduced because of loss of oxygen, and not by changes in protein levels of the responsible molecular machineries, histone lysine demethylase (HKDM) and histone lysine methyltransferase (HKMT) mRNA levels were measured. 
밈ำ

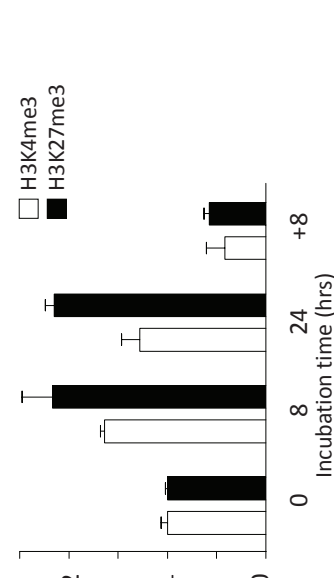

s|әләр u!әтолd әл!ฺерәд

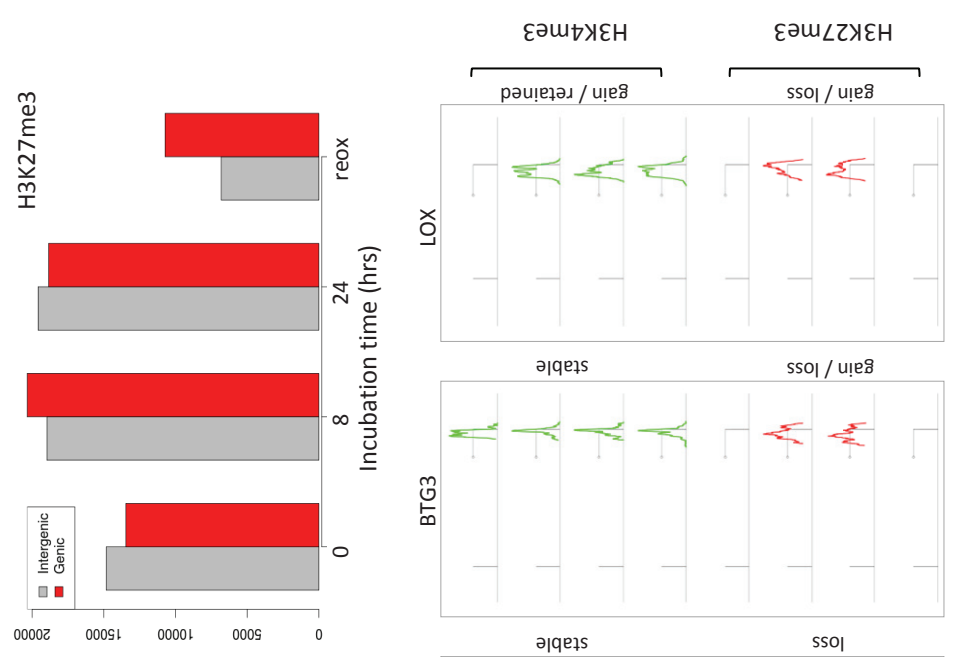

syeəd to ıəqunu

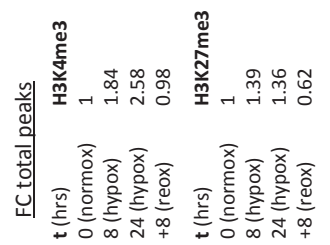

愛
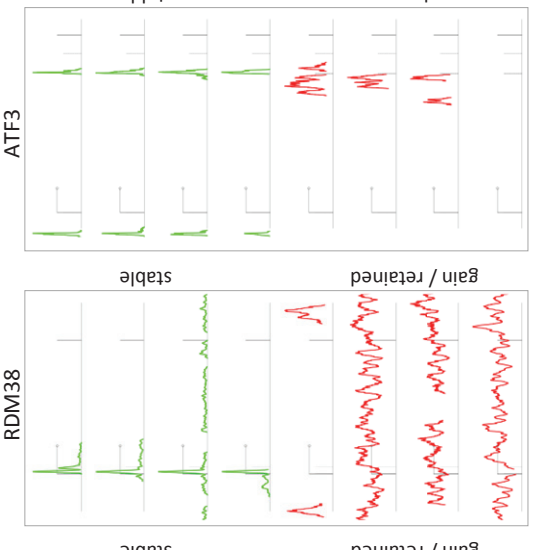

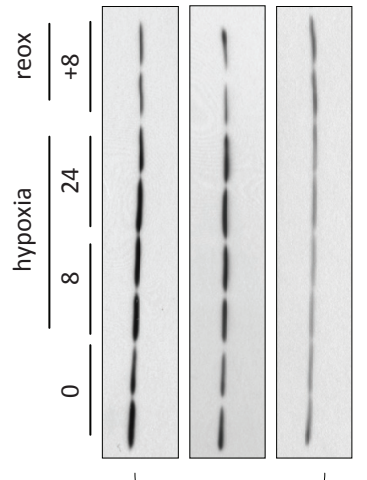

LAJW

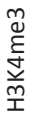
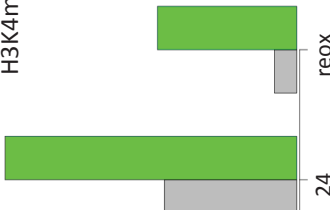

点

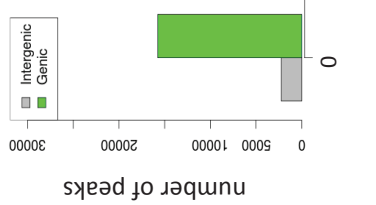

$\cup$

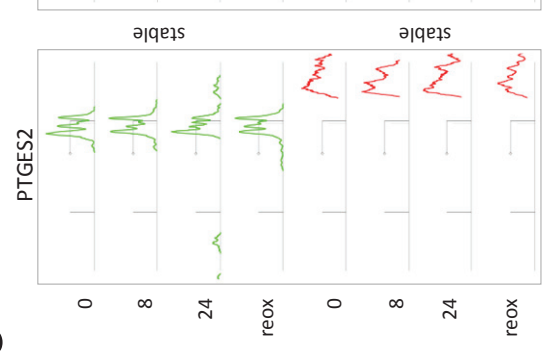


Figure 1. Reversible oxygen-dependent global changes of H3K4me3 and H3K27me3-levels. A; Immunoblot detection (IB) of epigenetic changes (H3K4me3 and H3K27me3) in MCF7 cells; right panel: quantification. B; H3K4me3 (left panel) and H3K27me3 (right panel) the number of peaks detected significantly above background level $(p<0.05)$ at different time points under hypoxic conditions $(t=8, t=24$ hrs) and after reoxygenation (reox; $\mathrm{t}=+8 \mathrm{hrs}$ ). C, Representative gene tracks of loci displaying (left to right): stable H3K27me3 and H3K4me3-marking, H3K27me3-gain/retained (at reox) and H3K4me3 gain/loss, H3K27me3gain/retained, loss of K27me3 (stable H3K4me3), H3K27me3-gain and loss (reox), gain of both marks (H3K4me3 retained; reox). Symbol indicates transcription-direction.

Expression of a number of relevant HKMDs including JARID2, JARID3 and JMJD3, show significantly increased expression in hypoxic cells (Figure S1B). In contrast, the H3K27me3-HMT EZH2 as well as numerous confirmed and putative H3K4me3 HMTs declined in response to oxygen deprivation or remained unaltered (Figure S1C). Hence, changes in levels of responsible writers or erasers could not explain the global increase in both activating and inactivating marks; instead the data is consistent with changes in enzymatic activity of KDM. Moreover, the sudden drop in H3K4me3 and H3K27me3 levels following reoxygenation is in agreement with activation of accumulated HKDM protein by molecular oxygen.

Previous studies have shown that expression of HKDM is controlled by HIF1a (Krieg et al., 2010; Yang et al., 2010; Zhou et al., 2010). To determine whether the effects of altered oxygenation on global trimethylation levels were dependent on HIF1a, HIF1adepleted MCF7 cells were exposed to hypoxia and analyzed for global changes in H3K4me3 (Figure S1D). No obvious differences with respect to hypoxia-induced increased histone trimethylation were observed between control and shHIF1a cells, indicating that the global epigenetic changes occurred in a HIF1a-independent fashion (data not shown). Combined, these data suggest that hypoxic stress induces a reversible increase in histone $\mathrm{H} 3$ trimethylation which is HIF1a-independent.

We next aimed to determine whether changes in oxygen regulate epigenetic states at specific genes. To this end ChIP-seq analysis was performed on H3K4me3 and H3K27me3-enriched sequences in normoxic ( $t=0)$, hypoxic ( $t=8$ and $24 \mathrm{hrs}$ ) and reoxygenated cells ( $\mathrm{t}=+8 \mathrm{hrs}$ ); the latter condition was considered as a transition point to restoration of the original epigenomic state under ambient conditions ( $21 \%$ oxygen). In parallel, RNA samples were collected for analysis at all four time points. ChIP-seq analysis confirmed enhanced global trimethylation of H3K4 and H3K27 in response to hypoxia, consistent with the immunoblotting findings: the total amount of H3K4me3 and $\mathrm{H} 3 \mathrm{~K} 27 \mathrm{me} 3$ peaks had increased \pm 2.6 and 1.4 fold, respectively, at 24 hours hypoxia (Figure 1B, cf. genome plots Figure S2A, B). Conversely, reoxygenated samples revealed a steep drop in the global number of trimethylation peaks: both the number of H3K4me3 and H3K27me3 peaks fell \pm 2.5 fold at 8 hours reoxygenation ( $t=+8$ ) to 0.98 
and 0.62 fold their original normoxic values, respectively (Figure 1B, cf. genome plots Figure S2A, B). Representative genome tracks illustrate examples of individual genes that displayed loss, gain or unchanged H3K4me3 and/or H3K27me3 levels in response to altered oxygenation (Figure $1 \mathrm{C}$ ). Consistent with the global decline of H3K4me3 and H3K27me3 ( $c f$. Figure 1A) enhanced trimethylation was lost upon reoxygenation at most of these loci. Reciprocal correlation analysis revealed a substantial overlap between enriched loci (for both H3 marks) under normoxic vs. hypoxic conditions (corr.coeff. at $\mathrm{t}=0$ vs. $\mathrm{t}=24 \mathrm{hrs}$ : $0.45 \mathrm{H} 3 \mathrm{~K} 4 \mathrm{me}, 0.53 \mathrm{H} 3 \mathrm{~K} 27 \mathrm{me} 3$ ) and vice versa (corr.coeff. at $\mathrm{t}=24$ vs. $\mathrm{t}=0 \mathrm{hrs}$ : $0.77 \mathrm{H} 3 \mathrm{~K} 4 \mathrm{me}, 0.60 \mathrm{H} 3 \mathrm{~K} 27 \mathrm{me} 3$ ). This data indicated that pre-existent (normoxic) $\mathrm{H} 3 \mathrm{~K} 4 \mathrm{me} 3$ and $\mathrm{K} 27$ me3-marking was generally retained under hypoxic conditions and that the increased enrichment was derived from hypoxiainduced de novo trimethylation. These findings validated the reliability of the comparative histone me3-marking analysis. H3K4me3-enrichment (i.e. sequences associated with peaks) returned to the normal situation normal (corr.coeff. at normoxia vs. reoxygenation and vice versa: 0.82). In contrast H3K27me3-marks show poor correlation between $\mathrm{t}=0$ and $\mathrm{t}=+8 \mathrm{hrs}$ (corr.coeff: 0.19 norm $v$ s. reox and 0.11 reox vs. norm) indicating that normoxic H3K27me3-distribution has not been restored at 8 hours after reoxygenation. Although a number of densly trimethylated genomic regions was relatively resistant to demethylation at reoxygenation, combined, these findings showed that hypoxia causes an overall reversible global increase of H3K4me3 and H3K27me3 and establishes that oxygen is required for constitutive activity of HKDM and maintenance of the basal epigenetic state in a large number of genomic regions.

\section{Preferred gain of histone methylation at genic regions}

The above findings provided us with a basis for comparative epigenomic and transcriptomic analysis. To relate epigenomic changes to transcriptional states, we first differentiated enriched sequences into genic (i.e. "genes" +/- 5000 bp) and intergenic regions. Under control conditions, trimethylation was highly associated to genic regions: $88 \%$ of all H3K4me3 peaks located to genes compared to $47 \%$ for $\mathrm{H} 3 \mathrm{~K} 27 \mathrm{me} 3$ peaks (Figure 1B). In normoxic cells, $46 \%$ of all genes carried H3K4me3-marks, whereas significantly less genes (9\%) were H3K27me3-enriched (Figure 2A, B/a).

Figure 2. Preferred gain of histone methylation at genic regions. A; a) number of H3K4me3-associated genes; b) H3K4me3-signal density distribution proximal to the TSS in relation to oxygen deprivation and reoxygenation; c) median gene expression in relation to H3K4me3-marking. B; a) number of H3K27me3associated genes; b) H3K27me3-distribution proximal to the TSS in relation to oxygen deprivation and reoxygenation; c) box-plots presenting median gene expression in relation to H3K27me3-marking; all data are presented at time points indicated ( $\mathrm{t}=8$ or 24 hours hypoxia; reoxygenation at $\mathrm{t}=+8$ hours). 


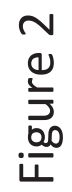

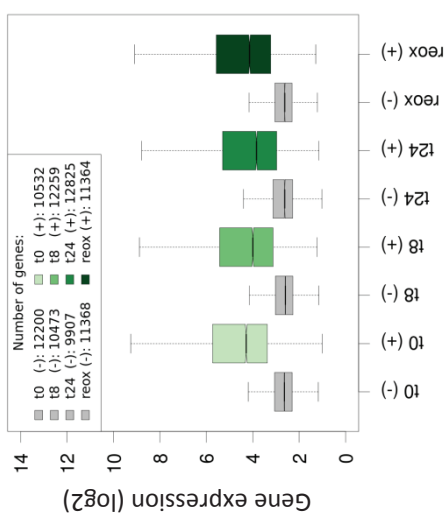

$\cup$

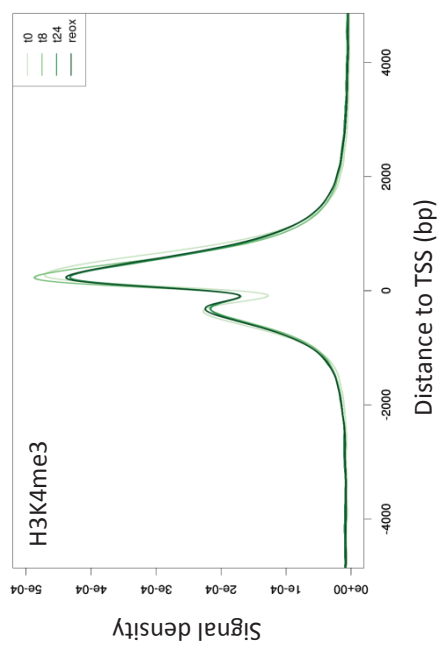

อ

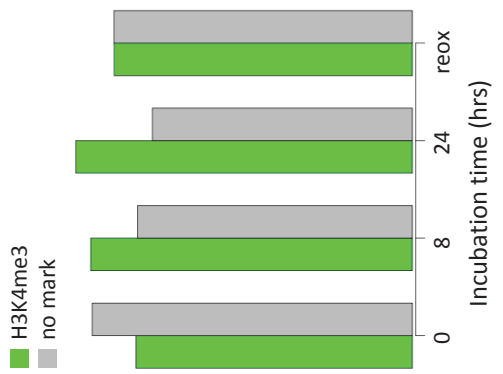

$000010008 \quad 0009 \quad 000 t \quad 0002 \quad 0$

$\varangle$
-) хоэл

†2)

$\downarrow 2$

(-) 81

+) 01

-) 01

$\frac{a}{2}$
范

م
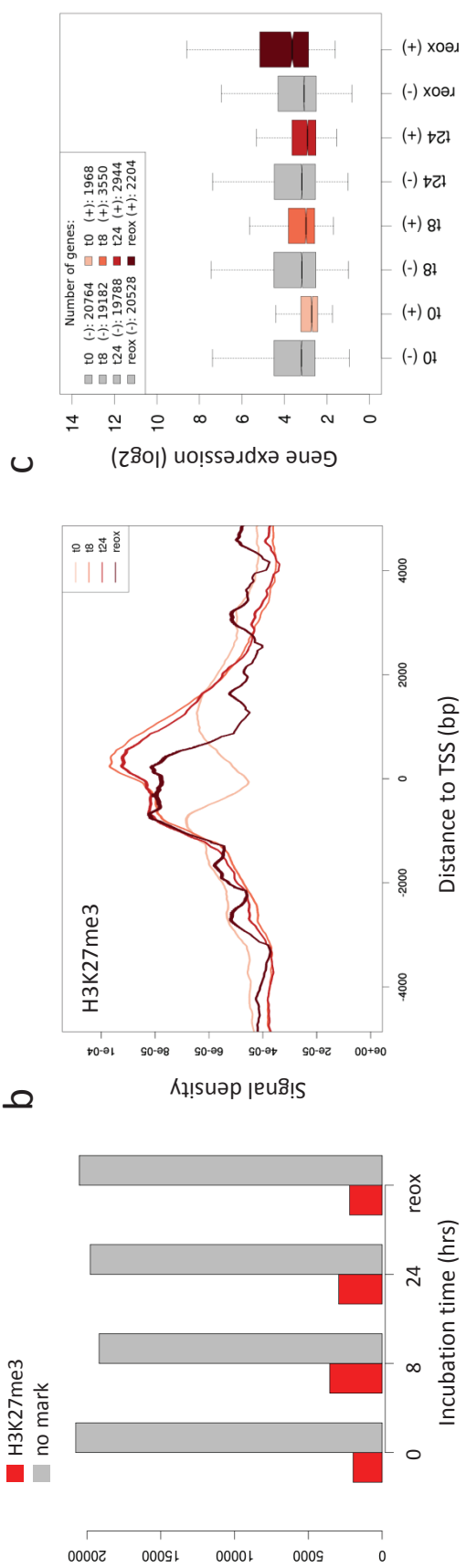

$\sigma$

səuə8 fo ıəqunN 
Intergenic $\mathrm{H} 3 \mathrm{~K} 4 \mathrm{me} 3$-association increased from $\pm 10 \%$ to $\pm 30 \%$; the relative genic/ intergenic H3K27me3-distribution did not change as a result of oxygen deprivation ( $\pm 50 \%$; Figure 1B). Although overall H3K4me3-peaks associated to genic regions decreased from $88 \%$ to $69 \%$ in low oxygen conditions (Figure 1B), the number of genes H3K4me3-associated genes increased from $46 \%$ to $56 \%$ under at 24 hours hypoxia (Figure S2Aa). In contrast to the rather stable relative number of H3K27me3 peaks associated with genic regions (48\% at normoxia vs. $49 \%$ at 24 hours hypoxia; Figure 1B), the percentage of genes associated with H3K27me3 nearly doubled at $16 \%$ after 8 hours of oxygen deprivation (Figure 2Ba). Thus, although the percentage of marked genes increased for both methyl marks (Figure 2A, B/a), the relative increase of H3K4me3-peaks was higher in intergenic regions, whereas relative gain H3K27me3 occurred mostly in genic regions (Figure 1B; Figure S3A, B). Alignment of all trimethylation-associated sequence tags to genic or intergenic regions, showed that the majority (>98\%) of trimethyl-marks was directed towards genic regions, irrespective of oxygen tension (Figure S3C, D). The combined above data confirms that genomic decoration with $\mathrm{H} 3 \mathrm{~K} 4 \mathrm{me} 3$ and $\mathrm{H} 3 \mathrm{~K} 27 \mathrm{me} 3$ relates to gene regulation and indicates that $\mathrm{H} 3$ trimethylation does not occur in a random fashion but is strongly directed towards genic regions.

Upon reoxygenation the overall number of trimethyl-marked genes reverted to that measured in normoxic cells (15752 genes, normox; 31919 genes, 24 hrs hypox vs. 15236 genes, reox, respectively) (Figure 1B, Figure 2A-B/a). The intergenic:genic ratio of trimethyl-mark distribution of $\mathrm{H} 3 \mathrm{~K} 4 \mathrm{me} 3$ was nearly fully restored to that initially observed at ambient oxygen levels $(0.14, \mathrm{t}=0 ; 0.45, \mathrm{t}=24$ vs. 0.16 , reox); in contrast, a relative genic-enrichment $\mathrm{H} 3 \mathrm{~K} 27 \mathrm{me} 3$ was apparent at the 8 hours reoxygenation time point (49\% vs. 61\%) (Figure 1B). This data is consistent with the poor H3K27me3-peak correlation between normoxic and reoxygenated samples (noted above) and shows that hypoxia-induced H3K27me3-marking at genic regions is relatively resistant to restored HKDM activity.

\section{Epigenetic profiles correlate with transcriptional state}

To profile the oxygen tension-induced enrichment of H3K4me3 and H3K27me3 at genic regions in more detail, enrichment data were visualized using TSS-centered plots. Under control conditions, H3K4me3 was prominently enriched around the TSS with a distinctive signal-depletion directly over the TSS; this finding is consistent with earlier reports showing reduced nucleosome presence at this site (Figure 2Ab) (Jiang and Pugh, 2009). In sharp contrast, H3K27me3-marking at normoxia coincided mainly within gene body-enrichment, consistent with the reported "blanketing" enrichment of 
H3K27me3 (Figure 2Bb) (Adriaens et al., submitted; Bracken et al., 2006; Pauler et al., 2009). The H3K4me3-enrichment profile did not significantly change as a result of hypoxia or reoxygenation (Figure 2Ab), whereas H3K27me3-enrichment was selectively enhanced over and around the TSS during oxygen deprivation (Figure 2Bb). Taken together, this data confirmed the genic bias of histone $\mathrm{H} 3$ trimethylation at $\mathrm{K} 4$ and $\mathrm{K} 27$ and revealed a pronounced TSS-directed hypoxia-induced increase of H3K27me3

To correlate H3K4 and H3K27-trimethylation to gene transcription, the ChIP-seq data were compared to expression data of corresponding genes. Expression array analysis revealed that a significant number of transcripts were down-regulated in response to upon hypoxic exposure (Figure S4A). Gene ontology (GO) classification confirmed regulation of expected processes in response to hypoxia (Figure S4C), consistent with published data (Chan and Giaccia, 2007). H3K4me3-marked genes were significantly higher expressed at all individual time points (Figure 2Ac) whereas H3K27me3-marked genes were transcribed at substantially lower levels as compared to non-marked genes, except for the reoxygenation time point (Figure 2Bc). H3K4me3enrichment showed a positive correlation with gene expression level at all time-points analyzed (data not shown). There was no apparent correlation between relative H3K27me3-enrichment and gene expression/repression at any time point suggesting that despite the overall increased H3K27me3-enrichment during oxygen deprivation, H3K27me3-enrichment was not repressive per se.

Reoxygenation only partially restored the hypoxia-induced epigenetic marking and expression levels, indicating that although many cellular processes are affected by reoxygenation, cells are at a transition phase at the 8 hours reoxygenation time point

(Figure S4B, D). Consistent with the earlier noted sustained H3K27me3-marking during reoxygenation, the TSS-associated H3K27me3-enrichment profile was not immediately reversed at $\mathrm{t}=+8$ hours (reox), confirming that specifically the $\mathrm{K} 27 \mathrm{me} 3$ signaldistribution at the TSS was relatively resistant to the effects of reoxygenation compared to overall H3K27me3-marking. The loss of genic H3K4me3-marking at reoxygenation, despite increase overall expression suggested functional uncoupling of epigenetic marking and gene expression in response to acute reoxygenation stress.

\section{Hypoxia induces bivalency}

As $46.3 \%$ of genes (22732) was already H3K4me3-enriched at normoxia (Figure 2Ab) and H3K27me3-enrichment specifically increased around the TSS (Figure $\mathbf{2 B b}$ ), the hypoxia-induced increase in trimethylation was likely to increase the frequency of co-occurrence of both trimethyl marks. Therefore, we selectively examined genes that showed double marking during hypoxia and calculated the Pearson correlation 
coefficient (pcc) at different time points to determine co-occurrence of trimethyl marks. Pearson correlation analysis in control samples (normoxia) indicated a negative correlation between 800 bp up- and downstream of the TSS, corresponding to H3K4me3 enrichment and H3K27me3 depletion around the TSS (Figure S5A, cf. Figure 2Ab). Regions more distant (up- or downstream) from the TSS and the TSS itself showed a positive pcc, which correlated with low presence of both marks (TSS) and absence of H3K4me3 (distant). H3K4me3-monovalency around the TSS at $\mathrm{t}=0$ was lost during hypoxia, consistent with the increased H3K27me3-enrichment around the TSS in response to hypoxia (Figure S5A, cf. Figure 2Bb). Restoration of ambient oxygen tension correlated with a sharp pcc decline at the TSS, correlating with a relative loss of one of two me3-marks at the TSS itself (Figure S5A, $c f$. Figure 2Ab, Bb).

To study the co-occurrence of H3K4me3 and H3K27me3 in further detail, all genes which were enriched for both trimethyl marks were plotted for each time point separately. We detected an almost 3 fold ( 821 genes, $t=0$ to $2200, t=24$ ) increase in the number of double-marked genes as a result of hypoxia. Remarkably, double-marking was maintained at more than $70 \%$ of genic loci upon reoxygenation ( $t=24$ hrs vs. reox; Figure S5B). This data demonstrated that hypoxia increases co-occurrence of H3K4 and H3K27-trimethylation marks, and suggested that this epigenetic state was selectively maintained upon reoxygenation despite global loss in trimethylation.

\section{Transcription at bivalent genes is primarily determined by $\mathrm{H} 3 \mathrm{~K} 4 \mathrm{me} 3$}

Expression of double-marked genes was marginally affected by hypoxia: $16 \%$ of double-marked genes were actively expressed in control samples vs. $15 \%$ and $13 \%$ at 8 and 24 hours hypoxia, respectively. To determine the consequences of dynamic epigenetic change, including the co-occurrence of H3K4me3 and H3K27me3, for geneexpression, genes were grouped based on their enrichment profile at normoxia (i.e. non-marked, $\mathrm{K} 4 \mathrm{me} 3$ only, $\mathrm{K} 27$ only, double marked); genes were then compared for changes in average expression levels in relation to changes in epigenetic marking. $90 \%$ of genes which were H3K4me3-marked at normoxia showed persistent H3K4me3marking well into hypoxia ( $\mathrm{t}=24 \mathrm{hrs}$ ) and remained transcriptionally active (Figure 3A).

Figure 3. Epigenetic profiles correlate with transcriptional state. A-D; Changes in epigenetic marking (left panels) at $\mathrm{t}=\mathbf{2 4}$ hours vs. $\mathrm{t}=0$ of genes that were A) H3K4me3-marked, B) double H3K4me3/H3K27me3marked, C) H3K37me3-marked or D) not marked under normoxic conditions $(t=0)$. Altered median gene expression in relation to changes in epigenetic marking (right panels). E-H Changes in epigenetic marking (left panels) at reoxygenation vs. $\mathrm{t}=24$ of genes that were $\mathrm{E}$ ) H3K4me3-marked, F) double H3K4me3/H3K27me3marked, G) H3K27me3-marked or $\mathbf{H})$ not marked under hypoxic conditions $(\mathrm{t}=24)$. Altered median gene expression in relation to changes in epigenetic marking (box-plots; right panels). 


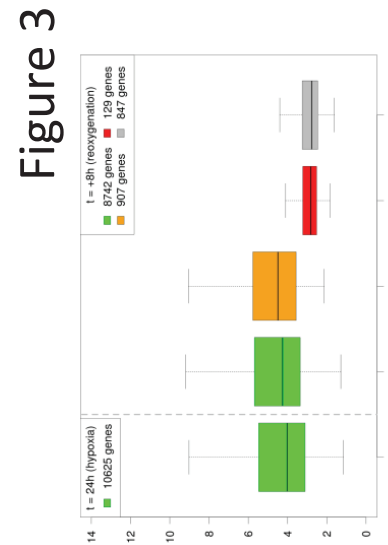

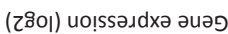
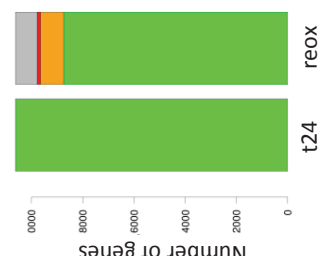

ப

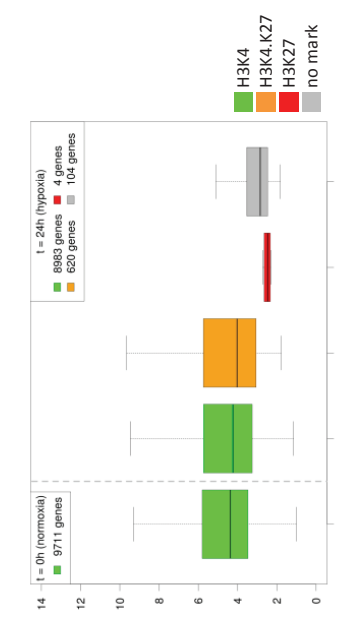

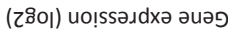
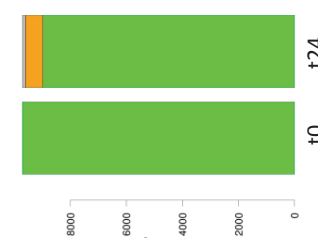

$<$

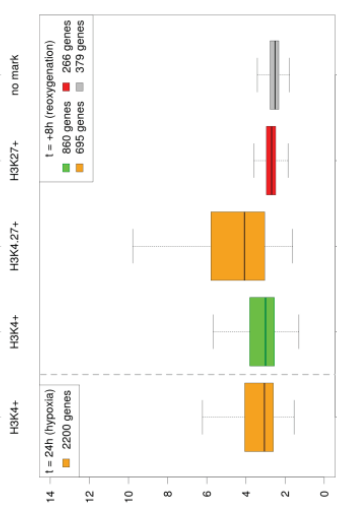

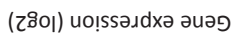

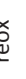

$\stackrel{\text { 品 }}{2}$
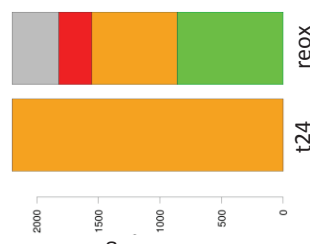

ᄂ

səuəร fo גəqunN

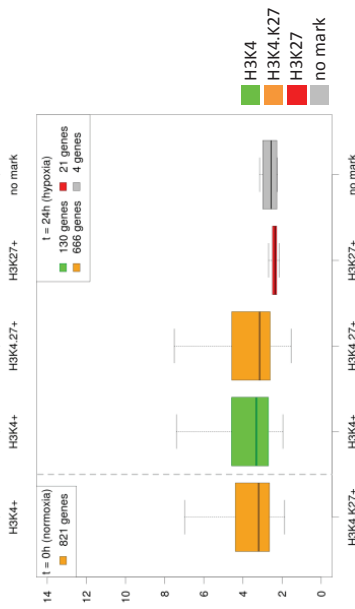

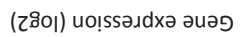
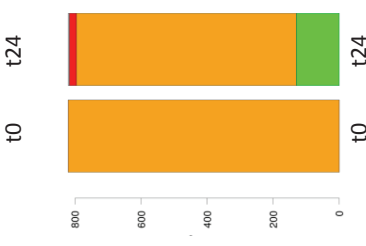

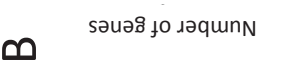

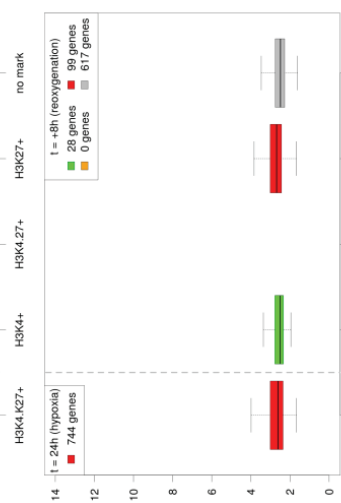

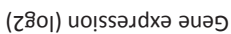

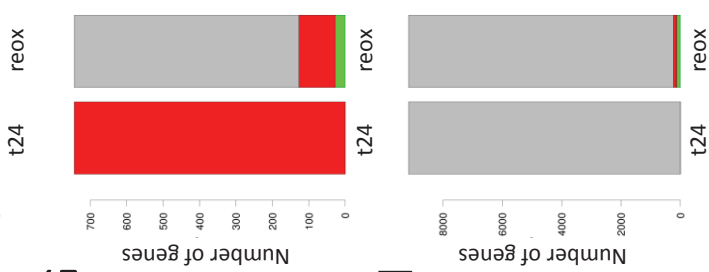

(1)

I
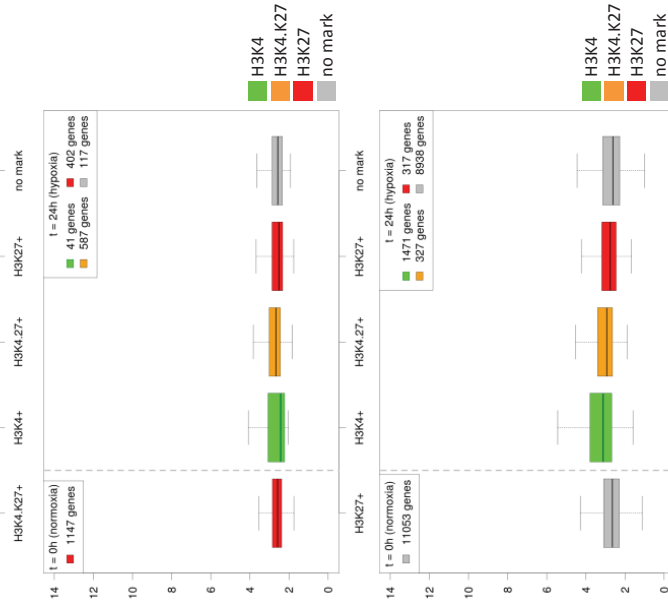

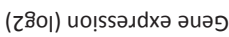
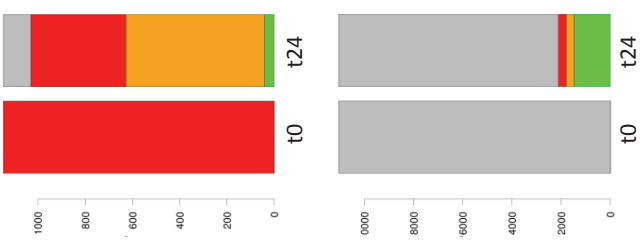

səuə8 fo גəqunN

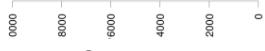

səuə8 fo גəqunN 
Notably, loci at which H3K4me3 was lost (single H3K27+ or non-marked at $\mathrm{t}=24 \mathrm{hrs}$ ) were transcriptionally silenced. In sharp contrast, H3K4me3 genes which gained $\mathrm{K} 27 \mathrm{me} 3+(\mathrm{K} 4 / \mathrm{K} 27+$ at $\mathrm{t}=24 \mathrm{~h})$ maintained their averages expression level. Genes that carried both trimethyl marks at $\mathrm{t}=0$ had an intermediate expression level compared to H3K4me3-marked (high expression) and H3K27me3 or non-marked genes (low expression) (Figure 3A-D; right panels). Approximately $20 \%$ of the double-marked genes either lost H3K4me3 or H3K27me3-enrichment in response to oxygen deprivation, while the majority of genes retained both marks. Loss of H3K27me 3 did not affect gene-expression, but loss of $\mathrm{H} 3 \mathrm{~K} 4 \mathrm{me} 3$ clearly reduced expression. In contrast, the H3K27me3-only pre-marked genes showed a strong shift toward gain of H3K4me3 in hypoxic conditions ( $\pm 55 \%$; Figure $3 \mathrm{C}$ ). However, gain of H3K4me3 did not parallel increased gene expression as all genes remained transcriptionally silent. Of all non-marked genes $(\mathrm{t}=0) \pm 81 \%$ maintained this status after 24 hours of hypoxia; genes that gained H3K4me3-single or both me3-marks (around 15\% in total) displayed slightly elevated median expression of $\mathrm{H} 3 \mathrm{~K} 4 \mathrm{me} 3 /$ single-marked genes; this mirrored the effect of H3K4me3-marking under oxygenated conditions, albeit with substantially lower expression levels (Figure 3D).

In the context of reoxygenation, the majority of H3K4me3-marked genes maintained some level of H3K4me3-marking, and gene expression was not changed upon acquisition of H3K27me3 (Figure 3E); non-marked genes ( $t=24 \mathrm{hrs}$ ) were slightly induced upon gain of H3K4me3 (independent of simultaneous gain of K27me3; Figure $3 \mathbf{H})$. The double-marked and the H3K27me3-only marked genes showed the most significant loss of $\mathrm{H} 3 \mathrm{~K} 27 \mathrm{me} 3$ upon reoxygenation: $56 \%$ and $87 \%$, respectively, which paralleled the global loss of H3K27me3 (Figure 3F, G, cf. Figure 1B). Approximately 30\% of the double-marked genes was significantly expressed at $\mathrm{t}=+8$ hours (Figure S5B). Double-marked genes that lost H3K4me3 (30\% and/or H3K27me3) showed a concomitant reduction of gene expression (Figure 3F). Remarkably, genes that maintained their double-marked status, showed higher transcription levels, whereas those that retained H3K4me3-only did not change their expression (Figure 3F), suggesting that gain of $\mathrm{H} 3 \mathrm{~K} 27 \mathrm{me} 3$ is regulatory. H3K27me3-only enrichment ( $\mathrm{t}=24 \mathrm{hrs}$ ) correlated with a transcriptionally silenced status that did not change in response to reoxygenation (Figure $\mathbf{3 G}$ ).

Thus, at any given time point H3K27me3-only or no-marking generally correlated with transcriptional silencing, whether the condition was pre-existent or acquired, and gain of H3K4me3 at these sites did not meaningfully change expression. H3K4me3 pre-marked genes were generally transcriptionally active, and maintained their expression level independent of gain of H3K27me3, as long as K4 marking was not lost. Changes of H3K4me3-trimethylation primarily control transcription in the context of 
adaptation to altered oxygenation. In combination with $\mathrm{H} 3 \mathrm{~K} 4 \mathrm{me} 3$, the H3K27me3-mark represented an important exception to the general trend that H3K27me3-marking correlates with silencing. The combined data identify H3K27me3 as the most transitory mark in response to hypoxia/reoxygenation appeared to be H3K27me3.

\section{Normoxic trimethylation profiles correlate with transcription status}

Since the (re)distribution of H3K27me3-enrichment appeared most affected by hypoxia and reoxygenation, we next determined the relation between the specific intragenic location of $\mathrm{H} 3 \mathrm{~K} 27 \mathrm{me} 3-$ marks and transcriptional regulation. For this purpose, the enrichment-profile of all genes was differentiated into three separate regions: promoter (promoter; $-3000 /-100$ bp TSS), transcription start site (TSS; $-100 /+1000$ bp), and gene-body (broad; $+1000 /$ last exon) as was defined before (Young et al., 2011). Genes were then assigned to one of three classes at each time point based on their H3K27me3-enrichment (Figure S6A/a): a distinct H3K27me3 peak upstream of the TSS (promoter class), ii) a distinct peak at the TSS (TSS-class) or iii) no peak, but instead the typical Polycomb-repression-associated 'blanketed' distribution over the gene body (broad class; Figure S6Aa). In control samples (normoxia) the majority of genes $( \pm 55 \%$ ) displayed the broad (gene body/blanketing) enrichment, whereas the TSS $( \pm 22 \%)$ and promoter $( \pm 23 \%)$ class were equally represented (Figure $4 \mathrm{Aa}$ ). Hypoxia induced a 1.7 fold increase in $\mathrm{H} 3 \mathrm{~K} 27$ me3-enrichment (total), and a clear shift toward TSS-class marking $( \pm 22 \%, \mathrm{t}=0$ to $\pm 33 \%, \mathrm{t}=8$ ), whereas relative promoter-class marking decreased $( \pm 23 \%, \mathrm{t}=0$ to $\pm 14 \%, \mathrm{t}=8$ ); this relative distribution was maintained for the duration of hypoxic exposure (Figure $4 \mathbf{B}, \mathbf{C} / \mathbf{a}$ ). TSS-class marking decreased in response to reoxygenation ( $\pm 31 \%$ to $\pm 16 \%$ ), whereas the percentage of $\mathrm{H} 3 \mathrm{~K} 27$ me3-blanketed genes increased to $\pm 75 \%( \pm 55 \%, t=0,24)$ (Figure $4 \mathrm{Da}$ ). This data indicated that hypoxia induces a reversible selective increase of $\mathrm{H} 3 \mathrm{~K} 27$ me3-marking at transcription start sites.

To gain insight into the biological relevance of H3K27me3-marking, average gene expression measurements were plotted for each individual subclass, and compared to the overall expression of all H3K27me3-enriched genes and non-marked genes. Distinctive TSS-marking and gene body-enrichment correlated with low expression/ repression, compared to the total pool of H3K27me3-marked genes (Figure S6A-D/b). Promoter H3K27me3-enrichment was associated with a significantly higher average gene expression level, compared to the total H3K27me3-marked gene population, and equaled expression levels of non-marked genes; consistent with the absence of transcriptional repression, gene body H3K27me3-marking was low at promoter-marked genes (Figure S6Aa,b). A similar correlation was observed between intragenic enrichment-profile and expression at acute or chronic hypoxia (Figure S6B-C/a, b). 
Chapter 5 |
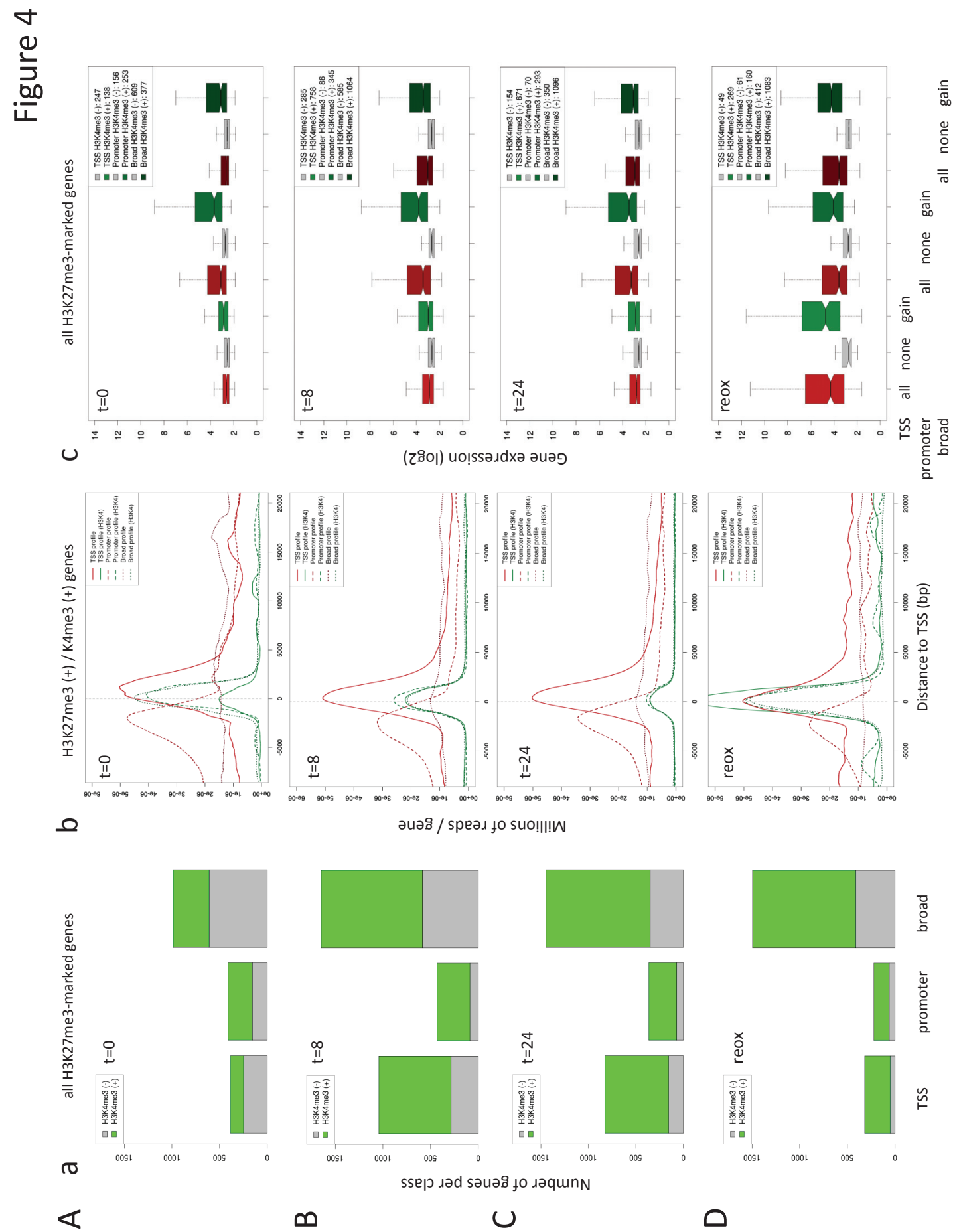

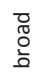
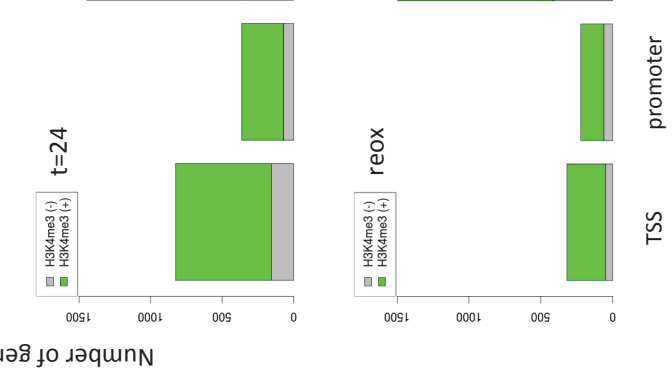

$\varangle$

$\infty$

$\cup$

口 
Figure 4. Transcription status is primarily controlled by H3K4me3. A-D; a panels) H3K27me3-enriched genes were classified as TSS-, promoter- and gene body (broad)-marked genes and differentiated according to their H3K4me3-status (marked (+) or non-marked (-); b panels) TSS-associated enrichment profiles (average number of reads) for the corresponding H3K27me3-classes (i.e. +/- gain of H3K4me3 as in a panel); red lines (solid/dashed/dotted) represent all single H3K27me3-marked genes, green lines represent all double H3K4me3/K27me3-marked genes (sold/dashed/dotted lines correspond to H3K27me3 classes in (a); c panels) box-plots: gain of H3K4me3-enrichment was plotted against median expression levels. Comparative analysis was done at $(A) t=0,(B) t=8,(C) t=24$ hours hypoxia and (D) after reoxygenation ( $t=+8$ hours). Bottom legend (c): subscripts correspond to all H3K27me3-marked genes (all), the H3K27me3-only (none) and H3K4me3/H3K27me3-marked sequences (gain), and are differentiated between promoter, TSS and gene body classes as indicated.

We next asked whether the presence of specific H3K4me3-enrichment within these 3 H3K27me3-classes could explain the observed increased expression at these loci. To this end, all H3K27me3-marked loci were divided into two categories: HK4me3-negative and H3K4me3-marked loci (Figure 4A-D/a). At normoxic conditions, all three H3K27me3-classes contained bivalent genes; the highest number of double-marked genes $(377 ; \pm 40 \%)$ was found in the gene body H3K27me3-class; whereas within the promoter-class loci, nearly $2 / 3^{\text {rd }}$ of all loci was marked (253 genes) (Figure 4Aa). Expression within these $\mathrm{H} 3 \mathrm{~K} 27 \mathrm{me3}$-classes positively correlated with the presence of the H3K4me3-mark (Figure 4Ac; red boxes marked "all"), and with relative H3K4me3/ TSS-enrichment (promoter and gene-body class; Figure 4Ab); TSS-double marked genes showed the lowest median expression. Under hypoxic conditions, the number of double-marked genes increased substantially in each H3K27me3-class (Figure 4B, C/a), yet the normoxic expression-ratio was maintained (i.e. promoter class $>$ gene body class > TSS class), despite progressive reduction of H3K4me3-marking at the TSS in all three H3K27me3-classes (Figure 4B-C/b, c).

The association between intragenic H3K27me3-marking and transcriptional regulation was lost in the context of reoxygenation stress (Figure S6Da, $\mathbf{b}$; $c f$. Figure 2Bc); of note: even silenced loci (TSS-peak or blanketing-type distribution; repressed at $\mathrm{t}=0$ and during hypoxia), showed a sudden rise of transcriptional activity (Figure S6Db). Loss of repression under these conditions was not explained by quantitative changes in H3K27me3-enrichment (data not shown). Following reoxygenation, expression was induced at all H3K4me3-marked loci irrespective of H3K27me3-subclass, but not at H3K27me3/single-marked loci (Figure 4Da-c). Of note, the relatively highest increase of H3K4me3-occupation within the H3K27me3/TSS-class correlated with the highest median expression induction (3,7 fold change; $t=24 \mathrm{vs}$. reox).

Thus, our comparative analysis of intragenic marking and gene activity identified H3K4me3-marking as the dominant determinant of expression of a locus. The data 
suggested that transcriptional status under hypoxic conditions was refractory to gain of H3K27me3-enrichment and revealed a clear association between defined promotertype H3K27me3-marking and a transcriptionally permissive state (vs. TSS or gene body-enrichment and transcriptional repression) under normoxic and hypoxic conditions. Reoxygenation induced massive transcriptional deregulation, irrespective of the H3K27me3-status of loci.

\section{Hypoxia-induced bivalency partially overlaps with bivalent genes in ES-cells}

More than 800 genes were classified as bivalently marked under normoxic conditions, based on co-occurrence of H3K4me3 and H3K27me3-marks within genic regions (Figure 3B). To determine whether bivalent genes specifically mapped to any of the intragenic H3K27me3-profile subclasses, we analyzed the promoter, TSS or gene body H3K27me3-marking groups for H3K4me3-presence. Approximately $80 \%$ (345 genes) of $\mathrm{H} 3 \mathrm{~K} 27 \mathrm{me} 3 /$ promoter-enriched genes was also enriched for H3K4me3 in hypoxic samples ( $c f$. Figure 4A-D/a). The broadly H3K27me3-enriched genes showed a relatively lower H3K4me3-enrichment ( $\pm 64 \%, 1064$ genes; $\mathrm{t}=8 \mathrm{hrs}$ ) compared to promoter or TSS-class genes (758 genes); of TSS-H3K 27 me 3 genes $\pm 72 \%$ was associated with $\mathrm{H} 3 \mathrm{~K} 4 \mathrm{me3}$, (cf. Figure 2Ab); the presence of H3K4me3 around the TSS (Figure A-D/ back) determined gene expression status, independent of the intragenic location of K3K27me3. Consistent with the earlier observed transcriptional deregulation at reoxygenation, like H3K27me3, H3K4me3-occupation failed to correlate with transcriptional status. This data indicated that bivalent genes are strongly represented among H3K27me3/TSS and promoter-marked genes.

To determine how bivalency was accomplished, the original epigenetic status of the bivalently marked genes (at $\mathrm{t}=8 \mathrm{hrs}$ hypoxia) was traced back to normoxia and displayed as a function of time spent at low oxygen. We first selected the TSSassociated bivalency (H3K4me3 and H3K27me3 at TSS) genes for further analysis. Bivalency increased approximately 3 fold (758 to 2157 genes) in response to loss of oxygen (Figure 5Aa).

Figure 5. Hypoxia-induced bivalency. A-C; a panels) Gain of epigenomic bivalency between A) control cells and hypoxic cells ( $\mathrm{t}=8 \mathrm{hrs}$ ); $\mathbf{B}$ ) during hypoxia ( $\mathrm{t}=24 \mathrm{vs}$. $\mathrm{t}=8 \mathrm{hrs}$ hypoxia) and $\mathrm{C}$ ) upon reoxygenation (reox vs. $\mathrm{t}=24 \mathrm{hrs}$ hypoxia); indicated are gene categories that acquired bivalency by: gain of H3K4me3, gain of H3K27me3, gain of both, or that had retained bivalency; left bars indicate bivalency at previous time point; bars to the right indicate loss of bivalency at time point analyzed; b panels) box-plots indicating median expression values of bivalently marked genes at indicated experimental time points; indicated are gene categories that acquired bivalency by: gain of H3K4me3, gain of H3K27me3, gain of both, or that had retained bivalency; indicated are also sites at which bivalency was lost (far right boxes); D) distribution of H3K4me3 and H3K27me3-marks at (top) high-CpG, (middle) intermediate and (bottom) low CpG-content promoters. 
ำ
$\frac{1}{2}$
$\frac{00}{4}$
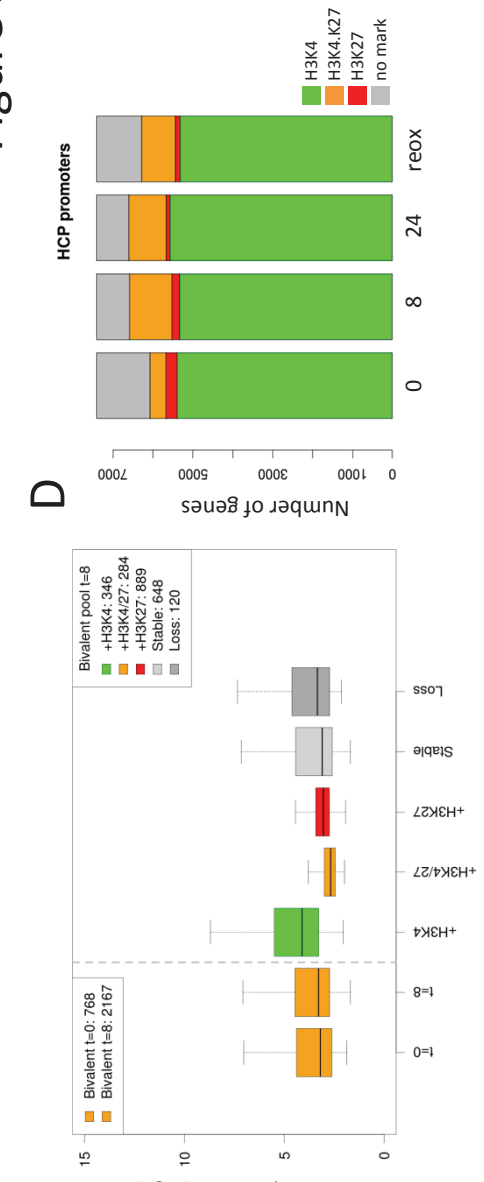

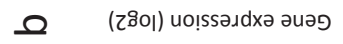

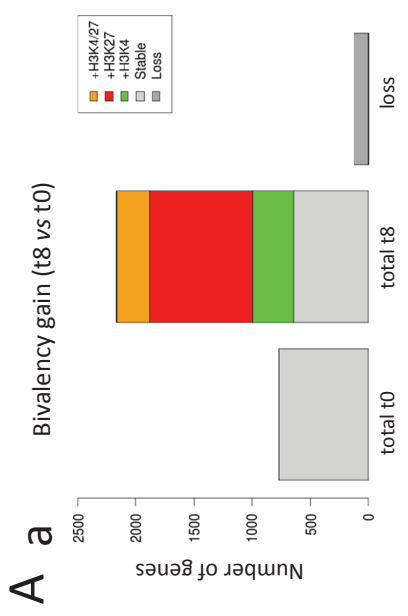

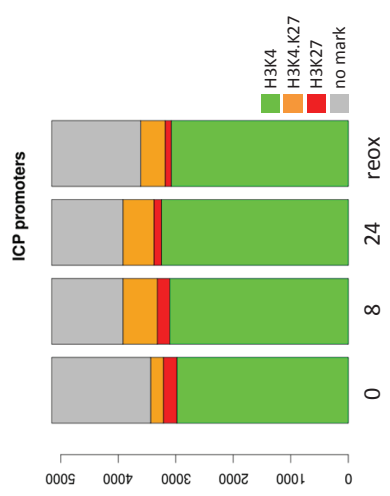

səuə8 fo ^əqunN

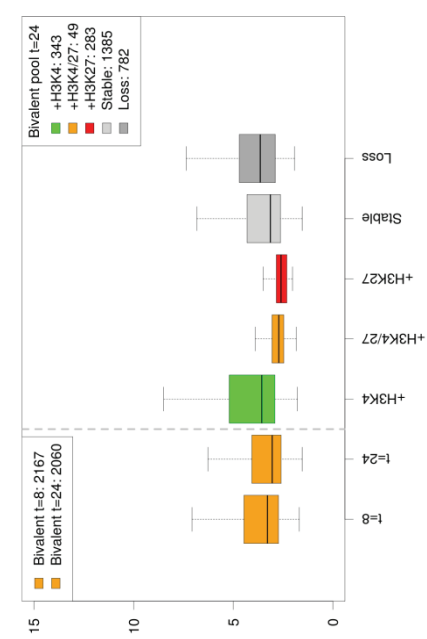

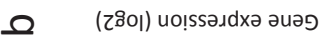
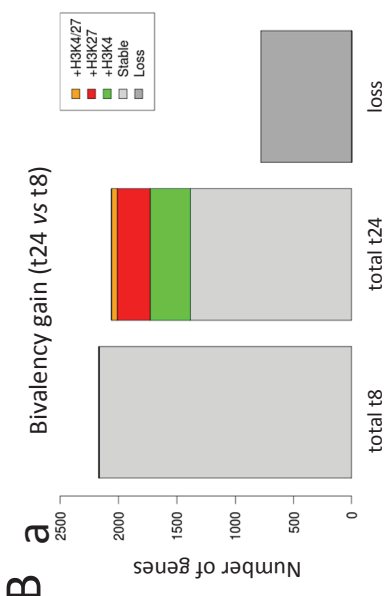
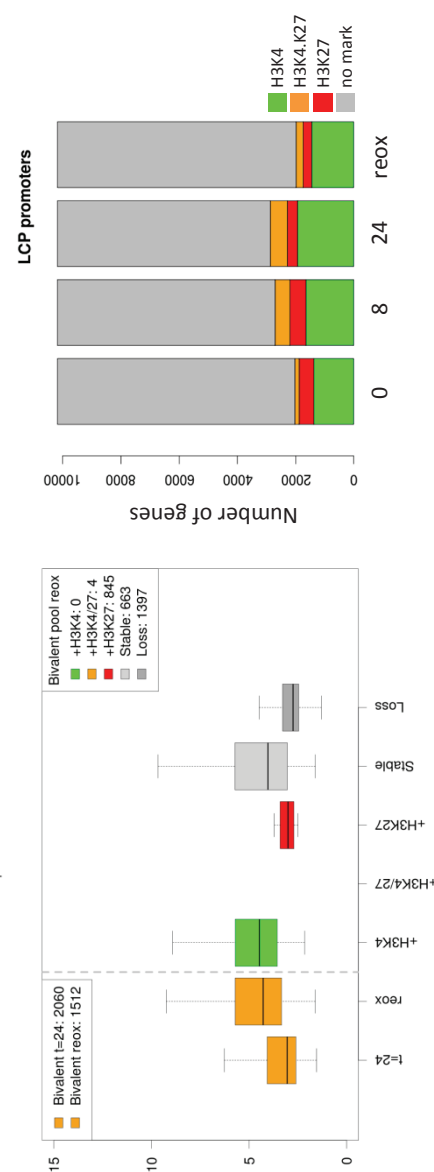

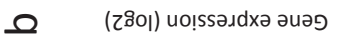

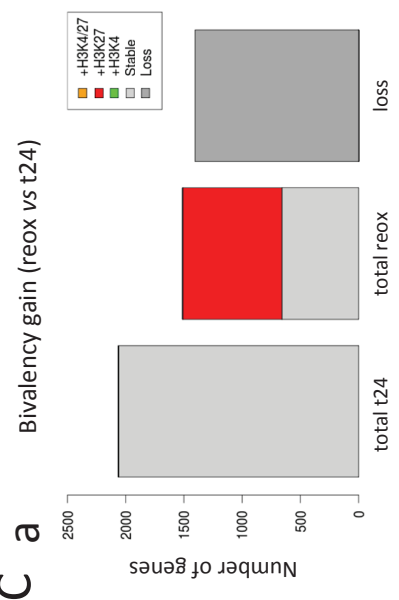


Gain of H3K4me3 appeared under-represented in regards to acquisition of bivalency, due to the fact that a many TSS genes had pre-existent H3K4me3-marking at baseline. More than half of the bivalent genes ( $\pm 54 \% ; 1173$ genes) at 8 hours of hypoxia had acquired H3K27me3-marking alone or in combination with H3K4me3-marking in response to oxygen withdrawal (Figure 5Aa). During prolonged hypoxia ( $t=8$ to $t=24$ hours) or at reoxygenation, again more than $\pm 50 \%$ of acquired bivalent loci showed gain of H3K27me3 (Figure 5Ba); this finding is in good agreement with the above proposed notion that H3K27me3-marking is most prone to dynamic modulation.

Expression levels of genes with acquired bivalency, largely reflected those corresponding to their original epigenetic status: for instance, genes that gained H3K27me3 were significantly higher expressed compared to stable bivalent genes (Figure 5Ac, BC). Although a possible contribution of mRNA stability could formally not be excluded, transcriptional changes should be detectable over an $8 \mathrm{hrs}$ interval and, moreover, we observed a similar trend for genes that gained H3K4me3.

Upon reoxygenation many bivalent genes were lost, and despite a substantial gain of bivalency, the number of bivalently marked loci dropped to $\pm 75 \%$ compared at $t=24$ and was still 2 fold higher compared to normoxia: more than 500 loci displayed bivalent epigenetic marking at the reoxygenation time point, suggesting that somehow these genes were shielded from acute HKDM activity (Figure $\mathbf{5 C a}$ ). Compared to loss of $\mathrm{H} 3 \mathrm{~K} 4 \mathrm{me} 3-$ marking in response to reoxygenation $( \pm 9 \%$ of $\mathrm{H} 3 \mathrm{~K} 4 \mathrm{me} 3 /$ single-marked genes; $t=24 \mathrm{hrs}$ vs. reox), H3K27me3 was considerably less stable $( \pm 87 \%$ at $\mathrm{H} 3 \mathrm{~K} 27 \mathrm{me} 3 /$ single-marked genes). At bivalent loci, the loss was relatively larger for H3K4me3 ( $\pm 29 \%)$, whereas H3K27me3-loss was substantially less ( $\pm 56 \%$ ) compared to single-marked genes.

In conclusion, hypoxia induces epigenetic bivalency, which correlates best with a TSS-specific increase of H3K27me3. Bivalent genes show transcriptional activity which overall resembles their original expressional status under normoxia.

Figure 6. Comparative analysis of hypoxia-induced bivalent genes in MCF7 cells and bivalent loci in ES cells. A; Heatmap indicating overlap of bivalent genes in this study with bivalently marked genes in embryonal stem cells (ES cells; orange = bivalently marked, red = H3K27me3 only, green $=\mathrm{H} 3 \mathrm{~K} 4 \mathrm{me} 3$ only, black = no marking), transcription factor genes (TF; blue = TF gene, black = not a TF gene), genes containing promoter CpG islands $(\mathrm{CpG} ;$ blue $=\mathrm{CpG}$ island present, black $=$ no $\mathrm{CpG}$ island $)$ and genes whose TSS coincides with transposonexclusion zones (TEZ; blue = located in TEZ, black = not TEZ associated). B; Gene Ontology enrichment analysis of bivalent genes; listed are $\mathrm{GO}$ analysis at $\mathrm{t}=0, \mathrm{t}=8$ and $\mathrm{t}=24$ hours hypoxia; same $\mathrm{GO}$-analysis was done on $\mathrm{ES}$ cell-bivalent markers published elsewhere (Bernstein et al., 2006). 

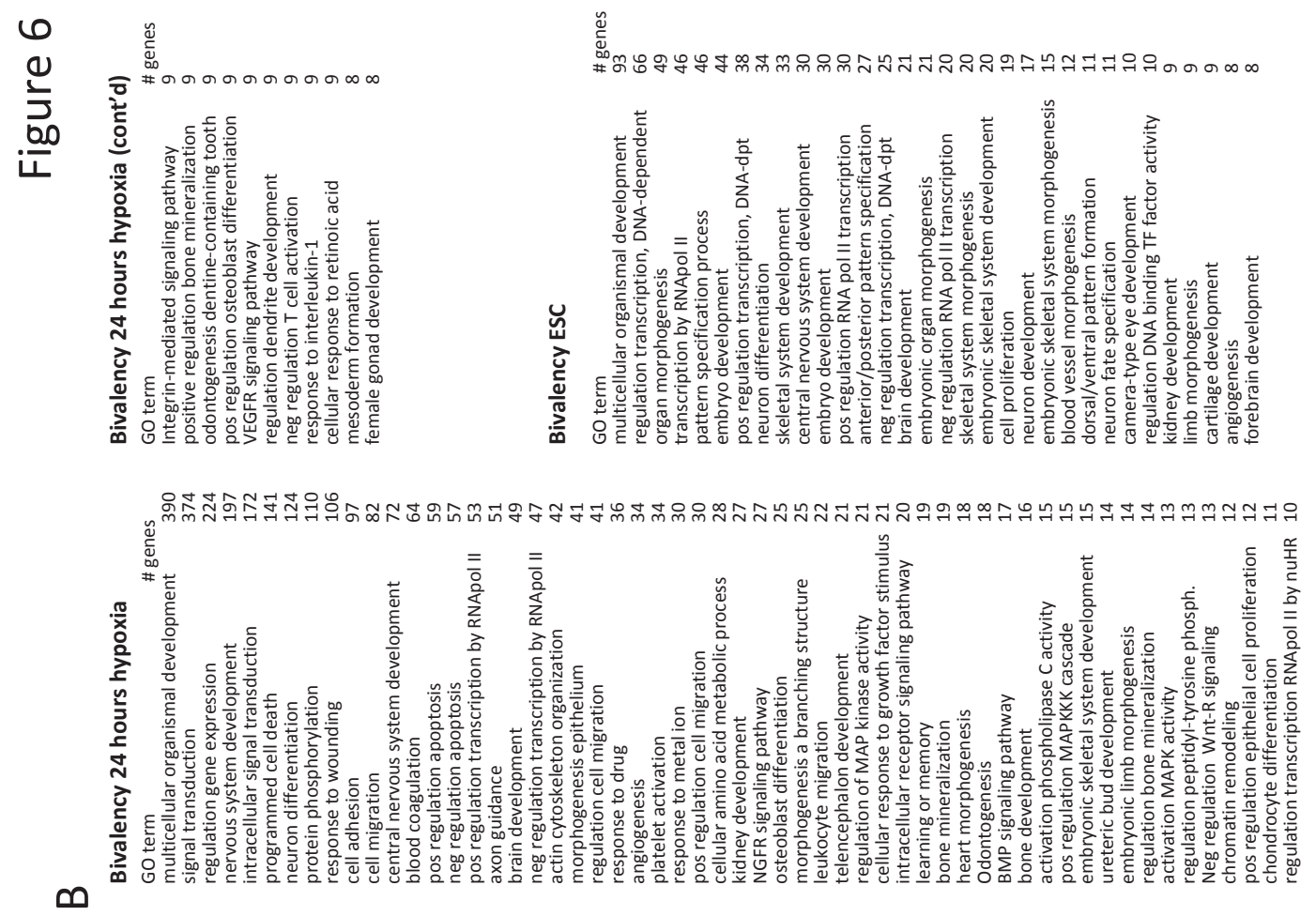

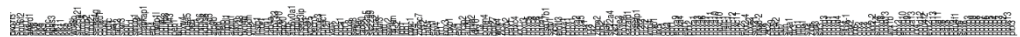

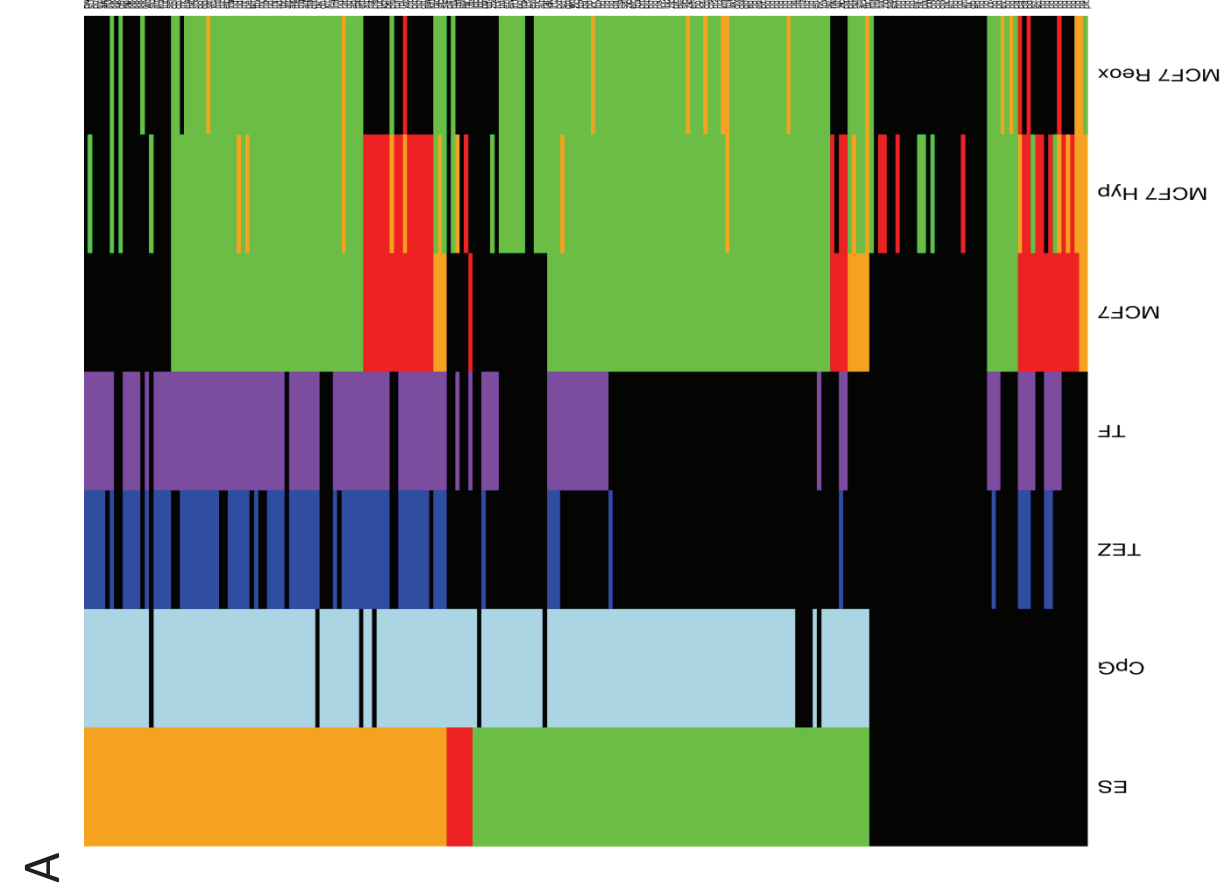


Bivalency in embryonal stem cells (ES cells) coincides with genomic CpG-content (i.e. CpG islands) (Ku et al., 2008). To verify whether this applied to bivalent epigenetic marking in MCF7 cancer cells as well, the prevalence of bivalently marked genes in high CpG-content promoter/enhancer regions (HCP) versus intermediate and low CpGregions (ICP and $L C P$, respectively) was determined. H3K4me3 preferentially localized to HCP regions; 5198 HCP, 2775 ICP and 1015 LCP promoters were positive for H3K4me3 at normoxia, corresponding to $70 \%$ of charted HCPs, $54 \%$ of ICPs and $10 \%$ of LCPs respectively (Figure 5D). Bivalently-marked genes showed a highly similar distribution pattern: $366 \mathrm{HCP}, 183$ ICP and 72 LCP promoters were positive for both trimethyl marks in control cells $(t=0)$. Relatively few promoters showed H3K27me3marking, consistent with the relative $\mathrm{H} 3 \mathrm{~K} 27$ me3-enrichment over gene bodies or at the TSS. H3K27me3/promoter distribution was opposite of that of H3K4me3: 310 HCP, 2720 ICP and 577 LCP promoters were enriched for H3K27m3; (Figure 5D, cf. Figure 4). Although the overall number of H3K4me3, H3K27me3 or H3K4me3/H3K27me3enriched genes increased in response to hypoxia, their relative association to HCP, ICP and LCP was maintained (data not shown).

In the context of stem/progenitor cells bivalently marked loci are often found among key developmental control genes (Bernstein et al., 2006; Mikkelsen et al., 2007). We next examined whether the hypoxia-induced bivalently marked gene set in MCF7 cancer cells showed overlap with bivalent genes previously identified in ES cells. Approximately 600 of the hypoxia-induced bivalent genes in MCF7 cells match a previously identified group of bivalent genes in ES cells (Bernstein et al in 2006) (Figure 6A). Comparative GO analysis of both bivalent gene pools revealed a distinctive overlap of functional pathways involved and confirmed the presence of bivalently marked developmental genes (Figure 6B). Many of these genes were already bivalently marked at the 8 hour hypoxia time point (Figure S7), suggesting that these loci are pre-set targets for bivalent marking under low oxygen. Hence, our findings suggest that oxygen deprivation in breast cancer cells induces bivalent epigenetic marking at genes which are also bivalently marked in ES cells and which control key processes during development.

\section{DISCUSSION}

We here report that oxygen deprivation induces massive genome-wide trimethylation at H3K4 and H3K27 through inhibition of JHDM function. Our data establish that oxygen-sensing by HKDM represents a direct link between the microenvironment and epigenetic regulatory mechanisms. Comparative analysis of ChIP- 
deep sequencing and expression array data sets revealed that H3K27me3-occupation at the TSS and over the gene body correlates with transcriptional repression, whereas H3K4me3 marking under all conditions correlates with gene activity. We establish H3K4me3-enrichment as the most relevant determinant of transcription status. H3K27me3-acquisition at the TSS with pre-existent H3K4me3 marking does not alter the expression status. We show that a subset of genes acquired bivalency, by gain of H3K27me3 (54\% 1173 genes). The bivalent subset represents genes previously identified as bivalently marked genes in ES cells.

Recent advances in the field of epigenetics suggest that chromatin state is more dynamic than originally anticipated. Comprehensive insight into transcriptional reprogramming in response to changing microenvironments requires systematical genome-wide mapping of epigenetic marks as a function of cell type, differential state and micro-environment (Berger, 2007). We here studied dynamic changes in histone trimethylation in the context of cellular adaptation to changed oxygenation (hypoxic stress, reoxygenation). To robustly detect H3K27me3-enrichment, we designed a novel enrichment-finding protocol including a normalization/summarization strategy. Importantly, this also allows us to obtain quantitative measurements of H3K27me3marking between samples (time points). Applying this novel strategy, we were able to reproduce reported correlation between gene-body associated 'blanketing' H3K27me3profile and transcriptional repression, thereby validating our analytical approach (Adriaens et al., submitted). The prominent TSS-centered H3K4me3-enrichment at expressed loci, and the marked low nucleosome abundance right over the TSS, is also consistent with earlier findings (Barski et al., 2007).

We detected a clear positive correlation between TSS/H3K4me3-enrichment and gene expression at all time-points studied. H3K27me3 displayed multiple distinct enrichment profiles, in line with previous publications (Hon et al., 2009; Young et al., 2011). H3K27me3-enrichment across the gene body followed the classical Polycombassociated repressive H3K27me3-enrichment profile, which is referred to as "blanketing" (Bracken et al., 2006; Pauler et al., 2009). Specific enrichment at the promoter region, in combination with a marked depletion of the signal within the gene body, overlaps with H3K4me3-enrichment profiles and appears permissive for active transcription; consequently bivalently-marked genes are expressed at low levels (Bernstein et al., 2006; Bracken et al., 2006; Mikkelsen et al., 2007). Interestingly, we observed enhanced H3K27me3-marking upon oxygen deprivation at the TSS, relative to promoter and gene-body; this resulted in bivalent marking of a substantial number of genes ( $\pm 950-1100 ; t=8$ and $24 \mathrm{hrs}$ combined). By inference, inhibition of HKDMs is likely to be responsible for the increased bivalency at TSS in the absence of molecular oxygen. The co-occurrence of both methyl marks was first described in embryonic stem 
cells (ES cells) and is thought to mark key developmental control genes as "poised" for transcriptional activation. We and others found a marked increase of bivalent marking at CpG-rich genomic regions (Young et al., 2011). CpG-islands are usually hypomethylated (at the DNA level), but instead are marked by trimethylation on histone 3 lysine 4 (H3K4me3) or lysine 27 (H3K27me3) (Azuara et al., 2006; Young et al., 2011); conversely, bivalent domains highly correlate with the presence of CpG-islands, and may reflect a competition between PcG-recruitment (silencing) and transcriptional activation (Lynch et al., 2011). A recent study in Xenopus showed that bivalency may, in reality, affect different alleles (Akkers et al., 2009). Hence, to conclusively prove that H3K4me3 and H3K27me3 occur on the same chromatin segments and are not merely a reflection of different cell pools, sequential ChIP for both marks needs to be performed.

Enhancers represent an important class of functional domains involved in transcriptional regulation. In contrast to promoter regions, enhancers operate as highly tissue-specific elements, and their (functional) existence cannot be directly inferred from underlying DNA-sequences (Heintzman et al., 2007). In addition, enhancers may be located thousands of base pairs removed in cis from their target genes, or even on a different chromosome, which makes it difficult to define and relate such regulatory regions to genes. No public MCF7 ChIP-seq data set is currently available, which includes TF/DNA-binding profiles and/or enhancer-associated epigenetic regulatory factors (e.g. p300) or associated histone modifications (e.g. H3K4me1 and H3K27ac). To probe for such histone modifications would be relevant in light of our current findings, as it was reported that bivalent promoters are associated to a specific subclass of enhancer elements which are H3K4me1/H3K27me3-marked instead of H3K4me1/ H3K27ac which is the case for active promoters (Rada-Iglesias et al., 2011).

The increased global methylation we observed could either be the result of increased histone methyltransferase activity or reduced histone demethylase activity. Transcriptional analysis of candidate enzymes involved in histone (de)methylation did not suggest a singular role for any of these factors in this respect. Expression of JHDM proteins is known to be controlled by HIF1a; likewise HTM expression is controlled by oxygen. Nevertheless, we showed that the hypoxia-increased trimethylation was HIF1a-independent. Most demethylases depend on molecular oxygen for their activity, making JHDM oxygen sensors

The exact relevance of Polycomb-associated marking and occupation at different intragenic positions is currently not fully understood. The H3K27me3-mark is bound by chromobox proteins belonging to Polycomb group proteins (Min et al., 2003). PcG proteins were shown to be present at active RPOL2 promoters in Drosophila and to interact with basal TF transcription factors (Breiling et al., 2001). Target-gene silencing 
via distant enhancer-looping to gene promoters has been shown in flies and in mammalian systems (Simon and Kingston, 2009). In addition, PcG complexes associate with splicing factors, revealing an as of yet poorly understood role in regulation of gene expression. In the context of our hypoxic-stress model, increased bivalency mostly correlated with gain of TSS-H3K27me3. It is also interesting to note that H3K27me3marking appeared to be the most prone to dynamic change. The transcriptional status of the corresponding loci, however, was generally maintained; hence, this finding suggests that, once marked for transcription, recruitment of HMT activity and concomitant H3K27me3-marking per se is not sufficient for transcriptional repression.

Despite the global demethylation following reoxygenation, a substantial number of loci were bivalently marked 8 hours into restoration of oxygen levels. It is currently unclear whether these loci are protected from HKDM activity and/or selectively methylated by K27-directed HMTs. It is conceivable that some other local epigenetic aspect (e.g. inhibitor recruitment of higher-order chromatin structure) prevents access and/or shields nucleosomes against demethylation. Of relevance, HKMTs are often found in close conjunction with HKDMs for opposing epigenetic marks; together these paired epigenetic modulators are thought to reinforce transcriptional decisions (co-stability) (Dodd et al., 2007). It is tempting to speculate about a causal role for similarly cooperative H3K4me3 HMTs/H3K27me3 HDMs in the establishment of bivalency under HKDM-inhibitory conditions. Irrespective of the exact underling mechanism, our findings confirmed our initial hypothesis and define a novel role for oxygen in epigenetic regulation of stress responses. Future experiments should address the question whether loss of UTX/JMJD3 abolishes the increase in H3K27me3 levels, both on the global as well as on individual gene level. Although the involvement of demethylases was recently addressed in the context of hypoxia-induced increased H3K4me3-marking (Zhou et al., 2010), our studies provide additional insight in to the effects of reoxygenation on epigenomic and transcriptomic responses.

Acquisition of bivalency under hypoxic conditions is a phenomenon which may have relevance in the context of tumor biology (i.e. plasticity, malignancy). TSSassociated H3K27me3 was previously shown to be prominent in embryonic stem cells (ES cells) (Young et al., 2011). As bivalency was proposed to represent an aspect of hierarchical differentiation, increased epigenomic bivalency may reflect acquisition of a more primitive chromatin state (Bernstein et al., 2007; Fisher and Fisher, 2011; Herz et al., 2009). Of relevance, stem cell niches are known to be hypoxic (Covello et al., 2006; Mohyeldin et al., 2010; Silvan et al., 2009; Welford and Giaccia, 2011). As cancer development is thought to be sustained by cancer stem cells (Clarke and Fuller, 2006; Hanahan and Weinberg, 2011; Lobo et al., 2007), it is tempting to speculate about a possible role for bivalent marking in re-establishing a 'poised' gene status, and that 
hypoxic microenvironment selects for or drives tumor plasticity through acquisition of a less differentiated epigenome. In this study, we applied severe hypoxic conditions $\left(<0.02 \% \mathrm{O}_{2}\right)$ to achieve a semi-synchronized cell population response. As tumor cells are subjected to constantly fluctuating oxygen concentrations due to poor vasculature, the effect of exposure to varying hypoxic as well as cycling hypoxia-reoxygenation conditions on epigenomic remodeling and associated transcriptional changes needs to be addressed (Chan et al., 2009). Repetitive exposure to hypoxia/reoxygenation-stress may impose a more realistic micro-environment and concomitant selection-pressure on a cancer cell population and support increased tumor plasticity and malignant progression. Our observations put forward the exciting possibility that repeated exposure to hypoxia may promote or select for stem-ness or cancer stem cell phenotypes.

To our knowledge, our study for the first time combines analysis of dynamic data sets on gene expression and histone methylation in the context of adaptation to acute environmental changes. The number of studies on altered chromatin states and the role of epigenetic modifiers, e.g. in the context of embryogenesis, spermatogenesis, metabolic disorders and tumorigenesis steadily increases (Bogdanovic et al., 2011; Fisher and Fisher, 2011). Combined epigenomic profiling and detailed analysis of transcriptional reprogramming will be crucial for fundamental understanding of pluripotency and clinical application of induced precursor cells.

\section{MATERIALS AND METHODS}

Cell Culture, expression vectors and viral infections MCF7 (human mammary adenocarcinoma) and DU145 (human prostate carcinoma) cells (ATCC) were cultured at $37^{\circ} \mathrm{C}, 5 \% \mathrm{CO}_{2}, 100 \%$ humidity in Dulbecco's Modified Eagle Medium: Nutrient Mixture F-12 (DMEM/F12 1:1; MCF7) and DMEM McCoy's 5A medium (DU145). The culture medium was supplemented with $10 \%$ fetal calf serum (FCS; Bodinco, Alkmaar, the Netherlands), 200 mM L-glutamine and antibiotics For hypoxic exposure, cells were transferred to a MACS VA500 microaerophilic workstation (Don Whitley Scientific, Shipley, UK) for the indicated duration. The atmosphere in the chamber consisted of $<0.02 \% \mathrm{O}_{2}, 5 \% \mathrm{H}_{2}, 5 \% \mathrm{CO}_{2}$, and $74 \% \mathrm{~N}_{2}$. For reoxygenation, cells were transferred back to the regular tissue culture inhibitor containing ambient oxygen levels (21\%). RNA interfering HIF1a sequences were obtained from Sigma (clone TRCN0000010819). Knock-down of HIF1alpha was achieved using lentiviral shRNA constructs. Lentiviral particles were generated by co-transfection of 293T cells with packaging plasmids pCMVdR8.74psPAX2 and PMD2.G together with shRNA vector pLKO.1. Virus supernatant was harvested 48 and 72 hrs post transfection. MCF7 cells were 
transduced with lentiviral supernatant in the presence of $8 \mu \mathrm{g} / \mathrm{ml}$ polybrene. Infected cells were selected for 2 days in $2 \mu \mathrm{g} / \mathrm{ml}$ puromycin containing media. Efficiency of plasmid constructs was verified by immunoblotting.

Protein isolation and Western blot analysis Cells were grown to around $70 \%$ confluency before they were transferred to the hypoxic chamber. Protein extraction was carried out using RIPA buffer supplemented with protease and phosphatase inhibitors. Lysates were further processed and protein concentrations were determined. Immunoblotting (IB) was performed as described previously, using antibodies raised against HIF1a (610958; BD Pharmingen ${ }^{\mathrm{TM}}$ /BD Biosciences, Franklin Lakes, NJ, USA), H3K4me3 (Ab8580; Abcam, Cambridge, UK), H3K27me3 (07-449; Upstate Biotechnology/Millipore, Waltham, MA, USA), H3K9/K14ac (Abcam, Cambridge, UK), H3 (ab1791; Abcam, Cambridge, UK), b-Actin (C4, 69100, MP Biomedicals, Solon, OH, USA), For more details, see Supplementary Materials and Methods.

Chromatin immunoprecipitation (ChIP) assays MCF7 cells were transferred to hypoxic culturing conditions for the indicated durations and immediately fixed to avoid reoxygenation. Cells were disrupted by sonication, yielding genomic DNA fragments ranging from 200-1000 bp, with a bulk size of 200-500bp. For each immunoprecipitation 10-20 million cells were used. $1 \%$ of the cell suspension was kept aside as input DNA to use as a reference. ChIPs were performed and analyzed as described previously with minor adjustments (Bracken et al., 2006), see Supplementary Materials and Methods. Antibodies used include: H3K4me3 (Ab8580; Abcam), H3K27me3 (07-449; Upstate), CBX8 (LAST; courtesy Klaus Hansen, Copenhagen, Denmark) and HA as a negative control (sc-805; Santa Cruz Biotechnology, Santa Cruz, CA, USA). The immunoprecipitated DNA was checked for enrichment using real-time PCR and quantified by fluorescence detection using Quant-iT ${ }^{\mathrm{TM}}$ Picogreen ${ }^{\circledR}$ dsDNA Reagent (Molecular Probers/Invitrogen, Eugene, OR, USA) before deep sequencing was applied.

Deep sequencing Input and ChIP samples were further processed and sequenced at the Ontario Institute for Cancer Research using the Illumina next generation sequencing platform. Processing involved size selection to enrich for mononucleosome-sized fragments, linker annealing and PCR amplification. Each resulting library was subsequently loaded onto individual lanes of a flow cell and sequenced using the 36 bp paired-end protocol on the Illumina Genome Analyser IIx (GAllx). In order to obtain sufficient sequencing depth additional lanes were sequenced if necessary. All data obtained from each individual sample was pooled. Data sets will be made publically available. 
Genome alignment, normalization, background correction; identification of enriched regions Image processing and base calling was performed using Illumina software tools provided by the manufacturer). Subsequent paired-end genome alignment was performed using Novoalign with human genome 18 (hg18) used as a reference genome. Only uniquely aligned reads were used for further analysis. To remove PCR artifacts all data were collapsed prior to peak calling. H3K27me3 data sets were normalized based on identification of regions with stable H3K27me3enrichment between all samples analyzed; the cumulative area under the curve for all peaks within these regions was scaled relative to the smallest value among the samples; the normalization strategy will be outlined in detail elsewhere (Adriaens et al., submitted). After normalization, a single cut-off value for all samples was set at the enrichment level (peak height; estimated by input sample data) above which H3K27me3 signal correlates with a known H3K27me3-binding protein under normoxic conditions ( $t=0$; data not shown). Signal intensities below this cut-off were not considered biologically relevant. To identify enriched regions in the ChIP samples relative to the input control, the peak caller Findpeaks (version 4.0) was used. For H3K4me3 the default settings were used, whereas for H3K27me3 the settings were adjusted in order to detect blanketing enrichment next to sharply defined peaks (Adriaens et al., submitted).

Microarrays RNA for microarray application was isolated using RNeasy mini kit (Qiagen, Hilden, Germany) according to manufacturer's protocol. Isolations were performed in triplicate. Total RNA samples were analyzed using the Affymetrics expression array platform (Affymetrix Gene Chip $1.0 \mathrm{ST}$ ). After scanning, data pre-processing and data analysis were done with $\mathrm{R}$ (http://www.R-project.org; version 2.12) using the Bioconductor (http://www.bioconductor.org; version 2.7). Data were backgroundcorrected and normalized using gcRMA (Wu et al., 2004). Microarray data will be made available at Gene Expression Omnibus (GEO; http://www.ncbi.nlm.nih.gov/geo/).

Integration with gene expression; gene ontology analysis To enable integration of expression data with our enrichment data, all Affymetrix probe IDs were converted into ENSEMBL gene identifiers. The expression level of an individual gene is defined as the average of all probe sets representing this gene on the array. Genes were considered expressed if expression exceeds 100 for at least one independent time-point. Genes were called regulated if genes are expressed ( $>100$ for 1 or more time-points) and the fold change between 2 independent time-points is $\geq 2$. All genes which were not represented on the micro-array were not included for further analysis. For the identification of enriched genes, we defined a gene as the region between its $5^{\prime}$ (most 
upstream TSS) and 3' (last exon) end plus $5 \mathrm{~kb}$ regulatory regions up and downstream respectively. A gene was called marked when there was a peak present within this region as determined by the enrichment finding procedure. Gene Ontology enrichment analysis was performed using topGO (Alexa et al., 2006; Ashburner et al., 2000). Comparative GO analysis with embryonal stem cell bivalent markers was based on published data (Bernstein et al., 2006).

Data visualization For data visualization several plots were created in $\mathrm{R}$, including histograms, gene tracks, genome plots, pie charts, TSS plots, boxplots, scatter plots and pie-charts. For the TSS plots all genes were considered in the same orientation (from $5^{\prime}$ to $3^{\prime}$ end), and the average signal intensity was depicted for a region defined in number of base pairs surrounding the TSS, as indicated. Box-plots show the $25^{\text {th }}$ and $75^{\text {th }}$ quartile (as indicated by the box), the median (indicated as a line within the box), and the whiskers indicate the $5 \%$ and $95 \%$ percentile respectively. Notches in box-plots indicate confidence intervals (5-95\%) of the median.

Data summarization To study different enrichment profiles for H3K27me3, each gene was assigned to the Promoter, the TSS or the Broad class in analogy to published analysis (Young et al., 2011). To properly classify all genes, each gene was first divided into three regions: the promoter region (-3000/-100 base pairs (bp) in relation to the TSS), the TSS region (-100/1000 bp), the broad region (+1000 bp to the last exon). Genes shorter than 4000 bp were excluded from the analysis, as they were too small to reliably assign them to either profile. Genes were allocated to the different classes based on which of the three regions contained the largest amount of signal scaled to the size of each region, provided it contains a peak which showed at least $25 \%$ more enrichment compared to any other peak within the gene (Promoter and TSS class), or it contains a enrichment above the average enrichment at more than $35 \%$ of the region (Broad class). Genes allocated to either region which did not meet above criteria, were not considered for profile analysis.

\section{ACKNOWLEDGEMENTS}

We are much indebted to many colleagues ( $c f$. Materials and Methods section) for sharing research materials and technical assistance; to Joep Geraedts and Ronit Sverdlov for critically reading the manuscript and to Chris Evelo, Lars Eijssen, Timothy Beck, Marianne Koritzinsky, members of the MAASTRO and Molecular Genetics departments for scientific discussions. These studies received financial support from the Dutch Science Organization (ZonMW-NWO): VIDI grant 016.046.362 (JWV); transnational University Limburg (tUL) grant (JWV/BGW). 


\section{REFERENCES}

Adriaens, M., Prickaerts, P., Chan-Seng-Yue, M., Beck, T., Wouters, B.G., Voncken, J.W., and Evelo, C. Capturing ChIP-seq profiles of H3K27me3 in dynamic biological systems (submitted).

Akkers, R.C., van Heeringen, S.J., Jacobi, U.G., Janssen-Megens, E.M., Francoijs, K.J., Stunnenberg, H.G., and Veenstra, G.J. (2009). A hierarchy of H3K4me3 and H3K27me3 acquisition in spatial gene regulation in Xenopus embryos. Dev Cell 17, 425-434.

Alexa, A., Rahnenfuhrer, J., and Lengauer, T. (2006). Improved scoring of functional groups from gene expression data by decorrelating GO graph structure. Bioinformatics 22, 1600-1607.

Ashburner, M., Ball, C.A., Blake, J.A., Botstein, D., Butler, H., Cherry, J.M., Davis, A.P., Dolinski, K., Dwight, S.S., Eppig, J.T., et al. (2000). Gene ontology: tool for the unification of biology. The Gene Ontology Consortium. Nat Genet 25, 25-29.

Azuara, V., Perry, P., Sauer, S., Spivakov, M., Jorgensen, H.F., John, R.M., Gouti, M., Casanova, M., Warnes, G., Merkenschlager, M., and Fisher, A.G. (2006). Chromatin signatures of pluripotent cell lines. Nat Cell Biol 8, 532-538.

Barski, A., Cuddapah, S., Cui, K., Roh, T.Y., Schones, D.E., Wang, Z., Wei, G., Chepelev, I., and Zhao, K. (2007). High-resolution profiling of histone methylations in the human genome. Cell 129, 823-837.

Berger, S.L. (2007). The complex language of chromatin regulation during transcription. Nature 447, 407-412.

Bernstein, B.E., Meissner, A., and Lander, E.S. (2007). The mammalian epigenome. Cell 128, 669-681.

Bernstein, B.E., Mikkelsen, T.S., Xie, X., Kamal, M., Huebert, D.J., Cuff, J., Fry, B., Meissner, A., Wernig, M., Plath, K., et al. (2006). A bivalent chromatin structure marks key developmental genes in embryonic stem cells. Cell 125, 315-326.

Bogdanovic, O., van Heeringen, S.J., and Veenstra, G.J. (2011). The epigenome in early vertebrate development. Genesis.

Bracken, A.P., Dietrich, N., Pasini, D., Hansen, K.H., and Helin, K. (2006). Genome-wide mapping of Polycomb target genes unravels their roles in cell fate transitions. Genes Dev 20, 1123-1136.

Breiling, A., Turner, B.M., Bianchi, M.E., and Orlando, V. (2001). General transcription factors bind promoters repressed by Polycomb group proteins. Nature 412, 651-655.

Brizel, D.M., Sibley, G.S., Prosnitz, L.R., Scher, R.L., and Dewhirst, M.W. (1997). Tumor hypoxia adversely affects the prognosis of carcinoma of the head and neck. Int J Radiat Oncol Biol Phys 38, 285-289.

Brock, H.W., and Fisher, C.L. (2005). Maintenance of gene expression patterns. Dev Dyn 232, 633-655.

Brown, J.M. (1999). The hypoxic cell: a target for selective cancer therapy--eighteenth Bruce F. Cain Memorial Award lecture. Cancer Res 59, 5863-5870.

Cao, R., Wang, L., Wang, H., Xia, L., Erdjument-Bromage, H., Tempst, P., Jones, R.S., and Zhang, Y. (2002). Role of histone H3 lysine 27 methylation in Polycomb-group silencing. Science 298, 1039-1043.

Chan, D.A., and Giaccia, A.J. (2007). Hypoxia, gene expression, and metastasis. Cancer Metastasis Rev 26, 333339.

Chan, N., Koch, C.J., and Bristow, R.G. (2009). Tumor hypoxia as a modifier of DNA strand break and cross-link repair. Curr Mol Med 9, 401-410.

Clarke, M.F., and Fuller, M. (2006). Stem Cells and Cancer: Two Faces of Eve. Cell 124, 1111-1115.

Cloos, P.A., Christensen, J., Agger, K., and Helin, K. (2008). Erasing the methyl mark: histone demethylases at the center of cellular differentiation and disease. Genes Dev 22, 1115-1140. 
Covello, K.L., Kehler, J., Yu, H., Gordan, J.D., Arsham, A.M., Hu, C.J., Labosky, P.A., Simon, M.C., and Keith, B. (2006). HIF-2alpha regulates Oct-4: effects of hypoxia on stem cell function, embryonic development, and tumor growth. Genes Dev 20, 557-570.

Dodd, I.B., Micheelsen, M.A., Sneppen, K., and Thon, G. (2007). Theoretical analysis of epigenetic cell memory by nucleosome modification. Cell 129, 813-822.

Esteller, M. (2007). Cancer epigenomics: DNA methylomes and histone-modification maps. Nat Rev Genet 8, 286-298.

Fisher, C.L., and Fisher, A.G. (2011). Chromatin states in pluripotent, differentiated, and reprogrammed cells. Curr Opin Genet Dev 21, 140-146.

Hanahan, D., and Weinberg, R.A. (2011). Hallmarks of cancer: the next generation. Cell 144, 646-674.

Hansen, K.H., Bracken, A.P., Pasini, D., Dietrich, N., Gehani, S.S., Monrad, A., Rappsilber, J., Lerdrup, M., and Helin, K. (2007). A model for transmission of the H3K27me3 epigenetic mark. Nat Cell Biol advanced online publication.

Heintzman, N.D., Stuart, R.K., Hon, G., Fu, Y., Ching, C.W., Hawkins, R.D., Barrera, L.O., Van Calcar, S., Qu, C., Ching, K.A., et al. (2007). Distinct and predictive chromatin signatures of transcriptional promoters and enhancers in the human genome. Nat Genet 39, 311-318.

Herz, H.-M., Nakanishi, S., and Shilatifard, A. (2009). The Curious Case of Bivalent Marks. Developmental Cell 17, 301-303.

Hockel, M., Schlenger, K., Aral, B., Mitze, M., Schaffer, U., and Vaupel, P. (1996). Association between tumor hypoxia and malignant progression in advanced cancer of the uterine cervix. Cancer Res 56, 4509-4515.

Hon, G.C., Hawkins, R.D., and Ren, B. (2009). Predictive chromatin signatures in the mammalian genome. Hum Mol Genet 18, R195-201.

Hou, H., and Yu, H. (2010). Structural insights into histone lysine demethylation. Curr Opin Struct Biol 20, 739748.

Ivan, M., Kondo, K., Yang, H., Kim, W., Valiando, J., Ohh, M., Salic, A., Asara, J.M., Lane, W.S., and Kaelin, W.G., Jr. (2001). HIFalpha targeted for VHL-mediated destruction by proline hydroxylation: implications for $\mathrm{O} 2$ sensing. Science 292, 464-468.

Jaakkola, P., Mole, D.R., Tian, Y.M., Wilson, M.I., Gielbert, J., Gaskell, S.J., Kriegsheim, A., Hebestreit, H.F., Mukherji, M., Schofield, C.J., et al. (2001). Targeting of HIF-alpha to the von Hippel-Lindau ubiquitylation complex by O2-regulated prolyl hydroxylation. Science 292, 468-472.

Jiang, C., and Pugh, B.F. (2009). Nucleosome positioning and gene regulation: advances through genomics. Nat Rev Genet 10, 161-172.

Johnson, A.B., Denko, N., and Barton, M.C. (2008). Hypoxia induces a novel signature of chromatin modifications and global repression of transcription. Mutat Res 640, 174-179.

Kenneth, N.S., and Rocha, S. (2008). Regulation of gene expression by hypoxia. Biochem J 414, $19-29$.

Kouzarides, T. (2007). Chromatin modifications and their function. Cell 128, 693-705.

Krieg, A.J., Rankin, E.B., Chan, D., Razorenova, O., Fernandez, S., and Giaccia, A.J. (2010). Regulation of the Histone Demethylase JMJD1A by Hypoxia-Inducible Factor 1a Enhances Hypoxic Gene Expression and Tumor Growth. Molecular and Cellular Biology 30, 344-353.

Ku, M., Koche, R.P., Rheinbay, E., Mendenhall, E.M., Endoh, M., Mikkelsen, T.S., Presser, A., Nusbaum, C., Xie, X., Chi, A.S., et al. (2008). Genomewide analysis of PRC1 and PRC2 occupancy identifies two classes of bivalent domains. PLoS Genet 4, e1000242.

Lobo, N.A., Shimono, Y., Qian, D., and Clarke, M.F. (2007). The Biology of Cancer Stem Cells. Annual Review of Cell and Developmental Biology 23, 675-699. 
Lohse, B., Kristensen, J.L., Kristensen, L.H., Agger, K., Helin, K., Gajhede, M., and Clausen, R.P. (2011). Inhibitors of histone demethylases. Bioorg Med Chem 19, 3625-3636.

Lynch, M.D., Smith, A.J., De Gobbi, M., Flenley, M., Hughes, J.R., Vernimmen, D., Ayyub, H., Sharpe, J.A., Sloane-Stanley, J.A., Sutherland, L., et al. (2011). An interspecies analysis reveals a key role for unmethylated CpG dinucleotides in vertebrate Polycomb complex recruitment. EMBO J 31, 317-329.

Mikkelsen, T.S., Ku, M., Jaffe, D.B., Issac, B., Lieberman, E., Giannoukos, G., Alvarez, P., Brockman, W., Kim, T.K., Koche, R.P., et al. (2007). Genome-wide maps of chromatin state in pluripotent and lineage-committed cells. Nature $448,553-560$.

Min, J., Zhang, Y., and Xu, R.M. (2003). Structural basis for specific binding of Polycomb chromodomain to histone H3 methylated at Lys 27. Genes Dev 17, 1823-1828.

Mohyeldin, A., Garzon-Muvdi, T.s., and Quinones-Hinojosa, A. (2010). Oxygen in Stem Cell Biology: A Critical Component of the Stem Cell Niche. Cell Stem Cell 7, 150-161.

Park, P.J. (2009). ChIP-seq: advantages and challenges of a maturing technology. Nat Rev Genet 10, 669-680.

Pauler, F.M., Sloane, M.A., Huang, R., Regha, K., Koerner, M.V., Tamir, I., Sommer, A., Aszodi, A., Jenuwein, T., and Barlow, D.P. (2009). H3K27me3 forms BLOCs over silent genes and intergenic regions and specifies a histone banding pattern on a mouse autosomal chromosome. Genome Res 19, 221-233.

Rada-Iglesias, A., Bajpai, R., Swigut, T., Brugmann, S.A., Flynn, R.A., and Wysocka, J. (2011). A unique chromatin signature uncovers early developmental enhancers in humans. Nature 470, 279-283.

Robertson, G., Hirst, M., Bainbridge, M., Bilenky, M., Zhao, Y., Zeng, T., Euskirchen, G., Bernier, B., Varhol, R., Delaney, A., et al. (2007). Genome-wide profiles of STAT1 DNA association using chromatin immunoprecipitation and massively parallel sequencing. Nat Methods 4, 651-657.

Schuettengruber, B., Chourrout, D., Vervoort, M., Leblanc, B., and Cavalli, G. (2007). Genome regulation by polycomb and trithorax proteins. Cell $128,735-745$.

Selak, M.A., Armour, S.M., MacKenzie, E.D., Boulahbel, H., Watson, D.G., Mansfield, K.D., Pan, Y., Simon, M.C., Thompson, C.B., and Gottlieb, E. (2005). Succinate links TCA cycle dysfunction to oncogenesis by inhibiting HIF-alpha prolyl hydroxylase. Cancer Cell 7, 77-85.

Semenza, G.L. (2003). Targeting HIF-1 for cancer therapy. Nat Rev Cancer 3, 721-732.

Shi, Y., Lan, F., Matson, C., Mulligan, P., Whetstine, J.R., Cole, P.A., Casero, R.A., and Shi, Y. (2004). Histone Demethylation Mediated by the Nuclear Amine Oxidase Homolog LSD1. Cell 119, 941-953.

Silvan, U., Diez-Torre, A., Arluzea, J., Andrade, R., Silia, M., and Arechaga, J. (2009). Hypoxia and pluripotency in embryonic and embryonal carcinoma stem cell biology. Differentiation 78, 159-168.

Simon, J.A., and Kingston, R.E. (2009). Mechanisms of polycomb gene silencing: knowns and unknowns. Nat Rev Mol Cell Biol 10, 697-708.

Smith, E.R., Lee, M.G., Winter, B., Droz, N.M., Eissenberg, J.C., Shiekhattar, R., and Shilatifard, A. (2008). Drosophila UTX is a histone H3 Lys27 demethylase that colocalizes with the elongating form of RNA polymerase II. Mol Cell Biol 28, 1041-1046.

Swigut, T., and Wysocka, J. (2007). H3K27 demethylases, at long last. Cell 131, 29-32.

Welford, S.M., and Giaccia, A.J. (2011). Hypoxia and Senescence: The Impact of Oxygenation on Tumor Suppression. Molecular Cancer Research 9, 538-544.

Wu, Z., Irizarry, R.A., Gentleman, R., Martinez-Murillo, F., and Forrest Spencer, F. (2004). A Model-Based Background Adjustment for Oligonucleotide Expression Arrays J Am Stat Assoc 99, 909-917.

Yang, J., Jubb, A.M., Pike, L., Buffa, F.M., Turley, H., Baban, D., Leek, R., Gatter, K.C., Ragoussis, J., and Harris, A.L. (2010). The histone demethylase JMJD2B is regulated by estrogen receptor alpha and hypoxia, and is a key mediator of estrogen induced growth. Cancer Res 70, 6456-6466. 
Young, M.D., Willson, T.A., Wakefield, M.J., Trounson, E., Hilton, D.J., Blewitt, M.E., Oshlack, A., and Majewski, I.J. (2011). ChIP-seq analysis reveals distinct H3K27me3 profiles that correlate with transcriptional activity. Nucleic Acids Res 39, 7415-7427.

Zhou, X., Sun, H., Chen, H., Zavadil, J., Kluz, T., Arita, A., and Costa, M. (2010). Hypoxia induces trimethylated H3 lysine 4 by inhibition of JARID1A demethylase. Cancer Res 70, 4214-4221.

Figure S1. (next page) Reversible oxygen-dependent global changes of H3K4me3 and H3K27me3 levels. A; Immunoblot detection (IB) of epigenetic changes (H3K4me3 and H3K27me3) in MCF7 and DU145 cells. B-C; expression levels changes of B) known and putative H3K4 (left) and H3K27 (right) methyltransferases (HKMT); C) known and putative H3K4 (left) and H3K27 (right) demethylases (HKDM).

Figure S2. (next page) Reversible oxygen-dependent global changes of H3K4me3 and H3K27me3 levels. A-B; Genome-wide presentation of A) H3K4me3 and B) H3K27me3 enrichment/peak locations at indicated experimental time points. Numbers in brackets indicate fold change in peak counts in respect to $t=0$. 


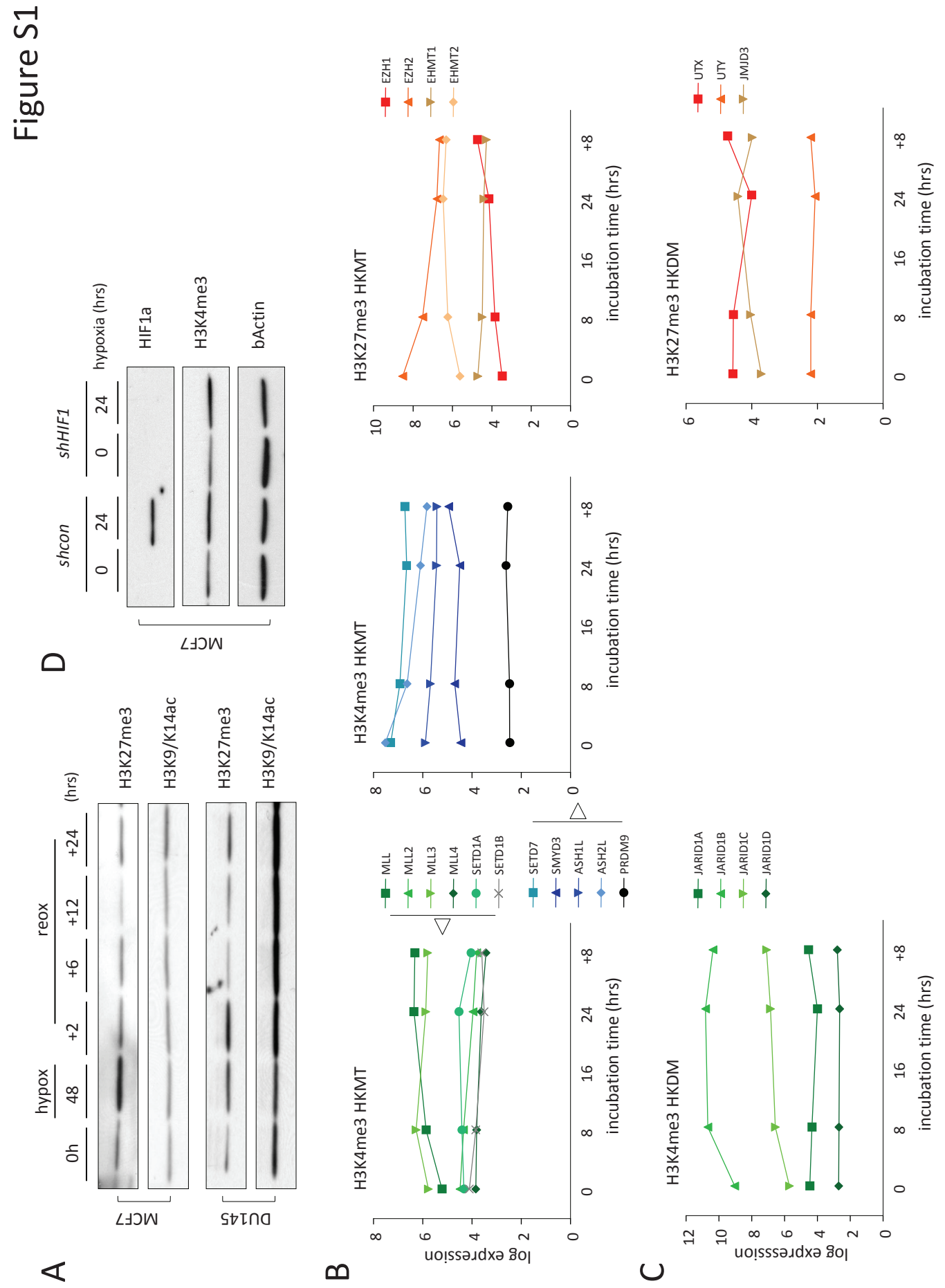



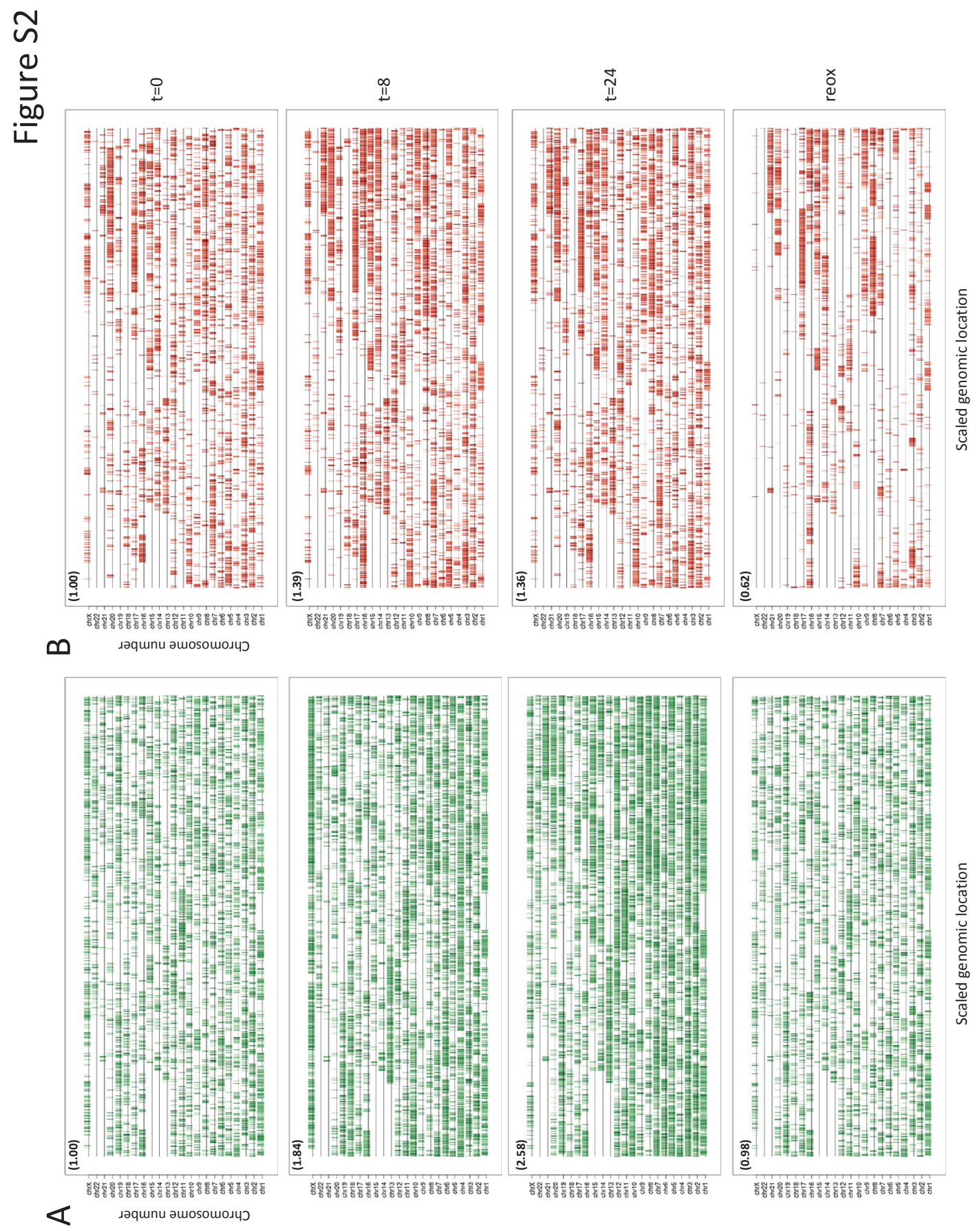
Chapter 5 |
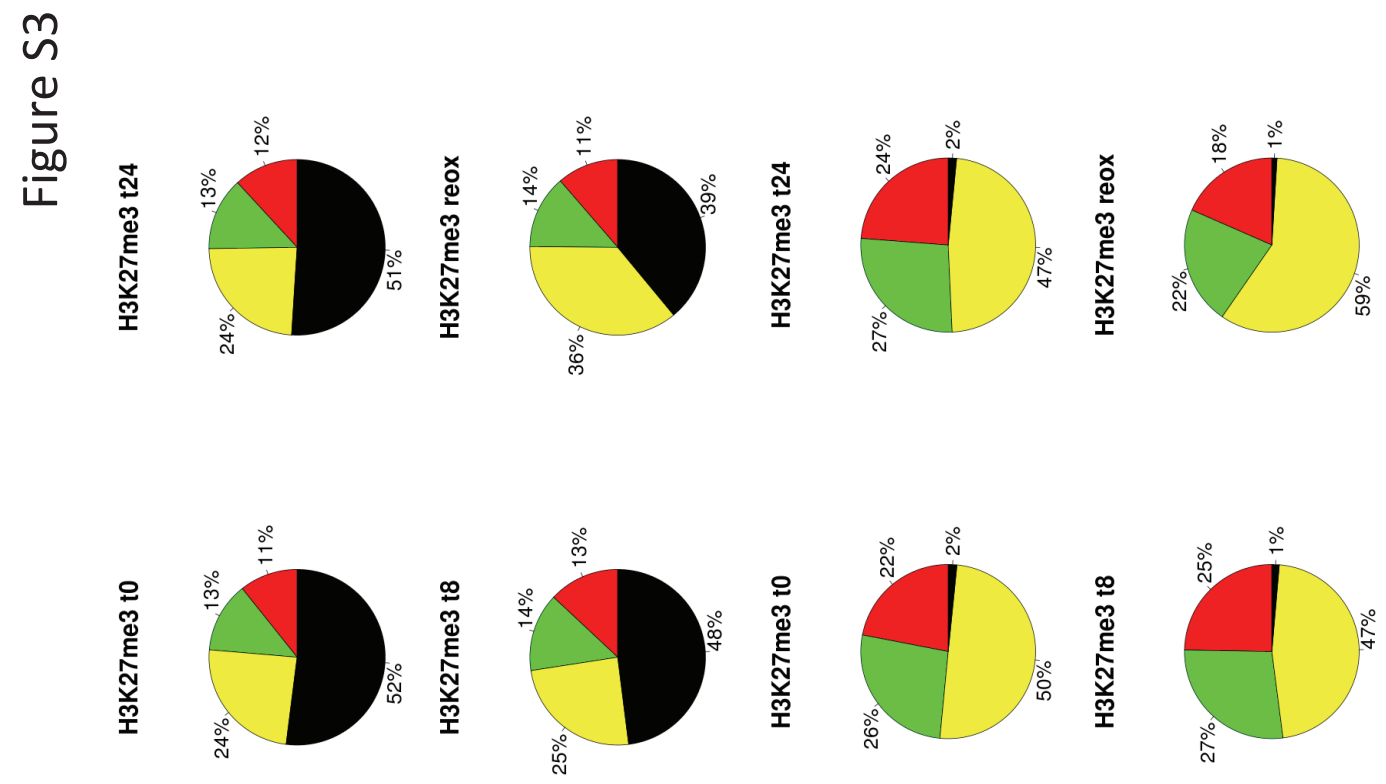

$\infty$

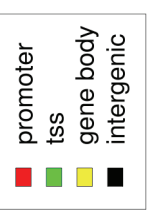

๑
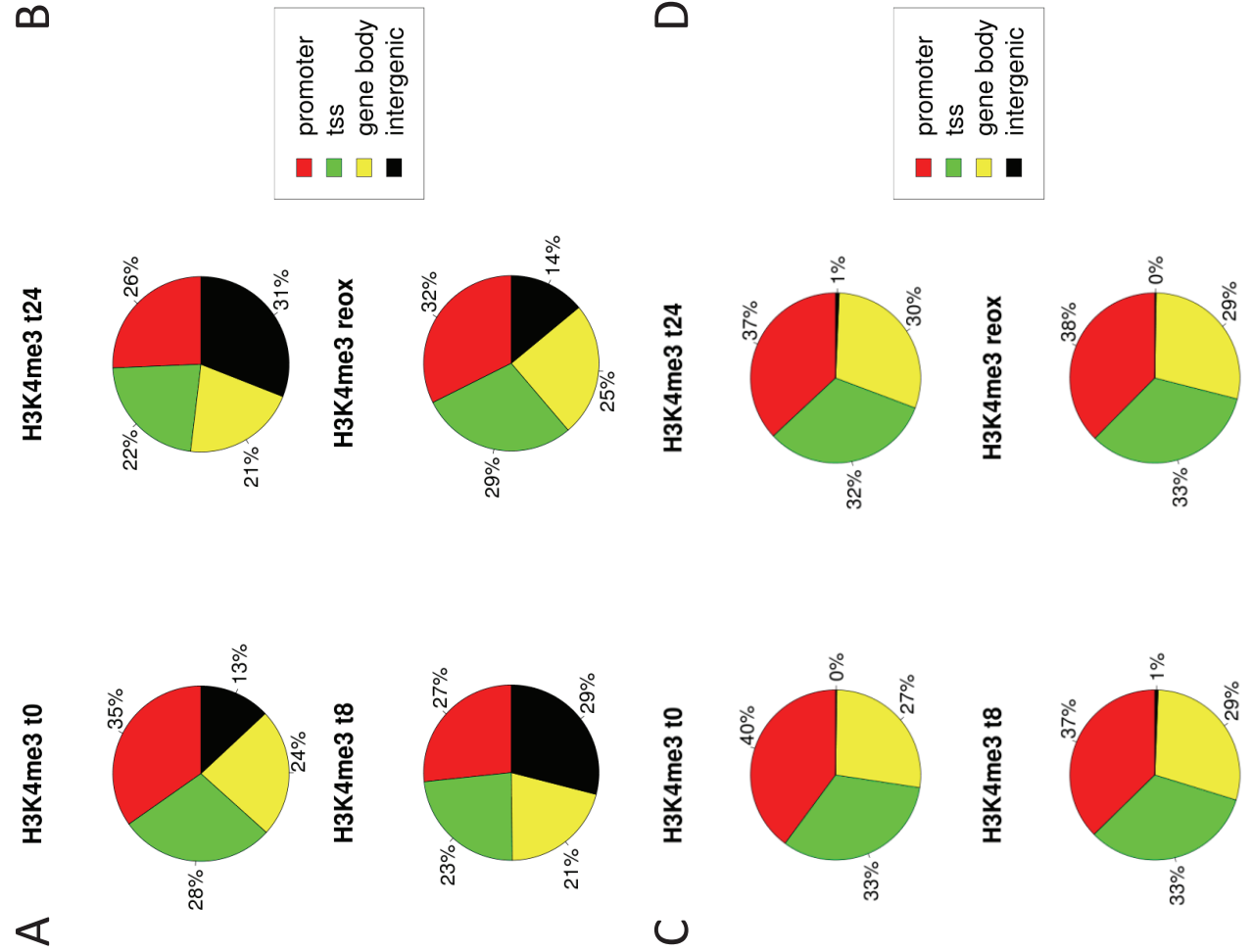
Figure S3. (on the left) Preferred gain of histone methylation at genic regions. A-B; Graphical representation of occurrence of A) H3K4me3-marks and B) H3K27me3-marks at genic (TSS, promoter, gene body) and intergenic regions. C-D; Graphical representation of relative distribution of sequences associated with C) H3K4me3-marks and D) H3K27me3-marks over genic (TSS, promoter, gene body) and intergenic regions.

Figure S4. (next page) Global expression changes in response to hypoxia and reoxygenation. A; Fold change (FC) of significantly regulated genes $(n=864)$ under hypoxia (pie charts; $t=8, t=24$ hrs vs. $t=0$ ); B; Fold change of significantly regulated genes $(n=864)$ following reoxygenation (pie charts; $t=+8$ hrs (reox) and $t=0$ vs. $t=24$ hrs hypoxia). Box-plots (right panels, A-B) show fold changes (FC) median expression levels for all genes up-regulated or down regulated at the indicated intervals. A regulated gene is defined as a gene with a minimum absolute expression of 100 (averaged across 3 replicates) at any experimental time point and expression level at least 2 fold changed in response to hypoxia. C; GO-analysis of genes and processes induced and down-regulated by hypoxia ( $\mathrm{t}=24 \mathrm{hrs}$ ). D; GO-analysis of genes and processes induced and reduced by reoxygenation $(\mathrm{t}=+8 \mathrm{hrs})$. 
Chapter 5 |
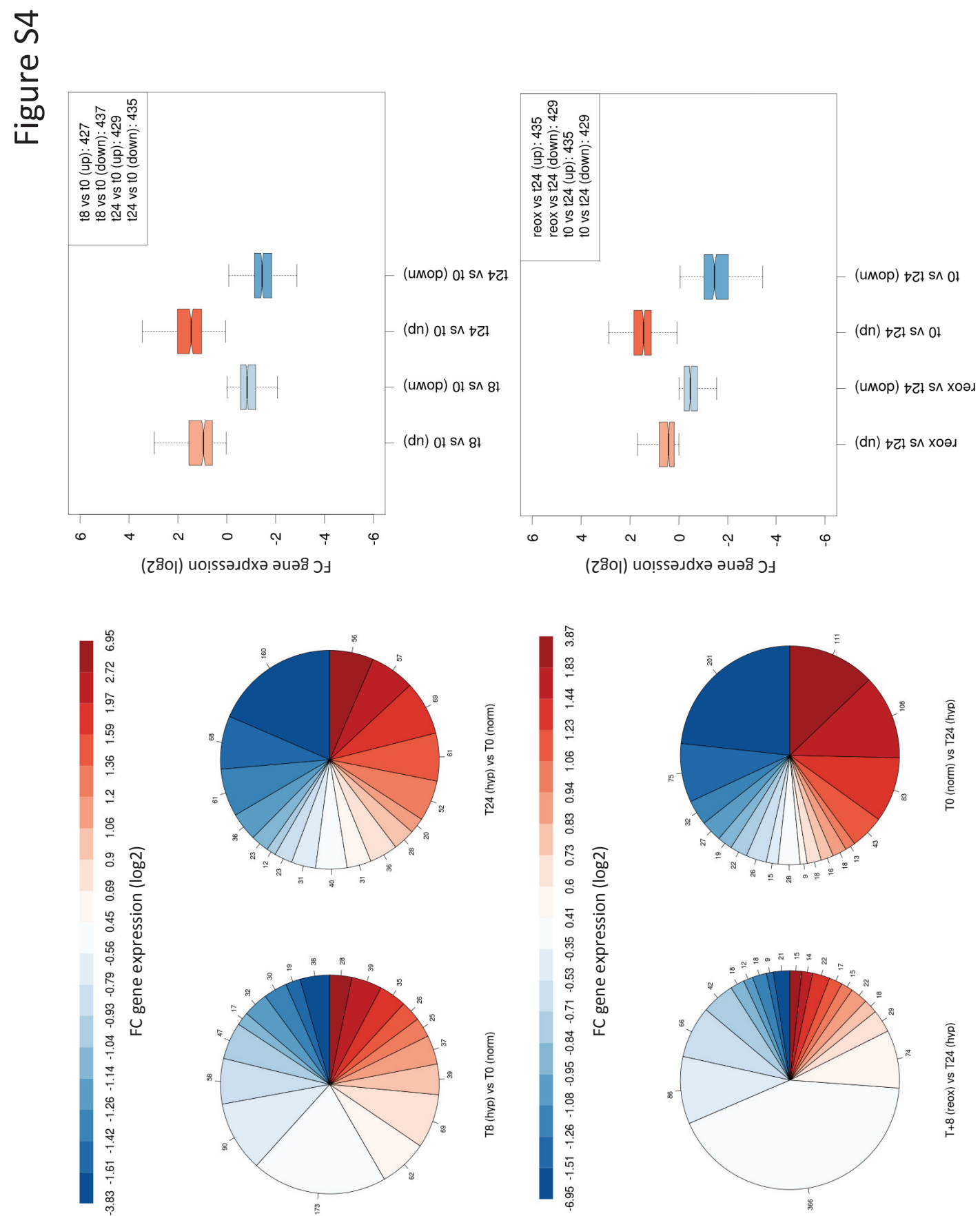

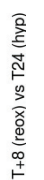

$\varangle$

$\infty$ 

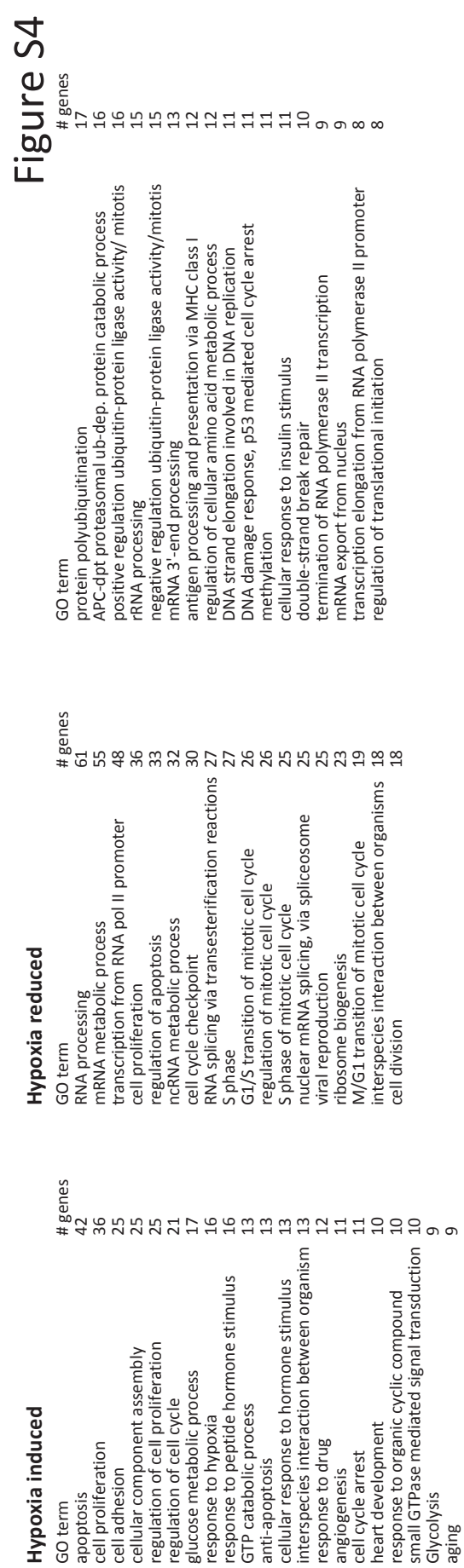
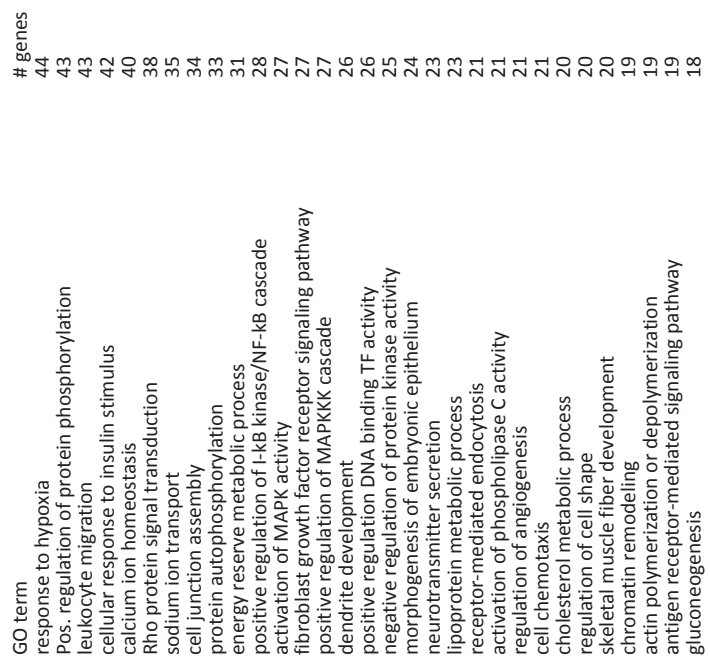

$\stackrel{\varrho}{凶}$
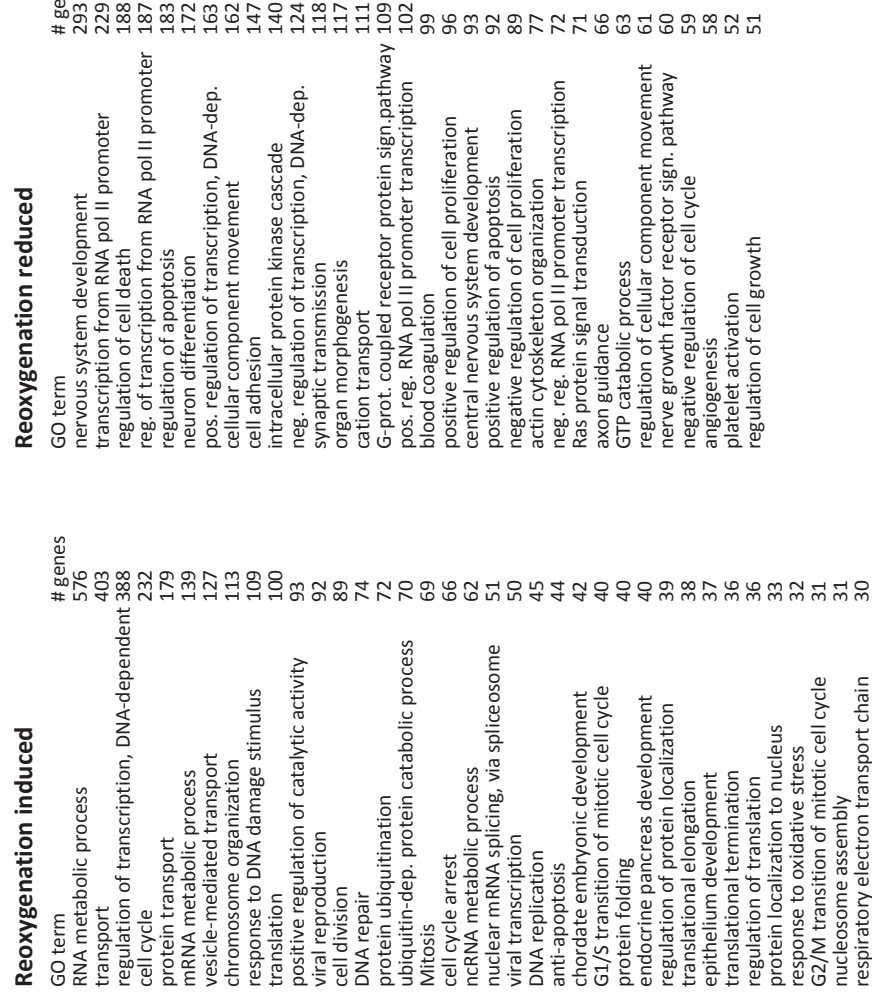


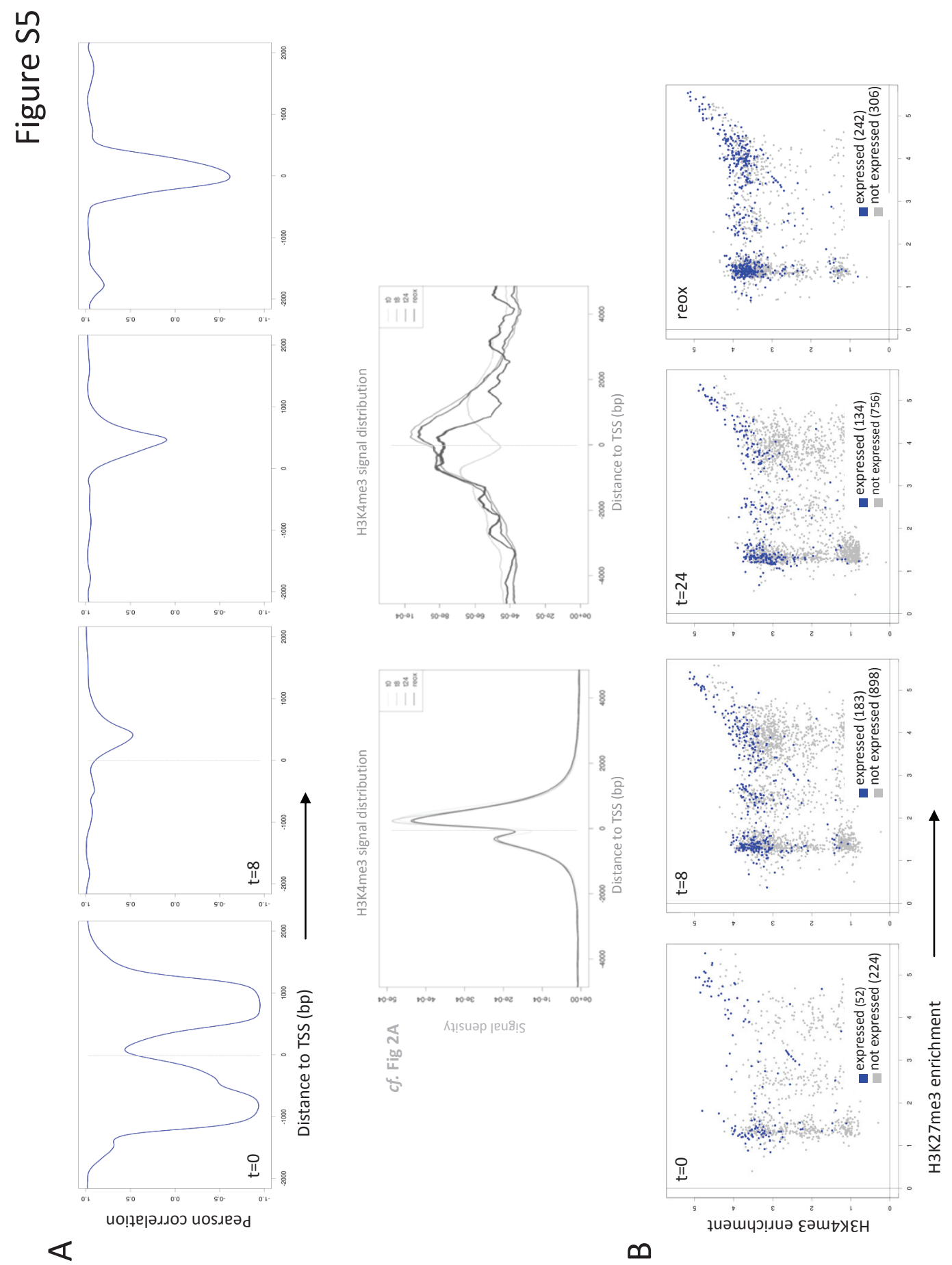


Figure S5. (on the left) Co-occurrence of H3K4me3 and H3K27me3 at TSS. A; Pearson correlation coefficients (ppc) were calculated for co-occupation by H3K4me3 and H3K27me3 in a region of +/- 2000 bp surrounding the TSS; a pcc of +1 is indicative of co-occurrence of the two marks, while a pcc of -1 indicates lack thereof. Pcc's were calculated at all indicated time points for all genes that carried associated H3K4me3 and H3K27me3-marks; lower panel - cf. Figure 2. B; double-marked genes are expressed; graphs depict genic H3K27me3-marking (x-axis) vs. H3K4me3-occupation (y-axis); expressed genes are indicated with blue dots.

Figure S6. (next page) Epigenetic profiles at H3K27me3-genes. A-D; a panels) TSS-associated enrichment profiles (average number of reads) for all H3K27me3-marked genes; b panels) box-plots depict median expression levels for each H3K27me3 class (a); c panels) TSS-associated enrichment profiles (average number of reads) for $\mathrm{H} 3 \mathrm{~K} 27 \mathrm{me} 3 /$ single-marked genes. Comparative analysis was done at (A) $t=0,(B) t=8,(C) t=24$ hours hypoxia and (D) after reoxygenation ( $t=+8$ hours).

Figure S7. (next page) GO-analysis of genes and processes associated with bivalent markers at $t=8, t=24$ hours hypoxia; reference lists: $t=0$ and processes associated with known bivalent markers in embryonal stem cells. 
Chapter 5 |
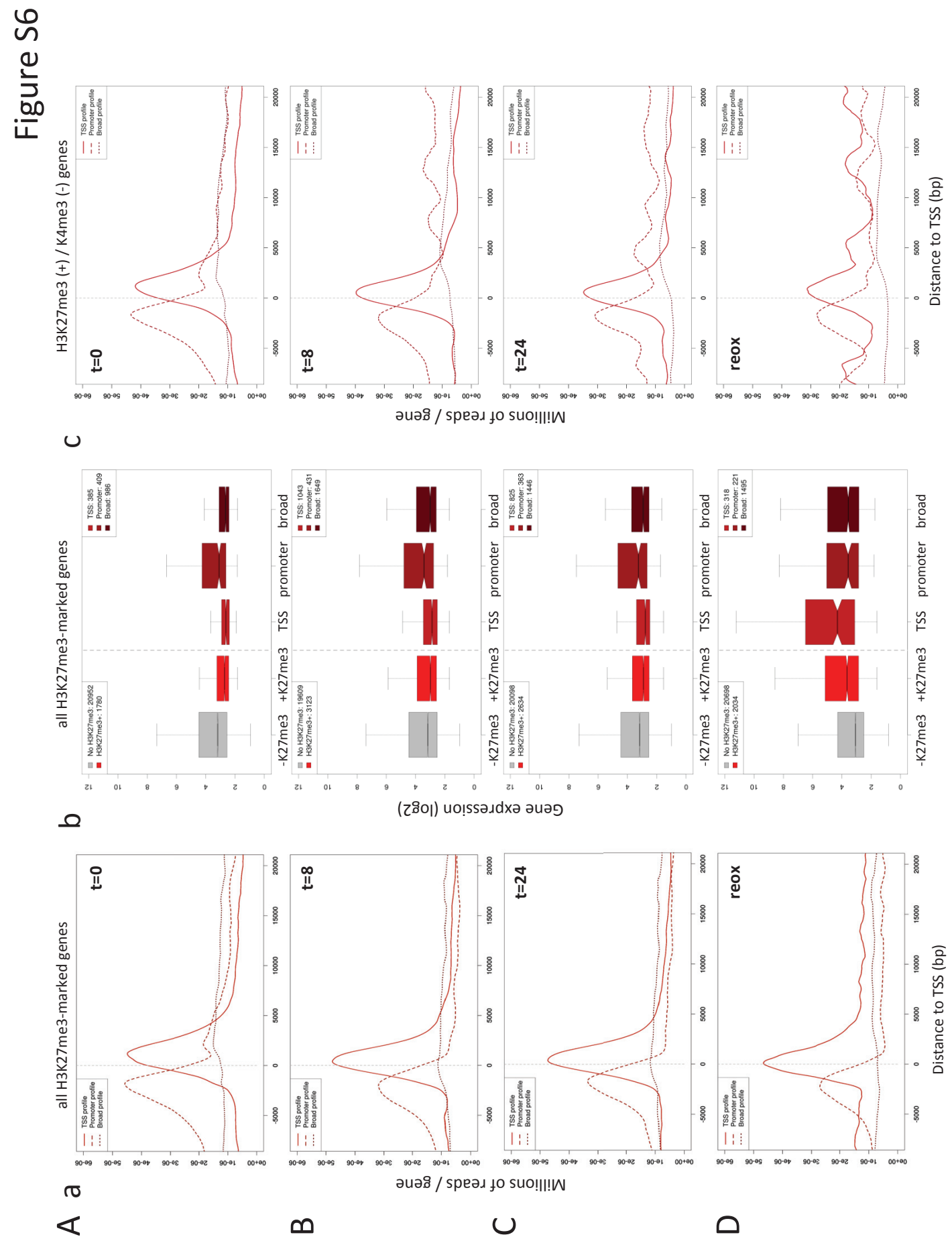


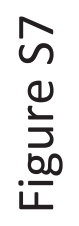

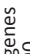

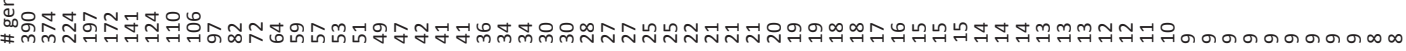

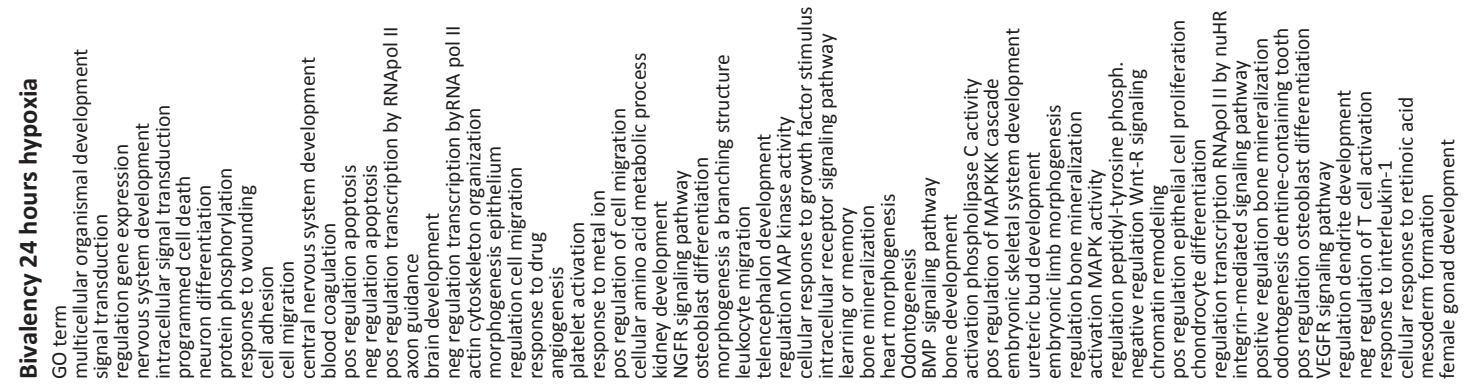

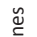

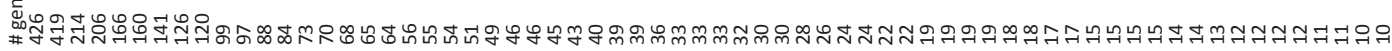

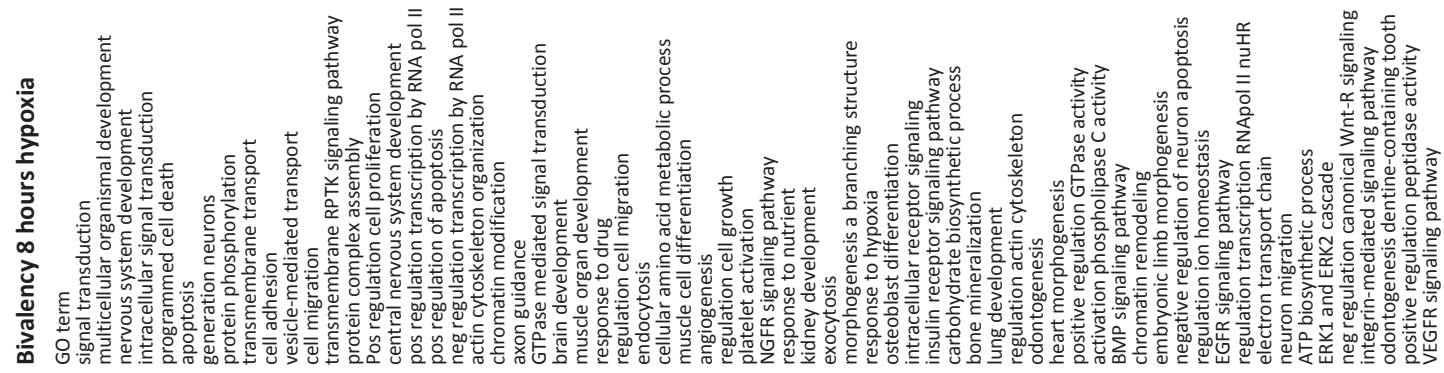

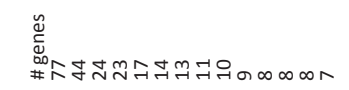

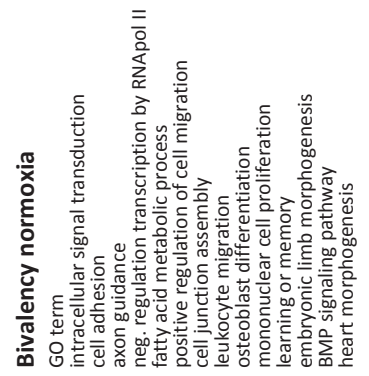

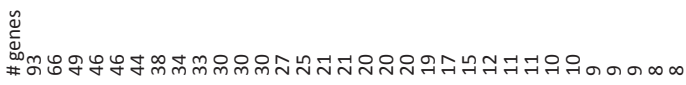

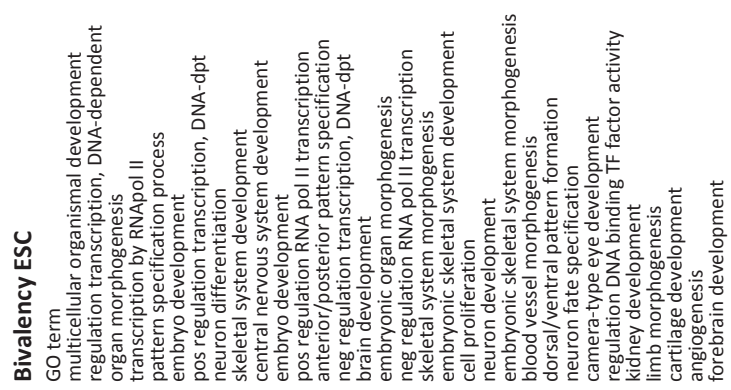




\section{SUPPLEMENTARY MATERIALS AND METHODS}

Chromatin immunoprecipitation (ChIP) assays Cells were fixed in Phosphate Buffered Saline (PBS) containing 1\% formaldehyde. Cross-linking was allowed to proceed for $10 \mathrm{~min}$ at room temperature and stopped by a 5 minute incubation with glycine at a final concentration of $0.125 \mathrm{M}$. Fixed cells were washed twice with PBS and harvested in SDS buffer (50 mM Tris at pH 8.1, 0.5\% SDS, $100 \mathrm{mM} \mathrm{NaCl}, 5 \mathrm{mM}$ EDTA), supplemented with protease inhibitors (Aprotinin, Antipain and Leupeptin all at $5 \mu \mathrm{g} / \mathrm{ml}$ and $1 \mathrm{mM}$ PMSF). Cells were pelleted by centrifugation, and suspended in IP buffer (100 mM Tris at pH 8.6, $100 \mathrm{mM} \mathrm{NaCl}, 0.3 \%$ SDS, 1.7\% Triton X-100, and 5 mM EDTA), containing protease inhibitors. Cells were disrupted by sonication, yielding genomic DNA fragments with a bulk size of 200-500 bp. For each immunoprecipitation, $1.2 \mathrm{ml}$ of lysate was pre-cleared by adding of $35 \mu \mathrm{l}$ of blocked protein $A$ beads (Protein A-Sepharose/ CL-4B, GE Healthcare, Piscataway, NJ, USA; $0.5 \mathrm{mg} / \mathrm{ml}$ fatty acid-free BSA, Sigma; and $0.2 \mathrm{mg} / \mathrm{ml}$ herring sperm DNA in TE), followed by centrifugation. $12 \mu \mathrm{l}$ aliquots of pre-cleared suspension were put aside as input DNA and kept at $4^{\circ} \mathrm{C}$. Samples were immunoprecipitated overnight at $4^{\circ} \mathrm{C}$ with primary antibodies. Immune complexes were recovered by adding $40 \mu \mathrm{l}$ of blocked protein A beads (GE Healthcare) and incubated for 4 hours at $4^{\circ} \mathrm{C}$. Beads were washed three times in $1 \mathrm{ml}$ of Mixed Micelle Buffer (20 mM Tris at $\mathrm{pH} 8.1,150 \mathrm{mM} \mathrm{NaCl}, 5 \mathrm{mM}$ EDTA, 5\% w/v sucrose, 1\% Triton X-100, and 0.2\% SDS), twice in $1 \mathrm{ml}$ of Buffer 500 (50mM HEPES at pH 7.5, 0.1\% w/v Sodium Deoxycholate, $1 \%$ Triton X-100, and $1 \mathrm{mM}$ EDTA), twice in $1 \mathrm{ml}$ of LiCl Detergent Wash Buffer (10 mM Tris at pH 8.0, 0.5\% Sodium Deoxycholate, 0.5\% NP-40, $250 \mathrm{mM} \mathrm{LiCl}$, and $1 \mathrm{mM}$ EDTA), and once in $1 \mathrm{ml}$ of TE. Immune complexes were eluted from beads in $250 \mu$ l elution buffer (1\% SDS; and $0.1 \mathrm{M} \mathrm{NaHCO3}$ ) for 2 hours at $65^{\circ} \mathrm{C}$ with continuous shaking at $1000 \mathrm{rpm}$, and after centrifugation, supernatants were collected. $250 \mu$ l elution buffer was added to input DNA samples and these were processed in parallel with eluted samples. Crosslinks were reversed overnight at $65^{\circ} \mathrm{C}$, followed by a 2 hours digestion with RNase $\mathrm{A}$ at $37^{\circ} \mathrm{C}$ and 2 hours proteinase $\mathrm{K}$ $(0.2 \mu \mathrm{g} / \mu \mathrm{l})$ at $55^{\circ} \mathrm{C}$. DNA fragments were recovered using QIAquick PCR purification columns (Qiagen, Hilden, Germany), according to manufacturers' instructions. Samples were eluted in $75 \mu \mathrm{l}$ EB buffer and checked for enrichment using qPCR before deep sequencing was applied on the immunoprecipitated DNA.

Protein isolation and Western blot analysis For protein extraction cells were washed twice with cold PBS and lysed in RIPA buffer $(150 \mathrm{mM} \mathrm{NaCl}, 1 \% \mathrm{NP}-40,0.5 \%$ w/v Sodium Deoxycholate, 0.1\% SDS, $50 \mathrm{mM}$ Tris at pH 8.0, $5 \mathrm{mM}$ EDTA) supplemented with protease and phosphatase inhibitors $(5 \mathrm{mM}$ Benzamidine, $5 \mu \mathrm{g} / \mathrm{ml}$ Antipain, 
$5 \mu \mathrm{g} / \mathrm{ml}$ Leupeptin, $5 \mu \mathrm{g} / \mu \mathrm{l}$ Aprotinin, $1 \mathrm{mM}$ Sodium Vanadate, $10 \mathrm{mM}$ Sodium Fluoride, $10 \mathrm{mM}$ Pyrophosphate, $10 \mathrm{mM}$ ß-glycerophosphate, $0.5 \mathrm{mM}$ DTT and 1mM PMSF). Lysates were subjected to two freeze-thow cycles in liquid nitrogen, followed by sonication on ice with a probe sonicator (Soniprep 150; MSE, London, UK) for 12 cycli ( $1 \mathrm{sec}$ ON, $1 \mathrm{sec}$ OFF) with amplitude 5 . After $10 \mathrm{~min}$ centrifugation at $13.200 \mathrm{rpm}\left(4^{\circ} \mathrm{C}\right)$, the supernatant was transferred to a fresh tube and protein concentration was determined using a BCA protein assay kit (Pierce/Thermo Fisher Scientific, Rockford, IL, USA) according to the manufacturer's protocols on a Benchmark 550 Micro-plate Reader (Bio-Rad).

For immunoblotting (IB) equal amounts of protein were boiled in Laemli buffer for 5 min and loaded on 9-15\% polyacrylamide gels. Following separation by SDS-PAGE, proteins were transferred onto polyvinylidene fluoride (PVDF) membranes (GE Healthcare). Ponceau S (Sigma) staining was used to check protein transfer. Subsequently, PVDF membranes were blocked with $3.4 \%$ non-fat dry milk (Protifar; Nutricia, Zoetermeer, the Netherlands) in PBS containing $0.1 \%$ Tween-20 (pH 7.5) for 1 hour at RT, followed by an overnight incubation at $4^{\circ} \mathrm{C}$ with the primary antibody (see Supplementary Table 2). After extensive washing with PBS/0.2\% Tween-20, membranes were probed with corresponding horseradish peroxidase conjugated secondary antibodies for 1 hour at RT: rat-anti-mouse (1:5000; DAKO, Glostrup, Denmark) and donkey-anti-rabbit (1:15.000; Jackson Lab, Bar Harbor, ME, USA), to detect monoclonal and polyclonal primary antibodies respectively. Signals were detected on autoradiograms using enhanced chemoluminescence (ECL; Pierce). Intensity of the bands was quantified with Quantity One software (Bio-Rad) and plots were generated using GraphPad Prism, version 4.03 for Windows (GraphPad Software, San Diego, CA, USA). Data was statistically analyzed by performing 2-tailed paired t-tests using Microsoft Excel. Data given is expressed as means \pm standard deviation (SD) and considered significant at $\mathrm{p}<0.05 .^{*}, * *$ and $* * *$ indicate $\mathrm{p}<0.05,0.01$ and 0,001 , respectively. 
Chapter 5 | 


\section{CHAPTER 6}

Capturing ChIP-seq profiles of H3K27me3 in dynamic biological systems

Michiel Adriaens*, Peggy Prickaerts*, Michelle Chan-Seng-Yue, Timothy Beck, Bradly G Wouters*, Jan Willem Voncken*, Chris Evelo*

*equal author contribution

Status: submitted 


\section{ABSTRACT}

Chromatin immunoprecipitation combined with high-throughput sequencing (ChIP-seq) has become the state-of-the-art technology for genome-wide mapping of epigenetic modifications. ChIP-seq has a much higher resolution than microarray based applications; this impacts on the requirements for analysis tools. Here we focus on applying ChIP-seq to study enrichment of tri-methylation of the lysine 27 residue on histone H3 (H3K27me3) in dynamic biological systems, where the majority of epigenetic markings are different between samples. A number of specific challenges exist with regards to studying histone modifications under dynamic conditions. Firstly, ChIP-seq enrichment-finding algorithms are optimized for identifying sharp peaks, whereas H3K27me3 marks tend to spread over a large number of nucleosomes (blanketing) and cover extended genomic regions. Thus, existing algorithms are unable to reliably identify H3K27me3 enriched regions. Secondly, ChIP-seq data requires normalization to enable quantitative comparison. In dynamic biological systems, both the total number of marks and their location is variable. A suitable normalization approach for this situation involves data-scaling of individual samples to genomic regions with relatively stable H3K27me3 marking. Definition of such stable regions depends heavily on the biological system. With these difficulties in mind, we have developed a standardized protocol for the processing of H3K27me3 ChIP-seq data. The protocol enables robust detection of H3K27me3 blanketing and allows for quantitative data comparison. As such, our protocol complements previous efforts to create a fully standardized analysispipeline for H3K27me3 enriched ChIP-seq data. 


\section{INTRODUCTION}

High-throughput sequencing (HTS) has enabled the study of biological systems on a greater scale and higher genomic resolution than ever before [1] and is prospected to soon replace microarrays as the platform of choice. Although the technology is constantly evolving, analysis approaches are lagging behind [2]. Most approaches are derivatives of approaches developed for the corresponding application on microarrays [3], which are characterized by lower genomic resolution and scale.

Epigenomics is the genome-wide study of epigenetic modifications, such as DNA methylation and modification of $\mathrm{N}$-terminal histone tails, which are heritable but nonetheless variable marks that influence gene expression and phenotypic plasticity. Well known examples of histone modifications are tri-methylation of lysine 4 of histone H3 (H3K4me3), which is associated with open chromatin and permissive for gene transcription, and tri-methylation of lysine 27 of histone H3 (H3K27me3), which is associated with closed chromatin and gene silencing. Chromatin immunoprecipitation (ChIP) combined with HTS technology (ChIP-seq) is a powerful approach to create genome-wide maps of such histone modifications. Here we focus specifically on H3K27me3 ChIP-seq data analysis in the context of biological systems where many epigenetic changes occur. Cancer cells exposed to fluctuating levels of oxygenation exemplify a model for such dynamic effects occurring in response to hypoxia in solid tumors.

The sequential steps involved in ChIP-seq analysis are summarized in figure 1. Each step is paralleled by important decisions and specific data formats. This flow scheme concludes with interpretation of the data in a biologically relevant context.

\section{Step 1: Sequencing}

The steps before enrichment finding are well established and are independent of the biological system studied. Yet a number of important considerations apply to the design of the sequencing experiment. The first is whether or not to include an input sample in parallel as a reference for analysis of ChIP-seq data. Although including input samples increases overall costs and reduces the number of experimental samples in one run, an input sample is absolutely essential for proper correction of background anomalies, such as amplified regions and variability in shearing of DNA [4]. Also the choice of sequencing technology, i.e. paired-end or single-end sequencing, requires consideration: paired-end sequencing yields paired reads corresponding to the start and the end of a fragment and thus allows for more robust alignment. For ChIP-seq experiments, paired-end technology is clearly the preferred method, provided it is available [5]. 


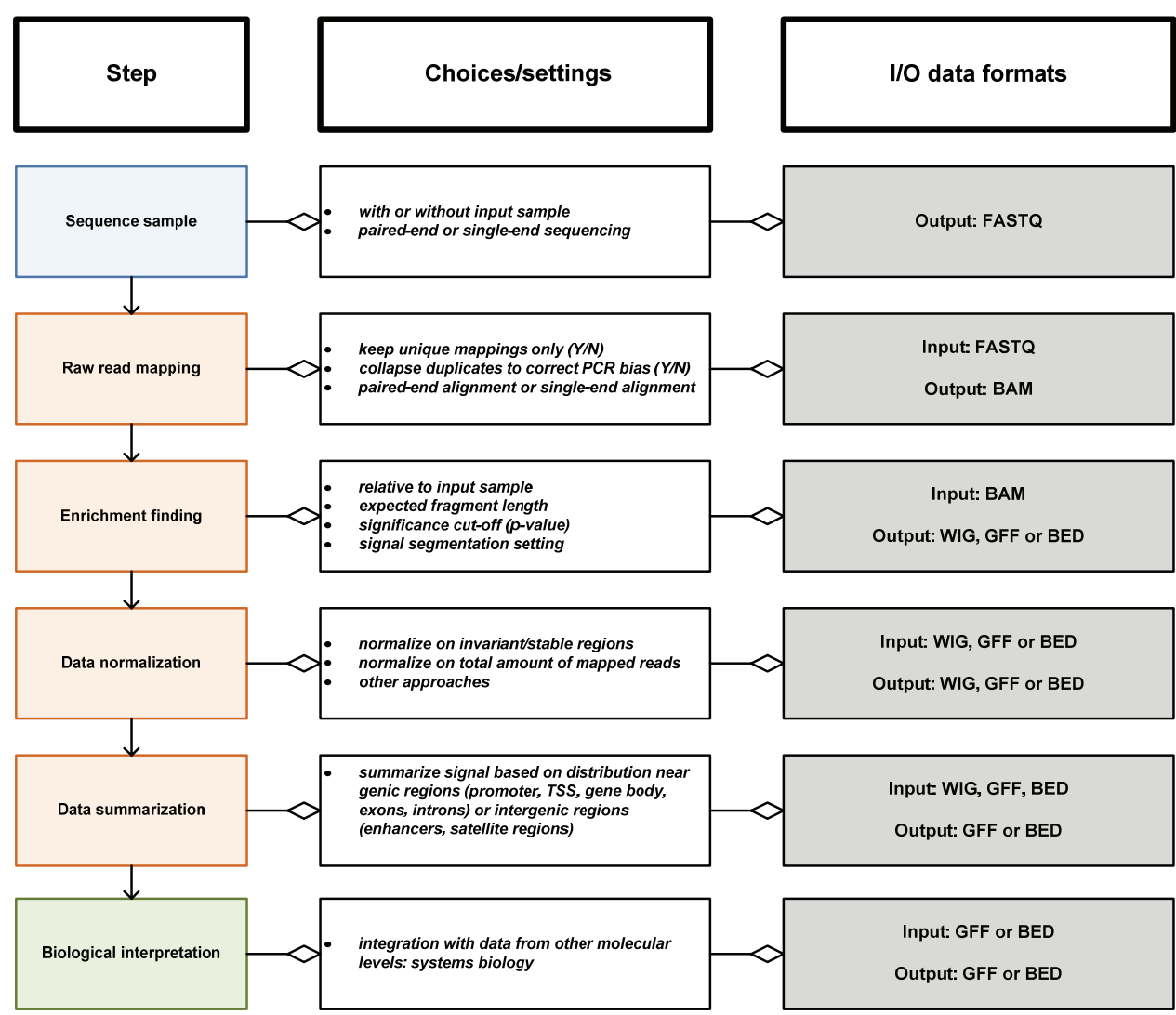

Figure 1: Simplified flow diagram of steps involved in the bioinformatics analyses of ChIP-seq data, from raw reads to biological interpretation.

\section{Step 2: Mapping reads}

The next step in the process is the mapping of the raw sequence reads to a reference genome build, most commonly performed using Needleman-Wunsch or BLAST algorithms [6]. Some are designed for rapid analysis, such as Eland, while others aim for sensitivity, such as Novoalign [7]. Important decisions to make at this step include exclusion of non-unique mappings and collapsing duplicate reads caused by PCR bias. In most cases, collapsing and proceeding with unique mappings only leads to more reliable, unbiased data [7]. Depending on the technology applied, raw reads consist of paired-end reads or single-end reads. As paired-end sequencing provides two connected DNA end-tags, it enables reliable identification of enriched regions. 
In addition, paired-end sequencing is more powerful in identifying enrichment in repeat regions, such as satellite DNA regions near centromeres. Single-end mappings from such regions will often be discarded from the analysis, because they do not map uniquely to one defined region. In case of paired-end reads, two close mappings, with ideally only one out of two reads mapping into a repeat region, prevent loss of such repeat regions. Since several histone modifications are associated with such regions and as such are of biological interest [8], paired-end mapping is always the algorithm of choice when studying histone modifications [5].

\section{Step 3: Identifying regions of enrichment}

Several tools are available for enrichment-finding in ChIP-seq data, of which FindPeaks [9], PeakSeq [10], USeq [11] and MACS [12] are the most popular. Although these tools produce comparable results, FindPeaks is most sensitive in distinguishing enrichment [13]. Enrichment-finding tools work by the assumption that regions enriched for a histone modification of interest will yield a higher number of reads representing the regions in ChIP samples relative to un-enriched regions. Hence, significantly enriched regions can be identified [14] against a null distribution, ideally in the form of a sequenced input sample. Additional modulations to support enrichmentfinding include modification of the expected fragment-length (post-sonication), resetting the significance cut-off (usually in the form of a percentile of the data above which a signal is considered enriched; e.g. 95\%), and varying the signal-segmentation setting (determines when a multi-modal signal should be split into separate peaks). Most of these distinct enrichment-finding approaches have been developed for lower resolution cistromics applications on microarray platforms. Although they work well for many histone modifications deposited over a small number of nucleosomes such as H3K4me3, such approaches are not optimized for detection of H3K27me3 enrichment. A potential underlying cause is that $\mathrm{H} 3 \mathrm{~K} 27 \mathrm{me} 3$ tends to spread over more nucleosomes to cover an entire locus, which is known as "blanketing" [15]. This results in data comprising spread-out enrichment signals covering large genomic regions $[15,16,17]$ instead of sharp peaks, which hampers their detection by existing peak-finding algorithms.

\section{Step 4: Data normalization}

To compare samples quantitatively, ChIP-seq data requires normalization, because signal intensity depends on sequencing depth and mapping efficiency. Several approaches have been developed, of which scaling based on the total number of aligned reads is most common $[18,19]$. This method starts with the assumption that there is only a small number of prospected differences between conditions, which does 
not hold for dynamic biological systems; for such systems normalizing on regions with stable enrichment seems the only valid approach. However, it is difficult to define $a$ priori genomic regions as candidates for such stable enrichment, as this relies heavily on the biological system as well as the studied histone modification [18].

\section{Step 5 \& 6: Data summarization and interpretation}

Although usually complex and mostly dependent on the biological system of choice and research question(s) at hand, the biological interpretation of the data is identical to comparable applications in microarrays [20]: integration of transcriptomics data is the minimal requirement for a meaningful analysis. Since experiments and research questions are extremely diverse, a flexible analysis tool is needed. Perhaps not surprising, several open source tools are available to enable robust analysis and biological interpretation of ChIP-seq data [21,22,23]. However, the sheer amount of sequencing information obstructs straight-forward data interpretation. Hence, most researchers choose to focus on genes and/or regulatory regions before committing to the biological interpretation phase. This requires summarization of the enrichment in genic and regulatory regions of interest. For $\mathrm{H} 3 \mathrm{~K} 27 \mathrm{me} 3$ data, such regulatory relevant regions have been defined successfully before [15].

Based on the considerations above, we have developed a protocol to handle H3K27me3 ChIP-seq data focused on (i) standardization of the enrichment finding, (ii) data normalization and (iii) data summarization steps, while building on the already standardized stages before enrichment finding.

\section{Procedure}

\section{(i) Finding regions of enrichment}

H3K27me3 is known to "blanket" over large genomic regions. In order to define such broad regions of enrichment, while still retaining all the advantages of using an input DNA sample as a reference, FindPeaks with adapted settings is used. The primary setting that needs to be changed in the FindPeaks algorithm is the expected sequence distribution setting. By increasing the expected fragment size (post-sonication), the algorithm successfully classifies broad regions of enrichment as peaks (figure 2).

Additional settings are tied to the normalization approach: for this method to work, the signal needs to be split in as many individual peaks as possible, which is controlled by the signal-segmentation setting. Furthermore, the approach requires retention of as much of the signal as possible, which is controlled by the significance cut-off setting. Although this will inevitably increase the size of the resulting data files, as the identified peaks will be broader, the signal sampling frequency can safely be decreased as a counter measure. 
A

B

- Expected enrichment profile:

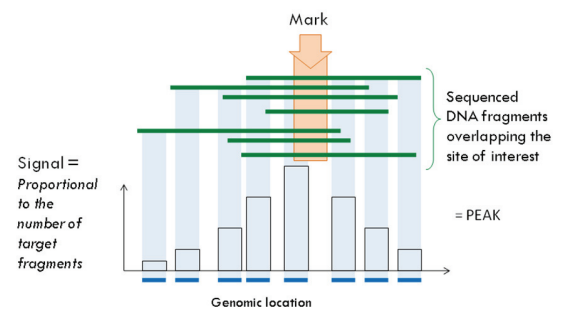

- Actual profile for H3K27me3:

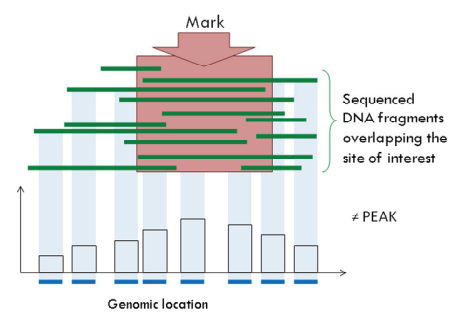

D

- Simulate same profile:

- broader distribution $\rightarrow$ longer average sequence length $\rightarrow$ peaks are expected to be broader

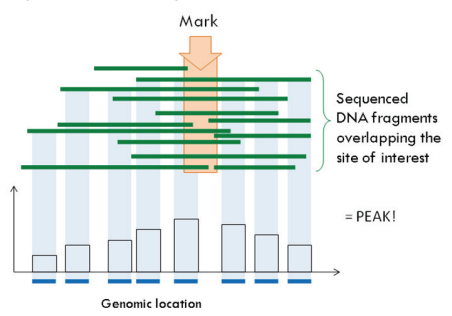

- Successful detection of H3K27me3 blanketing

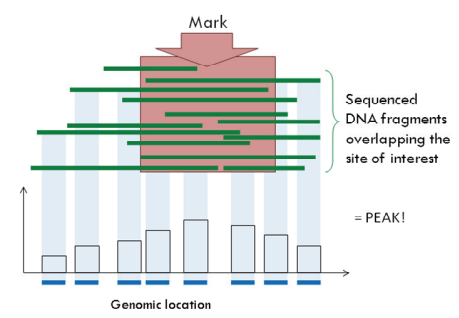

Figure 2: Illustration of the effect of increasing the expected fragment length-distribution to enable more robust detection of $\mathrm{H} 3 \mathrm{~K} 27 \mathrm{me} 3$ enriched regions.

Using the mapped input reads as control sample with flag "-control" and the immunoprecipitated sample as the experimental sample with the flag "-input", we use the following "broad-peak" settings:

- - aligner sam

- $\quad$-subpeaks 0.5

- -control_type 0

- $\quad$ alpha 0.2

- $\quad$-wig_step_size 10

- $\quad$-trim 0.2

- $\quad$-dist_type 1285600100 (aligner type)

(signal segmentation)

(control type: input DNA)

(significance setting)

(signal sampling frequency)

(peak trimming)

(expected sequence distribution) 


\section{Chapter 6}

As an example, using FindPeaks with these settings would lead to the following terminal command:

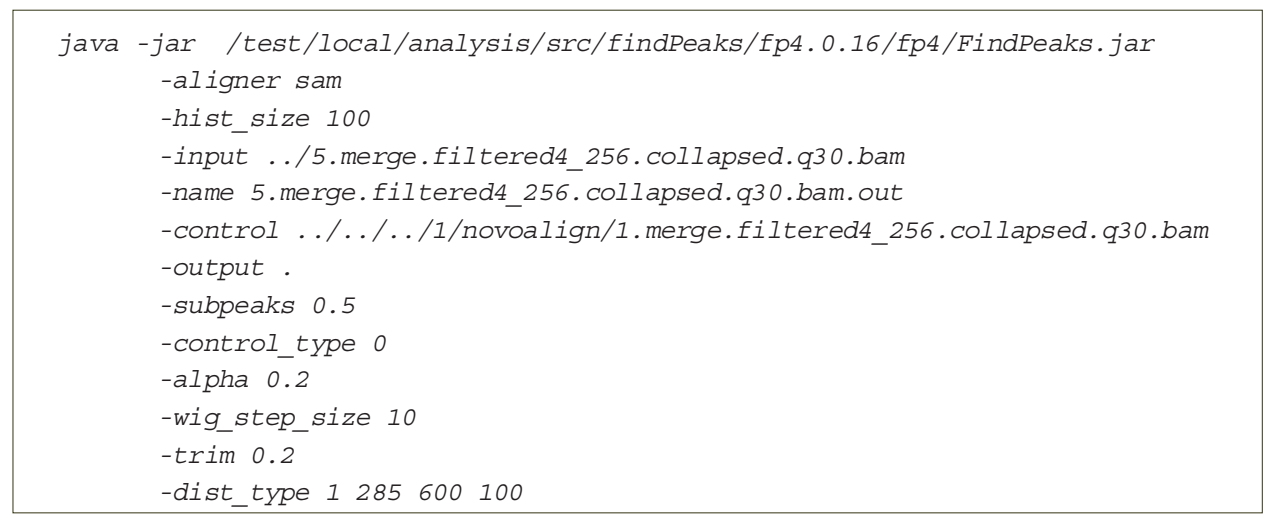

\section{(ii) Data normalization}

Normalization follows the identification of H3K27me3-enriched signals that are significantly above background level. The normalization strategy is based on identifying regions with stable H3K27me3-enrichment between two or more samples. The cumulative area under the curve (AUC) for all peaks in all these regions is determined for each condition, and based on those values, scaling factors are calculated relative to the smallest value among the samples (figure $\mathbf{3}$ ).

The most straightforward approach is to use the ACME package [14] in R, using the following approach:

- Import the files containing peak data in $\mathrm{R}$, including for each peak at least a genomic location and a height or AUC-value (GFF or BED format).

- Then for all samples/conditions:

- Create an ACME data object as described in the ACME documentation [14]

- Run ACME using a window size of at least $20000 \mathrm{bp}$ and a cut-off of 0.90 corresponding to the peak height value of the $90^{\text {th }}$ percentile. Peaks with a height above this value will be designated positive; those below this value will be designated negative (figure 3 ). The window size of $20000 \mathrm{bp}$ is chosen because it is much larger than the observed average peak width when using the settings specified in step (i), while being small enough to lead to a reasonable specificity. 
- Upon completion of the run, proceed only with regions with a chi-square test $\mathrm{p}$-value smaller than $1 \mathrm{E}-5$ in all samples/conditions. This value was shown empirically to yield good results.

- Add up all signals detected within the stable regions for each sample individually.

- Divide the individual sums by the smallest sum in the batch and use the resulting scaling factors to scale the peaks for each sample individually.

\section{Normalization strategy}

Peaks are positive or negative based on their height value

Test in sliding window of $20 \mathrm{~Kb}$ if significantly more positive peaks than expected

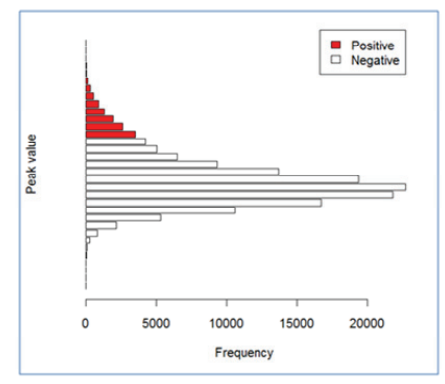

1
Check for each identified region in step 2 if this is the case under all studied conditions.

If yes, determine scaling factors based on peak value at each time point
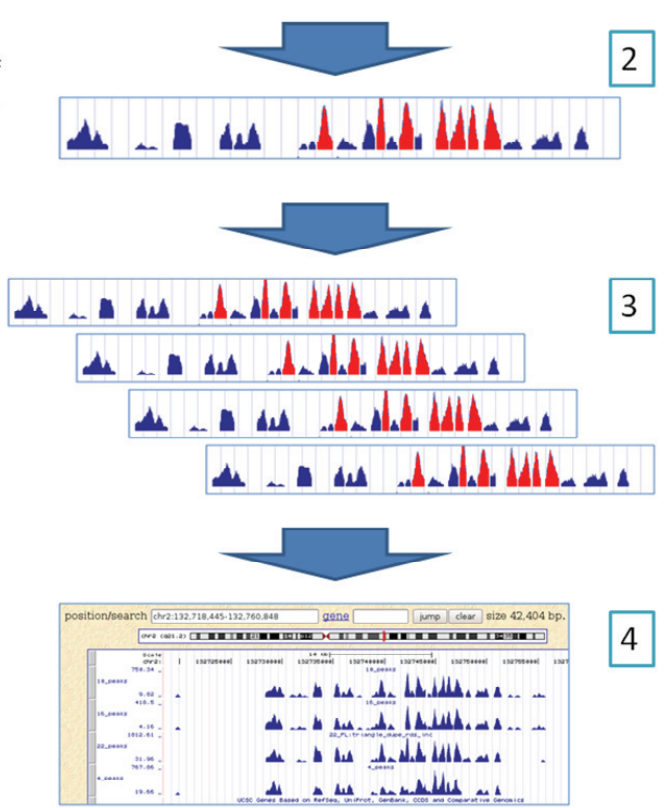

Figure 3: A schematic overview showing the steps involved in the normalization strategy based on finding regions with stable H3K27me3 enrichment. 


\section{(iii) Summarization at gene level}

To ease the interpretation of high-scale datasets such as obtained in ChIP-seq analysis, an excellent approach is to summarize the data based on relative location with respect to genes and gene regulatory regions. For H3K27me3, such regions have been defined successfully before, with clear associations to gene transcription regulation [15]:

- Promoter region: 3000 bp upstream of TSS to 100 bp upstream of TSS

- TSS region: $100 \mathrm{bp}$ upstream of TSS to $1000 \mathrm{bp}$ downstream of TSS

- Gene body: 1000 bp downstream of TSS to end of the last exon

Enrichment for $\mathrm{H} 3 \mathrm{~K} 27 \mathrm{me} 3$ that covers the entire gene body is associated with gene silencing. Enrichment within the TSS region contains candidates for so-called bivalency, i.e. nucleosomal regions marked with both gene-silencing-associated H3K27me3 and gene-activity-associated H3K4me3. Enrichment in the promoter region can be associated with gene silencing as well as active gene transcription, depending on the nearby presence of other histone modifications and the cellular context [15].

Other regions of interest (ROI) may be enhancers, which are known to be susceptible to various histone modifications [24]. A potential obstacle with these ROls is that a clear definition of what constitutes a functional enhancer is not always available, as these are functionally dependent on cellular context.

\section{Anticipated results}

We have tested the above protocol extensively on our own and published H3K27me3 ChIP-seq datasets. The enrichment-finding approach and the normalization approach are inseparably connected. The broad-peak settings for FindPeaks produce more returned signal, which is separated into more individual peaks (figure 4).

This is required for the normalization-approach to work properly, because it needs to integrate as much peak information as possible to reliably identify stable regions of enrichment within the genome. For the dataset used to develop the protocol, most of these stable regions were found near centromeres and in intergenic regions. It has been observed previously that centromeres are heavily enriched for H3K27me3 $[8,25,26,27]$. These regions are also known to contain a large number of repeats. Therefore, repeat region reads will inevitably end-up being mapped to these regions, regardless of their origin. Hence, the reason for finding high enrichment within these regions may reflect a technical artifact rather than biology. It is therefore essential to prevent accumulation of such reads leading to spurious enrichment in these regions, by keeping only uniquely mapped reads for normalization analysis. 


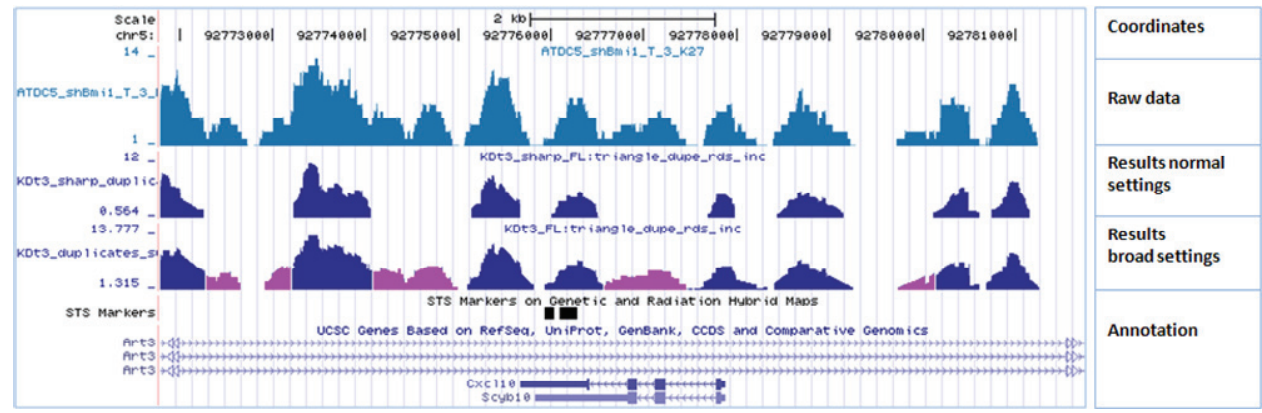

Figure 4: Results of the broad-peak settings for FindPeaks compared to the default settings.

We compared the results for our dataset and approach to published results [28]. In figure 5, condensed genome tracks are plotted for chromosome 1 and 19, showing very similar profiles, although our approach retains more signal.

Our results

chromosome 1

Published results

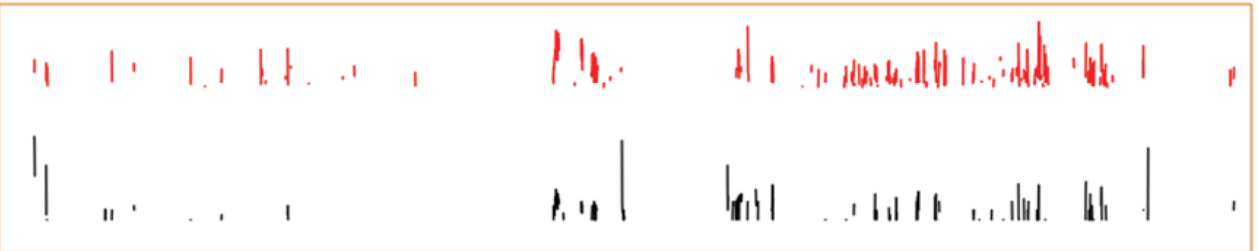

\section{chromosome 19}

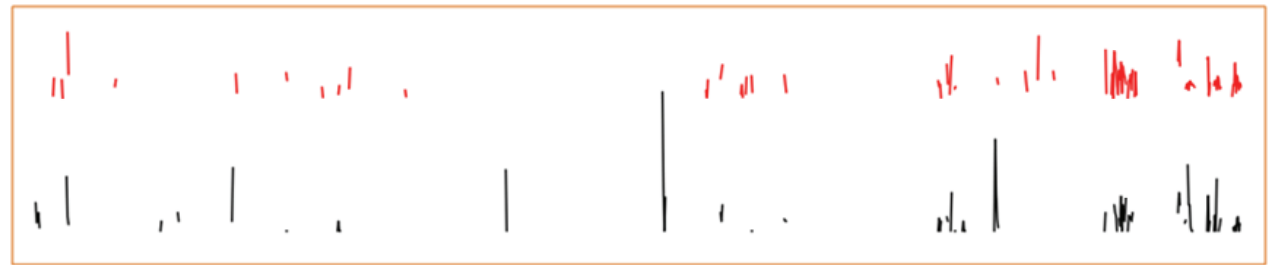

Figure 5: Comparison of published results with our results: a similar "fingerprint" is found, but more signal is retained using our H3K27me3 enrichment protocol. 


\section{DISCUSSION}

We here report on a straight forward, robust method to delineate non-uniform genomic H3K27me3-distributions. Our approach integrates a normalization method based on identifying regions with stable H3K27me3-enrichment between samples and a cumulative signal-retention strategy, which identifies broadly defined epigenetic marks, which would be lost with standard peak-find algorithms.

With respect to definition of H3K27me3-enrichment in ChIP-seq data multiple approaches are in use, which mostly build on limiting the enrichment-finding step to predefined regions and counting the number of reads in these regions. Some methods apply this to genic regions only [15,29], some to intergenic regions only [27] and others to both [30]. By limiting the enrichment-finding to regions with predefined start and end points, however, the enrichment analysis requires redefinition each time when novel regions of interest are probed, such as enhancers which are often located in the predefined intergenic regions. Moreover, when neither input sample data nor an estimated null-distribution is considered in the enrichment-finding strategy, which is the case for some published approaches $[29,30]$, this can result in spurious background enrichment, which limits the reliability of quantitative comparisons, regardless of normalization. Another downside of many of these approaches is that definition of enrichment is data driven, resulting in analytical parameter use that may not be applicable to other studies.

Genome-wide enrichment-finding methodology not limited by pre-defined static regions, include, for instance, a hidden Markov-model approach. Although some methods compare H3K27me3-enrichment between samples in a genome-wide fashion [31,32] and/or apply a genome-wide sliding window approach (i.e. summarizing the counts in each window; $[33,34])$, none of these approaches integrate input sample data or an estimated null-distribution to correct for background anomalies. Several studies report the use of standard enrichment-finding algorithms, like MACS [35], in combination with input sample data $[36,37,38]$. Using such algorithms without adapted settings, however, will inevitably result in significant loss of H3K27me3 signal.

With regard to data normalization, the most often reported approach is scaling by the total number of unique reads of each sample to the sample with the lowest number of reads [15,30,32]. A related approach is the dissection of nucleosome-filled genomic portions (or a subset thereof) into non-overlapping windows, followed by dividing the count-number in each window by the average number of counts for all windows $[27,29]$. Note that both normalization methods start from the assumption that the number of (any given) histone modifications does not vary between conditions. 
Whereas this may be satisfactory for specific experimental settings, it does not apply to dynamic biological systems. Strikingly, many published reports have omitted any form of data normalization as part of the data summarizing flow [33,34,36,37,38].

In conclusion, to our knowledge, the herein described approach is the only generic protocol that enables genome-wide, quantitative data comparison for H3K27me3 ChIP-seq data, while still retaining the robustness of using input sample data for enrichment-finding. Other histone modifications exist with similar distribution-profiles as H3K27me3, an example of which is the H3K36me3 modification; H3K36me3 is associated with transcription-elongation and typically covers the most if not all of the gene body. Hence, while our method is designed and optimized for studying H3K27me3-histone modifications, the protocol is readily adaptable for definition of other broad-type epigenetic enrichment profiles.

$\mathrm{R}$ scripts are available upon request from the authors. 


\section{REFERENCES}

1. Mardis ER: ChIP-seq: welcome to the new frontier. Nature methods 2007, 4(8):613-614.

2. Park PJ: ChIP-seq: advantages and challenges of a maturing technology. Nature reviews Genetics 2009, 10(10):669-680.

3. Ho JWK, Bishop E, Karchenko PV, Nègre N, White KP, Park PJ: ChIP-chip versus ChIP-seq: lessons for experimental design and data analysis. BMC genomics 2011, 12:134.

4. Kharchenko PV, Tolstorukov MY, Park PJ: Design and analysis of ChIP-seq experiments for DNA-binding proteins. Nature biotechnology 2008, 26(12):1351-1359.

5. Fullwood MJ, Wei C-L, Liu ET, Ruan Y: Next-generation DNA sequencing of paired-end tags (PET) for transcriptome and genome analyses. Genome research 2009, 19(4):521-532.

6. Altschul SF, Gish W, Miller W, Myers EW, Lipman DJ: Basic local alignment search tool. Journal of molecular biology 1990, 215(3):403-410.

7. Ruffalo $M$, LaFramboise $T$, Koyutürk $M$ : Comparative analysis of algorithms for next-generation sequencing read alignment. Bioinformatics (Oxford, England) 2011, 27(20):2790-2796.

8. Day DS, Luquette L, Park PJ, Kharchenko PV: Estimating enrichment of repetitive elements from high-throughput sequence data. Genome biology 2011, 11(6):R69.

9. Fejes AP, Robertson G, Bilenky M, Varhol R, Bainbridge M, Jones SJM: FindPeaks 3.1: a tool for identifying areas of enrichment from massively parallel short-read sequencing technology. Bioinformatics (Oxford, England) 2008, 24(15):1729-1730.

10. Rozowsky J, Euskirchen G, Auerbach RK, Zhang ZD, Gibson T, Bjornson R, Carriero N, Snyder M, Gerstein MB: PeakSeq enables systematic scoring of ChIP-seq experiments relative to controls. Nature biotechnology 2009, 27(1):66-75.

11. Nix DA, Courdy SJ, Boucher KM: Empirical methods for controlling false positives and estimating confidence in ChIP-Seq peaks. BMC bioinformatics 2008, 9:523.

12. Zhang Y, Liu T, Meyer CA, Eeckhoute Jrm, Johnson DS, Bernstein BE, Nusbaum C, Myers RM, Brown M, Li W et al: Model-based analysis of ChIP-Seq (MACS). Genome biology 2008, 9(9):R137.

13. Malone BM, Tan F, Bridges SM, Peng Z: Comparison of four ChIP-Seq analytical algorithms using rice endosperm H3K27 trimethylation profiling data. PloS one 2011, 6(9):e25260.

14. Scacheri PC, Crawford GE, Davis S: Statistics for ChIP-chip and DNase hypersensitivity experiments on NimbleGen arrays. Methods Enzymol 2006, 411:270-282.

15. Young MD, Willson TA, Wakefield MJ, Trounson E, Hilton DJ, Blewitt ME, Oshlack A, Majewski IJ: ChIP-seq analysis reveals distinct H3K27me3 profiles that correlate with transcriptional activity. Nucleic acids research 2011, 39(17):7415-7427.

16. Bracken AP, Dietrich N, Pasini D, Hansen KH, Helin K: Genome-wide mapping of Polycomb target genes unravels their roles in cell fate transitions. Genes \& development 2006, 20(9):1123-1136.

17. Pietersen $A M$, van Lohuizen $M$ : Stem cell regulation by polycomb repressors: postponing commitment. Current opinion in cell biology 2008, 20(2):201-207.

18. Huang W, Umbach DM, Vincent Jordan N, Abell AN, Johnson GL, Li L: Efficiently identifying genome-wide changes with next-generation sequencing data. Nucleic acids research 2011, 39(19):e130.

19. Cheung M-S, Down TA, Latorre I, Ahringer J: Systematic bias in high-throughput sequencing data and its correction by BEADS. Nucleic acids research 2011, 39(15):e103.

20. Adriaens ME, Romano A, Eijssen LMT, McKay JA, Mathers JC, Evelo CTA. enrichR: a Bioconductor toolbox for integrative analysis of ChIP-on-chip and DNA methylation microarray data. [submitted]

21. Taslim C, Huang T, Lin S: DIME: R-package for identifying differential ChIP-seq based on an ensemble of mixture models. Bioinformatics (Oxford, England) 2011, 27(11):1569-1570.

22. Muiño JM, Kaufmann K, van Ham RC, Angenent GC, Krajewski P: ChIP-seq Analysis in R (CSAR): An R package for the statistical detection of protein-bound genomic regions. Plant methods 2011, 7:11.

23. Mercier E, Droit A, Li L, Robertson G, Zhang X, Gottardo R: An integrated pipeline for the genome-wide analysis of transcription factor binding sites from ChIP-Seq. PloS one 2011, 6(2):e16432.

24. Heintzman ND, Hon GC, Hawkins RD, Kheradpour P, Stark A, Harp LF, Ye Z, Lee LK, Stuart RK, Ching CW et al: Histone modifications at human enhancers reflect global cell-type-specific gene expression. Nature 2009, 459(7243):108-112. 
25. Saurin AJ, Shiels C, Williamson J, Satijn DP, Otte AP, Sheer D, Freemont PS: The human polycomb group complex associates with pericentromeric heterochromatin to form a novel nuclear domain. The Journal of cell biology 1998, 142(4):887-898.

26. Voncken JW, Schweizer D, Aagaard L, Sattler L, Jantsch MF, van Lohuizen M: Chromatin-association of the Polycomb group protein BMI1 is cell cycle-regulated and correlates with its phosphorylation status. Journal of cell science 1999, 112 ( Pt 24):4627-4639.

27. Rosenfeld JA, Wang Z, Schones DE, Zhao K, DeSalle R, Zhang MQ: Determination of enriched histone modifications in non-genic portions of the human genome. BMC genomics 2009, 10:143.

28. Joseph R, Orlov YL, Huss M, Sun W, Kong SL, Ukil L, Pan YF, Li G, Lim M, Thomsen JS et al: Integrative model of genomic factors for determining binding site selection by estrogen receptor- $\alpha$. Molecular systems biology 2010, 6:456.

29. Chopra VS, Hendrix DA, Core LJ, Tsui C, Lis JT, Levine M: The polycomb group mutant esc leads to augmented levels of paused Pol II in the Drosophila embryo. Molecular cell 2011, 42(6):837-844.

30. Marks H, Chow JC, Denissov S, Françoijs K-J, Brockdorff N, Heard E, Stunnenberg HG: High-resolution analysis of epigenetic changes associated with X inactivation. Genome research 2009, 19(8):1361-1373.

31. Xu H, Sung W-K: Identifying Differential Histone Modification Sites from ChIP-seq Data. Methods in molecular biology (Clifton, NJ) 2012, 802:293-303.

32. Xu H, Wei C-L, Lin F, Sung W-K: An HMM approach to genome-wide identification of differential histone modification sites from ChIP-seq data. Bioinformatics (Oxford, England) 2008, 24(20):2344-2349.

33. Pauler FM, Sloane MA, Huang R, Regha K, Koerner MV, Tamir I, Sommer A, Aszodi A, Jenuwein T, Barlow DP: H3K27me3 forms BLOCs over silent genes and intergenic regions and specifies a histone banding pattern on a mouse autosomal chromosome. Genome research 2009, 19(2):221-233.

34. Akkers RC, van Heeringen SJ, Jacobi UG, Janssen-Megens EM, Françoijs K-J, Stunnenberg HG, Veenstra GJC: A hierarchy of H3K4me3 and H3K27me3 acquisition in spatial gene regulation in Xenopus embryos. Developmental cell 2009, 17(3):425-434.

35. Zhang Y, Liu T, Meyer CA, Eeckhoute Jrm, Johnson DS, Bernstein BE, Nusbaum C, Myers RM, Brown M, Li W et al: Model-based analysis of ChIP-Seq (MACS). Genome biology 2008, 9(9):R137.

36. Kim SW, Yoon S-J, Chuong E, Oyolu C, Wills AE, Gupta R, Baker J: Chromatin and transcriptional signatures for Nodal signaling during endoderm formation in hESCs. Developmental biology 2011, 357(2):492-504.

37. Li H, Bitler BG, Vathipadiekal V, Maradeo ME, Slifker M, Creasy CL, Tummino PJ, Cairns P, Birrer MJ, Zhang R: ALDH1A1 is a novel EZH2 target gene in epithelial ovarian cancer identified by genome-wide approaches. Cancer prevention research (Philadelphia, Pa) 2011.

38. Suzuki H, Takatsuka S, Akashi H, Yamamoto E, Nojima M, Maruyama R, Kai M, Yamano H-O, Sasaki Y, Tokino $T$ et al: Genome-wide profiling of chromatin signatures reveals epigenetic regulation of MicroRNA genes in colorectal cancer. Cancer research 2011, 71(17):5646-5658. 
Chapter 6 | 


\section{CHAPTER 7 \\ General Discussion}




\section{Control of PcG-function by the micro-environment}

This thesis focused on understanding the regulation of Polycomb-function in the context of gene-environment interaction; this was done by identifying and probing the function of novel interaction partners, by interrogating regulatory mechanisms and transcriptional regulation of PcG-target genes in response to changes in the microenvironment (e.g. kinase signaling, mitogenic stimulation, induction of cell stress and oxygen deprivation, see chapter 1, this thesis; Figure 5). In chapter 2 we described the role of the previously identified PRC1-associated kinase MK3 in relation to PcGfunction. We showed that overexpression of MK3 induced a P53-INK4A/ARF-dependent senescence phenotype, which could be bypassed by simultaneous overexpression of BMI1, indicating that MK3 controls proliferative life span via functional interaction with BMI1. Our data indicated that MK3-induced senescence resembles oncogene-induced senescence (OIS), suggesting MK3 acts oncogenic. In addition, our findings also indicated that MK3 overexpression activates dormant check points in cancer cells. In chapter 3 we identified pERK as an induced interaction partner of PRC1, downstream of mitogenic signaling; we characterize a regulatory signaling cascade in which the expression of a PRC1-target gene (ATF3) is induced through combined ERK and p38 signaling resulting in PRC1/chromatin-dissociation; we provide evidence that transcriptional re-silencing is controlled by MK3 via negatively feed-back on pERK most likely involving induction of DUSPs. The existence of a negative feedback loop involving MK3 and ERK was confirmed by in vivo experiments, in which we established a negative regulatory effect of $d M K$ in the context of ERK-induced ectopic wing vein formation. In chapter 4 we identified a novel stress-induced interaction between PRC1-protein Bmi1 and the heterochromatin protein KAP1. Both proteins were recruited to the chromatin upon induction of cell stress (by addition of arsenite or selenite) where KAP degrades Bmi1. Drosophila crosses between mutant $P c(d C B X 4)$ and bonus $(d K A P)$ alleles substantiated the genetic interaction of these two novel binding partners. In chapter 5 we report that hypoxia results in a massive, genome-wide induction of trimethylation at H3K4me3 (TrxG-associated, active mark) and H3K27me3 (PcG-associated, repressive mark). H3K27me3 showed a relative increase around the transcription start site, resulting in epigenetic bivalency (co-occurrence of K4me3 and K27me3), a subset of which coincide with genes that were previously identified as bivalently marked genes in embryonal stem cells. Our observations suggest molecular oxygen is a factor connecting the microenvironment directly to epigenetic regulatory mechanisms (oxygen-dependent demethylation). The hypoxia induced bivalent marking proposes the exciting possibility that oxygen-deprivation may induce or select for stem-ness or stem-cell like (epi)phenotypes. 
Chromatin-based silencing, including Polycomb-silencing and heterochromatin formation, consists of three major steps: 1) targeting silencing to specific DNA sequences (i.e. genes, chromosomal regions), 2) establishment of silenced chromatinstructure (i.e. chromatin state that restricts gene expression) and 3) the propagation of silent chromatin through cell division (i.e. epigenetic inheritance or cellular memory) (reviewed in Beisel and Paro, 2011; Simon and Kingston, 2009). All three individual steps are prone to environmental influences; chromatin-association of PcG complexes (i.e. recruitment or displacement) can be affected, chromatin state can be altered and cellular memory can become perturbed. Perturbation of cellular memory may result in inappropriate development (i.e. defects in anterior-posterior (AP) positioning, diminished stem cell renewal) or in disease (i.e. cancer). Furthermore, complex composition can also be affected by changes in the micro-environment, for instance by post-translational modification of complex members, and hence, complex function, localization and stability can be altered. Finally, trimethylation of H3K27 can be implicated in PcG-mediated silencing in various ways, depending on differentiation status, enrichment profile and cell cycle status.

\section{PcG complexes and chromatin association}

\section{Recruitment}

Almost two decades ago PRC1 and PRC2 proteins were found to specifically associate with defined elements termed Polycomb response elements (PREs) in Drosophila (Bloyer et al., 2003; Muller and Kassis, 2006; Ringrose and Paro, 2007). PREs constitute a group of DNA elements that vary in size, composition and distance to target genes. The functional relevance of PREs in relation to H3K37me3-marking had been drawn into question by the observation that PREs are relatively nucleosome-poor regions (Kahn et al., 2006). PREs, however, carry multiple sequence-motifs for various sequence-specific DNA-binding proteins (transcription factors) and hence function as remote (in cis- and/or in trans; Mateos-Langerak and Cavalli, 2008) regulatory elements (as enhancers or silencers); PREs recruit GAGA factor (GAF), Pipsqueak, Zeste, Dorsal switch protein 1 (DSP1), Grainyhead and Specificity protein 1 (SP1) (Beisel and Paro, 2011). The DNA binding protein Pleiohomeotic (PHO) plays a central role in the recruitment of PcG-complexes to PREs (Muller and Kassis, 2006; Ringrose and Paro, 2007): it complexes with SCM-related gene containing four MBT domains (SFMBT) which is termed $\mathrm{PHO}$ repressive complex (PHO-RC) and was identified as the third PcG-complex (Klymenko et al., 2006; Oktaba et al., 2008). Mammalian PREs were only 
recently discovered (Sing et al., 2009; Woo et al., 2010); they showed functional similarities to Drosophila PREs in that they contain GAGA and YY1 (homolog of PHO) binding motifs and are dependent on PcG-proteins for their repressive activity. In mammalian cells YY1 has been implicated in recruitment of PcG, however, in mammalian ES cells OCT4 is considered to be one of the major recruiters (Lee et al., 2006; Vella et al.). A recent genome-wide analysis of PRC1 and PRC2-occupancy indicated that the majority of PRC2-complexes are associated with CpG-rich regions including CpG-islands (Ku et al., 2008), suggesting that CpG-binding proteins might also be involved in PcG-recruitment.

In addition to transcription factors, non-coding RNAs (ncRNAs) have been implicated in PcG-recruitment in mammals. Examples include HOTAIR, a long intergenic ncRNA (lincRNA) which is involved in trans-silencing by recruiting PRC2 to the HOXD locus (Rinn et al., 2007), XIST, a long non-coding RNA (IncRNA) that binds PRC2 via its RepA RNA motif to accomplish in cis-silencing of the inactive X-chromosome (Brockdorff, 2011; Wutz et al., 2002; Zhao et al., 2008) and Kcnq1ot1, a paternally transcribed IncRNA implicated in in cis silencing of the maternally expressed Kcnql gene-cluster by recruitment of PRC2, G9a (generates repressive H3K9me1/me2) and DNA (cytosine-5)-methyltransferase 1 (DNMT1) (Mohammad et al., 2010; Pandey et al., 2008). As IncRNA represent a large portion of the mammalian transcriptome and their expression is tightly regulated (i.e. spatial/temporal expression), they may constitute a general targeting mechanism. Both the underlying RNA sequence as well as the secondary RNA structure could be involved in PcG-recognition (Beisel and Paro, 2011; Simon and Kingston, 2009).

Furthermore, histone modifications could provide an additional targeting mechanism for PcG. A hierarchical recruitment model was proposed to explain PRE-mediated silencing: PHO recruits the "writer" complex (PRC2) via direct proteininteraction, which subsequently establishes the H3K27me3-mark, which is recognized and bound by the "reader" complex (PRC1) (Fischle et al., 2003; Min et al., 2003). The contribution of H3K27me3 to PcG-targeting is discussed in detail below (see section "the role of H3K27me3 in PCG-mediated gene silencing"). Importantly, the affinity of PcG-complexes for histone modifications, like H3K27me3, may be fine-tuned by posttranslational modifications (PTMs) of specific subunits (Hatano et al.; Niessen et al., 2009). Close to 120 phospho-sites have been identified on PcG-proteins (Niessen et al., 2009) and there are likely to be additional PTMs which may be involved in fine-tuning of chromatin-binding; indeed CBX4-chromodomain/H3K27me3-binding appears also dependent on H2AK119ub1 (Wang et al., 2004a). Clearly, these findings imply a high 
level of complexity in regulation of PRC/chromatin-association and PRC-function. The availability of phospho-specific antibodies is limited and studies on the effect of phospho-mutants are only beginning to occur; such studies will be imperative to determine the functional implications of PTMs on PRC-proteins. Although we have not tested any specific phospho-mutants in these studies, we have observed that the relatively hyper-phosphorylated fraction of the PRC1-subunit BMI1 is nuclear (soluble and chromatin bound) (chapter 4, this thesis), even under serum-starved conditions during which PRC-phosphorylation levels are relatively low; although this may be an indication that phosphorylation generally promotes chromatin-association, enhanced phosphorylation could also relate to nuclear residence. In support of the hypothesis that specific PTMs are involved in chromatin-association, we observed increased chromatin-recruitment of BMI1 (and KAP1) upon induction of cell stress. As serum stimulation also induces phosphorylation, yet without detectable BMI1/chromatinrecruitment, it is possible that additional PTMs are involved and that chromatinrecruitment is determined by the nature of intensity of the stimulus applied in addition to the location (amino acid) and chemical nature of the PTMs. Some PTMs, like KAP1pS824, are induced by very specific stimuli, i.e. double-stranded DNA breaks (i.e. selenite exposure, ionizing radiation or ultra violet treatment; not arsenite or mitogenic stimulation), whereas KAP1pS473 is induced by both cell stress and mitogenic stimulation. Although such differential regulation has not been reported for PRCproteins, they are expected to be of significance for regulation of PRC-function. Taken together, the combinatorial effect of several, potentially redundant, independent targeting-mechanisms, including DNA-sequence-specific recruitment, association with ncRNAs, and post-translational modification of histone proteins (or other chromatin factors) and PcG-proteins, converge at the chromatin-level, and allow dynamic fine-tuning of PcG-repression in a cell context- and locus -dependent manner.

\section{Displacement - signaling to chromatin}

Most likely, chromatin displacement of PRC-complexes involves similar mechanisms as chromatin recruitment. From studies in Drosophila it is known that tissue-specific TATA-box binding protein (TBP) associated factors (TAFs) are able to displace PcG-proteins from chromatin during differentiation (Chen et al., 2005). Furthermore observations by us and other research groups suggest that H3S28phosphorylation interferes with PcG/chromatin-association (this thesis) (Gehani et al., 2010; Voncken et al., 2005). We here show that acute changes in the microenvironment, like mitogenic stimulation can also result in PRC1 displacement: both 
primary diploid fibroblasts and cancer cells respond to mitogenic stimulation by increasing expression of the immediate early gene (IEG) and PRC1-target gene ATF3 (chapter 3, this thesis). The use of select kinase inhibitors revealed that Polycombdissociation is dependent on both p38 and ERK-signaling. The biochemical trigger for PRC1/chromatin-dissociation is likely a combination of post-translational modification of histone proteins (p38 signaling is required for H3S28-phosphorylation) and of (multiple) subunits of the PRC1-complex itself. As we found that pERK is an induced interaction partner of PRC1, such putative PTMs may be a (direct or indirect) downstream effect of ERK-signaling. Relevantly, we previously identified MK3 as a putative PRC1-kinase/interaction partner; it is known that MK3 is a downstream target of pERK (Ludwig et al., 1996), which raises the possibility that pERK is recruited by MK3 to PRC1-targets, and activates MK3. Although we have indications that MK3 and pERK associate (data not shown), it is currently not clear whether this actually occurs at the chromatin level. Immunoprecipitations on fractionated cell extracts will provide more insight into where in the cell interaction occurs. In addition, MK3 may target PRC1 for phosphorylation and/or be involved in redirecting PRC1-proteins back to chromatin by inducing PTMs that favor chromatin -recruitment. It is clear that MK3 is involved in restoring PcG-repression: MK3 induces dual specificity phosphatases (DUSPs), which have recently been implicated in dephosphorylation of MAPK-family members (Patterson et al., 2009). In agreement with this, ERK-phosphorylation is sustained when MK3-function is disabled, either by RNA-interference or by chemical inhibition. Our loss of MK function studies in Drosophila confirms the negative regulatory loop on ERK, as $d M K$-depletion exacerbates the rolled $(d E R K)$ induced ectopic wing vein phenotype. A number of interesting questions remain: are Polycomb targets selectively targeted in response to mitogenic stimulation, or are most responses genome-wide (e.g. like H3S28-phosphorylation)? Or do specific PRC1-directed regulation and genome-wide responses co-exist? Does the presence of regulators, like MK3, at pre-determined loci provide a specific link with activated M/SAPK-signaling cascades? The presence of signal relay/recipient molecules at the chromatin level is an attractive model, as this would not only imply specificity, it would also allow for rapid responses. In line with this thought, immediate early genes are characterized by the presence of stalled RNA polymerase 2 (RPOL2) at the promoter which enable rapid transcriptional activation upon RPOL2-phosphorylation (Saha et al.) in addition, H2AK119-ubiquitylation by the PRC1 RINGfinger-proteins RNF2 and BMI1, is required for RPOL2 stalling (Breiling et al., 2001; Enderle et al., 2010; Stock et al., 2007; Zhou et al., 2008);. Hence, the PERK/PRC1/MK3 connection provides an operational model for specific, local transcriptional responses (see section "Role of H3K27me3 in Polycomb-mediated 
repression"). Of note, the presence of PC (D.mel CBX4-ortholog) at the TSS in a Drosophila-based model did not prevent transcription, underlining the notion that most likely an intact PRC1-complex is required for full repressive action (Breiling et al., 2001). Genome-wide responses, like H3S28-phosphorylation, could be required simultaneously, and would impact on PRC1-bound to the adjacent H3K27me3-mark in analogy to HP1 binding to H3K9me3 and H3S10 phosphorylation (Dormann et al., 2006; Fischle et al., 2005; Hirota et al., 2005; Lau and Cheung, 2011). Of note, the H3S29phosphorylation event in itself is probably insufficient for chromatin release as H3S28ph co-occurs with PRC1/chromatin-binding (chapter 3, this thesis); similar observations were reported for H3S10ph and HP1/chromatin binding (Vermeulen et al., 2010). The contribution of resident regulatory molecules, like MK3, pERK and/or pp38, and their chromatin-association in relation to mitogen-induced regulation of PRC1targte gene transcription requires detailed mapping by combined ChIP and transcriptional analysis.

\section{Role of H3K27me3 in Polycomb-mediated repression}

\section{PRC1 targeting}

The concept that H3K27me3 is an essential element in Polycomb-gene silencing is widely accepted. However the exact molecular and biological functions of H3K27me3 are still elusive. One of the earliest models positioned the H3K27me3-mark as an essential element in the initial recruitment of PRC1 to chromatin; in support of this model it was shown in Drosophila that upon disruption of H3K27me3, PRC1 is lost from its target genes (Cao et al., 2002; Wang et al., 2004b). However, these experiments did not rule out a contribution of the PRC2-complex itself (which was removed in order to disrupt H3K27me3). Increasing evidence indicates that PRC1/chromatin-association and H3K27me3 do not necessarily overlap, but can occur independently (Simon and Kingston, 2009). Probably the most convincing argument against initial recruitment by histone modifications is that, although the binding of the PRC1/CBX4 chromo-domain has a high specificity towards $\mathrm{H} 3 \mathrm{~K} 27 \mathrm{me} 3$, the binding affinity is relatively low; the binding affinity of chromo domains is within the micromolar range as compared to TF which bind in the nanomolar range (Fischle et al., 2003; Platero et al., 1995)In addition, binding of CBX4 was lost in RNF2-knock out cells, despite retention of H3K27me3, suggesting additional local requirements for optimal PRC1-recruitment(Wang et al., 2004a). It was recently shown that the PRC1 protein CBX7 chromo-domain also binds single-stranded RNA (ssRNA), like the IncRNA ANRIL, which is transcribed from the 
CDKN2A/INK4A-ARF locus and regulates expression of CDKN2B/p15INK4B (Bernstein et al., 2006b; Yap et al., 2010). In addition, CBX7 chromo-domain binds H3K9me3 (associated with (facultative) heterochromatin formation) with similar affinity as H3K27me3, yet H3K9me3-binding involves different residues of the chromo-domain (Bernstein et al., 2006b). These observations suggest that H3K27me3 in combination with ncRNAs may provide multiple binding options: numerous, relatively low affinity, interaction sites with histone modifications and RNA binding motifs may increase the binding-avidity of this apparently versatile interaction domain (Beisel and Paro, 2011). In the previous section we proposed that PRC1 PTM in combination with H3S28ph may be sufficient for PRC1/chromatin-release and transcriptional activation of the PRC1target gene ATF3. Thus far, we have found that H3K4me3 and/or H3K27me3-marking remain unaltered upon activation of the IEG ATF3. The multiple low-affinity binding-site concept provides an attractive model for rapid and dynamic regulation of PRC1mediated repression in response to environmental changes: complex-dissociation via PTMs can rapidly and reversibly switch the balance towards gene-activation. Recruitment of factors like H3K4me3 HMTs, H3K27me3 HDMs and/or transcriptional shut-down of IncRNAs may assist in reprogramming the local epigenome, and may be required to install long-lasting effects during differentiation and development.

\section{Re-establishment of silenced chromatin - bringing back PRC1}

An alternative model proposes that H3K27me3 acts in chromatin compaction by promoting the spreading of repressive chromatin. This model implies that chromatinlooping through interaction of PRC1 (associated with PREs/CpG-islands) with H3K27me3 allows close contact between both PRC2 and PRC1 with more distant nucleosomes. This could support spreading of H3K27me3 and H2AK119Ub through the gene body (Muller and Kassis, 2006; Schwartz and Pirrotta, 2007). Of note, the recruitment model and the chromatin-looping model are not mutually exclusive, but may well operate in parallel. The looping model also positions the effect of PRC1 in the gene-body: H3K27me3-marking in combination with PRC1-binding impedes transcriptional elongation. It is thought that H2AK119Ub is important in this respect (Cao et al., 2005; Stock et al., 2007; Zhou et al., 2008) by blocking transient displacement/disassembly of nucleosomes, thereby blocking transcriptional elongation of RPOL2 (Bondarenko et al., 2006). Of note: TrxG-proteins promote transcript elongation (Smith et al., 2011). Direct compaction of nucleosomes by PRC1 may also prevent elongation by stabilizing histone/DNA-interaction (Francis et al., 2004). We found that the presence of $\mathrm{H} 3 \mathrm{~K} 27 \mathrm{me} 3$ in itself does not obstruct transcriptional activity 
of underlying genes: mitogenic stimulation rapidly induces ATF3-transcription, yet H3K27me3 is maintained within the ATF3-promoter area (chapter 3, this thesis). Instead PRC1 is lost from chromatin. These findings are consistent with a transcriptionally repressive role for PRC1, rather than for H3K27me3. As we did not examine the consequences of mitogenic stimulation for epigenetic marking in the gene body, it is important to establish the enrichment profiles for both H3K27me3 and H2AK119ub1 levels under resting and stimulated conditions. Additional recent findings indicate that H3K27me3-marking may not preclude active transcription (Young et al). In line with this report, we also observed higher expression of H3K27me3/promoterenriched genes, compared to the TSS and gene body (broad)-enriched genes (chapter 5, this thesis). Our subsequent analysis revealed that transcriptional status did not correlate with presence of $\mathrm{f} \mathrm{H} 3 \mathrm{~K} 27 \mathrm{me} 3 \mathrm{per}$ se; also genes with a broad-enrichment profile were shown to be transcriptionally active. Instead, transcription status was determined by the presence of H3K4me3 (see section "Chromatin state"). In light of the physical presence of repressive PRC1, it is tempting to speculate that active genes are devoid of PRC1-binding whereas the silent genes are marked by PRC1-binding. Re-ChIP/deep sequencing analysis at PRC1-target loci is required to confirm whether PRC1-binding and H3K4me3 are indeed mutually exclusive.

\section{Propagation of Polycomb silencing}

New insights in the stable transmission of Polycomb-silencing information during cell division suggest a key role for H3K27me3: following DNA-replication PRC2 is recruited to the $\mathrm{H} 3 \mathrm{~K} 27 \mathrm{me} 3$ mark to re-establish trimethylation on the newly incorporated histone proteins, thereby restoring gene silencing (Hansen et al., 2007). Recruitment of PRC2 could be accomplished through direct interaction with H3K27me3 (most likely via the SUZ12/EED subunits) (Hansen et al., 2007; Margueron et al., 2009), or through transcription of ncRNAs (Chen et al., 2008; Kloc et al., 2008; Zaratiegui et al., 2011). In addition, PcG proteins themselves may remain bound at replicating DNA (Brock and Fisher, 2005; Francis et al., 2009). It was previously shown that bulk PcGbinding is lost at the end of S-phase; however, a low percentage of PRC complexes remained associated with chromatin (Buchenau et al., 1998; Voncken et al., 1999). Histone modifications and residual PRC-proteins could, in analogy to re-establishment of DNA-methylation on replicated DNA daughter strands by DNMTs, provide a means to re-establish PRC-mediated gene silencing after mitosis. In a broader context, given the paired presence of DNA-strands in the double helix and histones in nucleosomes, it is tempting to speculate about analogous mechanisms in duplicating genetic and 
epigenetic information. Taken together, the three models suggest, that although not sufficient for installing repression by itself, $\mathrm{H} 3 \mathrm{~K} 27 \mathrm{me} 3$ is involved in all three aspects of chromatin silencing; PcG recruitment (both PRC1 and PRC2), establishment of silenced chromatin (through chromatin looping) and cellular transcriptional memory (by marking genes that need to be repressed).

\section{Complex composition}

The discovery of additional PcG-proteins next to the core-subunits and the existence of multiple isoforms in mammals imply that alternative complexcompositions could confer distinct epigenetic functions (Simon and Kingston, 2009). For example the catalytic subunit of PRC2 (Czermin et al., 2002; Muller et al., 2002) has 2 paralogs in mammals: EZH1 and EZH2. Recent studies revealed striking differences between PRC2-variants containing EZH1 or EZH2, both in temporal/spatial expression and in biochemical activity; whereas EZH2 is predominantly expressed during embryogenesis and in proliferating tissues and has a high catalytic activity, EZH1 is mainly found in adult, non-dividing tissues and has a much lower catalytic activity; in fact the repressive (i.e. polynucleosome compaction in vitro) function of EZH1 appears independent of its SET domain (Margueron et al., 2008; Shen et al., 2008). Consistent with this notion, we interpreted the increased H3K27me3 (chapter 5, this thesis) in response to oxygen withdrawal, to be the result of decreased HDM-activity, rather than increased HMT-activity, as EZH2 mRNA levels decreased (despite increased EZH1 mRNA).

Like PRC2, PRC1 comes in different flavors: multiple paralogs of core-subunits give rise to multiple molecular combinations, which may differ in localization, expression profile, target genes or responses to external or internal stimuli. Like EZH1/2 RING1a/b (RING1/RNF2) are not redundant in embryogenesis, due to restricted expression of one of the two paralogs (Voncken et al., 2003). Although RNF2 and RING1 show some redundancy in cell systems, the presence of RING1 and BMI1 in PRC1 appears to specifically target Topoisomerase 2 (TOPO2) (Alchanati et al., 2009). In contrast to PRC2, some PRC1-family proteins may be part of complexes comprising core-subunits which are fundamentally different from PRC1 proteins and thus from the originally defined PRC1-complex (Shao et al., 1999). Examples include: the RING-associated factor (dRAF; Drosophila melanogaster) complex, which comprises of the core-subunits dRING, KDM2 and PSC (Cao et al., 2005; Lagarou et al., 2008; Wang et al., 2004a); the mammalian BCL6 co-repressor (BCOR) complex comprises RING1, RING1B, nervous 
system Polycomb 1 (NSPC1, also known as PCGF1), BCOR and F-box and leucine-rich repeat protein 10 (FBXL10, also known as JHDM1B and KDM2B (Gearhart et al., 2006; Sanchez et al., 2007). The dRAF/BCOR complex is able to efficiently ubiquitylate H2AK119 and demethylates H3K36me2 (an elongation mark; see section "Chromatin state"). Likewise, Polyhomeotic (PH) is can be part of non-PRC1 complexes (Hodgson et al., 2001). A recent study also indicated a PcG complex involved in deubiquitylation; the Polycomb repressive deubiquitinase (PR-DUB) (Scheuermann et al., 2010) comprising Calypso and ASX in Drosophila melanogaster (human homologs; BAP1 and ASXL1 respectively). Clearly, future studies should focus on charting overlapping and/or independent functions among these diverse complexes.

\section{Mitogenic stimulation and cell stress - signaling to Polycomb}

Complex composition can be dynamically regulated and even non-PcG proteins can become associated to PRC1-complexes as indicated in chapter 3 and 4 (this thesis). We identified pERK as an induced binding partner of PRC1, as it co-immunoprecipitated with the BMI1 subunit of PRC1 upon mitogenic stimulation. Although we do not know what the exact biochemical role is of this interaction, pERK appears functionally involved in the displacement of PRC1 from chromatin: inhibition of ERK signaling (using a MEKi) retains Polycomb at chromatin, and may contribute to the blocked transcriptional induction of ATF3 (chapter 3, this thesis; see section "PcG and chromatin association").

In chapter 4 we identified KAP1 as a novel stress-induced binding partner of the PRC1 complex using a combined immunoprecipitation (IP)/mass-spectrometry approach. We were not able to detect any stress-specific phosphorylation residues; as the applied detection method was non-quantitative, however, we cannot exclude any relative changes in phosphorylation at the detected residues. Stable isotope labeling by amino acids in cell culture (SILAC) should be applied to detect differences in abundance of specific phospho-residues. Nevertheless, our data confirmed that KAP/BMI1interaction is dependent on kinase signaling, because treatment with selective kinase inhibition affected the stress-induced association negatively or positively. KAP1 and PRC1 members clearly undergo mobility shifts in response to cell stress, indicating posttranslational modifications at different sites; however, the exact nature and location of PTMs that contribute to the establishment of the interaction are currently not known. KAP1 and BMI1 interact in the nucleoplasm and at the chromatin level; at this point it is not known whether this differential localization represents functionally different KAP1/BMI1 compartments, or merely reflects a different stage in an ongoing cellular 
process (e.g. KAP1/BMI1-interaction in one location followed by translocation). The biochemical consequence of the KAP1/BMI-interaction is evident though: BMI is targeted for proteolytic degradation by KAP1 in a RINGfinger dependent manner. The significance of BMI1/KAP1 interaction is further underscored by the genetic interaction of Drosophila bonus (D.mel ortholog KAP1) and polycomb in anterior-posterior patterning which is manifested as reduced expression of the $P C^{1}$-associated sex comb phenotype (chapter 1 , this thesis).

KAP1 and BMI1 have joined functions in DNA repair and/or transcriptional regulation of $\mathrm{PRC1}$-target genes. It is tempting to speculate about a functional connection between these two proteins in both processes. Chromatin-association of KAP1 is enhanced in the context of mitogenic signaling induced responses (MIR) and cell-stress responses, both of which have been associated with DNA-damage (Dinant et al., 2008; Valko et al., 2006). It could very well be that KAP1/BMI1-interaction serves a mechanistic purpose in DNA damage signaling: KAPS824-phosphorylation is required for chromatin decondensation at sites of double strand DNA breaks (DSBs); BMI1 is attracted to amplify local DNA-damage signals (by enhancing H2AK119Ub and incorporation of the histone variant H2A.X) (Ginjala et al., 2011; Ismail et al., 2010); hence KAP1-binding may be induced to halt the amplification process, through proteolytic degradation of BMI1 (possibly via ubiquitylation or SUMOylation). KAP1 is a transcriptional regulator through recruitment of the nucleosome remodeling and deacetylase (NuRD) complex and binding of HP1 (Chang et al., 2008; Ivanov et al., 2007; Lee et al., 2007; Mascle et al., 2007; Schultz et al., 2001). KAP1S473-phosphorylation was reported to release HP1-binding and its ability to repress transcription (Chang et al., 2008); KAP1pS473 also increases in response to DNA-damage, and may be required for expression of DNA-damage response genes (chapter 4, this thesis) (White et al., 2011). We observed that RNAi-mediated KAP1 depletion allows for a more robust transcriptional activation of ATF3 in the context of MIR, suggesting a role for KAP1 in PRC1-mediated repression. It is not known at this stage whether the effect of shKAP1 solely indicates more ATF3-induction as a result of loss of some aspect of local repression, or whether the expression kinetics are affected (i.e. premature induction and/or delayed re-silencing); in other words is the effect of KAP1-depletion a reflection of resident KAP1 being responsible for co-repression (local regulation) or is recruitment of KAP1 required to re-establish silencing of the PRC1-target gene? As PRC1 plays a major role in transcriptional repression (see section "role of H3K27me3 in Polycombmediated repression"), it would appear unlikely that an inability to remove BMI1 (through loss of the responsible BMI1 ubiquitin E3-ligase KAP1) would enhance gene expression. Published genome-wide KAP1-enrichment analysis suggested that ATF3 in 
no KAP1-target (O'Geen et al., 2007), hence the available data favors KAP1-recruitment and/or presence at the gene-body, although recruitment at the promoter/TSS cannot be ruled out at this point. The presence/recruitment of KAP1 may serve several, possibly combined purposes: balancing local DNA-damage responses (DDR) to transcription (e.g. removal of BMI1 as part of DDR/MIR, and/or local chromatin condensation to prevent DSBs (via recruitment of CHD3/HP1) (Chang et al., 2008; Ivanov et al., 2007), promotion of transcription initiation and/or elongation (by removal of BMI1/PRC1), re-instatement of PRC1-repression at the promoter/TSS or gene body (through recruitment of repressive factors like CHD3/HP1). It is likely that reduced DNAdamage responses as well as impeded re-silencing both contribute to enhanced gene expression. It is of note to recall the considerable mechanistic overlap between HP1- and PRC-mediated repression; also PRC1-silencing appears to be dependent on SUV39H1 (H3K9me3 HMT) (Sewalt et al., 2002) (see section “Chromatin state”).

Interestingly, our recent observations revealed an interaction between KAP1 and MK3 in serum starved cells, which was reduced in response to mitogenic signaling (data not shown). In concordance with a role for MK3 in re-silencing ATF3, it is conceivable that loss of MK3/KAP1-binding and/or KAPpS473 (p38/ERK-dependent) stimulates KAP1-translocation to chromatin to shut down transcriptional activity at the ATF3-locus. The observed chromatin recruitment of KAP1 (p38-dependent) following mitogenic stimulation, as well as increased ATF3 expression (i.e. reduced repression) in KAP1-depleted cells are consistent with such a model. We have observed that MK3overexpression (OE) increases KAP1pS473 (and bypasses the p38i-induced reduction of pS473 levels), whereas MK3 inhibition (Fluorescence-activated cell sorting) reduces KAP1pS473 levels. We propose that p38 is directly responsible for KAPS473phosphorylation, not MK3; these observation are in keeping with the reported (kinase-independent) stabilizing effect of MK3 on p38 (Ronkina et al., 2007); shKAP1 also bypasses the reducing effect of p38-inhibition on ATF3-expression. The combined data suggests that inhibition of p38-mediated KAPpS473-phosphorylation is functionally equivalent to loss of KAP1. Hence, p38/SAPK-mediated signaling may, besides H3S28-phosphorylation, fulfill additional tasks in regulation PRC1-target gene transcription, possibly through targeting KAP1 repressive function. Clearly, the exact effect of KAP1-phosphorylation and/or chromatin recruitment on PRC1-target gene expression requires phospho-mutant studies combined with gene expression analysis, in addition to detailed ChIP-analysis. Together these observations position ERK, p38 and MK3 as regulators of KAP-phosphorylation, chromatin-association and function in response to environmental cues (i.a. stress, mitogens). 


\section{Chromatin state}

As outlined in the introduction, chromatin has traditionally been subdivided into euchromatin and heterochromatin, of which the latter can be subdivided into constitutive and facultative heterochromatin. In line with recent advances in identification of among other issues, chromatin-associated proteins, histone modifications, gene regulation, and nuclear architecture, it should come to no surprise that based on these novel insights, chromatin is functionally further classified in multiple distinct chromatin subtypes. Indeed, a recent publication identified five principle chromatin types in embryonic Drosophila melanogaster cells, based on unique combinations of chromatin associated proteins (Filion et al., 2010). In concordance with the meaning of the Greek word "chroma", each type of chromatin was assigned a specific color. Three out of five colored subtypes classified as repressive chromatin, two of which closely resembled the previously identified classic heterochromatin (green) and Polycomb-silenced chromatin (blue), the third chromatin type (black) is characterized by lack of classic heterochromatin markers (like HP1) and PcG-proteins, and is, instead, enriched for linker histone H1, the AT-hook protein D1, Ipl1-aurora-like kinase (IAL), suppressor of underreplication (SUUR), SU(HW), Effete (EFE) and Lamin (LAM) (black). Although the exact nature and function of "black" chromatin awaits determination, the specific proteins which are relatively enriched in this chromatin subtype provide important clues towards the biological role of this chromatin: associated proteins have been implicated in the formation of higher order chromatin structures, transcriptional repression and replication control; LAM is a structural component of the peripheral nuclear lamina. Combined with the high prevalence of this chromatin subtype (it covers about half of the genome) this might suggest that "black" chromatin is not only important for gene repression, but most likely it also plays a prominent role in chromosome architecture, which is in close agreement with the notion that peripheral chromatin is silent (Towbin et al., 2009). The two remaining chromatin subtypes both classified as active chromatin (yellow and red) and although they had a high degree of similarity in respect to associated proteins, histone marks and expression level, they clearly differ in molecular organization and in H3K36 trimethylation status; "yellow" chromatin is enriched for H3K36me3, whereas "red" chromatin lacks H3K36me3. Other aspects differentiating these active chromatin subtypes include high occupancy by regulatory proteins, high occurrence of DNAbinding motifs, prominent representation of origin recognition complex (ORC) and an at least two-fold more frequently encountered highly conserved non-coding elements (HCNEs) found in "red" chromatin as opposed to "yellow" chromatin. In combination with the high prevalence of tissue-specific genes within "red" chromatin, it was 
suggested that the "yellow" chromatin harbors house-keeping genes which display relatively broad expression profiles, whereas "red" chromatin is mainly associated with dynamically regulated genes, in analogy to the chromatin organization in budding yeast (Tirosh and Barkai, 2008). Genomic regions displaying similar chromatin (associated) features as "red" chromatin have been described before in mouse ES cells (Chen and Daley, 2008), suggesting that the different chromatin subtypes may be evolutionary conserved. Although this study did not explore dynamic (ex)change within (or between) chromatin subtypes during the course of differentiation, tissue expression profiling data, in combination with the allocation data of genes into the five subclasses, suggest that (at least in some cell types) chromatin subtypes can be remodeled (Chintapalli et al., 2007; Filion et al., 2010).

\section{Silenced Polycomb chromatin - a role for KAP1?}

Although "black" chromatin is poorly characterized in regards to associated histone modifications, "green" and "blue" silenced chromatin have been extensively studied and show a high degree of similarity in targeting of silencing complexes, the establishment of silent chromatin structure and the maintenance of silencing (reviewed in (Beisel and Paro, 2011)). Analogies between Polycomb and heterochromatin silencing include: involvement of DNA-binding proteins and ncRNA in the initiation (and propagation) of silencing, the introduction of a histone methyl mark (H3K27me3 and H3K9me3/me3 respectively see Table 1, Figure 2, chapter 1, this thesis), binding of histone marks set by HKMTs (EZH2 and SUV39H1, respectively) through a conserved binding motif (chromodomain of PRC1 and HP1 respectively) and both are connected with DNA-methylation. The potentially similar phospho-methyl switch involvement in regulating chromatin-association of PRC1 and HP1 has been discussed above (see section "PcG-complexes and chromatin association") (Fischle et al., 2005; Hirota et al., 2005). HP1 binding to H3K9me2/3, can recruit additional SUV39H1 (Lachner et al., 2001; Schotta et al., 2002), similar to binding of SUZ12/EED (with associated EZH2) to H3K27me2/3 (Hansen et al., 2007; Margueron et al., 2009) which enables spreading/maintenance and/or propagation of the repressive histone mark via a selfreinforcing loop. Furthermore, the silencing of Krüppel-associated box domain zincfinger proteins (KRAB-ZFPs) shares striking similarities with the regulation of the HOX gene clusters: KRAB-ZFPs are often organized in multi-gene clusters and are transcriptionally auto-regulated (O'Geen et al., 2007), they recruit KAP1 as a scaffold protein which further contributes to the recruitment of heterochromatin inducing factors like HP1 and SETDB1, and subsequent spreading of heterochromatin into the 
gene body thereby mediating long range gene repression (Groner et al.; O'Geen et al., 2007; Vogel et al., 2006). This observation may provide an explanation for the chromatin recruitment of KAP1 we observed in response to cell stress (chapter 4, this thesis); it is thought that at euchromatic regions KAP1 may enhance chromatin compaction to prevent transcription from interfering with repair processes; its prolonged presence at heterochromatin in its p\$824-cinstitution is required to maintain an open state at relatively condensed regions to allow DNA repair (Goodarzi et al., 2011; Goodarzi et al., 2008). Of note, our finding that KAP1 functionally interacts with PRC1, in parallel to the reported interaction with HP1, indicates that there may be a partial functional overlap in PRC1 and HP1-mediated silencing through KAP1. Although some reports indicate that PcG and HP1 repressive chromatin are non-overlapping, other studies point to shared biology (de Wit et al., 2007; Filion et al., 2010; Sewalt et al., 2002). Although we provide evidence that KAP1 is involved in (re)silencing of PRC1target genes (chapter 4, this thesis; see section "Complex composition"), it is currently not known whether this is dependent on HP1 function/presence

Taken together, both the initiation of PcG-silencing and heterochromatin formation rely on DNA-binding proteins, suggesting that specificity and selectivity are ultimately generated by underlying DNA-sequences. Binding of these factors to their cognate sequences does not appear to be hampered by limited access to DNA (chromatin compaction); the exact nature and requirements for local interaction are currently poorly understood (Filion et al., 2010). Although DNA-sequences and DNA-binding proteins play a key role in the establishment of silent chromatin, local chromatin landscape (i.e. histone modifications and chromatin associated proteins) and establishment of higher-order states must involve control by an additional level of chromatin building mechanisms; this is illustrated by the observation that chimeric HP1-PcG proteins misdirect endogenous HP1 as well as PcG proteins to PcG-binding sites and heterochromatin respectively (Platero et al., 1995).

\section{Dynamic oxygen-dependent chromatin modulation - linking epigenetics to oxygen}

A limited number of studies has experimentally addressed dynamic changes of chromatin state, modulation of Polycomb-associated epigenetic marking was mainly studied in the context of development (Azuara et al., 2006a; Barski et al., 2007; Bernstein et al., 2006a; Mikkelsen et al., 2007), but not in response to acute changes in the micro-environment. One recent study reported on globally increased H3K27me3 marking in response to hypoxia along with additional repressive marks including H3K9me2, H3K9/27me2 (Johnson et al., 2008). Surprisingly, opposing active marks 
were simultaneously increased (H3R3me2, H3K4me2, H3K4me3, H3K79me2) (Johnson et al., 2008; Zhou et al., 2010). In line with these findings, we herein also report an increase of global histone trimethylation at $\mathrm{H} 3 \mathrm{~K} 4$ and $\mathrm{H} 3 \mathrm{~K} 27$ in response to hypoxic stress (chapter 5, this thesis). As (repeated) hypoxia/reoxygenation imposed oxidative stress on cells which is likely to include DNA damage, the increased methylation may, in concordance with increased methylation in response to different stressors, point to a general phenomenon in response to stress (Fullgrabe et al., 2010). A global response could imply that global histone methylation in some way contributes to chromatin protection; possibly through recruitment of factors that limit DNA-templated activity and as such prevent uncontrolled gene expression and thus the occurrence of doublestrand breaks (DSBs). In this respect it will be of interest to examine whether increased histone methylation represents a more generalized cellular reaction to environmental stress (i.e. arsenite/selenite exposure or mitogenic stimulation). We recently found that MK2 and presumably MK3 are activated under conditions of hypoxic stress (data not shown). MK3-depletion enhanced cell proliferation under hypoxic conditions. As M/SAPKs are known to govern cellular responses to oxygen stress (Cowan and Storey, 2003), this finding opens a novel avenue into studies of molecular regulatory mechanisms. KAP1 has thus far not been studied in the context of hypoxia/ reoxygenation. Given its pivotal involvement in DNA damage control and transcriptional regulation, it is conceivable that this protein will also play an important role in oxygen stress responses. It is tempting to speculate about a role for KAP1 in chromatin condensation during hypoxia in analogy to with the KAP1/chromatin-recruitment we detected upon induction of cell stress and mitogenic stimulation (chapter 4, this thesis; see section "PcG-complexes and chromatin association"). Higher order chromatin responses have not been studied in the context of hypoxia and reoxygenation. It is conceivable that KAP1-recruitment under oxygen stress conditions correlates with gene body accumulation and contributes to global transcriptional shut down and limiting transcription-associated DNA damage. This could be tested by measuring oxygen-stress induced DNA damage in KAP1-depleted cells. A caveat in this context may be the deregulation of many SUMO/Ub-mediated processes by hypoxia. Alternatively, increased trimethylation could be part of a default defense mechanism that replaces oxygen-dependent processes. The oxygen dependent activity of histone demethylases (Selak et al., 2005; Smith et al., 2007) could play a crucial role herein (further discussed below). 


\section{Bivalent chromatin state - connecting oxygen and cancer stem-ness?}

As outlined in the introduction, TrxG and PRC complexes counteract each other in the context of transcription by installing opposing histone marks: H3K4me3 and H3K27me3. Both marks play an important role in determining gene expression and thus cell fate during embryogenesis. H3K27me3 as well as $\mathrm{H} 3 \mathrm{~K} 4 \mathrm{me} 3$ profiles change dramatically during differentiation (Barski et al., 2007; Mikkelsen et al., 2007). Remarkably, in embryonic stem (ES) cells bivalent marking (both H3K4me3 and H3K27me3) is observed at key developmental control genes (Azuara et al., 2006b; Barski et al., 2007). Epigenomic comparison between ES cells and committed and/or fully differentiated cell types revealed that bivalent marking is lost during differentiation and mostly single-marked or non-marked loci remain (Bernstein et al., 2006a; Lee et al., 2006; Mikkelsen et al., 2007; Roh et al., 2007). Interestingly, we observed that oxygen deprivation resulted in gain of bivalent marking, and that bivalently marked genes overlapped with those reported in ES cells (chapter 5, this thesis). An interesting question is whether bivalent genes fall into one of the colored chromatin subtypes (by analogy with Drosophila) described earlier (Filion et al., 2010), and if so, do bivalent genes classify as "red", "yellow" (both enriched for H3K4me2) or "blue" (H3K27me3-enriched) chromatin? It is possible that bivalent chromatin represent a separate epigenetic subclass (perhaps in part distinguished by parental marks and/or factors) Or is bivalency merely a reflection of a co-existing allelic or cellular epigenomes (either enriched for K4me3 or H3K27me3 at the assumed genes) as is the case in Xenopus embryos, where apparent bivalency arises from differential allelic expression (i.e. allelic marking) in different parts of the embryo (Akkers et al., 2009). Although bivalency in mammalian ES cells is generally considered real (Herz et al., 2009), in light of our observations, the possibility exists that in vitro culturing conditions may contribute to bivalent marking. Notwithstanding this possibility, it is likely that bivalently marked genes would group into a mammalian "colored chromatin" equivalent in differentiated cells. Confirmation of bivalency (H3K4 and HK27me3 on the same allele) can be obtained by sequential ChIP. Parallel in situ expression analysis should then also answer the question whether the observed "intermediate" transcriptional activity at bivalent loci reflects expression of a subpopulation of cells or whether H3K4me3 or H3K27me3-enrichment directly affects expression.

An alternative model explaining the low transcriptional activity associated with bivalent genes, suggests that the simultaneous presence of H3K4me3 at Polycomb target genes generates short ncRNAs (Kanhere et al.). The short ncRNAs form structures similar to Xist-RepA stem-loops (see section "PcG chromatin association") 
which interact with PRC2 through SUZ12 and causes in cis gene repression by stabilizing PRC2-chromatin association. The short ncRNAs ( $\sim 50-200$ nucleotides) are transcribed from the 5-prime (5') side of PcG-target genes (promoters, introns and exons) of genes that are otherwise repressed (transcription of short RNAs can occur in the absence of mRNA transcription). ES cell differentiation resulted in loss of short ncRNAs at de-repressed PcG target loci, probably resulting in the destabilization of $\mathrm{PRC2}$ /chromatin-binding. It is tempting to speculate that the initiation configuration of RPOL2 (responsible for short RNA production) corresponds to the previously identified stalled RPOL2 at bivalent genes (Ser-5 phosphorylated RPOL2) (Bernstein et al., 2006a). This could suggest that destabilization of PRC2 during differentiation is accompanied by a release of an elongation block through loss of PRC1-RING proteins and concomitantly, reduced H2AK119Ub, and a switch in RPOL2 towards the elongating form (Stock et al., 2007; Zhou et al., 2008).

As bivalency tends to be associated with a less differentiated chromatin state, our observation that oxygen deprivation induces bivalent marking in a tumor cell line may reflect induction of potential cancer stem cell-like epigenotypes. Nevertheless, it remains to be determined what the exact molecular nature and biological relevance of this more "primitive chromatin" is. It is known that stem cells reside in relative poorly oxygenated parts of the body (Covello et al., 2006; Mohyeldin et al., 2010; Stock et al., 2007; Welford and Giaccia, 2011), it is conceivable that hypoxia disrupts a bi-stability modus (Dodd et al., 2007), i.e. coupled epigenetic activity targeted at functionally opposing epigenetic marks, by inactivating histone demethylase activity. Several lines of evidence indicate that PcG-complexes functionally interact with H3K4me3demethylases and vice versa, H3K27me3-demethylases associate with TrxG-complexes (Cho et al., 2007; De Santa et al., 2007; Issaeva et al., 2007; Pasini et al., 2008). Similarly, PcG-complexes are often associated with collaborating epigenetic activity, like histone deacetylases (HDACs), whereas TrxG-complexes bind histone acetyltransferase (HAT) activity (Beisel and Paro, 2011; Simon and Kingston, 2009). Concerted action of various histone modifying enzymes (i.e. readers and writers) by presence within the same complex or simultaneous recruitment has also been reported in respect to other combinations of post-translational modifications, like the coupled H2AK119 ubiquitylation and H3K36me2 demethylation accommodated by the dRAF/BCOR PcGcomplex (Cao et al., 2005; Gearhart et al., 2006; Sanchez et al., 2007; Wang et al., 2004a) and the co-recruitment of PRC2 and LSD1 by the ncRNA HOTAIR (Tsai et al., 2010) respectively. The fact that KDM2 mutations enhance Polycomb-mutations and suppress TrxG-mutations further underscores the biological significance of this cooperativity. 
The biological role of demethylating enzymes may reach beyond histone demethylation: demethylating enzymes were recently reported to be involved in PRC2recruitment. Five independent groups showed that the Jumonji-family member JARID2 co-localizes with PRC2 in ES cells (Landeira et al., 2010; Li et al., 2010; Pasini et al., 2010; Peng et al., 2009; Shen et al., 2009). The AT-rich interactive domain (ARID) of JARID2 directly binds DNA and is essential for PRC2-recruitment (Pasini et al., 2010). However, loss of JARID2 only had a mild effect on H3K27me3-levels and did not increase expression of PcG-target genes (in fact it even further reduced their expression) (Landeira et al., 2010). JARID2 appears also involved in recruiting RPOL2pS5 (Ser5phosphorylated, i.e. "stalled"), suggesting that JARID2 may be involved in establishing a "poised promoter state" (i.e. bivalent chromatin) (Li et al., 2010). Loss of JARID results in concomitant loss of stalled RPOL2, which accounted for the reduced expression compared to cells proficient for JARID2 function. Furthermore, this could also explain the requirement of JARID2 in early embryonic development (Loh et al., 2007; Pasini et al., 2010; Takeuchi et al., 1995). The bi-stability and the recruitment theory could very well co-exist. If this is indeed the case, physical absence of demethylases (by knock down) is expected to have an additive effect in comparison to the mere inactivation of its catalytic activity (by product or chemical inhibition), in analogy to p38-stabilization by the presence of (even chemically inhibited or kinase-dead) MKs (see section "mitogenic stimulation and cell stress"; chapter 3, this thesis; (Ronkina et al., 2007)).

In summary, the presence of bivalent chromatin might reflect the presence of a predefined epigenetic state of a set of genes, which are programmed to respond to specific changes in the micro-environment (i.e."poised"). The introduction of cell fate transcription factors and oxygen could create a shift in the equilibrium at bivalently marked genes by increasing the incidence of one histone methyl mark (i.e. by increased recruitment of HKMT activity) and simultaneously reducing the competing methyl mark (i.e. oxygen triggers activation of HKDM activity), allowing for robust switching of epigenetic state. As such this bi-stability modus could reinforce lineage commitment and differentiation (i.e. balance PcG-mediated silencing and TrxG-mediated activation) in response to environmental cues. Local environmental signals (including soluble factors, cell-cell contacts, waste products, $\mathrm{pH}$ ) can provide additional regulatory cues that directly tap into epigenetic programming and cell fate choices. Although still incompletely understood at the chromatin level, global reorganization of Polycomb/chromatin-association may relate to combined removal of local repressive recruitment-marks, like H3K27me3, and physical removal of PRC1-complexes by concerted action of MAPK/SAPK/MKs, which are all involved in development. It is currently unclear what the trigger is that initiates the release of bivalency. Obvious 
candidate mechanism include the recruitment of cell fate transcription factors, destabilization of PRC/chromatin-association (though loss of short RNAs), active removal of Polycomb within the gene body by stress signaling and/or KAP1-release (thereby releasing elongation block), the switch of RPOL2 to the elongation modus, the (de)activation of HKDMs (by oxygen availability) or (synergistic) cooperation of multiple events (i.e. concept of multi-stability).

\section{Conclusions \& Future Perspectives}

Exciting advances in the field of epigenetics have demonstrated that chromatin states are subject to dynamic modulation and that cell fate programming is flexible and in fact reversible (Zhou and Melton, 2008). This is illustrated ad optimum by the successful cloning of animals by somatic nuclear cell transfer; the remarkable reprogramming of adult fibroblasts into induced pluripotent stem cells (iPS) by expression of only four transcription factors (OCT4, SOX2, KLF4 and MYC), and the induction of trans-differentiation of various somatic cell types by deletion or insertion of key transcription factors (Hochedlinger and Plath, 2009; Stadtfeld and Hochedlinger, 2011; Takahashi et al., 2007; Wu and Hochedlinger, 2011; Yamanaka, 2008; Young, 2011).

Polycomb Group (PcG) proteins and their functional nemesis, the Trithorax Group (TrxG) proteins were among the first chromatin modifiers discovered; they act as epigenetic regulators of hundreds of genes, including cell fate transcription factors (CFTFs), and as such play a crucial role in stem cell maintenance, lineage commitment, cell fate choices and body patterning during development and differentiation (Simon and Kingston, 2009). During development, chromatin states are initially established by transcription factors and are subsequently propagated by balanced regulation of PcG and TrxG-function, in conjuntion with ncRNAs and DNA-methylation. Recent scientific progress revealed that PCG- and TrxG-proteins are also important in tissue homeostasis and disease (i.e. repair, senescence, apoptosis, and genome integrity) (Mills, 2010). Abnormal expression and/or mutation of PcG-genes has been associated with onset and malignant progression of cancer. In contrast, TrxG are generally considered to act tumor suppressive. Tumors exhibiting deregulated PcG/TrxG-function are thought to harbor "stem cell-like" characteristics (Klauke and de Haan; Richly et al.; Schuettengruber et al.); the cancer stem cell represents a biological concept which is held responsible for resistance to anti-cancer therapy evasion and malignant tumor 
progression. Stem-ness, analogous to acquisition of other cancer hallmarks, may be achieved in various ways, including deregulation of epigenetic homeostatic (Clarke and Fuller, 2006; Hanahan and Weinberg, 2000; Vogelstein and Kinzler, 2004). The epigenetic origin of cancer (stem) cells holds the promise of development of selective cancer therapy using epigenetic drugs and/or manipulation of the microenvironment (Baylin and Jones, 2011; Esteller, 2005; Herranz and Esteller, 2006). Epigenetic drug development receives much attention and some drugs have been approved for clinical application. However, many issues remain to be solved to enable full exploitation of the clinical potency of these and future epigenetic drugs. Some drugs have shown treatment efficacy for specific cancers, yet their mode of action is often inherently unspecific and the exact mechanisms by which clinical responses are produced are unknown. Epigenetic drugs may be applied to sensitize cancers to other therapeutic strategies; in addition, some HDAC-inhibitors were shown to overcome resistance to more conventional drugs, however, the underlying mechanisms remain unclear. Hence, it is imperative to increase fundamental knowledge of chromatin-associated regulation and gain critical insight into chromatin abnormalities.

In the context of Polycomb-biology several relevant questions remain: PcG-associated chromatin subtypes (including bivalent chromatin) need to be functionally characterized and compared in normal and abnormal cells to improve our understanding of epigenetic plasticity; linking epigenome-wide analysis of chromatinmodulation to transcriptomic, proteomic and metabolomic analyses are imperative to determine the exact relevance of PTMs for PcG-function, their interplay with additional epigenetic regulatory mechanisms (ncRNAs, DNA-methylation), and to fully understand and exploit the therapeutic potential of the micro-environment. Likewise, the nature and regulation of resident/recruited epigenetic regulatory molecules and/or switches, like the ones identified in the studies presented herein: ERK/MK3/PcG, p38/KAP/PcG, $\mathrm{O}_{2} / \mathrm{JHDM} / \mathrm{PCG}$, need to be better characterized to ultimately allow control over homeostasis, survival and cell death through manipulation of the environment.

We here provide evidence that important aspects of Polycomb-mediated silencing can be dynamically modulated by changing the cellular environment. Elucidation of the epigenetic mechanisms underlying dynamic physiological adaptation is expected to significantly impact on our understanding of normal development, maintenance of homeostasis and diseases such as cancer and trans-generational heritability of Western type diseases. 


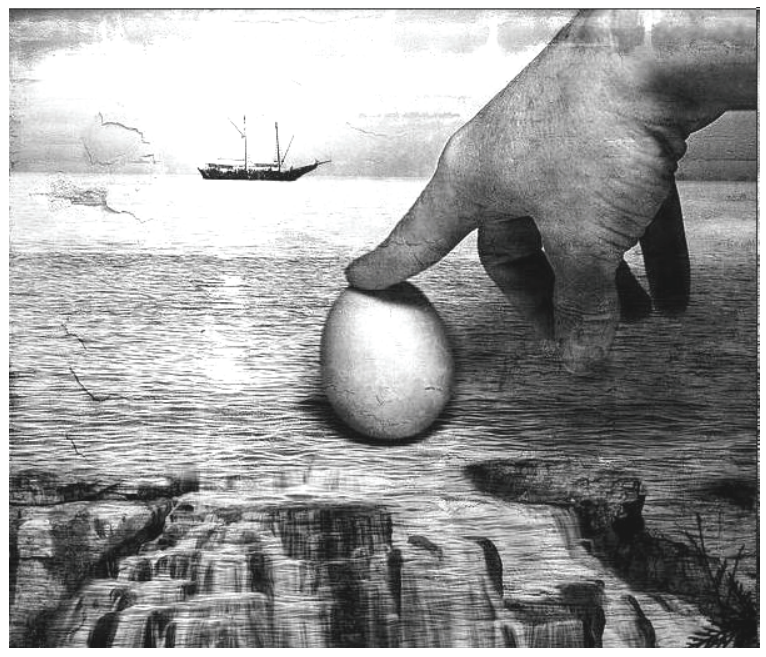




\section{REFERENCES}

Akkers, R.C., van Heeringen, S.J., Jacobi, U.G., Janssen-Megens, E.M., Francoijs, K.J., Stunnenberg, H.G., and Veenstra, G.J. (2009). A hierarchy of H3K4me3 and H3K27me3 acquisition in spatial gene regulation in Xenopus embryos. Dev Cell 17, 425-434.

Alchanati, I., Teicher, C., Cohen, G., Shemesh, V., Barr, H.M., Nakache, P., Ben-Avraham, D., Idelevich, A., Angel, I., Livnah, N., et al. (2009). The E3 ubiquitin-ligase Bmi1/Ring1A controls the proteasomal degradation of Top2alpha cleavage complex - a potentially new drug target. PLoS One 4, e8104.

Azuara, V., Perry, P., Sauer, S., Spivakov, M., Jorgensen, H.F., John, R.M., Gouti, M., Casanova, M., Warnes, G., Merkenschlager, M., and Fisher, A.G. (2006a). Chromatin signatures of pluripotent cell lines. Nat Cell Biol 8, 532-538.

Azuara, V., Perry, P., Sauer, S., Spivakov, M., Jorgensen, H.F., John, R.M., Gouti, M., Casanova, M., Warnes, G., Merkenschlager, M., and Fisher, A.G. (2006b). Chromatin signatures of pluripotent cell lines. Nat Cell Biol 8, 532-538.

Barski, A., Cuddapah, S., Cui, K., Roh, T.Y., Schones, D.E., Wang, Z., Wei, G., Chepelev, I., and Zhao, K. (2007). High-resolution profiling of histone methylations in the human genome. Cell 129, 823-837.

Baylin, S.B., and Jones, P.A. (2011). A decade of exploring the cancer epigenome â $€^{\prime \prime}$ biological and translational implications. Nat Rev Cancer 11, 726-734.

Beisel, C., and Paro, R. (2011). Silencing chromatin: comparing modes and mechanisms. Nat Rev Genet 12, 123-135.

Bernstein, B.E., Mikkelsen, T.S., Xie, X., Kamal, M., Huebert, D.J., Cuff, J., Fry, B., Meissner, A., Wernig, M., Plath, K., et al. (2006a). A bivalent chromatin structure marks key developmental genes in embryonic stem cells. Cell 125, 315-326.

Bernstein, E., Duncan, E.M., Masui, O., Gil, J., Heard, E., and Allis, C.D. (2006b). Mouse polycomb proteins bind differentially to methylated histone h3 and RNA and are enriched in facultative heterochromatin. Mol Cell Biol 26, 2560-2569.

Bloyer, S., Cavalli, G., Brock, H.W., and Dura, J.M. (2003). Identification and characterization of polyhomeotic PREs and TREs. Dev Biol 261, 426-442.

Bondarenko, V.A., Steele, L.M., Ujvari, A., Gaykalova, D.A., Kulaeva, O.I., Polikanov, Y.S., Luse, D.S., and Studitsky, V.M. (2006). Nucleosomes can form a polar barrier to transcript elongation by RNA polymerase II. Mol Cell 24, 469-479.

Breiling, A., Turner, B.M., Bianchi, M.E., and Orlando, V. (2001). General transcription factors bind promoters repressed by Polycomb group proteins. Nature 412, 651-655.

Brock, H.W., and Fisher, C.L. (2005). Maintenance of gene expression patterns. Dev Dyn 232, 633-655.

Brockdorff, N. (2011). Chromosome silencing mechanisms in X-chromosome inactivation: unknown unknowns. Development 138, 5057-5065.

Buchenau, P., Hodgson, J., Strutt, H., and Arndt-Jovin, D.J. (1998). The distribution of polycomb-group proteins during cell division and development in Drosophila embryos: impact on models for silencing. J Cell Biol 141, 469-481.

Cao, R., Tsukada, Y.I., and Zhang, Y. (2005). Role of Bmi-1 and Ring1A in H2A Ubiquitylation and Hox Gene Silencing. Mol Cell 20, 845-854.

Cao, R., Wang, L., Wang, H., Xia, L., Erdjument-Bromage, H., Tempst, P., Jones, R.S., and Zhang, Y. (2002). Role of Histone H3 Lysine 27 Methylation in Polycomb-Group Silencing. Science 26, 1039-1043. 
Chang, C.W., Chou, H.Y., Lin, Y.S., Huang, K.H., Chang, C.J., Hsu, T.C., and Lee, S.C. (2008). Phosphorylation at Ser473 regulates heterochromatin protein 1 binding and corepressor function of TIF1beta/KAP1. BMC Mol Biol 9, 61 .

Chen, E.S., Zhang, K., Nicolas, E., Cam, H.P., Zofall, M., and Grewal, S.I. (2008). Cell cycle control of centromeric repeat transcription and heterochromatin assembly. Nature 451, 734-737.

Chen, L., and Daley, G.Q. (2008). Molecular basis of pluripotency. Human Molecular Genetics 17, R23-R27.

Chen, X., Hiller, M., Sancak, Y., and Fuller, M.T. (2005). Tissue-specific TAFs counteract Polycomb to turn on terminal differentiation. Science 310, 869-872.

Chintapalli, V.R., Wang, J., and Dow, J.A. (2007). Using FlyAtlas to identify better Drosophila melanogaster models of human disease. Nat Genet 39, 715-720.

Cho, Y.W., Hong, T., Hong, S., Guo, H., Yu, H., Kim, D., Guszczynski, T., Dressler, G.R., Copeland, T.D., Kalkum, M., and Ge, K. (2007). PTIP associates with MLL3- and MLL4-containing histone H3 lysine 4 methyltransferase complex. J Biol Chem 282, 20395-20406.

Clarke, M.F., and Fuller, M. (2006). Stem Cells and Cancer: Two Faces of Eve. Cell 124, 1111-1115.

Covello, K.L., Kehler, J., Yu, H., Gordan, J.D., Arsham, A.M., Hu, C.J., Labosky, P.A., Simon, M.C., and Keith, B. (2006). HIF-2alpha regulates Oct-4: effects of hypoxia on stem cell function, embryonic development, and tumor growth. Genes Dev 20, 557-570.

Cowan, K.J., and Storey, K.B. (2003). Mitogen-activated protein kinases: new signaling pathways functioning in cellular responses to environmental stress. Journal of Experimental Biology 206, 1107-1115.

Czermin, B., Melfi, R., McCabe, D., Seitz, V., Imhof, A., and Pirrotta, V. (2002). Drosophila Enhancer of Zeste/ESC Complexes Have a Histone H3 Methyltransferase Activity that Marks Chromosomal Polycomb Sites. Cell 111, 185-196.

De Santa, F., Totaro, M.G., Prosperini, E., Notarbartolo, S., Testa, G., and Natoli, G. (2007). The histone H3 lysine-27 demethylase Jmjd3 links inflammation to inhibition of polycomb-mediated gene silencing. Cell 130, 1083-1094.

de Wit, E., Greil, F., and van Steensel, B. (2007). High-resolution mapping reveals links of HP1 with active and inactive chromatin components. PLoS Genet 3, e38.

Dinant, C., Houtsmuller, A.B., and Vermeulen, W. (2008). Chromatin structure and DNA damage repair. Epigenetics Chromatin 1, 9.

Dodd, I.B., Micheelsen, M.A., Sneppen, K., and Thon, G. (2007). Theoretical analysis of epigenetic cell memory by nucleosome modification. Cell 129, 813-822.

Dormann, H.L., Tseng, B.S., Allis, C.D., Funabiki, H., and Fischle, W. (2006). Dynamic regulation of effector protein binding to histone modifications: the biology of HP1 switching. Cell Cycle 5, 2842-2851.

Enderle, D., Beisel, C., Stadler, M.B., Gerstung, M., Athri, P., and Paro, R. (2010). Polycomb preferentially targets stalled promoters of coding and noncoding transcripts. Genome Res 21, 216-226.

Esteller, M. (2005). DNA methylation and cancer therapy: new developments and expectations. Curr Opin Oncol 17, 55-60.

Filion, G.J., van Bemmel, J.G., Braunschweig, U., Talhout, W., Kind, J., Ward, L.D., Brugman, W., de Castro, I.J., Kerkhoven, R.M., Bussemaker, H.J., and van Steensel, B. (2010). Systematic Protein Location Mapping Reveals Five Principal Chromatin Types in Drosophila Cells. Cell 143, 212-224.

Fischle, W., Tseng, B.S., Dormann, H.L., Ueberheide, B.M., Garcia, B.A., Shabanowitz, J., Hunt, D.F., Funabiki, H., and Allis, C.D. (2005). Regulation of HP1-chromatin binding by histone H3 methylation and phosphorylation. Nature 438, 1116-1122. 
Fischle, W., Wang, Y., Jacobs, S.A., Kim, Y., Allis, C.D., and Khorasanizadeh, S. (2003). Molecular basis for the discrimination of repressive methyl-lysine marks in histone $\mathrm{H} 3$ by Polycomb and HP1 chromodomains. Genes Dev 17, 1870-1881.

Francis, N.J., Follmer, N.E., Simon, M.D., Aghia, G., and Butler, J.D. (2009). Polycomb proteins remain bound to chromatin and DNA during DNA replication in vitro. Cell 137, 110-122.

Francis, N.J., Kingston, R.E., and Woodcock, C.L. (2004). Chromatin compaction by a polycomb group protein complex. Science 306, 1574-1577.

Fullgrabe, J., Hajji, N., and Joseph, B. (2010). Cracking the death code: apoptosis-related histone modifications. Cell Death Differ 17, 1238-1243.

Gearhart, M.D., Corcoran, C.M., Wamstad, J.A., and Bardwell, V.J. (2006). Polycomb group and SCF ubiquitin ligases are found in a novel BCOR complex that is recruited to BCL6 targets. Mol Cell Biol 26, 6880-6889.

Gehani, S.S., Agrawal-Singh, S., Dietrich, N., Christophersen, N.S., Helin, K., and Hansen, K. (2010). Polycomb group protein displacement and gene activation through MSK-dependent H3K27me3S28 phosphorylation. Mol Cell 39, 886-900.

Ginjala, V., Nacerddine, K., Kulkarni, A., Oza, J., Hill, S.J., Yao, M., Citterio, E., van Lohuizen, M., and Ganesan, S. (2011). BMI1 is recruited to DNA breaks and contributes to DNA damage-induced H2A ubiquitination and repair. Mol Cell Biol 31, 1972-1982.

Goodarzi, A.A., Kurka, T., and Jeggo, P.A. (2011). KAP-1 phosphorylation regulates CHD3 nucleosome remodeling during the DNA double-strand break response. Nat Struct Mol Biol 18, 831-839.

Goodarzi, A.A., Noon, A.T., Deckbar, D., Ziv, Y., Shiloh, Y., Löbrich, M., and Jeggo, P.A. (2008). ATM Signaling Facilitates Repair of DNA Double-Strand Breaks Associated with Heterochromatin. Molecular Cell 31, $167-177$.

Groner, A.C., Meylan, S., Ciuffi, A., Zangger, N., Ambrosini, G., Denervaud, N., Bucher, P., and Trono, D. KRABzinc finger proteins and KAP1 can mediate long-range transcriptional repression through heterochromatin spreading. PLoS Genet 6, e1000869.

Hanahan, D., and Weinberg, R.A. (2000). The hallmarks of cancer. Cell 100, 57-70.

Hansen, K.H., Bracken, A.P., Pasini, D., Dietrich, N., Gehani, S.S., Monrad, A., Rappsilber, J., Lerdrup, M., and Helin, K. (2007). A model for transmission of the H3K27me3 epigenetic mark. Nat Cell Biol advanced online publication.

Hatano, A., Matsumoto, M., Higashinakagawa, T., and Nakayama, K.I. (2010). Phosphorylation of the chromodomain changes the binding specificity of $\mathrm{Cbx} 2$ for methylated histone $\mathrm{H} 3$. Biochem Biophys Res Commun 397, 93-99.

Herranz, M., and Esteller, M. (2006). New therapeutic targets in cancer: the epigenetic connection. Clin Transl Oncol 8, 242-249.

Herz, H.-M., Nakanishi, S., and Shilatifard, A. (2009). The Curious Case of Bivalent Marks. Developmental Cell 17, 301-303.

Hirota, T., Lipp, J.J., Toh, B.H., and Peters, J.M. (2005). Histone H3 serine 10 phosphorylation by Aurora B causes HP1 dissociation from heterochromatin. Nature 438, 1176-1180.

Hochedlinger, K., and Plath, K. (2009). Epigenetic reprogramming and induced pluripotency. Development $136,509-523$.

Hodgson, J.W., Argiropoulos, B., and Brock, H.W. (2001). Site-specific recognition of a 70-base-pair element containing $d(G A)(n)$ repeats mediates bithoraxoid polycomb group response element-dependent silencing. Mol Cell Biol 21, 4528-4543.

Ismail, I.H., Andrin, C., McDonald, D., and Hendzel, M.J. (2010). BMI1-mediated histone ubiquitylation promotes DNA double-strand break repair. J Cell Biol 191, 45-60. 
Issaeva, I., Zonis, Y., Rozovskaia, T., Orlovsky, K., Croce, C.M., Nakamura, T., Mazo, A., Eisenbach, L., and Canaani, E. (2007). Knockdown of ALR (MLL2) reveals ALR target genes and leads to alterations in cell adhesion and growth. Mol Cell Biol 27, 1889-1903.

Ivanov, A.V., Peng, H., Yurchenko, V., Yap, K.L., Negorev, D.G., Schultz, D.C., Psulkowski, E., Fredericks, W.J., White, D.E., Maul, G.G., et al. (2007). PHD Domain-Mediated E3 Ligase Activity Directs Intramolecular Sumoylation of an Adjacent Bromodomain Required for Gene Silencing. Mol Cell 28, 823-837.

Johnson, A.B., Denko, N., and Barton, M.C. (2008). Hypoxia induces a novel signature of chromatin modifications and global repression of transcription. Mutat Res.

Kahn, T.G., Schwartz, Y.B., Dellino, G.I., and Pirrotta, V. (2006). Polycomb complexes and the propagation of the methylation mark at the Drosophila ubx gene. J Biol Chem 281, 29064-29075.

Kanhere, A., Viiri, K., Araujo, C.C., Rasaiyaah, J., Bouwman, R.D., Whyte, W.A., Pereira, C.F., Brookes, E., Walker, K., Bell, G.W., et al. (2010). Short RNAs are transcribed from repressed polycomb target genes and interact with polycomb repressive complex-2. Mol Cell 38, 675-688.

Klauke, K., and de Haan, G. (2011). Polycomb group proteins in hematopoietic stem cell aging and malignancies. International Journal of Hematology 94, 11-23.

Kloc, A., Zaratiegui, M., Nora, E., and Martienssen, R. (2008). RNA interference guides histone modification during the $\mathrm{S}$ phase of chromosomal replication. Curr Biol 18, 490-495.

Klymenko, T., Papp, B., Fischle, W., Kocher, T., Schelder, M., Fritsch, C., Wild, B., Wilm, M., and Muller, J. (2006). A Polycomb group protein complex with sequence-specific DNA-binding and selective methyl-lysinebinding activities. Genes Dev 20, 1110-1122.

Ku, M., Koche, R.P., Rheinbay, E., Mendenhall, E.M., Endoh, M., Mikkelsen, T.S., Presser, A., Nusbaum, C., Xie, X., Chi, A.S., et al. (2008). Genomewide analysis of PRC1 and PRC2 occupancy identifies two classes of bivalent domains. PLoS Genet 4, e1000242.

Lachner, M., O'Carroll, D., Rea, S., Mechtler, K., and Jenuwein, T. (2001). Methylation of histone H3 lysine 9 creates a binding site for HP1 proteins. Nature 410, 116-120.

Lagarou, A., Mohd-Sarip, A., Moshkin, Y.M., Chalkley, G.E., Bezstarosti, K., Demmers, J.A., and Verrijzer, C.P. (2008). dKDM2 couples histone H2A ubiquitylation to histone H3 demethylation during Polycomb group silencing. Genes Dev 22, 2799-2810.

Landeira, D., Sauer, S., Poot, R., Dvorkina, M., Mazzarella, L., Jorgensen, H.F., Pereira, C.F., Leleu, M., Piccolo, F.M., Spivakov, M., et al. (2010). Jarid2 is a PRC2 component in embryonic stem cells required for multilineage differentiation and recruitment of PRC1 and RNA Polymerase II to developmental regulators. Nat Cell Biol 12, 618-624.

Lau, P.N., and Cheung, P. (2011). Histone code pathway involving H3 S28 phosphorylation and K27 acetylation activates transcription and antagonizes polycomb silencing. Proc Natl Acad Sci U S A 108, 2801-2806.

Lee, T.I., Jenner, R.G., Boyer, L.A., Guenther, M.G., Levine, S.S., Kumar, R.M., Chevalier, B., Johnstone, S.E., Cole, M.F., Isono, K., et al. (2006). Control of developmental regulators by polycomb in human embryonic stem cells. Cell 125, 301-313.

Lee, Y.-K., Thomas, S.N., Yang, A.J., and Ann, D.K. (2007). Doxorubicin Down-regulates Kruppel-associated Box Domain-associated Protein 1 Sumoylation That Relieves Its Transcription Repression on p21WAF1/CIP1 in Breast Cancer MCF-7 Cells. J. Biol. Chem. 282, 1595-1606.

Li, G., Margueron, R., Ku, M., Chambon, P., Bernstein, B.E., and Reinberg, D. (2010). Jarid2 and PRC2, partners in regulating gene expression. Genes Dev 24, 368-380.

Loh, Y.-H., Zhang, W., Chen, X., George, J., and Ng, H.-H. (2007). Jmjd1a and Jmjd2c histone H3 Lys 9 demethylases regulate self-renewal in embryonic stem cells. Genes Dev. 21, 2545-2557. 
Ludwig, S., Engel, K., Hoffmeyer, A., Sithanandam, G., Neufeld, B., Palm, D., Gaestel, M., and Rapp, U. (1996). $3 \mathrm{pK}$, a novel mitogen-activated protein (MAP) kinase-activated protein kinase, is targeted by three MAP kinase pathways. Mol. Cell. Biol. 16, 6687-6697.

Margueron, R., Justin, N., Ohno, K., Sharpe, M.L., Son, J., Drury, W.J., 3rd, Voigt, P., Martin, S.R., Taylor, W.R., De Marco, V., et al. (2009). Role of the polycomb protein EED in the propagation of repressive histone marks. Nature 461, 762-767.

Margueron, R., Li, G., Sarma, K., Blais, A., Zavadil, J., Woodcock, C.L., Dynlacht, B.D., and Reinberg, D. (2008). Ezh1 and Ezh2 maintain repressive chromatin through different mechanisms. Mol Cell 32, 503-518.

Mascle, X.H., Germain-Desprez, D., Huynh, P., Estephan, P., and Aubry, M. (2007). Sumoylation of the transcriptional intermediary factor 1beta (TIF1beta), the Co-repressor of the KRAB Multifinger proteins, is required for its transcriptional activity and is modulated by the KRAB domain. J Biol Chem 282, 10190-10202.

Mateos-Langerak, J., and Cavalli, G. (2008). Polycomb group proteins and long-range gene regulation. Adv Genet 61, 45-66.

Mikkelsen, T.S., Ku, M., Jaffe, D.B., Issac, B., Lieberman, E., Giannoukos, G., Alvarez, P., Brockman, W., Kim, T.K., Koche, R.P., et al. (2007). Genome-wide maps of chromatin state in pluripotent and lineage-committed cells. Nature $448,553-560$.

Mills, A.A. (2010). Throwing the cancer switch: reciprocal roles of polycomb and trithorax proteins. Nat Rev Cancer 10, 669-682.

Min, J., Zhang, Y., and Xu, R.M. (2003). Structural basis for specific binding of Polycomb chromodomain to histone $\mathrm{H} 3$ methylated at Lys 27. Genes Dev 17, 1823-1828.

Mohammad, F., Mondal, T., Guseva, N., Pandey, G.K., and Kanduri, C. (2010). Kcnq1ot1 noncoding RNA mediates transcriptional gene silencing by interacting with Dnmt1. Development 137, 2493-2499.

Mohyeldin, A., Garzon-Muvdi, T.s., and Quinones-Hinojosa, A. (2010). Oxygen in Stem Cell Biology: A Critical Component of the Stem Cell Niche. Cell Stem Cell 7, 150-161.

Muller, J., Hart, C.M., Francis, N.J., Vargas, M.L., Sengupta, A., Wild, B., Miller, E.L., O'Connor, M.B., Kingston, R.E., and Simon, J.A. (2002). Histone Methyltransferase Activity of a Drosophila Polycomb Group Repressor Complex. Cell 111, 197-208.

Muller, J., and Kassis, J.A. (2006). Polycomb response elements and targeting of Polycomb group proteins in Drosophila. Curr Opin Genet Dev 16, 476-484.

Niessen, H.E., Demmers, J.A., and Voncken, J.W. (2009). Talking to chromatin: post-translational modulation of polycomb group function. Epigenetics Chromatin 2, 10.

O'Geen, H., Squazzo, S.L., Iyengar, S., Blahnik, K., Rinn, J.L., Chang, H.Y., Green, R., and Farnham, P.J. (2007). Genome-wide analysis of KAP1 binding suggests autoregulation of KRAB-ZNFs. PLoS Genet 3, e89.

Oktaba, K., Gutierrez, L., Gagneur, J., Girardot, C., Sengupta, A.K., Furlong, E.E., and Muller, J. (2008). Dynamic regulation by polycomb group protein complexes controls pattern formation and the cell cycle in Drosophila. Dev Cell 15, 877-889.

Pandey, R.R., Mondal, T., Mohammad, F., Enroth, S., Redrup, L., Komorowski, J., Nagano, T., Mancini-Dinardo, D., and Kanduri, C. (2008). Kcnq1ot1 antisense noncoding RNA mediates lineage-specific transcriptional silencing through chromatin-level regulation. Mol Cell 32, 232-246.

Pasini, D., Cloos, P.A., Walfridsson, J., Olsson, L., Bukowski, J.P., Johansen, J.V., Bak, M., Tommerup, N., Rappsilber, J., and Helin, K. (2010). JARID2 regulates binding of the Polycomb repressive complex 2 to target genes in ES cells. Nature 464, 306-310.

Pasini, D., Hansen, K.H., Christensen, J., Agger, K., Cloos, P.A., and Helin, K. (2008). Coordinated regulation of transcriptional repression by the RBP2 H3K4 demethylase and Polycomb-Repressive Complex 2. Genes Dev 22, 1345-1355. 
Patterson, K.I., Brummer, T., O'Brien, P.M., and Daly, R.J. (2009). Dual-specificity phosphatases: critical regulators with diverse cellular targets. Biochem J 418, 475-489.

Peng, J.C., Valouev, A., Swigut, T., Zhang, J., Zhao, Y., Sidow, A., and Wysocka, J. (2009). Jarid2/Jumonji coordinates control of PRC2 enzymatic activity and target gene occupancy in pluripotent cells. Cell 139, 12901302.

Platero, J.S., Hartnett, T., and Eissenberg, J.C. (1995). Functional analysis of the chromo domain of HP1. EMBO J 14, 3977-3986.

Richly, H., Aloia, L., and Di Croce, L. (2011). Roles of the Polycomb group proteins in stem cells and cancer. Cell Death and Dis 2, e204.

Ringrose, L., and Paro, R. (2007). Polycomb/Trithorax response elements and epigenetic memory of cell identity. Development 134, 223-232.

Rinn, J.L., Kertesz, M., Wang, J.K., Squazzo, S.L., Xu, X., Brugmann, S.A., Goodnough, L.H., Helms, J.A., Farnham, P.J., Segal, E., and Chang, H.Y. (2007). Functional demarcation of active and silent chromatin domains in human HOX loci by noncoding RNAs. Cell 129, 1311-1323.

Roh, T.Y., Wei, G., Farrell, C.M., and Zhao, K. (2007). Genome-wide prediction of conserved and nonconserved enhancers by histone acetylation patterns. Genome Res 17, 74-81.

Ronkina, N., Kotlyarov, A., Dittrich-Breiholz, O., Kracht, M., Hitti, E., Milarski, K., Askew, R., Marusic, S., Lin, L.L., Gaestel, M., and Telliez, J.B. (2007). The mitogen-activated protein kinase (MAPK)-activated protein kinases MK2 and MK3 cooperate in stimulation of tumor necrosis factor biosynthesis and stabilization of p38 MAPK. Mol Cell Biol 27, 170-181.

Saha, R.N., Wissink, E.M., Bailey, E.R., Zhao, M., Fargo, D.C., Hwang, J.Y., Daigle, K.R., Fenn, J.D., Adelman, K., and Dudek, S.M. (2011). Rapid activity-induced transcription of Arc and other IEGs relies on poised RNA polymerase II. Nat Neurosci $14,848-856$.

Sanchez, C., Sanchez, I., Demmers, J.A., Rodriguez, P., Strouboulis, J., and Vidal, M. (2007). Proteomic analysis of Ring1B/Rnf2 interactors identifies a novel complex with the Fbxl10/ Jmjd1B histone demethylase and the BcoR corepressor. Mol Cell Proteomics.

Scheuermann, J.C., de Ayala Alonso, A.G., Oktaba, K., Ly-Hartig, N., McGinty, R.K., Fraterman, S., Wilm, M., Muir, T.W., and Muller, J. (2010). Histone H2A deubiquitinase activity of the Polycomb repressive complex PRDUB. Nature $465,243-247$.

Schotta, G., Ebert, A., Krauss, V., Fischer, A., Hoffmann, J., Rea, S., Jenuwein, T., Dorn, R., and Reuter, G. (2002). Central role of Drosophila SU(VAR)3-9 in histone H3-K9 methylation and heterochromatic gene silencing. Embo J 21, 1121-1131.

Schuettengruber, B., Martinez, A.-M., lovino, N., and Cavalli, G. (2011). Trithorax group proteins: switching genes on and keeping them active. Nat Rev Mol Cell Biol 12, 799-814.

Schultz, D.C., Friedman, J.R., and Rauscher, F.J., 3rd (2001). Targeting histone deacetylase complexes via KRAB-zinc finger proteins: the PHD and bromodomains of KAP-1 form a cooperative unit that recruits a novel isoform of the Mi-2alpha subunit of NuRD. Genes Dev 15, 428-443.

Schwartz, Y.B., and Pirrotta, V. (2007). Polycomb silencing mechanisms and the management of genomic programmes. Nat Rev Genet 8, 9-22.

Selak, M.A., Armour, S.M., MacKenzie, E.D., Boulahbel, H., Watson, D.G., Mansfield, K.D., Pan, Y., Simon, M.C., Thompson, C.B., and Gottlieb, E. (2005). Succinate links TCA cycle dysfunction to oncogenesis by inhibiting HIF-alpha prolyl hydroxylase. Cancer Cell 7, 77-85.

Sewalt, R.G., Lachner, M., Vargas, M., Hamer, K.M., den Blaauwen, J.L., Hendrix, T., Melcher, M., Schweizer, D., Jenuwein, T., and Otte, A.P. (2002). Selective interactions between vertebrate polycomb homologs and the SUV39H1 histone lysine methyltransferase suggest that histone H3-K9 methylation contributes to chromosomal targeting of Polycomb group proteins. Mol Cell Biol 22, 5539-5553. 
Shao, Z., Raible, F., Mollaaghababa, R., Guyon, J.R., Wu, C.T., Bender, W., and Kingston, R.E. (1999). Stabilization of chromatin structure by PRC1, a Polycomb complex. Cell 98, 37-46.

Shen, X., Kim, W., Fujiwara, Y., Simon, M.D., Liu, Y., Mysliwiec, M.R., Yuan, G.C., Lee, Y., and Orkin, S.H. (2009). Jumonji modulates polycomb activity and self-renewal versus differentiation of stem cells. Cell 139, 13031314.

Shen, X., Liu, Y., Hsu, Y.J., Fujiwara, Y., Kim, J., Mao, X., Yuan, G.C., and Orkin, S.H. (2008). EZH1 mediates methylation on histone $\mathrm{H} 3$ lysine 27 and complements $\mathrm{EZH} 2$ in maintaining stem cell identity and executing pluripotency. Mol Cell 32, 491-502.

Simon, J.A., and Kingston, R.E. (2009). Mechanisms of Polycomb gene silencing: knowns and unknowns. Nat Rev Mol Cell Biol 10, 697-708.

Sing, A., Pannell, D., Karaiskakis, A., Sturgeon, K., Djabali, M., Ellis, J., Lipshitz, H.D., and Cordes, S.P. (2009). A vertebrate Polycomb response element governs segmentation of the posterior hindbrain. Cell 138, 885-897.

Smith, E., Lin, C., and Shilatifard, A. (2011). The super elongation complex (SEC) and MLL in development and disease. Genes Dev 25, 661-672.

Smith, E.H., Janknecht, R., and Maher, L.J., 3rd (2007). Succinate inhibition of alpha-ketoglutarate-dependent enzymes in a yeast model of paraganglioma. Hum Mol Genet 16, 3136-3148.

Stadtfeld, M., and Hochedlinger, K. (2011). Induced pluripotency: history, mechanisms, and applications. Genes Dev 24, 2239-2263.

Stock, J.K., Giadrossi, S., Casanova, M., Brookes, E., Vidal, M., Koseki, H., Brockdorff, N., Fisher, A.G., and Pombo, A. (2007). Ring1-mediated ubiquitination of H2A restrains poised RNA polymerase II at bivalent genes in mouse ES cells. Nat Cell Biol 9, 1428-1435.

Takahashi, K., Tanabe, K., Ohnuki, M., Narita, M., Ichisaka, T., Tomoda, K., and Yamanaka, S. (2007). Induction of pluripotent stem cells from adult human fibroblasts by defined factors. Cell 131, 861-872.

Takeuchi, T., Yamazaki, Y., Katoh-Fukui, Y., Tsuchiya, R., Kondo, S., Motoyama, J., and Higashinakagawa, T. (1995). Gene trap capture of a novel mouse gene, jumonji, required for neural tube formation. Genes Dev 9, 1211-1222.

Tirosh, I., and Barkai, N. (2008). Two strategies for gene regulation by promoter nucleosomes. Genome Res 18, 1084-1091.

Towbin, B.D., Meister, P., and Gasser, S.M. (2009). The nuclear envelope--a scaffold for silencing? Curr Opin Genet Dev 19, 180-186.

Tsai, M.-C., Manor, O., Wan, Y., Mosammaparast, N., Wang, J.K., Lan, F., Shi, Y., Segal, E., and Chang, H.Y. (2010). Long Noncoding RNA as Modular Scaffold of Histone Modification Complexes. Science, science.1192002.

Valko, M., Rhodes, C.J., Moncol, J., Izakovic, M., and Mazur, M. (2006). Free radicals, metals and antioxidants in oxidative stress-induced cancer. Chemico-Biological Interactions 160, 1-40.

Vella, P., Barozzi, I., Cuomo, A., Bonaldi, T., and Pasini, D. (2011). Yin Yang 1 extends the Myc-related transcription factors network in embryonic stem cells. Nucleic Acids Research.

Vermeulen, M., Eberl, H.C., Matarese, F., Marks, H., Denissov, S., Butter, F., Lee, K.K., Olsen, J.V., Hyman, A.A., Stunnenberg, H.G., and Mann, M. (2010). Quantitative Interaction Proteomics and Genome-wide Profiling of Epigenetic Histone Marks and Their Readers. Cell 142, 967-980.

Vogel, M.J., Guelen, L., de Wit, E., Peric-Hupkes, D., Loden, M., Talhout, W., Feenstra, M., Abbas, B., Classen, A.K., and van Steensel, B. (2006). Human heterochromatin proteins form large domains containing KRAB-ZNF genes. Genome Res 16, 1493-1504.

Vogelstein, B., and Kinzler, K.W. (2004). Cancer genes and the pathways they control. Nat Med 10, 789-799. 
Voncken, J.W., Niessen, H., Neufeld, B., Rennefahrt, U., Dahlmans, V., Kubben, N., Holzer, B., Ludwig, S., and Rapp, U.R. (2005). MAPKAP kinase 3pK phosphorylates and regulates chromatin-association of the polycombgroup protein Bmi1. J Biol Chem 280, 5178-5187.

Voncken, J.W., Roelen, B.A., Roefs, M., de Vries, S., VerhoFen, E., Marino, S., Deschamps, J., and van Lohuizen, M. (2003). Rnf2 (Ring1b) deficiency causes gastrulation arrest and cell cycle inhibition. Proc Natl Acad Sci U S A $100,2468-2473$.

Voncken, J.W., Schweizer, D., Aagaard, L., Sattler, L., Jantsch, M.F., and van Lohuizen, M. (1999). Chromatinassociation of the Polycomb group protein BMI1 is cell cycle- regulated and correlates with its phosphorylation status. J Cell Sci 112, 4627-4639.

Wang, H., Wang, L., Erdjument-Bromage, H., Vidal, M., Tempst, P., Jones, R.S., and Zhang, Y. (2004a). Role of histone H2A ubiquitination in Polycomb silencing. Nature 431, 873-878.

Wang, L., Brown, J.L., Cao, R., Zhang, Y., Kassis, J.A., and Jones, R.S. (2004b). Hierarchical recruitment of polycomb group silencing complexes. Mol Cell 14, 637-646.

Welford, S.M., and Giaccia, A.J. (2011). Hypoxia and Senescence: The Impact of Oxygenation on Tumor Suppression. Molecular Cancer Research 9, 538-544.

White, D., Rafalska-Metcalf, I.U., Ivanov, A.V., Corsinotti, A., Peng, H., Lee, S.-C., Trono, D., Janicki, S.M., and Rauscher, F.J. (2011). The ATM substrate KAP1 controls DNA repair in heterochromatin: Regulation by HP1 proteins and Serine 473/824 phosphorylation. Molecular Cancer Research.

Woo, C.J., Kharchenko, P.V., Daheron, L., Park, P.J., and Kingston, R.E. (2010). A region of the human HOXD cluster that confers polycomb-group responsiveness. Cell 140, 99-110.

Wu, S.M., and Hochedlinger, K. (2011). Harnessing the potential of induced pluripotent stem cells for regenerative medicine. Nat Cell Biol 13, 497-505.

Wutz, A., Rasmussen, T.P., and Jaenisch, R. (2002). Chromosomal silencing and localization are mediated by different domains of Xist RNA. Nat Genet 30, 167-174.

Yamanaka, S. (2008). Pluripotency and nuclear reprogramming. Philos Trans R Soc Lond B Biol Sci 363, 20792087.

Yap, K.L., Li, S., Munoz-Cabello, A.M., Raguz, S., Zeng, L., Mujtaba, S., Gil, J., Walsh, M.J., and Zhou, M.M. (2010). Molecular interplay of the noncoding RNA ANRIL and methylated histone H3 lysine 27 by polycomb CBX7 in transcriptional silencing of INK4a. Mol Cell 38, 662-674.

Young, R.A. (2011). Control of the Embryonic Stem Cell State. Cell 144, 940-954.

Zaratiegui, M., Castel, S.E., Irvine, D.V., Kloc, A., Ren, J., Li, F., de Castro, E., Marin, L., Chang, A.Y., Goto, D., et al. (2011). RNAi promotes heterochromatic silencing through replication-coupled release of RNA Pol II. Nature 479, 135-138.

Zhao, J., Sun, B.K., Erwin, J.A., Song, J.J., and Lee, J.T. (2008). Polycomb proteins targeted by a short repeat RNA to the mouse $X$ chromosome. Science 322, 750-756.

Zhou, Q., and Melton, D.A. (2008). Extreme makeover: converting one cell into another. Cell Stem Cell 3, 382388 .

Zhou, W., Zhu, P., Wang, J., Pascual, G., Ohgi, K.A., Lozach, J., Glass, C.K., and Rosenfeld, M.G. (2008). Histone $\mathrm{H} 2 \mathrm{~A}$ monoubiquitination represses transcription by inhibiting RNA polymerase II transcriptional elongation. Mol Cell 29, 69-80.

Zhou, X., Sun, H., Chen, H., Zavadil, J., Kluz, T., Arita, A., and Costa, M. (2010). Hypoxia induces trimethylated H3 lysine 4 by inhibition of JARID1A demethylase. Cancer Res 70, 4214-4221. 
Chapter 7 
To control the utilization of genetic information, a cell makes use of specific epigenetic regulatory mechanisms. These mechanisms operate at the level of DNA (DNA methylation), RNA (non-coding RNAs) and protein (covalent histone modifications), and determine which genes are active, and thus which proteins are expressed by a cell. As the specific combination of proteins within a cell determines its phenotype (cellular identity and function), tight maintenance of gene activity is important. The Polycomb complexes (PRC1/2) studied in this thesis play an important role in this respect; they are part of a cellular transcriptional memory system that maintains the inactive status of specific target genes (gene silencing). PRCs act on histone proteins, the structural proteins around which DNA is wrapped, and that are part of the macro-molecular structure in which nuclear DNA is organized (chromatin). Chemical modification of histone proteins either favors gene silencing or gene activation, depending on the type and combination of modifications as well as their exact location (i.e. the exact amino acid involved). Polycomb repressive complex 2 (PRC2) trimethylates lysine 27 of histone 3 (H3K27me3) through the catalytic activity of the EZH subunit; this epigenetic mark is generally associated with transcriptional repression. PRC1 recognizes and binds $\mathrm{H} 3 \mathrm{~K} 27 \mathrm{me} 3$ via a binding pocket embedded in the $\mathrm{CBX}$ subunit, and in doing so, is thought to maintain gene repression. A brief historical overview on genetics and epigenetics and the basic biological concepts involved in epigenetic regulation, with a focus on PcG biology, are described in chapter 1.

Despite significant progress over the last decennium on our understanding of PRC-function, the establishment and maintenance of repressive chromatin and the heritability of PRC-mediated silencing, numerous questions remain. How is Polycomb/ chromatin-association dynamically regulated during development and differentiation? What is the effect of post-translational modification (PTM) of PRC proteins and/or histone proteins? Does PTM act on chromatin recruitment, complex composition or regulation of catalytic activity? How is PRC-mediated silencing and TrxG-mediated gene activation balanced? How does the micro-environment control PRC-function and how does this contribute to development, differentiation and disease? Can any of these mechanisms be exploited for therapeutic purposes?

A steadily growing number of studies suggest that PRC-function is subject to control by intrinsic and external factors, however very little is currently known on the dynamic regulation of Polycomb-function by the micro-environment. As PRC proteins play an important role in both normal and abnormal development (cancer), the aim of this thesis is to increase our understanding on environment-mediated control of PRC-function. This is done by identifying and probing the function of novel 
interaction partners, by interrogating regulatory mechanisms, and transcriptional regulation of PRC target-genes in response to changes in the micro-environment; kinase signaling (chapter 2), mitogenic stimulation (chapter 3 ), induction of cell stress (chapter 4), and oxygen deprivation (chapter 5 \& 6).

Our previous observation that MAPKAP kinase 3 (MK3) is a binding partner for PRC1 provides a strong indication for a role of the microenvironment in regulation of Polycomb-function. MK3 integrates signaling through mitogen- as well as stressactivated protein kinases (M/SAPK). However, the exact role of MK3 within the Polycomb complex remained elusive. In chapter 2 we study the effect of MK3 on Polycomb-function and cell phenotype by increasing or reducing the expression of MK3. We show that MK3 overexpression induces a senescence-like cell-cycle arrest, characterized by stabilization of P53, induction of its downstream target P2 $1^{\text {CIP1/WAF1, }}$ and increased expression of CDKN2A/INK4A, a locus known to be epigenetically controlled by Polycomb- Group repressive complexes. Loss of Polycomb also induces a senescence-like state, suggesting that MK3 overexpression and PcG knockdown act in similar pathways. In good agreement with this we find reduced expression of the catalytic PRC2 subunit EZH2 and concomitant loss of epigenetic H3K27me3-marking and $\mathrm{CBX} 8 /$ chromatin-occupation at the CDKN2A/INK4A-locus. Furthermore we show that co-expression of the Polycomb protein Bmi1 bypasses MK3-induced senescence and restores global H3K27me3 levels, supporting a role for MK3 in control of proliferation and replicative life-span through concerted action with Bmi1. Relevantly, MK3 overexpression also induces senescence in cancer cells through apparent reactivation of 'dormant' senescence checkpoints. In contrast to the proposed role for MK3 as a potential tumor suppressor, the tumor-suppressive effect of MK3 overexpression we observe, suggests that MK3-overexpression evokes a condition similar to oncogene-induced senescence (OIS). Paradoxically, loss of MK3 also limits proliferation of primary fibroblasts. We show that (see: chapter 3) MK3 controls ERK, p38 and JNK expression and/or phosphorylation and that gain or loss of MK3-function results in senescence due to signaling imbalance in primary cells.

To further elucidate the mechanistic link between MK3 and PRC we test the hypothesis that activation of MAPK signaling cascades leads to phosphorylation and chromatin-dissociation of PRC proteins, thereby relieving transcriptional repression and allowing for gene activation. We and others have already shown that protein phosphorylation accompanies PRC-protein/chromatin-dissociation during cell cycle progression. In addition, previous work established that BMI1 phosphorylation is, at least in part, dependent on MK3. In chapter 3 we study the effect of MK3 on 
PRC/chromatin-association in the context of mitogenic stimulation. Mitogenic stimulation results in loss of chromatin-binding of certain PRC proteins; chromatindissociation is dependent on the activation of MAPK (ERK) and SAPK (p38) signaling cascades, both of which are upstream of MK3. We find that phosphorylated ERK directly interacts with PRC1 members upon activation and that H3S28-phosphorylation (H3S28ph), the serine immediately adjacent to the H3K27me3 mark, is controlled via P38-signalling. By using the immediate early gene ATF3 as a model system, we confirm that expression of PRC target genes is induced through combined ERK and p38 signaling. We establish that H3S28ph in itself is not sufficient for ATF3 induction, and that concomitant loss of local H3K27me3 promoter-marking is not required for ATF3 activation. In addition, we provide evidence that transcriptional re-silencing of ATF3 is controlled by MK3 via a negative feed-back mechanism on pERK and/or pMEK, most likely involving induction of DUSPs. The existence of a negative feedback loop involving MK3 and ERK is confirmed by in vivo experiments, in which we establish a negative regulatory effect of $d M K$ in the context of ERK-induced ectopic wing vein formation.

PcG proteins themselves are also targeted for post-translational modification (PTM). Currently, near 120 different PcG-phosphorylation sites have been documented, most of which have not been functionally defined. In chapter 4 we hypothesize that signaling through phosphorylation cascades affects the PcG-interactome. To study a possible role of PTMs in PRC-interactions, we ask whether cellular stress induces novel interactions with the mammalian PRC protein BMI1. This recently led to the identification of the heterochromatin protein KAP1; we show that both proteins are recruited to the chromatin in response to environmental stress (i.e. by addition of arsenite or selenite) where BMI1 is targeted by KAP1 for proteolysis. We show that minimally the KAP1-RBCC domain is required for the interaction and that KAP1 degrades BMI1 in a RINGfinger-dependent manner. Drosophila crosses between mutant Pc (dCBX4) and bonus (dKAP) alleles substantiate the genetic interaction between these two novel binding partners. We discuss the repercussions of our findings in the context of DNA-damage control and transcriptional regulation.

The biochemical counterparts of PcG-proteins, the Trithorax-Group (TrxG) proteins, are involved in maintenance of active gene transcription, thereby functionally antagonizing PcG function. Like PcG, TrxG function is also essential for normal development and it has been implicated in stem cell biology and cancer. As recent advances in the field of epigenetics suggest that chromatin state, including the post-translational histone modifications involved, is more dynamic than originally 
anticipated, we study dynamic changes in histone trimethylation in the context of cellular adaptation to changed oxygenation (hypoxic stress, reoxygenation) in chapter 5. To robustly detect H3K27me3-enrichment, we design a novel enrichmentfinding protocol to reliably identify H3K27me3 enriched regions based on chromatinimmunoprecipitation/deep-sequencing data, including a normalization/summarization strategy, as described in chapter 6. Importantly, this allows us to obtain quantitative measurements of H3K27me3-marking between samples (time points). Applying this novel strategy, we reproduced a previously reported correlation between gene-body associated 'blanketing' H3K27me3-profile (PRC mark) and transcriptional repression, thereby validating our analytical approach. The prominent TSS-centered H3K4me3enrichment (TrxG mark) at expressed loci, and the marked low nucleosome abundance right over the TSS, are also consistent with earlier findings. We charted the acute effects of changes in cell oxygenation on epigenomic distribution of PRC- and TrxGmediated marking. Our studies support the notion that in response to oxygendeprivation, global histone trimethyl-marking at genic regions is dramatically altered through inhibition of JHDM-function and that, as a consequence, chromatin acquires characteristics of a more primitive epigenomic state, in which H3K4me3 and H3K27me3-marking coincide (bivalency). Our data suggest that oxygen-sensing by HKDMs represents a direct link between the micro-environment and epigenetic regulatory mechanisms. We establish that H3K27me3-occupation not necessarily correlates with transcriptional repression and that, in fact, $\mathrm{H} 3 \mathrm{~K} 4 \mathrm{me} 3$-enrichment is the most relevant determinant of transcription status. The relevance of our findings is discussed in the context of cancer maintenance and progression.

Taken together these studies firmly establish a role for extracellular cues and signaling pathways in regulation of PRC-function and they provide a solid basis for future research into specific processes and molecular mechanisms in epigenetic regulation of PRC-associated chromatin. 

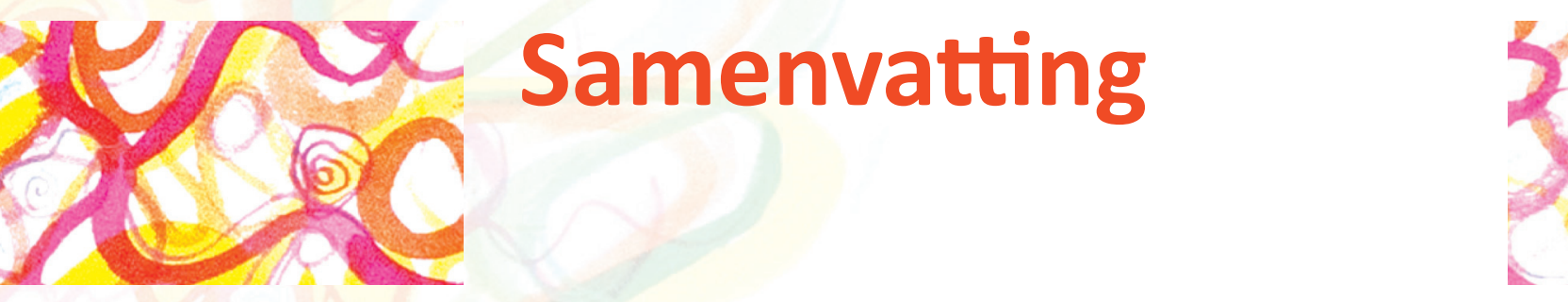
Om het gebruik van genetische informatie te reguleren, heeft een cel specifieke epigenetische regelmechanismen ter beschikking. Deze mechanismen opereren op het niveau van DNA (DNA methylering), RNA (niet-coderend RNA) en eiwitten (covalente histonmodificaties) en bepalen welke genen actief zijn en dus welke eiwitten tot expressie gebracht worden door een cel. Omdat de specifieke combinatie van eiwitten in een cel bepalend is voor het fenotype (cellulaire identiteit en functie), is het van belang dat genactiviteit strikt gecontroleerd wordt. De Polycomb complexen beschreven in dit proefschrift (PRC1/2) spelen een belangrijke rol in dit opzicht; ze maken deel uit van een cellulair transcriptioneel geheugensysteem dat de inactieve status van specifieke target genen in stand houdt (gene silencing). PRCs grijpen aan op het niveau van histoneiwitten, de structurele eiwitten waaromheen DNA verpakt wordt en die deel uit maken van de macromoleculaire structuur waarin nucleair DNA georganiseerd wordt (chromatine). Chemische modificatie van histoneiwitten bevordert het uitzetten dan wel het activeren van een gen, afhankelijk van het type en de combinatie van modificaties, evenals van de exacte locatie (i.e. het exacte aminozuur betrokken). Het Polycomb repressieve complex 2 (PRC2) trimethyleert lysine 27 van histon 3 (H3K27me3) door middel van de katalytische activiteit van het EZH eiwit; deze epigenetische markering wordt over het algemeen geassocieerd met transcriptionele repressie. PRC1 herkent en bindt H3K27me3 via een bindingsplaats op het CBX eiwit. Men denkt dat genrepressie op deze manier gehandhaafd wordt. Een kort historisch overzicht van genetica en epigenetica en de fundamentele biologische concepten die betrokken zijn bij epigenetische regulatie, met de focus op PcG biologie, worden beschreven in hoofdstuk 1.

Ondanks de aanzienlijke vooruitgang die in het laatste decennium geboekt is met betrekking tot het begrijpen van PRC-functie, het initiëren en behouden van repressief chromatine en de overerving van PRC-gemedieerde silencing, blijven veel vragen onbeantwoord. Hoe wordt Polycomb/chromatine-associatie tijdens ontwikkeling en differentiatie dynamisch gereguleerd? Wat is het effect van post-translationele modificatie (PTM) van PRC eiwitten en/of histoneiwitten? Hebben PTM invloed op chromatine recrutering, complex samenstelling of regulering van katalytische activiteit? Hoe worden PRC-gemedieerde gen silencing en TrxG-gemedieerde gen activering in evenwicht gehouden? Hoe wordt PRC-functie gereguleerd door de micro-omgeving en hoe draagt dit bij aan ontwikkeling, differentiatie en ziekte? Kan een van deze mechanismen worden benut voor therapeutische doeleinden?

Ondanks het feit dat een gestaag groeiend aantal studies suggereert dat PRCfunctie wordt gereguleerd door intrinsieke en externe factoren, is er momenteel nog weinig bekend over de dynamische regulering van Polycomb-functie door de 
micro-omgeving. Omdat PRC eiwitten een belangrijke rol spelen in zowel normale als abnormale ontwikkeling (kanker), is dit proefschrift gericht op het vergroten van onze kennis met betrekking tot omgevingsgemediteerde controle van PRC-functie. Dit is gerealiseerd door middel van het identificeren van nieuwe interactiepartners, door het bestuderen van regulerende mechanismen en transcriptionele regulering van PRC target-genen in reactie op veranderingen in de micro-omgeving; kinase signalering (hoofdstuk 2), mitogene stimulering (hoofdstuk 3 ), inductie van celstress (hoofdstuk 4) en zuurstof deprivatie (hoofdstuk 5 \& 6).

Onze eerdere bevinding dat MAPKAP kinase 3 (MK3) een bindingspartner van PRC1 is, geeft een sterke indicatie voor een rol van de micro-omgeving in de regulatie van Polycomb-functie. MK3 integreert signalering downstream van zowel mitogeen- als stressgeactiveerde eiwit kinases (M/SAPK). De exacte rol van MK3 in het Polycomb complex bleef echter onduidelijk. In hoofdstuk 2 is het effect van MK3 op Polycombfunctie en celfenotype bestudeerd door het verhogen of verlagen van de expressie van MK3. We laten zien dat MK3 overexpressie leidt tot een senescence-achtig cel cyclus arrest, dat gekenmerkt wordt door stabilisatie van P53, inductie van het downstream P2 $1^{\mathrm{CIP1} / \text { WAF1 }}$ eiwit en verhoogde expressie van CDKN2A/INK4A, een locus waarvan bekend is dat deze epigenetisch gecontroleerd wordt door Polycomb-Groep repressieve complexen. Verlies van Polycomb induceert eveneens een senescent fenotype, wat suggereert dat MK3 overexpressie en PcG knockdown in dezelfde signaleringsroute actief zijn. In overeenstemming hiermee vinden we verminderde expressie van het katalytische EZH2 eiwit en een gelijktijdig verlies van epigenetische H3K27me3-markering en CBX8/chromatine-occupatie op de CDKN2A/INK4A-locus. Verder tonen we aan dat co-expressie van het Polycomb-eiwit Bmi1 MK3-geïnduceerde senescence voorkomt en globale H3K27me3 niveaus herstelt, hetgeen een rol voor MK3 in de controle van proliferatie en replicatieve levensduur ondersteunt in combinatie met Bmi1. Relevant in dezen is dat MK3 overexpressie eveneens senescence induceert in kankercellen, wat gepaard gaat met reactivering van 'slapende' senescence-checkpoints. In tegenstelling tot de vermeende rol van MK3 als een tumorsuppressor, wijst het tumor suppressieve effect dat we zien erop dat MK3 overexpressie een conditie induceert die vergelijkbaar is met oncogen-geïnduceerde senescence (OIS). Ogenschijnlijk tegenstrijdig hiermee leidt verlies van MK3 ook tot verminderde proliferatie van primaire fibroblasten. We laten zien dat (zie: hoofdstuk 3 ) MK3 de expressie en/of fosforylering van ERK, p38 en JNK reguleert en dat verhoogde of verminderde MK3-functie resulteert in senescence als gevolg van onevenwichtige signalering in primaire fibroblasten. 
Om het mechanistisch verband tussen MK3 en PRC verder te verduidelijken, testen we de volgende hypothese: activering van MAPK signaleringscascades leidt tot fosforylering en chromatine dissociatie van PcG eiwitten, waardoor transcriptionele repressie wordt opgeheven en de mogelijkheid tot genexpressie ontstaat. Wij en anderen hebben al aangetoond dat eiwitfosforylering en PRC-eiwit/chromatinedissociatie samengaan tijdens celcyclus progressie. Bovendien is in eerder werk vastgesteld dat BMI1 fosforylering in ieder geval gedeeltelijk, afhankelijk is van MK3. In hoofdstuk 3 bestuderen we het effect van MK3 op PRC/chromatine-associatie in the context van mitogene stimulering. Mitogene stimulering resulteert in het verlies van chromatine-binding van bepaalde PRC eiwitten; chromatine-dissociatie is afhankelijk van de activering van MAPK (ERK) en SAPK (p38) signaleringscascades, die beide upstream van MK3 opereren. We zien dat gefosforyleerd ERK een directe interactie aangaat met het PRC1 eiwitcomplex en dat H3S28-fosforylering (H3S28ph), de serine direct naast de H3K27me3 markering, gecontroleerd wordt via p38-signalering. Door gebruik te maken van het "immediate early" gen ATF3 als modelsysteem, bevestigen we dat de expressie van PRC target genen geïnduceerd wordt door middel van gecombineerde ERK en p38 signalering. We stellen vast dat H3S28 fosforylering op zich onvoldoende is voor ATF3 inductie en dat gelijktijdig verlies van lokale H3K27me3 promoter-markering niet vereist is voor ATF3 activering. Daarnaast bewijzen we dat transcriptionele her-inactivatie van ATF3 gecontroleerd wordt door MK3 via een terugkoppelingsmechanisme op pERK en/of pMEK, waarschijnlijk via inductie van DUSPs. Het bestaan van een negatieve feedbackloop, waarbij MK3 en ERK betrokken zijn, is bevestigd door in vivo experimenten, waarin we een negatief regulerend effect van dMK vaststellen in de context van ERK-geïnduceerde ectopische adervorming in vleugels van de fruitvlieg Drosophila.

PcG eiwitten worden zelf ook post-translationeel gemodificeerd (PTM). Momenteel zijn er 120 verschillende PcG-fosforylatie-plaatsen beschreven, waarvan de meeste nog niet functioneel gedefinieerd zijn. In hoofdstuk 4 gaan we van de veronderstelling uit dat fosforylering van Polycomb eiwitten het PcG-interactoom beïnvloedt. Om de rol van PTMs in PRC-interacties te bestuderen, stellen we de vraag of cellulaire stress nieuwe interacties met het Polycomb eiwit BMI1 induceert. Dit leidde recent tot de identificatie van het heterochromatine eiwit KAP1; we laten zien dat beide eiwitten naar het chromatine gerecruteerd worden in reactie op omgevingsstress (bijv. na blootstelling aan arseen of seleen) waar BMI1 door KAP1 voor proteolytische afbraak gemarkeerd wordt. We tonen aan dat minimaal het KAP1-RBCC domein vereist is voor de interactie en dat afbraak van BMI1 door KAP1 RINGfinger afhankelijk is. Kruisingsexperimenten tussen fruitvliegjes mutant voor $\mathrm{Pc}(\mathrm{dCBX} 4)$ of bonus (dKAP) 
allelen onderbouwen de genetische interactie tussen deze twee nieuwe interactiepartners. De gevolgen van onze bevindingen worden besproken in het kader van DNA-schade controle en transcriptionele regulatie.

De biochemische tegenhangers van Polycomb Groep-(PcG) eiwitten, de TrithoraxGroep (TrxG) eiwitten, zijn betrokken bij het behoud van actieve gentranscriptie, waardoor ze functioneel tegengesteld werken aan PcG. Net als bij PcG, is TrxG-functie ook van essentieel belang voor een normale ontwikkeling en is het betrokken bij stamcelbiologie en kanker. Omdat recente ontwikkelingen op het gebied van epigenetica suggereren dat de chromatine status, waaronder de post-translationele histonmodificaties die hierbij betrokken zijn, dynamischer is dan aanvankelijk werd verondersteld, bestuderen we in hoofdstuk $\mathbf{5}$ dynamische veranderingen in histonmodificaties in de context van cellulaire aanpassingen aan gewijzigde oxygenatie (hypoxische stress, reoxygenering). $\mathrm{Om}$ een robuuste detectie van H3K27me3-verrijking mogelijk te maken, ontwerpen we een nieuw protocol, waaronder een normaliseringsstrategie, waarmee uit chromatine-immunoprecipitatie/ deep-sequencing experimenten verkregen H3K27me3-verrijking betrouwbaar bepaald kunnen worden; dit is beschreven in hoofdstuk 6. Belangrijk is dat dit ons in staat stelt om een kwantitatieve bepaling te doen van H3K27me3-markering tussen verschillende condities (tijdpunten). Gebruik makend van deze nieuwe strategie, waren we in staat de eerder gevonden correlatie tussen gen-brede H3K27me3-associatie (zogenaamde "blanketing profile" PRC markering) en transcriptionele repressie te bevestigen, hetgeen de validiteit van onze analytische aanpak onderstreept. De prominente TSS (transcriptionele start site)-gecentreerde H3K4me3-verrijking (TrxG mark) op actieve genen en de uitgesproken lage aanwezigheid van nucleosomen direct op de TSS zijn eveneens in overeenstemming met eerdere bevindingen. We brengen de acute effecten van veranderingen in cel oxygenering op epigenomische distributie van PRCen TrxG-gemedieerde markering in kaart. Onze studies ondersteunen het idee dat, in reactie op zuurstofonttrekking, globale histon trimethyl-markering rondom genen drastisch verandert door remming van JHDM-functie, als gevolg waarvan het chromatine een meer primitieve epigenomisch karakter verkrijgt, waarin H3K4me3 en H3K27me3 samen voorkomen (bivalentie). Onze data suggereren dat zuurstofafhankelijkheid van HKDMs een direct verband tussen de micro-omgeving en epigenetische regelmechanismen legt. We constateren dat H3K27me3-verrijking niet noodzakelijk correleert met transcriptionele repressie en dat in feite H3K4me3verrijking de meest bepalende factor van de transcriptionele status is. De relevantie van onze bevindingen wordt besproken in de context van overleving en kwaadaardige ontwikkeling van kanker. 
Samenvattend bevestigen deze studies een rol voor extracellulaire signalen en signaleringsroutes bij de regulatie van PRC-functie en leggen deze een solide basis voor toekomstig onderzoek naar specifieke processen en moleculaire mechanismen in de epigenetische regulatie van PRC-geassocieerd chromatine. 


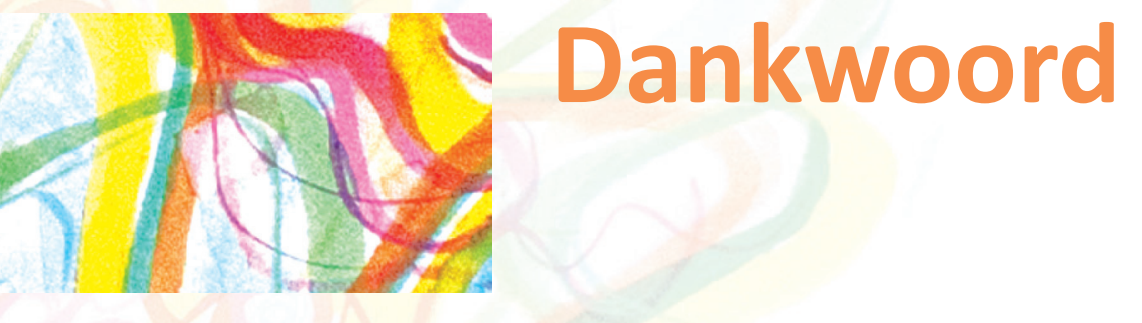


$\mathrm{Na}$ een reis van bijna 6 jaar (de pre-expeditie meegerekend) is de kust in zicht en ben ik toegekomen aan het schrijven van mijn laatste hoofdstuk, vooralsnog het hoofdstuk met de hoogste impact factor. Het was een mooie en interessante reis, met af en toe wat stormachtig weer, maar dankzij de bekwame bemanning heeft het schip de reis goed doorstaan. Een woord van dank is hier daarom op zijn plaats.

Noot vooraf: ik heb geprobeerd het kort te houden, dat dit niet gelukt is zal voor de meesten van jullie geen verassing zijn... (korte versie: dikgedrukt)

In de eerste plaats wil ik de kapitein van het schip bedanken, die de koers heeft uitgezet. Beste Willem, waar zal ik beginnen, of misschien een relevantere vraag; waar zal ik ophouden? We hebben elkaar leren kennen in het laatste jaar van mijn opleiding gezondheidswetenschappen. Voor de beoordeling van mijn eindstage op het NKI had ik een interne begeleider nodig, en wat bleek? Er werd ook onderzoek gedaan naar Polycomb in Maastricht. Niet veel later vroeg je of ik interesse had om een half jaartje naar Parijs te gaan en voor ik het wist hadden we een beurs aangevraagd en stond ik vanaf mijn balkon de Notre Dame te bewonderen. Het klikte goed tussen ons en na terugkomst werd ik voorgesteld aan Brad, de tweede kapitein. Ik werd aangesteld op een project waarbij ik epigenetische regulatie onder hypoxie zou bestuderen. In de jaren die volgden heb ik je leren kennen als een bevlogen wetenschapper met een brede interesse en een grote vakinhoudelijke kennis op (vrijwel alle) wetenschappelijk gebied(en). Je bent een (kei)harde werker en verwacht dit ook van je medewerkers. Daar staat tegenover dat je te allen tijde bereid bent te helpen, ook in de avond- en weekenduren (Monique, Sam en Robin: sorry dat ik de laatste tijd veel beslag op Willem heb gelegd). Je hebt me gestimuleerd om kritisch na te denken over mijn eigen onderzoek, maar ook om mee te denken en me te verdiepen in materie buiten mijn eigen onderzoeksveld. Dat heeft zeker een grote rol gespeeld in mijn wetenschappelijke vorming, maar ook in mijn persoonlijke ontwikkeling. Ik ben blij dat we de reis samen kunnen vervolgen!

Dear Brad, the Dutch saying "geen twee kapiteins op één schip" does not apply to my PhD study. You and Willem cooperate well; you are both inspiring and enthusiastic scientists with plenty of experimental ideas, and I feel privileged to work under your supervision! Most of the journey you followed me on the radar together with Marianne (e.g. videoconferences, phone calls, Skype), which involved some additional (technical) challenges and sometimes resulted in funny scenes $;$. I remember a dinner at Reube 
where you told me not to focus too much on other ships, but to sail my own course. Although it was difficult from time to time, it was certainly worthwhile. As you always say "the most difficult journeys are often the most rewarding ones". So far I only sailed the European seas, but now I'm ready to cross the Atlantic and join your fleet for a few months. I am looking forward to it!

Beste Joep, de eerste paar jaar sloeg je de koers van het schip gade vanuit het kraaiennest. Maar in de periode dat de eerste kapitein noodgedwongen veelal in zijn kajuit doorbracht, was je regelmatig op de brug te vinden om te zorgen dat alles op rolletjes bleef lopen. Naast het veilig stellen van de aankomst in de haven, heb je er ook zorg voor gedragen dat er voldoende tijd is om de lading van boord te halen. Hier ben ik je heel dankbaar voor! Beste Judith, Joep heeft het getroffen met jou! Ben benieuwd wat je van mijn kaft vindt...

Beste Jo, jij hebt er zorg voor gedragen dat het schip een kleurrijke entree maakt bij aankomst. Nu dekt de buitenkant van het schip de lading ervan; een mooiere balans had ik me niet kunnen wensen. Daarnaast was jouw aanwezigheid op het dek een bron van rust in de laatste weken op zee. Hartelijk dank daarvoor!

Beste leden van de leescommissie, Prof. Ramaekers, Prof. van Driel, Prof. de Haan, Prof. Kleinjans en Dr. Langen, bedankt dat jullie tijd wilden vrijmaken voor het beoordelen van mijn proefschrift!

Beste leden van de corona, Prof. Glatz, Prof. de Haan, Prof. Kleinjans, Dr. Langen, Dr. Logie, Prof. Peronnet, Dr. Sverdlov, Prof. Verfaillie, bedankt dat jullie zitting willen nemen tijdens mijn verdediging. Ik kijk ernaar uit om met jullie van gedachte te wisselen.

Lieve paranimfen, wat ben ik blij dat jullie 22 juni samen met mij op de boeg staan; de een aan stuurboord en de ander aan bakboord. Soms zijn juist de personen van wie de namen niet vooraan op een artikel staan, of überhaupt niet vermeld staan, degenen die onmisbaar zijn geweest tijdens het promotieonderzoek en die (soms achter de schermen) wel degelijk een heel belangrijke rol hebben gespeeld. Zonder jullie had ik de overtocht niet gered! Beste Vivian, met jou aan boord was er altijd voldoende wind in de zeilen en kon er op volle kracht gevaren worden. Jij bent voor mij een groot voorbeeld op het lab, en ook daarbuiten; je weet al je experimenten efficiënt te plannen, hebt mooie data (of zoals Willem zou zeggen "rete-strakke bandjes"), geeft waardevolle adviezen, en houdt daarbij voldoende tijd over voor Rick, Robin en Tim. 
Het is fijn om met jou een kamer te delen, samen experimenten op te zetten en uit te voeren en daarnaast hebben we ook veel steun aan elkaar als het even tegenzit, in de breedste zin van het woord. Gelukkig mag ik nog een jaartje blijven! Beste Nanda, als ik niet beter wist zou ik zeggen dat je mijn oudere zus bent en (jouw) Niels mijn schoonbroer. Je kent me als geen ander en weet me altijd op een ludieke en doeltreffende manier de spreekwoordelijke spiegel voor te houden. Dat maakt dat onze gesprekken vaak therapeutisch werken, gelukkig reken je mij geen consult! Hopelijk slagen we er in de toekomst ook in om af en toe een gaatje in onze overvolle agenda's te vinden om samen tot rust te komen onder het genot van een kopje koffie of een glaasje wijn, in de sauna, in de achtertuin of in de speeltuin.

Gelukkig ben je ook op zee nooit alleen en maak je deel uit van een grotere vloot: de WVO vloot in dit geval. Jullie hebben me allemaal enorm gemotiveerd (gehouden) en een prettige en leerzame werksfeer gecreëerd, bedankt daarvoor! Hanneke, jij hebt me vanaf de eerste dag op sleeptouw genomen en je over mij ontfermd en dat doe je nog steeds. Bedankt voor je steun tijdens mijn periodes van "krachttraining"! Ik hoop dat we deze zomer kunnen toasten op ons eerste gezamenlijke artikel, en dat de rest snel zal volgen. Want zoals je zelf altijd zegt: "Alles komt goed!" Frank, met jou in de buurt was er altijd wel wat te babbelen, of het nu over wetenschap ging of over voetbal, over Anunnaki's of Bonsai boompjes, overal kon je even enthousiast over vertellen. Jouw positieve instelling werkte dan ook vaak aanstekelijk. Ik hoop dat we ook in de toekomst nog veel leuke (wetenschappelijke) discussies zullen hebben! Claudia, je hebt me alle ins en outs van de kweek geleerd, waar we samen dan ook de nodige gezellige uurtjes hebben doorgebracht. Leuk dat we nog steeds contact hebben! Guus, je timmert aardig aan de weg met de ontwikkeling van je Nucleus pulposus en Annulus fibrosus cellijnen, de prijzen die je in de wacht gesleept hebt en al je congresbezoeken. En het skiën, ach dat krijg je ook nog wel onder de knie! Kan Anke goed skiën? (:) Nard, zoals je zelf al aangaf voelt het alsof we collega's waren, al is dit strict genomen nooit het geval geweest (imprinting?) Toronto en Washington liggen hemelsbreed ongeveer net zover uit elkaar als Munstergeleen en Wijlre, toch?! Daar moet iets op te vinden zijn. En onder 't mom van "beter goed gejat dan slecht bedacht" heb ik de promovenda versie van de stellingen gemaakt (mooi roze is niet lelijk!) (:). Céline, unfortunately we only worked together for one month, especially because we worked on a similar project. Raymond, jij had een sterke tweede plaats als het op koffie drinken aankwam, ik hoop dat je het naar je zin hebt bij Intervet. Juliette, the Drosophila expert in our lab, last time I saw you we didn't have a chance to catch up, see you again either in Maastricht or in Paris. 
Verder wil ik alle studenten die in de loop der jaren bij ons over de vloer gekomen zijn bedanken voor hun inzet op het lab, in het bijzonder Yvette, Caroline en Jolien. Yvette, jij was eigenlijk mijn eerste student, maar aangezien je gelijk met mij begon heeft Frank de begeleiding grotendeels op zich genomen. In die tijd is in feite de eerste aanzet gemaakt van wat uitgegroeid is tot het hypoxie hoofdstuk. Caroline, jouw komst was een schot in de roos; je werkte hard en met resultaat! Onder het pipetteren was er altijd genoeg te bespreken, en nog steeds, aan de lengte van je mails te zien. Jij ook veel succes met de laatste loodjes! Jolien, jij doet zeker niet onder voor Caroline wat hard werken en lange mails betreft. Waar anderen vaak moeite hebben studenten te motiveren, kreeg ik jou met veel moeite naar huis gestuurd. Veel succes met je promotie onderzoek en nog even en dan is jullie paleisje klaar! Ontzettend bedankt voor jullie hulp bij de totstandkoming van dit proefschrift!

Tijdens het laden en lossen in de thuishaven was het ook altijd plezierig vertoeven. Alle (ex) Molgen-ners bedankt voor al jullie hulp, interesse en de gezelligheid op het lab en in de koffiekamer! Arjen, Franky, Jana, Marten (Hofker), Veerle C, het was kort maar krachtig! Brigitte, ik hoop dat je bij GROW beter op je plek bent! Chantal, Laura, Lauran, Nadine, Nicole, Veerle B, bedankt voor alle creatieve en gezellige feest-cie activiteiten! Daniëlle, Inge, bedankt voor alle hulp met de FACS (en het leren skiën)! Daphna, ik ben de laatste tijd vaak langs je kamer gelopen, zal nu nog eens binnenlopen. Dietbert, impressive how you build your lab so quickly! Dipanjan, I'm curious how you will experience the Dutch traditions with respect to thesis defenses. Ellen D, wie weet worden we nog eens collega's, ik zal duimen voor je Kootstra! Ellen L, wat leuk dat we samen afronden! Is het eigenlijk een beetje ons feestje :-: Emiel, fijn hè dat plekje aan het raam?! Guillaume, (heel toepasselijk na de "E" van Ellen) nog eventjes en dan kun je samen met "mevrouw van Eys" (Jeanette) de bloemetjes buiten zetten! Ine, succes met het voortzetten van je PhD bij Pathologie! Jan, bedankt voor al je inzet om me als lid van de groep te behouden, dat heb ik enorm gewaardeerd! Joost, ik hoop dat het tij snel zal keren! Kristiaan, gezien de indrukken die je hebt achtergelaten, kan ik nog steeds niet geloven dat we eigenlijk maar zo kort samengewerkt hebben. Marie, nice to have someone in the lab who is more bioinformatics oriented! I think you will like chapter 6! Marion, ik moet (meestal) lachen met wat er allemaal uitflapt! Marjo, fijn dat jij voorlopig ook bij Molgen blijft! Marten, veel succes in San Diego! Menno en Will, wanneer drinken we weer een Val Dieu-tje samen? Mohammed, zien we elkaar volgend jaar weer in Rolduc? Monique, ik mis je nuchtere noot en het gemopper op het lab :). Patrick bedankt voor alle hulp, van pleisters plakken tot ICT ondersteuning! Petra, je bent echt een aanwinst voor onze afdeling! Pieter, jammer dat we de politie-inval gemist hebben, ik wens je veel succes in 
Marseille! Remco, het was altijd gezellig, of het nu pokeren, skiën of voetballen was. Robert, waar zullen we 13 juni afspreken? Neutraal terrein? Ronit, thanks a lot for our nice chats, I will cross my fingers for the VIDI grant! Sofia, ik hoop dat we onze "reunion dinners" in stand houden! Sofie, ik heb van horen zeggen dat jij wel van een feestje houdt, ik laat me verrassen de $22^{\text {ste }}$ ! Tim, hulde voor het in ere herstellen van de BBB (Bicky-Burger-Borrel)! Wilma, volgens mij ben je al heel Europa doorgecrost met de camper! Wino, wanneer zien we je nog eens in Wijlre? Yeliz, başarılarının devamını dilerim! Yvonne, de nieuwe wervelwind op het lab, volgens mij ben je vrijwel altijd als eerste en tevens als laatste op het lab te vinden. Werk niet te hard hè! Studenten, succes met het schrijven van jullie master-thesis en/of eindverslag! Wie weet zien jullie nog terug als AIO of analist... Mathijs, Maurice, Petra, Sander, Stijn, Susan, we komen elkaar vast nog wel eens tegen op de gang!

En dan niet te vergeten, onze handelspartners, om te beginnen met BiGCaT; Beste Michiel, we hebben de laatste jaren heel wat uurtjes samen doorgebracht, met name achter de computer waar je me wegwijs hebt gemaakt in de bioinformatica (als je nu je ogen dichtknijpt en door je wimpers de data bekijkt dan zie je.... (;)). In de eerste periode hebben we heel wat gediscussieerd en geoptimaliseerd, en vooral tijdens de laatste paar maanden heeft dropbox overuren gedraaid. Het was ontzettend leuk en leerzaam om met jou samen te werken en ik hoop dat we over niet al te lange tijd een borrel (whisky, roze olifant, ...) kunnen drinken op twee gezamenlijke publicaties! Maar eerst onze promotiefeestjes, succes met de allerlaatste loodjes! Lars, met jou in de buurt is er altijd wel wat te kletsen, liefst met een biertje erbij... als je behoefte hebt aan een praatje dan weet je me te vinden!

All co-authors, thanks for all your input during the set-up, the experimental phase as well as the synthesis of the manuscripts! A special word of thanks for the people in Toronto: Michelle and Tim, your input was extremely valuable for chapter 6 ! And Julie, thanks for all your help, I look forward to meet you in person!

Et nos compagnons français: Emmanuèle, Frédérique, Nicole, Sébastien et Valérie. Un grand merci pour le bon temps que j'ai passé à Paris. J'ai beaucoup aimé la façon de vivre en France. Le travail avec les mouches, les traditions et spécialités françaises; la Galette des Rois, le foie gras (je ne suis toujours pas la plus grande fan, mais celui de Séb est le meilleur que j' ai eu jusqu'à présent), la glace Berthillon, les macarons, etc. En revanche, les pizzas de la cantine étaient abominables. Merci pour toute aide au chapitre 3 et 4 ! 
Dan hebben we nog het schippersoverleg; EpigenUM, Genetica \& Celbiologie, GROW, MaastRO (Alexey, Arjan, Asferd, Barry, Carla, Chantal, Jan, Kasper, Kim P, Kim S, Hilda, Hugo, Janneke, Ludwig, Marco, Marc, Maud, Michael, Mieke, Natasja, Nele, Nejla, Philippe, Rianne, Renaud, Roger, Roland, Sanaz, Sarah, Sherry, Tom, Twan, Younan), Obstetrie en Gynaecologie (Aafke, Andrea, Bert, Josien, Sander), Orthopedie (Andy, Don, Marjolein, Tim) \& Pulmonologie (Alex, Astrid, Céline, Koen, Nicky, Ramon), bedankt voor al jullie waardevolle input tijdens onze gezamenlijke meetings!

Aan al mijn voormalige "collega's" van het NKI (Amsterdam) en afdeling Medische Microbiologie, bedankt voor de leuke en leerzame tijd!

Om een schip in optimale conditie te houden is onderhoud van essentieel belang. Een wijze vrouw zei ooit tegen mij: "vriendschap is als een huis, onderhoud is behoud, liefde is het fundament, maar trouw, vertrouwen aandacht en geduld maken het huis compleet, een huis van liefde waar het heerlijk thuiskomen is". Zonder jullie steun, vertrouwen, alle duwtjes in de goede richting en de ontspannen sfeer tijdens de talloze afspraken zou ik nooit zover gekomen zijn! Alma, Rimke, wat is het fijn om vriendinnen te hebben die in hetzelfde schuitje zitten! Even bijketsen met een kopje koffie op niveau 4 of bij de Starbucks in Palmersten North, alles kan! Ik hoop dat jullie al jullie ambities kunnen verwezenlijken en tegelijk een goede balans kunnen houden in de thuissituatie. Annemieke, ook ik draag je in mijn hart! En ik hoop dat jouw schip weer snel uit het dok komt. Chantal, ze zeggen wel eens van een bruiloft komt een bruiloft, in ons geval kwam van een bruiloft een vriendschap. Bedankt voor de gezellige etentjes! Claire, deze zomer weer eens een terrasje pikken? In Ulestraten, Slenaken, Wijlre of toch in Maastricht? Daiverke, precies 3 maanden na mijn promotie stap je in het huwelijksbootje, ik wens je alle geluk! En ik ben benieuwd wie dit keer het bruidsboeket zal vangen... Fabiënne, dit is de eerste reis die ik zonder jou gemaakt heb, maar desondanks bleef je steeds geïnteresseerd en begaan met mijn reis! Jouw reis is een andere koers ingeslagen dan je vooraf uitgestippeld had, maar je reeft de zeilen niet en maakt er een mooie reis van, daar heb ik heel veel respect voor! Jorien, de woensdagavond is onze avond geworden... eerst zwemmen, toen yoga. Heerlijk zo'n avondje ontspannen! Ik wens je een behouden vaart bij je nieuwe expeditie. Julie, jij bent iemand die haar dromen achterna gaat en daar ook risico's bij durft te nemen, hou dat vast, dan je kom er wel! Karin, de mannen hebben elkaar de laatste maanden vaker gezien dan dat wij elkaar zagen. De komende tijd zal ik je weer vaker zien, in de Spar of daarbuiten. Linda, ik hoop dat je met volle teugen zult genieten op (en liefst al voor) je trouwdag! Lisanne, ik ben benieuwd naar je (toekomstige) huis(dieren). Marian, weer terug op het oude nest $(;) ; i k$ ben heel benieuwd naar jullie kleine meid! 
Marianne, ik heb mijn biebmaatje wel gemist tijdens al die uren schrijven... Marion, hé bluts, we motte weer 'ns aafkalle! Mieke, kroegtijger, we gaan de $22^{\text {ste }}$ weer eens een ouderwets gezellig feestje bouwen! Myrte, wat is het mooi hè Down Under?! Ik hoop dat het je goed gedaan heeft! Sylvie, ik ken je ondertussen al 25 jaar! En wat hebben we een leuke tijden gekend samen. Succes met de huizenjacht! Noladida-dames, ik hoop jullie snel weer 'n keer te zien! ENEB '96 bedankt veur de gooje sjtimmung (in alle opzichte), mit euch is ummer waal get te beleave! "Praatgroep", we hebben weer genoeg te bespreken! "Mannen", jullie ook bedankt! Mijn kleine vriendjes en vriendinnetjes: Mathijs, Pepijn, Delaila, Niek, Lukas, Katrijn, Jens, Koen \& Max: ik zou het liefst uren met jullie spelen, helaas kan dit niet altijd...

Familie Prickaerts, familie Van Proemeren(-Creusen) \& familie Jehae: ik kan nu eindelijk de vraag beantwoorden wanneer ik ga promoveren: 22 juni! Bedankt voor alle leuke "get-togethers" tijdens de jaarlijkse NEND (neven en nichtendag), andere feestjes en partijtjes, de Jo-Ne wandeling in Vijlen of gewoon "in 't durrup" of "achterum". Marianne en Brand, in de familie zijn jullie degenen die mijn passie voor de wetenschap delen, bedankt voor jullie oprechte interesse! Familie Maas, ook jullie maken deel uit van mijn familie, bedankt voor jullie steun en vertrouwen!

Lieve oma, als kind zei ik altijd dat mijn oma 100 zou worden. Ik moet zeggen dat je al een aardig eind op weg bent... Mijn promotie mag je ieder geval nog meemaken en ik hoop dat je, net als jouw moeder, nog deel mag uitmaken van de vierde generatie!

Beste Trautje, lieve ma, vanaf onze eerste ontmoeting werd ik warm onthaald in de familie Van Proemeren en dat is niet veranderd sindsdien. Ik hoop na de promotie weer wat meer tijd te hebben, zodat we weer wat vaker samen koffie kunnen drinken!

Daarnaast heb ik er twee (schoon)zussen, twee (schoon)broers, een neefje en een aankomend nichtje? bijgekregen. Mariëlle, Jo, Veronique, Maarten, Ruben (\& ...): ik ben trots dat ik jullie nu ook officieel tot mijn familie mag rekenen! Toch jammer dat Pater Gelissenstraat 78 nooit wat geworden is :-). Ruben, binnenkort gaan we d'n dieke in het echt bekijken!

Lieve pap \& mam ("het moederschip"), het te water laten van een schip is altijd een spannend moment, zowel voor degenen die de kiel gelegd hebben, als voor de opvarenden. Gelukkig heb ik me nooit hoeven afvragen of de kiel van mijn schip stevig genoeg is; het is immers Prickaerts' vakmanschap en kan dus wel tegen een stootje :). Jullie staan altijd met raad en daad voor mij klaar: dag en nacht, jaar in, jaar uit! 
En ondanks dat ik vaak te laat ben voor het eten en ook meteen daarna weer weg moet zijn jullie altijd blij om mij te zien (en ik jullie). Ik ben jullie heel dankbaar voor alles voor jullie voor me gedaan hebben, weet dat jullie heel veel voor mij betekenen!

Lieve Sas, jouw schip is samen met Johannes uitgevaren naar de Deense wateren en waar jullie daarna koers heen zetten is nog onduidelijk. Zolang onze scheepjes elkaar maar geregeld kruisen is er geen wolkje aan de lucht. We weten precies wat we aan elkaar hebben en ik hoop dat dat ook in de toekomst zo blijft. Ik zal in ieder geval altijd een oogje in jouw zeil houden!

En als laaste Niels, het anker van mijn schip... jij bent mijn rots in de branding, het is niet voor niets dat niemand jou omver loopt in het veld; je bent groot, sterk en je hebt in tegenstelling tot wat je zelf altijd zegt een enorm groot hart en ik ben heel blij dat ik daar een plekje in heb mogen veroveren! De afgelopen tijd heeft het schip op volle koers moeten varen en was er weinig tijd om aan te meren, maar na 22 juni gooien we het anker uit! Ik hoop dat we samen nog heel lang gezond en gelukkig blijven...

En nu maar hopen dat ik niemand vergeten ben...

Bedankt allemaal en hopelijk zullen we in de toekomst nog veel mooie en leerzame reizen maken samen, zowel in de wetenschap als daarbuiten!

\section{Bedankt!}
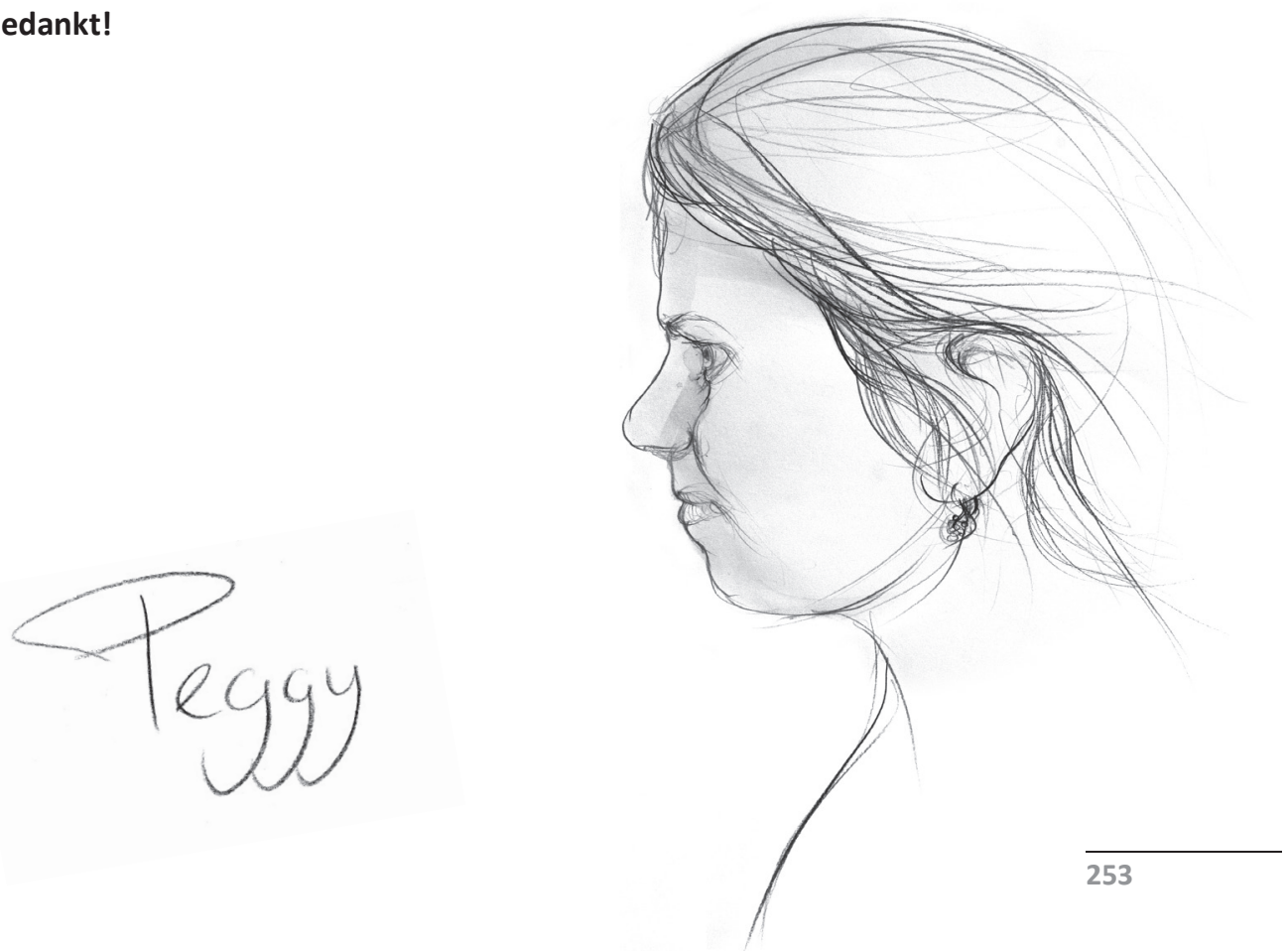
Love is like quicksilver in the hand.

Leave the fingers open and it stays. Clutch it, and it darts away.

-Dorothy Parker- 


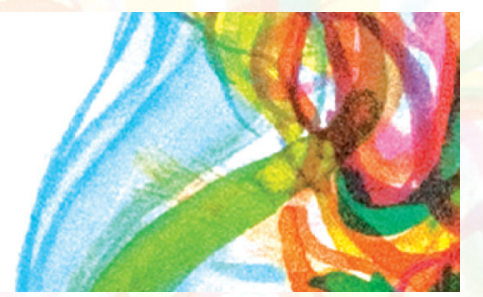

Curriculum Vitae \& List of Publications 


\section{Curriculum Vitae}

Peggy Prickaerts was born on September $30^{\text {th }}, 1983$ in Heerlen. She attended the Stella Maris College in Meerssen, where she graduated with honors in 2001. The same year she started the study Health Sciences at Maastricht University. In her last year, she did an internship at the department of Molecular Genetics at the Netherlands Cancer Institute in Amsterdam (NKI) on the role of Polycomb genes in normal breast development and breast cancer. After graduation with honors in Biological Health Sciences in 2006, she went to the lab of Frédérique Peronnet at Université Pierre et Marie Curie in Paris to study MAPKAP kinase and Polycomb function in Drosophila melanogaster on a personal VSBfonds scholarship for a period of 6 months. In February 2007 she started her PhD at the department of Molecular Genetics at Maastricht University in the lab of Jan Willem Voncken where she focused on Polycomb function in gene-environment interactions. She will continue her work at the department of Molecular Genetics as a post-doctoral fellow, combined with a work visit to the lab of Prof. Brad Wouters at the Ontario Institute for Cancer Research (OICR) in Toronto, Canada, which was made possible by a René Vogels travel grant. During her postdoctoral training she will follow up on her in vitro hypoxia studies with the aim to establish novel insights in Polycomb biology in the context of pre-clinical tumor models. 


\section{List of Publications}

Peggy Prickaerts*, Hanneke EC Niessen*, Vivian EH Dahlmans, Juliette Salvaing, Frank Spaapen, Jolien Vanhove, Claudia Geijselaers, Stefanie JJ Bartels, Yoshihiro Takihara, Bradly G Wouters, UIf R Rapp, Jan Willem Voncken. MK3 controls replicative life-span through interaction with BMI1. Submitted / provisionally accepted (Oncogene)

Peggy Prickaerts, Hanneke EC Niessen ${ }^{*}$, Emmanuèle Mouchel-Vielh ${ }^{*}$, Vivian EH Dahlmans, Claudia Geijselaers, Michiel Adriaens, Frank Spaapen, Yoshihiro Takihara, Ulf R Rapp, Frédérique Peronnet, Jan Willem Voncken. MK3 controls Polycomb-target gene expression via negative feedback on ERK. Submitted / under review (Epigenetics \& (chromatin)

Peggy Prickaerts*, Hanneke EC Niessen*, Vivian EH Dahlmans, Frank Spaapen, Claudia Geijselaers, Sheng Chung Lee, Bradly G Wouters, Frédérique Peronnet, Jan Willem Voncken. Signaling-induced degradation of BMI1 by chromatin-associated KAP1.

In preparation

Peggy Prickaerts*, Michiel Adriaens*, Twan van den Beucken, Caroline Gits, Vivian EH Dahlmans, Philip Zuzarte, John McPherson, Bradly G Wouters*, Jan Willem Voncken*. Hypoxia induces bivalent chromatin domains by specific gain of H3K27me3.

\section{In preparation}

Michiel Adriaens*, Peggy Prickaerts*, Michelle Chan-Seng-Yue, Timothy Beck, Bradly G Wouters*, Jan Willem Voncken*, Chris Evelo*. Capturing ChIP-seq profiles of H3K27me3 in dynamic biological systems. Submitted (Genome Biology)

Frank Spaapen, Guus van den Akker, Marjolein Caron, Peggy Prickaerts, Hanneke EC Niessen, Vivian EH Dahlmans, Don AM Surtel, Yvette Paulis, Finja Schweizer, Céline Rofel, Tim Welting, Lars Eijssen, Jan Willem Voncken. The immediate early gene EGR1 controls epigenetic reprogramming through Polycomb Group proteins during chondrogenesis. In preparation

Frank Spaapen, Lars Eijssen, Tim Welting, Juliette Salving, Peggy Prickaerts, Vivian EH Dahlmans, Don AM Surtel, H Koseki, M Vidal, Yoshihiro Takihara, Bradly G Wouters, Jan Willem Voncken. Polycomb repressive complex 1 coordinates enhanced replication and transcription in transit amplifying chondrogenic progenitors. In preparation

\footnotetext{
* equal contribution
} 
Curriculum Vitae \& List of Publications | 


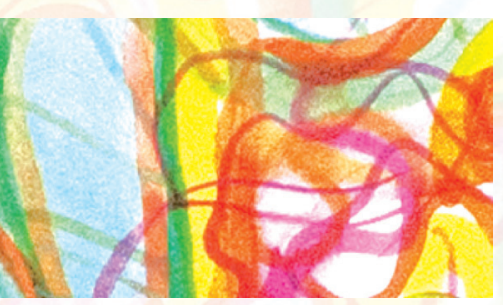

List of Abbreviations 


$\begin{array}{ll}\text { 2Py(-tag) } & \text { polyoma virus antigen (tag) } \\ 3^{\prime} & \text { 3-prime } \\ \text { 3pK } & \text { chromosome 3p kinase } \\ 5^{\prime} & \text { 5-prime }\end{array}$

A

A alanine (amino acid) or adenine (base)

ACME algorithms for calculating microarray enrichment

ADP adenosine diphosphate

AKT v-akt murine thyoma viral oncogene homolog 1

ANRIL antisense non-coding RNA in the INK4 locus

AOF1 amine oxidase (flavin containing) domain 1

AP anterior-posterior

ARF alternate reading frame

ARID AT-rich interactive domain

As arsenite/arsenic

$\mathrm{As}_{2} \mathrm{O}_{3} \quad$ arsenic trioxide

ASH1L ash1 (absent, small, or homeotic)-like (Drosophila)

ASH2 absent, small, or homeotic discs 2

ASX

additional sex combs

ASXL1 additional sex combs like 1

ATCC

global nonprofit bioresource center (BRC)

ATF3 activating transcription factor 3

AT-hook DNA-binding motif (binds to the minor groove of AT rich DNA

ATM(i) ataxia telangiectasia mutated (inhibitor)

AUC area under the curve

B

BAM binary sequence alignment/map

BAP1 BRCA1 associated protein 1

B-box zinc finger domain

BCA bicinchoninic acid

BCL6 B-cell lymphoma 6

BCOR BCL6 co-repressor

BED browser extensible data

BJ human foreskin fibroblasts

BMI1 B-cell-specific Moloney murine leukemia virus integration site 1

bp base pairs 


\begin{tabular}{|c|c|}
\hline BrdU & bromodeoxyuridine \\
\hline BROMO & protein domain that recognizes acetylated lysine \\
\hline BSA & bovine serum albumin \\
\hline \multicolumn{2}{|l|}{ C } \\
\hline $\mathrm{CBX}(\mathrm{n})$ & chromobox homolog (n) \\
\hline $\mathrm{CC}$ & coiled-coil \\
\hline CCNA2 & cyclin-A2 \\
\hline CDKN2A/B & cyclin-dependent kinase inhibitor $2 \mathrm{~A} / \mathrm{B}$ \\
\hline cDNA & complement DNA \\
\hline$c f$ & compare for \\
\hline CFTF(s) & cell fate transcription factor(s) \\
\hline CHD3 & chromodomain helicase DNA binding protein 3 \\
\hline ChIP & chromatin immunoprecipitation \\
\hline ChIP-seq & ChIP-sequencing \\
\hline CIP1 & CDK-interaction protein 1 \\
\hline $\mathrm{CO}_{2}$ & carbon dioxide \\
\hline CpG & cytosine-phosphate-guanine \\
\hline CPT & camptothecin \\
\hline c.q. & casu quo \\
\hline C-terminal & carboxyl-terminal \\
\hline Cyto & cytoplasmic \\
\hline \multicolumn{2}{|l|}{ D } \\
\hline D & aspartic acid \\
\hline (k)Da & (kilo) dalton \\
\hline DAPI & 4'-6-diamidino-2-phenylindole \\
\hline DDR & DNA-damage response \\
\hline$d K A P$ & (D.mel) KRAB-associated protein \\
\hline D.mel & Drosophila melanogaster \\
\hline DMEM & Dulbecco's Modified Eagle Medium \\
\hline DNA & deoxyribonucleic acid \\
\hline DNMT(s) & DNA methyltransferase(s) \\
\hline DOC & deoxycholate \\
\hline$D-p 38 b$ & D.mel p38 ortholog \\
\hline $\mathrm{DSB}(\mathrm{s})$ & double strand break(s) \\
\hline dsDNA & double-stranded DNA \\
\hline DSP1 & dorsal switch protein 1 \\
\hline
\end{tabular}




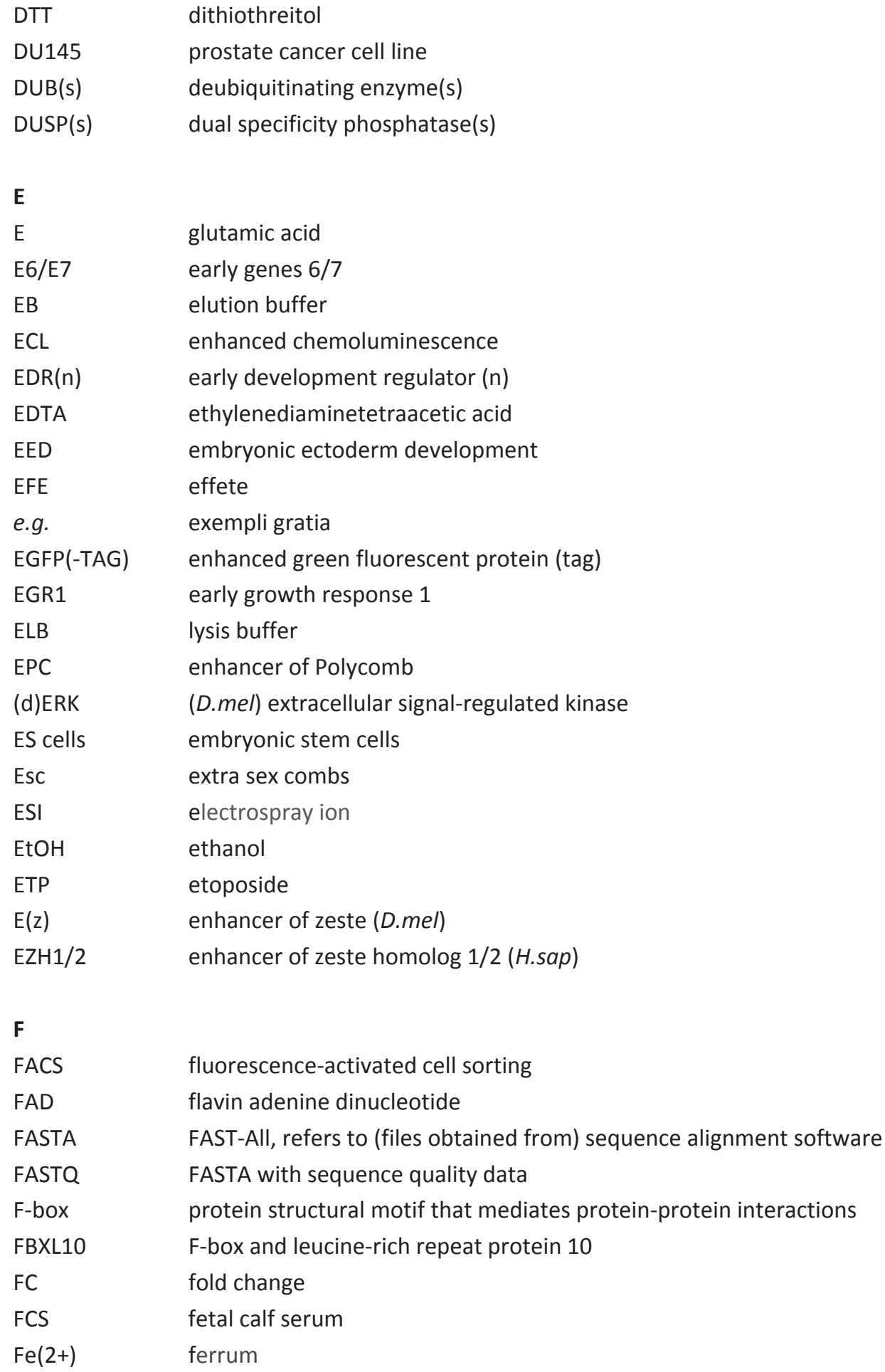




$\begin{array}{ll}\text { Fig } & \text { figure } \\ \text { FITC } & \text { fluorescein isothiocyanate } \\ \mathrm{fl} & \text { full-length } \\ \text { FLAG-tag } & \text { polypeptide protein tag }\end{array}$

\section{G}

G

glycine

G0/G1-arrest

gap 0/1 phase arrest

G9a

G9a histone methyltransferase

GAllx Genome Analyser IIx

Gadd45 $\alpha$

Growth arrest and DNA-damage-inducible, alpha

GAF

GAGA-factor

Gal4 transcription activator protein

gcRMA

$\mathrm{GC}$ robust multi-array average

GEO gene expression omnibus

GFF generic feature format

GFP(-tag)

green fluorescent protein (tag)

GLP

G9a like protein

GO gene ontology

GOF gain-of-function

GSK3ß(i)

glycogen synthase kinase $3 ß$ (inhibitor)

GST-tag glutathione $S$-transferase (tag)

GTP(ase) guanosine-5'-triphosphate (enzyme that can bind and hydrolyze GTP)

H

$H(n)$

histone ( $n$ )

$\mathrm{H}_{2}$

hydrogen

$\mathrm{H} 2 \mathrm{~A}$

histone $2 \mathrm{~A}$

H2AK119ub1

histone $\mathrm{H} 2 \mathrm{~A}$ mono-ubiquitylation of $\mathrm{K} 119$

H2A.X

histone 2 variant $X$

$\mathrm{H} 2 \mathrm{~B}$

histone $2 \mathrm{~B}$

H3K9/K14ac

acetylation on lysine 9 and 14 of histone 3

H3K(n)me1 mono-methylation on lysine $(\mathrm{n})$ of histone 3

H3K(n)me2

di-methythylation on lysine (n) of histone 3

H3K(n)me3

tri-methylation on lysine ( $n$ ) of histone 3

H3S28ph

phosphorylation on serine 28 of histone 3

H4K(n)me3

trimethylation on lysine ( $\mathrm{n}$ ) of histone 4

HA(-tag)

hemagglutinin (tag) 


$\begin{array}{ll}\text { HAT(s) } & \text { histone acetyltransferase(s) } \\ \text { HCl } & \text { hydrogen chloride } \\ \text { HCNE(s) } & \text { highly conserved non-coding element(s) } \\ \text { HCP } & \text { high CpG content } \\ \text { HDAC(s) } & \text { histone deacetylase(s) } \\ \text { HDM(s) } & \text { histone demethylase(s) } \\ \text { HeLa } & \text { cervical cancer cells derived from Henrietta Lacks } \\ \text { HEPES } & \text { 4-(2-hydroxyethyl)-1-piperazineethanesulfonic acid } \\ \text { hg18 } & \text { human genome 18 } \\ \text { HIF1a } & \text { hypoxia-inducible factor 1a } \\ \text { HKDM(s) } & \text { histone lysine demethylase(s) } \\ \text { HKMT(s) } & \text { histone lysine methyltransferase(s) } \\ \text { HMT(s) } & \text { histone methyltransferase(s) } \\ \text { hMW } & \text { high molecular weight } \\ \text { Hom-C } & \text { Homeotic complex } \\ \text { HOTAIR } & \text { HOX antisense intergenic RNA } \\ \text { HOXA-D(n) } & \text { homeobox A-D (n) } \\ \text { HP1 } & \text { heterochromatin protein 1 } \\ \text { HPC1/2 } & \text { homolog of polycomb 1/2 } \\ \text { HPH(n) } & \text { homolog of polyhomeotic (n) } \\ \text { HPV } & \text { human papillomavirus } \\ \text { H.sap } & \text { Homo sapiens } \\ \text { hTERT } & \text { human telomerase reverse transcriptase } \\ \text { HTS } & \text { high-throughput sequencing } \\ \text { HYPB } & \text { huntingtin yeast partner B } \\ \text { I } & \\ \text { i.a } & \text { inter alia } \\ \text { IAL } & \text { ipl1-aurora-like kinase } \\ \text { IB } & \text { immunoblot(ting) } \\ \text { ICC } & \text { immunocytochemistry } \\ \text { ICP } & \text { intermediate CpG content } \\ \text { ID(s) } & \text { identifier(s) } \\ \text { i.e. } & \text { id est } \\ \text { IEG(s) } & \text { immediate early gene(s) } \\ \text { IF } & \text { immunofluorescence } \\ \text { IgG } & \text { immunoglobulin G } \\ \text { IgH } & \\ & \end{array}$




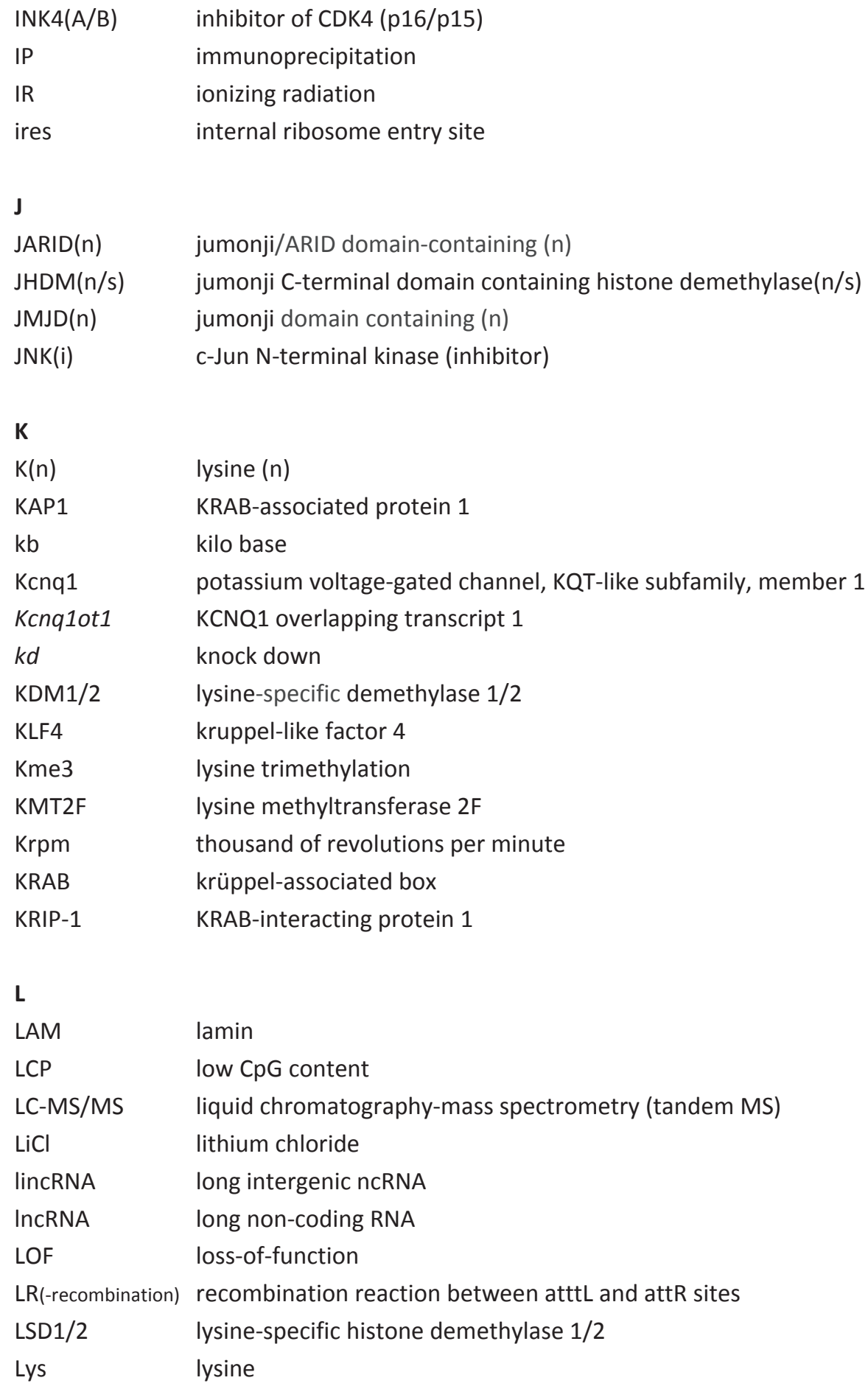


M

$\mathrm{M}$

mAb

MACS

MAPK

MAPKAP

MAPKAPK

MCF7

MDM2

MEK1/2

MEKi

MEL18

$\mathrm{MetOH}$

MG132

$\mathrm{Mi} 2$

MIR

(d)MK

$\operatorname{MK}(n)$

MKi

MLL

MNK1/2

$\mathrm{MOI}$

mRNA

MS

MSK1

Myc(-tag)

$\operatorname{molar}(\mathrm{mol} / \mathrm{L})$

monoclonal antibody

model-based analysis for ChIP-seq

mitogen-activated protein kinase

mitogen-activated protein kinase-activated protein

mitogen-activated protein kinase-activated protein kinase

breast cancer cell line, acronym of Michigan Cancer Foundation - 7

murine double minute

mitogen-activated protein kinase kinase $1 / 2$

MEK inhibitor

melanoma nuclear protein 18

methanol

proteasome inhibitor

mi2-alfa

mitogen-induced immediate-early responses

(D.mel) MAPKAP kinase

MAPKAP kinase $(\mathrm{n})$

MK inhibitor

myeloid/lymphoid or mixed-lineage leukemia

MAPK-interacting kinase $1 / 2$

multiplicity of infection

messenger RNA

mass spectrometry

mitogen and stress-activated protein kinase 1

v-myc myelocytomatosis viral oncogne homolog (tag)

N

(n)

$\mathrm{N}$

$\mathrm{N}_{2}$

$\mathrm{NaCl}$

NAD

$\mathrm{NADH}$

$\mathrm{NaHCO}_{3}$

$\mathrm{NB}(\mathrm{s})$

NCBI

number (1,2,3 etc)

normal (mol/L* number of ions formed)

nitrogen

sodium chloride

nicotinamide adenine dinucleotide

hydrolysed form of NAD

sodium bicarbonate / sodium hydrogen carbonate

nuclear bodie(s)

national center for biotechnology information

ncRNA

non-coding RNA 


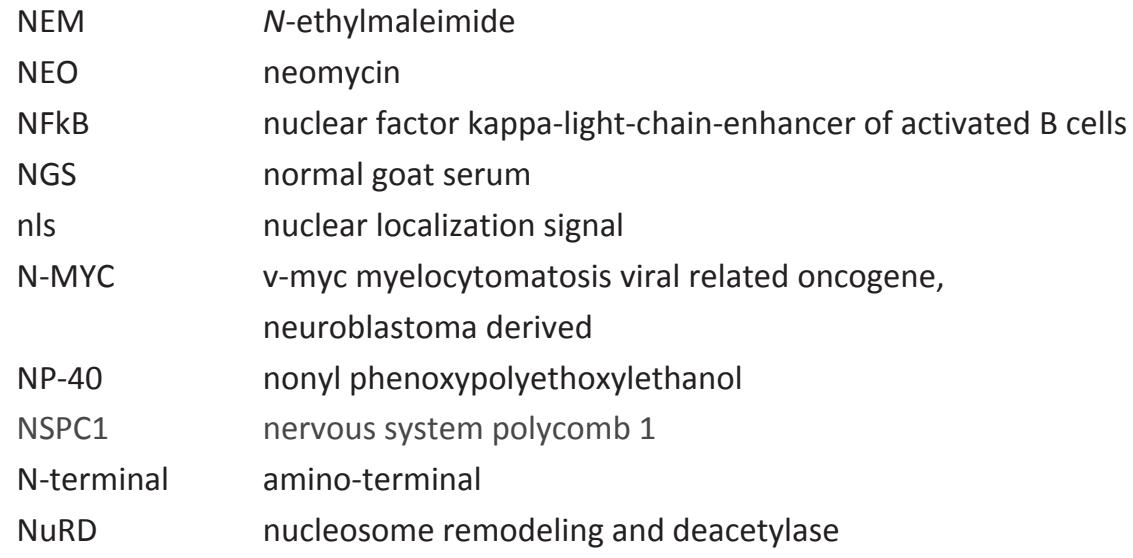

0

$\mathrm{O}_{2} \quad$ oxygen

OCT4 octamer-binding transcription factor 4

OE overexpression

OIS oncogene-induced senescence

ORC origin recognition complex

ORF open reading frame

$\mathbf{P}$

$\mathrm{p}(\mathrm{n}) \quad$ protein (n) or short arm of chromosome (n)

p38i p38 inhibitor

$\mathrm{pAb} \quad$ polyclonal antibody

PBS phosphate buffered saline

Pc Polycomb

pcc Pearson correlation coefficient

PcG Polycomb Group

PCGF(n) Polycomb group ring finger ( $n$ )

pCHK2 phospho CHK2 (checkpoint kinase 2)

PCR polymerase chain reaction

PERK phospho ERK

$\mathrm{PH}$ polyhomeotic

$\mathrm{pH} \quad$ potential hydrogen

$\mathrm{PHC}(\mathrm{n}) \quad$ polyhomeotic-like (n)

PHD (finger/domain) plant homeo domain

PHD prolyl hydroxylase

$\mathrm{PHO} \quad$ Pleiohomeotic 


\begin{tabular}{|c|c|}
\hline PHO-RC & $\mathrm{PHO}$ repressive complex \\
\hline p.i. & proteasome inhibitor \\
\hline PI3K & phosphatidylinositol 3-kinase \\
\hline pJNK & phospho JNK \\
\hline PKB & protein kinase $B$ \\
\hline PKC(i) & protein kinase C (inhibitor) \\
\hline pKS & pBluescript \\
\hline PMEK & phospho MEK \\
\hline PML & promyelocytic leukemia \\
\hline PMSF & phenylmethanesulfonylfluoride \\
\hline PTM(s) & post-translational modification(s) \\
\hline pp38 & phospho p38 \\
\hline ppm & parts per million \\
\hline PP-ase & protein phosphatase \\
\hline PRAK & p38-regulated/activated protein kinase \\
\hline PRC1/2 & Polycomb repressive complex $1 / 2$ \\
\hline PR-DUB & Polycomb repressive deubiquitinase \\
\hline $\operatorname{PRE}(s)$ & Polycomb response element(s) \\
\hline Prot $A / G$ & (immunoglobulin binding bacterial) protein $A / G$ \\
\hline Psc & posterior sex combs \\
\hline$p$-st & post stimulation \\
\hline PURO & puromycin \\
\hline PVDF & polyvinylidene fluoride \\
\hline \multicolumn{2}{|l|}{$\mathbf{Q}$} \\
\hline qPCR & quantitative PCR \\
\hline \multicolumn{2}{|l|}{$\mathbf{R}$} \\
\hline $\mathrm{R}$ & arginine or " $\mathrm{R}$ " (programming language) \\
\hline Rae28 & retinoic acid induced embryonic clone 28 \\
\hline (d)RAF & (D.mel) rapidly accelerated fibrosarcoma \\
\hline RAR & retinoic acid receptor \\
\hline RAS(V12) & rat sarcoma (G12V mutation) \\
\hline RB & retinoblastoma protein \\
\hline RBCC & RING, B-box, coiled-coil \\
\hline REN & restriction endonuclease \\
\hline $\operatorname{RepA}($ motif) & replication protein $\mathrm{A}$ ( $\mathrm{N}$-terminal motif) \\
\hline RET & rearranged during transfection \\
\hline
\end{tabular}


RF

RFP

(d)RING

RING1A/B

RIPA

RNA

RNase

RNAi

RNF(n)

RO52

ROI

ROS

rp49

RPA-70

RPOL2

$\operatorname{RSK}(n)$

RT

rtPCR

RYBP

\section{S}

$\mathrm{S}$

S2

SA-bGal

SAM

SAM

SAPK

SB201290

Sce

$\mathrm{Scm}$

SD

SDS

SDS-PAGE

Se

Ser

SET

SET1

SETD
RINGfinger

RET finger protein

(D.mel) really interesting new gene

really interesting new gene $1 \mathrm{~A} / \mathrm{B}$

radio immunoprecipitation assay

ribonucleic acid

ribonuclease

RNA interference

ring finger protein $(n)$

Ro protein, $52 \mathrm{kDa}$

regions of interest

reactive oxygen species

ribosomal protein 49

replication protein $\mathrm{A} 1,70 \mathrm{kDa}$

RNA polymerase 2

ribosomal $\mathrm{S} 6$ protein kinase (n)

room temperature

real-time PCR

RING1 and YY1 binding protein

serine

D. mel cell line, acronym of Schneider 2 cells

senescence-associated b-Galactosidase

sequence alignment/map

(sterile alpha motif) self association motif

stress-activated protein kinase

p38 inhibitor

Sex combs extra

Sex comb on midleg

standard deviation

sodium dodecyl sulfate

sodium dodecyl sulfate polyacrylamide gel electrophoresis

selenite/ selenium

serine

protein domain, acronym of Su(var)3-9, Enhancer of Zeste, Trithorax

SET domain containing $1 \mathrm{~A}$

SET domain containing 2 
SETDB1

SFMBT

sh(RNA)

SILAC

SIM

SIRT1

SMCX

sol nuc

SOX2

SP1

$S$ phase

spt6

ssDNA

stim

SU(HW)

SUMO

suppl

SUUR

SUV39H1

Su(z)12

SUZ12

$\mathbf{T}$

$\mathrm{T}$

T1

$\mathrm{T} 2$

T3

TAF(s)

TAP(-tag)

TBP

TE

TEZ

Thr

TIF1 $\beta$

TIG3

$\mathrm{TiO} 2$

TOPO2

(T)P53
SET domain, bifurcated 1

SCM-related gene containing four MBT domains

short hairpin (RNA)

stable isotope labeling by amino acids in cell culture

single-minded protein

sirtuin (silent mating type information regulation 2 homolog) 1

selected mouse cDNA on $\mathrm{X}$

soluble nuclear

SRY (sex determining region Y)-box 2

specifity protein 1

synthesis phase

suppressor of Ty 6 homolog

single-stranded DNA

stimulation

suppressor of hairy-wing

small ubiquitin-like modifier

supplemental

suppressor of underreplication

suppressor of variegation 3-9 homolog 1

suppressor of zeste 12 (D.mel)

suppressor of zeste 12 (H.sap)

threonine (amino acid) or thymine (base)

prothoracic

mesothoracic

metathoracic

TBP associated factor(s)

tandem affinity purification (tag)

TATA-box binding protein

Tris-EDTA

transposon-exclusion zones

treonine

transcription(al) intermediary factor 1 beta

primary fibroblasts

titanium dioxide

topoisomerase 2

tumor protein $\mathrm{p} 53$ 
TPA

TRIM

$\operatorname{TRIM}(\mathrm{n})$

Tris

TRX

TrX

TrxG

TSS

TUB

TXRD

Tyr

U

U2-OS

Ub

UTX

UTY

UV

V

vs.

W

W

WAF1

WD40

WIG

X

Xist

$Y$

$Y$

YY1

Z

ZFP(s)
12-O-tetradecanoylphorbol-13-acetate

tripartite motif

tripartite motif containing (n)

tris(hydroxymethyl)aminomethane

Trithorax

Triton X-100

Trithorax Group

transcription start site

tubulin

TexasRed

tyrosine

osteosarcoma cells

ubiquitin (ubiquitous immunopoietic polypeptide)

ubiquitously transcribed tetratricopeptide repeat, $\mathrm{X}$ chromosome ubiquitously transcribed tetratricopeptide repeat gene, $Y$ linked ultraviolet

versus

tryptophan

wild-type p53-activated fragment 1

structural motif of 40 amino acids often terminating in a WD dipetide wiggle genome track format

X-inactive specific transcript

tyrosine

yin-yang transcription factor 1

zinc-finger protein(s) 
Helduheid, keunn̈kheid, adaptatie, ust in teweging Kompositie in rust. Fa'sen (perioden en geleidelïkheid Lijn on losmakelijke tegrippen gewarden in of tijdens het ontrtaansproces van haar werk.

hij vereen relft fich mot het microkeinaat van haar weck. Vamist mïn actiticke visie 2ore ik dit een organische Climax willen noemen.

De dynamiek van de cel is als metafoor gefruikt in rook is het leven weergegeven. De D.N.A strengen geven de toe voer krachten aan met daain de profiel schetsen van de personen die vor have leven en wreck see belangrijk. ä̈n

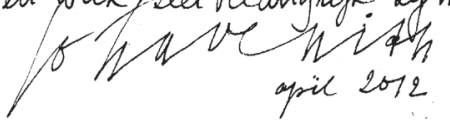

